\title{
Prospecting the insect model, Galleria mellonella, for gut-related pathobiology
}

\author{
By \\ Helena Emery \\ Doctor of Philosophy
}

A thesis submitted to Swansea University, in fulfilment of the requirements for the degree of

August 2020

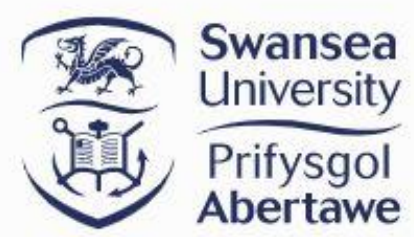

Swansea

Prifysgol Abertawe
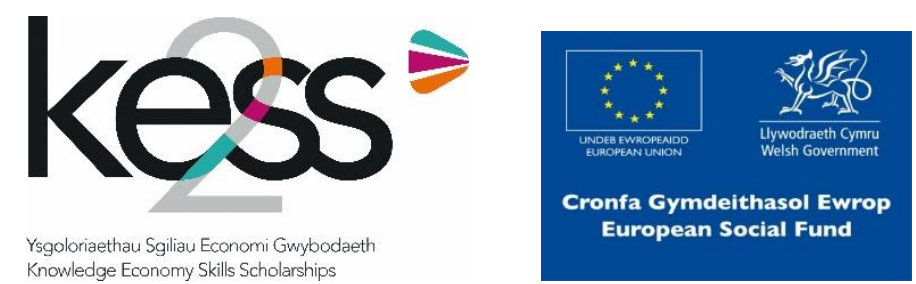


\section{Declaration of originality}

\section{DECLARATION}

This work has not previously been accepted in substance for any degree and is not being concurrently submitted in candidature for any degree.

Signed: Helena Emery

Date: $27 / 08 / 2020$

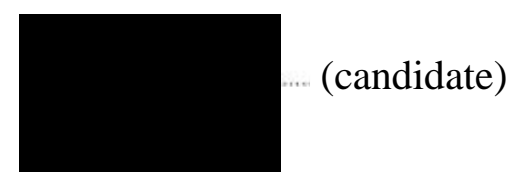

\section{STATEMENT 1}

This thesis is the result of my own investigations, except where otherwise stated. Where correction services have been used, the extent and nature of the correction is clearly marked in a footnote(s).

Other sources are acknowledged by footnotes giving explicit references. A bibliography is appended.

Signed: Helena Emery

Date: $27 / 08 / 2020$

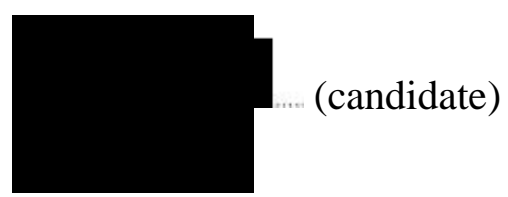

\section{STATEMENT 2}

I hereby give consent for my thesis, if accepted, to be available for photocopying and for inter-library loan, and for the title and summary to be made available to outside organisations.

Signed: Helena Emery

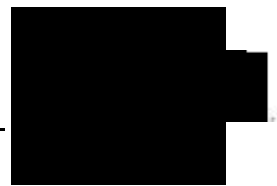
(candidate)

Date: $27 / 08 / 2020$ 


\section{Abstract}

Animal research has contributed immensely to medical and scientific advances over the last century, and continues to play important roles in enhancing our understanding of infectious and non-communicable disease development, and the search for treatments. The mouse, for example, shares $\sim 95 \%$ of human genes and is the most widespread vertebrate model in use. Since the late 1980s, there has been several UK and EU directives (e.g., 2010/63/EU) to improve the welfare of animals considered essential for experimentation, and to link directly with the principle of the $3 \mathrm{Rs}$, to Replace, Reduce and Refine animal use. Additionally, animal maintenance, husbandry, compliance with legislation and licencing, and staff training are costly and time-consuming. Hence, there is much to gain from developing alternative in vivo models and complementary in vitro, in chemico and in silico tools.

Larvae of the wax moth Galleria mellonella represent one such surrogate to rodents, and have been used successfully to study microbial isolates for virulence traits, putative antibiotic therapies, and more recently, toxicological assessment. There is an abundance of practical and biological advantages to selecting G. mellonella over rodents and traditional non-mammalian fruit flies and nematodes (which are described in Chapter 1), but one area lacking in knowledge is their applicability for studies of gut pathobiology. Therefore, the aim of this thesis was to evaluate the usefulness and accuracy of $G$. mellonella larvae as a model for gut specific toxins and pathogens when administered through an oral route (gavage).

A series of whole-organism (phenotype), cellular, biochemical, microbiological and microscopy methods were used to interrogate the gastrointestinal tract of G. mellonella in the absence and presence of chemicals and microbes known to cause gastropathy in rodents and humans. First, the transferability of the indomethacin restraint/ulcer assay was established in G. mellonella, with levels of tissue deterioration and enhanced leakiness reminiscent of rodents (Chapter 2). Second, the rearing of insects on nutraceuticals Cordyceps sinensis and bovine colostrum alleviated gut damage caused by indomethacin, and improved survival outcomes when challenged with the enteric pathogen Campylobacter jejuni (Chapter 3). Third, oral administration of shellfish poisoning toxins (okadaic acid and azaspiracids 1-3) to G. mellonella, interfered with tissue integrity and microbial stability of the gastrointestinal tract, and produced comparable $\mathrm{LD}_{50}$ levels to their rodent counterparts (Chapter 4).

The results presented here go beyond establishing synonymous damage phenomena between $G$. mellonella larvae and vertebrates (Chapter 5), but adds new knowledge to the structure and function of the lepidopteran alimentary canal, the cytopathology of emerging marine toxins, and how diet invariably influences a host's capacity to recover from subacute chemical and microbial disruptors. 


\section{Acknowledgements}

I would like to show my appreciation to all those who have helped me throughout this $\mathrm{PhD}$. In particular, I wish to thank my supervisor Dr Christopher Coates for his continued support, guidance and patience. Without him, this $\mathrm{PhD}$ would not have been possible, and I am very grateful for all that he has taught me.

A special thanks to all those in my laboratory, Sophie Malkin, Jessica Bevan, Charlotte Davies and Emma Quinn (AKA the Bluefish Babes) for their advice and assistance with histology and NGS and most importantly all the laughs and singing to keep me going. You have made it a truly enjoyable experience. Thank you to the MSc and year in industry students for helping me rear the larvae and helping with histology. I cannot forget to thank all the thousands of Galleria that have sacrificed themselves for my research.

Thank you to Professor Andrew Rowley for teaching me and helping with histology. Thank you to Hillary Williams for always being a helping hand whenever something broke, went missing or lending equipment. I wish to thank all those I shared an office with for always being there to help and give advice.

Finally, I wish to thank my Parents, Grandparents, Hannah, Mike and Sam and all my friends and family for their constant support and love. For putting up with me coming home stressed and boring you with "interesting" things I have learnt. Thank you for your guidance and help, for always managing to cheer me up, make me laugh and inspiring me to do this $\mathrm{PhD}$. 


\section{Funding}

This research was funded through the KESS II scheme (supported Mr John Rolfs, The Golden Dairy Ltd.) and supplemented by start-up funds (College of Science) assigned to Dr Coates.

Ysgoloriaeth Sgiliau Economi Gwybodaeth (KESS) yn Gymru gyfan sgiliau lefel uwch yn fenter a arweinir gan Brifysgol Bangor ar ran y sector AU yng Nghymru. Fe' i cyllidir yn rhannol gan Gronfeydd Cymdeithasol Ewropeaidd (ESF) cydgyfeirio ar gyfer Gorllewin Cymru a'r Cymoedd.

Knowledge Economy Skills Scholarships (KESS) is a pan-Wales higher-level skills initiative led by Bangor University on behalf of the HE sector in Wales. It is partfunded by the Welsh Government's European Social Fund (ESF) convergence programme for West Wales and the Valleys.
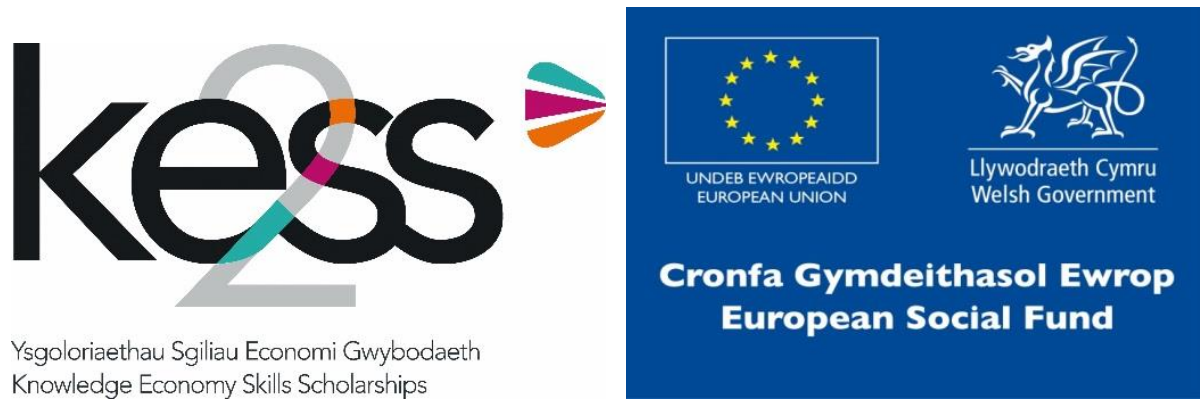

Ysgoloriaethau Sgiliau Economi Gwybodaeth Knowledge Economy Skills Scholarships 


\section{Table of Contents}

Declaration of originality............................................................................................ i

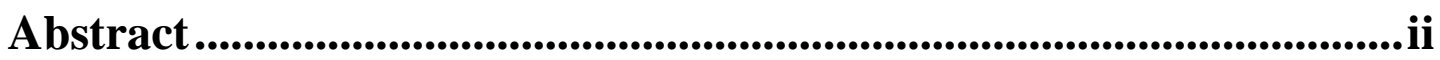

Acknowledgements.....................................................................................................iii

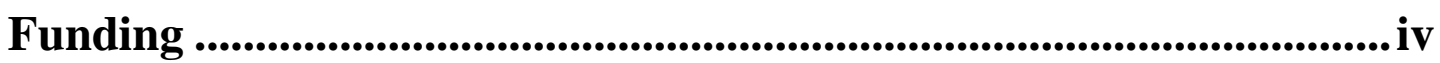

Table of Contents ........................................................................................................ v

List of Figures ............................................................................................................ $\mathrm{x}$

List of Tables .........................................................................................................xiii

List of Abbreviations............................................................................. xiv

Chapter 1: Introduction ............................................................................................... 1

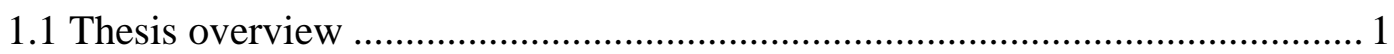

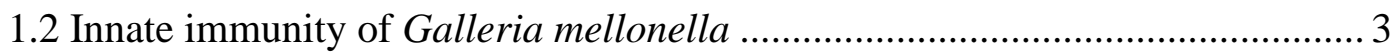

1.2.1 Cellular immune response in Galleria mellonella ................................... 4

1.2.2 Galleria mellonella humoral immune response ........................................ 6

1.3 Galleria mellonella gastrointestinal morphology .......................................... 6

1.4 Methods of infecting Galleria mellonella ................................................... 10

1.5 Existing evidence for Galleria mellonella as a candidate model for gut pathology

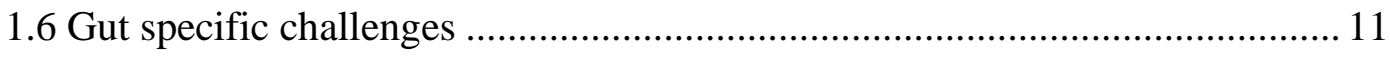

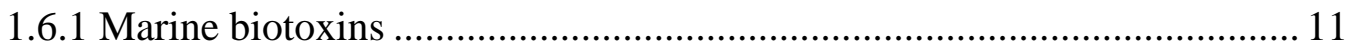

1.6.2 Campylobacter jejuni ...................................................................... 14

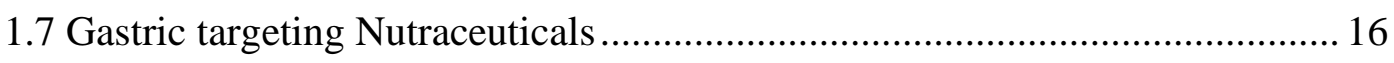

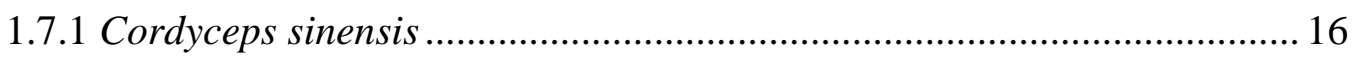

1.7.1.1 C. sinensis in gut repair and protection ............................................ 16

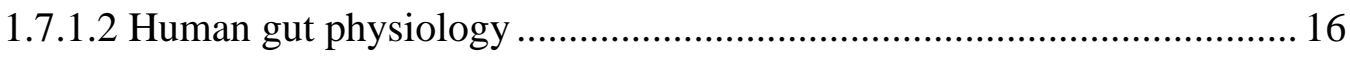

1.7.1.3 Properties for gut restitution .......................................................... 17 
1.7.1.4 Properties to inhibit apoptosis

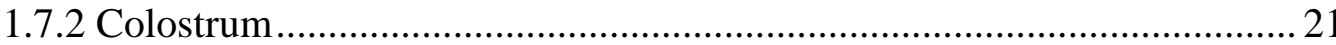

1.7.2.1 Colostrum anti-inflammatory properties.......................................... 21

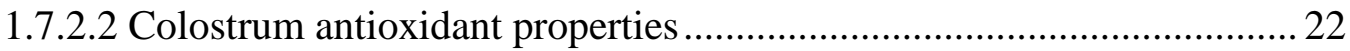

1.7.2.3 Colostrum gastric protective and reparative properties ....................... 23

1.7.2.4 Colostrum growth factors and hormones ......................................... 23

1.7.2.5 Colostrum impact on gastric microbiome ......................................... 24

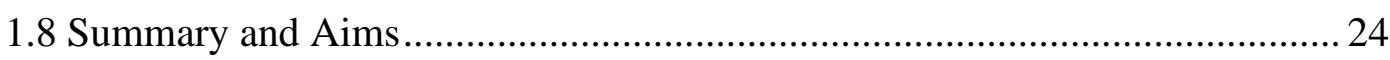

Chapter 2: Developing an alternative in vivo (insect) model to replace the indomethacin restraint/ulcer assay in rodents..........................26

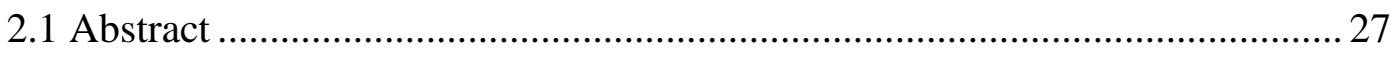

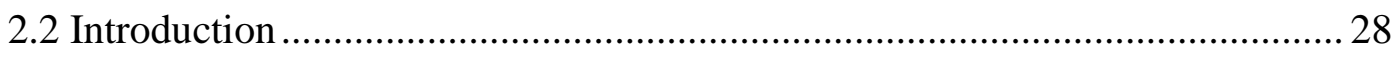

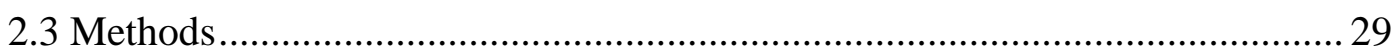

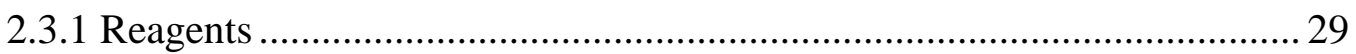

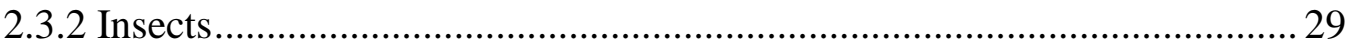

2.3.3 Larval survival and pupation studies .................................................... 30

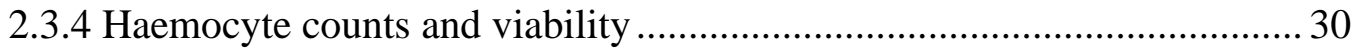

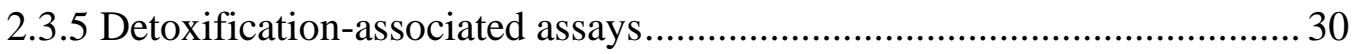

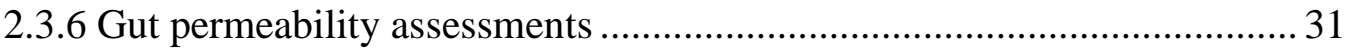

2.3.7 Quantifying bacterial colony forming units in larval faeces .................... 32

2.3.8 Histopathology of the insect alimentary canal ....................................... 32

2.3.9 X-ray microtomography/microscopy of Galleria mellonella ................... 33

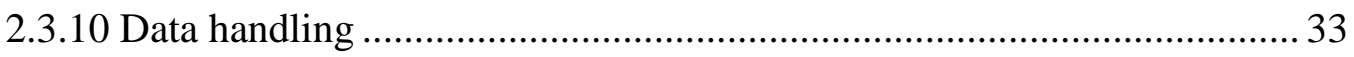

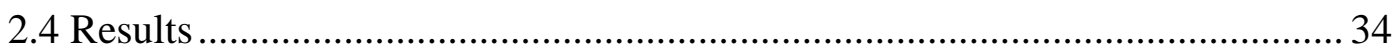

2.4.1 Evaluating the relative toxicity of indomethacin on Galleria mellonella. 34

2.4.2 Characterising the effects of indomethacin on the midgut of Galleria mellonella 39 
2.5 Discussion

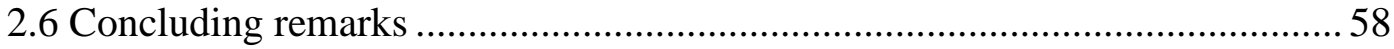

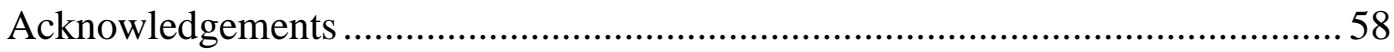

Chapter 3: Evaluating the protective properties of 'nutraceuticals', Cordyceps sinensis and colostrum, against bacterial- and chemicallyinduced gastric damages.............................................................................55

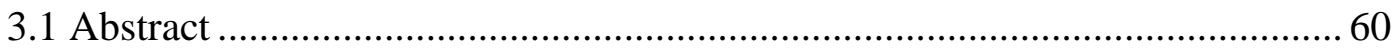

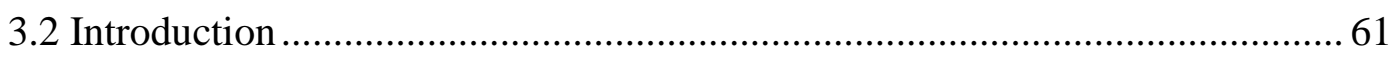

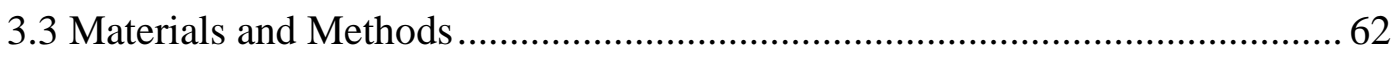

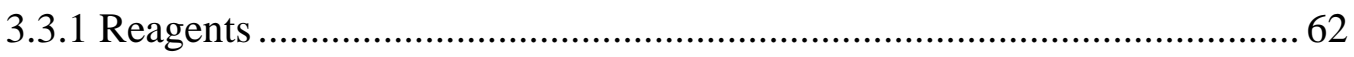

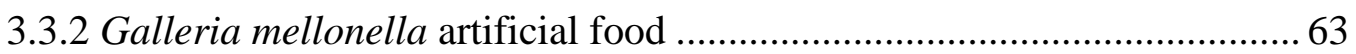

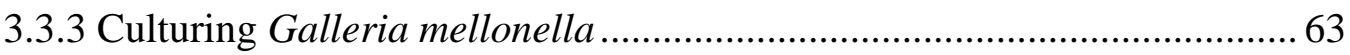

3.3.4 Health indices of larvae exposed to biopharmaceuticals ......................... 64

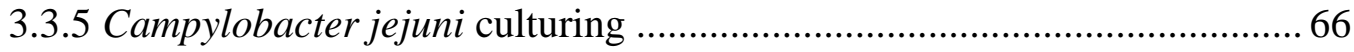

3.3.6 Health indices of Galleria mellonella larvae during bacterial infection... 66

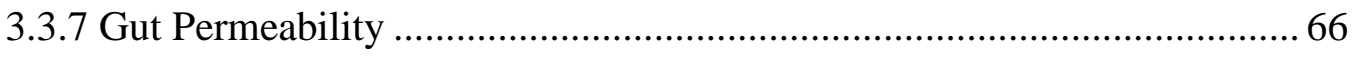

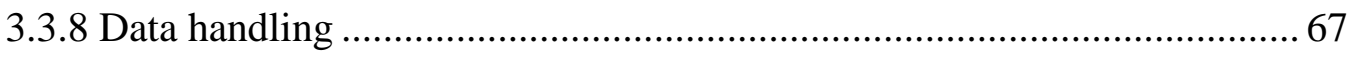

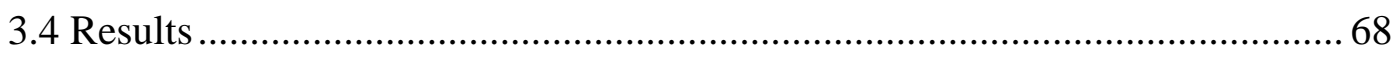

3.4.1 Larval development across nutraceutical-enhanced diets ....................... 68

3.4.2 Larval development and survival after nutraceutical inoculations ........... 71

3.4.3 Larval development and survival after pharmaceutical inoculations........ 73

3.4.4 Effect of nutraceuticals on maintaining larval health ........................... 75

3.4.5 Effect of nutraceuticals on improving gut permeability ........................ 77

3.4.6 Impact of nutraceuticals on Campylobacter jejuni infection outcomes.... 81

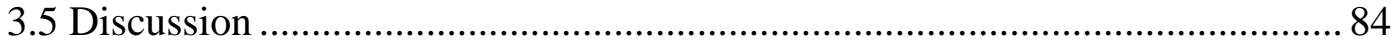

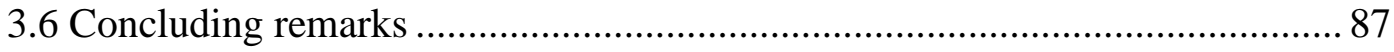

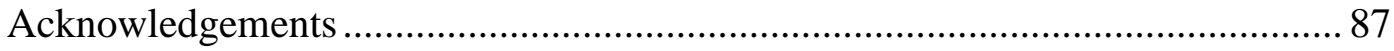




\section{Chapter 4: Relative toxicity and cytopathology of the emerging marine toxins, Azaspiracids, screened in Galleria mellonella larvae88}

4.1 Abstract 89

4.2 Introduction 90

4.3 Methods 92

4.3.1 Azaspiracid solution preparation 92

4.3.2 Assessment of Azaspiracid toxicity using a Galleria mellonella health index 92

4.3.3 Total haemocyte counts and cell sizes 93

4.3.4 Quantifying faecal microbes 93

4.3.5 Gut microbiome analysis of AZA 1 and okadaic acid-intoxicated larvae 93

4.3.6 Tissue histology of AZA and OA inoculated larvae. 94

4.3.7 Culturing of Campylobacter jejuni .95

4.3.8 Infecting Galleria mellonella larvae with Campylobacter jejuni .95

4.3.9 Pre-treatment of Galleria mellonella with AZA or OA, followed by Campylobacter jejuni .96

4.3.10 Data handling .96

4.4 Results

4.4.1 Survival, responsiveness (activity) and melanisation levels of intoxicated larvae 97

4.4.2 Total haemocyte counts and size(s) within the haemolymph of intoxicated larvae. 100

4.4.3 Impact of azaspiracid exposure on CFUs within insect faecal loads 102

4.4.4 Histological examination of the insect midgut post-azaspiracid exposure 104

4.4.5 Impact of azaspiracid-1 and okadaic acid inoculation on gut microbiota of the Galleria mellonella larvae 
4.4.6 Susceptibility of insect larvae to Campylobacter jejuni in the absence and presence of azaspiracid 1

4.5 Discussion

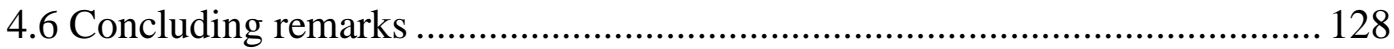

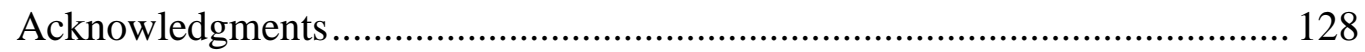

Chapter 5: Overall Summary and General discussion........................129

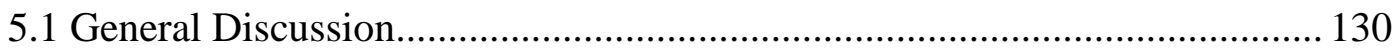

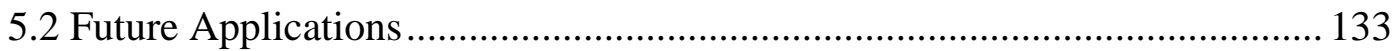

5.2.1 Galleria mellonella's role in pharmaceutical development ................... 133

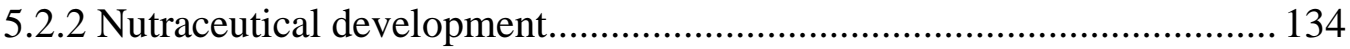

5.2.3 Galleria mellonella as a biotoxin model .............................................. 136

Appendices .................................................................................................................. 158

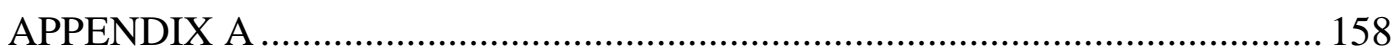

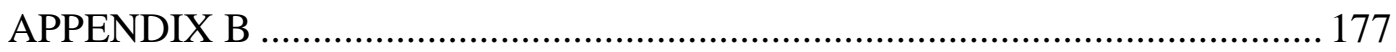

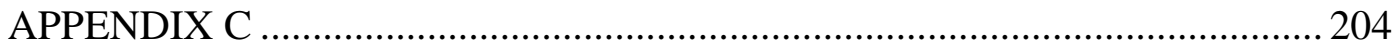




\section{List of Figures}

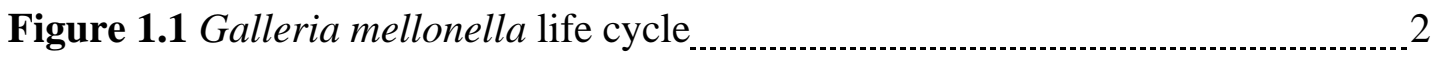

Figure 1.2 The general structure of okadaic acid (Dietrich et al. 2019) _............... 13

Figure 1.3 Chemical structures of AZA toxins 1, 2 and 3 (Twiner et al. 2012a) _........ 14

Figure 1.4 Effects of $C$. sinensis on rat gastric indomethacin induced damage _....... 19

Figure 1.5 Effect of $C$. sinensis extract on cell migration in HT29 cell culture _.......2 20

Figure 2.1 Survival of Galleria mellonella larvae following intrahaemocoelic injection (a) or force-feeding (b) of indomethacin, 0.5-7.5 $\mu \mathrm{g} / \mathrm{larva} \ldots 36$

Figure 2.2 Development (pupation) of Galleria mellonella larvae following inoculation with indomethacin, 0.5-7.5 $\mu \mathrm{g} / \mathrm{larva}$

Figure 2.3 Haemocyte responses of Galleria mellonella larvae following inoculation of indomethacin, $1-7.5 \mu \mathrm{g} / \mathrm{larva}$

Figure 2.4 Cross-sectional X-ray microtomography of the upper segment of a Galleria mellonella larva

Figure 2.5 X-ray microtomography of Galleria mellonella. A 3-dimensional render of a representative insect larva stained with Lugol's iodine

Figure 2.6 Gut permeability of Galleria mellonella larvae following force-feeding of indomethacin, $0-7.5 \mu \mathrm{g} /$ larva

Figure 2.7 Latex microspheres $(6 \mu \mathrm{m})$ in the haemolymph of Galleria mellonella coinoculated with indomethacin $(7.5 \mu \mathrm{g} / \mathrm{larva})$

Figure 2.8 Tissue (longitudinal) section of the Galleria mellonella midgut 46

Figure 2.9 Gut permeability of Galleria mellonella larvae following force-feeding of microspheres $(0.5-6 \mu \mathrm{m})$

Figure 2.10 Culturable colony-forming units from faeces of indomethacin-inoculated Galleria mellonella larvae. 48

Figure 2.11 Gross histopathology of the midgut tissues from control and indomethacin-treated Galleria mellonella... .49-50

Figure 2.12 The extent of midgut tissue damage in Galleria mellonella force-fed indomethacin $(7.5 \mu \mathrm{g} / \mathrm{larva})$ or PBS

Figure 2.13 Histopathology of compromised midgut tissues from Galleria mellonella force-fed $(7.5 \mu \mathrm{g})$ indomethacin

Figure 2.14 Detoxification-associated activities within Galleria mellonella following force-feeding of indomethacin, $0-7.5 \mu \mathrm{g} / \mathrm{larva}$

Figure 2.15 Detoxification-associated activities within Galleria mellonella following intrahaemocoelic injection of indomethacin, $0-7.5 \mu \mathrm{g} / \mathrm{larva}$ 55

Figure 3.1 Standard set-up for rearing Galleria mellonella 66 
Figure 3.2 Representative Galleria mellonella larvae before (left panels) and after (right panels) rearing on nutraceutical enhanced food.

Figure 3.3 Weights of Galleria mellonella larvae after 4 days of being reared on nutraceutical-enhanced food. 71

Figure 3.4 Pupation levels of Galleria mellonella larvae after 7 days of being reared on nutraceutical-enhanced food.

Figure 3.5 Galleria mellonella larval development and survival post-nutraceutical force-feeding.

Figure 3.6 Galleria mellonella larval pupation (A, C and E) and larval survival 0-10 days post force-feeding (B, D and F).

Figure 3.7 Health indices of Galleria mellonella larvae reared on nutraceuticalenhanced foods and exposed to indomethacin and/or lansoprazole. 77

Figure 3.8 Gut permeability of Galleria mellonella larvae reared on standard (A and B) and nutraceutical supplemented diets 79

Figure 3.9 Total microsphere loads of Galleria mellonella reared on standard (A and B) and nutraceutical supplemented diets 81

Figure 3.10a Health indices of Galleria mellonella larvae reared on supplemented food and force fed with Campylobacter jejuni (1x106 and 3x106 CFU/larva). 83

Figure 3.10b Health indices of Galleria mellonella larvae reared on supplemented food and injected with Campylobacter jejuni (1x106 and 3x106 CFU/larva). 84

Figure 4.1 Health status of Galleria mellonella larvae following intrahaemocoelic injection of azaspiracids, $5-200 \mathrm{ng} / \mathrm{larva}$. 99

Figure 4.2 Health status of Galleria mellonella larvae following force-feeding (gavage) of azaspiracids, $5-200 \mathrm{ng} / \mathrm{larva}$. 100

Figure 4.3 Haemocyte responses of Galleria mellonella larvae following intrahaemocoelic injection of azaspiracid 1 across the concentration range, $5-50$ ng/larva.

Figure 4.4 Typical appearance of bacterial colonies cultured from Galleria mellonella faeces.

Figure 4.5 Numbers of bacterial colony forming units in the faeces of azaspiracidintoxicated insects.

Figure 4.6 The extent of midgut tissue damage in Galleria mellonella after forcefeeding AZA1 (5 and $37.5 \mathrm{ng} / \mathrm{larva}$ ), okadaic acid (6.25 and $62.5 \mathrm{ng} / \mathrm{larva}$ ), or PBS.

Figure 4.7 Tissue sections of the Galleria mellonella midgut when force-fed PBS (2\% v/v ethanol).

Figure 4.8 Tissue sections of the Galleria mellonella midgut when force-fed azaspiracid 1 . 108

Figure 4.9 Tissue sections of the Galleria mellonella midgut when force-fed okadaic acid. 109-110 
Figure 4.10 Agarose gel electrophoresis of PCR amplicons after 16S V4 region amplification from Galleria mellonella whole gut tissue

Figure 4.11 Microbial (16S) composition of the gut tissue from Galleria mellonella larvae exposed to OA and AZA-1.

Figure 4.12 Microbial (16S) composition of the gut tissue from Galleria mellonell larvae exposed to OA and AZA-1.

Figure 4.13 Health indices of Galleria mellonella larvae following force-feeding or intrahaemocoelic injection of Campylobacter jejuni.

Figure 4.14 Susceptibility of Galleria mellonella to Campylobacter jejuni after azaspiracid-1 pre-treatment. 122 


\section{List of Tables}

Table 1.1 Comparison of gastric features in humans, rats and Galleria mellonella larvae

Table 2.1 Proportions of haemocytes staining positively for Trypan-blue (i.e., cell death) when treated with indomethacin $(1-7.5 \mu \mathrm{g} / \mathrm{larva})$ or PBS

Table 3.1 Nutritional content of $C$. sinensis and colostrum powder (Data based on product packaging)

Table 3.2 Health index scoring system for Galleria mellonella (developed by Loh et al., 2013) 66

Table 4.1 Re-suspended azaspiracid stock concentrations 93

Table 4.2 Survival analyses of Galleria mellonella larvae exposed to azaspiracids ....98

Table 4.3 Diversity indices of the gut microbiome of Galleria mellonella larvae after azaspiracid and okadaic acid exposure 113

Table 4.4 Statistical differences of the gut microbiome structure in Galleria mellonella larvae after azaspiracid and okadaic acid exposure 114

Table 4.5 Statistical differences of the gut microbiome structure in Galleria mellonella larvae after azaspiracid and okadaic acid exposure when comparing 4, 24 and 48hr within each treatment 115

Table 4.6 Azaspiracid administration, toxicology, and pathology in mice 125 


\section{List of Abbreviations}

\begin{tabular}{|c|c|}
\hline$A J$ & Adherence junctions \\
\hline$A L S$ & Amyotrophic lateral sclerosis \\
\hline$A M P$ & Antimicrobial peptides \\
\hline$A Z A$ & Azaspiracids \\
\hline Caco-2 & Caucasian colon adenocarcinoma cells \\
\hline$C A T$ & Catalase \\
\hline$C F U$ & Colony forming units \\
\hline $\operatorname{COX}$ & Cyclooxygenase \\
\hline DMSO & Dimethyl sulfoxide \\
\hline$D N C B$ & 2,4-Dinitrochlorobenzene \\
\hline$D S P$ & Diarrheic shellfish poisoning \\
\hline$D T T$ & DL-Dithiothreitol \\
\hline EDTA & Ethylenediaminetetraacetic \\
\hline GI & Gastro-intestinal \\
\hline$G N B P$ & Gram-negative binding protein \\
\hline$G S T$ & Glutathione S-transferase \\
\hline$I B D$ & Inflammatory bowel diseases \\
\hline$I M B$ & Immune deficiency \\
\hline ISCs & Intestinal stem cells \\
\hline$L D$ & Lethal dose \\
\hline$L P S$ & lipopolysaccharides \\
\hline MAMP & Microbe-associated molecular patterns \\
\hline$M D A$ & Malondialdehyde \\
\hline$N A D P H$ & Nicotinamide adenine dinucleotide phosphate \\
\hline$N B T$ & Nitroblue tetrazolium \\
\hline NSAIDs & Nonsteroidal Anti-inflammatory Drugs \\
\hline$O A$ & Okadaic acid \\
\hline$O M V s$ & Outer membrane vesicles \\
\hline PAMPS & Pathogen associated molecular patterns \\
\hline$P B S$ & Phosphate buffer solution \\
\hline
\end{tabular}




$\begin{array}{ll}\text { PGRP } & \text { Peptidoglycan recognition protein } \\ \text { PO } & \text { Phenoloxidase } \\ \text { PP } & \text { Protein phosphatases } \\ \text { proPO } & \text { Prophenoloxidase } \\ \text { PRR } & \text { Pattern recognition receptors } \\ \text { RNS } & \text { Reactive nitrogen species } \\ \text { ROS } & \text { Reactive oxygen species } \\ \text { SOD } & \text { Superoxide dismutase } \\ \text { TBA } & \text { Thiobarbituric acid } \\ T I R & \text { Toll-interleukin receptor } \\ T I R A P & \text { TIR-domain containing adaptor protein } \\ T J & \text { Tight Junctions } \\ T L R & \text { Toll like receptor } \\ T N F & \text { Tumour necrosis factor } \\ T R A M & \text { TRIF-related adaptor molecule } \\ T R I F & \text { TIR containing interferon- } \beta\end{array}$




\section{Chapter 1: Introduction}

\subsection{Thesis overview}

In 2019, some 3.4 million procedures were carried out on living animals in Great Britain alone with rats, mice and fish representing 93\% of all test subjects (Home Office, Annual Statistics of Scientific Procedures on Living Animals 2019). Over half (1.73 million) were for experimental procedures, which includes 437,000 for regulatory purposes (33\% of these were classified as toxicity and safety testing for medicine, dentistry and veterinary). Partially due to the efforts of the National Centre for Replacement, Refinement and Reduction of Animals in Research (NC3Rs; https://www.nc3rs.org.uk/) and the Fund for the Replacement of Animals in Medical Experimentation (FRAME; https://frame.org.uk/), the numbers of living animals used has decreased annually, for example, 4.14 million were approved for use in 2015. Although it is unlikely that all (vertebrate) animal experimentation will be eliminated, there are alternative non-vertebrate models available as well as emerging in vitro and in silico approaches. For example, the fruit fly Drosophila melanogaster and the nematode worm Caenorhabditis elegans have fulfilled the role of alternative models to mammals for almost a century. Another insect, namely the wax moth (Galleria mellonella), has been in use as an infection model for at least 40 years (Ratcliffe and Gagen, 1976). G.mellonella's popularity has increased dramatically over the last 5 years, in part, due to the availability of molecular resources and the genome (Lange et al., 2018a). Now, G. mellonella larvae represent a common surrogate model for the investigation of microbial pathogenicity, virulence, and antibiotic screening. Relatively low maintenance costs, limited ethical considerations and functional similarities to the human innate immune system are some of the key features that attract lab users to adopting G. mellonella (Wojda, 2017, Tsai et al., 2016). 
Wax moths have a relatively short life cycle, which can be split into four stages: (1) eggs that incubate for 5 to 8 days; (2) caterpillars/larvae that grow for 6 to 7 weeks (up to $23 \mathrm{~mm}$ long over 8 to 10 moults); (3) pupate (form brownish, hardened cocoons) for 1 to 8 weeks before (4) emerging as an adult moth (final stage) (Figure 1.1). The female moths are lighter in colour and slightly larger than the males, and females have a bifurcated proboscis. Females lay between 10-50 eggs at a time (Jorjão et al., 2018). This closed life cycle can be easily maintained in laboratory settings and manipulated for experimental purposes. A further benefit of using G. mellonella larvae is their culturing parameters. Larvae naturally encounter temperatures between $24-32^{\circ} \mathrm{C}$ (infesting beehives) and survive comfortably at $37^{\circ} \mathrm{C}$ (Tsai et al., 2016, Jorjão et al., 2018), thereby enabling researchers to use wax moth larvae to study pathogens at human relevant temperatures.

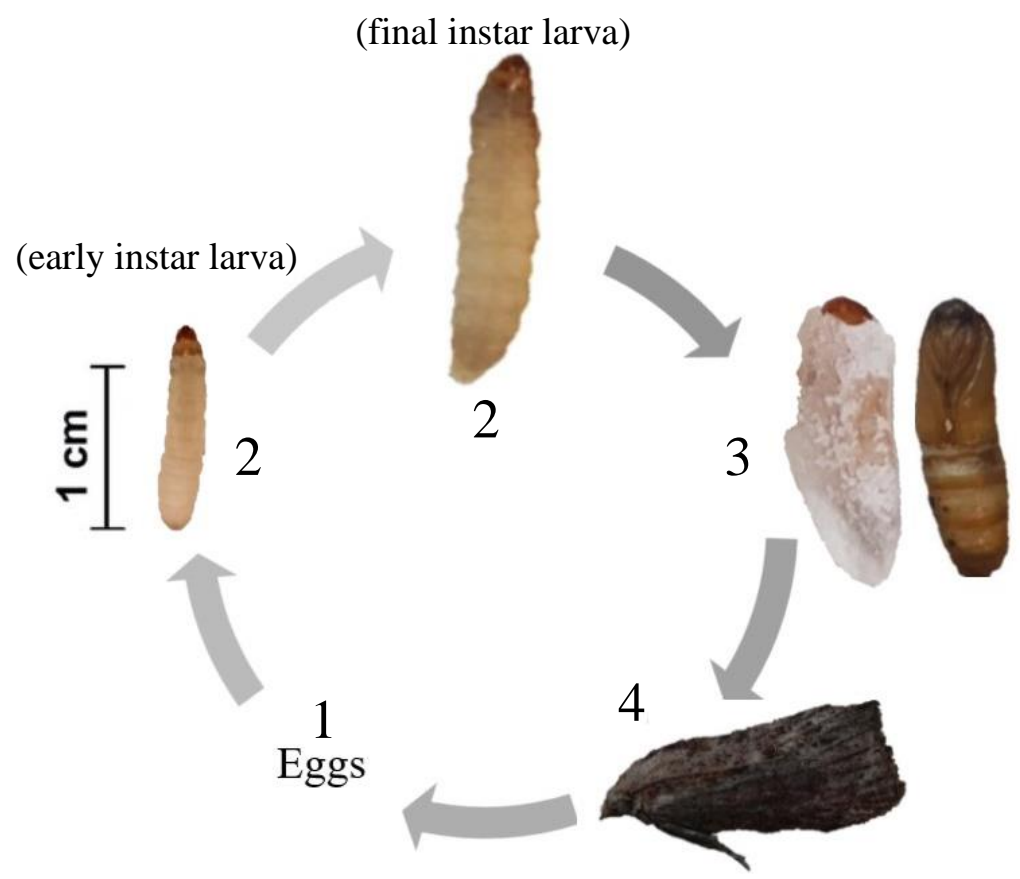

Figure 1.1 Complete life cycle of the wax moth Galleria mellonella

As an invertebrate, G. mellonella does not possess an adaptive immune system, i.e., appears incapable of producing immunoglobulins clonally (Rowley and Powell, 2007), but does share broad structural/functional innate immune mechanisms with mammals (Browne et al., 2013). For example, pathogen discrimination from self, intracellularisation, and subsequent destruction by NADHP-dependent oxidative burst is highly conserved among insect haemocytes and human professional phagocytes (Bergin et al., 2005). Beyond frontline 
defences, lepidopteran insects appear to have tissue organisation and structural features reminiscent of the human gastrointestinal (GI) tract, such as the epithelial layer and mucosa akin to the intestines, and an organ called the fat body that acts as the liver (Vallet-Gely et al., 2008). With that said, comparatively little is known regarding the gut physiology in $G$. mellonella and its putative suitability as a model for the human GI tract disorders, specifically, enteric pathogens or toxins encountered orally. Inoculation of G. mellonella with test compounds/microbes is predominantly carried out through intrahaemocoelic injection, with precious few studies attempting gavage (force-feeding). Research within this thesis endeavours to address these shortfalls, and in doing so, provide a more in-depth account of G. mellonella's alimentary canal, as well as the optimisation of techniques for investigating tissue damage, permeability, and restitution that mimic most closely those methods developed in rodents.

The overall aim of this thesis was to evaluate the usefulness and accuracy of Galleria mellonella larvae as a substitute in vivo model for gut-related pathobiology. To achieve the overall aim, the presented thesis is constructed around three primary objectives:

1. To test whether the established indomethacin gastric damage (ulcer) assay of rodents can be recapitulated in an insect (Chapter 2)

2. To determine if dietary intervention with nutraceuticals, Cordyceps sinensis and colostrum, can alleviate gastric damage caused by chemicals and/or microbes (Chapter 3)

3. To screen emerging marine toxins (associated with food poisoning) for signs of gastropathy and dysbiosis, assessing how generalisable the model is for future applications.

\subsection{Innate immunity of Galleria mellonella}

G. mellonella's immune system has similarities to that of humans with haemolymph being the functional equivalent of blood. Insect haemocytes found within the haemolymph can carry out phagocytosis and produce antimicrobial peptides (AMPs). The larval innate immune system is comprised of two broad lines of defence known as the humoral and cellular responses, however they lack the acquired immune system of humans. The humoral response incorporates AMPs, enzyme cascades and reactive oxygen and nitrogen species, instigating haemolymph clotting and melanisation (Browne et al., 2014). The cellular response is mediated by a heterogeneous 
population of haemocytes and involves various processes such as phagocytosis, nodulation and encapsulation - with evidence of chromatin trap release (Browne et al., 2013, Nathan, 2014).

\subsubsection{Cellular immune response in Galleria mellonella}

The cellular immune response of insects is complex relying on several distinct classes of haemocytes - including plasmatocytes, granular haemocytes, oenocytoids, spherule cells and prohaemocytes. However, some researchers argue that there are other classes of haemocytes called coagulocytes and adipoheamocytes (Ribeiro et al., 2016, Arteaga Blanco et al., 2017). The cellular response bares resemblance to that in humans whereby the immune cells are capable of phagocytosis, degranulation and produce superoxide to destroy pathogens akin to mammalian neutrophils and monocytes (Browne et al., 2014). Haemocytes also express encapsulating proteins that have been found to have high homology to human calreticulin, which is involved in protein folding and immunomodulation (Choi et al., 2002, de Bruyn et al., 2015). Haemocytes are found freely circulating in the haemolymph and/or adhered (sessile) to organs. Levels of haemocytes fluctuate throughout the lifespan of the insect and circulating levels drop during infection (Marmaras and Lampropoulou, 2009, Choi et al., 2002, Browne and Kavanagh, 2013). There are up to 8 different types of haemocytes characterised among insects, however it is unusual that all 8 are found within a single species.

Plasmatocytes are the most commonly found haemocyte and along with granular cells are involved in phagocytosis to remove pathogens in a similar way to mammalian macrophages and neutrophils (Lamprou et al., 2007). Mammalian neutrophils are involved in the formation of NADPH required for oxidative burst to kill internalised pathogens. Homology of neutrophil proteins $\mathrm{p} 47^{\text {phox }}$ and $\mathrm{p} 67^{\text {phox }}$ has been identified in G. mellonella haemocytes $67-\mathrm{kDa}$ and 47 $\mathrm{kDa}$ proteins which contained peptides matching those of $\mathrm{p} 67^{\text {phox }}$ and $\mathrm{p} 47^{\text {phox }}$ and are thought to be involved with the production of an oxidative burst (Bergin et al., 2005).

Encapsulation in G. mellonella occurs when larger foreign bodies are identified such as parasites and protozoa. During this process granular cells bind to, and lyse, the parasite and release plasmatocyte spreading protein, driving further recruitment and attachment of plasmatocytes (Butt et al., 2016). Multiple layers of bound plasmatocytes release cytoplasmic adhesion molecules onto their surface, forming a capsule around the parasite. Granular cells then adhere to the capsule and undergo apoptosis to form an inert outer layer. The production of reactive oxygen or nitrogen species then assist in killing the intruder (Nappi and Ottaviani, 2000, Butt et al., 2016). 
Nodulation is a process unlike any in the human body and occurs when clusters of bacteria are bound by multiple haemocytes, forming a shell and immobilising them inside. Once contained, the process can be completed by the oenocytoids undergoing cell lysis to release prophenoloxidase (proPO) into the haemolymph for melanin biosynthesis. The activation of proPO is regulated by proteases (serpins) and initiated by haemocyte bound pattern recognition receptors (PRRs) interacting with pathogen associated molecular patterns (PAMPS) (Sideri et al., 2008, Browne et al., 2013, Hoffmann, 1995, Kim et al., 2018, Butt et al., 2016, Whitten and Coates, 2017). Inactive proteolytic enzyme precursors, known as zymogens are activated leading to the cell to burst and release activated PO and the production of melanin, killing the pathogen (Sideri et al., 2008, Browne et al., 2013, Hoffmann, 1995, Kim et al., 2018, Butt et al., 2016).

The immune response in G. mellonella predominantly involves AMPs, proPO cascade and opsonins (Tsai et al., 2016). Toll and Immune deficiency (IMD) signalling pathways mediate responses from PRRs detecting PAMPs such as peptidoglycans within the pathogens cell wall. When host PRR detect peptidoglycan recognition protein (PGRP) or Gram-negative binding protein (GNBP) a signal cascade is activated within the fat body through toll receptor pathway leading to the expression of AMPs. AMPs are then synthesised within the haemocytes, epithelia and fat body and secreted into the haemolymph (Wojda et al., 2009, Mak et al., 2010, Nehme et al., 2007). Toll pathway relays signals intracellularly when presented with Gram-positive bacteria and fungi, whereas the IMD pathway is active against Gram-negative bacteria. This separation gives a level of specificity in the insect immune response, resulting in the production of specific AMPs (Wang and Ligoxygakis, 2006).

Melanisation is a vital part of $G$. mellonella's clotting and immune response. Once a pathogen is detected by PRRs, the proPO cascade is initiated. Serine protease inhibitors such as serpin regulate the proPO cascade, proPO is proteolytically cleaved during the response to infection to form the active phenoloxidase (PO) (Kanost and Gorman, 2008, Sheehan et al., 2018, Butt et al., 2016). PO then enables the oxidation and hydroxylation of phenols to create quinones, which are further processed a series of enzymatic and spontaneous reactions to form melanotic polymers (Sheehan et al., 2018, Butt et al., 2016). Melanin is deposited on pathogen surfaces and recruits' haemocytes to encapsulate or engulf the pathogen. This process also produces toxic reactive oxygen species (ROS) intermediates that further damage bacterial cell walls (Tsai et al., 2016). 


\subsubsection{Galleria mellonella humoral immune response}

G. mellonella have a wide range of humoral responses to infection, these include the production of ROS, PO and AMPs. There have been at least 18 AMPs identified in G. mellonella such as cecropin, gallerimycin and galiomicin are (Wojda et al., 2009, Moghaddam et al., 2016). AMPs are found the haemolymph and in the midgut of pre-pupae and are vital in the immune defence. Interestingly the AMP lysozyme found in G. mellonella is structurally similar to lysozymes identified in chicken and is active against gram-positive bacteria through the hydrolysis of peptidoglycan, lysozyme is also somewhat effective against gram-negative bacteria and fungi (Jiang et al., 2010). Gallerimycin and galiomicin are cysteine-rich defensin AMPs, which are homologous to human cysteine-rich peptides hBD1, 2, 3 and 4. Gallerimycin and galiomicin are the most effective AMP against fungal infection, studies infecting G. mellonella with Candida found an upregulation of both defensins within the first hour of inoculation and a maximum regulation 24 hours post-inoculation (Lee et al., 2004). This suggests that gallerimycin and galiomicin are involved with the initial antimicrobial response.

Another branch of the humoral immune response is the production of ROS and reactive nitrogen species (RNS) which are essential antimicrobial and signalling molecules (Butt et al., 2016). ROS such as superoxide $\left(\mathrm{O}_{2}-\right)$ and hydrogen peroxide $\left(\mathrm{H}_{2} \mathrm{O}_{2}\right)$ are produced in haemocytes and released into the haemolymph within the first 30 minutes of a foreign body entering the hemocoel and during the encapsulation and melanisation process (Dubovskii et al., 2010). The haemocyte production of ROS is thought to be mediated by an NADPH oxidase complex, and there is evidence to suggest that $\mathrm{O}_{2}-$ is involved in the activation of cationic granule enzymes that kill the pathogens (Bergin et al., 2005, Reeves et al., 2002). ROS can be harmful to the host and pathogen, damaging DNA, causing protein degradation and lipid peroxidation therefor the host also produces an array of antioxidants such as superoxide dismutase (SOD), catalase and glutathione-S-transferase (GST) (Dubovskii et al., 2010).

\subsection{Galleria mellonella gastrointestinal morphology}

Generally, the insect gastrointestinal tract can be divided into three sections; foregut, midgut, and the hindgut. The larval gut has several similarities to that in mammals, including microvilli, natural flora and epithelial cells (Table 1.1). G. mellonella larvae are of interest due to its tolerance of temperatures up to $37^{\circ} \mathrm{C}$ and its large size, making them more suitable for accurate injection of microbes into the gut, compared to other insect models such as Drosophila melanogaster (Vogel et al., 2011, Fedhila et al., 2010, Tsai et al., 2016). 
The gut of insect larvae also contains smooth septate junctions between epithelial cells which act similarly to tight junctions found in mammals, whereby they control the movement of molecules in and out of the gut (Green et al., 1980). Additionally, within the midgut of $G$. mellonella larvae there is the peritrophic matrix, which lines the digestive tract acting similarly to the mucus layer, protecting the epithelial cell layer from pathogens and aiding food digestion. The peritrophic matrix is formed of proteins, glycoproteins and chitin, making a thin and semi-permeable barrier (Campbell et al., 2008). The midgut is where the majority of digestion and absorption of nutrients occurs and contains specialised cells such as goblet and columnar cells for the secretion of enzymes and ion transport (Tanigawa et al., 2016). Interestingly, the midgut also contains intestinal stem cells (ISCs) which are vital for repair and replacement of damaged tissue, and during larval moulting. The differentiation of ISCs is controlled by the secretion of hormones from endocrine cells within the epithelial layer of the midgut (Franzetti et al., 2016). The foregut of larvae contains sclerotized microspines to assist digestion, little to no absorption of nutrients occurs here but serves to start the physical degradation of the food and passage of the bolus into the midgut (Wang et al., 2018).

Recent research shows melanisation occurring within the gut as a mode of protection against ingested pathogens (Whitten and Coates, 2017). However, there is debate as to whether the proPO is produced in the haemolymph then transported into the gut before becoming activated by gut trypsin acting as serine protease (Joseph, 2014). Further research indicates that in silkworms proPO is expressed in the hindgut where PO melanises faeces (Whitten and Coates, 2017). Interestingly, there is evidence the gut of G. mellonella larvae can differentiate between symbiotic and pathogenic bacteria through microbe-associated molecular patterns (MAMPs) and elicit different intensities of immune response - similar to both mice and humans (Lange et al., 2018b).

Other than ultrastructural diagrams of the G. mellonella gut, there is little information of the cellular/molecular arrangements, and much of what is indicated is based on other insect models and assumptions. To improve the data on the structure and morphology of the G. mellonella gut, a computer-assisted 3D render would be helpful. The natural microbiota of G. mellonella larva consists of some bacterial species found routinely in human guts such as Proteobacteria, Bacteroidetes and Firmicutes, all of which are associated with syndromes such as Crohn's disease (Dubovskiy et al., 2016, Ley et al., 2006b, Carvalho et al., 2012, Holmes et al., 2011). 
Table 1.1 Comparison of gastric features in humans, rats and Galleria mellonella larvae

\begin{tabular}{|c|c|c|c|c|}
\hline Feature & Human & Rat & $\begin{array}{l}\text { G. mellonella } \\
\text { larvae }\end{array}$ & Supporting evidence \\
\hline Peristalsis & $\checkmark$ & $\checkmark$ & $\checkmark$ & $\begin{array}{l}\text { (Sláma and Lukáš, } \\
\text { 2011, Grider et al., } \\
\text { 1998) }\end{array}$ \\
\hline Mucosal layer & $\checkmark$ & $\checkmark$ & $\begin{array}{l}\text { Peritrophic } \\
\text { membrane }\end{array}$ & $\begin{array}{l}\text { (Campbell et al., 2008, } \\
\text { DeSesso and Jacobson, } \\
2001 \text { ) }\end{array}$ \\
\hline Tight Junctions & $\checkmark$ & $\checkmark$ & $\begin{array}{l}\text { Septate } \\
\text { junctions }\end{array}$ & (Green et al., 1980) \\
\hline Villi & $\checkmark$ & $\checkmark$ & $\checkmark$ & $\begin{array}{l}\text { (Wang et al., 2018) } \\
\text { (Maguire et al., 2016) }\end{array}$ \\
\hline $\begin{array}{l}\text { Plicae circulares } \\
\text { (Folds in } \\
\text { mucosal layer) }\end{array}$ & $\checkmark$ & $x$ & $x$ & $\begin{array}{ll}\text { (DeSesso } & \text { and } \\
\text { Jacobson, 2001) }\end{array}$ \\
\hline $\begin{array}{l}\text { Diverse } \\
\text { microbiome } \\
\text { (Several species } \\
\text { of bacteria, fungi } \\
\text { and virus) }\end{array}$ & $\checkmark$ & $\checkmark$ & $\begin{array}{l}\text { Limited; } \\
\text { Predominantly } \\
\text { Enterococcus } \\
\text { mundtii }\end{array}$ & $\begin{array}{l}\text { (Ignasiak and Maxwell, } \\
\text { 2018, Zhang et al., } \\
\text { 2017) }\end{array}$ \\
\hline pH & $\begin{array}{l}\text { Stomach: } \\
2-5 \\
\text { Intestine: } \\
6-7.5\end{array}$ & $\begin{array}{l}\text { Stomach: } \\
3-4 \\
\text { Intestine: } \\
5-6.6\end{array}$ & $\begin{array}{l}\text { Whole gut: } \\
8-11\end{array}$ & $\begin{array}{l}\text { (Coddington } \\
\text { Chamberlin, } \\
\text { McConnell et al., 2008, } \\
\text { Nugent et al., 2001) }\end{array}$ \\
\hline
\end{tabular}

Gastric $\mathrm{pH}$ is an important factor for digestion and the metabolic processing of toxins and pharmaceuticals, therefore it must be considered when modelling the human gut. The $\mathrm{pH}$ of the stomach in both rats and humans are very acidic (Table 1.1) and the $\mathrm{pH}$ of the intestine is 
close to neutral (Nugent et al., 2001, McConnell et al., 2008). However, the gut of G. mellonella larvae is very alkaline with a $\mathrm{pH}$ range of 8-11 (Coddington and Chamberlin, 1999). This indicates a physiological limitation of G. mellonella's ability to act as a model for the human gut, as it could result in the production of different metabolites and changes to pharmacokinetics during the digestion of toxins and pharmaceuticals (Hughes et al., 1989). It is understood that stomach $\mathrm{pH}$ can alter the absorption and bioavailability of drugs. For example, when tyrosine-kinase inhibitors are co-administered with proton-pump inhibitors, the stomach acid $\mathrm{pH}$ increases, causing tyrosine-kinase inhibitors to become the non-ionised form, which is less soluble and reduces the bioavailability (van Leeuwen et al., 2014). Pharmaceuticals are designed with a drug delivery method, many of which include $\mathrm{pH}$ due to difference of $\mathrm{pH}$ within tissues eg., slightly acidic tumours and areas of the GI tract (Cao et al., 2019).

Similar to rodents and humans, G. mellonella larvae utilise digestive enzymes to break down large, complex macromolecules. Serine proteases, $\beta$-glucosidases and $\alpha$-amylase are some of the digestive enzymes identified in both G. mellonella larvae and humans (Bulushova et al., 2011b, Kara et al., 2014, Bulushova et al., 2011a). Serine proteases are a large group of enzymes that make up one third of all proteases that break peptide bonds of proteins. Of these serine proteases, chymotrypsin, trypsin and pancreatic elastase are categorised as digestive enzymes (Hedstrom, 2002). Interestingly, several serine proteases including three anionic and two cationic forms of trypsin, one anionic and one cationic form of chymotrypsin and one elastase-like protease have been identified in midgut of G. mellonella larvae. Although they are analogues to the mammalian equivalent, their optimum $\mathrm{pH}$ is between 9.5-11 (Bulushova et al., 2011a).

$\beta$-glucosidases are enzymes that hydrolyse glycosidic linkages present in disaccharides, oligosaccharides and glucosides (Yeoman et al., 2010). Most $\beta$-glucosidases, including human analogues have an optimum $\mathrm{pH}$ between 4-7.5, whereas those present in the midgut of $G$. mellonella larvae are active under alkaline conditions (Ketudat Cairns and Esen, 2010, Kara et al., 2014). $\beta$-glucosidases are adapted to the diet of the species, therefore it is unsurprising that those present in G. mellonella larvae differ to those in rats and humans. The natural diet of $G$. mellonella is limited to bees wax, honey, pollen and pupal skins, therefore most of the midgut $\beta$-glucosidases target $\beta$-glucosides (Kara et al., 2014, Jorjão et al., 2018). A potential limitation of using G. mellonella larvae as a model for the human gut is differences in gastric enzymes. Although $\beta$-glucosidases are present in midgut of $G$. mellonella, they may lack some key 
analogues. Currently, there has not been a study comparing gastric enzymes between $G$. mellonella and mammalian models, this would be beneficial to further develop G. mellonella larvae as a gastric model.

A potential limitation of $G$. mellonella larvae as a model is the comparison between hepatic metabolism and the functional equivalent, which is insect the fat body. The larval fat body is an important energy store and produces AMPs similarly as in humas, however the fat body's metabolism of drugs compared to the liver is likely to differ. Research shows that the fat body contains a number of enzymes involved in drug detoxification, including several cytochrome P450s and sulfo-, glutathione- or glucose-conjugation enzymes (Maguire et al., 2017). A recent study determined that force feeding G. mellonella larvae caffeine produced similar metabolites theobromine and theophylline to those usually detected in humans. Additionally, the same study found the larvae to have reduced movement and development (Maguire et al., 2017). Although there are differences in the specific enzymatic profiles, there are analogues active at a high $\mathrm{pH}$ resulting in similar metabolites and metabolic pathways, therefore G. mellonella larvae may be a broad model for the human gut but could be limited in the assessment of pharmaceuticals that rely on a specific $\mathrm{pH}$ range for their mode(s) of action. Therefore, although there are differences between G. mellonella's and humans digestive enzymes and $\mathrm{pH}$, there are enough functional similarities to utilise $G$. mellonella as a model in this manner

\subsection{Methods of infecting Galleria mellonella}

There are several methods to administer bacteria or compounds to G. mellonella larvae, directly injecting into the body cavity (haemocoel) through a pro-leg, topical application, submersion in particulate suspensions, and feeding (Kavanagh and Reeves, 2004, Salamitou et al., 2000, Grizanova et al., 2014, Maguire et al., 2016, Coates et al., 2019). Most studies inject the compound directly through the last proleg, however, Fedhila et al. (2010) argue that this method bypasses the usual oral route and therefore force-feeding the larvae could give a more accurate response to the pharmaceutical or microbe administered. Further research found that delivering saline solution alone via gavage did not result in mortality and delivered comparable results to direct injection (Coates et al., 2019). Maguire et al. (2017) provided some evidence that caffeine is similarly metabolised in G. mellonella as in humans (Maguire et al., 2017). Considering pathogens and toxins that lead to gastropathy are delivered through an oral route, then studies directly injecting such compounds into insects or mice (intraperitoneal injection) may not accurately characterise the effects or targets of these factors. 


\subsection{Existing evidence for Galleria mellonella as a candidate model for gut pathology}

Although some similarities between the gut of G. mellonella and the human are documented, there is a knowledge deficit regarding the specific physiology and importance of microbiota that colonise it, and there are significant gaps as to whether they are a good model for gut properties such as leakiness, inflammation, apoptosis and restitution. G. mellonella larvae have shown to be useful models for studying the pathogenicity of food poisoning associated bacteria, e.g., Bacillus cereus upon oral administration (Jensen et al., 2003, Salamitou et al., 2000), Campylobacter jejuni, Clostridium perfringens, Vibrio spp., via subcutaneous injection (Senior et al., 2011, Wagley et al., 2018, Bokhari et al., 2017, Kay et al., 2019). Interestingly, some probiotic bacteria such as Lactobacillus rhamnosus GG were screened in G. mellonella for their potential protective properties against gut-damaging microbes (e.g., Salmonella enterica), but these were not studied through an oral route (Scalfaro et al., 2017).

\subsection{Gut specific challenges}

\subsubsection{Marine biotoxins}

To test how generalisable G. mellonella larvae is as a model for gut specific damage and disease, using a gastrointestinal-specific marine biotoxins such as okadaic acid and azaspiracid is an additional proof of concept approach. Okadaic acid has previously been used in $G$. mellonella to study toxicological end point $\left(\mathrm{LD}_{50}\right)$, therefore its usefulness can be extended to marine toxins that are poorly understood and have not yet been screen in a non-mammalian model eg., azaspiracids (Coates et al., 2019).

\subsubsection{Okadaic acid}

Okadaic acid (OA) is a lipophilic phycotoxin (Figure 1.2) found in shellfish and causes diarrheic shellfish poisoning (DSP) upon human consumption of contaminated tissue and is produced by dinoflagellates of the genera Dinophysis and Prorocentrum (Dietrich et al., 2019). The consumption of $<50 \mu \mathrm{g}$ per $\mathrm{kg}$ of OA contaminated tissue can result in DSP causing stomach cramps, diarrhoea and vomiting which begin within 4 hours of ingestion and can last several days (Dominguez et al., 2010). OA is an inhibitor of protein phosphatases that play various roles in cellular metabolism, which not only affects the GI tract but also contributes to neuronal toxicity and tumour promotion. These combined make OA highly toxic, and therefore, 
current upper regulatory limits with the UK/EU are set at $160 \mu \mathrm{g}$ OA equivalents per $\mathrm{kg}$ of shellfish (Munday, 2013).

OA causes damage to the villi and the epithelial layer, increasing permeability of the intestine and erosion of the mucosal layer in mice (Ito and Terao, 1994). The increased permeability could be caused by OA increasing paracellular permeability through the disruption of tight junctions (TJ) and adherens junctions (AJ). TJ and $\mathrm{AJ}$ are regulated through protein phosphatases 1 and 2A (PP1 and PP2A) and protein kinases in rats and humans. The exact mode of OA's increased permeability remains unclear, it has been suggested to involve inhibiting PPA2 and PP1 (Bialojan and Takai, 1988) or through disrupting F-actin cytoskeleton with is involved in TJ control (Fiorentini et al., 1996, Dietrich et al., 2019).

$\mathrm{OA}$ is known to increase tumour necrosis factor $\alpha(\mathrm{TNF} \alpha)$ release from host cells which then increased paracellular permeability of epithelial cells in culture (Munday, 2013). OA's increase in permeability could be a result of cellular damage rather than a targeted effect (Turner, 2006, Ito and Terao, 1994, Berven et al., 2001). The microbiome of rats that were administered with a low oral dose of OA ( $80 \mu \mathrm{g} / \mathrm{kg}$ tissue) daily for 30 days was assessed. This study found that although the rats appeared healthy with no weight change, or no changes to microbiome richness or diversity, however there was a significant change in individual abundance in the gastric microbiota, with an increase in Clostridiales (Liu et al., 2020). Interestingly, Clostridiales include many pathogenic bacteria such as Clostridium difficile, which is associated with inflammation and tissue damage (Smits et al., 2016).

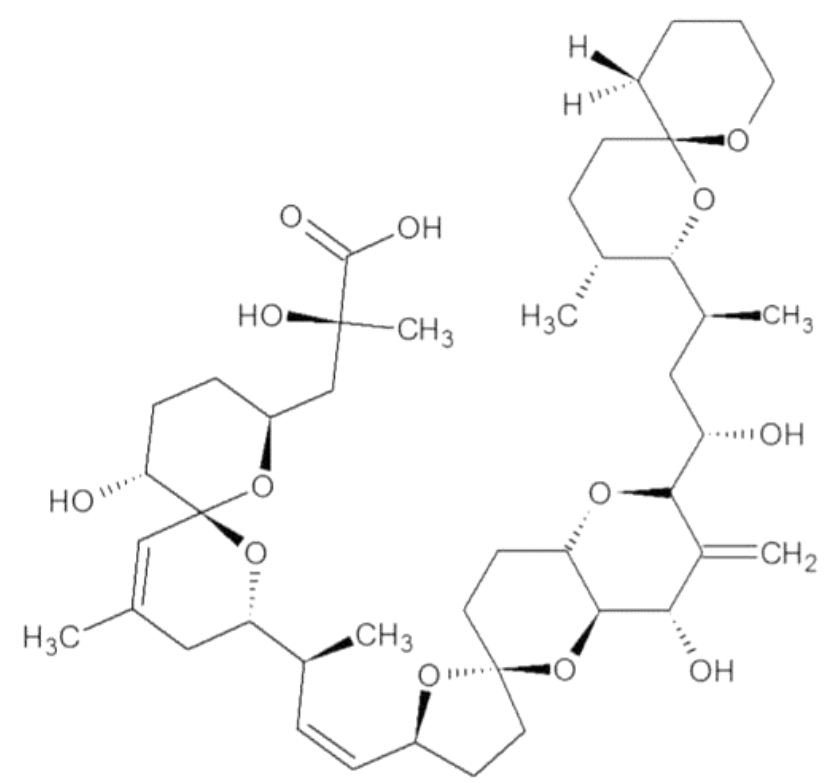

Figure 1.2 The general structure of okadaic acid (Dietrich et al., 2019) 


\subsubsection{Azaspiracids}

Azaspiracids (AZAs) are also lipophilic polyether toxins - first detected in 1995 from a contaminated batch of mussels on the West coast of Ireland. AZAs represent one of the most potent marine toxins, which induce similar gastrointestinal distress as observed in OA (Román et al., 2002). Since their discovery in 1995 there has been over 30 analogues identified globally (Krock et al., 2019). Currently, there are four known producers of AZAs; Azadinium spinosum, Azadinium dexteroporum, Azadinium poporum and Amphidomataceae languida all of which are dinoflagellates (Krock et al., 2014, Rossi et al., 2017, Tillmann et al., 2017, Kilcoyne et al., 2014). Studies in mice demonstrated that AZA not only affects the GI tract but also targetsthe liver, lung, pancreas, thymus and spleen (Furey et al., 2010). Additionally, AZAs induce breathing difficulties and paralysis of limbs in mice, which show clear indications of neurotoxicity and separates AZA poisoning from other diarrheic shellfish poisoning (DSP) (Román et al., 2002).

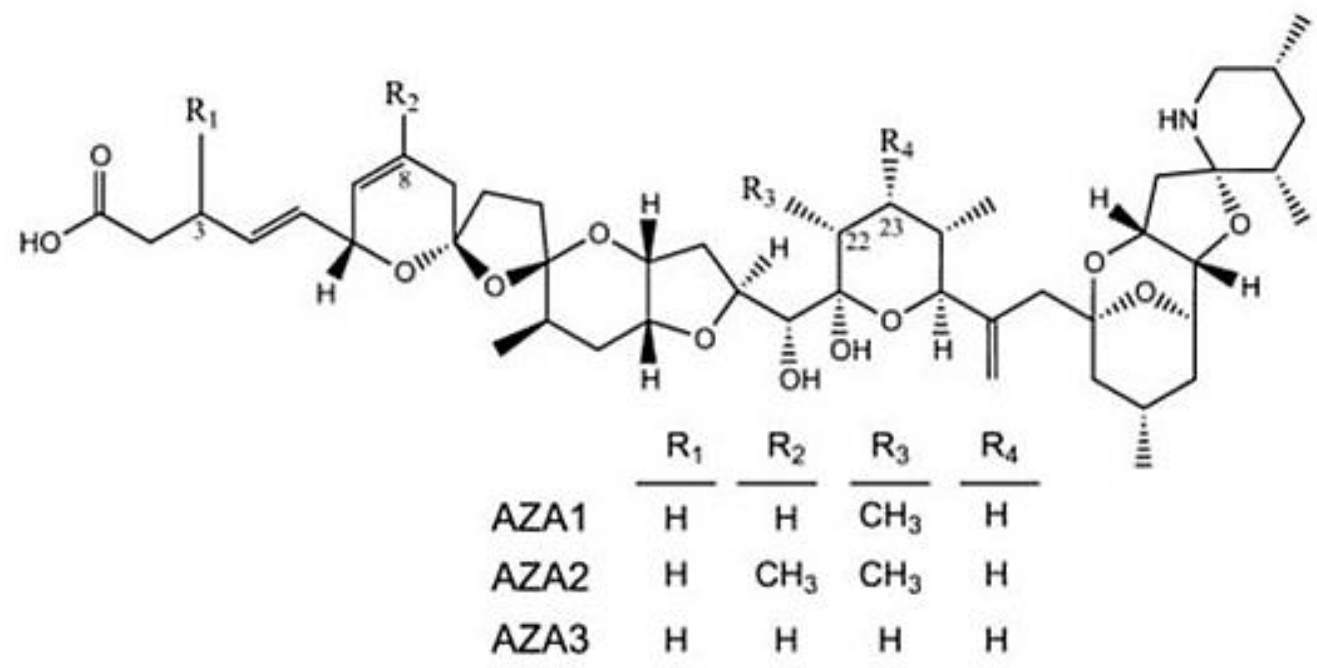

Figure 1.3 Chemical structures of AZA toxins 1, 2 and 3 (Twiner et al., 2012a)

AZA consumption results in increased permeability of the intestine, leading to fluid accumulation in the small intestine and diarrhoea. The mode of action of AZA is unclear, studies conducted on Caco-2 cells that model the human intestine found that exposure to AZA1 interfered with the protein occludin, and leading to disruption in paracellular permeability (Abal et al., 2017). A further observation of AZA-1 exposure to Caco-2 cells was a disorganisation of actin cytoskeleton and a decrease in adherence, this could be a factor involved in increased intestinal permeability (Vilariño et al., 2006, Vilariño, 2008). AZA-1 exposure also results in an increase in caspase activity. For example, in $\mathrm{T}$ lymphocytes an 
increase in caspase- 2 was observed, which may go on to induce caspase -3 via the release of cytochrome $c$ in mitochondria through the caspase -9 independent pathway leading to apoptosis (Twiner et al., 2012b).

Neurotoxicity tests conducted on cell cultures, found that AZA-1 neurotoxic effect appears to require very low dosages of the nanomolar range to cause toxic effects. In neocortical neurons, AZA-1 had a concentration and time dependent effect causing maximum toxicity 48 hours after a dosage of $\mathrm{EC}_{50}$ value $31.7 \mathrm{nM}$. It is thought that AZA inhibits the spontaneous $\mathrm{Ca}^{2+}$ oscillations in neocortical neurons which could alter the synaptic signalling between neurons and found that AZA-3 was more neurotoxic than AZA-1 and AZA-2 in neocortical neurons (Cao et al., 2010). A study using PC12 cells which are derived from rat pheochromocytoma cells in the adrenal medulla assessed the effects of AZA-1 (Hjørnevik et al., 2015). This study found that exposure to AZA-1 resulted in a down-regulation of the intermediate filament protein peripherin and an increase in differentiated phenotype of PC12 cells which had protrusions similar to neurites (Hjørnevik et al., 2015).

A change in the regulation of peripherin has been linked to amyotrophic lateral sclerosis (ALS), a neurodegenerative disease and is important for neuronal repair after injury (McLean et al., 2010, Robertson et al., 2003) these findings could elude to some of the neurotoxicity properties of AZA. The full mechanisms of AZA are unclear however AZA-1 is thought to induced changes in cell morphology by acting on F-actin resulting in cytoskeleton destruction in neuroblastomas and $\mathrm{T}$ cells also causing an increase in $\mathrm{Ca}^{2+}$ in cells and causing villi erosion (Ito et al., 2006). A study in epithelial colorectal adenocarcinoma cells known as Caco-2 cell line show AZA-1 caused increased permeability in a concentration dependent manor. This study found that there was a decrease in trans-epithelial electric resistance which indicates a reduction in barrier stability which could be linked to an increase in permeability (Abal et al., 2017).

\subsubsection{Campylobacter jejuni}

Campylobacter jejuni is a gram-negative bacteria and is one of the most significant bacterial causes of diarrheal illness in humans, and is most commonly transmitted through the consumption of chicken (Harris et al., 1986, Taylor et al., 2017). Estimated to cause more than 2 million cases of gastrointestinal illness annually in America and can have complications such as reactive arthritis and Guillain-Barre' syndrome (a type of acute peripheral neuropathy), 
making it an important pathogen to model (Kelly et al., 2003). Most infections result in diarrhoea, stomach cramps and fever which can persist for a week, however some patients can continue for several weeks (Acheson and Allos, 2001). In three quarters of patients, red blood cells are found in infected patients' faeces and the colon becomes inflamed with is similar to the initial stages of IBD and occasionally leads to a miss diagnosis (Blaser et al., 1979, Lawson et al., 1999). Due to $C$. jejuni's gut specific pathogenicity it is a suitable candidate to assess $G$. mellonella's ability to model a microbes, toxins and pharmaceuticals.

The molecular pathogenesis of $C$. jejuni in the GI is unclear, as C. jejuni does not secrete typical virulence factors, rather it is hypothesised that the mechanism includes outer membrane vesicles (OMVs) to deliver virulence factors to the host. It has been found that they produce OMVs between $10-250 \mathrm{~nm}$ diameter and are of different density suggesting differences in their contents (Elmi et al., 2012). It is understood that $C$. jejuni produce three different OMVs containing different virulence factors including serine proteases, these $\operatorname{are~} \mathrm{HtrA}, \mathrm{Cj} 0511$, and $\mathrm{Cj} 1365 \mathrm{c}$. These serine proteases are involved with cleaving epithelial cells in the intestine and binds to the junction proteins occludin thus promoting further invasion of epithelial cells (Elmi et al., 2018). C. jejuni uses invasive antigens to infect cells, and produce cytolethal distending toxins (CdtA, B, C). Cytolethal distending toxins are the only toxins produce by this bacterium and it appears to be involved in the stimulation of host cell apoptosis which is a main factor of C. jejuni pathogenicity (Dasti et al., 2010). Other developments in understanding C. jejuni pathogenesis have identified the type VI secretion system (T6SS) to be involved in virulence and could be associated with more severe infections. It is postulated that T6SS is involved in a contact dependent secretion of effector proteins into host cell (Bleumink-Pluym et al., 2013).

Lipopolysaccharides (LPS) are important in maintaining outer membrane stability and protecting the bacterium against host defences. Studies have found that LPS present on the $C$. jejuni cell surface are pivotal in immunopathogenesis of the infection, resulting in increased inflammation and is linked to the development of Guillain-Barre' syndrome, IBS and reactive arthritis as a result of prolonged C. jejuni infection (Naito et al., 2010, Zarantonelli et al., 2006, Mortensen et al., 2009, Godschalk et al., 2007). LPS binds to TLR-4 receptors on host cell surface, this stimulates an intracellular signalling cascade via the Toll-interleukin receptor (TIR) containing interferon- $\beta$ (TRIF) and TRIF- related adaptor molecule (TRAM), this is known as the TRIF-TRAM pathway which upregulates type one interferons and triggers tumour necrosis factor (TNF)- $\alpha$ production and secretion. The other signalling cascade known as the TIRAP-MyD88-pathway which involves the TIR-domain containing adaptor protein 
(TIRAP) and myeloid differentiation primary response 88 (MyD88), resulting in the activation of $\mathrm{NF}_{-} \mathrm{B}$ cells and upregulation of inflammatory cytokines such as IL-12 (Mousavi et al., 2020). Interestingly, one of the difficulties in understanding C. jejuni infection is due to the murine model's intrinsic resistance to $C$. jejuni infection. This is partly due to rats having 10,000-fold increase in resistance to LPS than in humans (Mousavi et al., 2020). Other animal models such as G. mellonella and ferrets have been used to study $C$. jejuni infection but are limited (Senior et al., 2011, Nemelka et al., 2009), therefore an insect can help to improve the understanding of $C$. jejuni infection.

\subsection{Gastric targeting Nutraceuticals}

To study G. mellonella's ability to act as a model for gastric protection and assess the therapeutic potential of selected nutraceuticals. Suitable nutraceuticals to study gastric protection against pharmaceutical, toxin and microbial induced damage are those that have anti-inflammatory, anti-apoptotic and antioxidant properties, i.e., Cordyceps sinensis extract and colostrum.

\subsubsection{Cordyceps sinensis}

Cordyceps sinensis is an entomopathogenic fungus, for which its uses in traditional medicine can be dated back to the late 1400's in Tibet. Spores from the fungus penetrate through the cuticle layer of insect larvae, e.g., Hepialus armoricanus, which then infect and grow within the host, progressively taking control of the insect before eventually resulting in its death and the fruiting body protruding from the cadaver (Chen et al., 2013).

\subsubsection{1 $C$. sinensis in gut repair and protection}

There has been increasing interest towards $C$. sinensis due to the discovery of numerous therapeutic effects in cancer treatment, cardiovascular disease, diabetes among others (Li et al., 2006). It has been shown to have both protective and reparative properties against gastric damage, therefore it is important to investigate the specific effects $C$. sinensis has on the human gut and its potential applications as a functional food (Marchbank et al., 2011).

\subsubsection{Human gut physiology}

The human GI tract is a continuous layer of cells from the lower oesophageal sphincter to the anus with the purpose of delivering nutrients and removing waste from the body. Controlled absorption and secretion whilst maintaining a barrier against large molecules, pathogenic 
material and microorganisms are essential functions of the gut. The complexity of both cellular and molecular physiology supports these essential functions. The GI epithelial layer is comprised of paneth cells, enterocytes, goblet cells, dendritic cells, apoptotic enterocytes, tight junctions and mucosal layer; in addition microvilli on the cell surface increase surface area of the cell to improve absorption. Within the Mucus contains secreted defensins, bacteria and $\operatorname{IgA}$, all of which perform a vital role in GI tract function (Michielan et al., 2015).

The mucus layer that is secreted by goblet cells acts as a protective barrier against microorganisms, reducing the opportunity for pathogens to cause to enter the blood steam via the epithelial layer and cause infection. The gut generally has a wide range of microbial flora with one study finding 395 different bacterial phylotypes present which act as competition to pathogenic microbes and aid in the digestion of some material (Eckburg et al., 2005). However, if the gut becomes damaged some of the commensal bacteria can become pathogenic and cause illness and disease. It has been demonstrated that natural population variation reduces through antibiotics use and the gut can become prone to infection due to reduced competition for resources (Hooper and Gordon, 2001).

\subsubsection{Properties for gut restitution}

Gut restitution is typically characterised as a healing process involving cell migration, invasion, proliferation and remodelling. This often occurs in response to damage caused by infection, intolerances, and disorders such as Crohn's disease (Waller et al., 1988). Although a lot of research has focussed on therapeutic properties of $C$. sinensis, there has been little research into its potential in treating gastric issues such as gut restitution.

Marchbank et al. (2011) investigated $C$. sinensis reparative properties in rat gastric damage induced by the NSAID indomethacin. In that research, rats were randomly given saline or $C$. sinensis extract by oral administration. All rats were then subcutaneously administered $20 \mathrm{mg} / \mathrm{kg}$ of indomethacin and placed in restraint cages for 3 hours and then killed. Their stomachs were removed, internal $\mathrm{pH}$ determined and then filled with neutral buffer formalin overnight. Dissecting microscopes were used to determine macroscopic internal injury of the stomach, depth of damage was recorded as a scale from $0-4$ where $0=$ no damage, $1=$ one small erosion, $2=$ two small or one large erosion area greater than $0.5 \mathrm{~mm}, 3=$ two or more large erosions and 4=extensive damage where ulceration extends to the muscularis mucosa (Marchbank et al., 2011). 
Marchbank et al. (2011) found that rats administered 20mg protein $/ \mathrm{ml}$ of $C$. sinensis extract 2 had $58 \%$ less damage and $20 \mathrm{mg}$ protein $/ \mathrm{mL}$ of $C$. sinensis extract 3 resulted in $60 \%$ less damage than those given saline alone (Figure 1.3). The results obtained are promising as a significant reduction in gut damage, suggests $C$. sinensis may have gastric-protective properties. However, the authors do not discuss results from $C$. sinensis extract 1 administered group and it is unclear how the extract is acting within the gut to protect from indomethacin damage (Marchbank et al., 2011).

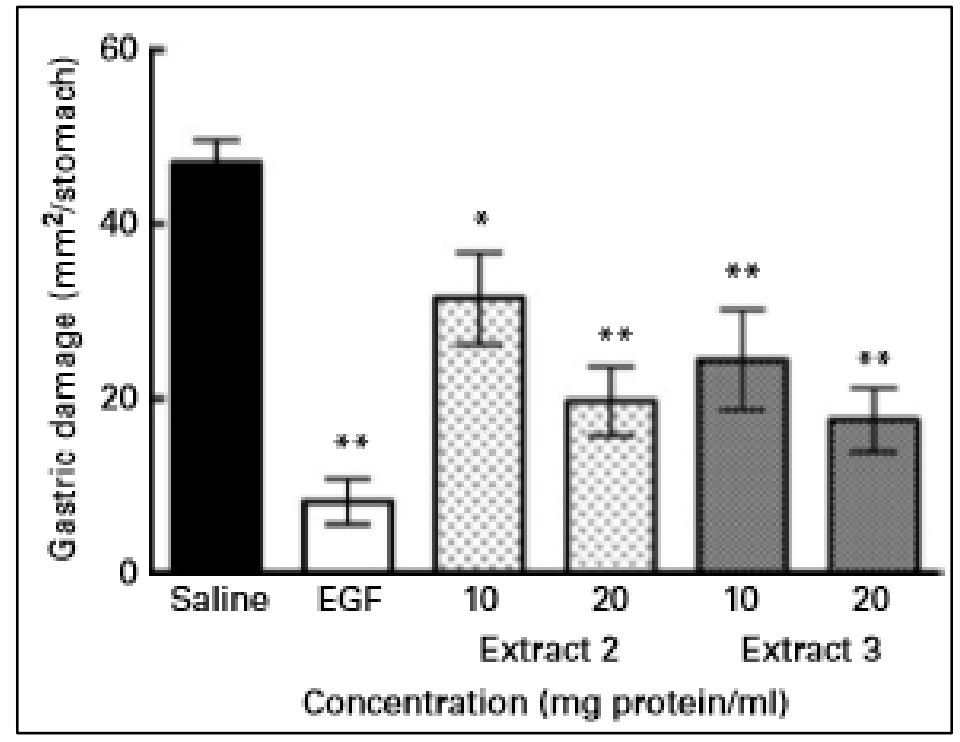

Figure 1.4 Effects of $C$. sinensis on rat gastric indomethacin induced damage. Ground rice and potato grown extract (Extract 2) Liquid medium grown extract (Extract 3), Negative control is saline. Epidermal growth factor $(\mathrm{EGF})$ is the positive control. $(* \mathrm{P}<0.05) \quad\left(\mathrm{P}^{*}<0.01\right)$ (Marchbank et al., 2011).

Marchbank et al. (2011) discovered that hot water extract of both whole and ground rice and potato grown $C$. sinensis had pro-migratory effects in human colonic carcinoma HT29 tissue culture post mechanical damage. Although the liquid grown fungi had a pro-migratory effect in a similar response, it was at a much lower level of efficacy. (Figure 1.4). This may suggest that this liquid culturing method may lack certain qualities required to produce compounds involved in initiation cell migration. Additionally, extracts 1 and 2 administered in excess of $10 \mathrm{mg}$ protein $/ \mathrm{ml}$ resulted in a reduced level of cell migration compared to a $5 \mathrm{mg}$ protein $/ \mathrm{mL}$ dose. It is unclear why the decrease was observed, it is possible that at this concentration the extract has an inhibitory effect, however migration was still increased in comparison to the negative control and extract 3 showed an increase in migration at the same concentration. The 
method of culturing likely impacts the compounds present in the extract, however the mode of action is unclear.

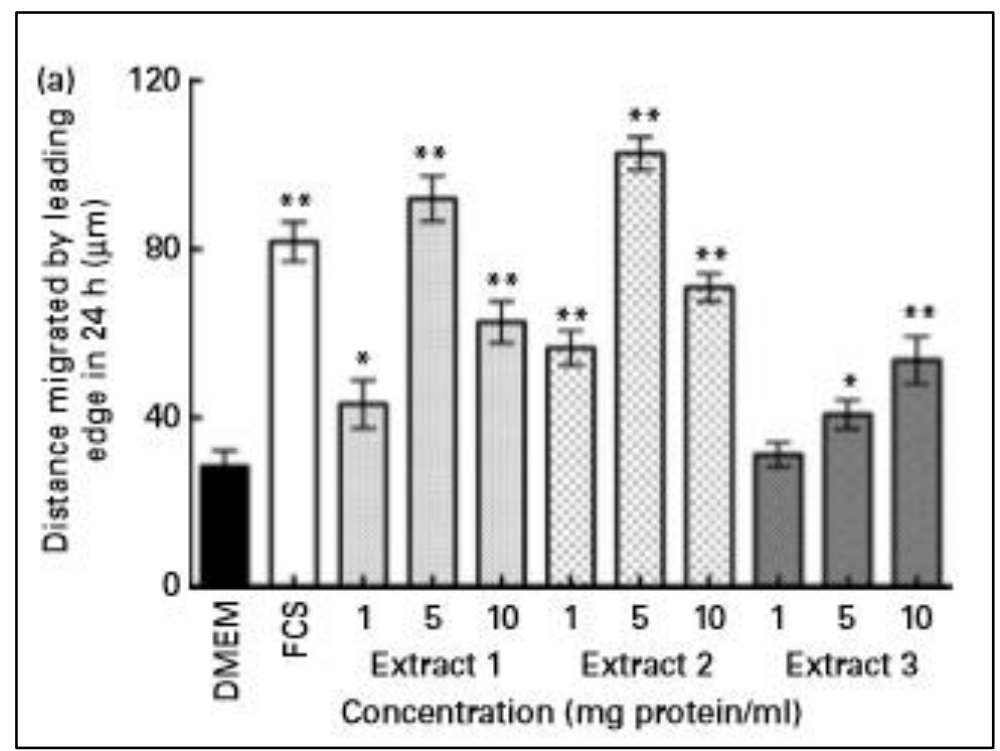

Figure 1.5 Effect of $C$. sinensis extract on cell migration in HT29 cell culture. Whole rice and potato grown extract (Extract 1), Ground rice and potato grown extract (Extract 2) Liquid medium grown extract, (Extract 3) positive control: 10\% Fetal calf serum (FCS), Negative control: Dulbecco's modified Eagle's medium (DMEM). $(* \mathrm{P}<0.05)(* * \mathrm{P}<0.01)($ Marchbank et al., 2011).

\subsubsection{Properties to inhibit apoptosis}

Apoptosis is a natural and necessary process of programmed cell death. It can be separated into two paths, the extrinsic whereby death receptors on the cell surface are stimulated, inducing a signalling cascade of ligands such as TNF, and caspases. The intrinsic pathway, which is initiated by intracellular stimuli such as DNA damage and hypoxia, causes the outer mitochondrial membrane to become permeable. Pro-apoptotic proteins such as Bax are dimerised on the cell surface and trigger a signalling cascade, resulting in the formation of an apoptosome and the stimulation of caspase proteins. Inhibitory proteins are then cleaved, allowing caspases to carry out apoptosis (Mukhopadhyay et al., 2014). If it is possible to inhibit apoptosis with $C$. sinensis it could be used therapeutically to help treat ulcerative colitis where excessive apoptosis in active disrupts the epithelial cells, thereby exacerbating the disease (Hagiwara et al., 2002).

Several studies have explored $C$. sinensis anti-apoptotic properties and found that it reduces the number of apoptotic cells from various causes of injury; this suggests that $C$. sinensis may 
influence signalling pathways, gene regulation and reduction of apoptosis and damage. (Buenz et al., 2005).

Shahed et al. (2001) tested the effect of $C$. sinensis extracts on rat kidney after ischemia leading to reperfusion. The group found that fungal extracts inhibited the expression of apoptotic genes Fas and FasL, furthermore, they found that inflammatory gene expression for TNF- $\alpha$, which is involved in extrinsic cell death, had also reduced. Bax and Bcl2 expression in the intrinsic pathway were not altered, however caspase 3 activity was lowered with the extract suggesting that $C$. sinensis targets the extrinsic pathway sufficiently to reduce apoptosis (Shahed et al., 2001).

Other research groups have investigated $C$. sinensis ability to inhibit apoptosis via the intrinsic pathway. It is considered that $C$. sinensis compounds may act as reactive oxygen species (ROS) scavengers, thus preventing the initiation of apoptosis via oxidative stress. Conversely, there is conflicting evidence from Yamaguchi et al. (2000) who investigated $C$. sinensis extract's antioxidant activity by superoxide dismutase (SOD) assays, hydroxyl radical scavenging, free cholesterol analysis and macrophage culture and oxidation of low-density lipoprotein. SOD assays show that the extracts had some mimetic activity in a concentration dependent inhibition of malondialdehyde production. A lower malondialdehyde indicates a reduction in radical concentration available to form malondialdehyde. In addition, macrophage culture tests showed that $C$. sinensis does possess antioxidant properties and inhibited peroxidation of lipid and cholesterol ester accumulation in cells in a concentration dependent manner (Yamaguchi et al., 2000).

Although the results discussed suggest that $C$. sinensis does indeed inhibit apoptosis, research carried out by Buenz et al. (2004) indicated this is not that case. In their study, Jurkat T lymphocytes were used, and apoptosis was triggered by agonistic anti-Fas antibody or hydrogen peroxide. They found that neither water nor ethanol extracted $C$. sinensis inhibited hydrogen peroxide, or agonistic anti-Fas antibody induced apoptosis. This implies that $C$. sinensis extract does not interfere with the Fas signalling pathway, as the Shahed's group postulated and that it does not inhibit apoptosis initiation via the reduction the ROS present as proposed by the Yamagucghi's group (Buenz et al., 2004, Shahed et al., 2001). More recently, Gu et al., (2015) used rats to investigate preservative effects of $C$. sinensis extracts on the intestinal mucosal barrier in the presence of endotoxin-induced sepsis. This study found that 
rats treated with $C$. sinensis extracts had a significantly lower percentage of apoptotic cells (Gu et al., 2015)

\subsubsection{Colostrum}

Colostrum is the first milk produced after birth and contains all the vital compounds required to support the development of an effective (early) immune system. It is also of high nutritional value and also contains AMPs such as lactoferrin (Khan et al., 2002). Colostrum has many potential therapeutic properties and has been researched as a treatment for cancer due to its lactoferrin contents. It is thought that lactoferrin extracted from colostrum stimulates natural killer cells, modulates carcinogen-metabolising enzymes as well has having iron-binding properties which would reduce the number of free irons which is a mutagenic promoter. All of these have a combined effect of increasing apoptosis and reducing tumour growth (KIM et al., 2009). Colostrum has also been the subject of research into the protective and reparative properties against isoproterenol-induced myocardial infarction in rats due to its contents of growth hormones, which could stimulate repair of damaged cardiac muscle and blood vessels. Colostrum was found to reduce lipid peroxidation and free radicals that otherwise would go on to cause further damage to cardiac tissue (Kaur et al., 2014). These findings make colostrum a suitable candidate for studying protective and reparative properties of the gut against pharmaceutical and microbial damage in G. mellonella.

\subsubsection{Colostrum anti-inflammatory properties}

Colostrum's anti-inflammatory properties have gained interest in recent years with a studies conducted in human cell lines and rats focusing on applications against NSAID and gastrointestinal diseases such as Crohn's disease (Playford et al., 2001, Chae et al., 2017). A study carried out in human Caucasian colon adenocarcinoma cells (Caco-2 and HT-29 cells) found that bovine colostrum reduced the production of interleukin-8 (IL-8), which is a proinflammatory mediator after Escherichia coli and tumour necrosis factor $\alpha$ (TNF- $\alpha$, a cytokine which increases IL-8 expression) stimulation. These data suggest that colostrum may prevent inflammation by inhibiting TNF- $\alpha$ binding to epithelial cells (Chae et al., 2017, Hechtman et al., 1991).

An experiment conducted in rats found that rats which were administered bovine colostrum had lower markers of inflammation (TNF- $\alpha$, IL-1 $\beta$, and IL-6) after intestinal ischemia had been surgically induced compared to the rats administered saline. This result indicates an antiinflammatory property of colostrum, however in the same study the serum anti-inflammatory 
cytokine interleukin 10 (IL-10) was not significantly different to the saline administered rats (Kwon et al., 2010). This supports the idea that colostrum's anti-inflammatory effect is due to it inhibiting TNF- $\alpha$ rather than a stimulation of anti-inflammatory cytokines such as IL-10.

Another study using HT-29 cells found supporting evidence of bovine colostrum to having anti-inflammatory effects via the inhibition of IL-1 $\beta$ induced IL-8 (An et al., 2009). Moreover, the same study found that bovine colostrum inhibited the nuclear factor $\kappa \mathrm{B}(\mathrm{NF}-\kappa \mathrm{B})$ signalling pathway by inhibiting the phosphorylation of I $\mathrm{KB}$ enzyme, which in turn prevents the binding of NF- $\kappa \mathrm{B}$ DNA, and translocation of NF- $\kappa \mathrm{B}$ from its inactive state in the cytoplasm to its active state in the nucleus (An et al., 2009, Karin, 1999). A further mode of action of colostrum's antiinflammatory effect postulated by An et al. (2009) is the inhibition of COX-2 expression by inhibiting IL-1 $\beta$ activity.

\subsubsection{Colostrum antioxidant properties}

Human colostrum is known to have antioxidant properties although it is unclear what components are responsible for this but are thought to include vitamins A, D and E as well as carotenoids and catalase (Hanna et al., 2004). A study conducted in neonatal calves found that bovine colostrum contained the antioxidant $\mathrm{Cu} / \mathrm{Zn}$-Superoxide dismutase ( $\mathrm{Cu} / \mathrm{Zn}-\mathrm{SOD}$ ), and calves that were fed colostrum had higher serum levels of SOD than calves fed tank milk. Additionally, calves reared on colostrum had significantly lower serum concentration of malondialdehyde (MDA) which is correlates to damage caused by free radicals which suggests calves reared on colostrum have a more established anti-oxidative defence than those reared on tank milk and that the higher concentration of SOD may decrease oxidation by reducing MDA production (Yang et al., 2015).

A study inducing toxicity with paracetamol in rats, found that rats administered colostrum alone had higher SOD concentration in brain tissue. Those having received paracetamol and colostrum had lower kidney MDA concentrations and higher catalase (CAT) concentrations than those in receipt of paracetamol only. This indicates that colostrum has anti-oxidative properties which may be due to radical scavenging and gives the recipient improved defence against toxins (Karabacak et al., 2018). In a subsequent study which induced intestinal ischemia in rats found that when rats received colostrum after the ischemic event a reduction in the levels of lipid peroxidation and an increase anti-oxidative activity was observed with greater SOD activity and higher CAT concentration compared to those which received saline (Kwon et al., 2010). 


\subsubsection{Colostrum gastric protective and reparative properties}

Due to colostrum's high content of immunoglobulins (such as $\mathrm{IgG}$ ) and AMPs, it has been researched for its protective properties against gastric bacterial induced septic shock in rats. Rats that were infected with Escherichia coli and orally administered colostrum, had a reduction in endotoxins produced compared to the control (Döhler and Nebermann, 2002). Oral delivery of colostrum has also been found to reduce intestinal permeability induced by NSAID in rat studies, reduce bacterial translocation and reduce the number of intestinal lesions caused by NSAID oral administration in rats (Kim et al., 2005). This indicates that colostrum has protective properties to pharmaceutical induced damage and would be a suitable nutraceutical test on G. mellonella larvae against NSAIDs. To date, there appears to be no evidence of such experiments being replicated in insect larvae.

Research using hyper-immune bovine colostrum (HBC), which contains higher concentration of immunoglobulins, has indicated that it may protect against and treat GI infection of C. difficile and enterotoxigenic E. coli. Freedman et al., (1998) found that an oral dose of HBC in tablet form prevented diarrhoea in 93\% of human subjects orally infected with $106 \mathrm{CFU}$ Enterotoxigenic E. coli. They were however unable to identify the molecular basis for the observed effects (Freedman et al., 1998). Additional research found defatted colostrum protected the intestine against NSAID induced permeability and intestinal erosion in humans and rats (Macdonald et al., 1998, Playford et al., 1999). In Playford et al. (1999), that authors state the presence of recombinant transforming growth factor $\beta$ (TGF- $\beta$ ) in colostrum is involved in the gastro-protective effect against NSAID induced damage

\subsubsection{Colostrum growth factors and hormones}

One of the reasons of interest in colostrum is its bioactive contents such as growth factors and hormones. These include insulin-like growth factor 1 (IGF-1), transforming growth factor (TGF- $\beta 2$ ) and growth hormones (Elfstrand et al., 2002), as well as cortisol, androstenedione, testosterone, estradiol, cortisone, progesterone and estrone (Xu et al., 2011). IGF-1 and TGF$\beta 2$ are important factors for cell division, homeostasis, maturation of the gut and wound repair. Additionally IGF-1 has been associated with increased weight gain in rats, stimulating muscle gain and the burning of fat stores (Uruakpa et al., 2002). This indicates that both growth factors could play a vital role in gastric repair and increased larval growth rate. Although TGF- $\beta 2$ supports growth and repair, 90\% of TGF- $\beta 2$ present in colostrum is inactive and becomes activated through changes in ionic strength, acidification or by enzymes such as cell-associated plasmin (Kojima and Rifkin, 1993, Elfstrand et al., 2002). 


\subsubsection{Colostrum impact on gastric microbiome}

It is understood that colostrum is important in the early development of a healthy gastric microbiome, calves fed colostrum immediately after birth have higher proportions of Bifidobacterium and lower proportion of opportunistic bacteria such as Escherichia and Shigella compared to those which did not receive colostrum (Song et al., 2018). There is evidence to suggest that the early establishment of a healthy gut microbiome is important in the long-term health of an individual, preventing allergies and affecting the metabolites with infants fed on formula milk instead of colostrum having a higher concentration of short-chain fatty acids and lower concentrations of lactate than infants breast fed (van den Elsen et al., 2019). A study on mature dogs found that the administration of bovine colostrum led to an increase in gut microbial diversity and a more stable population (Satyaraj et al., 2013).

\subsection{Summary and Aims}

G. mellonella larvae are useful screening tools for pharmaceutical toxicity and bacterial/fungal pathogenicity. Although they do not possess adaptive immune mechanisms, they have a highly effective and complex innate immunity with broad functional similarities to humans, e.g., production of AMPs, use of PRRs and signalling cascades to stimulate the phagocytosis of foreign bodies Wojda, Kowalski and Jakubowicz, 2009, Mak, Zdybicka-Barabas and Cytryńska, 2010, Nehme et al., 2007). However, a vital aspect of G. mellonella immunity (as well as invertebrates in general) is the melanisation and encapsulation of pathogens using PO, which is not used in this manner in humans or rat models (Butt et al., 2016). Although the use of PO (i.e., tyrosinase) to kill pathogens in this way is not observed in humans, it remains a measurable response to infection when using G. mellonella as a model.

Similarities in larval GI tissue architecture such as the presence of a protective mucous-like layer over the epithelial cells, goblet cells and septate junctions to control permeability (Tanigawa et al., 2016) allow the observation of putative pathological changes caused by bacterial and pharmacological damages (Uwo et al., 2002, Endo and Nishitsutsuji-Uwo, 1981, Lane et al., 1996). Previous work has promoted the use of G. mellonella larvae to study the protective properties of probiotics against enteropathogenic E. coli, through simple experimental endpoints such as larval survival and total immune cell (haemocyte) counts (Scalfaro et al., 2017). That study, and many others discussed here, suggest it to be a suitable 
model to study $C$. jejuni infection and nutraceutical protective properties. Simple cell counts and detoxification assays are useful, but to fully establish the potential of $G$. mellonella as an immunotoxicology model, it is essential to also look at tissue-specific changes in the gut (e.g., histology), understand the gastric structure /function relationship, as well as observations that go beyond survival to look at overall health (development, movement, appearance).

NSAIDs are one of the most commonly prescribed drugs found to cause GI distress, usually in the form of increased permeability, breakdown of the mucosal and epithelial layers, and enhanced levels of apoptosis (García-Rayado et al., 2018). Indomethacin and Aspirin are the basis of substantial research addressing GI-related damages and their potential modes of action (Seo et al., 2012), suggesting they are suitable pharmaceuticals to assess G. mellonella's ability as a pathological model. To greater test the applicability and variety of applications that can incorporate $G$. mellonella as a model or oral toxicity, marine biotoxins (OA and AZA) represent an exciting opportunity to study toxicity and microbial dysbiosis - to be compared to existing, extensive information available from rats. 


\section{Chapter 2: Developing an alternative in vivo (insect) model to replace the indomethacin restraint/ulcer assay in rodents}

A version of this chapter has been published:

Helena Emery, Rich Johnston, Andrew F. Rowley, Christopher J Coates. (2019).

Indomethacin-induced gut damage in a surrogate insect model, Galleria mellonella. Archives of toxicology, 93(8), 2347-2360.

*RJ and AFR assisted with x-ray microtomography/microscopy and histology, respectively. 


\subsection{Abstract}

Indomethacin is a non-steroidal anti-inflammatory drug that causes gastric ulceration and increased 'leakiness' in rat models and is used routinely as a toxicology assay to screen novel compounds for repair and restitution properties. This chapter aims to establish conditions for indomethacin-induced gut damage in wax-moth (Galleria mellonella) larvae with a view to reducing the need for rodents in such experimentation. Indomethacin $(0.5-7.5 \mu \mathrm{g} / \mathrm{larva} ; 2-$ $30 \mathrm{mg} / \mathrm{kg}$ ) was administered to $G$. mellonella via intrahaemocoelic injection and gavage (force-feeding). Larval survival and development, blood cell (haemocyte) numbers, and changes in gut permeability were monitored in the absence and presence of this NSAID. Increased levels of gut leakiness were observed within the first 4-24 hours by tracking fluorescent microspheres in the faeces and haemolymph (blood equivalent). Additionally, varying levels of tissue damage were recorded in histological sections of the insect midgut, including epithelial sloughing and cell necrosis. Degeneration of the midgut was accompanied by significant increases in detoxification-associated activities (superoxide dismutase and glutathione-S-transferase) and episodes of dysbiosis. Herein, the reader will find evidence that G. mellonella larvae force-fed indomethacin display broad signs of gastric damage similar to their rodent counterparts. 


\subsection{Introduction}

When considering more carefully the use of vertebrates in disease and toxicology experimentation, there is constant need to develop alternative model systems in vitro, in vivo or in silico. One such in vivo alternative is the larvae of the greater wax-moth, Galleria mellonella. These insects are now used widespread as 'mini-hosts' for the investigation of microbial pathogenicity (Mowlds et al., 2010, Kloezen et al., 2015, Lim et al., 2018, Cools et al., 2019) screening of xenobiotics/toxins (Allegra et al., 2018, Coates et al., 2019) and functional characterisation of virulence factors (Altincicek et al., 2007, Champion et al., 2016). Larvae of G. mellonella are designated a non-animal technology (Allegra et al., 2018), which means there are fewer ethical restrictions and regulations compared to vertebrates. Additionally, practical advantages include low maintenance costs, thermal tolerance to $37{ }^{\circ} \mathrm{C}$, ease of use (accurate dosages) and high turnover results can be obtained within $72 \mathrm{~h}$ in contrast to vertebrates. Although G. mellonella have been used to study the infectivity of gut pathogens, e.g., Campylobacter jejuni (Senior et al., 2011) Listeria monocytogenes (Mukherjee et al., 2013a), Vibrio spp. (Wagley et al., 2018), Shigella spp. (Barnoy et al., 2017) and Salmonella enterica (Card et al., 2016), there remains a distinct lack of information regarding the alimentary canal of this insect and its role in pathogenesis.

From mouth to rectum, the digestive system of lepidopteran larvae (like G. mellonella) consists of three distinct regions: the foregut (stomatodaeum), midgut (mesenteron) and hindgut (proctodaeum) (Engel and Moran, 2013, Linser and Dinglasan, 2014). The midgut is the primary site of digestion and absorption in many insects, and importantly, it lacks the exoskeletal/chitin lining seen in the fore- and hind-guts. The basic tissue architecture of the midgut is similar to those found in the human intestine, such as epithelial arrangements of columnar cells and smooth septate junctions that control permeability-analogous to tight junctions (Green et al., 1980). The insect peritrophic matrix is the functional equivalent to the mammalian mucus layer, which acts as a barrier for the epithelial cells and impedes pathogen movement into the body cavity (i.e., the haemocoel) (Campbell et al., 2008, Kuraishi et al., 2011). Moreover, some microbial communities characterised in the midgut of $G$. mellonella are similar to those found in crypts of the human intestine (Mukherjee et al., 2013b).

Non-steroidal anti-inflammatory drugs (NSAIDs) are used extensively to alleviate pain by inhibiting the activities of cyclooxygenase isozymes (COX1, COX2), yet known side effects 
manifest in gastrointestinal injury (Playford et al., 1999, Brune and Patrignani, 2015). In particular, indomethacin causes ulceration by inducing apoptosis, reducing gastric blood flow and activating innate immune cells (neutrophils), which all contribute to mucosal secretion, maintenance and defence (Marchbank et al., 2011, Matsui et al., 2011). Gastric complications arising from indomethacin exposure have been developed into a standard rodent restraint/ulcer assay to screen novel compounds and health food supplements for putative therapeutic properties - tissue repair and restitution (Aguwa, 1985, Playford et al., 1999, Mahmood et al., 2007). The adverse effects of indomethacin, notably inflammation, permeability and REDOX imbalance, have been studied thoroughly in humans and rodents (Basivireddy et al., 2003, Bjarnason and Takeuchi, 2009, Sigthorsson et al., 2000, Perron et al., 2013). Therefore, the approach of this chapter was to interrogate the effects of indomethacin on the alimentary canal of G. mellonella. First, two inoculation methods (intrahaemocoelic injection and gavage) were utilised to assess the relative toxicity of indomethacin in insect larvae across the concentration range, $0.5-7.5 \mu \mathrm{g} /$ larva. Second, histopathology screening, X-ray microtomography microscopy (XRM) and enzyme activities were combined to assess the integrity of the midgut tissues in the absence and presence of this NSAID. Indomethacin treatments were established by extrapolating from Marchbank et al. (2011), wherein a dose of $20 \mathrm{mg} / \mathrm{kg}$ was administered to induce gastric damage in rats - the equivalent being $5.0 \mu \mathrm{g} / \mathrm{larva}$.

\subsection{Methods}

\subsubsection{Reagents}

Unless stated otherwise, all chemicals and reagents were sourced from Sigma Aldrich (Dorset UK) in their purest form listed. Green and blue fluorescent (latex) microspheres ranging from 0.5 to $6 \mu \mathrm{m}$ in diameter were purchased from Polysciences, Inc. (Fluoresbite ${ }^{\circledR}$ ). Stock solutions of indomethacin were prepared in 5\% dimethyl sulfoxide (DMSO; v/v) and diluted in filtersterilized $(0.2 \mu \mathrm{m})$ phosphate-buffered saline (PBS) pH 7.4.

\subsubsection{Insects}

Larvae of G. mellonella (final instar) were purchased from Livefoods Direct Ltd (Sheffield UK). Upon arrival, each larva was inspected for signs of damage, infection and melanisation. Healthy larvae weighing between $0.25 \mathrm{~g}$ and $0.35 \mathrm{~g}$ were retained and stored at $15^{\circ} \mathrm{C}$ in the dark for no more than 7 days. 


\subsubsection{Larval survival and pupation studies}

Larvae were assigned randomly to each treatment ( $n=10$ per replicate) and placed in $90 \mathrm{~mm}$ petri dishes lined with Whatman filter paper and wood shavings from the commercial supplier. Indomethacin was administered by intrahaemocoelic injection (INJ) or force feeding (FF; gavage; Figure 2.1) using a sterile 27-gauge hypodermic needle across the concentration range, 0 to $7.5 \mu \mathrm{g} /$ larva. The volume of each inoculum was standardised to $10 \mu \mathrm{L}$. The negative control consisted of PBS pH 7.4 containing 5\% dimethyl sulfoxide (DMSO). Post-inoculation, larvae were incubated at $30{ }^{\circ} \mathrm{C}$ and assessed for mortality (response to prodding) and development (cocoon formation) for up to 10 days.

\subsubsection{Haemocyte counts and viability}

Larvae treated with indomethacin, PBS (containing 5\% DMSO), or untreated (i.e., no inoculation) were assessed for haemocyte numbers within 4- and 72-hours post-inoculation. Insects were chilled on ice for $\sim 3$ min prior to injection of $100 \mu \mathrm{L}$ anti-coagulant $(15 \mathrm{mM}$ $\mathrm{NaCl}, 155 \mathrm{mM}$ trisodium citrate, $30 \mathrm{mM}$ citric acid, $20 \mathrm{mM}$ disodium EDTA, pH 5.5). Larvae containing anti-coagulant were placed back on ice for $2 \mathrm{~min}$ prior to piercing the integument above the head using a 27-gauge hypodermic needle. Haemolymph was collected into prechilled, sterile microcentrifuge tubes. For haemocyte viability, haemolymph was extracted in the absence of anti-coagulant and mixed in a ratio of 1:5 with $0.4 \%$ trypan blue (w/v in PBS) and incubated at room temperature for 1 to $2 \mathrm{~min}$. In all cases, haemolymph samples were applied to an improved Neubauer haemocytometer for cell enumeration using bright-field optics of a compound microscope. Two technical replicates were performed per sample. Haemocytes were considered dead when the cytoplasm stained positively for trypan-blue (Strober, 2015).

\subsubsection{Detoxification-associated assays}

Haemolymph was extracted (as stated above) and pooled from three larvae at 4, 24, 48 and $72 \mathrm{~h}$ after being force-fed indomethacin. Haemolymph was centrifuged at $500 \times g$ for $5 \mathrm{~min}$ at $4{ }^{\circ} \mathrm{C}$ to pellet the haemocytes. Approximately, $2.5 \times 10^{5}$ haemocytes from each treatment/time point were added to the lysis buffer $(50 \mathrm{mM}$ potassium phosphate, $2 \mathrm{mM}$ EDTA, $1 \mathrm{mM}$ DTT and a proteinase inhibitor cocktail (Roche cOmplete ${ }^{\mathrm{TM}}$ Mini kit)) and centrifuged at $14,000 \times g$ for $10 \mathrm{~min}$ at $4{ }^{\circ} \mathrm{C}$. Supernatants were retained and stored at $-80{ }^{\circ} \mathrm{C}$. Midgut tissues from three larvae per treatment/time point were dissected out and washed in PBS. Approximately, $30 \mathrm{mg}$ of tissue was placed in $1 \mathrm{ml}$ lysis buffer and homogenised for $\sim 1 \mathrm{~min}$ (using a borosilicate mini glass homogeniser) prior to centrifugation and storage as described 
above. Protein concentrations of haemolymph and midgut samples were determined using the Biuret method with bovine serum albumin (BSA, 0-20 mg/ml) as a standard (Gornall et al., 1949).

\section{Superoxide dismutase (SOD) activity}

SOD activity (EC 1.15.1.1) was determined using the method described by Dubovskiy et al. (2008). Briefly, $80 \mu \mathrm{L}$ of sample (haemolymph or homogenised midgut) was mixed with reaction solution (500 $\mu \mathrm{L}$ PBS containing $70 \mu \mathrm{M}$ NBT, $125 \mu \mathrm{M}$ xanthine), followed by the addition of $20 \mu \mathrm{L}$ xanthine oxidase solution ( $5 \mathrm{mg}$ BSA, $15 \mu \mathrm{L}$ xanthine oxidase (20 units $/ \mathrm{mL}$ ) per $\mathrm{ml}$ PBS), and incubated for $20 \mathrm{~min}$ at $28{ }^{\circ} \mathrm{C}$. Total assay volume was $600 \mu \mathrm{L}$. Xanthine oxidase catalyses xanthine to produce superoxide anions $\left(\mathrm{O}_{2}^{-}\right)$, which reduce NBT to a formazan dye. The inhibition of NBT reduction is indicative of SOD activity, which is monitored spectrophotometrically at $560 \mathrm{~nm}$. SOD activity is presented as the increase in absorbance $(560 \mathrm{~nm})$ per min per mg protein.

\section{Glutathione S-transferase (GST) activity}

GST activity (EC 2.5.1.18) was determined using the method described by Dubovskiy et al. (2008). Briefly, $20 \mu \mathrm{L}$ of sample was mixed with $500 \mu \mathrm{L}$ GST assay solution (1 mM glutathione, $1 \mathrm{mM}$ 1-chloro-2,4-dinitrobenzene (DNCB) dissolved in PBS) and incubated at $28{ }^{\circ} \mathrm{C}$ for $5 \mathrm{~min}$. The reaction of DNCB and glutathione is catalysed by GST, producing 5-(2,4dinitrophenyl)-glutathione - detectable at $340 \mathrm{~nm}$. GST activity is presented as the increase in absorbance $(340 \mathrm{~nm})$ per min per $\mathrm{mg}$ protein.

\section{Lipid peroxidation (malondialdehyde levels)}

Oxidative damage of lipids leads to the formation of malondialdehyde (MDA), which was monitored using a colorimetric kit (Abcam [Cat. No. ab118970], Cambridge UK). Midgut homogenates $(30 \mu \mathrm{l})$ and/or haemocyte $\left(2.5 \times 10^{5}\right)$ samples were mixed with $300 \mu 1$ MDA lysis solution for 5 - 10 minutes at room temperature. Approximately $200 \mu 1$ of lysed sample was added to $600 \mu \mathrm{l}$ thiobarbituric acid and further incubated at $95^{\circ} \mathrm{C}$ for 1 hour. Lipid peroxidation was recorded as an increase in absorbance $(532 \mathrm{~nm})$ per $\mathrm{mg}$ protein and converted to concentration (nmol) of MDA (molar coefficient $1.56 \times 10^{5} \mathrm{M}^{-1} \mathrm{~cm}^{-1}$ ) using the standard curve recommend by the supplier.

\subsubsection{Gut permeability assessments}

Fluorescently tagged, carboxylate-modified latex microspheres $\left(1 \times 10^{6}\right)$ of $0.5 \mu \mathrm{m}, 1 \mu \mathrm{m}$, $2 \mu \mathrm{m}$ and $6 \mu \mathrm{m}$ in diameter were resuspended in $10 \mu \mathrm{L}$ PBS (control) or $10 \mu \mathrm{L}$ indomethacin 
solution ( 1 or $7.5 \mu \mathrm{g}$ dose) to form co-inoculates (thereby avoiding piercing an insect twice). Larvae were force-fed $10 \mu \mathrm{L}$ of each co-inoculate and incubated at $30^{\circ} \mathrm{C}$ until haemolymph and faeces were collected from each treatment group at 4, 24, 48 and $72 \mathrm{~h}$. Faeces were homogenised in $1 \mathrm{~mL}$ PBS $\mathrm{pH}$ 7.4. The number of microspheres in the haemolymph/faeces was enumerated using a fluorescent microscope (Olympus Bx43f).

\subsubsection{Quantifying bacterial colony forming units in larval faeces}

The faecal loads of control and indomethacin-inoculated larvae $(0-7.5 \mu \mathrm{g} /$ larva $)$ were collected from Petri dishes at 4, 24, 48 and 72 hours post-treatment. Faeces were weighed prior to homogenisation in PBS ( $\mathrm{pH} 7.4$ ) to achieve $0.1 \mathrm{mg}$ of faeces $\mathrm{mL}^{-1}$. Approximately $200 \mu \mathrm{L}$ of each homogenate were spread across pre-poured nutrient agar (Thermo Scientific) and incubated at $30^{\circ} \mathrm{C}$ for up to 48 hours prior to counting.

\subsubsection{Histopathology of the insect alimentary canal}

Larvae force-fed indomethacin (7.5 $\mu$ g per insect) or PBS (negative control) were killed at 4, 24, 48 and $72 \mathrm{~h}$ post-inoculation by intrahaemocoelic injection of $100 \mu \mathrm{L} 10 \%$ buffered formalin, immediately prior to submersion in the same solution for $24 \mathrm{~h}$. Larvae were cut into three sections, head, middle and posterior (anus), and stored in $70 \%$ ethanol prior to wax embedding. Briefly, each sample was dehydrated using an ethanol series, 70\%, 80\% and 90\% for $1 \mathrm{~h}$ each, followed by $3 \times 1 \mathrm{~h} \mathrm{100 \%}$ ethanol washes. Dehydrated samples were washed twice in HistoClear or HistoChoice (Sigma Aldrich) for $1 \mathrm{~h}$ each to remove any remaining residues. Samples were resuspended in 50:50 HistoChoice: paraffin wax for $1 \mathrm{~h}$ prior to complete wax embedding. Embedded samples were cut into $\sim 6 \mu \mathrm{m}$ sections using a microtome, adhered to glass slides with egg albumin solution $(\sim 1 \% \mathrm{w} / \mathrm{v})$ and dried for $24 \mathrm{~h}$ before staining. Loaded slides were stained using Cole's haematoxylin and eosin.

Slides were dewaxed using Histochoice/Histoclear for 15 mins prior to washing in absolute ethanol for $2 \mathrm{~min}$, followed by $1 \mathrm{~min}$ in $90 \%$ ethanol and $1 \mathrm{~min}$ in $70 \%$ ethanol before staining in Cole's haematoxylin for $13 \mathrm{~min}$. Once stained in haematoxylin, slides were then washed in Scotts' solutions for 2 min to stain nuclear material blue. Samples were washed in water for 10 seconds to remove any excess stain. At this point, several slides were selected and viewed under a microscope to check the staining before proceeding with Eosin. Samples were dehydrated in $70 \%$ ethanol for $1 \mathrm{~min}$, followed by Eosin for $6 \mathrm{~min}$. Once stained in Eosin, samples were dipped in $70 \%$ ethanol for a few seconds to remove excess stain. Slides were viewed under a microscope at this point to check the stain was sufficient. Slides were further 
dehydrated in $90 \%$ ethanol for 10 seconds and then in absolute ethanol for 5 min twice. Finally, slides were washed three times in fresh Histochoice/Histoclear each for $5 \mathrm{~min}$ three times prior to coverslip mounting using DPX. Mounted slides were transferred to a heating rack for $10 \mathrm{~min}$ before being placed in an incubator at $40{ }^{\circ} \mathrm{C}$ until dry, and the DPX had set.

\subsubsection{X-ray microtomography/microscopy of Galleria mellonella}

Larvae force-fed PBS were killed at $72 \mathrm{~h}$ and fixed with $10 \%$ formalin for $30 \mathrm{~h}$, followed by dehydration in $100 \%$ ethanol for $24 \mathrm{~h}$. Samples were submerged in Lugol's iodine (PRO.LAB Diagnostics) for 2 weeks and washed with $70 \%$ ethanol prior to microscopy. Insect samples were analysed via X-ray microscopy (XRM) using a lab-based Zeiss Xradia 520 (Carl Zeiss XRM, Pleasanton, CA, USA) X-ray microscope attached to a CCD detector system with scintillator-coupled visible light optics, and tungsten transmission target. The specimen was placed in a plastic screw-top microcentrifuge tube and submerged in $75 \%$ ethanol to prevent the tissues from drying out. To achieve a higher resolution over the entire organism, the insect was imaged along its $\sim 25 \mathrm{~mm}$ length at high resolution, using an overlap-scan and stitching procedure including five individual scans, with $44 \%$ overlap between each scan. An X-ray tube voltage of $80 \mathrm{kV}$ and a tube current of $87 \mu \mathrm{A}$ were used with an exposure of $1000 \mathrm{~ms}$ and a total of 3201 projections. An objective lens giving an optical magnification of 0.4 was selected with binning set to 2 , producing an isotropic voxel (3-D pixel) size of $8.5635 \mu \mathrm{m}$. The individual tomograms were reconstructed from 2-D projections and stitched into a single large volume using a Zeiss commercial software package (XMReconstructor, Carl Zeiss), utilising a cone-beam reconstruction algorithm based on filtered back-projection. XMReconstructor was also used to produce 2-D grey scale slices for subsequent analysis. Drishti and Drishti Paint software V2.6.4. (Limaye, 2012) was employed to highlight regions of interest and digitally segment the alimentary canal.

\subsubsection{Data handling}

All data were gathered from experiments carried out on at least three independent occasions and are represented by mean values \pm standard error (see individual figure legends for sample sizes). D'Agostino and Pearson normality tests were performed, and if necessary, data were square-root transformed. Results from survival studies were analysed using the log-rank (Mantel-Cox) test for comparing curves, whereas larval pupation levels, total haemocyte counts, cell deaths, and faecal/haemolymph microsphere loads were analysed using 2- or 3way ANOVA with Tukey's multiple comparison tests in GraphPad Prism v7. A Pearson's Correlation test was employed to assess the relationship between microsphere size $(0.5-6 \mu \mathrm{m})$ 
and their presence in the haemolymph of control larvae. Enzyme assays, GST and SOD, were analysed in ' $R$ ' studio using General Liner Hypotheses (ghlt). In all cases, differences were considered significant when $P \leq 0.05$. Histology slides were single-blind assessed using paired treatment $(n=13)$ and control $(n=13)$ samples from 4 to $72 \mathrm{~h}$. A grading system (1-4) was used to categorise the extent of tissue damage(s) or lack thereof by modifying the guidelines developed by Watanabe et al. (2017):

1) discrete change with no more than $0-2$ tissue aberrations per slide

2) discrete changes of 3 to 5 tissue aberrations per slide

3) spatial change representing $\geq 25 \%$ damage (the alteration is dramatic)

4) global change of $>50 \%$ of a specific tissue type or the entire slide.

Drishti and Drishti Paint V2.6.4. (Limaye, 2012) and ImageJ (Abràmoff et al., 2004) software were used to process/present microscopy findings. Images were adjusted for colour balance and contrast/brightness only.

\subsection{Results}

\subsubsection{Evaluating the relative toxicity of indomethacin on Galleria mellonella}

Survival

Intrahaemocoelic injection of indomethacin had no measurable negative impacts on larval survival across the concentration range $0.5-7.5 \mu \mathrm{g} / \mathrm{larva}$ ( $0 \%$ mortality (Figure $2.1 \mathrm{a}$ ), which is the equivalent to $2-30 \mathrm{mg} / \mathrm{kg}$ in rodents. Oral administration of indomethacin (force-feeding) in excess of $2.5 \mu \mathrm{g} /$ larva led to a $7-10 \%$ decline in survival within $72 \mathrm{~h}$ (Figure $2.1 \mathrm{~b}$ ) but was not found to be statistically significant overall (log-rank (Mantel-Cox) test; $\left.X^{2}(6)=11.71, P=0.0688\right)$. Further inspection of the survival curves revealed no statistical differences between the PBS/DMSO controls and either $2.5 \mu \mathrm{g} / \mathrm{larva}$ $\left(X^{2}(1)=2.034, P=0.1538\right)$ or $5-7.5 \mu \mathrm{g} / \mathrm{larva}\left(X^{2}(1)=3.105, P=0.0780\right)$.

\section{Development}

Approximately $87 \%$ of untreated larvae transitioned into pupae after 6 days incubation at $30{ }^{\circ} \mathrm{C}$ (experimental temperature) - increasing to $97 \%$ by day 10 (Figure 2.2). No more than $40 \%$ of $G$. mellonella force-fed indomethacin (0.5-7.5 $\mu \mathrm{g} / \mathrm{larva})$ or PBS (+5\% DMSO, absent indomethacin) were observed pupating at day 6. By day 10 , numbers of pupae increased to 80 
$90 \%$ for injected larvae (Figure 2.2a) and $\leq 63 \%$ for force-fed larvae (Figure $2 b$ ). Using a threeway ANOVA, we determined time (Days 6 and 10) and inoculation method (FF and INJ) to account for $38 \%(F(1,24)=50.02, P<0.0001)$ and $41 \%(F(1,24)=54.19, P<0.0001)$ of the variation, respectively. Less than $0.5 \%$ of the variation within the data can be attributed to treatment: PBS $+5 \%$ DMSO (negative control), $1 \mu \mathrm{g}$ (low dose) and $7.5 \mu \mathrm{g}$ (high dose) indomethacin per larva $(F(2,24)=0.0833, P=0.92)$. Tukey's multiple comparison (post hoc) tests revealed statistical differences between the untreated insects and those force-fed indomethacin or PBS only (Figure 2.2b), with no differences detected between mean values of injected insects at any time-point. These data suggest the route of administration alone can delay the onset of pupation, and it is unlikely due to indomethacin exposure.

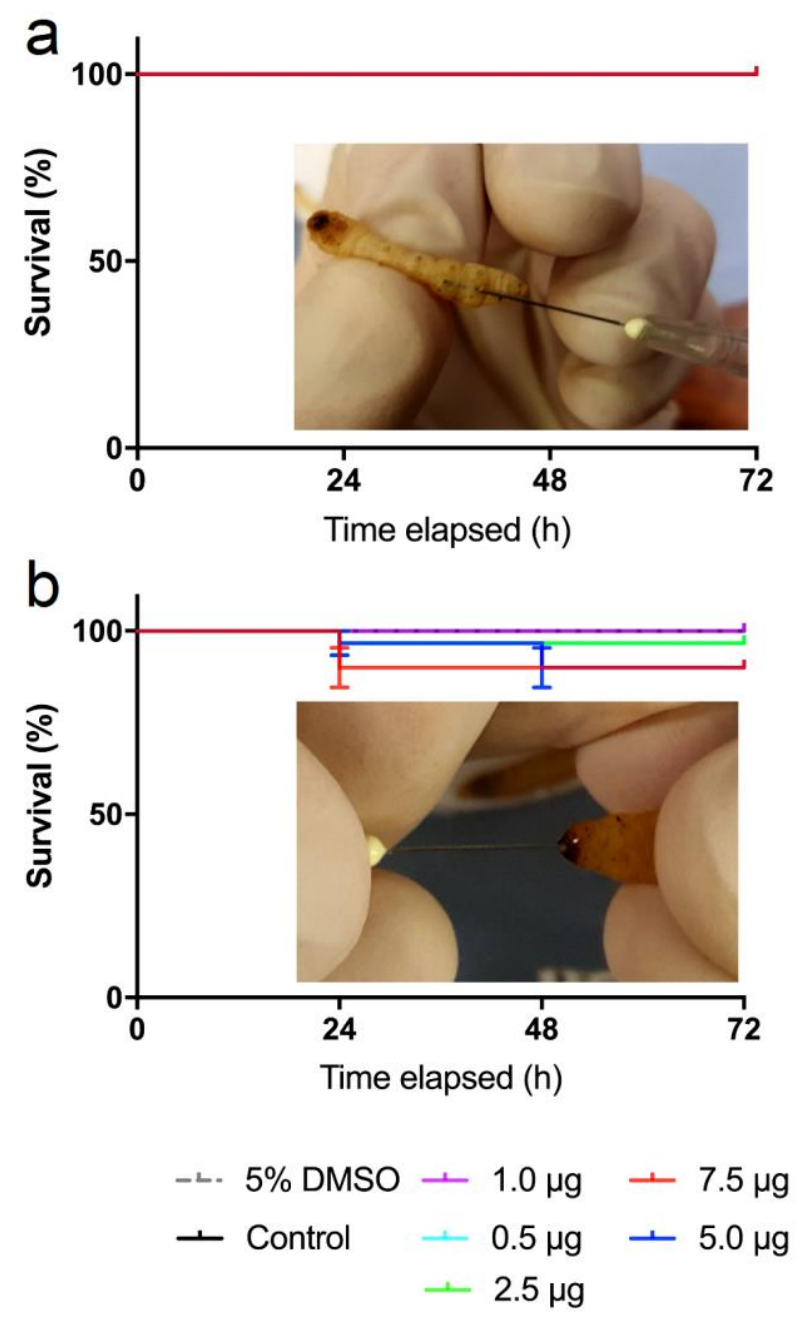

Figure 2.1 Survival of Galleria mellonella larvae following intrahaemocoelic injection (a) or force-feeding (b) of indomethacin, 0.5-7.5 $\mu \mathrm{g} /$ larva.Post-inoculation, larvae were maintained in darkness at $30{ }^{\circ} \mathrm{C}$ for $72 \mathrm{~h}$. Larvae that were unresponsive to being rolled over or prodded were considered dead. Values are expressed as mean $\pm \operatorname{SE}(n=30$ per treatment, 420 in total). Inset: images demonstrate the inoculation method for each $G$. mellonella larva. It should be noted that direct injection of indomethacin or PBS with 5\% DMSO (control) did not lead to any insect mortality 

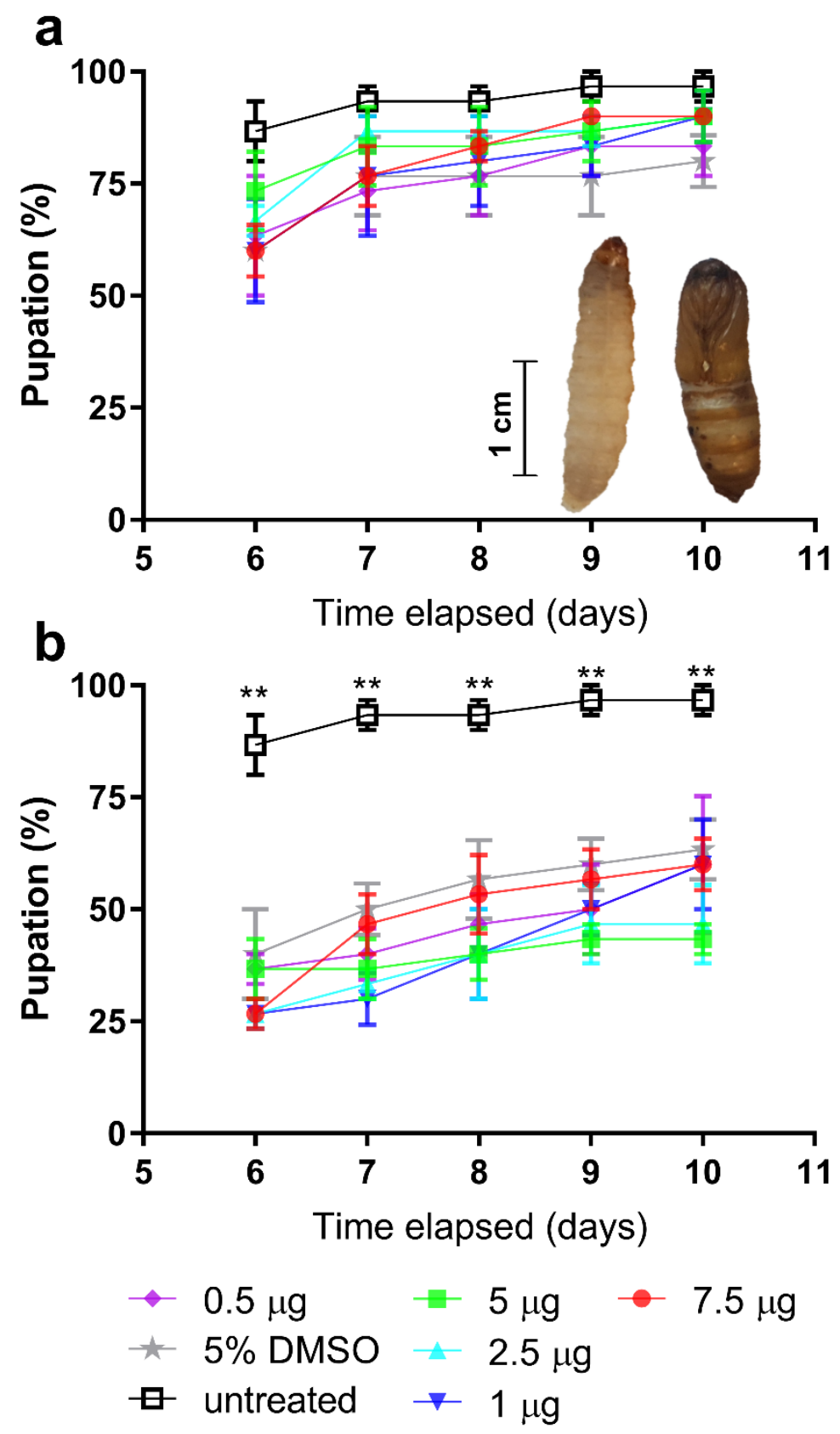

Figure 2.2 Development (pupation) of Galleria mellonella larvae following inoculation with indomethacin, 0.5-7.5 $\mu \mathrm{g} / \mathrm{larva}$. Larvae received indomethacin via intrahaemocoelic injection (a) or force feeding (b) and were maintained subsequently in darkness at $30{ }^{\circ} \mathrm{C}$ for 10 days. The number of larvae undergoing pupation was recorded. Inset (a): typical appearances of a healthy larva (left image) and a moth pupa (right image). Values are expressed as mean $\pm \mathrm{SE}$ ( $n=30$ per treatment, 390 in total across both inoculation methods). Symbol: $* * P<0.01$ when comparing untreated to all other treatments at the respective time points. The negative control consisted of force feeding or injecting the insect with PBS containing 5\% DMSO 


\section{Circulating blood cells (haemocytes)}

To investigate further any potential non-target effects of indomethacin, immune cell (haemocyte) numbers were monitored as well as levels of cell death within the insect haemolymph (i.e., blood). Total haemocyte counts varied little in untreated and PBS $(+5 \%$ DMSO) control insects over the 72-h experimental period, $2.5-3 \times 10^{6} / \mathrm{mL}$ and $2.7-$ $3.7 \times 10^{6} / \mathrm{mL}$, respectively (Figure 2.3). At $24 \mathrm{~h}$ post-inoculation, all concentrations of indomethacin injected into the haemolymph led to substantial increases in haemocyte numbers (up to $5 \times 10^{6} / \mathrm{mL}$ ) compared to the untreated/control $\left(<2.8 \times 10^{6} / \mathrm{mL}\right.$; Figure 2.3a). Forcefeeding indomethacin led to increased circulating haemocyte numbers at the highest dose of $7.5 \mu \mathrm{g} /$ larva only $\left(4.4 \times 10^{6} / \mathrm{mL}\right.$; Figure $\left.2.3 \mathrm{~b}\right)$. In all cases, haemocyte numbers returned to control levels by $48 \mathrm{~h}$. A 2-way ANOVA revealed treatment to account for 15-19\% of the variation within the data $(\mathrm{INJ}-F(4, \quad 40)=4.488, P=0.0043 ; \quad \mathrm{FF}-F(4$, $40)=2.988, P=0.030)$. Time accounted for $<5 \%$ of the variation in force-fed insects (Time, $F(3, \quad 40)=1.033, P=0.3884) \quad$ but $\sim 30 \%$ for injected insects (Time, $F(3$, $40)=11.84, P<0.0001)$. These data likely reflect the unimpeded exposure of haemocytes to indomethacin when it is administered directly into the haemocoel (body cavity), whereas the gut presents a natural barrier when administered via force-feeding.

Concerning cytotoxicity, proportions of haemocytes staining positively for trypan-blue (i.e., dead or dying) ranged from 7 to $12 \%$ for the duration of the experiment-regardless of the inoculation method used or a dose of indomethacin (Figure 2.3; Table 2.1). 


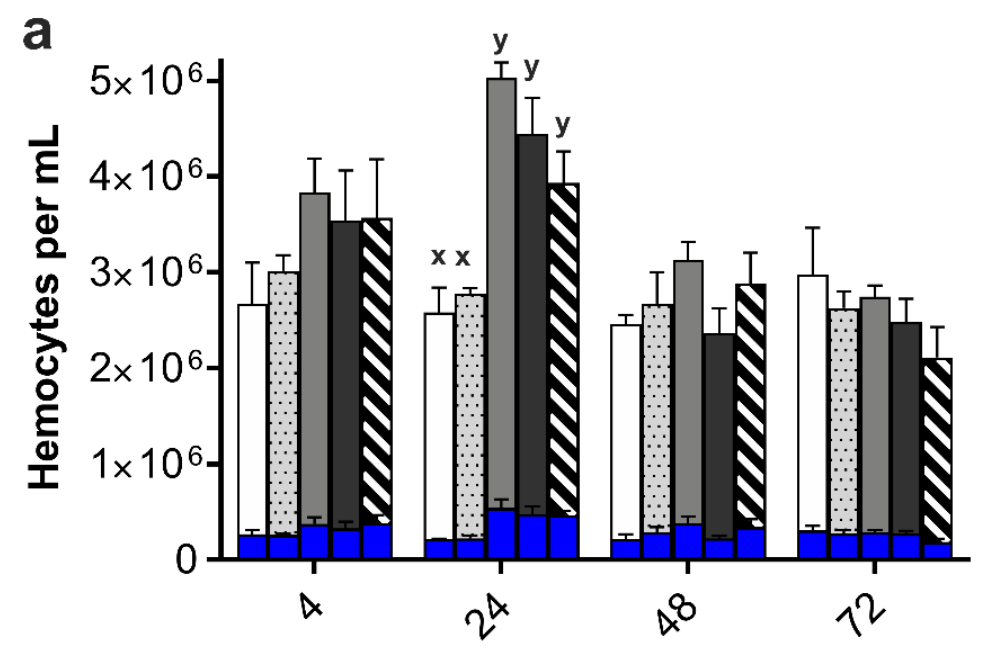

Time (h)

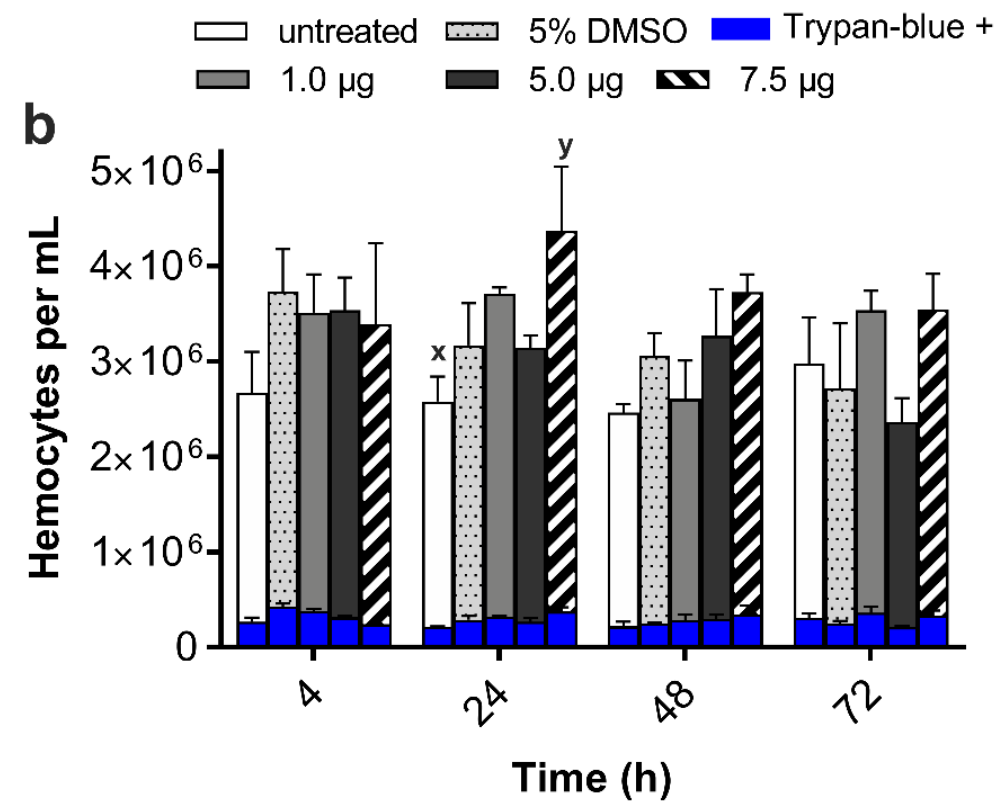

Figure 2.3 Haemocyte responses of Galleria mellonella larvae following inoculation of indomethacin, 1-7.5 $\mu \mathrm{g} / \mathrm{larva}$. Larvae received indomethacin via intrahaemocoelic injection (a) or force feeding (b) and were maintained subsequently in darkness at $30^{\circ} \mathrm{C}$ for 3 days. Total haemocyte counts were performed, and haemocyte viability was determined using the trypan blue exclusion assay (dead cell numbers are represented by the blue bars in $\mathbf{a}$ and $\mathbf{b}$ ). Values are presented as the mean \pm SE $(n=36$ per treatment, 324 in total across both inoculation methods). Unshared letters $(\mathrm{x}, \mathrm{y})$ represent significant differences $(P \leq 0.05)$ determined by Tukey's multiple comparison tests. The negative control consisted of force feeding or injecting the insect with PBS containing 5\% DMSO. 
Table 2.1 Proportions of haemocytes staining positively for Trypan-blue (i.e., cell death) when treated with indomethacin $(1-7.5 \mu \mathrm{g} /$ larva) or PBS.

\begin{tabular}{lllll}
\hline & 4 hours & 24 hours & 48 hours & 72 hours \\
\hline untreated & $9.9 \%$ & $8.3 \%$ & $9 \%$ & $10.2 \%$
\end{tabular}

Injection

\begin{tabular}{lllll}
\hline PBS + 5\% DMSO & $8.5 \%$ & $8 \%$ & $10.6 \%$ & $10.3 \%$ \\
$1 \mu \mathrm{g} /$ larva & $9.8 \%$ & $10.7 \%$ & $12.1 \%$ & $10.5 \%$ \\
$5 \mu \mathrm{g} /$ larva & $9.2 \%$ & $10.7 \%$ & $9.4 \%$ & $11 \%$ \\
$7.5 \mu \mathrm{g} /$ larva & $10.8 \%$ & $11.9 \%$ & $11.9 \%$ & $8.8 \%$
\end{tabular}

Force feeding

\begin{tabular}{lllll}
\hline PBS + 5\% DMSO & $11.3 \%$ & $9 \%$ & $8.1 \%$ & $9.4 \%$ \\
$1 \mu \mathrm{g} /$ larva & $10.8 \%$ & $7.2 \%$ & $10.8 \%$ & $10.3 \%$ \\
$5 \mu \mathrm{g} /$ larva & $8.9 \%$ & $8.5 \%$ & $9.2 \%$ & $9 \%$ \\
$7.5 \mu \mathrm{g} /$ larva & $7.1 \%$ & $8.6 \%$ & $9.3 \%$ & $9.2 \%$ \\
\hline
\end{tabular}

\subsubsection{Characterising the effects of indomethacin on the midgut of Galleria mellonella}

Alimentary canal mapping

A simple 3-step process of fixing whole insects for $<30$ hours in $10 \%$ unbuffered formalin, dehydration in $100 \%$ ethanol for 24 hours and staining in 100\% Lugol's iodine for two weeks, was developed for G. mellonella (see Appendix A). The accumulation of iodine within the various tissue structures enabled whole organ identification and segmentation (Figure 2.4). Using X-ray microtomography, the alimentary canal and integumentary musculature of $G$. mellonella larvae $(n=3)$ were mapped over their entire $\sim 25 \mathrm{~mm}$ lengths with a resolution of $8.6 \mu \mathrm{m}$ (Figure $2.5 \mathrm{a}-2.5 \mathrm{c}$ ). Like most insects, the alimentary canal can be sub-categorised into three regions: foregut, midgut and hindgut (Figure 2.5b). The midgut is distinguishable from the fore- and hind-guts due to the absence of a cuticle lining, and these measurements indicate that the midgut tissues make-up $>50 \%$ of the alimentary canal $(n=3)$ in this species. The midgut is arranged into pleats or folds of columnar epithelial cells and goblet cells that produce and maintain the peritrophic matrix (functional equivalent to the human mucus layer). 
Visceral (striated) muscles surround the gut tissues (anchoring the cellular arrangements in place) and represent the final layer between the gut contents and the haemolymph.

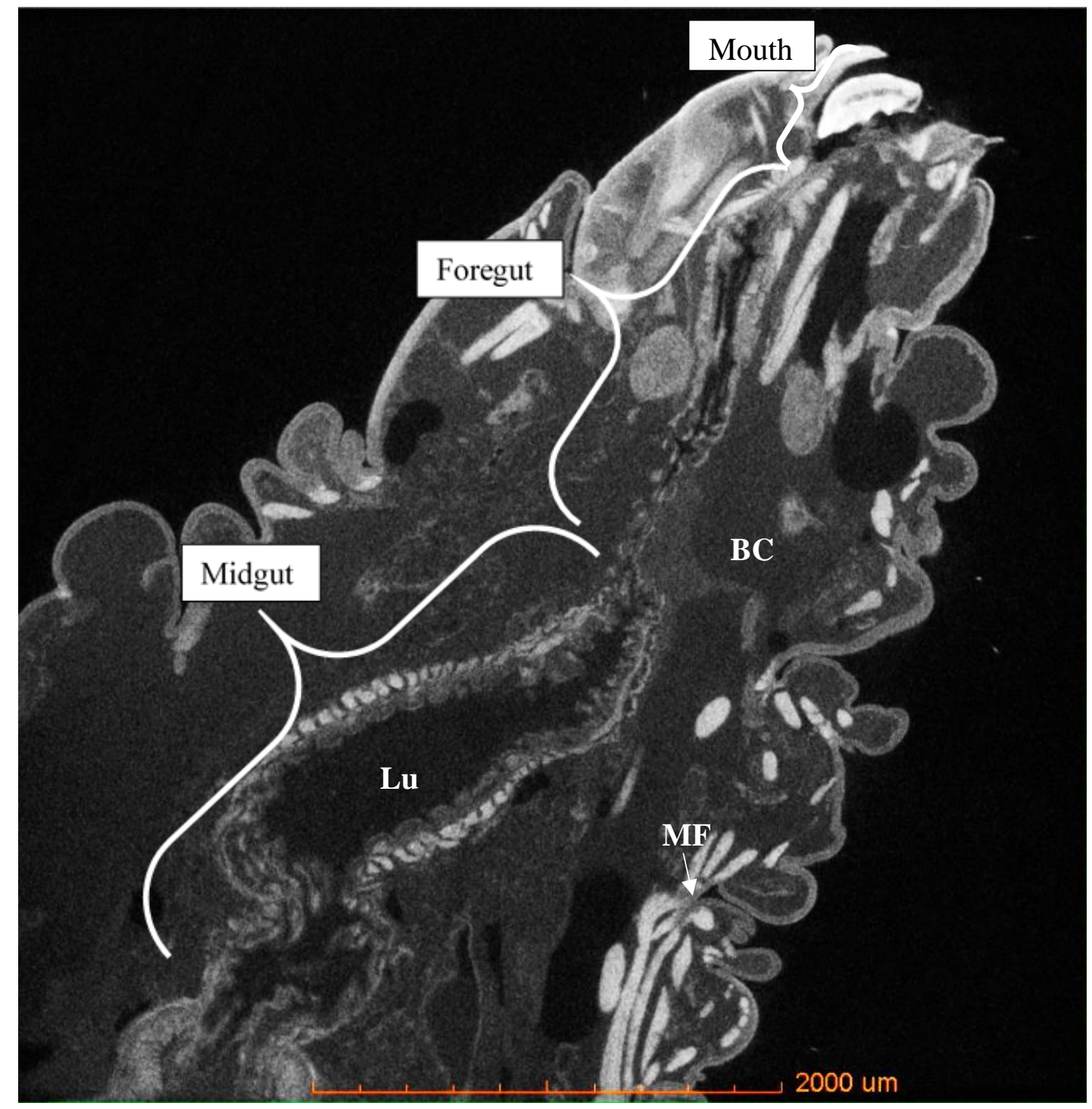

Figure 2.4 Cross-sectional X-ray microtomography of the upper segment of a Galleria mellonella larva. The image is an example output of the fix/dehydrate/stain protocol developed here. The musculature of the insect foregut is visible as is the gut lumen (Lu), body cavity (BC) and muscle fibres (MF). 


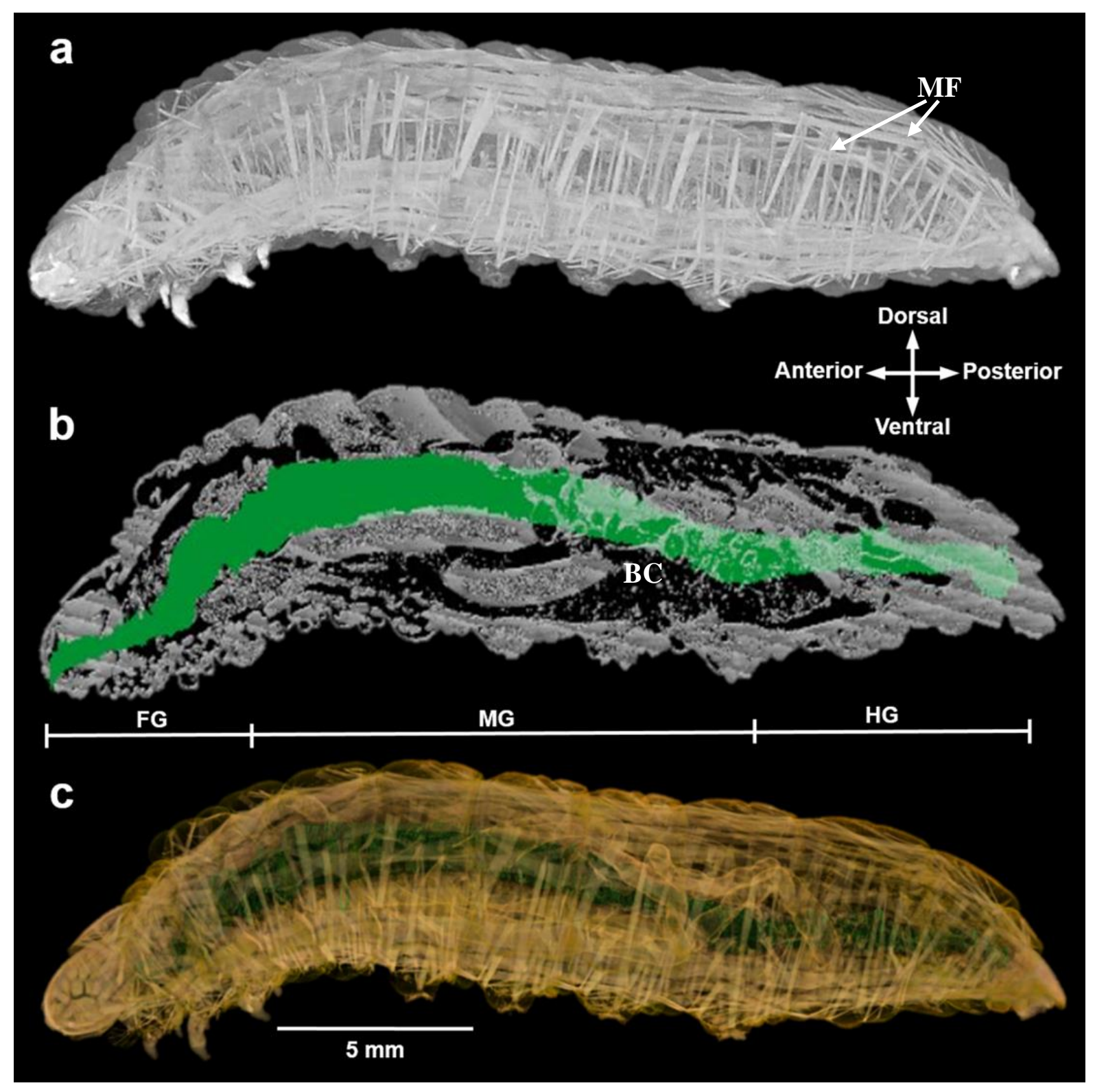

Figure 2.5 X-ray microtomography of Galleria mellonella. a 3-dimensional render of a representative insect larva stained with Lugol's iodine. Striated muscle fibres are distributed throughout the insect - muscles are attached to the integument and run through the cuticle layers. b The entire alimentary canal of the insect larva was highlighted by manually inspecting and colouring 1014 slices using Drishti software (Limaye, 2012). FG, foregut; MG, midgut; HD, hindgut. c Combined 3-dimensional render with the integument architecture coloured yellow and the alimentary canal coloured green. The body cavity (BC) and muscle fibres (MF) are visible as well as the highlighted alimentary canal. 


\section{Gut permeability}

Indomethacin and NSAIDs broadly increase gastrointestinal 'leakiness' in humans and rodents, with tissue damage located in the small intestine and colon (Smecuol et al., 2001). In order to test whether indomethacin caused similar pathological symptoms in the insect alimentary canal, G. mellonella were force-fed inocula containing indomethacin $(0,1$ or $7.5 \mu \mathrm{g} / \mathrm{larva})$ and a selection of microspheres, $0.5-6 \mu \mathrm{m}$ in diameter (Figure 2.6). In the absence of indomethacin (PBS [+5\%DMSO] only), the majority of $6 \mu \mathrm{m}$ and $2 \mu \mathrm{m}$ spheres (36-58\%) made their way down the alimentary canal and were defecated within $24 \mathrm{~h}$ (Figure 2.6a and 2.6b), whereas $1 \mu \mathrm{m}$ spheres (33\%) were released later at $48 \mathrm{~h}$ (Figure 2.6c). The presence of indomethacin (1 or $7.5 \mu \mathrm{g} / \mathrm{larva})$ led to substantial increases in the number of microspheres $(0.5-6 \mu \mathrm{m})$ detected in the haemolymph (blood) and concomitantly fewer were recovered from faeces. At 4 and $24 \mathrm{~h}$ post-indomethacin treatment, $2.7-3.1 \times 10^{4}(6 \mu \mathrm{m})$ spheres leaked from the gut into the haemolymph in comparison to the control, 1.0-1.4 $\times 10^{4}$ (Figure 2.6a). Notably, the presence of microspheres in the haemolymph was recognised by phagocytic haemocytes and subsequently internalised (Figure 2.7). Independent of microsphere size, indomethacin exacerbated gut leakiness by 1.4 - to 3-fold: $6 \mu \mathrm{m}(F(2,24)=5.801, P=0.0042), 2 \mu \mathrm{m}(F(2$, $24)=11.04, P<0.0001), \quad 1 \mu \mathrm{m} \quad(F(2, \quad 24)=21.48, P<0.0001), \quad$ and $\quad 0.5 \mu \mathrm{m} \quad(F(2$, $24)=22.75, P<0.0001)$ and accounted for $9-30 \%$ of the variation in haemolymph loads. In contrast, time accounted for the majority of variation, $25-51 \%$, in faecal microsphere loads: $6 \mu \mathrm{m} \quad(F(3,24)=17.38, P<0.0001), 2 \mu \mathrm{m} \quad(F(3,24)=3.57, P=0.0288), \quad 1 \mu \mathrm{m} \quad(F(3$, $24)=3.94, P=0.0204)$, and $0.5 \mu \mathrm{m}(F(3,24)=8.378, P=0.0006)$.

Overall, significantly fewer $0.5 \mu \mathrm{m}$ spheres made their way into the faeces within $24 \mathrm{~h}$ and beyond (Figure 2.6d). This is likely due to the small size enabling movement across the gut barrier, and those that remain within the midgut may get caught-up in tissue folds (Figure 2.8), thereby delaying passage through the hindgut/rectum. By analysing the control data (absent indomethacin) across the 72-h period (Figure 2.9), a strong inverse correlation was found between microsphere size and haemolymph loads (Pearson Correlation; $r=-0.988, P=0.0115, R^{2}=0.9771$ ) in support of this theory. Moreover, in the absence of indomethacin $\sim 715,000$ microspheres were found in the faeces over the 72 hours and a further $\sim 154,000$ from the haemolymph - this indicates a recovery rate of $87 \%$. 


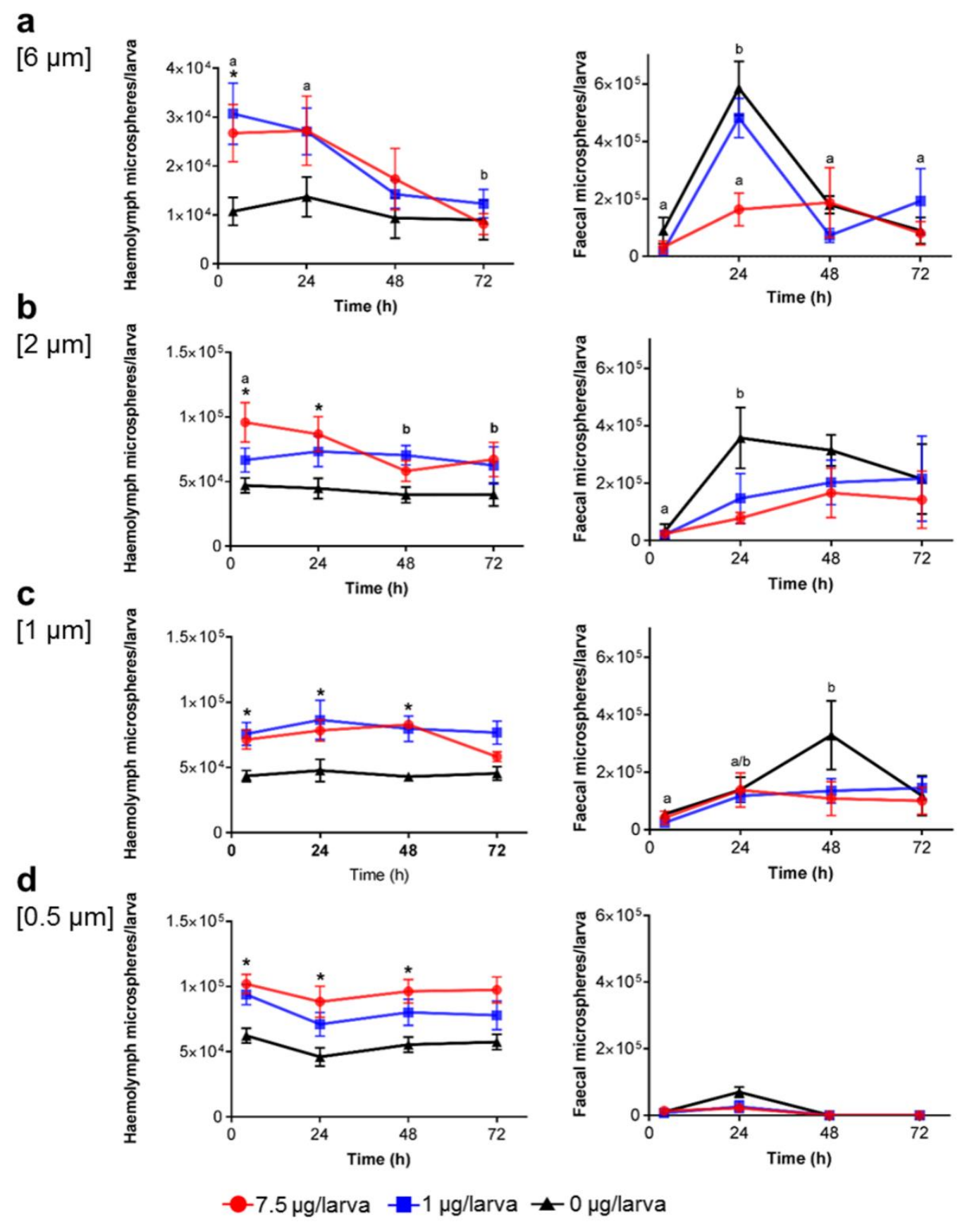

Figure 2.6 Gut permeability of Galleria mellonella larvae following force-feeding of indomethacin, $0-7.5 \mu \mathrm{g} / \mathrm{larva}$. Permeability (or leakiness) was determined by the number of $6 \mu \mathrm{m}(\mathbf{a}), 2 \mu \mathrm{m}(\mathbf{b}), 1 \mu \mathrm{m}$ (c) and $0.5 \mu \mathrm{m}$ (d) spheres found in the haemolymph and faeces between 4 and $72 \mathrm{~h}$ post-inoculation. Each larva was co-inoculated with $1 \times 10^{6}$ microspheres and indomethacin $(1,7.5 \mu \mathrm{g} / \mathrm{larva})$ or PBS and incubated in the dark at $30^{\circ} \mathrm{C}$. Data represent the mean $\pm \operatorname{SE}(n=36,432$ in total across all four microsphere sizes). Symbol: $* P<0.05$ when comparing PBS to indomethacin treatments at each respective time point. Unshared letters (a, b) represent significant differences $(P<0.05)$ determined by Tukey's multiple comparison tests. The negative control consisted of force-feeding the insect with PBS (+5\% DMSO) in the absence of indomethacin $(0 \mu \mathrm{g} / \mathrm{larva})$. 

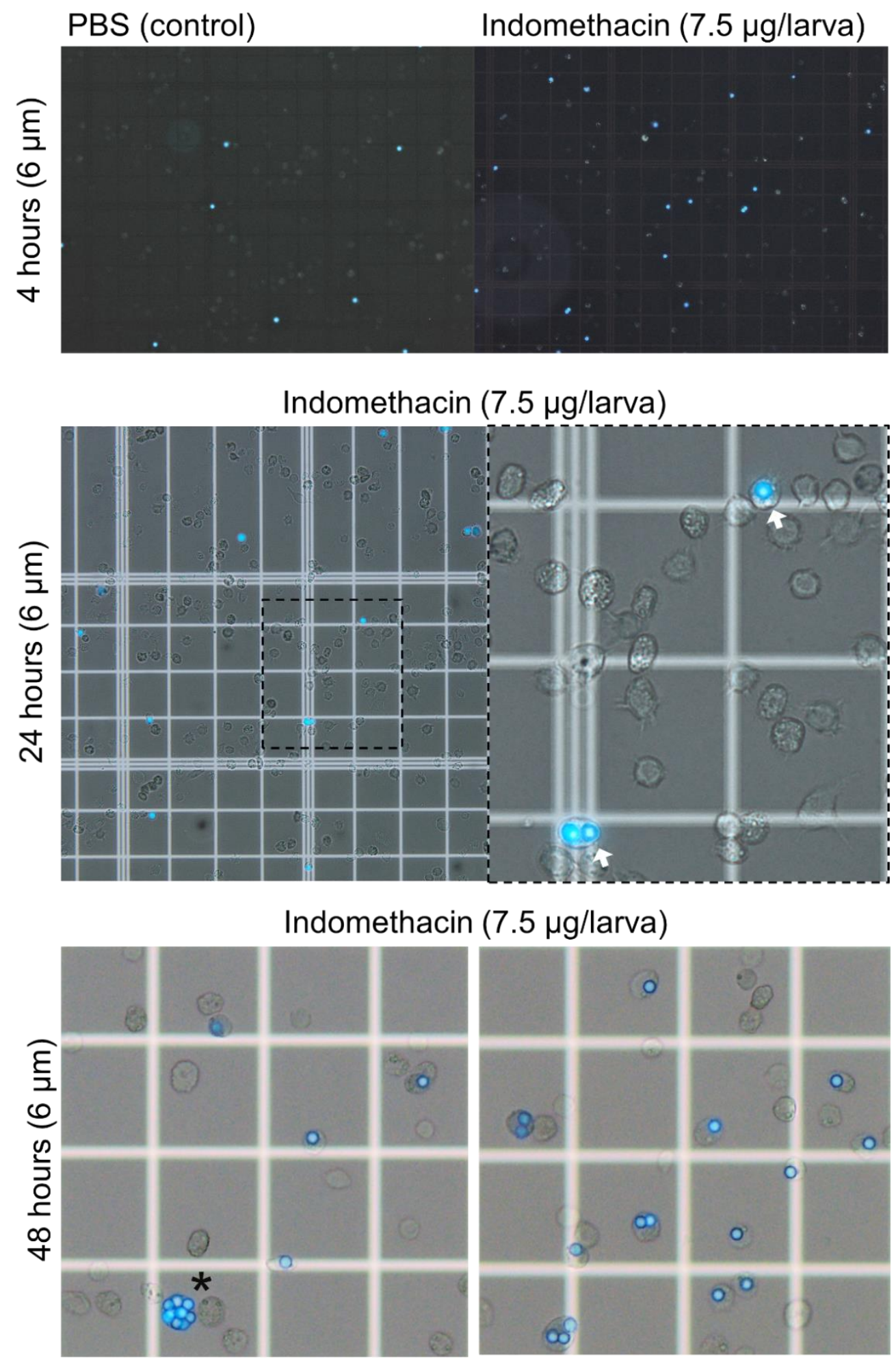

Figure 2.7 Latex microspheres $(6 \mu \mathrm{m})$ in the haemolymph of Galleria mellonella co-inoculated with indomethacin $(7.5 \mu \mathrm{g} / \mathrm{larva})$. The top, middle and bottom panels represent images taken from 4, 24 and 48-hours post-inoculation, respectively. The white arrows point to spheres within the cytoplasm of phagocytic haemocytes (immune cells). The asterisk (*) signifies a haemocyte with eight internalised microspheres. 


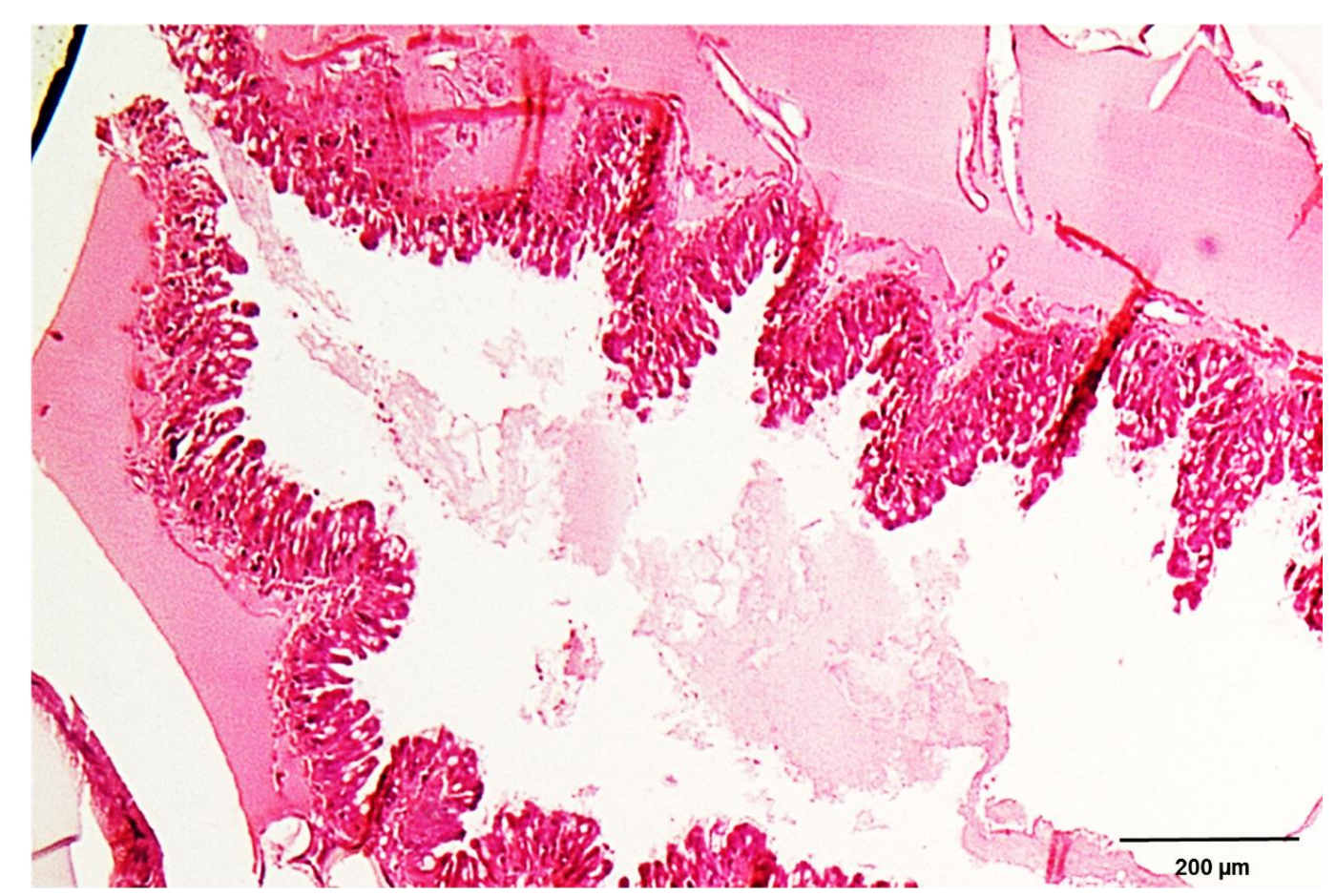

Figure 2.8 Tissue (longitudinal) section of the Galleria mellonella midgut. The photomicrograph depicts the series of folds/pleats that make-up the midgut.

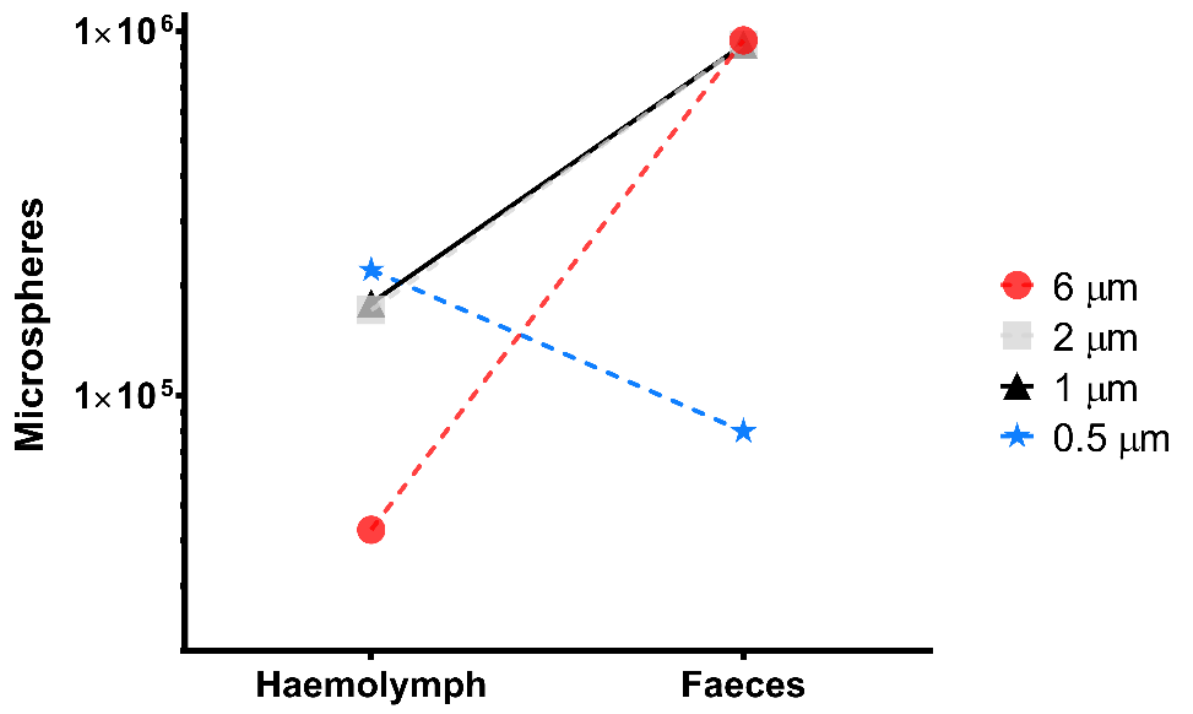

Figure 2.9 Gut permeability of Galleria mellonella larvae following force-feeding of microspheres $(0.5-6 \mu \mathrm{m})$. The data presented are from the control (no indomethacin) larvae only over the entire experimental period ( 72 hours), i.e., the total microspheres recovered. There is an inverse correlation between microsphere diameter and haemolymph load - the larger a microsphere is the less likely it will make its way through the gut wall and into the body cavity (haemocoel). 


\section{Gastric damage of the midgut}

Faecal samples collected from G. mellonella larvae were assessed for putative dysbiosis upon exposure to indomethacin via force-feeding and/or intrahaemocoelic injection. Colony forming units (CFUs) increased dramatically at 24 hours post-inoculation of $7.5 \mu \mathrm{g}$ when force-fed ( 18,000 CFUs) and injected ( 11,500 CFUs) into larvae when compared to untreated/control larvae $(<5,500)$ (Figure 2.10). The increased microbial load may be due to a change in permeability or the gut being flushed out. Overall, CFUs recovered from insect faeces at 4 hours were rather low, $<1,300$. These data mirror the transition time of microspheres through the alimentary when administered orally (Figure 2.6). Despite the apparent dose-dependent increases in CFUs observed in treated larvae (independent of inoculation route), time alone was revealed to be a significant factor $(F(3,72)=4.23, P=0.0082)$ and accounted for $>12 \%$ of the variation within these data, whereas treatment was non-significant $(F(8,72)=1.01, P=$ 0.433). By 72 hours, the levels of CFUs in faeces returned to similar levels observed at 4 hours (Figure 2.10).

The effects of force-feeding indomethacin (7.5 $\mu \mathrm{g} / \mathrm{larva})$ and PBS (+5\%DMSO) on $G$. mellonella larvae were further examined using wax (H\&E) histology. The larval midgut in the presence of PBS did not show any clear symptoms of damage or altered tissue morphology (Figure 11-upper panels). Uniform cellular arrangements of epithelial and goblet cells were observed, in addition to an intact basement membrane and visceral (striated) muscle layer. All control slides were considered grade 1 (0-2 discrete changes), with the exception of a single section at the 48-h time point that was assigned grade 2 (Figure 2.12). Following indomethacin treatment, varying levels of tissue damage and midgut degradation were visible within 4-48 h of all insects surveyed (Figure 2.11-lower panels). Tissue slides were assigned damage grades 2 to 4 at $4 \mathrm{~h}$, and grades 2 or 3 at 24 and $48 \mathrm{~h}$ (Figure 2.12) - representing at least five aberrations per slide (discrete, localised changes) to $>50 \%$ compromised tissue (global damage; Figure 2.13). Deterioration of the larval gut manifested as sloughed epithelial cells, increased vacuolisation, partial/complete displacement of the gut lining into the lumen, cellular debris (or potential apoptotic bodies), membrane blistering/blebbing, nuclear condensation (pyknosis) and fragmentation (karyorrhexis) (Figures 2.11 and 2.13). By $72 \mathrm{~h}$, several slides were graded 1 and 2 (Figure 2.12), which suggests the tissue is being repaired. 


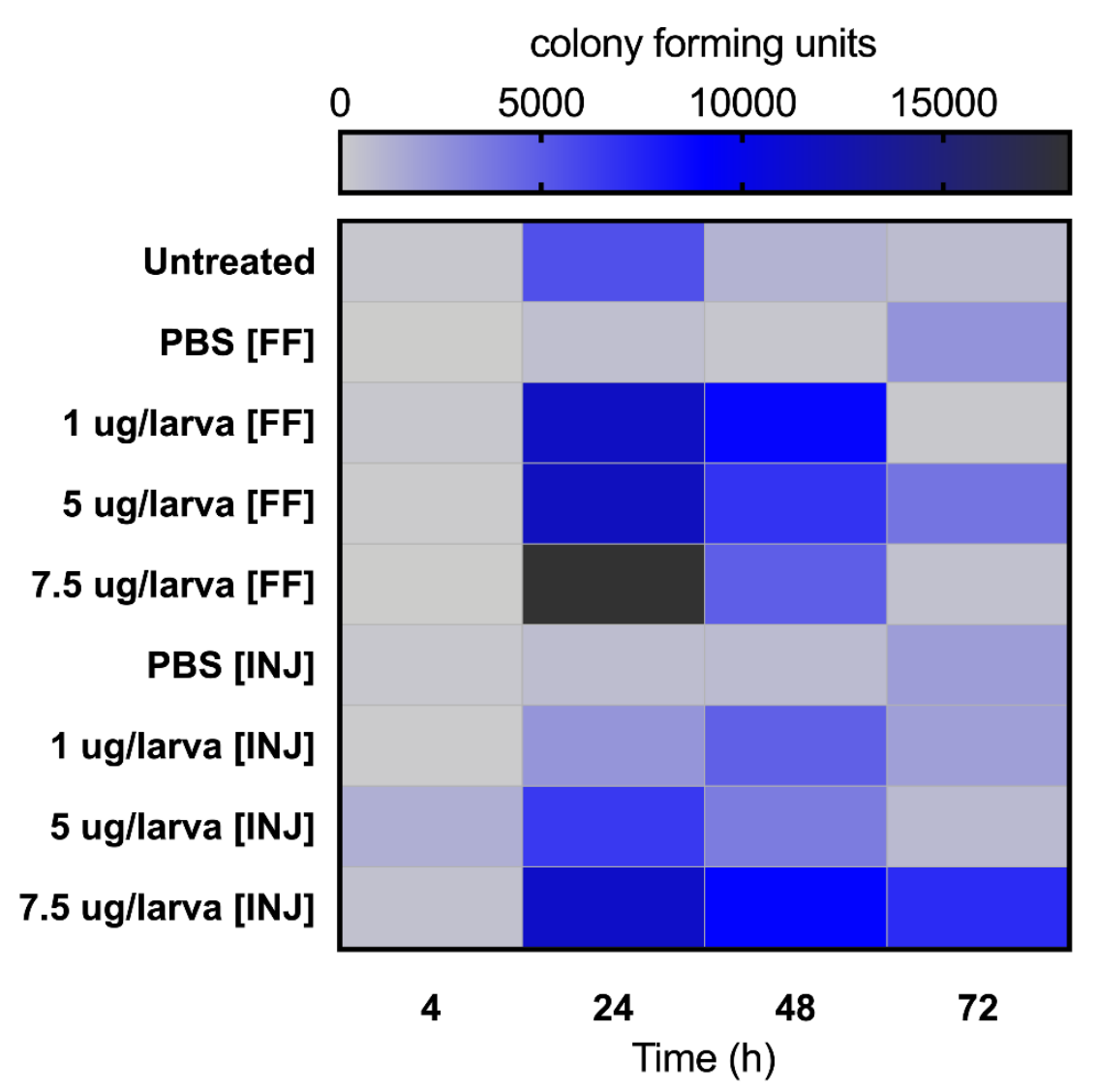

Figure 2.10 Culturable colony-forming units from faeces of indomethacin-inoculated Galleria mellonella larvae. The heat map depicts mean values of CFUs visible on nutrient agar. Insects were force-fed or injected with indomethacin across the concentration range $1-7.5 \mu \mathrm{g}$ or PBS (+ 5\% DMSO). Faeces were collected at each time point, homogenised, standardised to $0.1 \mathrm{mg}$ per treatment group, and plated on nutrient agar. Faeces were collected on three independent occasions from petri dishes containing at least 10 insects each. FF, force-fed; INJ, intrahaemocoelic injection. 


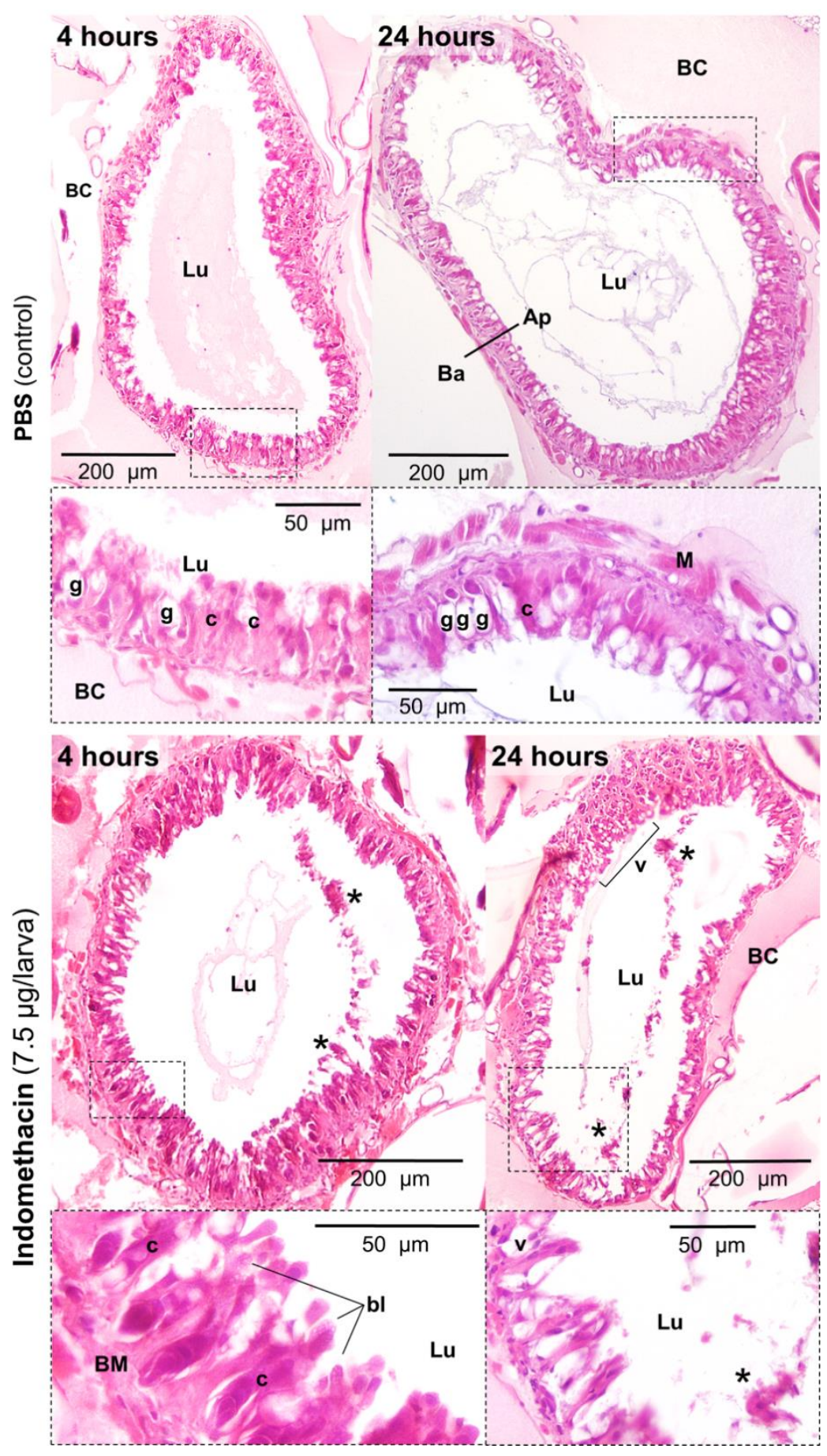

Figure 2.11 Gross histopathology of the midgut tissues from control and indomethacin-treated Galleria mellonella. Photomicrographs depict transverse sections of the midgut architecture at 4, 24, 48 and $72 \mathrm{~h}$ after force-feeding PBS (control—upper panels) or indomethacin ( $7.5 \mu \mathrm{g}-$ lower panels). Black boxes (broken lines) are used to highlight magnified regions of interest. Ap, apical; Ba, basolateral; BB, brush border; BC, body cavity; bl, blebbing/blistering of the cells; BM, basement membrane; c, columnar epithelial cell; g, goblet cell Lu, lumen; M, muscle; v, vacuole. An asterisk (*) denotes cellular damage and displacement into the lumen, and black arrows point to haemocytes (immune cells) within the body cavity (haemocoel). The negative control consisted of force-feeding the insect with PBS (+5\% DMSO) in the absence of indomethacin $(0 \mu \mathrm{g} / \mathrm{larva})$ 

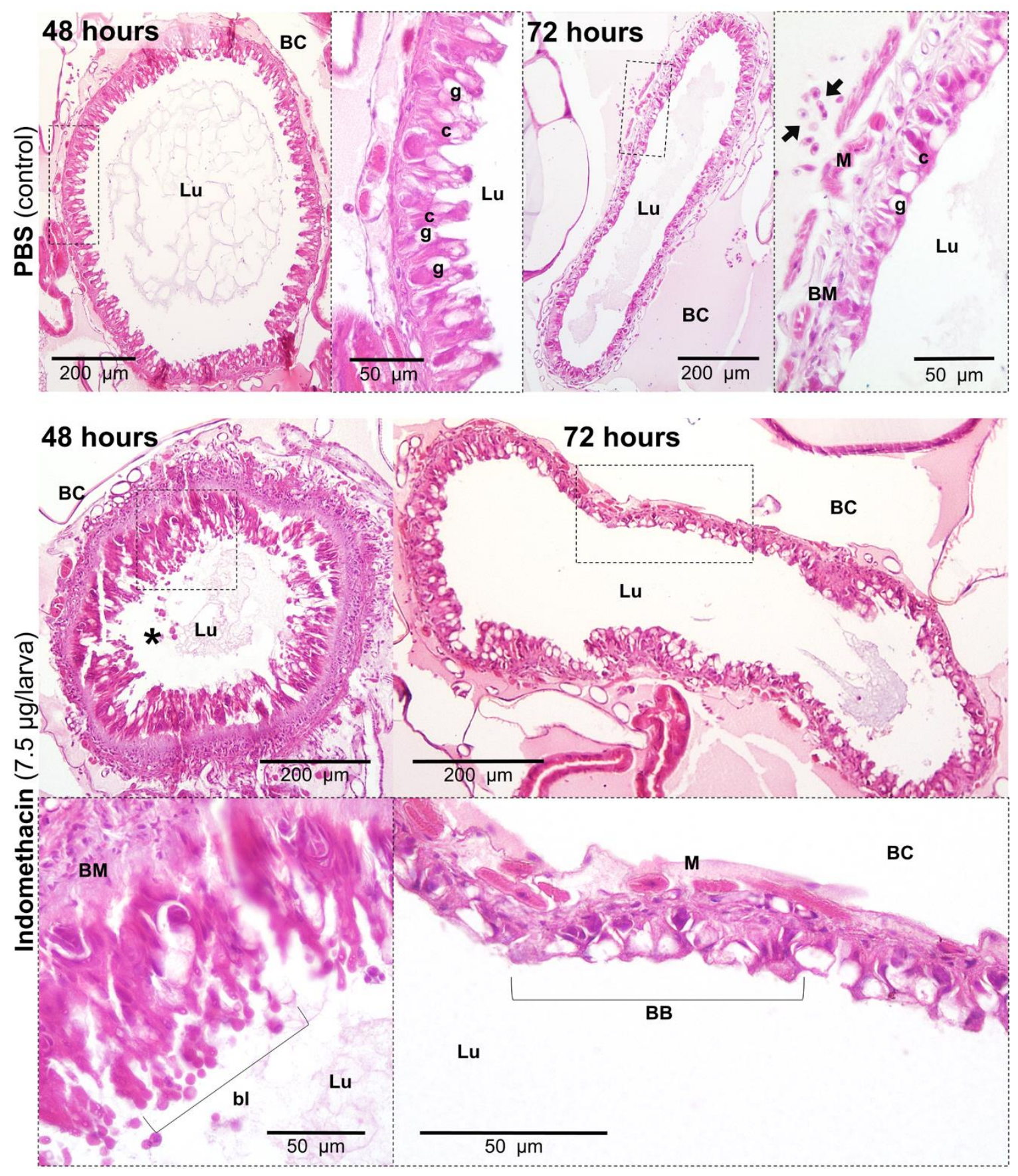

Figure 2.11 continued... 


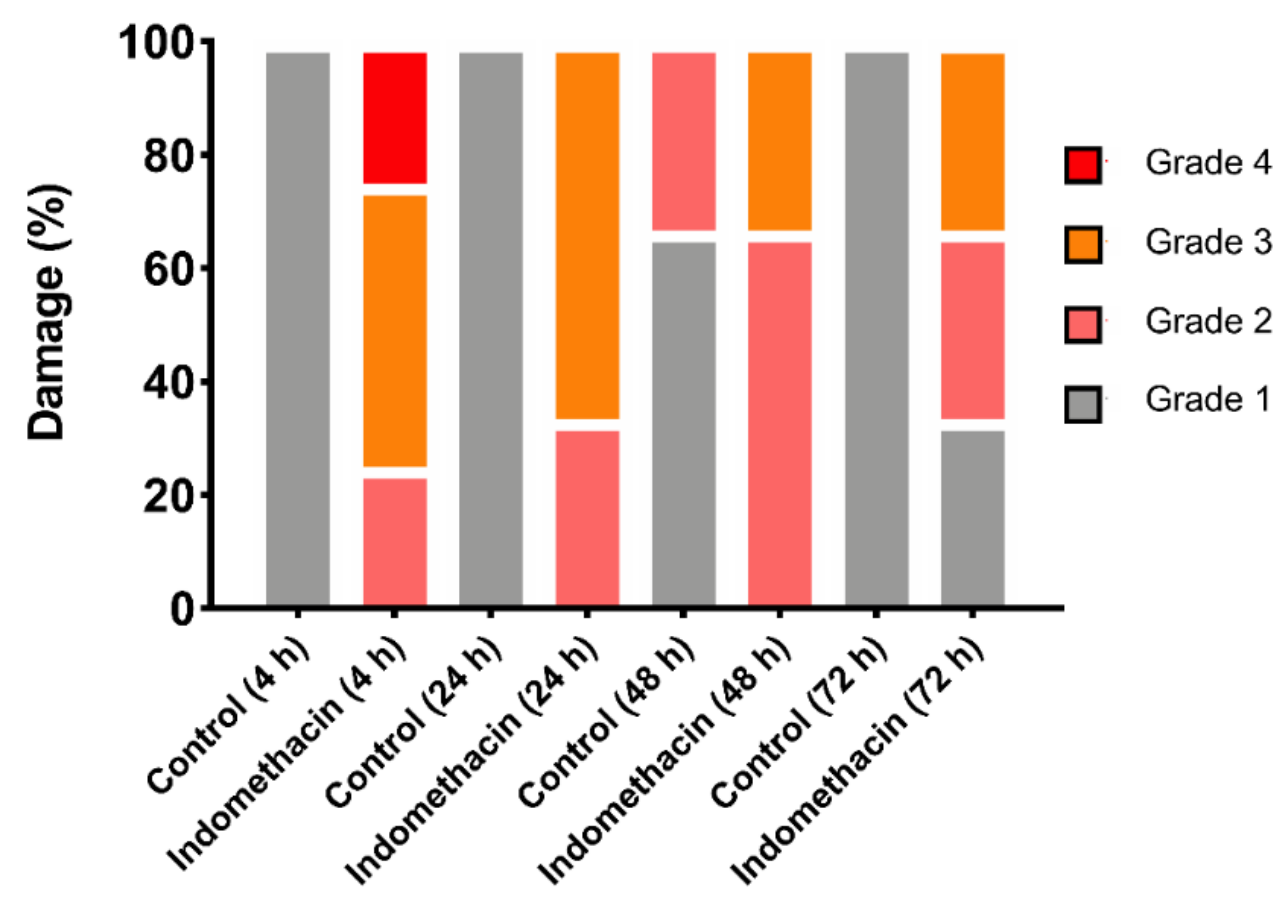

Figure 2.12 The extent of midgut tissue damage in Galleria mellonella force-fed indomethacin (7.5 $\mu \mathrm{g} / \mathrm{larva}$ ) or PBS. Histology slides were single blind assessed in pairs (treatment vs control) and subsequently assigned a grade $(1-4)$ based on damage(s). Grade 1 indicates little to no damage, whereas Grade 4 represents global damage affecting $>50 \%$ of tissue. Data have been compiled from assessments carried out at $4(n=8), 24(n=6), 48(n=6)$ and $72(n=6)$ hours post-inoculation. 


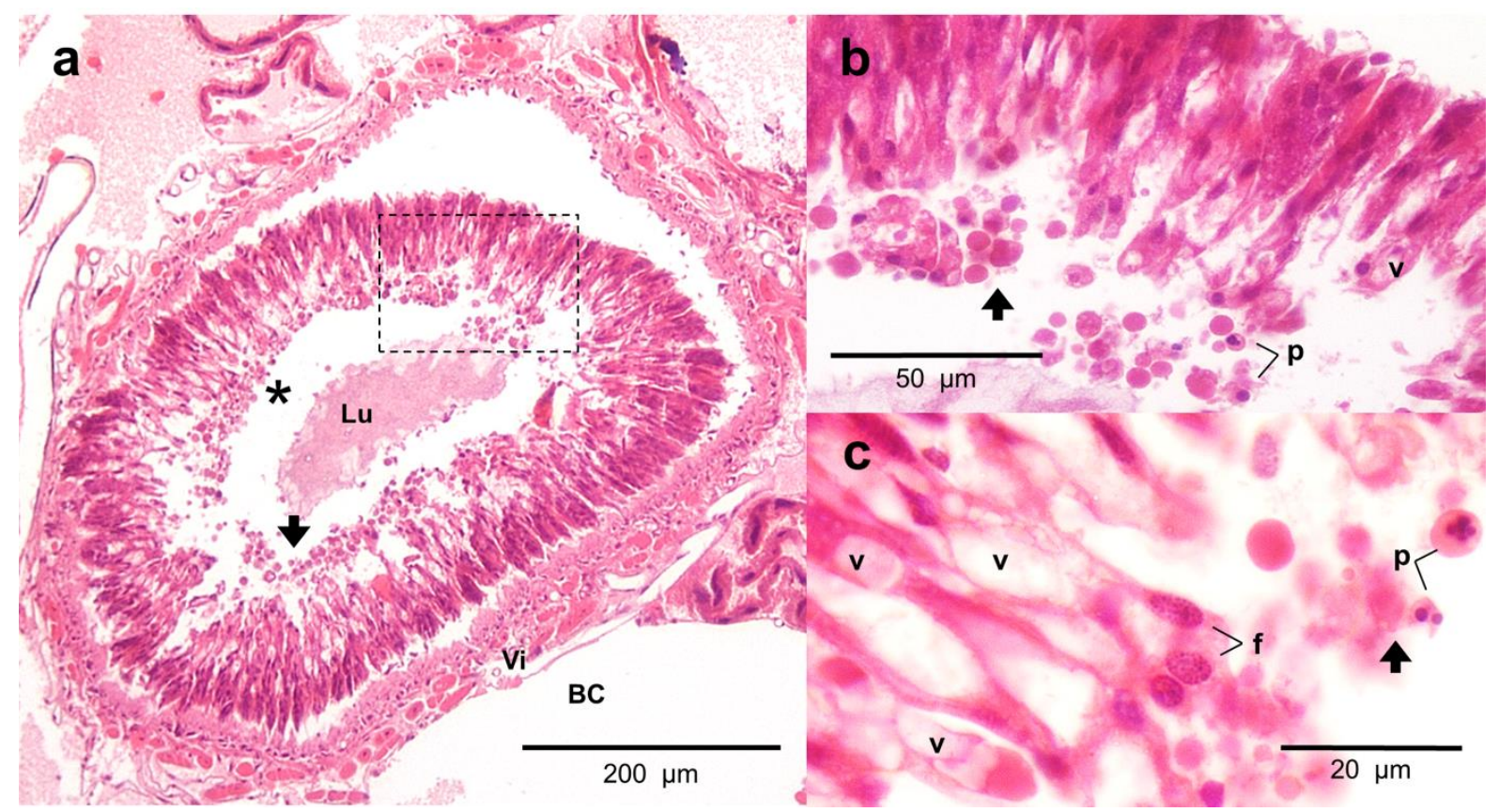

Figure 2.13 Histopathology of compromised midgut tissues from Galleria mellonella forcefed $(7.5 \mu \mathrm{g})$ indomethacin. a Transverse section of the larval midgut at $4 \mathrm{~h}$ post-treatment. The photomicrograph displays severe global tissue damage, where almost the entire epithelium has dissociated (sloughed) from the basement membrane and visceral (Vi) muscle. The black arrows (all panels) signify the large number of cells displaced into the lumen (Lu). BC, body cavity (or haemocoel). $\mathbf{b}, \mathbf{c}$ These photomicrographs are magnified regions of the black box and asterisk, respectively. The epithelial cells are showing clear signs of death: nuclear pyknosis (p) and cytoplasmic fragmentation (f), distinct vacuolisation (v) and cell disintegration/debris (potential apoptotic bodies).

\section{Gut repair (detoxification)}

Administration of indomethacin by force feeding led to significant increases in glutathione- $S$ transferase $\quad(F(4,40)=7.35, P=0.0002)$ and superoxide dismutase (F (4, $40)=2.635 P=0.0481)$ activities within the gut, but not in the haemolymph (GST; $F(4$, $40)=0.5449, P=0.704$ and SOD; $F(4,40)=0.801, P=0.515$; Figure 2.14). Larvae that were inoculated with $7.5 \mu \mathrm{g}$ indomethacin demonstrated consistently higher levels of GST activity in the gut, 2.3-3.2 Abs[340 nm]/min/mg, when compared to all other treatments/controls and timepoints (Figure 2.14a), whereas SOD activity peaked at 24 and $72 \mathrm{~h}, \sim 0.4$ Abs[560 nm]/min/mg (Figure 2.14c). A two-way ANOVA revealed time to be a significant factor regarding SOD activity $(F(3,40)=4.266, P=0.0105)$, but this was not the case for GST $(F(3,40)=2.584, P=0.067)$. These patterns of enzyme activity complement the restored nature of the midgut tissues seen at $72 \mathrm{~h}$ in the histology (Figure 2.11). Detoxification- 
associated activities in the haemolymph remained below $0.32 \mathrm{Abs}[340 \mathrm{~nm}] / \mathrm{min} / \mathrm{mg}$ for GST and $0.15 \mathrm{Abs}[560 \mathrm{~nm}] / \mathrm{min} / \mathrm{mg}$ for SOD (Figure 2.14b and 2.14d) - indicating that the adverse effects of indomethacin were restricted to the gut. Levels of malondialdehyde (MDA) - a byproduct of lipid peroxidation - varied considerably in the gut between 4 and 72 hours (Figure 2.14e). The highest level of $0.29 \mathrm{nmol}$ [MDA] $\mathrm{mg}$ [protein] was recorded in the untreated insects at 24 hours. Neither time $(P=0.0629)$ nor treatment $(P=0.165)$ were considered significant factors and accounted for $<24 \%$ combined of the variation within the MDA data. In the haemolymph (Figure 2.15f), MDA levels remained consistently below $2.3 \mathrm{nmol}$ [MDA] $\mathrm{mg}[$ protein], except for indomethacin treatments 5 and $7.5 \mu \mathrm{g} / \mathrm{larva}$ at 4 hours, where levels rose to 3.5 and $4.1 \mathrm{nmol}$ [MDA] $\mathrm{mg}$ [protein], respectively. Although time $(P=0.428)$ and/or treatment $(P=0.952)$ were not considered significant factors, and accounted for $\sim 7 \%$ combined of the variation within the data, the higher levels of MDA in the haemolymph at 4 hours do complement the initial 'leakage' of microspheres seen at the equivalent dose of indomethacin (Figure 2.6). Despite recording sizeable variation amongst samples, intrahaemocoelic injection of indomethacin did not lead to significant changes (explained by time or treatment) in glutathione- $S$-transferase (gut, $0.4-4.4$; haemolymph, $0.15-0.4$ ) and superoxide dismutase (gut, $0.04-0.2$; haemolymph, 0.1 - 0.4) activities, or levels of MDA (gut, $0.14-0.31$; haemolymph, 1.12 - 2.65) (Figure 2.15). However, a dose of $1 \mu \mathrm{g}$ indomethacin induced the largest increase in GST and SOD activities ( 0.4 Abs/min/mg each) within 24 hours in the haemolymph (Figure $2.15 \mathrm{~b}$ and $2.15 \mathrm{~d}$ ). These data are likely outliers. 

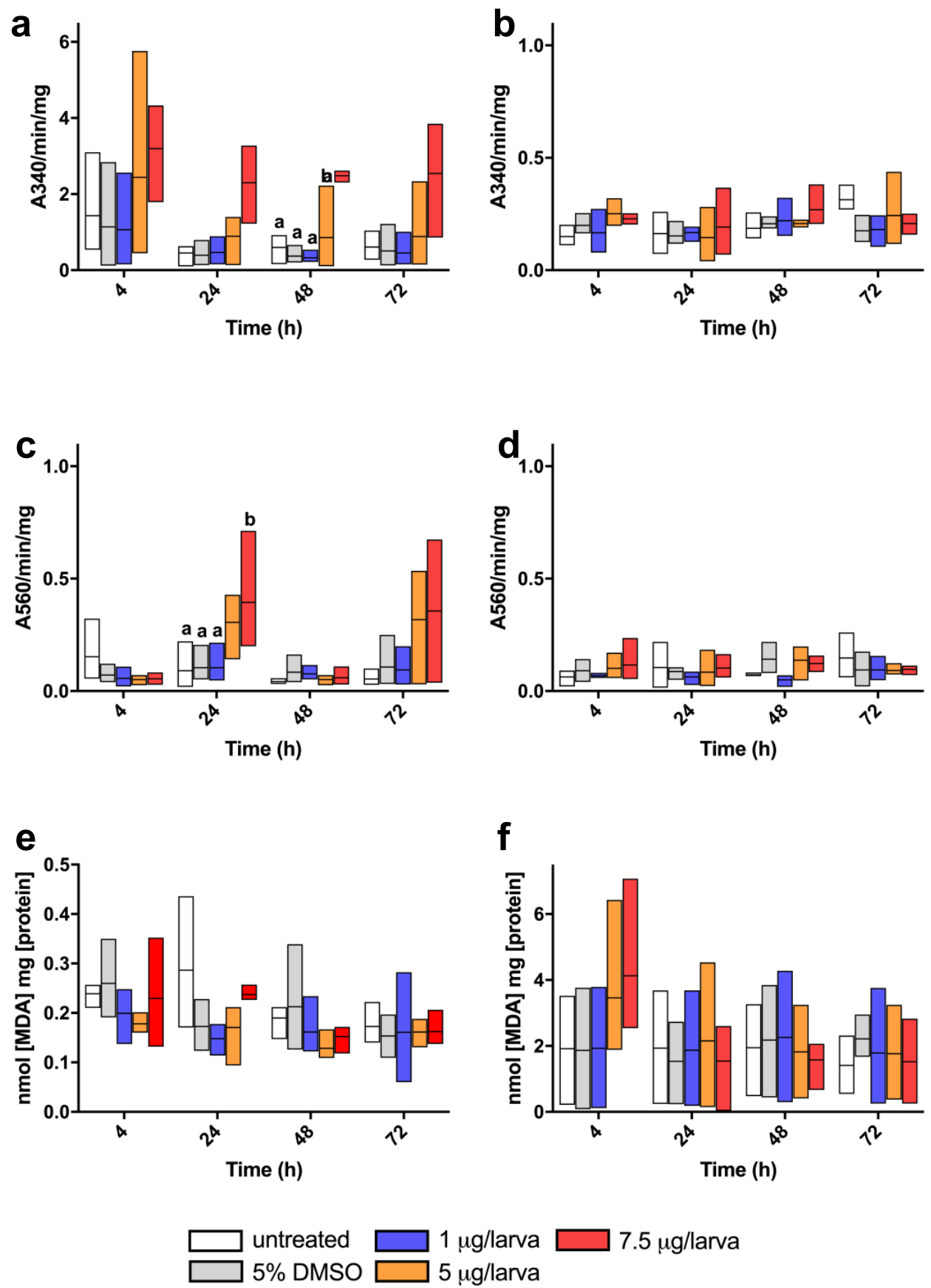

Figure 2.14 Detoxification-associated activities within Galleria mellonella following forcefeeding of indomethacin, $0-7.5 \mu \mathrm{g} / \mathrm{larva}$. Glutathione $S$-transferase activity was determined in the midgut (a) and haemolymph (b) by the change in 5-(2,4-dinitrophenyl)-glutathione accumulation (A340 $\mathrm{nm}$ ). Superoxide dismutase activity was determined in the midgut (c) and haemolymph (d) via the inhibition of NBT reduction $($ A560 $\mathrm{nm})$. Malondialdehyde (MDA) levels were determined was determined in the midgut (e) and haemolymph (f) via increases in A532 $\mathrm{nm}$. Data are presented as floating bars (min, max) with mean lines shown $(n=36$ per treatment, 180 in total; 3 insects were pooled at each time point). Unshared letters (a, b) represent statistical differences $(P<0.05)$ as determined by Tukey's multiple comparison tests. The negative control consisted of force feeding the insects with PBS $+5 \%$ DMSO in the absence of indomethacin $(0 \mu \mathrm{g} / \mathrm{larva})$. 

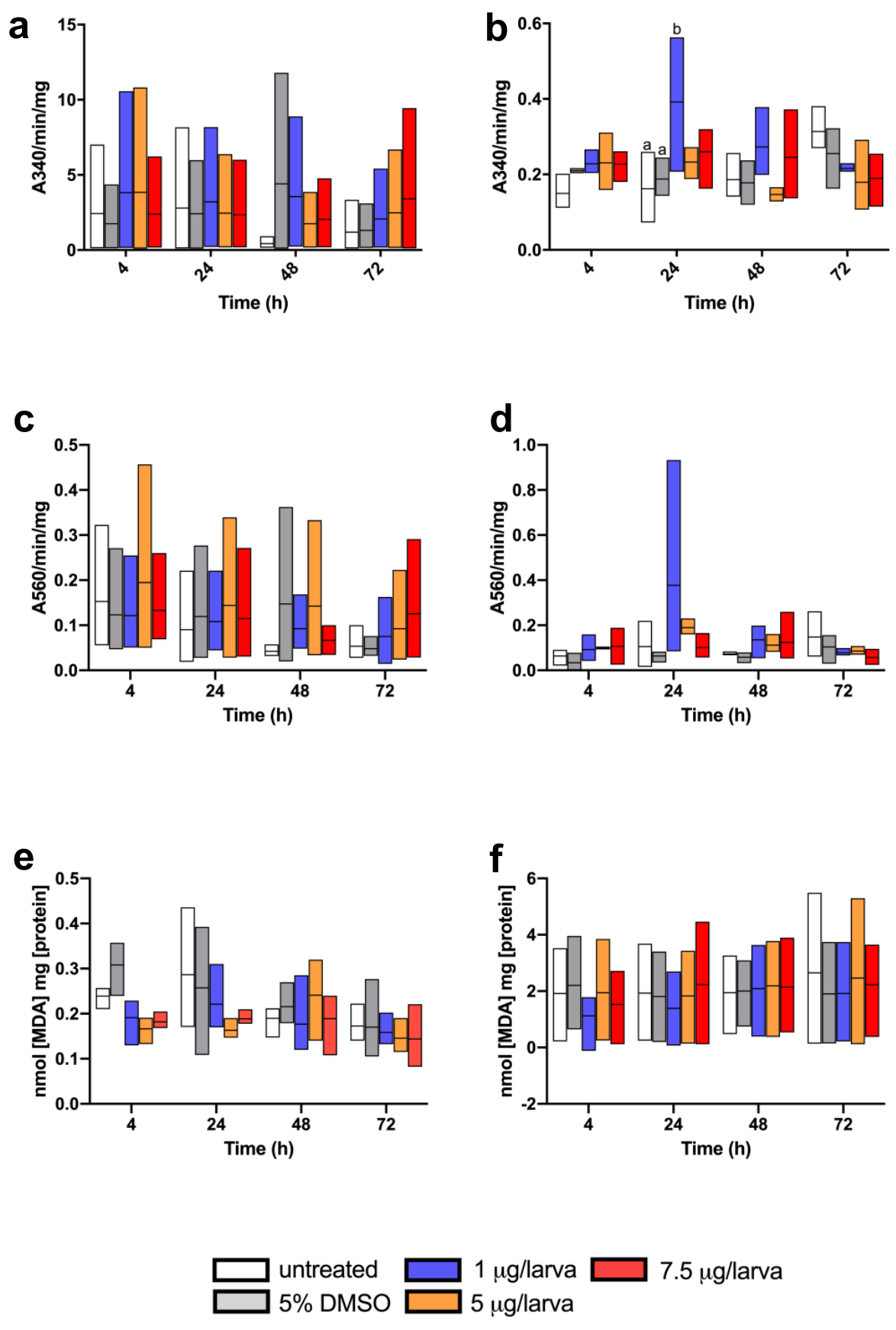

Figure 2.15 Detoxification-associated activities within Galleria mellonella following intrahaemocoelic injection of indomethacin, $0-7.5 \mu \mathrm{g} / \mathrm{larva}$. Glutathione $S$-transferase activity was determined in the midgut (a) and haemolymph (b) by the change in 5-(2,4-dinitrophenyl)glutathione accumulation (A340 nm). Superoxide dismutase activity was determined in the midgut (c) and haemolymph (d) via the inhibition of NBT reduction (A560 nm). Malondialdehyde (MDA) levels were determined was determined in the midgut (e) and haemolymph (f) via increases in A532 $\mathrm{nm}$. Data are presented as floating bars (min, max) with mean lines shown ( $n=36$ per treatment, 180 in total; 3 insects were pooled at each time point). Unshared letters $(\mathrm{a}, \mathrm{b})$ represent statistical differences $(P<0.05)$ as determined by Tukey's multiple comparison tests. The negative control consisted of injecting insects with PBS $+5 \%$ DMSO in the absence of indomethacin ( $0 \mu \mathrm{g} /$ larva $)$. 


\subsection{Discussion}

The relative toxicity of indomethacin was assessed in insect larvae via gavage (force-feeding) and intrahaemocoelic injection across a concentration range relevant to rodent models, $2-$ $30 \mathrm{mg} / \mathrm{kg}$ (0.5-7.5 $\mathrm{g} / \mathrm{larva})$, and found negligible side effects in terms of survival, development or immune cytotoxicity (Figures 2.1, 2.2 and 2.3). When force-fed, larvae displayed broad signs of injury to the alimentary canal (Figures $2.6-2.13$ ), e.g., a threefold increase in gut permeability and tissue degradation. Herein, considerable evidence confirms that the integrity of the midgut is compromised by indomethacin within 4 to $24 \mathrm{~h}$, causing sufficient damage to activate repair/detoxification mechanisms (GST and SOD). The onset of indomethacin-associated gut leakiness has also been recorded within $24 \mathrm{~h}$ in humans and mice, alongside ulceration and epithelial cell shrinkage (Playford et al., 1999, Bjarnason and Takeuchi, 2009). The combined use of X-ray microtomography and wax histology provides novel insight into the gross anatomy of the G. mellonella digestive tract-highlighting the suitability of the midgut for comparative pathobiology.

Indomethacin is a broad inhibitor of cyclooxygenase isozymes (COX1 and COX2), which are responsible for the initiation of prostaglandin synthesis $\left(\mathrm{PGG}_{2}\right)$, and ultimately, the maintenance of inflammatory programmes and gastrointestinal mucosa (reviewed by Brune and Patrignani, 2015). A possible limitation of using indomethacin in G. mellonella larvae is the mode of action through the inhibition of COX-1 and 2 which may be different in $G$. mellonella (Summ and Evers, 2013). Although the therapeutic effects of indomethacin are not being investigated in this research, it is unclear what receptors or enzymes indomethacin may bind to in the larvae and thus what other effects it may have and how this could contribute to gut damage. Additionally, as COX-1 and 2 are involved in mucosal maintenance of the intestines and inflammation (Lanas and Sopeña, 2009), it is possible that indomethacin is binding to an alternative receptor or enzyme in the larvae and contributing to disruption of the gut integrity through alternative methods. For example, indomethacin induced disruption of intestinal epithelial cells through the inhibition of macroautophagy (Chamoun-Emanuelli et al., 2019). Autophagy in G. mellonella larvae has been observed, and is an important part of homeostasis, therefore when this is disrupted it can result in dysbiosis, increased risk of infection, inflammation and cell death (Kazek et al., 2020). This could account for some of the effects observed in the histology slides. 
In a previous study by Büyükgüzel et al., (2007), eicosanoid presence in the haemolymph was deemed essential for mediating nodule formation - a cellular defence reaction — during viremia (Büyükgüzel et al., 2007). When they injected $50 \mu \mathrm{g}$ of indomethacin into the haemocoel, there was a significant reduction in the number of nodules formed. No measurable reduction in the phagocytic capacity of haemocytes in insects force-fed $7.5 \mu \mathrm{g}$ indomethacin and latex microspheres (Supp. Figure 1) was recorded here, which is also in contrast to earlier findings by Mandato et al., (1997). The authors reported on a reduction in the phagocytic index of $G$. mellonella haemocytes when exposed to $10 \mu \mathrm{M}$ indomethacin in vitro (Mandato et al., 1997). A likely explanation for this discrepancy is that the majority of force-fed indomethacin remains in the midgut of our insects, despite the microspheres leaking into the surrounding haemolymph (where they are targeted by haemocytes; Figure 2.7). The immune cells of insects, namely the haemocytes, share many mechanistic and structural similarities with the innate immune cells of vertebrates, including pathogen recognition receptor signalling and phagocytosis-associated respiratory burst (Renwick et al., 2007, Browne et al., 2013, Butt et al., 2016). Mandato et al., (1997) and Büyükgüzel et al., (2007) describe the immune-interference of indomethacin, and in addition to my observations of indomethacin-induced gut impairment, eicosanoid-like signalling should be added to the list of functional similarities between the innate immune systems of insects and vertebrates. Indomethacin is a weak acid and becomes soluble in a $\mathrm{pH}$ neutral or alkaline solution, when indomethacin is formed as a pharmaceutical to ensure correct drug delivery $\mathrm{pH}$ dependent polymers is used to control drug release (Akhgari et al., 2017, Tres et al., 2016). To avoid this interfering with experiments in G. mellonella larva $98.5 \%$ pure indomethacin was used and dissolved in 5\% DMSO prior to inoculations.

Galleria mellonella as an alternative animal model

The fruit fly Drosophila melanogaster is a superior genetic resource and over the past decade has been manipulated to gain novel insight into stem cell fate, immunity, antibiosis and homeostasis in the gut (Buchon et al., 2009, Chandler et al., 2011, Broderick and Lemaitre, 2012, Miguel-Aliaga et al., 2018). Only recently, the genome of G. mellonella was made available (Lange et al., 2018a), and the annotated version remains incomplete. Furthermore, despite wax-moth larvae being used widespread as a screening tool for novel therapeutics, pathogenicity, and toxicology (reviewed by (Tsai et al., 2016)), there remains a historical lag in molecular resources with the exception of some transcriptomic and miRNA data (Vogel et al., 2011, Mukherjee and Vilcinskas, 2014). The financial and ethical incentives for using 
insect larvae over rodents and zebrafish are attractive, and so, I propose that vertebrates could be replaced partially for gut pathobiology. Waxmoth larvae are larger than traditional models like nematodes and drosophilids - this has two distinct advantages: (1) accurate doses can be administered orally (force-feeding), and (2) gram quantities of gut tissue can be obtained easily for downstream processing. Unlike other insect orders, the midgut of lepidopteran larvae represent the majority of tissue along the alimentary canal (mouth to anus; Figure 2.5) and contains a specialised cell type, namely the goblet cell, which is also found in the human intestine (Engel and Moran, 2013, Linser and Dinglasan, 2014).

The most common larval inoculation technique is intrahaemocoelic injection of test compounds/microbes; however, force-feeding $G$. mellonella (i.e., gavage) is an emerging practice. When screening common food preservatives (e.g., potassium nitrate), Maguire et al., (2016) obtained comparable toxicology data $\left(\mathrm{LD}_{50}\right)$ between insect larvae, human cell lines (HEp-2) and rats. Recently, Coates et al. (2019) provided evidence that larvae can also be used to assess the lethality and putative immune-toxicological effects of shellfish poisoning toxins (e.g., okadaic acid) at FDA-regulated levels in contaminated foods. Okadaic acid that was force-fed to insects disrupted midgut homeostasis, leading to detrimental levels of lipid peroxidation (malondialdehyde accumulation) in a dose-dependent manner-resembling symptoms found often in the standardised mouse bioassay. Lange et al., (2018b) proved that $G$. mellonella could differentiate between an enteric symbiont (Bacteroides vulgatus) and pathobiont (Escherichia coli), and mount a strong immune response involving reaction oxygen/nitrogen species. Oral administration of the pathogen stimulated the up-regulation of immune-recognition genes in insects (e.g., apolipophorin III) and mice (e.g., Cd14) alike. Interestingly, oral exposure of $G$. mellonella larvae to caffeine led to elevated levels of theobromine and theophylline in the haemolymph — suggesting that caffeine metabolism in this insect is similar to the process in mammals (Maguire et al., 2017).

A better understanding of G. mellonella's alimentary canal should assist insecticide development (e.g., boric acid and biopesticides, (Büyükgüzel et al., 2013, Grizanova et al., 2014) as lepidopteran insects represent a sizeable number of devastating agricultural pests (e.g., Spodoptera littoralis; Linser and Dinglasan, 2014). A key difference between the digestive systems of vertebrates and insects is the presence of phenoloxidase (PO) enzymes (Whitten and Coates, 2017). Phenoloxidases are responsible for the early processing of pigment 
precursors (quinones) into melanin, which plays several roles in development and immunity. Insect faeces are melanised upon release, and although the function of this is unclear, presumably it is due to gut phenoloxidase-activities and their oxidising/nitrosative by-products maintaining resident microbial populations from over-growing (Whitten \& Coates, 2017). The gut microbiomes of insects are diverse and tend to be species specific influenced invariably by diet and environmental factors (Engel and Moran, 2013). Few studies have focussed on the $G$. mellonella gut microbiome, yet representatives of the Bacteroidetes, Firmicutes and Proteobacteria are homologous to the biota on microvilli of the human intestinal crypts (Mukherjee et al., 2013b; Dubovskiy et al., 2016). Further work is needed to profile the residents of the insect gut, including fungi, viruses and Archaea. This is a timely topic, as waxmoth larvae are capable of degrading plastic (polypropylene; (Bombelli et al., 2017) likely facilitated by the microbial consortium of their alimentary canal.

\subsection{Concluding remarks}

The physiological effects of indomethacin on G. mellonella were investigated, providing compelling evidence that indomethacin exposure leads to tissue damage, cell death, gut leakiness, and REDOX imbalance in insect larvae. This mimics closely the pathological symptoms of their rodent counterparts. Herein, the functional/structural similarities of lepidopteran midgut tissues to those found in regions of the human gastrointestinal tract are described. These data reinforce the use of G. mellonella as a surrogate toxicology model, with a focus on screening nutraceuticals and food additives.

\section{Acknowledgements}

This chapter was released as a pre-print (Emery et al. 2019) and was available at bioRxiv; https://www.biorxiv.org/content/10.1101/606319v1, prior to publication. I would like to thank Mrs Sophie Malkin (Bluefish Technical Officer, Swansea University) for her assistance with histology preparations, and Elizabeth Evans and Ria Mitchell (AIM Facility) for helpful tips with image data/segmentation. 


\section{Chapter 3: Evaluating the protective properties of}

'nutraceuticals', Cordyceps sinensis and colostrum, against bacterial- and chemically-induced gastric damages

A version of this chapter is in preparation for publication:

Helena Emery, Tariq M. Butt, Christopher J Coates (20xx). Nutraceutical intervention fortifies the insect (Galleria mellonella) gut against chemical and microbial disruptors.

*TMB provided some resources. 


\subsection{Abstract}

The entomopathogenic fungus Cordyceps sinensis and bovine colostrum are considered nutraceuticals (health food supplements) due to their anti-inflammatory, anti-apoptosis and gut restitution properties in rodent models. Crude extracts of $C$. sinensis have been used in traditional Chinese medicines for many years but the effective ingredients remain largely uncharacterised. Herein, the capacity of these nutraceuticals to alleviate gastric damages caused by indomethacin and/or the Gram-negative bacterial pathogen, Campylobacter jejuni, were investigated using the insect model, Galleria mellonella. Insect larvae were reared from eggs on diets supplemented with $10 \%(\mathrm{w} / \mathrm{w})$ colostrum, $10 \%(\mathrm{w} / \mathrm{w})$ C. sinensis (powder), or 5\% colostrum and 5\% C. sinensis combined. Fifth instar larvae from each diet regime were forcefed indomethacin $\left(7.5 \mu \mathrm{g} /\right.$ larva) or $C$. jejuni $\left(1 \times 10^{6}-3 \times 10^{6} \mathrm{CFU} / \mathrm{larva}\right)$, and monitored for survival, development and gastric permeability.

Insects reared on a $C$. sinensis-supplemented diet demonstrated reduced gut leakiness after indomethacin inoculation (by tracking fluorescent (latex) microspheres) and higher health indices after $C$. jejuni challenge between 24- and 72-hours post-treatment. Insects reared on a colostrum-supplemented diet, weighed significantly more than those receiving a 'standard diet' and some enhanced resistance to infection. The combined diet (5\% of each nutraceutical) also yielded improved outcomes to the usually detrimental side effects of indomethacin, but was not as effective as either supplement alone. Herein, the reader will find evidence that the gut of G. mellonella was reinforced against chemical (indomethacin) and microbial (C. jejuni) damages by nutraceuticals. 


\subsection{Introduction}

'Nutraceuticals' is a broad term used to describe any dietary supplement (non-pharmaceutical) considered to contain bioactive compound(s) that can improve health (often available in tablet form). There is no specific regulation of nutraceuticals within Europe and the term can often overlap with 'functional foods' (Coppens et al., 2006), which can be described as enriched or fortified foods that possess properties beyond nourishment to include named health-benefits or disease prevention (Espín et al., 2007). The practice of consuming specific foods to improve health and treat ailments dates back to around 460 - 377 BC. For over 2,000 years, the study of relationships between specific foods and their apparent medicinal properties have helped to drive innovation in health and quality of life (Andlauer and Fürst, 2002). This has resulted in the production of drugs such as Aspirin (acetylsalicylic acid), which was extracted as salicylic acid from the Spiraea ulmaria plant (Cheng, 2007).

One nutraceutical, Cordyceps sinensis, is an entomopathogenic fungus and its use can be dated back to the late 1400s in Tibet (Chen et al., 2013). Research conducted into the bioactive properties of $C$. sinensis extracts has revealed many therapeutic links, including anti-cancer and cardiovascular disease treatments ( $\mathrm{Li}$ et al., 2006). Crucially, evidence points to both protective and reparative properties of Cordyceps spp. against NSAID-induced gastric damage and renal interstitial fibrosis (diabetic nephropathy) in rodent models (Marchbank et al., 2011, Liu et al., 2020). An emerging nutraceutical, bovine colostrum, is the first milk produced after birth, and is vital to the early development of new-borns as it contains antimicrobial peptides (AMPs), growth factors, and fats (Bagwe et al., 2015). Studies conducted within the last decade have linked colostrum intake to positive cancer outcomes, increased repair rates of cardiac muscle/vessels after induced myocardial infarction in rats (Kaur et al., 2014), and to ameliorate the effects of exercised-induced permeability of the gut (Davison et al., 2016). Prior to this, Döhler and Nebermann (2002) noted a 67\% reduction in plasma endotoxin levels from rats infected orally with Escherichia coli and then treated with colostrum compared to those subjects that received a negative control (albumin). Similarly, (Kim et al., 2005) pre-treated rats with colostrum (for 5 days) prior to exposure to the ulcer-causing drug diclofenac. Diclofenac-induced damage, e.g., gut permeability, dysbiosis and bacterial translocation, were less pronounced in colostrum-treated rats and even more so when colostrum was coadministered with glutamine.

The vast majority of exploratory and clinical studies of gastrointestinal damage and integrity are carried out in rodents. After performing an extensive literature search for articles on Google 
Scholar, between 2009 and 2018, using the key terms 'indomethacin', 'ulcer', 'gut' and 'rodents', over 15,000 papers were highlighted. Within the first fifty listed results, an average of 64 mice was calculated (ranging from 8 to 250) per study. These were studies of the general pathology and toxicology of indomethacin and other toxins (e.g. see Chapter 4) on the rodent gut, and the screening of novel compounds for anti-oxidant and gut-repair properties. By extrapolation, this is an average of $\sim 98,000$ to 114,000 rodents per year. Therefore, there are substantial cost, time, and ethical incentives to find an alternative, non-mammalian model for studies of gastric damage and repair. The following chapter sought to enhance further the use of $G$. mellonella as a surrogate to rodents, and to build upon the success of Chapter 2 in establishing its potential.

Firstly, the putative gut fortification properties of nutraceuticals were assessed in G. mellonella, by raising insects on diets supplemented with $C$. sinensis or bovine colostrum and subsequently exposing them to bacterial (Campylobacter jejuni) and chemical (indomethacin) disruptors. The enteric pathogen, C. jejuni, is commonly transmitted through contaminated foods and can lead to diarrhoea, fever, abdominal cramps, and in extremis, gastroenteritis (van Putten et al., 2009). Wax moth larvae can be maintained at human relevant temperature, $37^{\circ} \mathrm{C}$, and so previous work has used this mini host to study the pathogenicity of $C$. jejuni isolates/strains (Senior et al., 2011). Secondly, the use of G. mellonella to triage existing and emerging pharmacological demulcents will be investigated using lansoprazole when co-administered orally with indomethacin. Previous work has described the therapeutic effects of this proton pump inhibitor (lansoprazole) in reducing the severity of indomethacin-associated gastropathy in rats (Kuroda et al., 2006, Maity et al., 2008, Rai et al., 2011).

\subsection{Materials and Methods}

\subsubsection{Reagents}

Unless stated otherwise, all chemicals and reagents were sourced from Sigma Aldrich (Dorset UK) in their purest form listed. Green and blue fluorescent (latex) microspheres ranging from 0.5 to $6 \mu \mathrm{m}$ in diameter were purchased from Polysciences, Inc. (Fluoresbite ${ }^{\circledR}$ ). Stock solutions of indomethacin were prepared in 5\% dimethyl sulfoxide (DMSO; v/v) and diluted in filtersterilized $(0.2 \mu \mathrm{m})$ phosphate-buffered saline (PBS) pH 7.4. Mr John Rolfs (Pembrokeshire 
Dairy Ltd., Wales UK) provided colostrum. Oxford Vitality, part of the Oxford Health Company Ltd., provided the C. sinensis extract.

\subsubsection{Galleria mellonella artificial food}

Galleria mellonella larvae were cultured on artificial food adapted from Cherkova et al., (2018). Components all purchased from Sainsbury's unless stated otherwise: Corn meal (90 g), Wheat flour (50 g), Dried milk (50 g), Beeswax (50 g) (Thorne natural beeswax F0000), Honey (50 g), Glycerol (50 g) (Sigma Aldrich G5516), Dry yeasts (22 g) and Distilled water (50 g) (Chertkova et al., 2018). Dry components (Corn meal, wheat flour, dried milk and yeast) were combined and sterilised under UV for 15 minutes. Water, glycerol and beeswax were mixed and microwaved until wax is completely melted (every 30 second steps with regular stirring). Once melted, the honey is added and mixed thoroughly before being poured into the dry components and mixed until smooth. Once mixed, the food is spread into a tray and stored at $4{ }^{\circ} \mathrm{C}$ for no more than 2 weeks. For nutraceutical studies, four diets were prepared: (1) standard recipe, (2) $10 \%(\mathrm{w} / \mathrm{w})$ C. sinensis enhanced recipe, (4) 10\% (w/w) colostrum enhanced recipe (extrapolated from (Davis et al., 2007), and (4) 5\%:5\% (w/w) colostrum: Cordyceps recipe.

Table 3.1. Nutritional content of $C$. sinensis and colostrum powder (Data based on product packaging)

\begin{tabular}{lll}
\hline & $\begin{array}{l}\text { Cordyceps sinensis } \\
\text { powder } \mathbf{( 5 0 g )}\end{array}$ & Colostrum Powder (50g) \\
\hline Kilo calories (kcal) & 194 & 180 \\
\hline Protein (g) & 18.22 & 27.5 \\
\hline Carbohydrate (g) & 6.8 & 16.5 \\
\hline Fat $(\mathbf{g})$ & 7.4 & 0.4 \\
\hline
\end{tabular}

\subsubsection{Culturing Galleria mellonella}

To establish a G. mellonella culture, fifteen final instar larvae purchased from Livefoods Direct Ltd. were placed into a glass jar containing $20 \mathrm{~g}$ of food. Filter paper was fixed to the lid of the jar to aid egg collections. Larvae were maintained at $28-30{ }^{\circ} \mathrm{C}$ under a 12 -hour light, 12 hour dark cycle (Figure 3.1). Larvae were allowed to pupate (form cocoons), transition to adults (moths) and subsequently lay eggs. Food was added ad hoc, filter paper in jars was replaced weekly, and moths emerged, the jars were covered to protect the insects. Filter paper with eggs attached were transferred into a separate jar containing $20 \mathrm{~g}$ food, where larvae were allowed 
to hatch and grow undisturbed (food was added $a d$ hoc). Once larvae reached the $3^{\text {rd }}$ or $4^{\text {th }}$ instar stage, the populations were divided between two new jars in order to avoid overcrowding and spoilage. Dead or melanised larvae were removed daily (if necessary). These steps were repeated until sufficient numbers were provided for experimentation (Jorjão et al., 2018).

When up-scaling for nutraceutical studies, larvae were reared on the standard diet until they reached 75-100 mg per larva (between 30 and 35 days old), then placed into new jars containing one of the four diet regimes mentioned above. Larvae were further maintained for 4 to 7 days on the supplemented diets, weighed, inspected and assigned randomly to Petri dishes for experimentation (chemical and microbial inoculations).

\subsubsection{Health indices of larvae exposed to biopharmaceuticals}

Aspirin is a common NSAID that is known to cause gastric damage in rats across the concentration range, $10-100 \mathrm{mg} / \mathrm{kg}$ (Wallace et al., 2004, Sanyal and Kaushal, 2005). The equivalent dosages for a $250 \mathrm{mg}$ larva are $2.5-25 \mu \mathrm{g}$. Diclofenac sodium is another commonly used NSAID to treat arthritis and is understood to induce gastric ulcers, with some research suggesting colostrum can aid in reducing the side effects of diclofenac sodium (Kim et al., 2005). Accordingly, diclofenac sodium was also incorporated into $G$. mellonella experimentation at concentrations ranging from $2.5-10 \mu \mathrm{g} / \mathrm{larva}$ (the equivalent being $10-$ $40 \mathrm{mg} / \mathrm{kg}$ in rodents; (Khazaeinia and Jamali, 2003, Reuter et al., 1994, van der Vijver et al., 2013, Shaheen et al., 2013). To compare the effective ness of nutraceuticals, lansoprazole which is often prescribed alongside an NSAID as a neutralising agent, was used also. A concentration range of $20-100 \mathrm{mg} / \mathrm{kg}$ has been successfully administered to rats, therefore larvae were administered 5 - $25 \mu$ g (Al-Qarawi et al., 2005, Rodríguez et al., 2006, Higuchi et al., 2009).

Healthy final instar G. mellonella larvae reared for $4-7$ days on supplemented diets $(10 \% C$. sinensis, $10 \%$ colostrum and 5\% each) were inoculated with indomethacin, lansoprazole, diclofenac salt and/or aspirin by intrahaemocoelic injection (INJ) or force-feeding (FF; gavage) using a sterile 27-gauge hypodermic needle (as described in chapter 2). The negative control consisted of PBS pH 7.4 containing $5 \%$ DMSO. Post-inoculation, larvae were incubated in the dark at $30^{\circ} \mathrm{C}$. Individual larval health was assessed using a scoring system of 0 (dead) and 10 (healthy, full pupation) (Table 3.1) over a 72 hours period. 


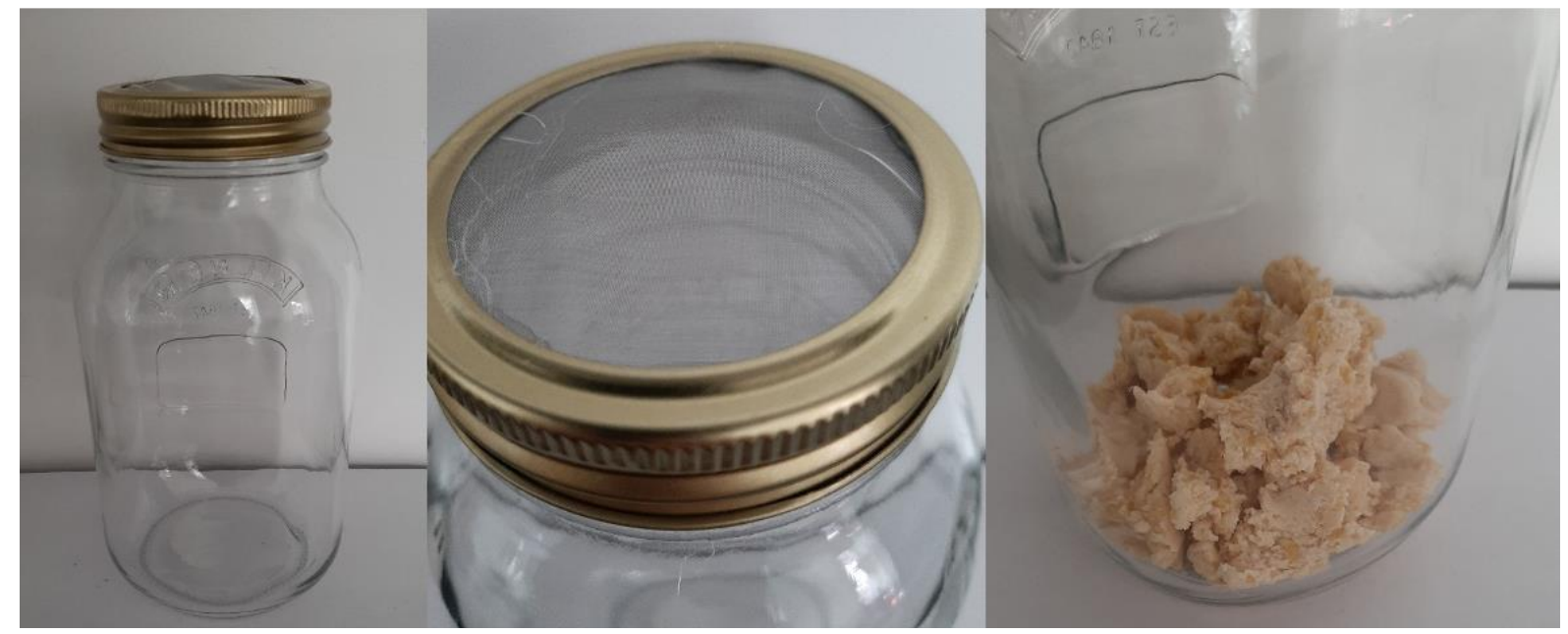

Figure 3.1 Standard set-up for rearing Galleria mellonella. Jar lids are stainless steel with fixed meshes, $0.125 \mathrm{~mm}$ diameter holes. Standard honey-based diet is visible in the far right.

Table 3.2 Health index scoring system for Galleria mellonella (developed by Loh et al., 2013)

\begin{tabular}{|c|c|c|}
\hline Category & Feature & Score \\
\hline \multirow[t]{4}{*}{ Activity } & Movement without stimulation & 3 \\
\hline & Moves once stimulated & 2 \\
\hline & $\begin{array}{l}\text { Minimal movement when stimulated } \\
\text { (slow, moves } \leq 2 \mathrm{~cm} \text { ) }\end{array}$ & 1 \\
\hline & No movement & 0 \\
\hline \multirow[t]{5}{*}{ Melanisation } & No melanisation & 4 \\
\hline & $<3$ Beige spots & 3 \\
\hline & $\geq 3$ Beige spots & 2 \\
\hline & Whole larvae brown with black spots & 1 \\
\hline & Black larvae & 0 \\
\hline \multirow{3}{*}{$\begin{array}{l}\text { Cocoon } \\
\text { formation }\end{array}$} & Full cocoon & 1 \\
\hline & Incomplete cocoon & 0.5 \\
\hline & No cocoon & 0 \\
\hline \multirow[t]{2}{*}{ Survival } & Alive & 2 \\
\hline & Dead & 0 \\
\hline
\end{tabular}




\subsubsection{Campylobacter jejuni culturing}

Campylobacter jejuni from ATCC - LGC Standards (Campylobacter jejuni subsp. jejuni (ATCC ${ }^{\circ}$ BAA-224 $\left.{ }^{\mathrm{TM}}\right)$ ) was reconstituted with $1 \mathrm{ml}$ Mueller Hinton broth (Sigma Aldrich). The bacterial suspension was aliquoted in sterile micro-centrifuge tubes with $15 \%(\mathrm{v} / \mathrm{v})$ glycerol and stored at $-70^{\circ} \mathrm{C}$. Approximately, $100 \mu \mathrm{l}$ of bacterial suspension was added to 300 ml Mueller Hinton broth in a Corning® Erlenmeyer cell culture flask with vented cap and incubated at $37^{\circ} \mathrm{C}$ with constant agitation, $200 \mathrm{rpm}$ overnight. Bacteria $(100 \mu \mathrm{l})$ were subcultured onto Mueller Hinton agar $(2 \%$ w/v) and maintained anaerobically using a gas generation pack (Merck Anaerocult ${ }^{\circledR} \mathrm{P}$ ) at $37^{\circ} \mathrm{C}$ for 48 hours before colony forming units (CFUs) were counted.

\subsubsection{Health indices of Galleria mellonella larvae during bacterial infection}

Healthy larvae reared for $4-7$ days on supplemented food (10\% C. sinensis, $10 \%$ colostrum and $5 \%$ each of $C$. sinensis and colostrum combined) were inoculated with $C$. jejuni diluted in PBS $\left(1 \times 10^{6} \mathrm{CFU} / \mathrm{larva}\right.$ or $3 \times 10^{6} \mathrm{CFU} /$ larva) by intrahaemocoelic injection (INJ) or forcefeeding (FF; gavage) using a sterile 27-gauge hypodermic needle (as described in chapter 2). The negative control consisted of PBS pH 7.4 containing 50\% Muller-Hinton broth. Postinoculation, larvae were incubated in the dark at $30{ }^{\circ} \mathrm{C}$. Individual larval health was assessed using a scoring system of 0 (dead) and 10 (healthy, full pupation) (Table 3.1) over a 72 hour period.

\subsubsection{Gut Permeability}

Fluorescently tagged, carboxylate-modified latex microspheres $\left(1 \times 10^{6}\right)$ of $2 \mu \mathrm{m}$ and $6 \mu \mathrm{m}$ in diameter were re-suspended in $10 \mu \mathrm{L}$ PBS (control) or $10 \mu \mathrm{L}$ indomethacin solution $(7.5 \mu \mathrm{g}$ dose) to form co-inoculates (thereby avoiding piercing an insect twice). Larvae were reared on nutraceutical enhanced food (10\% colostrum, $10 \%$ C. sinensis or combined 5\% colostrum with $5 \%$ C. sinensis) for $4-7$ days before being force-fed $10 \mu \mathrm{L}$ of each co-inoculate and incubated at $30{ }^{\circ} \mathrm{C}$ until haemolymph and faeces were collected from each treatment group at 4, 24, 48 and 72 hours. Faeces were homogenised in $1 \mathrm{~mL} \mathrm{PBS} \mathrm{pH} \mathrm{7.4.} \mathrm{To} \mathrm{assess} \mathrm{the} \mathrm{microsphere} \mathrm{load}$ in the haemolymph, insects were chilled on ice for $\sim 3 \mathrm{~min}$ prior to injection of $100 \mu \mathrm{L}$ anticoagulant $(15 \mathrm{mM} \mathrm{NaCl}, 155 \mathrm{mM}$ trisodium citrate, $30 \mathrm{mM}$ citric acid, $20 \mathrm{mM}$ disodium EDTA, pH 5.5). Larvae containing anti-coagulant were placed back on ice for 2 min prior to 
piercing the integument above the head using a 27-gauge hypodermic needle. Haemolymph was collected into pre-chilled, sterile micro-centrifuge tubes. The number of microspheres in the haemolymph/faeces was enumerated using a fluorescent microscope (Olympus Bx43f).

\subsubsection{Data handling}

All experiments were completed in triplicate on three separate occasions (refer to individual figure legends for sample sizes). Results are expressed as mean \pm SE unless stated otherwise. Data were handled in GraphPad PRISM v8 and analysed using one- or two-way ANOVAs (or Kruskal-Wallis) with Tukey's multiple comparison (post-hoc) tests. Statistical significance was determined when $P \leq 0.05$. 


\subsection{Results}

\subsubsection{Larval development across nutraceutical-enhanced diets}

Regardless of the food recipe, standard or supplemented with nutraceuticals, larvae continued to grow and weighed significantly more with time (Figures 3.1 and 3.2). The average weight of $G$. mellonella reared on colostrum-supplemented foods [10\% w/w] was $>200 \mathrm{mg}$, which equates to a 2.5 -fold $(>150 \%)$ increase over the 4-day period. Insects reared on the unaltered honey-based diet (control) increased in weight by 2 -fold $(\sim 104 \% ; P<0.0001)$, whereas those reared on the $C$. sinensis diet $[10 \% \mathrm{w} / \mathrm{w}]$ were 1.8 -fold heavier $(\sim 85 \% ; P<0.0001)$. The combined supplement, $5 \%$ C sinensis $+5 \%$ colostrum $[\mathrm{w} / \mathrm{w}]$, resulted in the second highest gain of $\sim 120 \%$ (2.2 fold; $P=0.0221$ ) (Figure 3.3). These data indicate that the colostrumenhanced food may have greater nutritional value, containing more carbohydrates and protein than the standard food or the $C$. sinensis enhanced food (Table 3.1). There appears to be a dosedependent increase in larval weight when exposed to colostrum, full [10\%] colostrum supplement versus the combined [5\%/5\%] colostrum/Cordyceps. Weight gain alone is not sufficient to assign health benefits to nutraceuticals, so further studies on larval development and susceptibility to infection were performed.

The trend of increased weights of larvae (Figure 3.3) could not be used as a predictor of metamorphosis at day 7, i.e., numbers of pupating (or cocooned) larvae (Figure 3.4). Overall, diet supplementation appeared to significantly influence pupation levels (Kruskal-Wallis test; $\mathrm{P}=0.0286$ ). At $\sim 16 \%$, pupation levels of the colostrum/Cordyceps combination were significantly less than the standard diet (28.6\%), or individual supplements (25-33\%). Enhancing the food with $C$. sinensis results in much smaller larvae but a higher rate of metamorphosis, however the $5 \%$ each of $C$. sinensis/colostrum resulted in a much lower rate of metamorphosis than $C$. sinensis alone. 


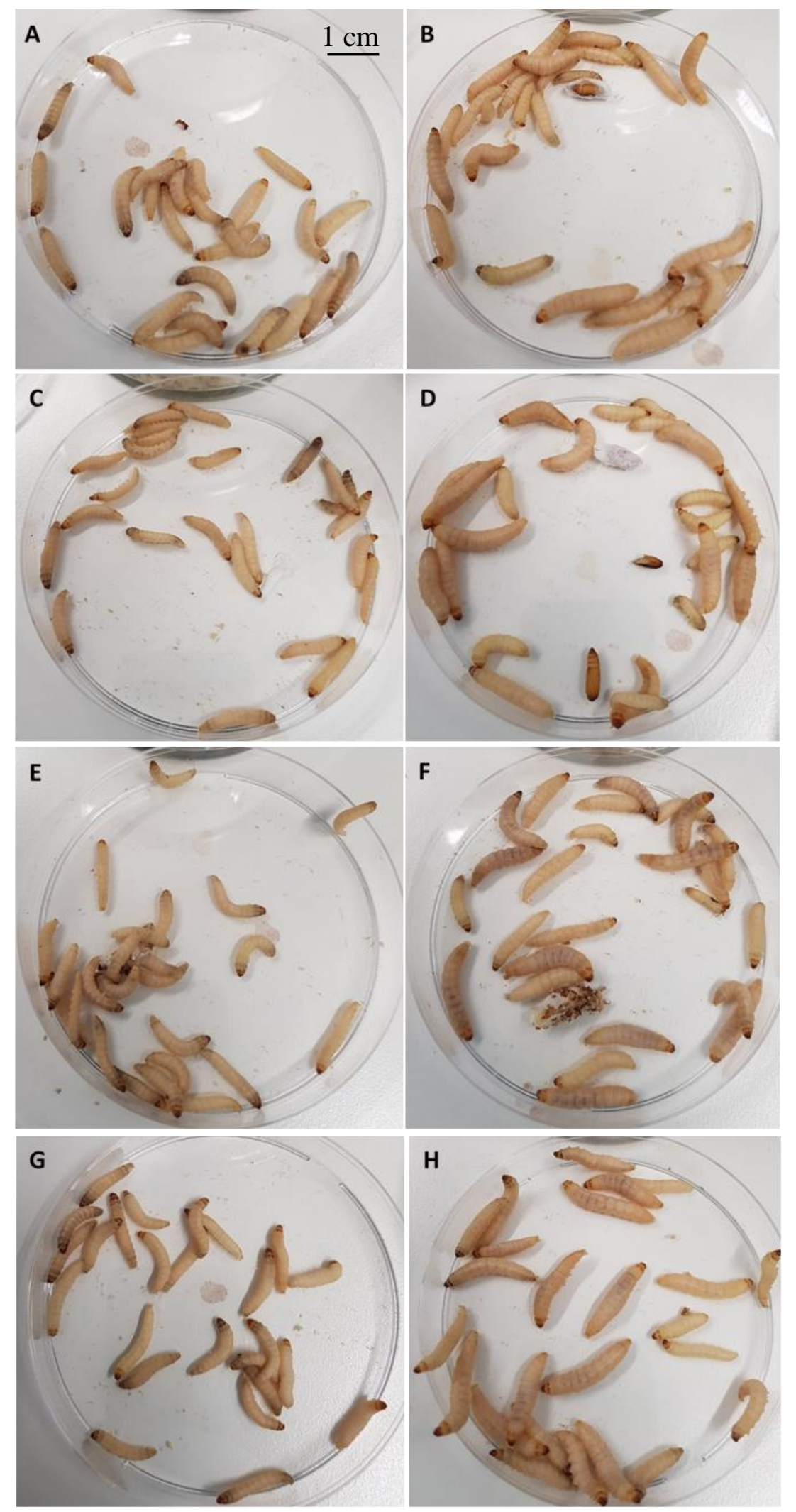

Figure 3.2 Representative Galleria mellonella larvae before (left panels) and after (right panels) rearing on nutraceutical enhanced food. Normal diet (A and B), 10\% colostrum diet (C and $\mathrm{D}, 10 \%$ C. sinensis diet $(\mathrm{E}$ and $\mathrm{F}), 5 \%$ C. sinensis $+5 \%$ colostrum combined diet $(\mathrm{G}$ and $\mathrm{H})$. 


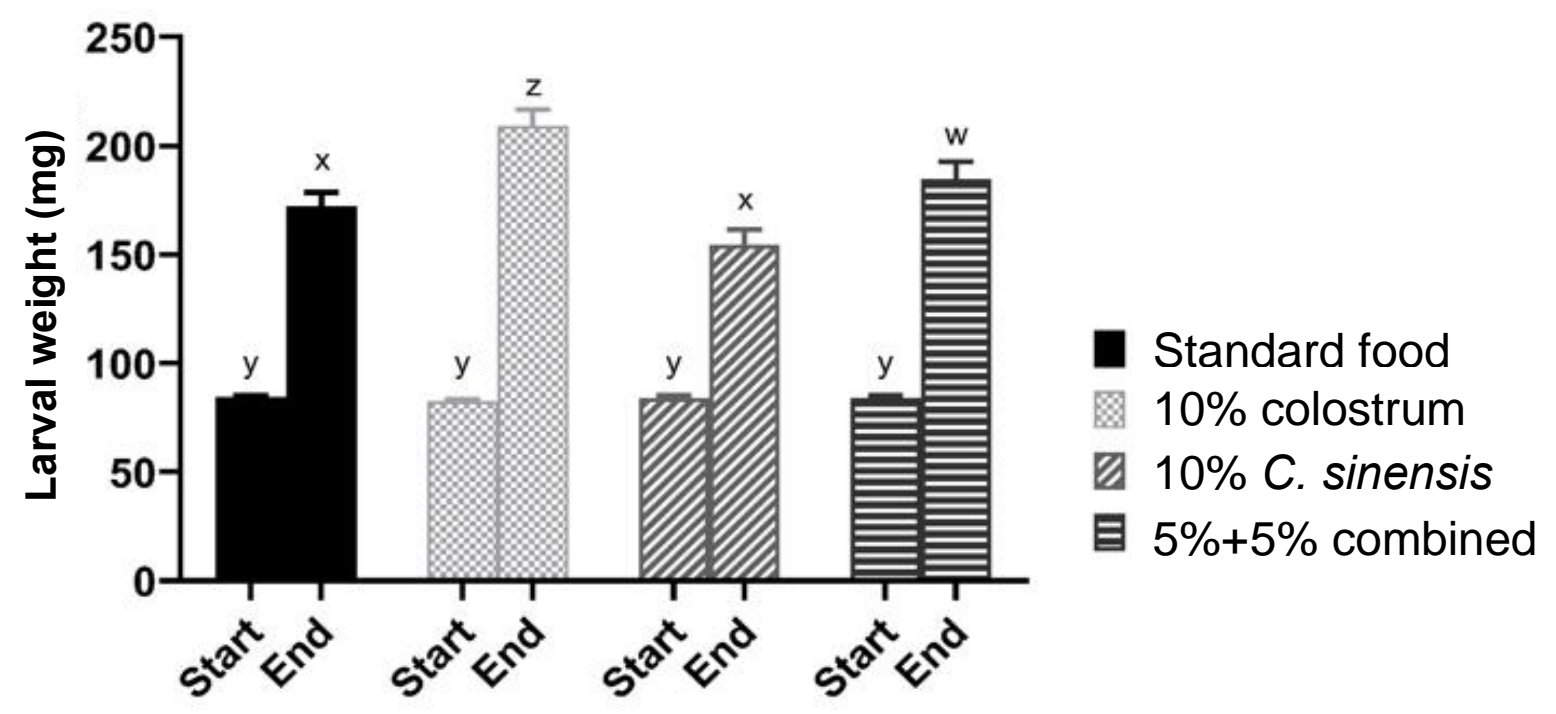

Figure 3.3 Weights of Galleria mellonella larvae after 4 days of being reared on nutraceuticalenhanced food. (10\% colostrum, 10\% Cordyceps sinensis and 5\% colostrum with 5\% C. sinensis). Larvae were maintained in darkness at $30{ }^{\circ} \mathrm{C}$. Values are expressed as mean $\pm \mathrm{SE}$ $(n=60$ per treatment, 240 in total). Unshared letters represent significant differences determined using Tukey's post-hoc tests.

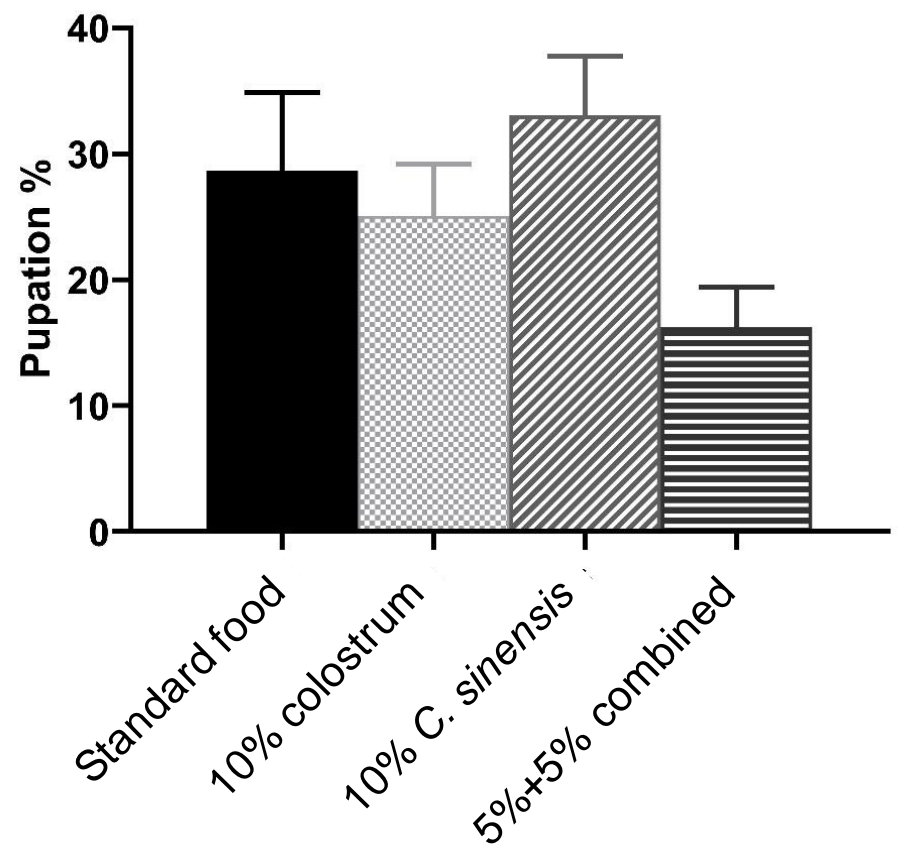

Figure 3.4 Pupation levels of Galleria mellonella larvae after 7 days of being reared on nutraceutical-enhanced food. (10\% colostrum, 10\% Cordyceps sinensis and 5\% colostrum with $5 \%$ C. sinensis). Larvae were maintained in darkness at $30{ }^{\circ} \mathrm{C}$ for 7 days. Values are expressed as mean $\pm \operatorname{SE}(n=400$ per treatment, 1,600 in total $)$. 


\subsubsection{Larval development and survival after nutraceutical inoculations}

To investigate further the effects of nutraceuticals on G. mellonella, larvae were directly forcefed a range of $C$. sinensis or colostrum extracts across a concentration range of $0.5-8 \mathrm{mg}$ (protein) $/ \mathrm{ml}$ (PBS), which is 5 to $80 \mu \mathrm{g} /$ larva (or, $20-320 \mathrm{mg} / \mathrm{kg}$ ). As demonstrated in Chapter 2 with indomethacin (Figure 2.2), fewer larvae were pupating by day 10 after force-feeding regardless of the dose or nutraceutical used (Figure 3.5A and 3.5C). It is worth noting that the force-feeding approach appears to have some non-target effects (this is touched upon with relation to 'flushing' the gut in Chapter 4). Although, the $6 \mathrm{mg} / \mathrm{ml} \mathrm{C}$. sinensis treatment was determined to be significantly different to the untreated larvae by day 10 (50\% versus $93 \%$, respectively), there was no clear dose-dependent response. For example, 73\% had pupated when exposed to the highest dose of $8 \mathrm{mg} / \mathrm{ml}$ (Figure 3.5A). The majority of variation in the data for both $C$. sinensis and colostrum can be attributed to time (58\% and 71\%, respectively), whereas treatment accounts for $9.7 \%$ to $<20 \%$.

Both doses of 6 and $8 \mathrm{mg} / \mathrm{ml}$ of $C$. sinensis extract decreased larval survival by 3-7\% (Figure 3.4B), but overall, survival was not significantly negatively affected by $C$. sinensis $\left(X^{2}(8)=\right.$ 9.21, $\mathrm{P}=0.3246)$ or colostrum (100\% survival; Figure 3.5D) inoculations. 
A

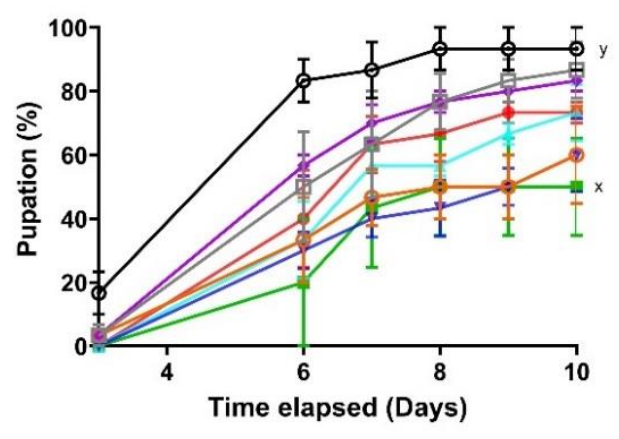

C
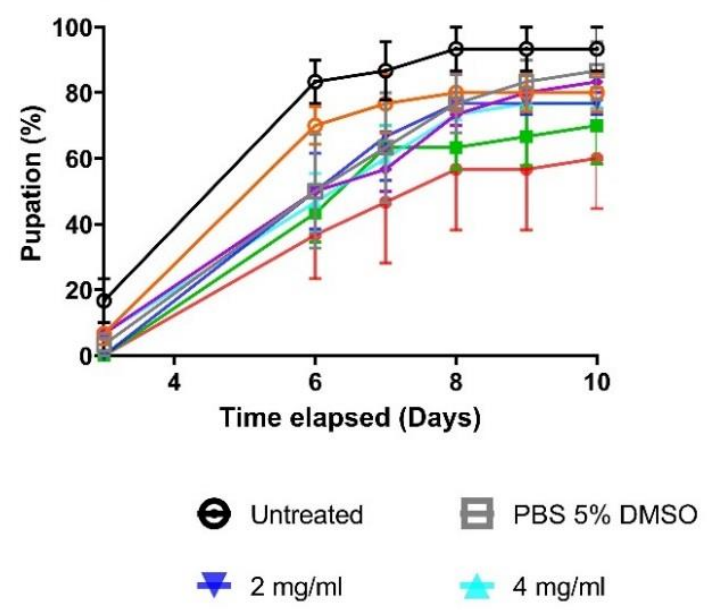

B

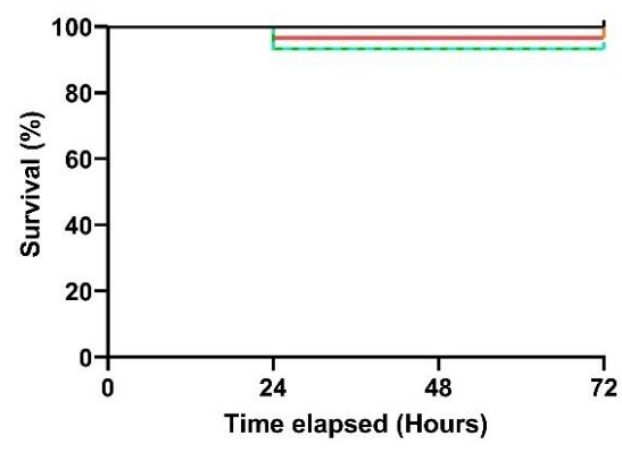

D

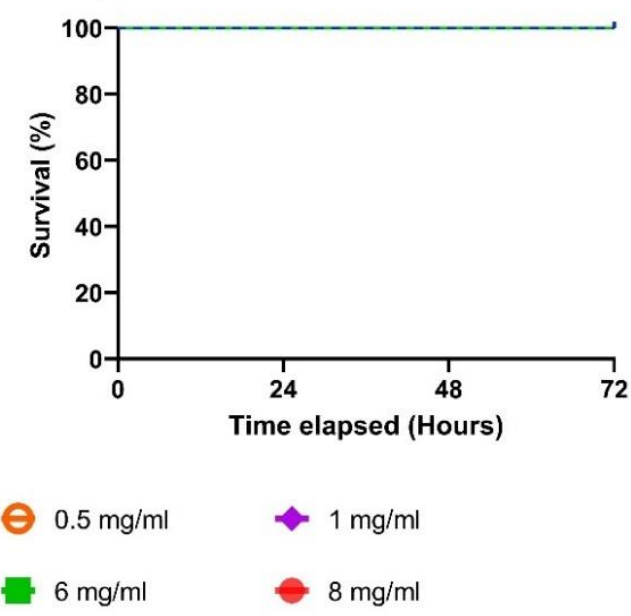

Figure 3.5 Galleria mellonella larval development and survival post-nutraceutical forcefeeding. Larvae were force-fed $0.5 \mathrm{mg}$ to $8 \mathrm{mg}$ protein per $\mathrm{ml}$ (PBS) of Cordyceps sinensis (A and B) and colostrum (C and D). Larvae were maintained in darkness at $30^{\circ} \mathrm{C}$ for $72 \mathrm{~h}$. Insects that were unresponsive to being rolled over or prodded were considered dead. Values are expressed as mean $\pm \operatorname{SE}$ ( $n=30$ per treatment, 420 in total). It should be noted that direct forcefeeding of colostrum or PBS+5\% [v/v] DMSO (control) did not lead to any insect mortality. 


\subsubsection{Larval development and survival after pharmaceutical inoculations}

To investigate if nutraceuticals have any protective properties against pharmaceutically induced gastric damage in G. mellonella (in addition to indomethacin; Chapter 2), a further assessment of NSAIDs known to cause gastric damage, and a common demulcent (lansoprazole) known to protect against gastric inflammation, was conducted for added value. As observed previously (Figure 3.5), force-fed larvae had an overall reduction in pupation rates compared to the untreated control (Figure 3.6A, C, and E), however, the smallest effect was observed in lansoprazole-treated larvae (Figure 3.6A). The only apparent significant difference in development between untreated and Lansoprazole treated was at day 6 using $2.5 \mu \mathrm{g} / \mathrm{larva}(\mathrm{P}$ $=0.0033)$ (Table B1 - Appendix). This is possibly due to damage that may have been caused by the operator's handling at the lower dose.

Both NSAIDs, aspirin and diclofenac salt, had significantly reduced development from Day 6 onwards compared to the untreated control (Figure 3.6 C and E) - determined from ANOVA and Tukey's post-hoc tests. Importantly, no discernible dose-dependent impact on development was recorded. The lowest pupation level, when force-fed diclofenac salt, was observed at 5 $\mu \mathrm{g} /$ larva with an average of $50 \%$ by day 10 compared to the control of $96 \%(\mathrm{P}=0.0009)$. However, this dose was not significantly different to any other NSAID dose or the negative control (PBS alone), in which 76\% of larvae had pupated by day 10 (Table B1 - Appendix and Figure 3.6). Larvae force-fed aspirin demonstrated the lowest rate of pupation with $56 \%$ positive by day 10 in those inoculated with $2.5 \mu \mathrm{g} /$ larva $(2.5 \mu \mathrm{g} /$ larva versus control; $\mathrm{P}=0.0113$ ). Although not statistically significant at the highest concentration of $25 \mu \mathrm{g} / \mathrm{larva}$, pupation was $7 \%$ higher on average than larvae inoculated with $2.5 \mu \mathrm{g} / \mathrm{larva}$.

For NSAIDs, the highest dose of $10 \mu \mathrm{g} /$ larva reduced survival by $17 \%$ for aspirin and $7 \%$ for diclofenac by 72 hours (Figure 3.6). However, increasing the dose of aspirin to $25 \mu \mathrm{g} / \mathrm{larva}$ led to $100 \%$ survival; therefore, although survival was reduced overall $\left(X^{2}(6)=21.25 ; \mathrm{P}=0.0017\right)$, no dose-dependent trend was observed $\left(X^{2}(1)=1.308 ; \mathrm{P}=0.253\right)$. Survival trends for and diclofenac were also found to be non-significant $\left(X^{2}(6)=5.944 ; \mathrm{P}=0.43\right)$ and $X^{2}(61)=2.0 ; \mathrm{P}$ $=0.157$, respectively) . 

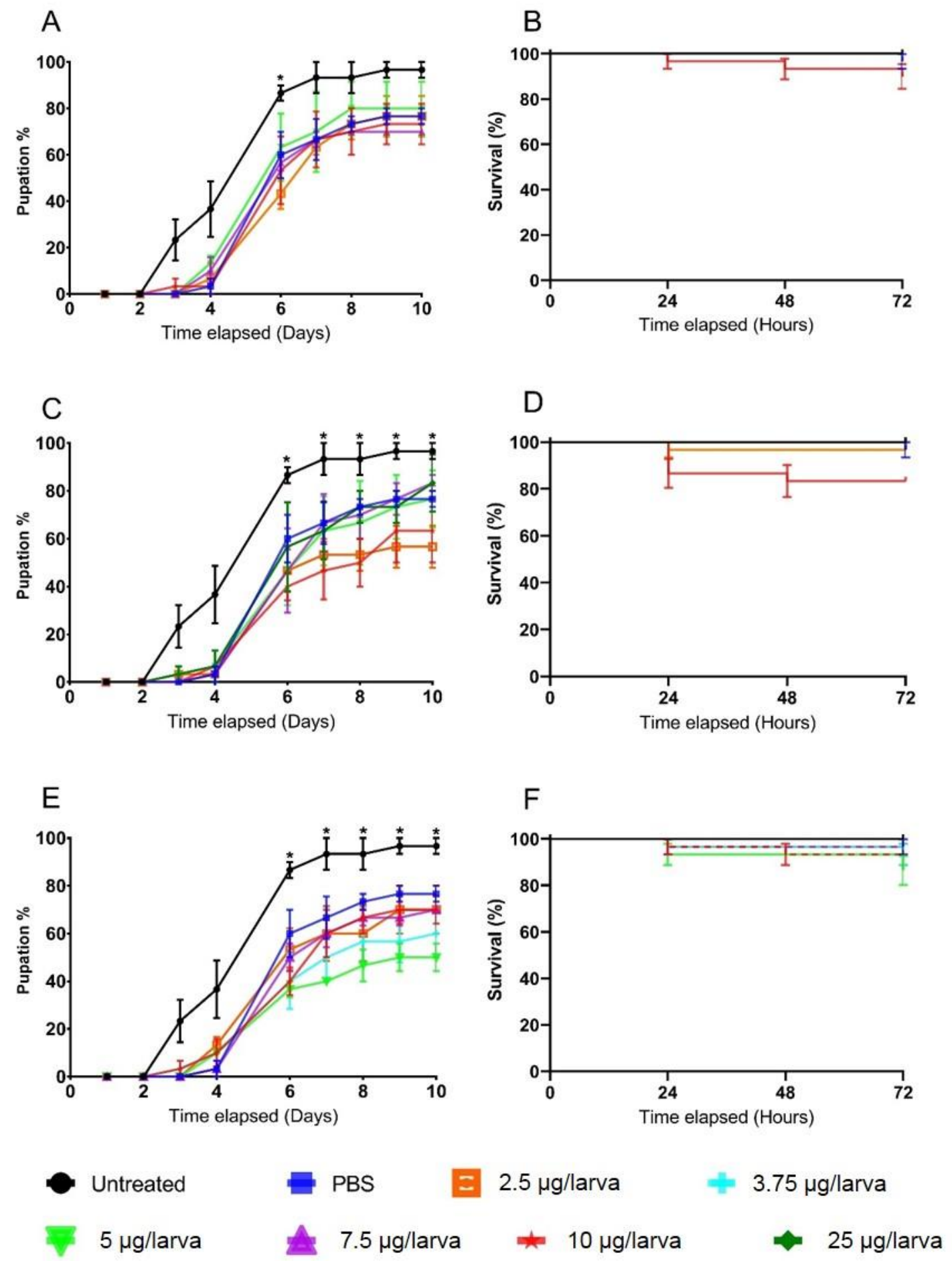

Figure 3.6 Galleria mellonella larval pupation (A, C and E) and larval survival 0-10 days post force-feeding (B, D and F). [Lansoprazole (A, B), Aspirin (C, D) and Diclofenac sodium (E, F)]. Larvae were maintained at $30{ }^{\circ} \mathrm{C}$ in darkness for 10 days post inoculation. The number of larvae undergoing pupation was recorded. Values are expressed as mean $\pm \mathrm{SE}(\mathrm{n}=30$ per treatment, 480 in total). Symbol: $* \mathrm{P}<0.03$ when comparing untreated to all other treatments at the respective time points. The negative control consisted of force-feeding the insect with PBS containing 5\% [v/v] DMSO. 


\subsubsection{Effect of nutraceuticals on maintaining larval health}

No additional value was identified in replacing indomethacin (Figures 2.1 and 2.2) with aspirin or diclofenac salt based on the insect survival and pupation data here (Figure 3.6), so indomethacin $(7.5 \mu \mathrm{g} / \mathrm{larva})$ was selected to investigate the putative repair properties of nutraceuticals. Lansoprazole $(7.5 \mu \mathrm{g} / \mathrm{larva}, \sim 30 \mathrm{mg} / \mathrm{kg})$ was also established to be a suitable (non-toxic) dose to compare to the potency of a pharmacological agent to the benefits of nutraceuticals.

Larvae that were maintained on nutraceutical enriched foods were force-fed either indomethacin alone, lansoprazole alone, a co-inoculation of indomethacin and lansoprazole, or PBS (5\% DMSO), and assessed for health (described in Table 3.1). Indomethacin treatment returned similar health indices for all rearing diets with the standard diet resulting in marginally higher average scores of 2.7 for activity and 3.7 for melanisation, followed by the combined colostrum/Cordyceps diet $(5 \%+5 \%)$, which scored 2.7 for activity and 3.6 for melanisation (Figure 3.7). Larvae force-fed a combination of lansoprazole and indomethacin when reared on the standard food, displayed significant decreases in both activity $(2.5 ; P=0.0047)$ and melanisation $(3.2 ; P=0.0018)$ levels at 72 hours compared to the untreated control, which scored 3 and 4, respectively (Figure 3.67A). Surprisingly, the combination of indomethacin/lansoprazole led to the largest reduction in activity and melanisation - which may suggest that insects cannot handle the chemical load on their regular diet. Moreover, the PBS (negative control) inoculum did lead to decreases in activity and melanisation indices (e.g., Figure 3.7A, B, C) in line with indomethacin alone.

Importantly, the co-inoculation of indomethacin and lansoprazole resulted in higher average health indices for both melanisation and activity over the 72 hours period compared to indomethacin alone for all supplemented foods (Figure 3.7 C to $\mathrm{H}$ ). Larvae maintained on the combined diet of colostrum/Cordyceps (Figure $3.7 \mathrm{G}$ and $\mathrm{H}$ ) appeared to be more resilient to indomethacin across both indices when compared to $10 \%$ of either nutraceutical alone - this may indicate a synergistic effect of the two combined but at heal the dose each. 

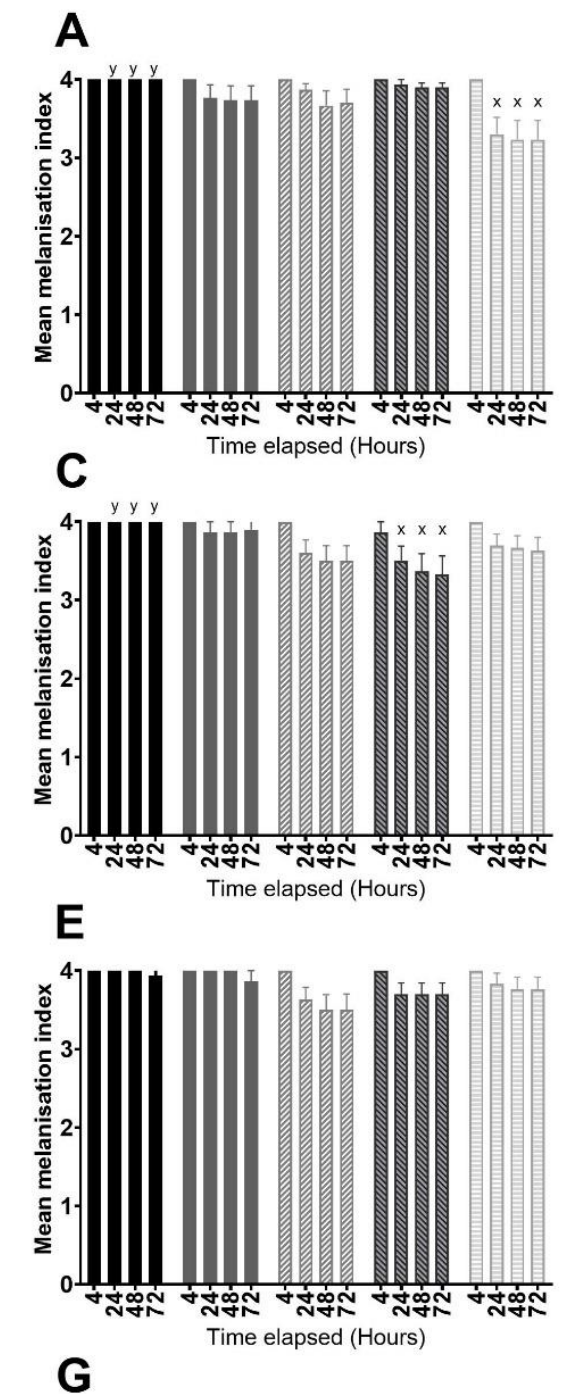

G
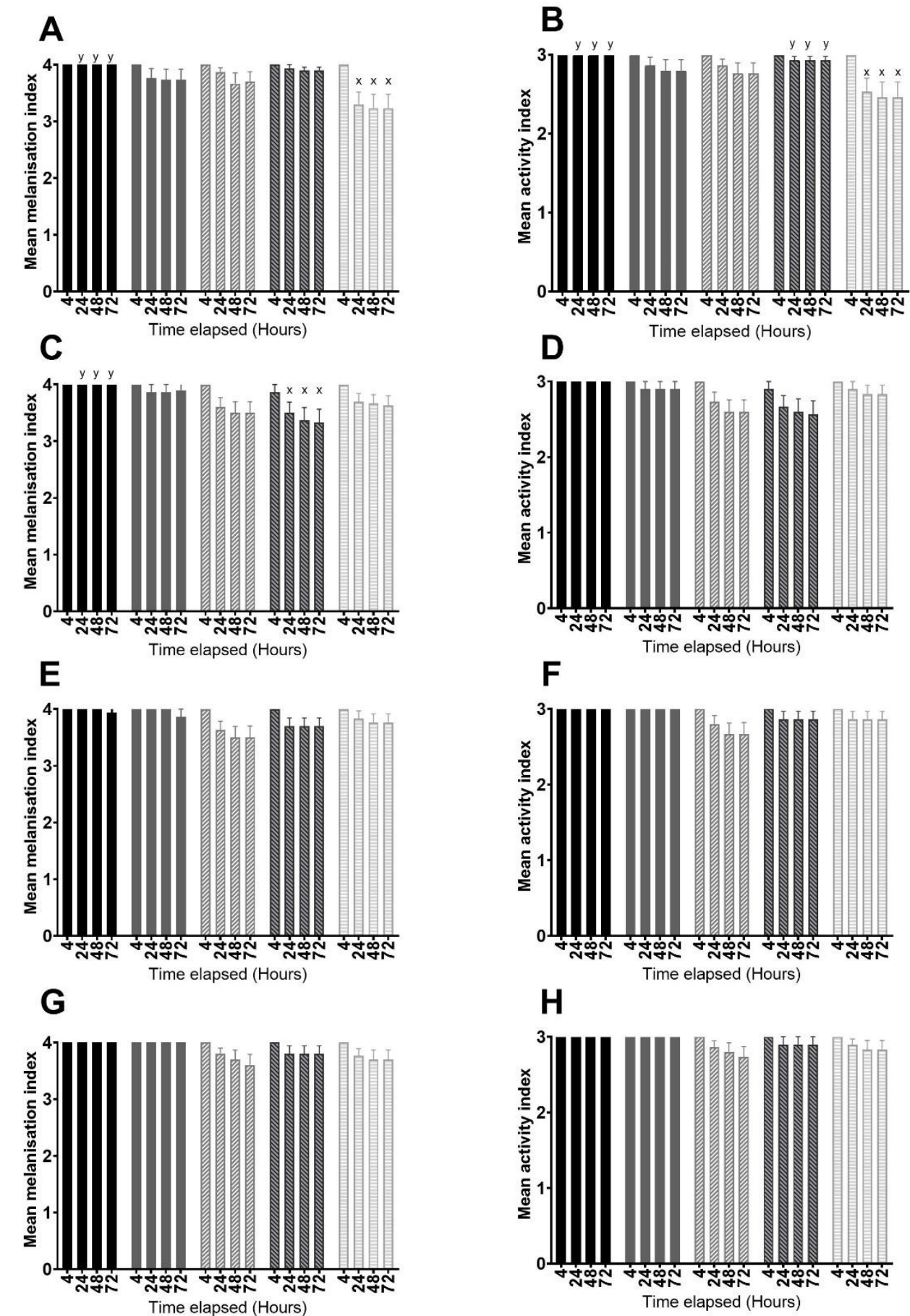

Untreated PBS (5\% DMSO)
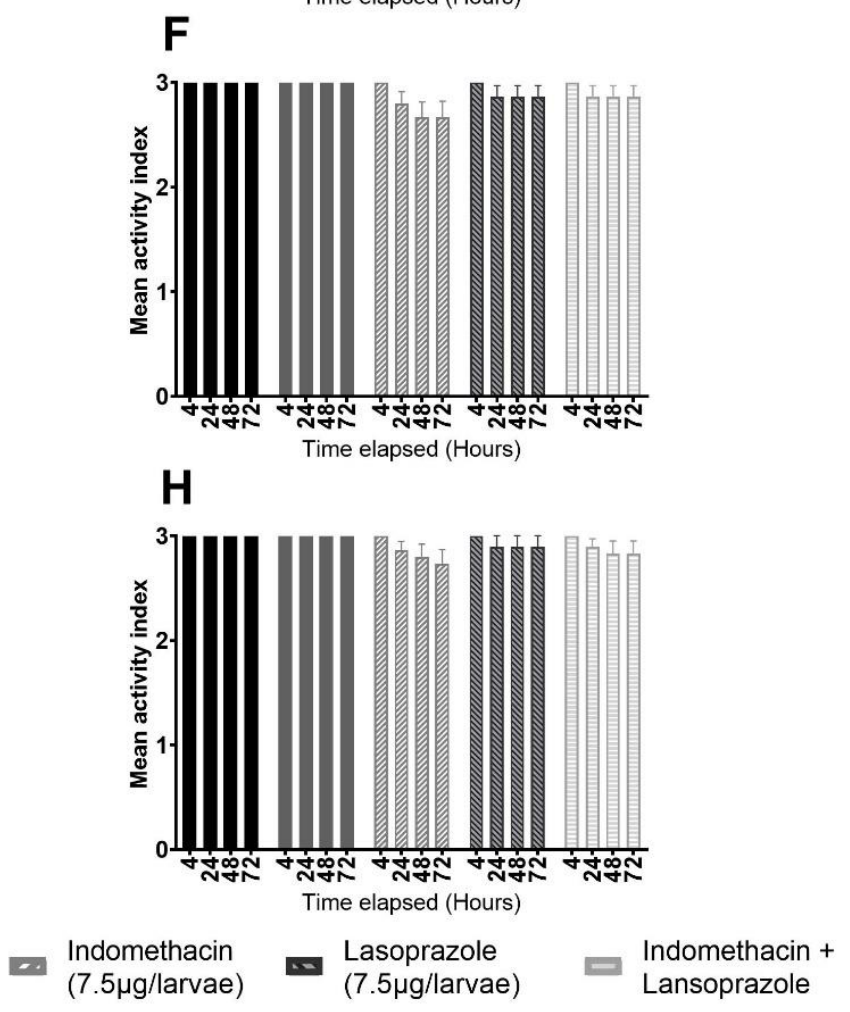

Figure 3.7 Health indices of Galleria mellonella larvae reared on nutraceutical-enhanced foods and exposed to indomethacin and/or lansoprazole. Larvae were reared on normal food (A and B), 10\% colostrum enhanced (C and D), 10\% Cordyceps sinensis enhanced (E and F) and 5\% colostrum with 5\% C. sinensis enhanced ( $\mathrm{G}$ and $\mathrm{H}$ ). Larval health was scored (Table 3.1) by assessing larvae movement (A, C, E and G), melanisation (B, D, F and H). Values are presented as mean $\pm \operatorname{SE}(n=30$ per treatment, 600 total). Unshared letters represent significant differences $(\mathrm{P} \leq 0.05)$ determined by Tukey's multiple comparisons tests. The negative control consisted of force-feeing larvae with PBS (+5\% DMSO). Unshared letters represent statistical differences. 


\subsubsection{Effect of nutraceuticals on improving gut permeability}

As observed in chapter 2 , indomethacin can increase gut permeability through tissue damage (Figure 2.6), therefore it was important to assess if nutraceuticals could tackle the negative impacts of indomethacin on the gut. Larvae reared on the standard diet had more microspheres ( $6 \mu \mathrm{m}$ and $2 \mu \mathrm{m}$ ) detectable in the haemolymph at $4-24$ hours after indomethacin force-feeding compared to the PBS control, equating to an increase of $\sim 1 \times 10^{5}$ to $1.5 \times 10^{5}$ microspheres (Figure $3.8 \mathrm{~A}$ and B). For all treatments, the highest number of spheres present in the haemolymph occurs within the first 4 hours (for $6 \mu \mathrm{m}$ ) and 24 hours (for $2 \mu \mathrm{m}$ ), suggesting the initial damage allows spheres to become displaced from the gastrointestinal tract. Larvae reared on each enhanced diet had a more gradual flow of $6 \mu \mathrm{m}$ microspheres into the haemolymph over the 72 hours, and crucially, no measurable differences were detected in the numbers of haemolymph microspheres in any nutraceutical diet in the absence or presence of indomethacin (Figure $3.8 \mathrm{C}, \mathrm{G}, \mathrm{E}$ ). These data indicate that the nutraceuticals may have a positive impact on gut resilience to NSAID-associated leakage. This could also suggest that the nutraceuticalsenhanced diets are superior to the standard diet used in this chapter, and the bait-shop larvae used in Chapter 2 (Figure 2.6).

Larvae inoculated with indomethacin had large variation of $6 \mu \mathrm{m}$ spheres found in the faeces for all treatments (Appendix B, figure B3). However, larvae that were reared on $10 \% \mathrm{C}$. sinensis had a peak of 87,500 microspheres/larva $(6 \mu \mathrm{m})$ in the faeces at 24 hours post inoculation, whereas other indomethacin force-fed larvae peaked at 48 hours. Furthermore, larvae that were reared on $10 \%$ C. sinensis and inoculated with PBS peaked at 48 hours $\left(1.2 \times 10^{5}\right.$ microspheres/larva), whereas all other larvae inoculated with PBS had a gradual increase from 4-48 hours followed by a peak at 72 hours (see Appendix B). This could indicate that the $C$. sinensis enhanced diet increases the rate of defecation in larvae (possibly through increased gut motility (Table B2, B3 a and B3 b-Appendix B).

Larvae inoculated with $2 \mu \mathrm{m}$ spheres and indomethacin had higher numbers of microspheres in the haemolymph for the first 24 hours than those inoculated with PBS. Larvae maintained on a standard diet prior to indomethacin inoculation, had the highest number of spheres in the haemolymph for the first 24 hours, $\sim 2.8 \times 10^{5}-3 \times 10^{5}$ per larva, with a gradual decline over the following 48 hours (Figure 3.8B). Larvae maintained on a $10 \%$ colostrum or the $5 \% / 5 \%$ combined diets had fewer microspheres in the haemolymph at 4 hours $\left(2.4 \times 10^{5}-2 \times 10^{5}\right.$ microspheres/larva), similar in number to larvae inoculated with PBS $\left(2 \times 10^{5}-1.7 \times 10^{5}\right.$ microspheres/larva) (see Figure 3.8 D, F, H). 
A

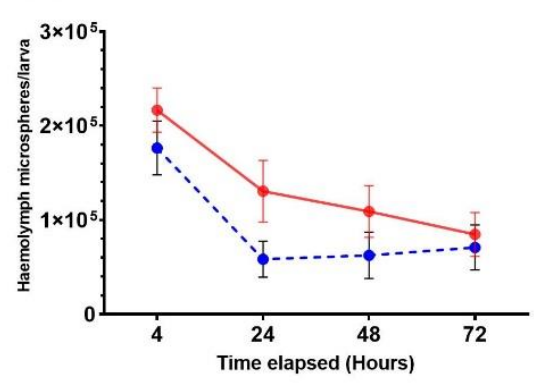

C

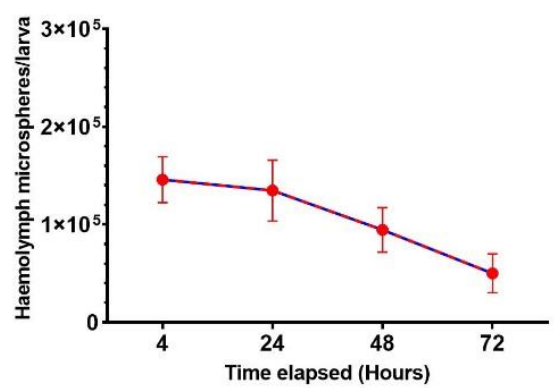

$E$

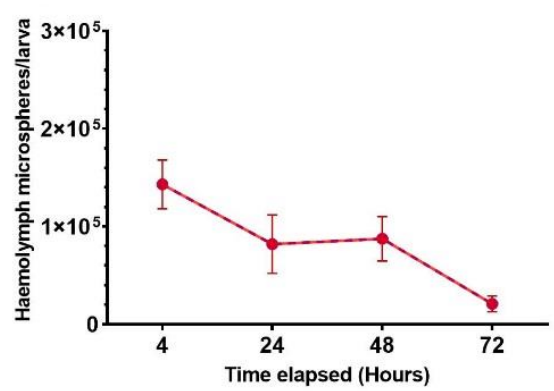

G

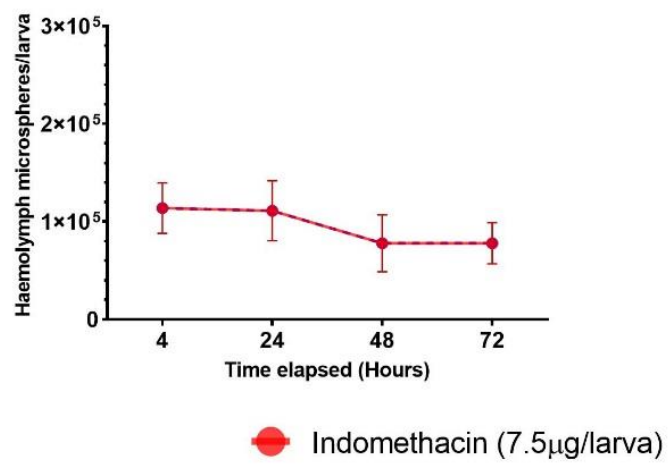

B

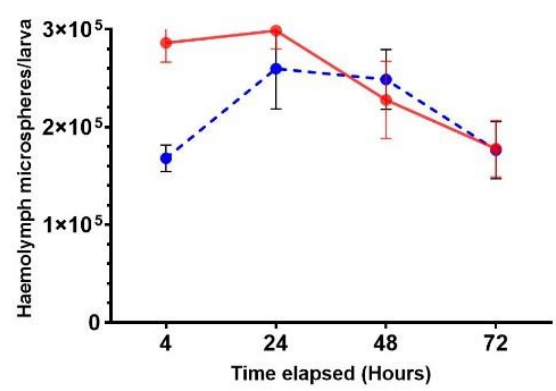

D

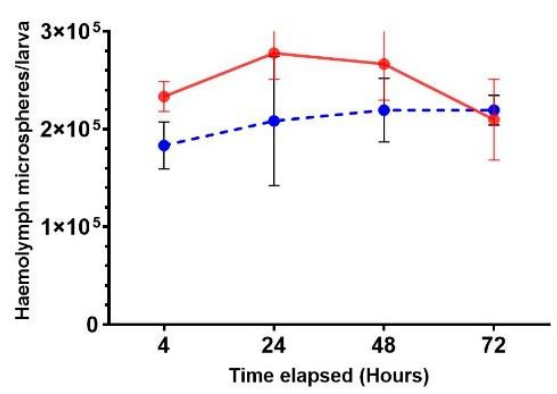

$\mathbf{F}$

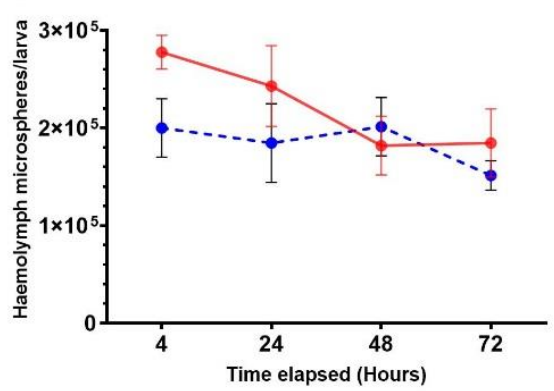

H

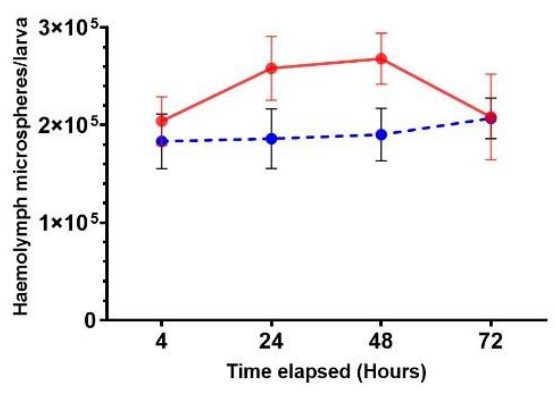

PBS (5\% DMSO)

Figure 3.8 Gut permeability of Galleria mellonella larvae reared on standard (A and B) and nutraceutical supplemented diets: $10 \%$ Colostrum (C and D), 10\% C. sinensis (E and F) and $5 \%$ C. sinensis with $5 \%$ colostrum $(\mathrm{G}$ and $\mathrm{H})$. Larvae were force-feed indomethacin $(7.5 \mu \mathrm{g})$ or PBS (control). Permeability was determined by the number of $6 \mu \mathrm{m}$ (A, C, E, G-left panels) and $2 \mu \mathrm{m}(\mathrm{B}, \mathrm{D}, \mathrm{F}, \mathrm{H}$ - right panels) spheres found in the haemolymph between 4 and $72 \mathrm{~h}$ post-inoculation. Each larva was co-inoculated with $1 \times 10^{6}$ microspheres and indomethacin or PBS and incubated in the dark at $30^{\circ} \mathrm{C}$. Data represent the mean $\pm \operatorname{SE}(n=192,768$ in total across both microsphere sizes). The negative control consisted of force-feeding the insect with PBS (+5\% DMSO) in the absence of indomethacin ( $0 \mu \mathrm{g} / \mathrm{larva})$. 
Cumulatively (over the 72 hour experimental period), the total number of $6 \mu \mathrm{m}$ spheres found in the haemolymph of larvae reared on a standard diet followed by indomethacin inoculation was $5.4 \times 10^{5}$ - compared to $\sim 3.7 \times 10^{5}$ microspheres/larva when inoculated with PBS alone (Figure 3.9A). This indicates indomethacin enhanced leakiness by $>40 \%$. A total of $1.67 \times 10^{5}$ microspheres/larva were found in the faeces of those reared on the standard diet and exposed to indomethacin, which is similar to the $1.70 \times 10^{5}$ microspheres (faecal load) found in PBSinoculated larva (Figure B1 - Appendix).

The fewest number of $6 \mu \mathrm{m}$ spheres found in the haemolymph was from larvae reared on $10 \%$ C. sinensis enhanced food, $\sim 3.3 \times 10^{5}$ microspheres/larva for both indomethacin and PBS inoculated larvae (Figure 3.9 E). Larvae reared on this diet and inoculated with PBS had the most $6 \mu \mathrm{m}$ spheres found in the faeces with a total average of $2.4 \times 10^{5}$, compared to indomethacin-treated where a total of $1.7 \times 10^{5}$ microspheres/larva (Figure $3.9 \mathrm{E}$ ). It is interesting to note that larvae reared on Cordyceps-supplemented diets and exposed to $2 \mu \mathrm{m}$ spheres (Figure $3.9 \mathrm{~F}, \mathrm{H}$ ), had consistently more spheres detected in the haemolymph and faeces compared to the PBS controls. Larvae reared on the combined nutraceutical diet (Figure $3.9 \mathrm{G})$, resulted in almost identical numbers of $6 \mu \mathrm{m}$ spheres between the treatment and control (in the haemolymph and faeces). 
A

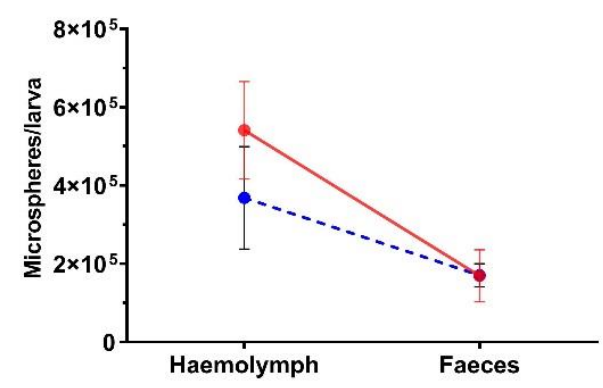

C

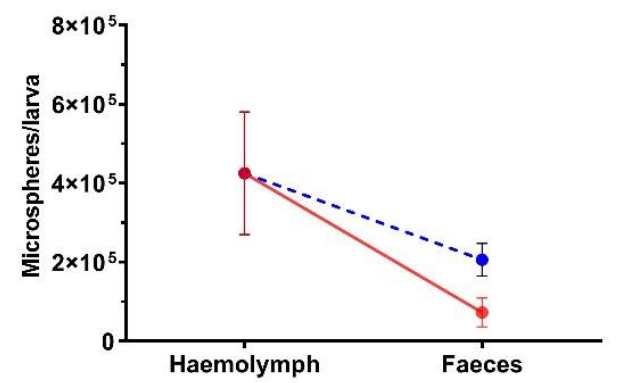

E

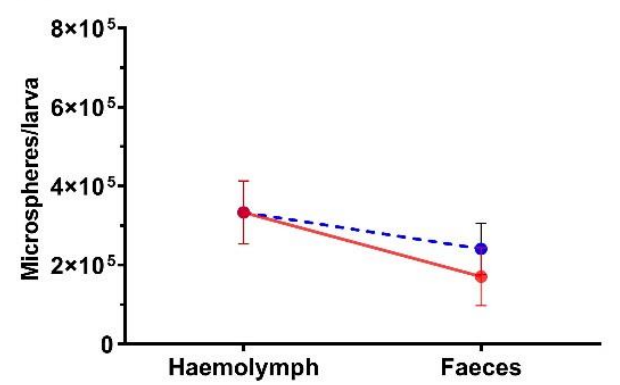

G

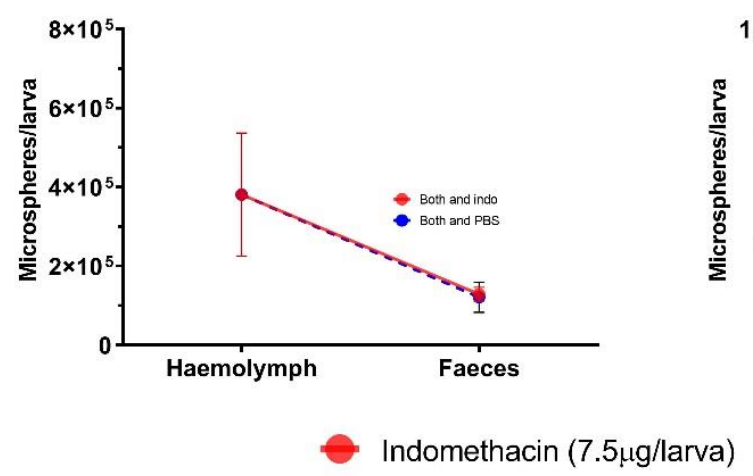

B

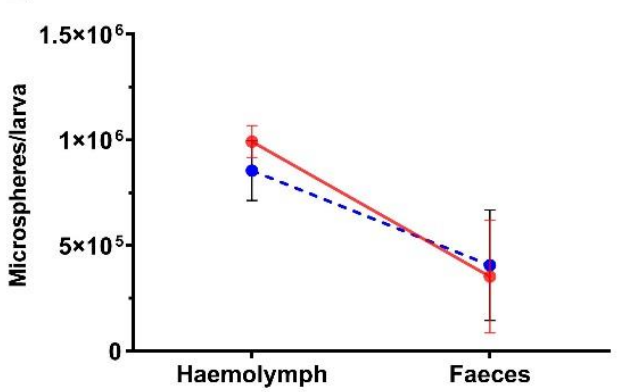

D

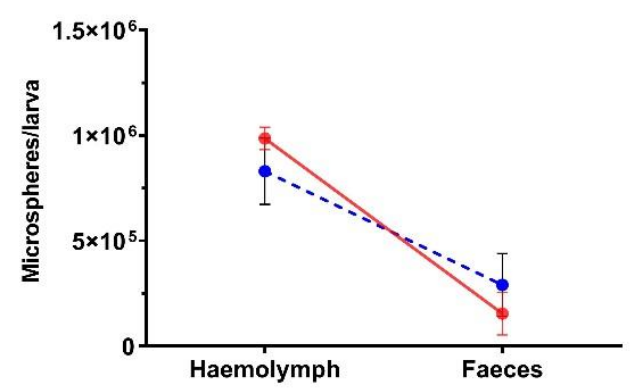

$\mathbf{F}$

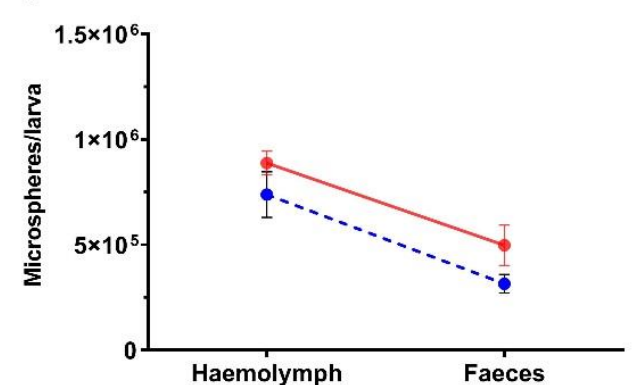

H

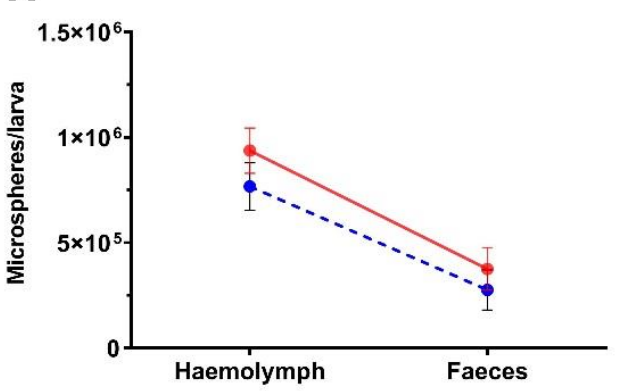

Figure 3.9 Total microsphere loads of Galleria mellonella reared on standard (A and B) and nutraceutical supplemented diets; $10 \%$ Colostrum (C and D), 10\% C. sinensis (E and F) and $5 \%$ C. sinensis with 5\% colostrum (G and $\mathrm{H})$. Larvae were force-fed either $6 \mu \mathrm{m}(\mathrm{A}, \mathrm{C}, \mathrm{E}, \mathrm{G}$ -left panels) or $2 \mu \mathrm{m}$ (B, D, F, H - right panels) with indomethacin or PBS (control). The data presented is the total microspheres recovered across the 72-hour period displayed in Figure 3.8. There is an inverse correlation between microsphere diameter and haemolymph load - the larger a microsphere is the less likely it will make its way through the gut wall and into the body cavity (haemocoel). 


\subsubsection{Impact of nutraceuticals on Campylobacter jejuni infection outcomes}

Insect larvae - reared on all diet regimes - were exposed to Campylobacter jejuni and assessed for health outcomes (according to Table 3.1). Increasing the dose of C. jejuni from 1 to $3 \times 10^{6}$ CFUs per larva led to a dose-dependent deterioration in health - regardless of inoculation route (Figure 3.10).

Larvae reared on either the standard (Figure 3.10a A-C and Figure 3.10b A-C) or Cordyceps/colostrum combined (Figure 3.10a J-L and Figure 3.10b J-L) diets were most sensitive to the bacterium $\left(3 \times 10^{6} \mathrm{CFU} /\right.$ larva) when force-fed, resulting in lower activity values (1.8 and 1.23, respectively), melanisation levels (1.43 and 0.93 , respectively) and overall survival (average $\sim 63 \%$ and $\sim 33 \%$ at 72 hours, respectively). Ostensibly, the least impacted group were those reared on the 10\% Cordyceps diet (Figure 3.10a and b G-I), survival levels were $>65 \%$, whereas the other diets, $10 \%$ colostrum and 5\%-each Cordyceps/colostrum had reduced survival rates between $33 \%$ and $50 \%$ by 72 hours (Figure $3.10 \mathrm{a}$ and b, F and I).

Force-feeding the gut-specific pathogen leads to higher survival rates, up to $80 \%$, in the single supplemented diets (10\% colostrum; Figure 3.10a F and 10\% C. sinensis; Figure 3.10a I). These larvae also appear to be more sensitive to intrahaemocoelic injection of the gut pathobiont - having lower activity $(\sim 1.5$ and $\sim 2.1)$ and melanisation indices $(\sim 1.3$ and $\sim 1.7)$ when compared to those force-fed. The opposite trend is observed for the standard and combined Cordyceps/colostrum diets where the lowest survival rates were recorded when the pathogen was force-fed (Figures 3.9C and 3.9L, respectively). This is also the case for their activity and melanisation levels. These data are intriguing as they suggest investment in fortifying the gut may be at the expense of haemolymph-based defences (i.e., a trade-off).

Although C. sinensis reared larvae are more resistant to $C$. jejuni when administered orally, it is nearly as resistant to injection as the normal diet larvae, having activity index of just 0.13 lower and survival of $7 \%$ lower, they also have a melanisation index of 0.7 higher than those on a normal diet and are not statistically different from one another. Therefore, larvae on a normal diet and a $C$. sinensis diet may have similar immune defence but larvae reared on $C$. sinensis could have a more resistant gut to infection than on a normal diet although not statistically significant.

[For a complete list of statistical outputs, see Appendix Table B2 
A
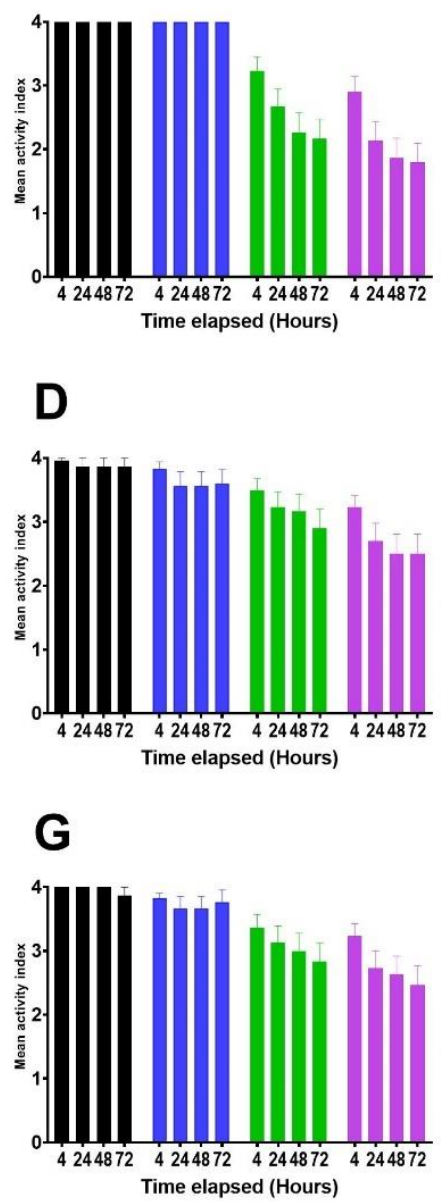

J

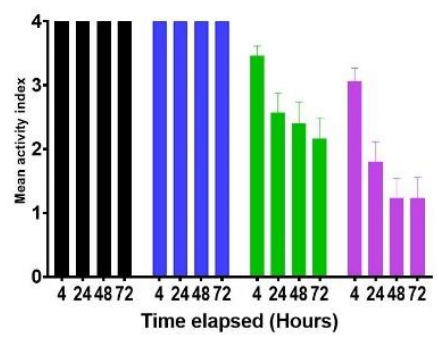

B

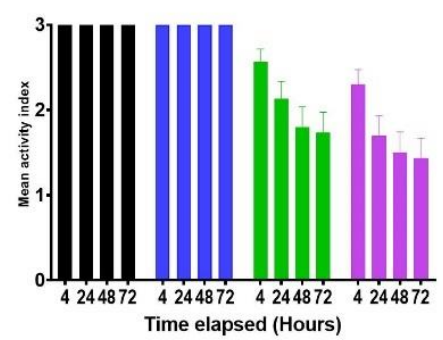

E
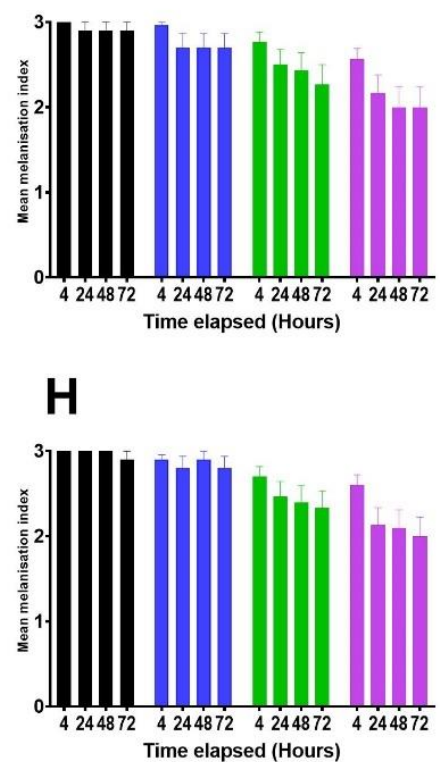

K

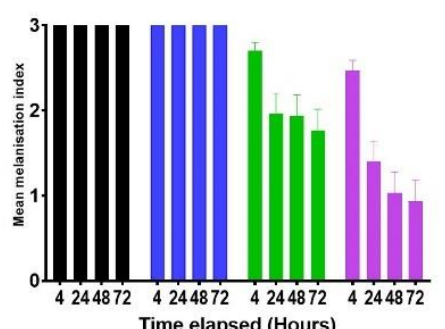

Time elapsed (Hours)

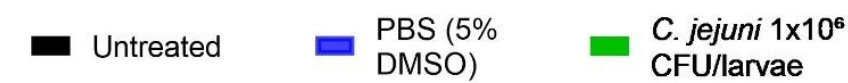

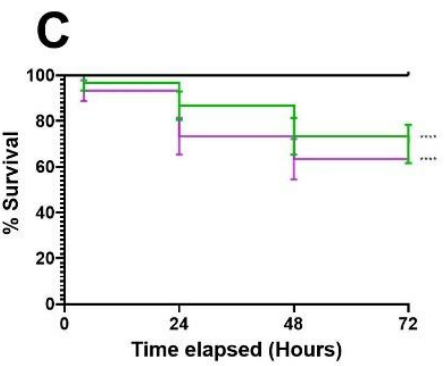

F

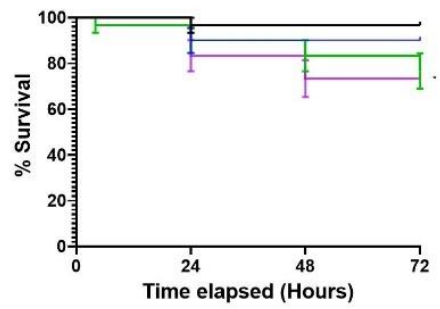

I

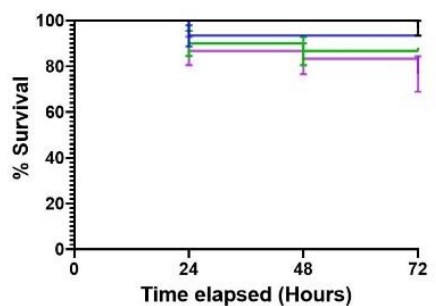

L

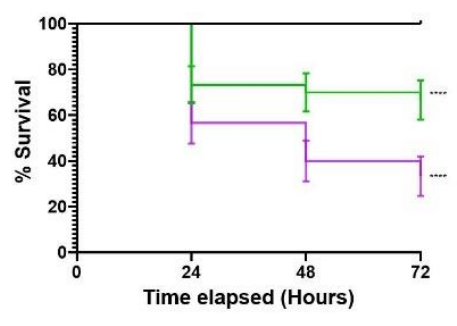

C. jejuni $3 \times 10^{6}$

CFU/larvae

Figure 3.10a Health indices of Galleria mellonella larvae reared on supplemented food and force-fed with Campylobacter jejuni $\left(1 \times 10^{6}\right.$ and $\left.3 \times 10^{6} \mathrm{CFU} / \mathrm{larva}\right)$. Larvae were reared on standard food (A, B and C), 10\% colostrum (D, E, and F), 10\% Cordyceps sinensis (G, H, I) or 5\% each Cordyceps/colostrum combined (J, K, and L). Larvae were maintained subsequently in darkness at $30{ }^{\circ} \mathrm{C}$ for 72 hours. Larval health was recorded using movement (A, D, G and J), melanisation (B, E, H and K), and viability (C, F, I and L). Values are expressed as mean $\pm \mathrm{SE}$ ( $n=30$ per treatment, 390 in total across both inoculation methods). The negative control consisted of force feeding the insect with PBS containing 50\% Muller Hinton broth $[\mathrm{v} / \mathrm{v}]$. 
A
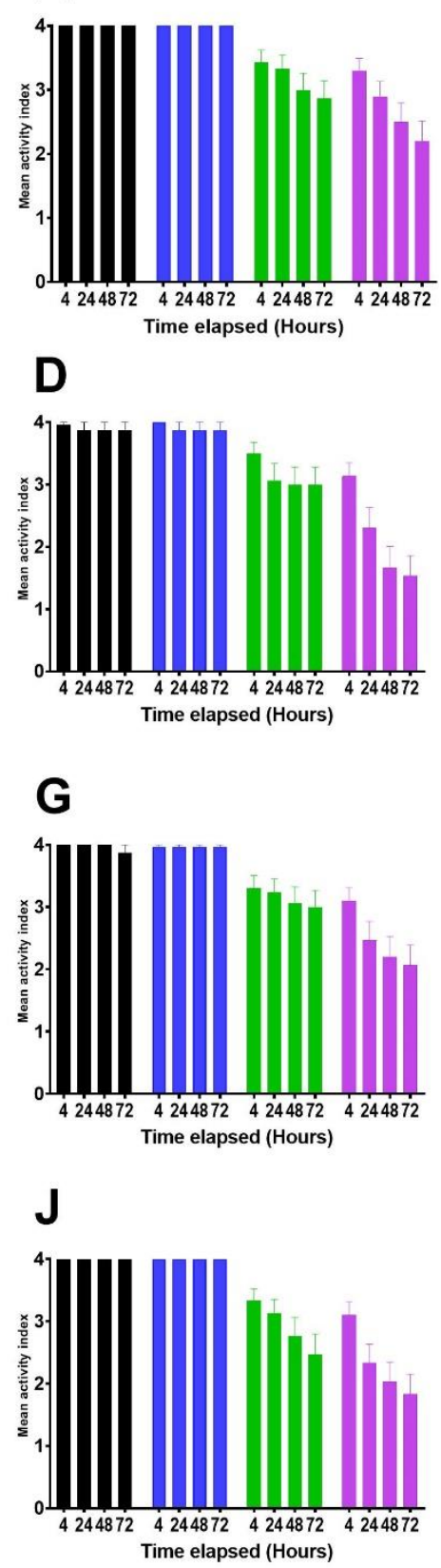

Untreated
B

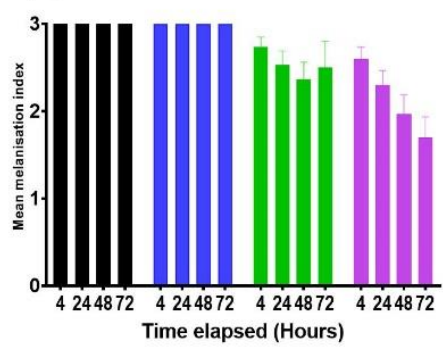

E

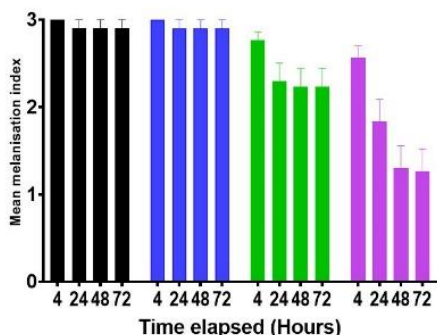

H

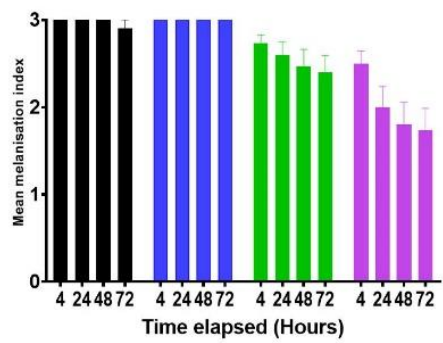

K

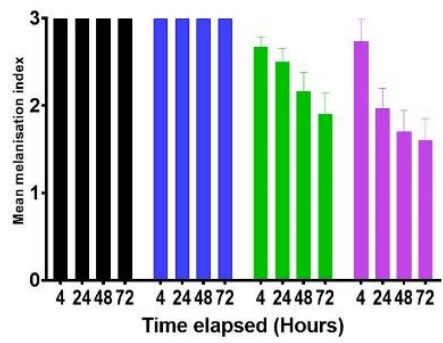

Time elapsed (Hours)

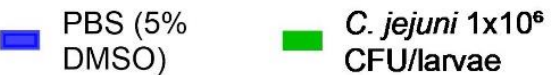

\section{C}

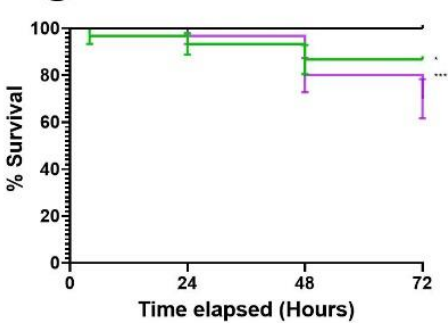

F
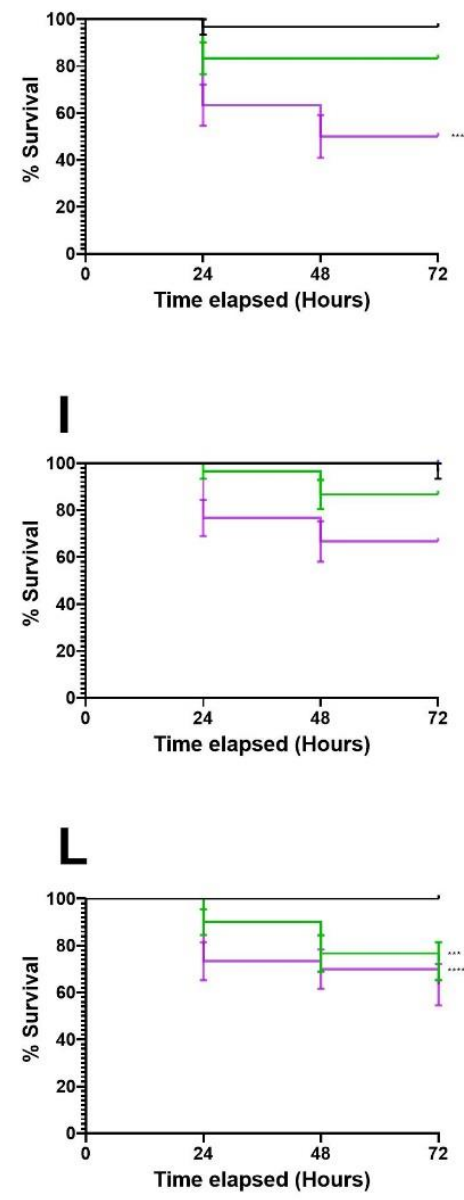

C. jejuni $3 \times 10^{6}$

CFU/larvae

Figure 3.11b Health indices of Galleria mellonella larvae reared on supplemented food and injected with Campylobacter jejuni $\left(1 \times 10^{6}\right.$ and $3 \times 10^{6}$ CFU/larva). Larvae were reared on standard food (A, B and C), 10\% colostrum (D, E, and F), 10\% Cordyceps sinensis (G, H, I) or $5 \%$ each Cordyceps/colostrum combined (J, K, and L). Larvae were maintained subsequently in darkness at $30{ }^{\circ} \mathrm{C}$ for 72 hours. Larval health was recorded using movement (A, D, G and J), melanisation (B, E, H and K), and viability (C, F, I and L). Values are expressed as mean $\pm \mathrm{SE}$ ( $n=30$ per treatment, 390 in total across both inoculation methods). The negative control consisted of injecting the insect with PBS containing 50\% Muller Hinton broth [v/v]. 


\subsection{Discussion}

Rat models have been used successfully to characterise the protective and reparative properties of colostrum and $C$. sinensis against NSAID-induced gastric damage (Marchbank et al., 2011). Herein, the putative therapeutic properties of nutraceuticals, $C$. sinensis and colostrum extracts, were assessed using an insect model (G. mellonella) for the first time.

NSAIDs are prescribed for managing arthritis and musculoskeletal injuries, however long term use of causes about $4 \%$ of patients to develop gastrointestinal injuries such as ulceration (MacDonald et al., 1997, Paulus, 1988, Langman et al., 1985). Lansoprazole is a proton pump inhibitor often used alongside an NSAID to prevent GI damage (Higuchi et al., 2009, Maity et al., 2008). Specifically, indomethacin-induced damage in rats can be abated by lansoprazole attributed to a reduction in oxidative stress of the mucosa (Kuroda et al., 2006, Yoda et al., 2010). There is some literature describing localised inflammation (colitis) and diarrhoea associated with lansoprazole treatment ( 3-8\%), but these are rare (Matsukawa et al., 1997, Mukherjee, 2003, Sugano et al., 2012). Therefore, it is preferable to find other treatments to assist in managing NSAID induced gastric damage. The neutralising effects of lansoprazole on indomethacin were not apparent in insects reared on the standard or colostrum-supplemented diets. Surprisingly, the co-inoculum of indo/lansop led to significantly reduced activity and melanisation indices (Figure 3.7A and B).

Lansoprazole, as with most proton pump inhibitors, requires protonation to become activated therefore activates in an acidic environment where it goes on to interact with cysteine residues Cys813 and Cys321 of the membrane transporters (Shin and Kim, 2013, Bosnjak et al., 2019). This is a potential limitation of using G. mellonella larvae as a gastric model for assessing pharmaceutical protection as their gut is very alkali and unlikely to provide protonation for lansoprazole to activate. This could be why there is no significant improvement on larval health when co-inoculating with lansoprazole and indomethacin compared to indomethacin inoculation alone (Figure 3.7). An additional consideration of using G. mellonella and lansoprazole is its mode of action, and whether it targets Cys813 and Cys321 on relevant proteins (Cytryńska et al., 2007).

The relative toxicity of additional NSAIDs, aspirin and diclofenac acid, were assessed in $G$. mellonella larvae via gavage, with doses of $10 \mu \mathrm{g} / \mathrm{larva}(40 \mathrm{mg} / \mathrm{kg})$ resulting in a significant decrease in survival. Diclofenac administration produced similar results in larvae compared to indomethacin across the same dose range (Figures 3.5 and 2.1). Inspired by Chapter 2, by co- 
inoculating $6 \mu \mathrm{m}$ spheres with indomethacin, levels of gut leakiness were determined among insects reared on all diet regimes (Figure 3.8). Compared to the standard diet, larval guts appeared to be less leaky when reared on each nutraceutical-supplemented diet (Figure 3.8 and 3.8). For some of the supplemented diets, there were still higher levels of microspheres overall in control larval faeces versus indomethacin-treated (Figure $3.9 \mathrm{C}, \mathrm{E}$ ), but numbers recovered from the haemolymph showed no differences between treatment and control. From Chapter 2, it was apparent that phagocytes targeted microspheres displaced into the haemolymph (Figure 2.7), and the smaller the microsphere the longer they will take to make their way through the GI tract and be defecated (Figures 2.8 and 2.9).

Larvae were inoculated directly with increasing concentrations of nutraceutical extracts (standardised to protein levels across $0-80 \mu \mathrm{g} /$ larva) to determine any potential toxic sideeffects. Beyond $60 \mu \mathrm{g} / \mathrm{larva}$, the Cordyceps extract appeared mildly toxic - reducing survival up to $7 \%$ at 24 hours, but no further mortalities were recorded. Perhaps some residual bioactive factors are present in the extract and may be antagonising the insect. Cordyceps spp. are distinct entomopathogens, and some species like $C$. militaris are associated with necrotic haemocyte death, oxidative imbalance, and developmental arrest (Kryukov et al., 2015, Kryukov et al., 2020). The latter observation is interesting as doses of 60 and $80 \mu \mathrm{g} / \mathrm{larva}$ resulted in the lowest pupation levels of those insects exposed to either colostrum or Cordyceps ( $<50 \%$ by day 10$)$, whereas untreated and PBS force-fed insect pupation levels reached $>90 \%$. That being said, rearing insects on Cordyceps-supplemented diets yielded similar levels of pupation compared to larvae reared on the standard and colostrum-supplemented diets (Figure 3.4). Previously, dipping leaves in methanol extracts of $C$. militaris and allowing larvae of diamondback moth (Plutella xylostella) to feed on them resulted in high mortalities rates (Kim et al., 2002). The insecticidal activity was attributed to cordycepin - a 3'-deoxyadenosine - found to be in high concentration in the extracts. Due to the intimate host-pathogen interactions described for some insects and Cordyceps spp., future studies using Cordyceps extracts for nutraceutical assessments should proceed with caution in this non-mammalian model.

Rearing larvae on diets supplemented with nutraceuticals led to weight gain, with colostrum proving superior (Figure 3.3), and differential rates of development (i.e., pupation; Figure 3.3) with the combined colostrum/Cordyceps [5\%-5\%] diet yielding the fewest pupated larvae. This is possibly due to the presence of growth factors in colostrum as well as the increased levels of protein and carbohydrates present in colostrum (Table 3.1). Previous studies have described so-called 'melanic' G. mellonella that have evolved enhanced resistance to fungal infection by 
investing in frontline immune defences (thicker cuticles, higher levels of enzyme activity), however, these defences have a direct cost - melanic larvae are smaller, have a reduced lifespan and produce fewer offspring (Dubovskiy et al., 2013, Grizanova et al., 2019). Here, larvae reared on a Cordyceps-supplemented diet weighed the least (Figure 3.4) but demonstrated enhanced resistance to infection from $C$. jejuni, when administered by force-feeding. Conversely, larvae reared on the same diet are more susceptible to infection by intrahaemocoelic injection - this would suggest that fortifying the gut due to the perceived threat of Cordyceps signals in the diet, may have a trade-off with size and/or haemolymph based defences. By using both intrahaemocoelic injection and force-feeding, it allows the assessment of the dietary effects on susceptibility to infection. When injecting directly into the haemocoel, the assessment focuses on the immune response against $C$. jejuni. Conversely, when $C$. jejuni is delivered via force-feeding the experiment focusses on the overall defence to infection, including the physical defence of the gut and subsequent immune response. After rearing on a $C$. sinensis supplemented diet, the increased susceptibility to $C$. jejuni via intrahaemocoelic injection, and a reduced susceptibility when force-fed, therefore by comparing the results from inoculation methods it appears the $C$. sinensis extracts benefit the gut but does not give an overall enhanced defence to infection. $C$. jejuni can be cultured between $30-44{ }^{\circ} \mathrm{C}$ and has been studied using several in vivo models, including both $G$. mellonella land rats, making it a suitable pathogen to assess protective properties of nutraceuticals against bacterial infection (Senior et al., 2011). Rearing larvae on a single nutraceutical diet, either colostrum or $C$. sinensis, seemed to have prophylactic effects when force-fed an NSAID (known to cause gastric ulcers) and an enteric pathogen (C. jejuni). In fact, larvae raised on both diets containing Cordyceps extract (Figure $3.7 \mathrm{E}-\mathrm{H}$ ) were the least sensitive to chemical damage (indomethacin), based on activity and melanisation endpoints.

Colostrum has been identified as a potent nutraceutical, which can provide protection against gastrointestinal bacteria-induced septic shock in rats and to reduce the detrimental side effects of common NSAIDs in long-term pain sufferers (Playford et al., 2000, Playford et al., 2001, Playford et al., 1999). The apparent benefits observed in larvae did vary, with colostrum increasing larval weight and reducing some of the impact of $C$. jejuni infection on health indices (Figure 3.3 and 4.9). This may be due to the increased protein and carbohydrate content in colostrum compared to the fungal extract. Additionally, colostrum contains growth hormones which encourage damage repair. Interestingly, G. mellonella larvae reared on a low nutritional diet resulted in expedited pupation (Coskun et al., 2006). In contrast, work conducted in $G$. 
mellonella larvae infected with the entomopathogenic fungus, Beauveria bassiana, reported that larvae on a low nutrition diet were more resistant to the fungal infection compared to the high nutritional diet (Kangassalo et al., 2015).

A final point to note is that NSAID usage is linked intimately with disease outcomes of clinical importance, e.g., Campylobacter (formerly Helicobacter) pylori and group A streptococcal infections (Bryant et al., 2015, Stack et al., 2002). As G. mellonella has been proven to be a suitable host for studying the infectivity of such pathogens (Olsen et al., 2011, Loh et al., 2013, Giannouli et al., 2014), and NSAID-related damages (Emery et al., 2019), they could be used to for high- throughput screening nutraceutical and other antimicrobial blends prior to testing in rodents.

\subsection{Concluding remarks}

Common NSAIDs and nutraceuticals used in the study of gastroenterology in rats and humans appear to have similar broad symptoms of damage and repair seen in $G$. mellonella larvae either by gavage or by raising larvae on supplemented diets. Both colostrum and $C$. sinensis extracts had positive impacts on larval health compared to a standard diet or control inoculum (PBS), but $C$. sinensis was superior when assessing resistance to oral infection using a gut specific pathogen, i.e., C. jejuni.

Considering insects like G. mellonella are usually targeted by intact Cordyceps spp., it is necessary to investigate further how active compounds from fungal extracts of this traditional Chinese medicine could be leading to gut-fortification, and is it a similar mechanism to the therapeutic properties observed in vertebrates. Do these prophylactic properties extend to food poisoning toxins or idiopathic gastropathy?

\section{Acknowledgements}

I would like to thank Mr Ryan Poole (Bluefish Research Intern) and Mr James Taylor (ARCHUK Hub Manager) at Swansea University for assistance and advice with insect rearing. 


\section{Chapter 4: Relative toxicity and cytopathology of the emerging marine toxins, Azaspiracids, screened in Galleria mellonella larvae}

A version of this chapter is in preparation for publication:

Helena Emery, Jane Kilcoyne, Philipp Hess, Andrew F. Rowley, Christopher J Coates. (20xx). Resolving the relative toxicities and cytopathology of Azaspiracids 1, 2 and 3 in a nonmammalian insect model, Galleria mellonella.

* JK and PH provided toxins; AFR assisted with histology. 


\subsection{Abstract}

Azaspiracids are a collection of marine lipophilic biotoxins that accumulate in edible shellfish tissues. Human consumption of contaminated shellfish leads to a gastrointestinal disorder referred to as azaspiracid shellfish poisoning - symptoms include nausea and stomach cramps. The cellular targets of azaspiracids are not well defined, and further compounded by a high rate of false negatives when screened in mice. In the following chapter, Galleria mellonella larvae were tested as an alternative toxicology model to determine the (1) differential toxicities of azaspiracids (AZA1 - AZA3), (2) their pathobiological effects, and (3) whether pre-exposure to a sublethal toxin dose enhances susceptibility to gastric infection (e.g., Campylobacter jejuni). A comparison is also made between AZA1 and the causative agent of diarrheic shellfish poisoning, namely okadaic acid. Physiologically relevant amounts of azaspiracids (5 - 200 ng/larva; $20-800 \mu \mathrm{g} / \mathrm{kg}$ ) were administered via intrahaemocoelic injection (subcutaneous) and gavage (force-feeding). Health indices (survival, movement, and melanisation), tissue histology, and gut microbiome analysis (16S profiles) were used to assess intoxicated larvae between 4- and 96-hours. Each toxin - regardless of the route of inoculation, impacted larval survival negatively. Histopathology of the midgut depicted epithelial degeneration and cell death, including distinct pathologic symptoms between AZA1 and okadaic acid. A sub-lethal dose of AZA-1 (25 ng/larva) enhanced susceptibility to infection by the Gram-negative bacterium $C$. jejuni. Herein, the reader will find evidence that G. mellonella larvae can distinguish between the different azaspiracid congeners, and offer novel insight into the mechanism(s) of action of these toxins. 


\subsection{Introduction}

The azaspiracids (AZA) are a group of cyclic, polyether toxins produced by the dinoflagellate genera Azadinium and Amphidoma. These dinoflagellates are distributed globally, and are associated with $>40$ AZA analogues (Pelin et al., 2018). During bloom events, AZA1 and AZA2 are released routinely and accumulate in the tissues of commercially important shellfish, predominantly bivalves (reviewed by Hess et al., 2015), but crustacean (edible crab Cancer pagurus) intoxication has also been recorded (Torgersen et al., 2008). A study in bivalves found that during blooming events, the hosts become immune suppressed and more susceptible to disease and subsequent infection. It is possible that bioaccumulation of the toxins produced in blooms could affect negatively the responsiveness of the immune cells to subsequent infection (Lassudrie et al., 2020). Human consumption of contaminated shellfish can lead to a gastrointestinal disorder known as azaspiracid shellfish poisoning (AZP), which manifests as diarrhoea, stomach cramps and vomiting. Although these symptoms are similar to those caused by diarrheic shellfish poisoning (DSP), AZP pathobiology is distinct; necrosis and erosion of the small intestine, neurotoxicity, fatty acid build-up in the liver, and lymphopenia in the spleen (Ito et al., 2000; Abal et al., 2017). It is understood that a disruption to the intestinal epithelial layer increases the susceptibility to infection (Antonissen et al., 2014), therefore it is possible that even a transient disruption to the intestines cellular organisation could increase the chance of subsequent infections. Accordingly, AZP represents a substantial threat to human health and financial risk to shellfish producers. AZA-1, 2 and 3 are the only analogues subsumed by existing regulation (EFSA, 2008; FSA(UK), 2018) - the other 40 AZA congeners are considered metabolic derivatives.

The mode of action of azaspiracids is currently unknown; some studies have shown AZA-2 to inhibit human Ether-à-go-go-Related Gene (hERG) and a hERG trafficking effect on cell surfaces. hERG encodes cardiac potassium channels and is considered to be one of the modes of action resulting in arrythmias and cardiotoxicity in rat models (Ferreiro et al., 2014). Using Caco-2 cells to study AZA-1, the toxin appeared to target steps in glycolysis and the Krebs cycle, resulting in an increased NADH concentration within cells, and eventually, cell death (Bodero et al., 2018). AZA are often compared to okadaic acid (OA), another lipophilic toxin that accumulates in edible shellfish tissues and causes diarrheic shellfish poisoning (DSP). OA is produced mainly by the dinoflagellate genera Prorocentrum and Dinophysis (Franchini et al., 2010). OA consumption causes diarrhoea, vomiting, stomach cramps and chills within 4 hours of exposure - leading to the inhibition of serine-threonine phosphatases, and in extreme 
cases, the erosion of the small intestine and oedema (Dounay and Forsyth, 2002, Ito et al., 2002b). OA inhibition of serine-threonine phosphatases 1 and 2A (PP1 and PP2A) disrupts phosphorylation and dephosphorylation events, which are important for maintaining gap junctions and regulating the permeability of the intestine (Dietrich et al., 2019).

The practice of using mice for general toxin detection (OA or AZA) is routine, involving tens of thousands of mice per year. Since 2011, the UK has moved towards highly sensitive mass spectrometry-based methods, however, rodents are still needed to characterise mechanisms of toxicity (cellular targets) and symptoms of intoxication because in vitro approaches do not provide this important information. As established in Chapter 2, wax moth larvae (Galleria mellonella) have increasingly replaced and reduced the need for mammals in infectious disease research for several reasons, including fewer ethical restrictions, experimental veracity and affordability. In a pilot study by Coates et al. (2019), okadaic acid lethality (LD 50 values), cytotoxicity and REDOX imbalance - measured through malondialdehyde (MDA) accumulation - were highly similar between insects (G. mellonella) and mice. Like OA, the permitted upper regulatory limit for AZP contamination of shellfish products is $160 \mu \mathrm{g}$ (equivalents) per kg tissue (EFSA, 2008; FSA(UK), 2018), and so, toxin doses were prepared across the range $5-200 \mathrm{ng} / \mathrm{larva}$ (i.e., $20-800 \mu \mathrm{g} / \mathrm{kg}$ ).

The aims of this chapter were to:

(1) Establish the sensitivity of insect larvae to distinguish between different congeners of closely related toxins (Azaspiracids 1-3) using two inoculation methods (force-feeding and injection) as achieved in mice. Both inoculation methods are utilised due to the natural method of intoxication through ingestion and the traditional method of screening via injection.

(2) Determine the extent of gut-specific damage induced by AZA1 compared to okadaic acid using a combination of tissue histopathology and microbial profiling (16S NGS sequencing)

(3) Assess the effects of low-dose (sub-lethal) toxin exposure, and subsequent susceptibility to a human gut-specific pathogen, Campylobacter jejuni. 


\subsection{Methods}

\subsubsection{Azaspiracid solution preparation}

Azaspiracid (AZA-1, 2 and 3) stocks (prepared in 100\% methanol) were provided by Dr Jane Kilcoyne, Marine Institute, Ireland (Table 4.1). Methanol was evaporated under a stream of nitrogen, prior to toxins being re-suspended in filter-sterilised $(0.2 \mu \mathrm{m})$ phosphate buffered saline (PBS) pH 7.4 with 2\% (v/v) ethanol. Biologically relevant doses ranging from 5 to 200 $\mathrm{ng} / \mathrm{larva}(20-800 \mu \mathrm{g} / \mathrm{kg})$ were prepared, which span the upper regulatory limit of $160 \mu \mathrm{g} / \mathrm{kg}$ (EFSA, 2008; FSA(UK), 2018) - the equivalent being 40 ng/larva.

Table 4.1 Re-suspended azaspiracid stock concentrations

\begin{tabular}{llll}
\hline Toxin & ID & Concentration $(\boldsymbol{\mu g} / \mathbf{m L})$ & Volume $(\mathbf{m L})$ \\
\hline AZA-1 & AIL-08-03 & $111.4 \pm 2.2$ & 1 \\
AZA-2 & AIL-10-01 & $86.2 \pm 0.9$ & 0.5 \\
AZA-3 & AIL-10-01 & $107.4 \pm 0.9$ & 0.5 \\
\hline
\end{tabular}

\subsubsection{Assessment of Azaspiracid toxicity using a Galleria mellonella health index}

Healthy, final instar larvae (see section 2.3.2) were administered with AZA-1, 2 and 3 by intrahaemocoelic injection (INJ) or force-feeding (FF; gavage) using a sterile 27-gauge hypodermic needle (see section 2.3.3) across the concentration range 5 - $50 \mathrm{ng} / \mathrm{larva}$ for AZA1 and AZA2, and $20-200 \mathrm{ng} / \mathrm{larva}$ for AZA3 ( $n=30$ per treatment/time point, $n=990$ in total). The negative control consisted of PBS pH 7.4 containing $2 \%(\mathrm{v} / \mathrm{v})$ ethanol. Post-inoculation, larvae were incubated in the dark at $30{ }^{\circ} \mathrm{C}$. The health of each larva was assessed over a 72hour period using a scoring system of 0 (dead) and 10 (healthy, pupated/cocooned) (Table 3.1) developed by Loh et al. (2013) for this insect species. The volume of each inoculum was standardised to $10 \mu \mathrm{l}$ per insect (regardless of gavage/injection or whether the contents were chemical/microbial). 


\subsubsection{Total haemocyte counts and cell sizes}

To further investigate the potential effects of AZA on larvae, haemocyte (immune cell) counts and sizes were assessed. Larvae were sacrificed at 4, 24, 48 and 72-hours post-injection with 5,25 , or $50 \mathrm{ng} / \mathrm{larva}$ of AZA-1. This was conducted by cooling each larva on ice for $\sim 3$ minutes before injecting $100 \mu \mathrm{l}$ of de-coagulant solution (section 2.3.4) into the last left proleg. Larvae were then chilled for two minutes prior to using a 27-gauge needle to pierce the head and exsanguinate into a sterile microcentrifuge tube. Approximate $20 \mu \mathrm{l}$ of haemolymph was used to conduct a total cell count on an improved Neubauer haemocytometer. Haemocyte diameters/sizes were measured using an Olympus Bx43f microscope and Olympus Soft Imaging Solutions cellSens software. Twenty cells/larva were measured alongside each total haemocyte count ( $n=36$ per treatment, 180 in total).

\subsubsection{Quantifying faecal microbes}

Microbial loads, i.e., culturable colony forming units (CFU), were assessed from the faeces of larvae that were force-fed AZA 1 to AZA 3 (5 - 200 ng/larva). Faeces were collected at 4, 24, 48- and 72-hours post treatment. Faecal pellets were homogenised in $500 \mu \mathrm{l}$ PBS, and standardised to $1 \mathrm{mg} / \mathrm{ml}$. Approximately, $200 \mu \mathrm{l}$ of faecal homogenate were spread across nutrient agar and incubated at $30^{\circ} \mathrm{C}$ for up to 48 hours. After incubation, colonies were counted (section 2.3.7).

\subsubsection{Gut microbiome analysis of AZA 1 and okadaic acid-intoxicated larvae}

Randomly selected larvae ( $\mathrm{n}=3$ per timepoint/treatment, 54 in total), inoculated with either AZA1, okadaic acid, or PBS (control), were sacrificed across 4 to 48 hours. The gut tissues (mouth to anus) were dissected from each larva, weighed, snap frozen in liquid nitrogen, and stored at $-80^{\circ} \mathrm{C}$ before genomic DNA extraction using a Qiagen DNeasy Blood and Tissue Kit by following the manufacturer's protocol. DNA yields were quantified using the Qubit high sensitivity assay kit, and standardised to $50 \mathrm{ng} / \mu \mathrm{l}$ prior to sequencing. A sub-set of samples were chosen for 16S PCR amplification and visualisation through [2\%] gel electrophoresis. For amplification of $16 \mathrm{~S}$ V4 region the forward 515fmod (GTGYCAGCMGCCGCGGTAA) (Parada et al., 2016), and reverse 806rmod (GGACTACNVGGGTWTCTAAT) (Apprill et al., 2015) primers were used. The PCR cycling conditions were modified from Helgesen (2017) and Walters et al (2015). Briefly, each PCR reaction mixture (25 $\mu \mathrm{l})$ contained molecular-grade water $(6.5 \mu \mathrm{l})$, Taq 2X master mix (New England Biolabs: M0270L) (12.5 $\mu \mathrm{l})$, forward primer $(1 \mu \mathrm{M}, 2.5 \mu \mathrm{l})$, reverse primer $(1 \mu \mathrm{M}, 2.5 \mu \mathrm{l})$, and DNA $(1.0 \mu \mathrm{l})$. PCR amplifications were carried out using a BioRad thermocycler under the following conditions: $96^{\circ} \mathrm{C}$ for 2 min for 1 
cycle; 30 cycles of $96^{\circ} \mathrm{C}$ for $15 \mathrm{~s}, 50^{\circ} \mathrm{C}$ for $30 \mathrm{~s}$, and $70^{\circ} \mathrm{C}$ for $90 \mathrm{~s}$, and a final single cycle at $70^{\circ} \mathrm{C}$ for $10 \mathrm{~min}$ (Walters et al., 2015, Helgesen, 2017). Approximately, $5 \mu \mathrm{l}$ of PCR product was combined with $1 \mu \mathrm{l}$ of Gel loading buffer (sigma G2526-5ML), and loaded into a 1X TBE agarose $[2 \% \mathrm{w} / \mathrm{v}]$ gel $\left(10 \mathrm{ml}\right.$ UltraPure ${ }^{\mathrm{TM}}$ TBE Buffer, 10X Fisher Scientific diluted in 90ml ultrapure water, and $5 \mu \mathrm{l}$ of green safe premium (NZYtech)), The gel was run for 1 hour at $80 \mathrm{~V}$.

Purified DNA ( $25 \mu \mathrm{l}$ of sample) with a concentration of $50 \pm 2 \mathrm{ng} / \mu \mathrm{l}$ was used for sequencing. Eurofins Genomics (UK Limited) performed the next generation sequencing using an Illumina MiSeq platform targeting the V4 16S ribosomal RNA region ( 2 x $250-300 \mathrm{bp})$ using the following primers: Forward: TACGGGAGGCAGCAG (Turner et al., 1999) Reverse: CCAGGGTATCTAATCC (Kisand et al., 2002), with no less than 100,000 reads per library. Amplicon generation, adapter addition, quality control, size selection, pooling, demultiplexing, removing of the primers and advanced bioinformatics were performed by Eurofins (raw data processing, read merging, quality filtering and chimera removal). De-multiplexing was carried out on all reads, which were approved by the standard Illumina chastity filter. Read merging was completed using the FLASH algorithms to create a consensus sequence using the greatest quality value and considered all overlaps and produced merged readings with the longest targeted region (Magoč and Salzberg, 2011). Where merging was not possible the forward reading was used, merged regions were then filtered, removing reads which were too long or short. Initial Microbiome profiling to remove chimeric reads was conducted using UCHIME followed by entropy decomposition analysis to form data sets of partition marker genes within operational taxonomic units (OTUs) (Schloss et al., 2011, Eren et al., 2015). Each OTU was then assigned to a taxonomy with a minimum of $70 \%$ sequence identity across a minimum representative sequence of $80 \%$ using DC-Megablast (Altschul et al., 1990). Finally, OTUs were processed using QIIME software (version 1.9.1) (Caporaso et al., 2010) using 0.97\% homogeneity threshold for OTU selection. OTUs which had no taxonomic matches were categorised as "unclassified" and taxonomic units which had fewer than $0.1 \%$ reads were considered "other). OTUs at Phylum and Genus levels were visualised and analysed in R studio (see 4.2.10)

\subsubsection{Tissue histology of AZA and OA inoculated larvae}

First, larvae were force-fed low or high doses of AZA-1 (5 ng/larva and $37.5 \mathrm{ng} / \mathrm{larva}$ ) and OA (6.25 ng/larva and $62.5 \mathrm{ng} / \mathrm{larva})$. OA doses were determined previously by Coates et al. (2019). Second, larvae were sacrificed at 4, 24 and 48 hours post gavage and fixed in 10\% 
formalin (optimised in Appendix A) for 24 hours at room temperature. Third, fixed larvae were cut into three parts; head, middle and rear in preparation for paraffin wax embedding. Samples were dehydrated in $70 \% 80 \%$ and $90 \%$ each for one hour followed by three 1 -hour $100 \%$ ethanol washes.

Dehydrated samples were incubated in histoclear twice for 1 hour to remove any remaining fixative, followed by $50 \%$ histoclear: $50 \%$ paraffin wax for one hour. Samples were positioned in cartridges and filled with molten paraffin wax before being placed in a vacuum to drive the wax into the tissue and remove all air. Once vacuumed, samples were then orientated correctly and topped up with molten wax and allowed to set overnight. Once completely set, the wax block containing the samples were trimmed to the minimise cutting and staining areas. Trimmed blocks were sliced into $5-7 \mu \mathrm{m}$ sections using a microtome. Samples were loaded onto glass slides with water, and dried on a heating block to allow the wax ribbon to fully expand and stick to the surface of the glass slide - ready for hematoxylin and eosin staining.

\subsubsection{Culturing of Campylobacter jejuni}

Campylobacter jejuni from ATCC - LGC Standards (Campylobacter jejuni subsp. jejuni (ATCC ${ }^{\circ}$ BAA-224 ${ }^{\mathrm{TM}}$ )) was reconstituted with $1 \mathrm{ml}$ Mueller Hinton broth (Sigma Aldrich). The bacterial suspension was aliquoted in sterile microcentrifuge tubes with $15 \%$ (v/v) glycerol and stored at $-70^{\circ} \mathrm{C}$. Approximately, $100 \mu \mathrm{l}$ of bacterial suspension was added to $300 \mathrm{ml}$ Mueller Hinton broth in a Corning Erlenmeyer cell culture flask with vented cap and incubated at $37^{\circ} \mathrm{C}$ with constant agitation, $200 \mathrm{rpm}$ overnight. Bacteria $(100 \mu \mathrm{l})$ were sub-cultured onto Mueller Hinton agar $(2 \% \mathrm{w} / \mathrm{v})$ and maintained anaerobically using a gas generation pack (Merck Anaerocult ${ }^{\circledR} \mathrm{P}$ ) at $37^{\circ} \mathrm{C}$ for 48 hours before colony forming units (CFUs) were counted.

\subsubsection{Infecting Galleria mellonella larvae with Campylobacter jejuni}

Larvae were inoculated with $1 \times 10^{4}, 1 \times 10^{5}, 1 \times 10^{6}$ or $3 \times 10^{6}$ CFUs of $C$. jejuni per larva by intrahaemocoelic injection (INJ) or force-feeding (FF; gavage) using a sterile 27-gauge hypodermic needle. Bacterial inocula (CFU/larva) were extrapolated from Senior et al. (2011) where $10^{6}$ CFUs injected into G. mellonella resulted in loss of gastric integrity within 24 hours. This level of damage within 24 hours is too severe for a susceptibility study, so a broader range of doses was selected $\left(1 \times 10^{4}-3 \times 10^{6}\right)$ to gauge sub-lethal effects. The negative control consisted of PBS pH 7.4 containing 50\% (v/v) Mueller Hinton broth. Post-inoculation, larvae 
were incubated in the dark at $30^{\circ} \mathrm{C}$ and assessed for health indicators as stated above (Table $4.2)$.

\subsubsection{Pre-treatment of Galleria mellonella with AZA or OA, followed by Campylobacter jejuni}

Larvae were pre-injected with a sublethal dose of AZA-1 (25 ng/larva) using a sterile 27-gauge hypodermic needle and incubated at $30{ }^{\circ} \mathrm{C}$ in darkness. After 24 hours, the same larvae were force-fed $C$. jejuni $\left(1 \times 10^{4}, 1 \times 10^{5}, 1 \times 10^{6}, 3 \times 10^{6}\right.$ CFUs each). Controls consisted of injection/force-feeding the following combinations: PBS (2\% ethanol) + PBS (50\% Mueller Hinton broth); PBS (2\% ethanol) $+1 \times 10^{4}$ CFU C. jejuni; AZA1 (25 ng/larva) + PBS (50\% Mueller Hinton broth). Individual larval health was assessed as stated above (Table 4.2).

\subsubsection{Data handling}

Each experiment was repeated on at least three separate occasions. Results are expressed as mean \pm SE (unless stated otherwise), and samples sizes can be found within the respective figure legends. Statistical analyses were completed using GraphPad PRISM v8 (except for the microbiome data); log-rank (Mantel-Cox) tests were carried out on survival curves, whereas two-way ANOVAs (with Turkey's multiple comparison tests) were applied to cellular (haemocyte and CFUs) data. Statistical significance was determined when $P \leq 0.05$. Histology slides were visualised and singled-blind assessed as outlined in section 2.3.10.

Next generation sequencing (16S microbiome) data were analysed in $\mathrm{R}$ studio using the microbiome and vegan packages to carry out Chao-1 and Shannon indices and the randomised permutation test; adonis permanova (Bray- Curtis method with 999 permutations) was conducted to asses significance between microbiomes (Cree et al., 2016). Stress and dispersion tests were conducted and plotted to ensure the model was a good fit. All samples were filtered to remove contamination by using the reads present in the extraction blank. OTU reads in the extraction blank ( $\leq 200$ reads) were removed from the samples to leave the remaining read abundance and all Eukaryotic OTUs such as those associated with arthropod signals and Chlorophyta were removed. Replicates were merged to create average OUT profiles for each time point and treatment. Two samples (untreated $24 \mathrm{~h}$ and PBS $48 \mathrm{~h}$ ) were unsuccessfully sequenced, this is highlighted in the results (Tables $4.4-4.6$ and Figure 4.11 B and C, Figure 4.12 B and C). 


\subsection{Results}

\subsubsection{Survival, responsiveness (activity) and melanisation levels of intoxicated larvae}

Exposure of larvae to an azaspiracid, either by intrahaemocoelic injection or by gavage, had clear negative impacts on health indices (Figures 4.1 and 4.2, respectively). Both AZA1 and AZA3 reduced survival levels significantly (Table 4.2), whereas AZA2 did not appear as toxic/lethal across the concentration range $5-50 \mathrm{ng} / \mathrm{larva}$, with no less than $93 \%$ survival (Figures $4.1 \mathrm{~F}$ and $4.2 \mathrm{~F}$ ). It was the highest concentrations of AZA1 (37.5 and $50 \mathrm{ng} / \mathrm{larva})$ that killed between 17 and $20 \%$ of insects by 96 hours. A clear dose-dependent response was observed for AZA3 lethality (20 - $200 \mathrm{ng} / \mathrm{larva})$, resulting in 47\% and 67\% mortality by 96 hours, through injection and force-feeding, respectively (Figures 4.1I and 4.2I). Decreases in both melanisation and activity indices at 4 hours-post inoculation for AZA1 and AZA3, and at 48 hours for AZA2, were good predictors of survival outcomes (Figures 4.1 and 4.2). It is worth noting that a score of 4 indicates pale, non-pigmented insect integument, whereas a score of 1 or 0 indicates highly melanised (black-brown), gross discolouration. Unsurprisingly, reduced movement and increased darkening (melanisation) correlated broadly with reduced survival. For example, in larvae forced-fed AZA3, doses of 100, 150 and 200 ng substantially reduced activity when compared to untreated and control (PBS) larvae responsiveness across the entire 96-hour experimental period (Figure 4.1G, H and 4.2G, H). It was not until 48 hours postinoculation that survival rates dropped significantly between AZA3 doses 150 and $200 \mathrm{ng} / \mathrm{larva}$ and the untreated/control larvae, regardless of the inoculation route (Figures 4.1I and 4.2I). By force-feeding AZA3 at 20 - $200 \mathrm{ng} / \mathrm{larva}(40-800 \mu \mathrm{g} / \mathrm{kg})$, an LD $\mathrm{L}_{50}$ value of $881.4 \mu \mathrm{g} / \mathrm{kg}(773.1$ - 1028.2) at 48 hours post-inoculation was calculated.

Table 4.2 Survival analyses of Galleria mellonella larvae exposed to azaspiracids. Values represent outputs from Log-rank [Mantel-Cox] tests.

\begin{tabular}{|c|c|c|c|}
\hline & AZA1 & AZA 2 & AZA 3 \\
\hline $\begin{array}{l}\text { Intrahaemocoelic } \\
\text { injection }\end{array}$ & $X^{2}(6)=20.9, \mathbf{P}=\mathbf{0 . 0 0 1 9}$ & $X^{2}(6)=12.2, \mathrm{P}=0.0576$ & $X^{2}(6)=60.9, \mathbf{P}<\mathbf{0 . 0 0 0 1}$ \\
\hline Force feeding & $X^{2}(6)=26.8, \mathbf{P}=\mathbf{0 . 0 0 0 2}$ & $X^{2}(6)=4.04, P=0.6708$ & $X^{2}(6)=85.1, \mathbf{P}<\mathbf{0 . 0 0 0 1}$ \\
\hline
\end{tabular}


A

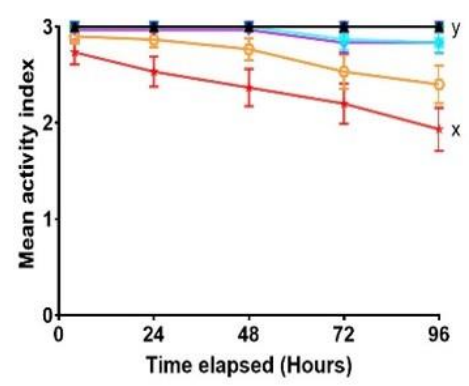

D

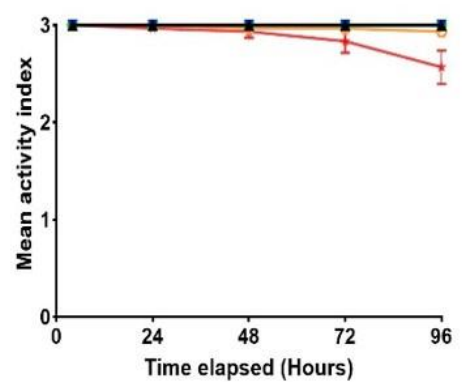

G

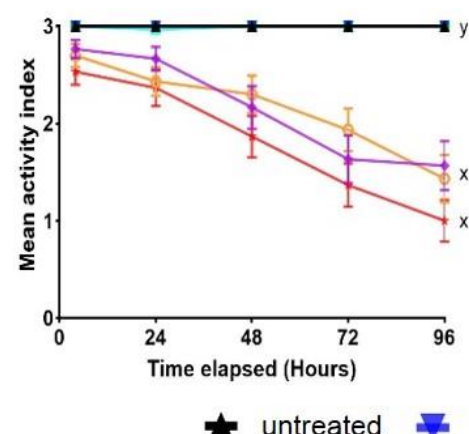

25 or $100 \mathrm{ng} / \mathrm{larva}$
B
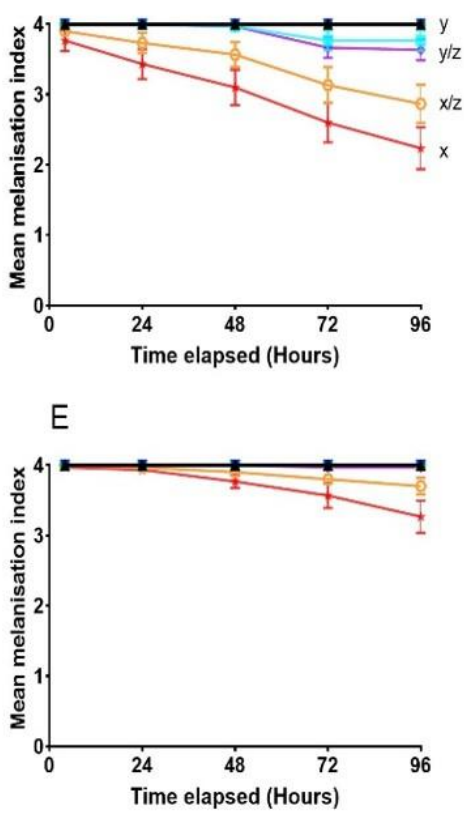

$\mathrm{H}$

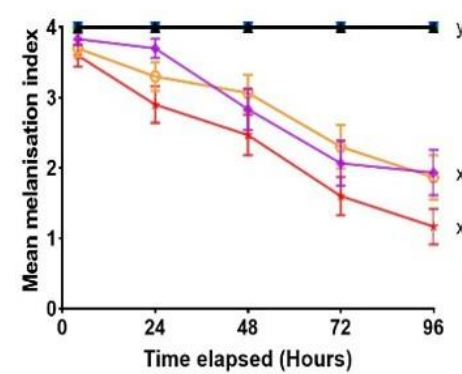

5 or 20 ng/larva

37.5 or 150 ng/larva
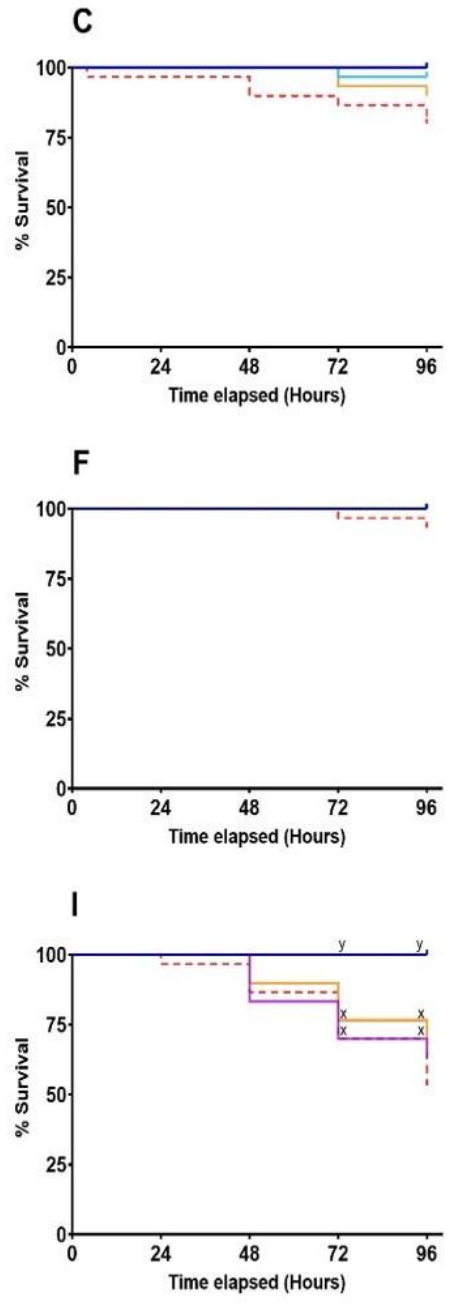

12.5 or 50 ng/larva 50 or 200 ng/larva

Figure 4.1 Health status of Galleria mellonella larvae following intrahaemocoelic injection of azaspiracids, 5 - $200 \mathrm{ng} / \mathrm{larva}$. Larvae were inoculated with AZA-1 (panels A-C), 2 (panels DF) and 3 (panels G-I) and maintained in darkness at $30^{\circ} \mathrm{C}$ for 96 hours. Larval health was scored (see Table 4.2; Loh et al., 2013) by assessing movement (A, D and G), melanisation (B, E and $\mathrm{H})$ and survival $(\mathrm{C}, \mathrm{F}$ and $\mathrm{I})$. Values are presented as mean $\pm \mathrm{SE}(\mathrm{n}=30$ per treatment, 510 in total). Unshared letters represent significant differences $(P \leq 0.05)$ determined by Tukey's multiple comparisons tests. The negative control consisted of injecting larvae with PBS containing $2 \%$ ethanol. 

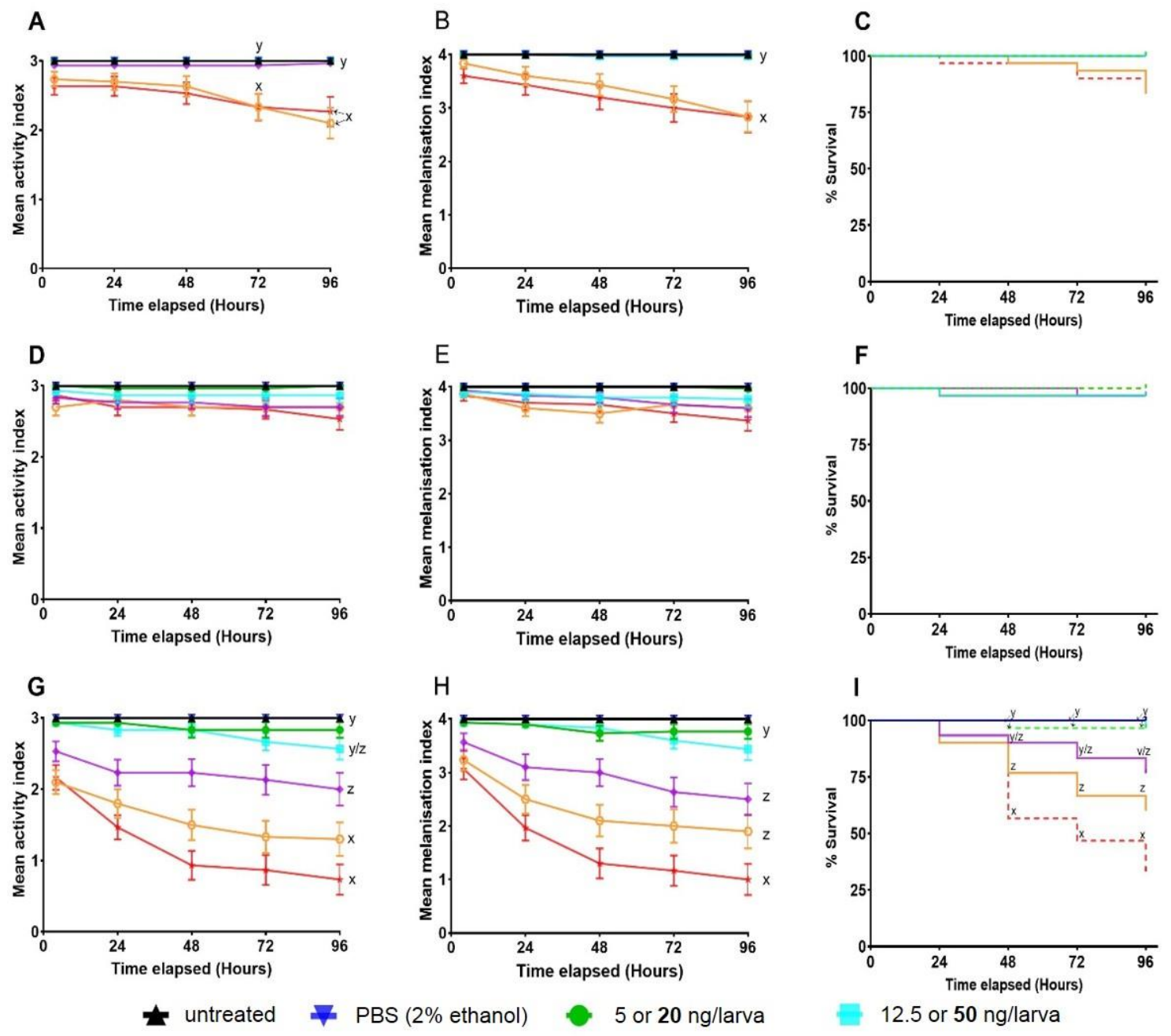

25 or $100 \mathrm{ng} / \mathrm{larva}$

37.5 or $150 \mathrm{ng} / \mathrm{larva}$

50 or $200 \mathrm{ng} / \mathrm{larva}$

Figure 4.2 Health status of Galleria mellonella larvae following force-feeding (gavage) of azaspiracids, 5 - $200 \mathrm{ng} / \mathrm{larva}$. Larvae were inoculated with AZA-1 (panels A-C), 2 (panels DF) and 3 (panels G-I) and maintained in darkness at $30^{\circ} \mathrm{C}$ for 96 hours. Larval health was scored (see Table 4.2; Loh et al., 2013) by assessing movement (A, D and G), melanisation (B, E and $\mathrm{H})$ and survival $(\mathrm{C}, \mathrm{F}$ and $\mathrm{I})$. Values are presented as mean $\pm \mathrm{SE}(\mathrm{n}=30$ per treatment, 510 in total). Unshared letters represent significant differences $(P \leq 0.05)$. The negative control consisted of force-feeding larvae with PBS containing $2 \%$ ethanol. 


\subsubsection{Total haemocyte counts and size(s) within the haemolymph of intoxicated larvae}

Injecting AZA-1 (10, 50 and $100 \mathrm{ng} / \mathrm{larva})$ directly into the insect body cavity (haemocoel) induced significant changes in haemocyte numbers within 4 hours (Figure 4.3A), with time $\left(F_{(3,156)}=3.28, \mathrm{P}=0.0225\right)$ and treatment $\left(F_{(4,156)}=3.84, \mathrm{P}=0.0052\right)$ accounting for $\sim 5 \%$ and $\sim 7 \%$ of the variation within the data, respectively. Importantly, a reciprocal increase was observed also with the negative control (PBS), indicating the route of inoculation is likely responsible for this cellular response. Untreated insects had $\sim 2.4 \times 10^{6}$ haemocytes $/ \mathrm{ml}$ at 4 hours, whereas all three AZA1 doses and PBS had between 5.2 and $5.7 \times 10^{6}$ haemocytes $/ \mathrm{ml}$. Haemocyte numbers continued to fluctuate between 24 and 72 hours. At 48 and 72 hours, cell numbers remained higher for AZA doses 37.5 and $50 \mathrm{ng} / \mathrm{larva}\left(4.2-5.2 \times 10^{6} / \mathrm{ml}\right)$ compared to the untreated and negative control $\left(3.2-3.8 \times 10^{6} / \mathrm{ml}\right)$, but these were not significantly different (Figure 4.3A).

To assess any putative changes in cell size, swelling or shrinkage, due to the presence of AZA1, haemocyte diameters were recorded (Figure 4.3B). The injection of AZA-1 did lead to an increase in the average diameter of haemocytes, with time $\left(F_{(3,3458)}=60.6, \mathrm{P}<0.0001\right)$ and treatment $\left(F_{(4,3548)}=12.8, \mathrm{P}<0.0001\right)$ considered significant factors. When referring to the highest dose of AZA1 (50 ng/larva), and untreated/negative control (PBS) insects from 4-48 hours post injection, haemocytes were between $0.75 \mu \mathrm{m}$ and $1.22 \mu \mathrm{m}$ larger $(P=0.026-$ 0.001). Larger haemocytes tended to be granular or spherule cells (Figure 4.3C), and likely represented a switch in haemocyte sub-type composition. Interestingly, at 72 hours postinoculation, the haemocytes in untreated insects were $0.89 \mu \mathrm{m}$ larger than those injected with 5 ng/larva AZA-1 $(P=0.0127)$ (Figure 4.3B) - this could be attributed to some untreated larvae initiating the break-down of the fat body for metamorphosis. 


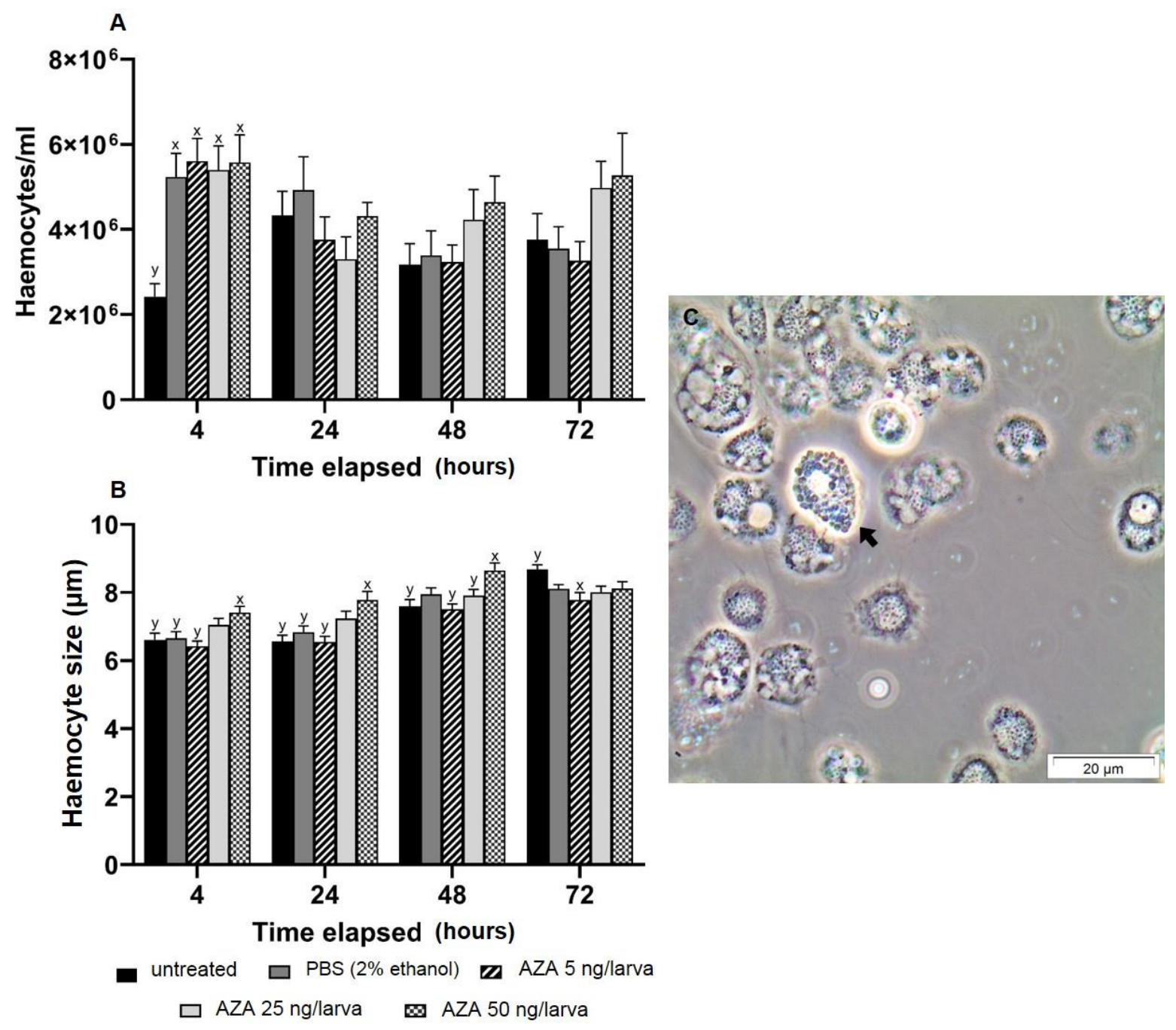

Figure 4.3 Haemocyte responses of Galleria mellonella larvae following intrahaemocoelic injection of azaspiracid 1 across the concentration range, $5-50 \mathrm{ng} / \mathrm{larva}$. Haemocyte counts (A) and sizes (B) were measured between 4- and 72-hours post-injection. Values represent means $\pm \operatorname{SE}(n=36$ per treatment, 180 in total). Unshared letters represent significant differences $(P \leq 0.05)$ determined by Tukey's multiple comparisons tests. The negative control consisted of injecting larvae with PBS containing $2 \%$ ethanol. (C) phase contrast image of freshly withdrawn haemocytes without any de-coagulant. Several different morphotypes are visible, including spherule cells (black arrow). There are two plasmatocytes below the labelled spherule cell (either side), and one small spherule cell above. Note, spherule cells do not attach and spread and the nucleus is never visible except for the absence of the spherules in the cytoplasm. Most of the other cells visible are de-granulated granular cells (occurs when in contact with a surface in vitro). 


\subsubsection{Impact of azaspiracid exposure on CFUs within insect faecal loads}

There is large variation in total CFU numbers from G. mellonella faecal samples, however, two distinct colonies appear consistently: (1) small, punctate, yellowish colonies, and (2) larger cream coloured colonies (Figure 4.4). For insects exposed to AZA-1 and 2, there were significant increases in the numbers of CFUs from 4 to 48 hours, ranging from $\sim 7,000-20,000$ to $28,500-33,500$ in faecal loads (AZA1, time $\left[F_{(2,42)}=32.5, \mathrm{P}<0.0001\right]$, treatment $\left[F_{(6,42)}=\right.$ $2.44, \mathrm{P}<0.041]$; AZA2, time $\left[F_{(2,42)}=35.9, \mathrm{P}<0.0001\right]$, treatment $\left[F_{(6,42)}=2.51, \mathrm{P}<0.036\right]$; Figure 4.5A and 4.5B). For both AZA1 and AZA2, time accounted for $~ 48 \%$ of the variation within the data, whereas treatment accounted for $\sim 11 \%$. Conversely, it was only the lower doses of AZA3, 20 - $100 \mathrm{ng} /$ larva that led to large increases in CFU numbers (Figure 4.5C). For AZA3, time was revealed as a significant factor $\left(F_{(2,42)}=5.02, \mathrm{P}=0.011\right)$ and accounted for $\sim 14 \%$ of the variation within the data, unlike treatment $\left(F_{(6,42)}=1.41, \mathrm{P}=0.235\right)$.

Untreated larvae yielded consistent levels of CFUs between 16,000 and 19,000. The negative control (PBS $+2 \%$ ethanol) did show increased CFU levels over time, but not to the same extent as azaspiracid-treated larvae.

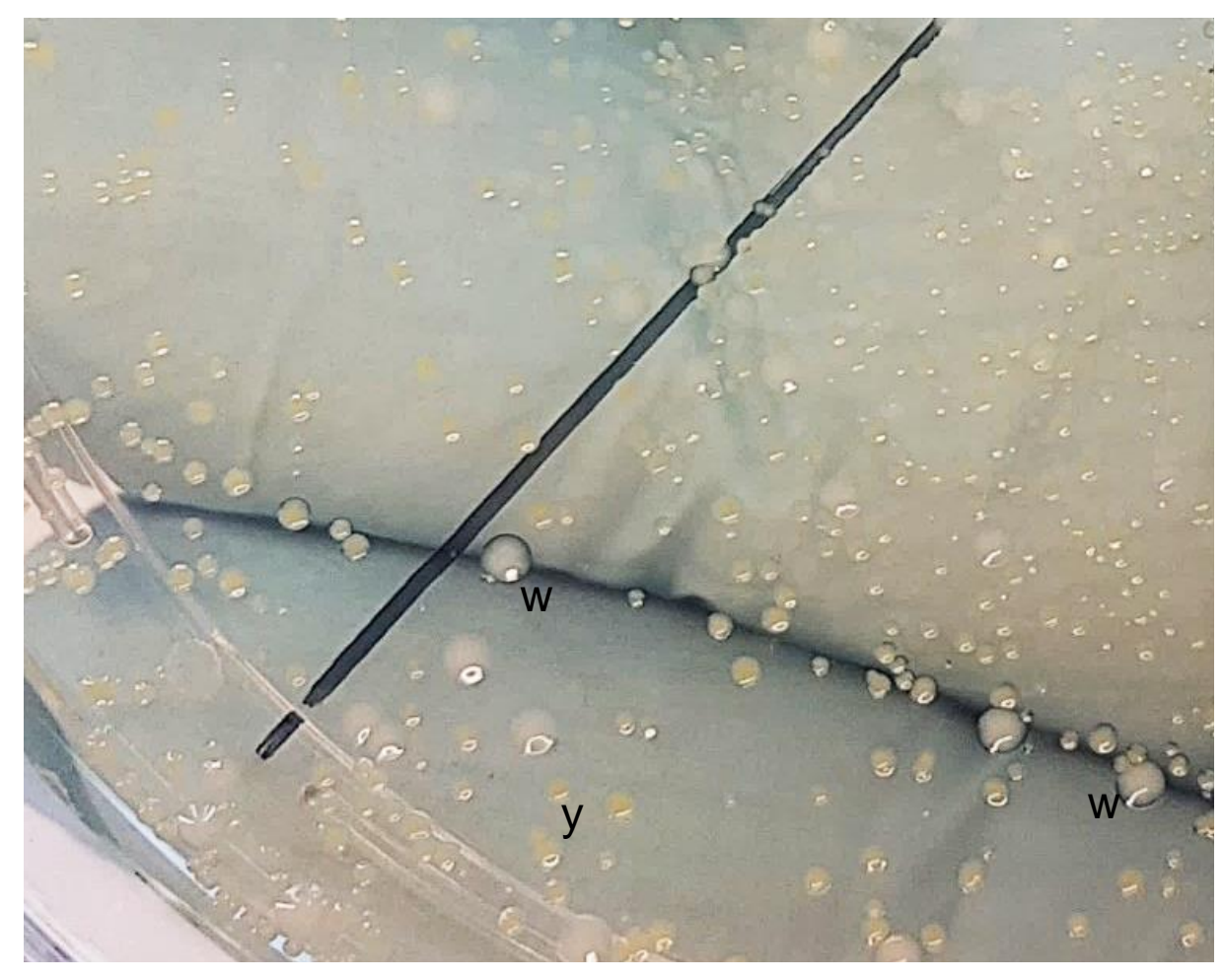

Figure 4.4 Typical appearance of bacterial colonies cultured from Galleria mellonella faeces. Faeces were collected from larvae, homogenised in PBS pH7.4 and inoculated onto nutrient agar, followed by incubation at $30{ }^{\circ} \mathrm{C}$ for 48 hours. W refers to larger white colonies. Y refers to smaller yellow colonies. 

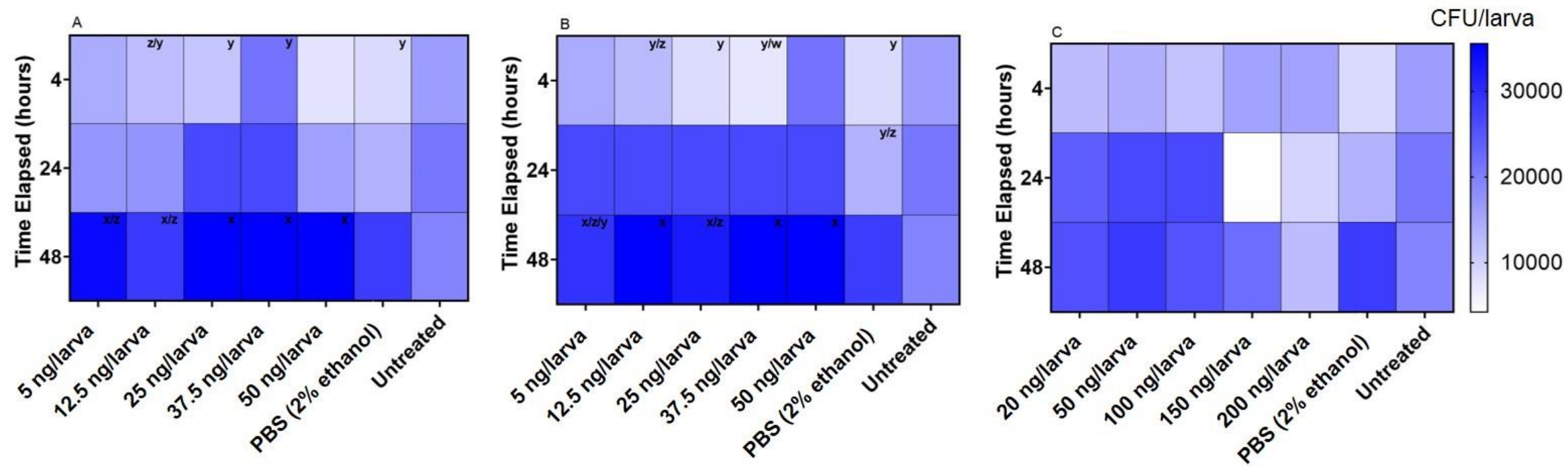

Figure 4.5 Numbers of bacterial colony forming units in the faeces of azaspiracid-intoxicated insects. Larvae received AZA-1 (A), 2 (B) and 3 (C) across the concentration range $5-200 \mathrm{ng} /$ larva, and were maintained in darkness at $30{ }^{\circ} \mathrm{C}$ for 72 hours. Faecal samples were collected, homogenised in PBS and streaked onto nutrient agar, and incubated for 48 hours at $30^{\circ} \mathrm{C}$ before counting. Values are presented as mean $\pm \mathrm{SE}$ ( $\mathrm{n}$ $=9$ per treatment, 171 in total). Unshared letters represent significant differences $(P \leq 0.05)$ determined by Tukey's multiple comparisons tests. The negative control consisted of force-feeing larvae with PBS (2\% ethanol). CFUs are expressed as numbers per individual (larval) faecal load. 


\subsubsection{Histological examination of the insect midgut post-azaspiracid exposure}

Histology slides were prepared from insects force-fed PBS or AZA1, and single-blind assessed using the same modified grading system developed for chapter 2 (Figure 4.6). Midgut tissues examined from untreated and negative controls (PBS $+2 \%$ ethanol) showed some variation in tissue architecture but contained few clear signs of damage; all slides were assigned grade 1 ( $<2$ discrete changes), apart for two PBS slides graded 2 (on each at 4 and 48 hours; Figure 4.6). High doses of OA $(62.5 \mathrm{ng} / \mathrm{larva} .(P<0.0001)$ and AZA-1 $(37.5 \mathrm{ng} / \mathrm{larva} . P=0.008)$ caused significant damage to the larval mid-gut within the first 4 hours of force-feeding. Damage worsened during the 48 hours incubation although only significantly more for the high dose of OA (62.5 ng/larva). Lower doses of both OA (6.25 ng/larva) and AZA (5 ng/larva) inflicted discrete (or localised) damage, however, they were statistically significant compared to the untreated and negative control after 24 hours of OA and 48 hours for AZA1.

Transverse and longitudinal sections along the midgut of PBS-treated larvae revealed an intact arrangement of epithelial cells, including columnar, goblet and regenerative cells (Figure 4.7 A-H). Epithelial folds, involved in nutrient absorption, were identified easily (Figure 4.7B and 4.7D), as were the brush borders (with peritrophic matrix), basement membrane, and underlying muscle tissue (Figures 4.7C, G, H). The midgut lumen appeared mostly free of cellular debris (Figure 4.7A, D, E), and there were few signs of haemocyte presence within the body cavity surrounding the midgut (Figure 4.7.C). Administration of higher doses of AZA1 (37.5 ng/larva; Figure 4.8) or okadaic acid (62.5 ng/larva; Figure 4.9) resulted in moderate to extensive tissue damages within 4 hours post-inoculation (and were still obvious after 48 hours). Common features of tissue degradation included displacement of cells into the midgut lumen, membrane blebbing/blistering, loss of cellular morphology, and nuclear aberrations (pyknosis, karyolysis, and karyorrhexis) associated with cell death.

In AZA1 treated insects, extensive vacuolisation, elongation and desquamation of epithelia were observed (Figure 4.8A to 4.8I). Epithelial folds that were clearly intact at 4 hours (Figure 4.8D) had deteriorated by 48 hours - almost complete tissue distortion (Figure $4.8 \mathrm{H}$ ). There were signs of haemocyte recruitment (Figure 4.8C), presumably due to damage detection. However, the basement membrane and visceral muscle remained mostly intact. For insects treated with okadaic acid, entire regions sloughed into the lumen by 4 and 24 hours (Figure 4.9A -D). In the body cavity, haemocytes were damaged and fragmented (Figure 4.9E), which indicates the toxin made its way across the protective layers of the midgut. By 48 hours, global tissue damage was apparent, and in extremis, the integrity of the entire midgut was 
compromised by necrosis and gross melanisation (Figure 4.9G, H). Okadaic acid appears more toxic to the gastrointestinal tract of larvae.

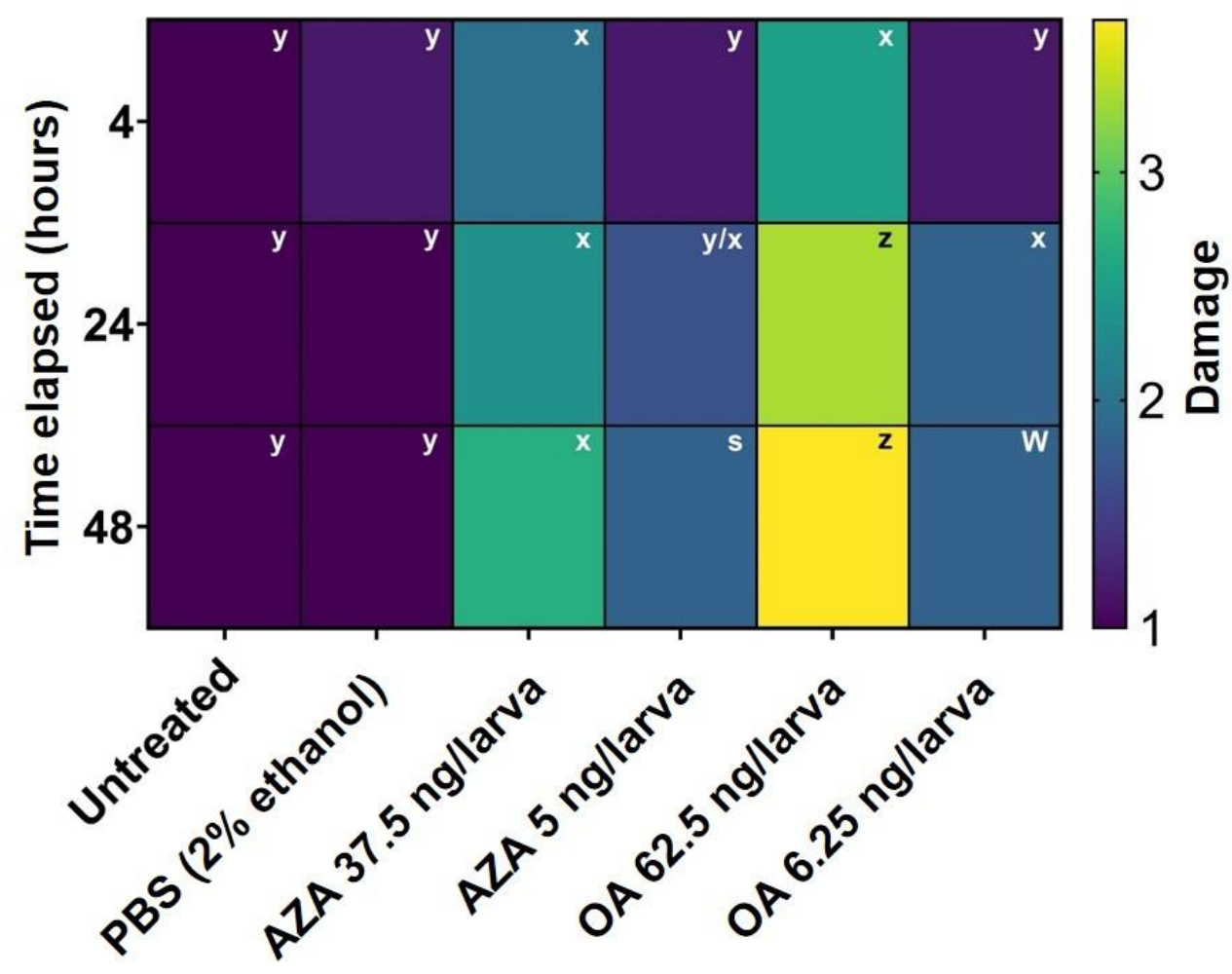

Figure 4.6 The extent of midgut tissue damage in Galleria mellonella after force-feeding AZA1 (5 and $37.5 \mathrm{ng} / \mathrm{larva}$ ), okadaic acid (6.25 and $62.5 \mathrm{ng} / \mathrm{larva})$, or PBS. Histology slides were single blind assessed in pairs (treatment vs control) and subsequently assigned a grade (1 - 4) based on damage(s). Grade 1 indicates little to no damage, whereas Grade 4 represents global damage affecting $>50 \%$ of tissue. Data have been compiled from assessments carried out at $4(n=9), 24(n=9)$ and $48(n=9)$ hours post-inoculation. Unpaired letters indicate significant differences $(\mathrm{P}<0.05)$, determined by ANOVA and Tukey's multiple comparison tests. 


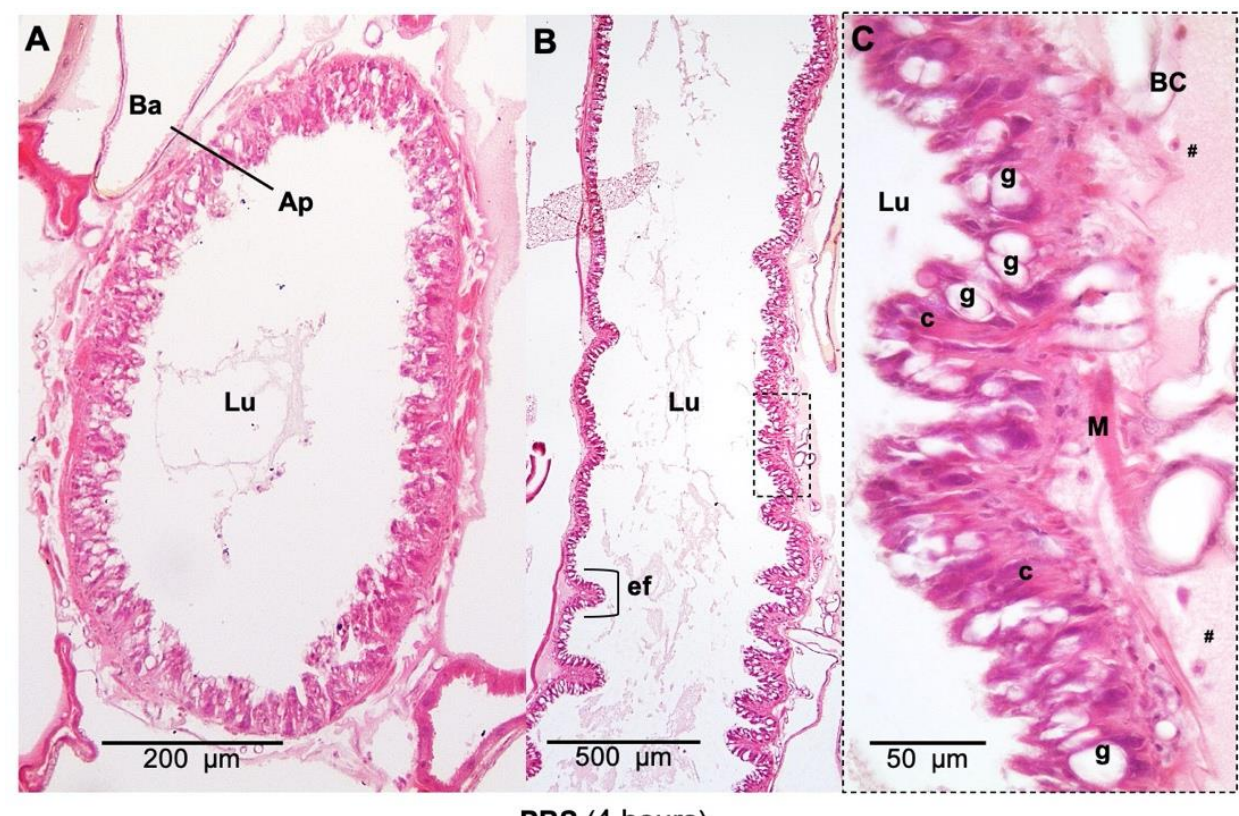

PBS (4 hours)
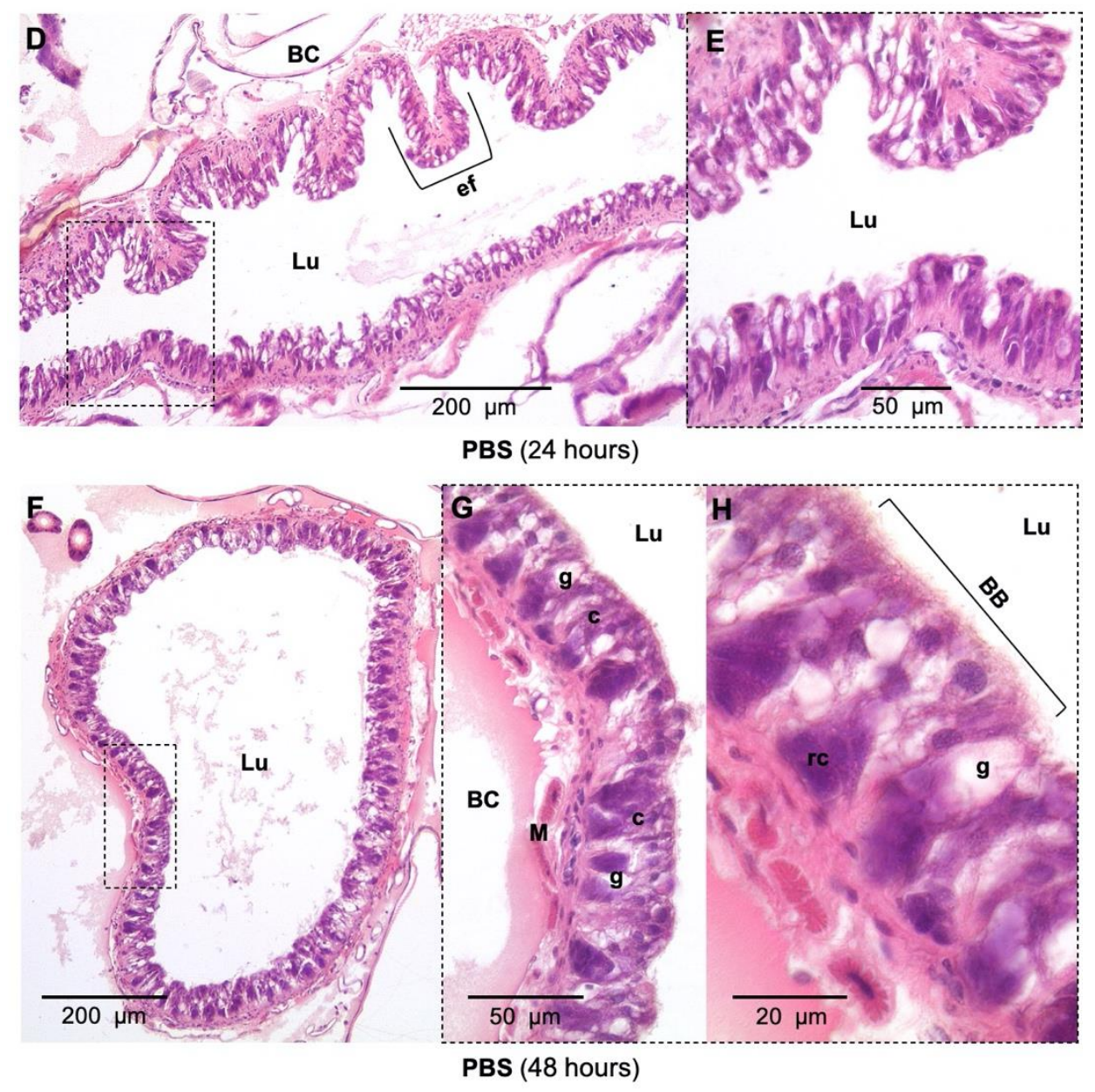

Figure 4.7 Tissue sections of the Galleria mellonella midgut when force-fed PBS (2\% v/v ethanol). Photomicrographs depict transverse and longitudinal sections at 4 (panels A, B, C), 24 (panels D, E) and 48 (panels F, G, I) hours post-inoculation. Ap, apical; Ba, basolateral; BB, brush border; BC, body cavity; c, columnar (epithelial) cell; ef, epithelia fold(s); g, goblet cell; Lu, lumen; M, muscle; rc, regeneration cell. Hashtags (\#) denote haemocytes. 

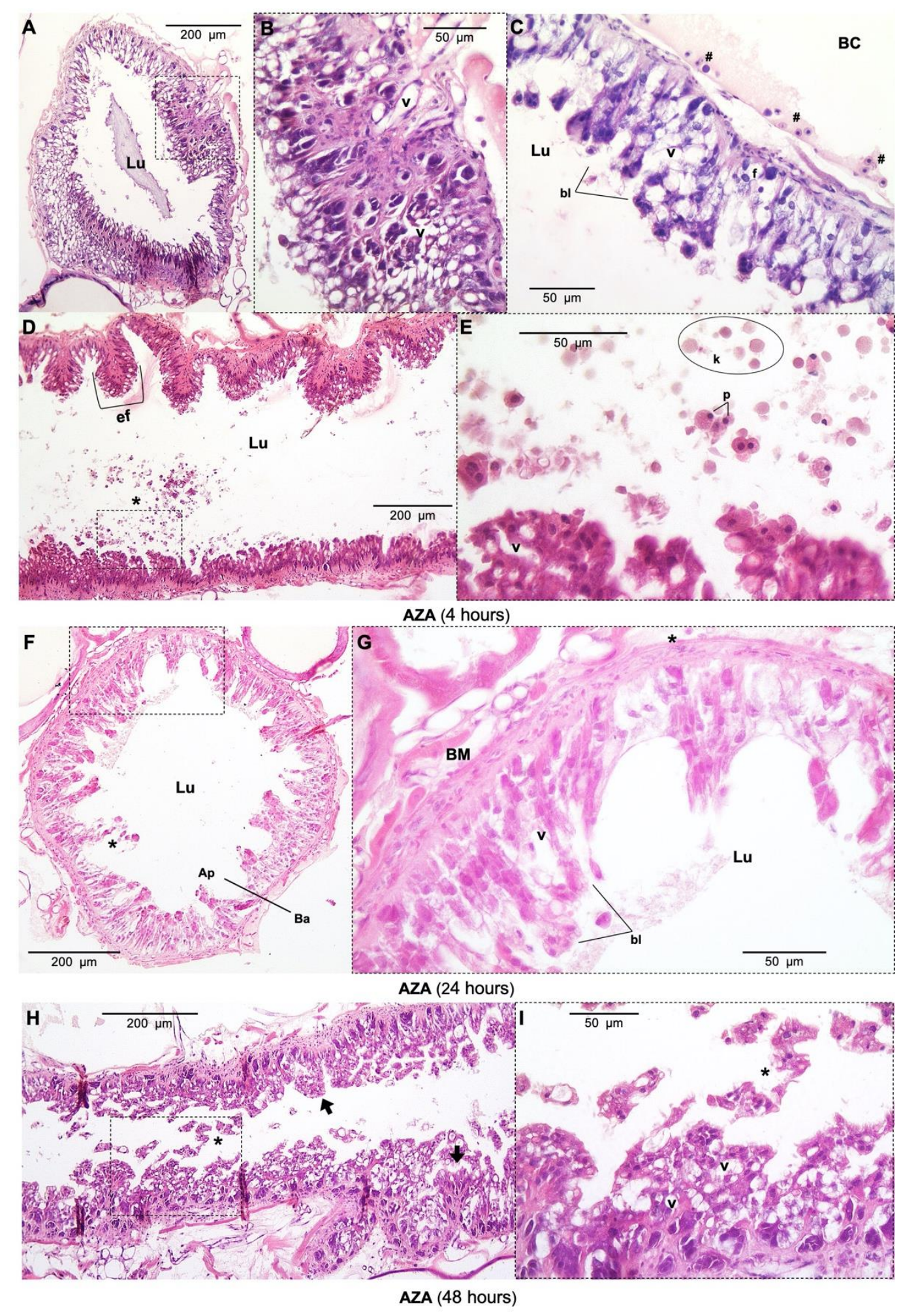

Figure 4.8 Tissue sections of the Galleria mellonella midgut when force-fed azaspiracid 1. Photomicrographs depict transverse and longitudinal sections at 4 (panels A, B, C, D, E), 24 (panels F, G) and 48 (panels H, I) hours post-inoculation. Ap, apical; Ba, basolateral; BC, body cavity; BM, basement membrane; bl, blebbing/blistering; ef, epithelia fold(s); f, fragmentation; k, karyolysis; Lu, lumen; p, pyknosis; rc, regeneration cell; v, vacuolisation. Hashtags (\#) denote haemocytes. Black arrows point to distorted architecture of epithelial folds. An asterisk (*) indicates cellular displacement. 

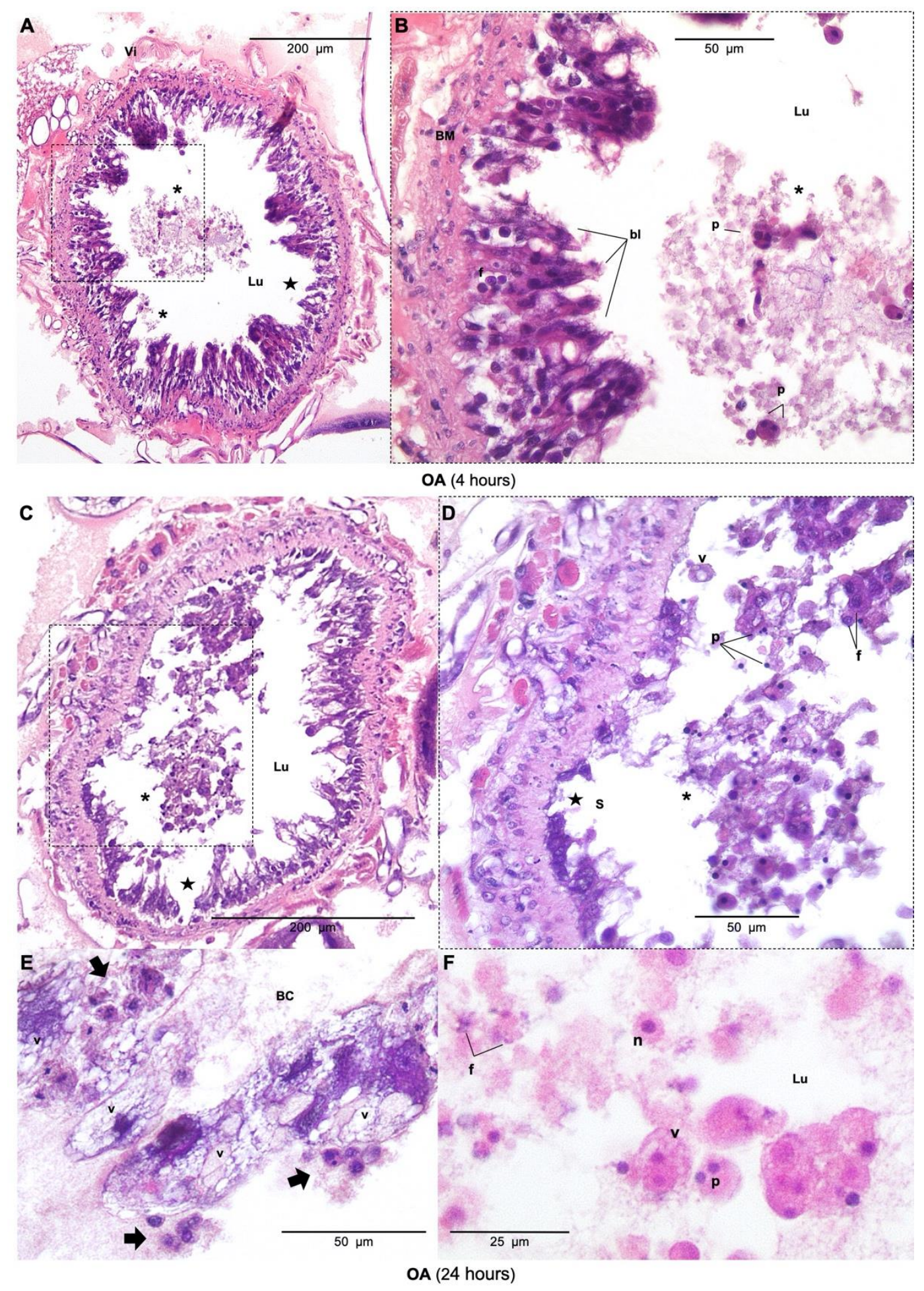

Figure 4.9 Tissue sections of the Galleria mellonella midgut when force-fed okadaic acid. Photomicrographs depict transverse sections at 4 (panels A, B), 24 (panels C, D, E, F) and 48 (panels $\mathrm{G}, \mathrm{H}$ ) hours post-inoculation. BC, body cavity; BM, basement membrane; bl, blebbing/blistering; ef, epithelia fold(s); f, fragmentation (karyorrhexis); k, karyolysis; Lu, lumen; $n$, normal nucleus; $p$, pyknosis; rc, v, vacuolisation; vi, visceral muscle tissue. An asterisk (*) indicates cellular displacement. Black stars highlight areas where damage has completely removed epithelia and exposed underlying muscle. Black arrows point to compromised haemocytes. 

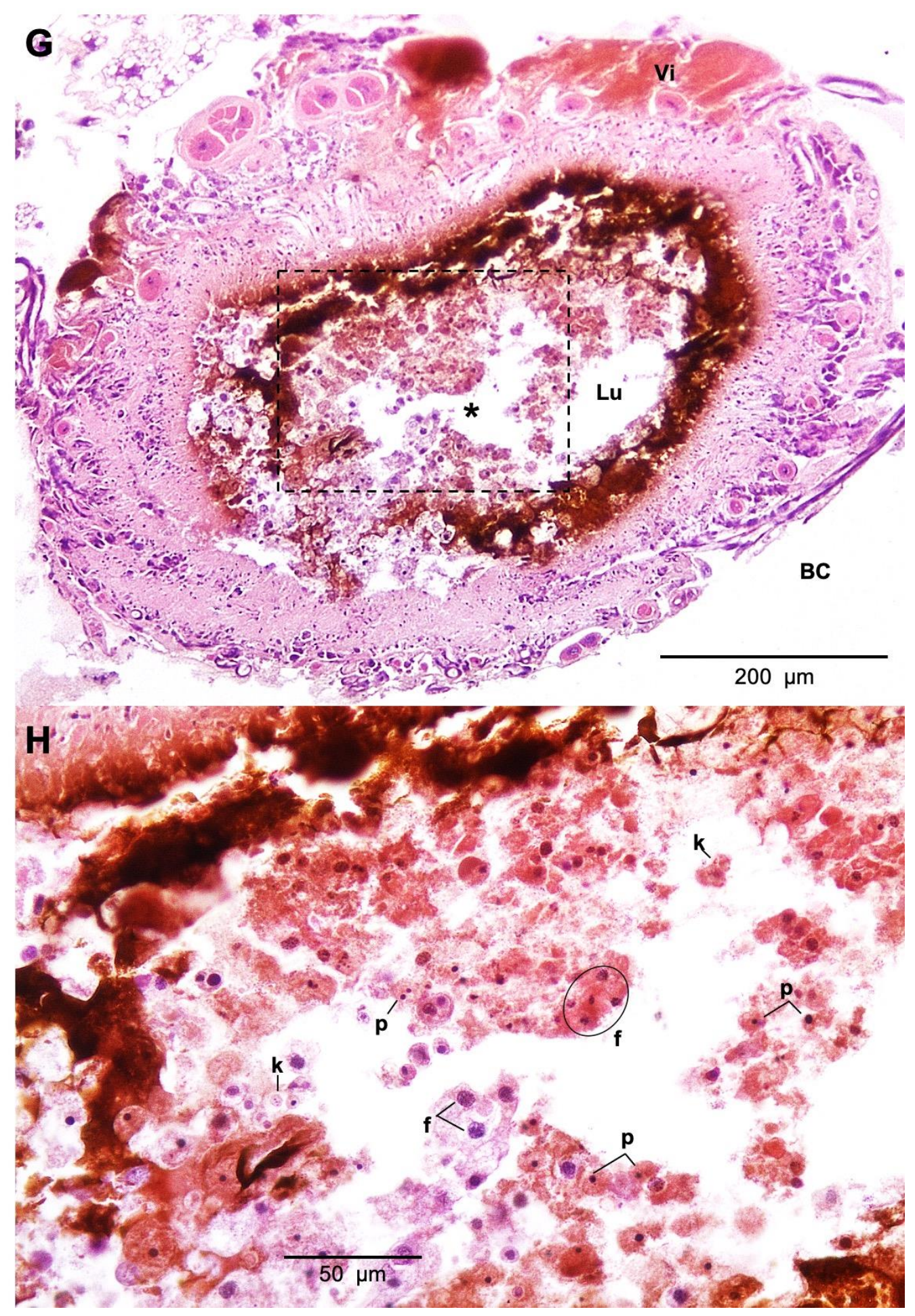

\section{Figure 4.9 continued...}

[Panels $\mathrm{G}$ and $\mathrm{H}$ represent tissues at 48 hours post-inoculation of okadaic acid (62.5 ng/larva)] 


\subsubsection{Impact of azaspiracid-1 and okadaic acid inoculation on gut microbiota of the}

\section{Galleria mellonella larvae}

Prior to next generation sequencing of the gut tissues, a subset of extracted DNA samples were amplified for the $16 \mathrm{~S}$ V4 region through PCR and visualised on a agarose gel (for quality control). PCR amplicons from insect gut tissues were $\sim 500$ bp compared to the positive control (+ve) which was $\sim 300$ bp (Figure 4.10). A possible explanation for this difference might be that the primers are binding non-specifically or to host DNA, which may also explain the presence of a double band for the OA High sample. This nonspecific binding could be due to the annealing temperature being too low or requiring more specific primers.

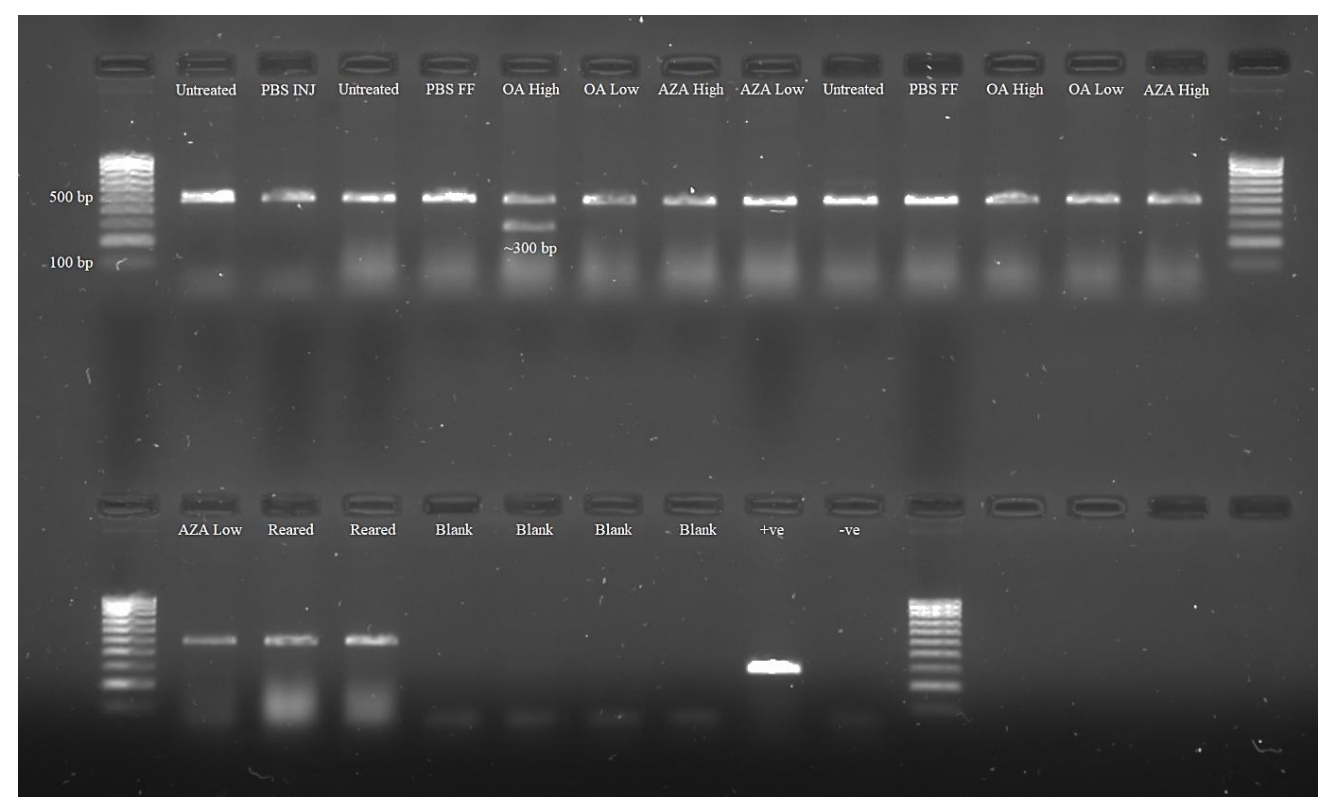

Figure 4.10 Agarose gel electrophoresis of PCR amplicons after 16S V4 region amplification from Galleria mellonella whole gut tissue.

Exposure of AZA-1 or low OA did not cause gross changes in diversity when comparing Chao1 (richness) and Shannon (diversity) indices of microbial signals; however PBS and OA high have lower than average diversity indices of 354.3 and 336.3, respectfully (Table 4.3). For the PBS, this could be an effect of "flushing" due to the force feeding method or a direct effect of the high OA dose, however if this is the case you would expect all samples to have lower indices than the untreated control. OA high Shannon and Chao-1 indices are consistently lower during the entire sampling period, with a gradual decrease from 372 to 300 for the Chao-1 index and 4.083 to 3.496 for the Shannon index. Such changes are not observed in the OA low dose or AZA-1 high dose. A similar trend is observed after AZA-1 low exposure, causing the Chao- 1 index to decrease by 115 to an index of 423 by 48 hours. Conversely, AZA-1 high dose has an initial Chao-1 index of 366, which gradually increases to 461 after 48 hours. This data 
may indicate that AZA-1 at the high dose causes a loss of diversity within the first 4 hours of exposure followed by a gradual improvement/recovery.

At 48 hours, the OA low dose has a much higher Chao-1 index of 603 compared to all other samples. The untreated control and OA low have large variation over the 48-hour experiment for both Shannon and Chao-1 indices. Liu et al. (2020) report that OA-treated mice depict similar fluctuations in microbial diversity and richness over a ten-day period, becoming higher and then lower than the control. This fluctuation is observed here in both the low and high dose when comparing time points, however the fluctuation observed here is much greater than in Liu study. The lowest recorded values were for PBS 48 hour and untreated control 24 hour, these could be due to only having 2 replicates received as a result of complications during sequencing. 
Table 4.3 Diversity indices of the gut microbiome of Galleria mellonella larvae after azaspiracid and okadaic acid exposure. $(n=3$ per treatment per time. Total $=52$ technical repeats, $*=$ two technical repeats)

\begin{tabular}{|c|c|c|c|c|c|}
\hline Treatment & $\begin{array}{l}\text { Hours post } \\
\text { inoculation }\end{array}$ & $\begin{array}{l}\text { Diversity } \\
\text { Chao-1 } \\
\text { index }\end{array}$ & $\begin{array}{l}\text { Chao-1 } \\
\text { index } \\
\text { average }\end{array}$ & $\begin{array}{l}\text { Diversity } \\
\text { Shannon } \\
\text { index }\end{array}$ & $\begin{array}{l}\text { Shannon } \\
\text { index } \\
\text { average }\end{array}$ \\
\hline \multirow{3}{*}{$\begin{array}{l}\text { AZA-1 } \\
\text { High }\end{array}$} & 4 & 366 & & 3.894 & \multirow{3}{*}{4.411} \\
\hline & 24 & 462 & 429.667 & 4.647 & \\
\hline & 48 & 461 & & 4.693 & \\
\hline \multirow{3}{*}{$\begin{array}{l}\text { AZA-1 } \\
\text { Low }\end{array}$} & 4 & 528 & & 4.756 & \multirow{3}{*}{4.519} \\
\hline & 24 & 417 & 452.667 & 4.282 & \\
\hline & 48 & 413 & & 4.521 & \\
\hline \multirow{3}{*}{ OA High } & 4 & 372 & & 4.083 & \multirow{3}{*}{3.739} \\
\hline & 24 & 337 & 336.333 & 3.639 & \\
\hline & 48 & 300 & & 3.496 & \\
\hline \multirow{3}{*}{ OA Low } & 4 & 322 & & 3.615 & \multirow{3}{*}{4.225} \\
\hline & 24 & 406 & 443.667 & 4.277 & \\
\hline & 48 & 603 & & 4.783 & \\
\hline \multirow{3}{*}{ PBS } & 4 & 379 & & 4.163 & \multirow{3}{*}{3.981} \\
\hline & 24 & 438 & 354.333 & 4.530 & \\
\hline & $* 48$ & 246 & & 3.251 & \\
\hline \multirow{3}{*}{ Untreated } & 4 & 472 & & 4.593 & \multirow{3}{*}{4.383} \\
\hline & $* 24$ & 273 & 403.000 & 4.124 & \\
\hline & 48 & 464 & & 4.432 & \\
\hline
\end{tabular}


Although PBS and OA high have the lowest diversity indices, AZA-1 high and OA high are the only treatments to have a statistically significant difference in microbiome structure when compared to the untreated control (adonis $P=0.032$ and adonis $P=0.003$, respectfully; Table 4.4). These results may indicate that the high oral dose of AZA-1 (75 ng/larva) and OA (62.5 ng/larva) does in fact cause changes to the microbiome. As there is no statistical difference between time points within each treatment (Table 4.5), it suggests that the changes in microbiome in AZA-1 High and OA High occurs within the first 4 hours of inoculation and no significant subsequent alterations in microbiome are detected up to 24 hours.

Table 4.4 Statistical differences of the gut microbiome structure in Galleria mellonella larvae after azaspiracid and okadaic acid exposure. Data represented as permutation test for adonis under reduced model and permutation test for homogeneity of multivariate dispersions following the Bray-Curtis method. $(n=9$ per treatment, total $=52)(P B S$ and untreated $n=8$ per treatment) (PBS $=2$ repeats at $48 \mathrm{hr}$, untreated $=2$ repeats at $24 \mathrm{hr}$ )

\begin{tabular}{lll}
\hline & Adonis Pr $(>\mathrm{F})$ & Dispersion Pr $(>\mathrm{F})$ \\
\hline Untreated vs & 0.8 & 0.9083 \\
PBS & & \\
Untreated vs & $0.032 *$ & 0.084 \\
AZA-1 High & & \\
Untreated vs & 0.062 & 0.149 \\
AZA-1 Low & & \\
Untreated vs & $0.003 *$ & $0.001 *$ \\
OA High & & 0.223 \\
Untreated vs & 0.067 & $0.001389 * *$ \\
OA Low & & \\
AZA-1 High & & \\
vs AZA-1 & 0.639 & \\
Low & & 0.3 \\
OA High vs & & \\
OA Low & & \\
\hline
\end{tabular}


Table 4.5 Statistical differences of the gut microbiome structure in Galleria mellonella larvae after azaspiracid and okadaic acid exposure when comparing 4, 24 and 48hr within each treatment. Data represented as permutation test for adonis under reduced model and permutation test for homogeneity of multivariate dispersions following the Bray-Curtis method. $(\mathrm{n}=3$ per treatment per time, total $=52)(\mathrm{PBS}=2$ repeats at $48 \mathrm{hr}$, untreated $=2$ repeats at $24 \mathrm{hr}$ )

\begin{tabular}{lll}
\hline & Adonis $\operatorname{Pr}(>\mathrm{F})$ & Dispersion $\operatorname{Pr}(>\mathrm{F})$ \\
\hline Untreated & 0.778 & $0.001^{*}$ \\
PBS & 0.49 & 0.122 \\
AZA-1 High & 0.742 & 0.392 \\
AZA-1 Low & 0.757 & 0.675 \\
OA High & 0.976 & 0.887 \\
OA Low & 0.327 & 0.085 \\
\hline
\end{tabular}

Firmicutes are Gram-positive bacteria and often represent the most predominant phylum found in mouse and human gastric microbiomes (Ley et al., 2006a). When assessing the effects of AZA and OA on the individual populations of bacteria within the larval gut, the untreated control and AZA-low appear to have a much lower abundance of Firmicutes at 4 hours, making up less than half of the overall abundances and remains constant for the duration of the experiment (Figure 4.11). On the other hand, Firmicutes is the most prevalent phylum in all other treatments throughout the experiment, with OA Low being almost entirely Firmicutes 4 hours post inoculation with a gradual decline to account for $\sim 50 \%$ of the total population by 48 hours. Conversely, the proportion of Firmicutes fluctuates largely in larvae exposed to PBS, accounting for $\sim 60 \%$ at 4 hours, $\sim 40 \%$ at 24 hours and increasing again to almost $100 \%$ by 48 hours post inoculation (Figure 4.11). Another notable difference in Firmicutes proportion is in larvae inoculated with a high dose of OA, which have a sustained high proportion of Firmicutes accounting for $75 \%-85 \%$ of the total microbiome from $4-48$ hours.

Untreated larvae have a much higher abundance of Bacteroidetes than all other treatments at 4 hours (Figure $4.11 \mathrm{~A}$ ), making up just over a quarter of the total abundance becoming the second most prevalent phylum for that group. In all other treatments, Bacteroidetes is the third (PBS and AZA High) or forth (OA High, AZA low and AZA High) most abundant phylum at 4 hours accounting for less than $10 \%$ of the total proportion, with Proteobacteria being the 
second most abundant. Interestingly, AZA-1 high exposed larvae have a notable increase in Bacteroidetes from $\sim 7 \%$ at 4 hours to $\sim 15 \%$ at 48 hours after inoculation. All other OA and AZA exposed larvae have a small increase in Bacteroidetes at 24 hours, which then return to similar levels as at 48 hours (Figure 4.11).

Proteobacteria is the second or third most abundant phylum, however there is a large amount of variation as to what proportion it holds between each treatment group. OA High exposed larvae have a consistently low proportion of Proteobacteria, between $\sim 9-14 \%$. This is consistently lower than the proportion observed in the untreated and AZA low treated larvae. Surprisingly, PBS inoculated larvae have a similar proportion as to the untreated at 4 hours $(\sim 17 \%)$ and $24(30 \%)$ hours post inoculation (Figure $4.11 \mathrm{~A}$ and B). However, 48 hours after inoculation Proteobacteria is almost entirely gone in the microbiome. This is not observed in any other treatment groups, conversely OA low exposed larvae have a very low proportion of Proteobacteria of $\sim 6 \%$ which then increase to $\sim 30 \%$ by 48 hours post exposure. The presence of Actinobacteria and Acidobacteria are likely to be due to bacteria in the culturing environment or food substrate, which the larvae are reared on (Figure 4.11). 


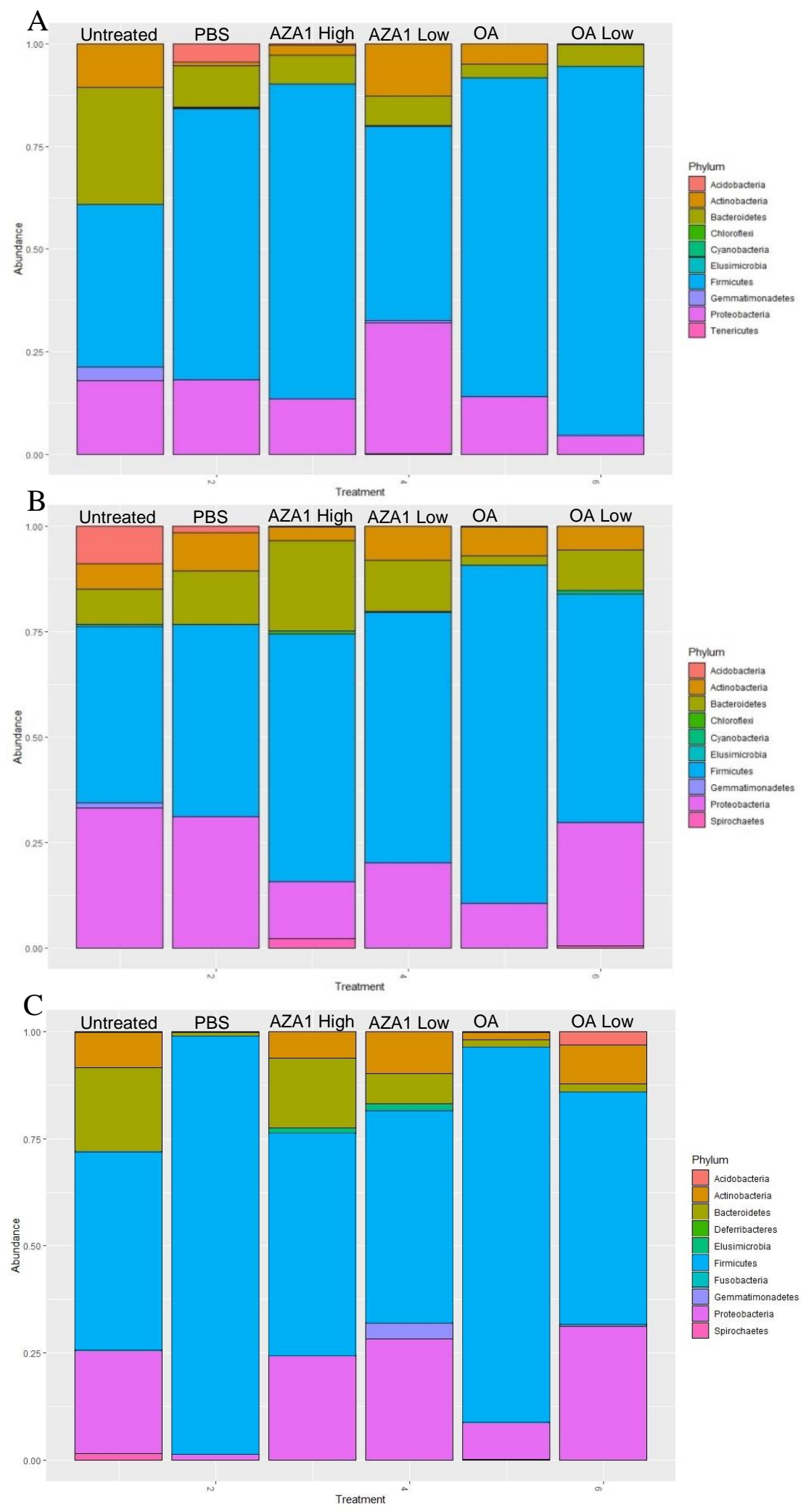

Figure 4.11 Microbial (16S) composition of the gut tissue from Galleria mellonella larvae exposed to OA and AZA-1. A comparison of the top 10 most abundant phyla depicted as relative abundance 4 hours (A), 24 hours (B) and 48 hours $(C)$ after force-feeding. (n $=3$ per treatment per time point, total $=52)$. $(\mathrm{PBS}=2$ repeats at $48 \mathrm{hr}$, untreated $=2$ repeats at $24 \mathrm{hr})$. 
Untreated larvae - no PBS or toxin inoculation - displayed a different microbiome structure at the Genus level, when compared to all others, with a much lower proportion of Enterococcus (between $40-50 \%$ of total). Enterococcus accounts for $>50 \%$ for all time points except for OA low at 48 hours where the proportion decreases to $40 \%$ (Figure 4.12). Interestingly, OA high exposed larvae have the largest proportion of Enterococcus accounting for $\sim 85 \%$ of the total populations at 4 hours (Figure $4.12 \mathrm{~A}$ ) and remains between $80-95 \%$ for the duration of this experiment. This observation could explain the statistical difference between the untreated and OA high adonis p-value (Table 4.5) and clearly shows no substantial change over the 48-hour period.

The untreated larvae also have a high proportion of Flavobacterium across all time points making up between $14-25 \%$ of the total abundance compared to the other treatments, which range between 1-14\% (Figure 4.12). Interestingly, larvae treated with AZA have a larger proportion of Flavobacterium than OA high or low which could suggest different effects of the two toxins. Another notable difference is the presence of Prevotella in untreated and the AZA low treated larvae at 4 hours (Figure $4.12 \mathrm{~A}$ ), which declines in 24 and 48 hour time points but increases in AZA high at 24 hours post inoculation (Figure 4.12 B). Prevotella is a member of the Bacteroidetes and can be a sign of microbiome imbalance - its presence in humans can be associated with a plant rich diet in humans (Ley, 2016, Si et al., 2017). Therefore, it's presence here could be as a result of the larval diet or a disruption in the normal microbiome.

Another notable genus is Staphylococcus at 4 and 24 hours (Figure 4.12 A and B), which is present at low levels in the untreated and PBS larvae at 4 hours and then appears in all groups at a low level (less than $10 \%$ of total abundance) and then does not appear at 48 hours. Some species of Staphylococcus are pathogenic and are identified in Lepidoptera gut microbiota in other studies (Paniagua Voirol et al., 2018). This could indicate a subsequent infection or an imbalance in the microbiome. 


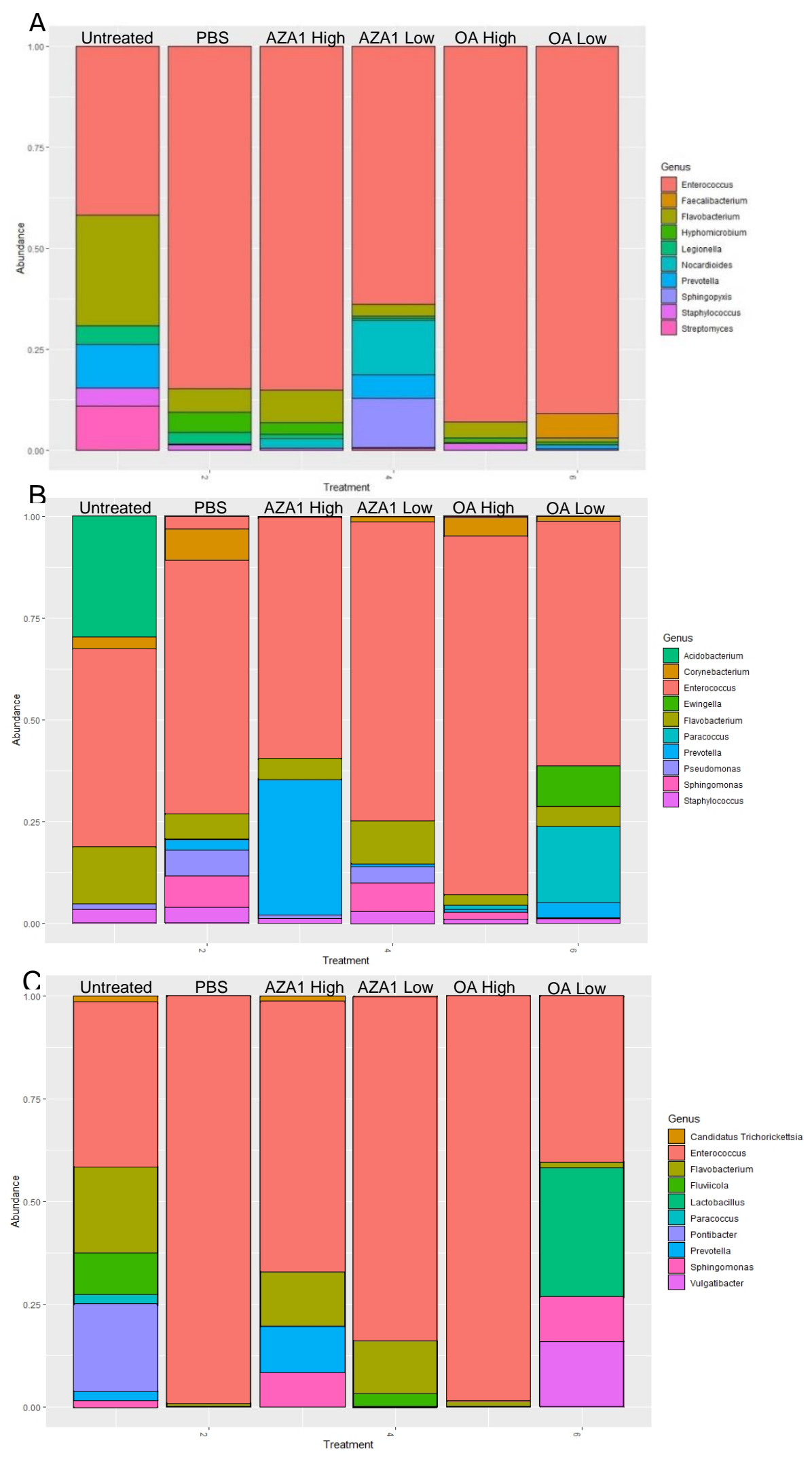

Figure 4.12 Microbial (16S) composition of the gut tissue from Galleria mellonella larvae exposed to OA and AZA-1. A comparison of the top 10 most abundant genus depicted as relative abundance 4 hours (A), 24 hours (B) and 48 hours (C) after force feeding. ( $n=3$ per treatment per time, total $=52)(\mathrm{PBS}=2$ repeats at $48 \mathrm{hr}$, untreated $=2$ repeats at $24 \mathrm{hr}$ ) 


\subsubsection{Susceptibility of insect larvae to Campylobacter jejuni in the absence and presence of azaspiracid 1}

To determine a suitable inoculum of Campylobacter jejuni for insect susceptibility studies, initial viability tests were performed using $1 \times 10^{4}$ to $3 \times 10^{6}$ CFUs per larva (Figure 4.13). Exposure of insects to $C$. jejuni led to significant decreases in survival, either by force-feeding $\left(X^{2}(5)=50.96, \mathrm{P}<0.0001 ;\right.$ Figure 4.13C), or intrahaemocoelic injection $\left(X^{2}(5)=60.4, \mathrm{P}<\right.$ 0.0001; Figure 4.13F). For force-fed insects, lower doses of $1 \times 10^{4}$ and $1 \times 10^{5}$ bacteria reduced survival by no more than $7 \%$ at 72 hours post-inoculation. Conversely, higher doses of $1 \times 10^{6}$ and $3 \times 10^{6}$ reduced survival rates by $40 \%$ (Figure $4.13 \mathrm{C}$ ). Similar survival trends were observed for insects infected through injection - however, no mortalities were recorded for lower doses, and $1 \times 106$ reduced mortality by $20 \%$ at 72 hours (Figure $4.13 \mathrm{~F}$ ). Force-feeding of $1 \times 10^{6}$ $\mathrm{CFU} /$ larva caused a more rapid reduction in survival with $\sim 67 \%$ alive after 24 hours compared to $\sim 85 \%$ alive in larvae injected the same dose.

Exposure of larvae to $C$. jejuni also yielded reciprocal decreases in movement and melanisation indices (Figure 4.13 A, B, D, E). Both injection and force feeding produced a similar decrease in activity to a mean of 1.2 after $3 \times 10^{6}$ CFU/larva, which is significantly lower than the untreated and negative control (PBS) values of 3 and 4, respectively. By 24 hours post forcefeeding there is dose dependent reduction in melanisation and movement indices for $3 \times 10^{6}$ (1.97 and 1.53) $(P<0.0001)$ and $1 \times 10^{6}(2.37$ and $1.97 ; P<0.0001$ and 0.0002 , respectively). These data reveal a clear, continuous deterioration in health over the experimental period, with an obvious dose-dependent response in larvae injected with the bacterium (Figure 4.13, D-F).

The injection of insects with $25 \mathrm{ng} /$ larva of AZA1, followed 24 hours later by force-feeding PBS (with 2\% ethanol) did not cause any mortality (Figure 4.14C), which is in agreement with toxicity studies performed above (Figure 4.1C). Survival rates do not differ significantly when larvae are pre-treated with AZA-1 (25 ng/larva) followed by $1 \times 10^{4} \mathrm{CFUs}$ when compared to PBS followed by $1 \times 10^{4}$ CFUs $\left(X^{2}(3)=5.2, \mathrm{P}=0.157\right.$; Figure 4.14C). AZA-1 (25 ng/larva) alone did not cause any significant reductions in either health index, however 4 hours after force-feeding $C$. jejuni there were significant reductions in movement and melanisation for all doses used, $1 \times 10^{4}$ to $3 \times 10^{6}(P<0.0001$; Figure 4.14A, B). All larvae pre-treated with AZA deteriorated faster and to a greater extent, compared to those that received $C$. jejuni alone (Figure 4.13). For example, doses of $1-3 \times 10^{6}$ alone killed $40 \%$ of larvae by 72 hours (Figure 4.13C), whereas pre-exposure to a sublethal dose of AZA1 (25 ng/larva), killed $40 \%$ by 4 
hours and $\sim 60 \%$ of larvae by 48 hours (Figure $4.14 \mathrm{C}$ ). There is a $23 \%$ decrease in survival when larvae are pre-exposed to AZA1.

There is some evidence to suggest the double inoculation approach (initial injection followed by force-feeding 24 hours later) did cause a decrease in the melanisation index, however this is a small, non-significant difference of 0.75 when compared to untreated larvae (Figure 4.14B).
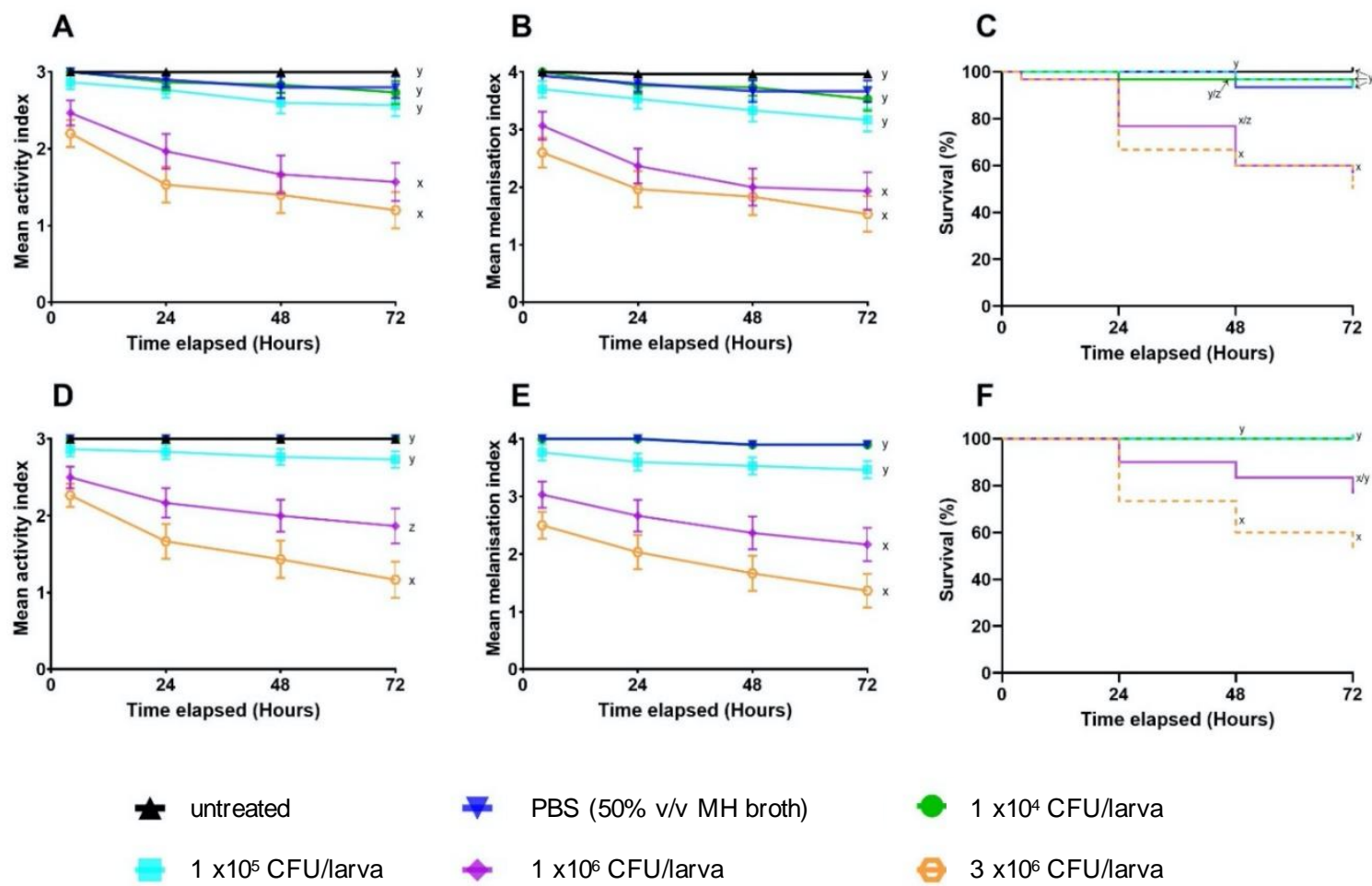

Figure 4.13 Health indices of Galleria mellonella larvae following force-feeding or intrahaemocoelic injection of Campylobacter jejuni. Insects received C. jejuni $1 \times 10^{4}$ to $3 \times 10^{6}$ CFU/larva via force-feeding (A, B and C) or injection (D, E and F) and were maintained in darkness at $30{ }^{\circ} \mathrm{C}$ for 96 hours. Larval health was scored (see Table 4.2) by assessing movement (A and D), melanisation (B and E) and survival ( $\mathrm{C}$ and F). Values are presented as mean $\pm \mathrm{SE}$ ( $\mathrm{n}=30$ per treatment, 330 in total across both inoculation methods). Unshared letters represent significant differences $(P \leq 0.05)$ determined by Tukey's multiple comparisons tests. The negative control consisted of force-feeing or injecting larvae with PBS containing 50\% Mueller Hinton broth. 


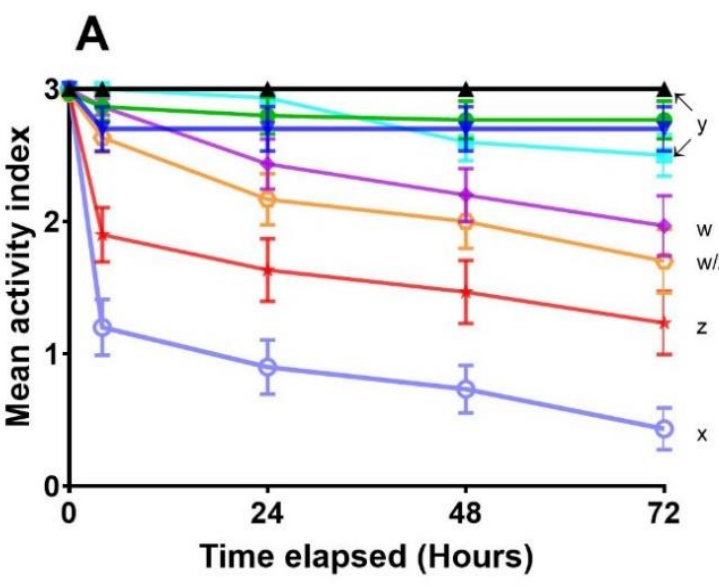

Control NT

$A Z A+1 \times 10^{4}$
B

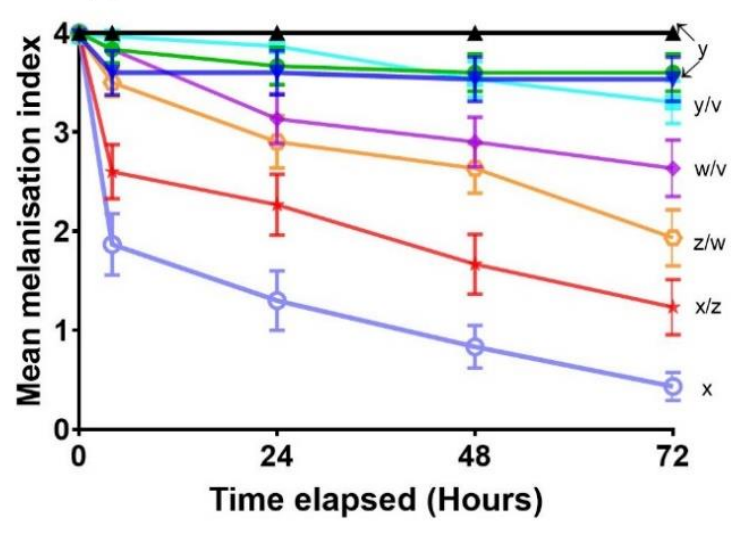

PBS+PBS

$A Z A+1 \times 10^{5}$
PBS $+1 \times 10^{4}$

$A Z A+1 \times 10^{6}$
C

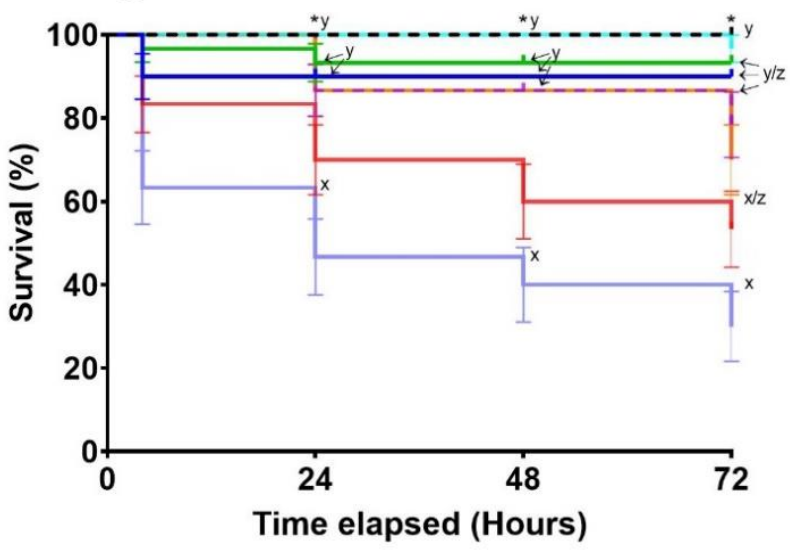

$\mathrm{AZA+PBS}$

$A Z A+3 \times 10^{6}$

Figure 4.14 Susceptibility of Galleria mellonella to Campylobacter jejuni after azaspiracid-1 pre-treatment. Larvae received a pre-treatment of AZA-1 (25 ng/larva) via injection, after 24 hours larvae then received $C$. jejuni $\left(1 \times 10^{4}-3 \times 10^{6} \mathrm{CFU} /\right.$ larva) via force feeding and were maintained in darkness at $30{ }^{\circ} \mathrm{C}$ for a further 72 hours. Larval health was scored (Table 4.2) by assessing movement (A), melanisation (B) and survival (C). Larvae were considered dead when they no longer responded to being rolled over. Values are presented as mean \pm SE $(n=30$ per treatment, 240 total). Unshared letters represent significant differences $(P \leq 0.05)$ determined by Tukey's multiple comparisons tests. The negative control consisted of with PBS (2\% ethanol) + PBS (50\% Mueller Hinton broth). 


\subsection{Discussion}

Marine biotoxins, such as azaspiracids and okadaic acid, are known to induce gastric damage such as epithelial degeneration, ulcers and increased permeability in both humans and rodent models (Abal et al., 2017, Franchini et al., 2010) - yet, their impacts on non-target tissues (e.g., immune cells) are largely unknown. The aim of this chapter was to further investigate how generalizable G. mellonella is as a model for studying marine toxins, and whether a sub-lethal dose of AZA-1 increases larval susceptibility to gut specific bacterial infection $(C$. jejuni). The latter has not been determined in rodents, but represents an important question of human health. Toxicities of AZA-1, 2 and 3 were assessed successfully in G. mellonella larvae via intrahaemocoelic injection and gavage (force-feeding) across two biologically relevant concentration ranges of 5-50 ng/larva for AZA1/AZA2 and 20-200 ng/larva for AZA3. It is important to use both inoculation methods to assess if the effects are localised to the gut when force-feeding compared to intrahaemocoelic injection. By utilising both methods it also allows a comparison of effects between the traditional injection (screening toxicities) and the natural route of ingestion. Azaspiracid toxicological endpoints, e.g., LD50 value, differ depending on the route of administration in rodents - Table 4.6, however, a common observation is the AZA1 is the most toxic of the three. AZA1 appears to be more toxic than AZA2 across a similar concentration range, and this is in agreement with a recent assessment of AZA oral toxicities ranking them AZA1>AZA2>AZA3 (Pelin et al., 2018b). Further still, the same authors calculated an $\mathrm{LD}_{50}$ value of $875 \mu \mathrm{g} / \mathrm{kg}$ for AZA3 at 24 hours post-gavage in mice - which is in close agreement with the $\mathrm{LD}_{50}$ value of $881.4 \mu \mathrm{g} / \mathrm{kg}$ that was calculated for AZA3 here. The azaspiracids are lipophilic toxins, and cause similar symptoms of gastrointestinal stress as another lipophilic toxin, okadaic acid. In a proof of concept study, Coates et al. (2019) demonstrated the capacity of $G$. mellonella larvae to assess the toxicity of okadaic acid, and calculated an oral $\mathrm{LD}_{50}$ value of $\sim 239 \mu \mathrm{g} / \mathrm{kg}$, which falls within the range of values obtained in mammalian models, 206-242 $\mu \mathrm{g} / \mathrm{kg}$. Although Coates et al. (2019) established several oxidative stress markers associated with okadaic acid intoxication, namely superoxide dismutase activity and malondialdehyde levels (i.e., lipid peroxidation) in the midgut, they did not assess tissue damage directly using histology. Here, the gut tissues of insect larvae were assessed histologically after exposure to $62.5 \mathrm{ng}$ okadaic acid (i.e., $\sim 250 \mu \mathrm{g} / \mathrm{kg}$ ), and revealed severe damage to the midgut within 24 hours, and evidence of haemocyte cytotoxicity in the body cavity surrounding the GI tract (Figures 4.7 - 4.9). Force-feeding AZA-3 resulted in a higher rate of fatalities and greater reduction in health indices for the same concentrations 
administered by intrahaemocoelic injection. The inoculation of AZA-2 did not cause a significant reduction in viability or health indices with this concentration range $(5-50$ ng/larva), suggesting this is not as toxic as AZA1 in G. mellonella. Differences in health indices between inoculation methods also indicate the importance of using a biologically relevant method of administration to get more realistic responses. 
Table 4.6 Azaspiracid administration, toxicology, and pathology in mice.

\begin{tabular}{|c|c|c|c|c|}
\hline Model & Administration & Toxicity/Lethality & Pathologic features & Reference \\
\hline $\begin{array}{l}\text { CD-1 mice } \\
\text { [female] }\end{array}$ & Gavage & $\begin{array}{l}\text { AZA } 1 ; \mathrm{LD}_{50}=443 \mu \mathrm{g} / \mathrm{kg} \\
\text { AZA } 2 ; \mathrm{LD}_{50}=626 \mu \mathrm{g} / \mathrm{kg} \\
\text { AZA } 3 ; \mathrm{LD}_{50}=875 \mu \mathrm{g} / \mathrm{kg}\end{array}$ & $\begin{array}{l}\text { Enlarged pale liver; } \\
\text { increased levels of } \mathrm{K}^{+} \\
\text {ions in blood; tissue } \\
\text { alterations in the GI tract } \\
\text { and spleen }\end{array}$ & $\begin{array}{l}\text { (Pelin et al., } \\
2018 b)\end{array}$ \\
\hline $\begin{array}{l}\text { ddY mice } \\
\text { [male, 14- } \\
17 \mathrm{~g}]\end{array}$ & Intraperitoneal & $\begin{array}{l}\text { AZA1 }=200 \mu \mathrm{g} / \mathrm{kg} \\
\text { AZA2 }=110 \mu \mathrm{g} / \mathrm{kg} \\
\text { AZA3 }=140 \mu \mathrm{g} / \mathrm{kg}\end{array}$ & Not described & $\begin{array}{l}\text { (Satake et al., } \\
\text { 1998) } \\
\text { (Ofuji et al., } \\
\text { 1999) }\end{array}$ \\
\hline $\begin{array}{l}\text { ICR mice } \\
\text { [male, } \sim 25 \mathrm{~g}]\end{array}$ & Gavage & $\begin{array}{l}\text { AZA1; } 1-3000 \mu \mathrm{g} / \mathrm{kg} \\
\text { AZA1; } 2 \text { doses of } 300- \\
450 \mu \mathrm{g} / \mathrm{kg}\end{array}$ & $\begin{array}{l}\text { Cell degeneration on } \\
\text { villi; atrophic lamina } \\
\text { propria; vacuolisation of } \\
\text { epithelial cells; crypt } \\
\text { irregularities; necrotic } \\
\text { (B and T) lymphocytes } \\
\text { in thymus, spleen and } \\
\text { Peyer's patches; } \\
\text { bleeding, oedema, and } \\
\text { cell infiltration of } \\
\text { alveolar cells; interstitial } \\
\text { pneumonia }\end{array}$ & $\begin{array}{l}\text { (Ito et al., } \\
\text { 2006, Ito et al., } \\
\text { 2000, Ito et al., } \\
\text { 2002a, Hess et } \\
\text { al., 2015, } \\
\text { Panel, 2008, } \\
\text { UK, 2018) }\end{array}$ \\
\hline $\begin{array}{l}\text { NMRI mice } \\
\text { [female, 18- } \\
22 \mathrm{~g}]\end{array}$ & Gavage & $\begin{array}{l}\text { AZA } 1 ; \mathrm{LD}_{50}=775 \mu \mathrm{g} / \mathrm{kg} \\
{\left[\mathrm{LD}_{10}=570 \mu \mathrm{g} / \mathrm{kg}\right]}\end{array}$ & $\begin{array}{l}\text { Pale liver; broader villi } \\
\text { in duodenum with some } \\
\text { neutrophil } \\
\text { accumulation; apoptosis } \\
\text { in lamina propria }\end{array}$ & $\begin{array}{l}\text { (Aune et al., } \\
2012 \text { ) }\end{array}$ \\
\hline $\begin{array}{l}\text { NMRI mice } \\
\text { [female, 19- } \\
22.5 \mathrm{~g}]\end{array}$ & Gavage & AZA $1 ; 100-300 \mu \mathrm{g} / \mathrm{kg}$ & $\begin{array}{l}\text { Cellular detachment in } \\
\text { the duodenum villi; } \\
\text { crypt expansion; } \\
\text { necrosis of lamina } \\
\text { propria }\end{array}$ & $\begin{array}{ll}\text { Aasen } & \text { et al. } \\
(2010 & \text { and } \\
2011) & \end{array}$ \\
\hline
\end{tabular}


Although there is no significant difference in total haemocyte counts upon exposure to AZA1, there was a significant increase in haemocyte size between 4 - 48 hours after the injection of AZA-1 (50 ng/larva). This effect has been observed in $G$. mellonella upon fungal (Conidiobolus coronatus ) infection and can be a sign cell swelling associated with apoptosis (Boguś et al., 2018). Larvae force-fed AZA-1 (37.5 ng/larva) and okadaic acid (62.5 ng/larva) displayed significant midgut damage with obvious nuclear aberrations - condensation, fragmentation and dissolution. Similar to histopathology after indomethacin (Chapter 2), there is variation in the level of damage observed from the severe total degradation of the epithelial layer to minor/regional abrasions and disorganisation. These changes to the larval midgut are similar to those found in rats with increased apoptosis and erosion of the epithelial layer (Ito et al., 2002b, Dounay and Forsyth, 2002). In fact, AZA1 force-feeding of mice depicts similar displacement of duodenum villi, and cell death (Aasen et al., 2010, Aasen et al., 2011).

An open question for marine toxicologists is the impact of chronic exposure to sub-lethal doses, or doses below the acceptable upper regulatory limits on human health. For azaspiracids and okadaic acids, the upper regulatory limit in the UK and EU is $160 \mu \mathrm{g} / \mathrm{kg}$ shellfish tissue. Recently, Ferreiro et al. (2016) exposed rats to AZA1 up to $55 \mu \mathrm{g} / \mathrm{kg}$ on four occasions across 15 days, and observed structural and functional deterioration of cardiac tissue. In a follow-on study, Ferreiro et al. (2017) exposed rats to another lipophilic toxin, yessotoxin, via intraperitoneal injection on four occasions across 15 days (50 or $70 \mu \mathrm{g} / \mathrm{kg}$ ), which resulted in immunotoxicity (lower lymphocyte counts, splenic damage/apoptosis viewed through histology, and reduced interleukin-6 levels in plasma). These two studies are evidence of subacute toxicity, that do not lead to the typical symptoms of shellfish poisoning, however, there does not appear to be any study reporting on the putative link between intoxication and increased risk of infection (Ferreiro et al., 2016, Ferreiro et al., 2017). Injecting G. mellonella with a sublethal dose of AZA-1 (25 ng/larva) increased their susceptibility to $C$. jejuni infection administered 24 hours later. Pre-exposure caused a significant decrease in larval health with lower activity and melanisation indices compared to $C$. jejuni force feeding alone (Figure 4.14). These results indicate the larval gut and/or immune system may be compromised and are less able to contain the bacterium. This is supported by the histopathology (Figures 4.7-4.9), where displacement and damage to the epithelium may facilitate pathogen movement across the gut and into the body cavity - causing septicaemia.

To further investigate the effects of AZA and OA has on the G. mellonella gut it is important to assess the bacterial microbiome as it is understood that changes to the natural microbiome 
can result in increased risk of infection, disease and alter the breakdown of food (Becattini et al., 2016). From studying colony forming units following AZA-1, 2 and 3 force feeding showed that there were significant changes from 4-48 hours after inoculation but only compared to PBS and not the untreated control. AZA-3 force feeding did not causing any significant difference compared to the control or concentrations, which is unexpected as the disruption to the midgut observed in histology it is likely to observe changes in the microbiome from bacteria being "flushed out" of the gut through cellular sloughing (if all azaspiracid congeners have similar modes of toxicity). It appears that there is an overall increase in CFU from 4-48 hours in all treatments with AZA-2 causing the biggest differences. This could be due to AZA-2 being less toxic to the larvae, but causing subtle changes to the gut without causing the degradation of the epithelial layer but enough to alter the microbiome. Next-generation sequencing of the $16 \mathrm{~S}$ rRNA (v4) region was used to investigate the composition of the gastric microbiota.

Similarly to observations in the mouse and human gastric microbiomes (Ley et al., 2006a), most of Firmicutes present is attributed to the genus Enterococcus, which also dominated the haemolymph and skin of bait and research grade G. mellonella larvae (Allonsius et al., 2019). In this chapter, G. mellonella larvae were found to have a large proportion of Firmicutes in the microbiome with larvae exposed to high doses of OA having a consistent higher proportion of Firmicutes. Interestingly, Firmicutes presence is associated with diet in humans, with diets high in sugar and fat and in undernourished diets associated with greater proportions of Firmicutes than those having a balanced diet (Méndez-Salazar et al., 2018). This could suggest that larvae with high proportions of Firmicutes are storing more fat from their diet than the other larvae. Both OA low and high treatments impacted the abundances of Firmicutes and Bacteroidetes with initial low levels of Bacteroidetes and high levels of Firmicutes at 4 hours were similar to observations in rats when repeatedly exposed to low concentrations of OA (Liu et al., 2020). Low does exposure of rats to okadaic acid ( $80 \mu \mathrm{g} / \mathrm{kg}$ via gavage) over 30 days caused damage to the epithelium, and increased the relative abundance of bacterial pathobionts in the colon, compared to control rats (Liu et al., 2020).

The genus Prevotella is one represented population of Bacteroidetes and has been linked with $\mathrm{T}$ helper cell mediated inflammation via the activation of Toll-like receptors (Larsen, 2017). Interestingly, G. mellonella larvae exposed to high dose of AZA-1 have a notable increase in Prevotella 24-48 hours after inoculation. This coincides with the period of observed histological damage in AZA-1 high inoculations, however there is no marked increase in Prevotella in OA exposed larvae. Perhaps this reflects the differential impacts of marine toxins. 
A high proportion of Firmicutes and a low proportion of Bacteroidetes is an indicator of dysbiosis, in studies assessing dysbiosis in patients suffering from the neurodegenerative disorder Amyotrophic lateral sclerosis found all patients were affects by dysbiosis with a high proportion of Firmicutes and low proportion of Bacteroidetes (Rowin et al., 2017). This is also observed in other neurological disorders such as Parkinson's disease (Sampson et al., 2016) and in gastric disorders such as IBD (Tamboli et al., 2004). AZA and OA both induce diarrhoea with erosion of the epithelial layer of the gut similar to that in IBD patients and indicate that AZA and OA exposure induce dysbiosis, which could in turn exacerbate inflammation within the gut.

A high proportion of Proteobacteria is observed in patients with IBD and Crohn's disease, although the mechanisms is unknown, it is considered to be as a result of inflammation of epithelial cells and increased oxygenation, favouring Proteobacteria (Rizzatti et al., 2017). Additionally, studies conducted in G. mellonella infected with the bacterium Bacillus thuringiensis found an increase in Proteobacteria and a reciprocal decrease in Firmicutes and Bacteroidetes (Dubovskiy et al., 2016) compared to uninfected control larvae. Surprisingly, AZA high and OA exposed larvae had lower proportions of Proteobacteria than untreated and PBS larvae at 4 and 24 hours post inoculation, which indicate that dysbiosis and associated inflammation are different for chemical disruptors versus microbial disruptors of the gut.

Sphingomonas is a proteobacterium present in AZA-1 low and PBS-treated insects at 24 hours post inoculation and AZA-1 high and OA low at 48 hours post inoculation. Sphingomonas is associated with inflammation via $\mathrm{T}$ helper cells activation and is theorised to be involved in the induction of natural killer cells in germ free mice once orally inoculated with conventional mouse faecal suspension (El Aidy et al., 2014). This could indicate that the larvae exposed to AZA and OA undergo dysbiosis associated with inflammation followed by re-colonisation driven by commensals such as Sphingomonas, which may promote inflammation.

Although there are notable differences between intoxicated (AZA1, OA) larvae and the untreated larvae, there is rather a lot of variation across time/treatments. The overall observations supported with adonis statistical analysis indicate that both AZA and OA high have statistically significant microbiome structures that may in part be attributed to the high proportions of Prevotella and Sphingomonas as well as much higher proportions of Enterococcus. Surprisingly, PBS is not statistically different to untreated when conducting an adonis analysis, however, when comparing populations to the untreated, PBS appears less 
diverse at 48 hours being almost entirely Enterococcus similarly as OA high. This may represent a flushing to the GI tract.

\subsection{Concluding remarks}

Work presented in this chapter has demonstrated that G. mellonella larvae are sensitive to physiologically relevant doses of AZA and OA, and that it is possible to asses toxicity through several endpoints. To further investigate the larval' $s$ ability to model human intestinal damages, and act as an alternative model it is important to also asses its ability to repair and respond to the protective effects of nutraceuticals against toxins such as AZA and pharmaceuticals such as indomethacin.

Azaspiracids, notably AZA3, caused a dose dependent reduction in larval health indices such as movement and melanisation as well as increasing susceptibility to gastric bacteria $C$. jejuni resulting in further morbidity and in exitus. Additionally, AZA-1 and okadaic acid forcefeeding caused significant damage and disruption to the larval mid gut, similarly to those observed in indomethacin treated larvae. IN an experimental first, marine toxins appear to disrupt microbial community structure in the larval gut that mimics okadaic acid-induced changes in the rat colon. These findings expand the utility of G. mellonella as a platform for investigating subacute or chronic impacts of toxin exposure.

\section{Acknowledgments}

I would like to thank Mrs Sophie Malkin (Bluefish Technical Officer, Swansea University) for her assistance with processing histology samples, and Dr Jessica Thomas (Bevan) (Bluefish Scientific Officer, Swansea University) for the valuable assistance with NGS data analyses. 
Chapter 5: Overall Summary and General discussion 


\subsection{General Discussion}

This body of work represents an evaluation of the usefulness and accuracy of Galleria mellonella larvae as a substitute in vivo model for gut-related pathobiology. It was established that the indomethacin gastric damage (ulcer) assay of rodents could be recapitulated in $G$. mellonella (Chapter 2), dietary intervention with two nutraceuticals, Cordyceps sinensis and colostrum, can alleviate some gastric damage caused by chemicals and/or microbes (Chapter 3 ), and gastropathy/toxicity of closely related marine toxins, okadaic acid and azaspiracids 1,2,3 can be distinguished (Chapter 4).

The approach in chapter two sought to replicate studies conducted in murine models (e.g., by Marchbank et al., 2011) to G. mellonella larvae using an NSAID known to cause gastric damage, allowing a direct comparison to mice, to optimise methodology, and to assess limitations of using an insect in this manner. Data presented in chapter two reveal G. mellonella larvae to have similar histopathological signatures to an equivalent oral dose of indomethacin as observed in rats, with sloughing of epithelial cells, apoptosis and membrane blebbing/ulceration (figures 2.11 - 2.13) all recorded. Moreover, increased gut permeability was seen within 24 hours in the larvae, which has also been documented in both humans and mice (Playford et al., 1999 and 2001; Bjarnason and Takeuchi, 2009). Such information positions G. mellonella as a suitable model for tracking damage and pathology induced by common pharmacological agents. However, the model is limited to observations within a 72hour period, as metamorphosis began from 72 hours after inoculation of the control saline, thus longer-term studies in excess of five days (final instar larvae) are less likely to produce reliable results. To overcome this, it could be possible to rear larvae and use them soon after they moult into the final instar; however, for the purposes of screening compounds for reparative properties, the 3-day window is sufficient.

Another limitation for utilising G. mellonella in pharmaceutical studies are the differences in gut $\mathrm{pH}$ compared to human and murine models. The lepidopteran gut is alkaline, therefore it is perhaps not reliable for studying acid dependent pharmaceuticals, such as proton pump inhibitors which require acidic conditions to become their active forms (Shin and Kim, 2013, Bosnjak et al., 2019). Although the focus on indomethacin in this study was in relation to the induction of gastric damage, due to the lack of COX-1 and 2 receptor characterisation in $G$. mellonella is not a fully developed platform yet for assessing the therapeutic properties of COX-1 and 2 targeting drugs such as NSAIDs (Summ and Evers, 2013). However, as the larvae demonstrates similar metabolic processes and digestive enzymes, resulting in similar metabolic 
profiles (e.g., caffeine processing Maguire et al. (2017)), the larvae remain a suitable screening tool to asses gastric damage, toxicity, gut permeability and changes to microbial populations.

The outcome of chapter 3 broadened the scope of G. mellonella to include testing the protective properties of $C$. sinensis and colostrum against indomethacin and $C$. jejuni infection. Larvae reared on enhanced diets with $10 \%$ C. sinensis or $10 \%$ colostrum, provided ostensible survival benefits with respect to indomethacin induced health decline. Some protection against gut permeability was provided when reared on the $10 \%$ C. sinensis enhanced diet, indicating a a greater capacity to recover. Larvae reared on a $10 \%$ colostrum enhanced diet showed protection against the initial increase in permeability within the first 4 hours of indomethacin gavage. These findings indicate the $C$. sinensis and colostrum may reinforce the gut against pharmaceutical- associated tissue degeneration (Figures 3.7 and 3.8), however, the dose range and potential for additive effects need to be investigated further.

In the same chapter (3), larvae reared on the $10 \%$ colostrum enhanced diet were significantly heavier and more resistant to the adverse effects of $C$. jejuni infection - determined using an expanded health index rather than survival alone. Larvae reared on $10 \%$ C. sinensis supplemented diet were not as heavy, yet they were also more resistant to $C$. jejuni compared to those reared on a normal diet (figures 3.2 and 3.9). This is not the case when larvae are injected directly into the haemocoel with $C$. jejuni, which suggests that nutraceuticals (notably Cordyceps) improves gut resistance (specifically) to bacterial infection. The next step would be to investigate the potential immune-modulation properties of these nutraceuticals.

Chapter four describes the gut-specific damage caused by two groups of toxins known to induce diarrhoea-associated symptoms when consumed by humans, the azaspiracids $(1-3)$ and okadaic acid. This chapter is unique, as it is the first to compare all these toxins simultaneously in a model system, and measure pathology and dysbiosis. Reassuringly, the G. mellonella proved sensitive enough to distinguish the toxicities of AZA-1 and AZA-2, which is in agreement with the available literature (Pelin et al., 2018a). The $\mathrm{LD}_{50}$ value calculated for AZA-3 is highly similar to mice: $881.4 \mu \mathrm{g} / \mathrm{kg}$ and $875 \mu \mathrm{g} / \mathrm{kg}$, respectively. Histological studies found larvae orally administered AZA-1 (37.5 ng/larva) and okadaic acid (62.5 ng/larva) experienced gastric damage similar to murine models and some common features with indomethacin-treated insects (figures 4.4 and 4.9), with clear signs of nuclear aberrations, loss of tissue structure, and epithelial cell erosion (Ito et al., 2002b, Aasen et al., 2010). This supports the use of G. mellonella larvae as a generalised model for marine toxicology, and 
more broadly, hints at a conserved damage-response pathway along the alimentary canals of insects and mammals, including the maintenance of the mucosa (i.e., peritrophic matrix) by eicosanoids and derivatives (Emery et al., 2019; Chapter 2). Profiling the larval gut microbiome highlighted Enterococcus as the dominant OUT, which is comparable to mouse and human intestinal studies (Ley et al., 2006a), and a recent microbiome atlas of G. mellonella larvae from bait-shop grade and scientific grade (Allonsius et al., 2019). Interestingly 4-24 hours after force-feeding AZA-1 and OA, the larvae had a larger proportion of Firmicutes coupled with a low abundance of Bacteroidetes compared to the untreated control. This imbalance is an indicator for dysbiosis (Rowin et al., 2017). Additionally, larvae force-fed a high concentration of AZA and OA had significantly different microbiome composition (figures 4.11 and 4.12). These findings demonstrate that G. mellonella is a suitable model, with the ability to observe not only phenotypic effects, i.e., survival as a single toxicological endpoint, but also changes in microbiome composition (which can be further expanded by profiling the faeces).

Rats repeatedly exposed to AZA-1 at sub-lethal (below regulatory limits) showed deterioration of the cardiac tissue (Ferreiro et al., 2016), however no such test appears to have been conducted to review the susceptibility to infection. A key objective of chapter four was to assess the effect of a subacute dose of AZA-1 on the susceptibility to C. jejuni infection. Larvae that were pre-injected with AZA-1 below the regulated limits were more susceptible to C. jejuni infection within 24 hours, resulting in decreased activity and increased melanisation of the integument compared to larvae force-fed $C$. jejuni alone. This is an important finding as it suggests that regular consumption of shellfish with acceptable levels of AZA-1 toxin, and without showing symptoms of shellfish poisoning, are increasing their risk of collateral infection from a common enteric microbe. Consequently, further research into the potential long-term effects of the repeated consumption of "safe" concentrations of marine toxins (AZAs and $\mathrm{OA}$ ) and bioaccumulation potential among tissues within humans is needed. An appropriate study design to utilise the G. mellonella model by rearing larvae on an artificial diet containing AZA or OA (similar to Chapter 3), and observing their microbiome, changes to movement, resistance to infection and larval development. Additionally, microdissection of larval organs such as the brain, gut and fat body and quantitating levels of AZA and OA would be highly valuable.

Data presented here represent strong evidence for the use of G. mellonella as an in vivo tool for assessing established and emerging marine toxins at the tissue level - what remains unclear, and the natural next step for this work, is are the molecular targets of the toxins conserved 
across insects, mice and humans? One novel approach would be to focus on cellular (haemocyte) bioenergetics, as it is an important factor for understanding the pathophysiology of cancer, cardiovascular disease and diabetes (Bradford et al., 2012). Changes in cellular energetics, and mitochondrial functioning can indicate general declines in organism health (Bradford et al., 2012). Therefore, experiments studying the bioenergetics of extracted $G$. mellonella haemocytes in the absence/presence of AZA, could reveal modes of action based on cellular respiration, and as mitochondria are inextricably linked to apoptosis - this may go some way to explain the cytotoxicity of AZA observed in the tissue histology (Figure 4.9). Mitochondrial membrane integrity could be assessed fluorescently using indicator dyes like MitoTracker ${ }^{\circledR}$ CMTMRos, or individual components of the electron transport chain could be assessed using the Agilent Seahorse mitochondrial stress test (e.g., used for Drosophila brains; (Neville et al., 2018).

Future experiments to explore the understanding of AZA mode of action would be to dissect targeted tissues such as the gut, liver and brain and study transcriptional signatures of stress and damage. A study by Manfrin et al. (2010) used transcriptomics to identify mRNAs encoding stress-associated proteins in the mussel Mytilus galloprovincialis which contained OA (Manfrin et al., 2010). Additionally, studies in Caco-2 cells have used transcriptomics to elude to modes of action of AZA and OA which found that AZA exposure resulted in an increase in expression for genes associated with cholesterol synthesis whereas OA exposure led to an increase in expression of genes involved in hypoxia (Bodero et al., 2018). This established technique could be used to investigate changes in gene expression within individual organs of $G$. mellonella larvae after being exposed to AZA to gain insight into its mode of action.

\subsection{Future Applications}

\subsubsection{Galleria mellonella's role in pharmaceutical development}

The research presented in this thesis has demonstrated G. mellonella larvae to be a strong tool for assessing a tissue-level response to pharmaceutical inoculation. G. mellonella larvae are currently used for toxicology studies, often those derived from fungi and bacteria, and used as virulence factors (Pereira et al., 2018, Fuchs et al., 2010, Reeves et al., 2004). Recently there has been increasing interest in using G. mellonella larvae to test antibacterial drugs (Cutuli et al., 2019). Results in this thesis indicate that G. mellonella larvae may also play a role in the 
development of other pharmaceuticals such as anti-inflammatories; this would therefore reduce the use of mammalian models whilst providing a high-throughput and less expensive option. As G. mellonella are susceptible to the gastric damage caused by NSAID, larvae could be an effective screening tool for the assessment of risk of gastric damage induced by newly developed NSAID prior to administration in murine models. This would enable pre-clinical trials to evaluate the risk of gastric damage and quantify the amount of damage caused by the new drug at ranging concentrations quickly, cheaper and reliably (triaging sizeable libraries of compounds to a few promising candidates).

There are limitations to the use of G. mellonella as a screening model in the pharmaceutical development process such as larval metamorphosis. One constraint is the lack of molecular and immunological resources. The genome of G. mellonella was sequenced recently (Lange et al., 2018a), which is a major step forward, but CRISPR and RNAi based genetic manipulations, as well as established wild-type and transgenic lines are not available. Moreover, there are at least two G. mellonella morphotypes - a melanic strain and a non-melanic strain. These are geographic variants, with the melanic strain appearing more resistant to entomopathogens (Dubovskiy et al., 2013, Grizanova et al., 2019). "Research grade" larvae, to improve reproducibility are now available from a company called BioSystems Technology, which was founded in 2015. These hormone-free larvae are more expensive than bait grade larvae and were found to have a lower microbiome diversity in the gut compared to the bait grade larvae (Allonsius et al., 2019). Additionally, there are no standardised protocols of rearing or utilising G. mellonella as a screening model (each research group using their in-house protocols for diet), which limits uptake from the biomedical industry as there seems to be a lack of standardisation.

\subsubsection{Nutraceutical development}

Nutraceuticals such as $C$. sinensis and colostrum appear to improve health outcomes in larvae when challenged with chemical and microbial disruptors (Chapter 3). These results are supported by experiments conducted in murine models (Playford et al., 1999, Marchbank et al., 2011), it would therefore be advantageous to isolate the active compounds and cellular targets responsible for these attributes. In order to detect active compounds, various extraction processes could be used. Currently hot water extraction (HWE) is commonly used in the food and pharmacology industry due to it being nontoxic and lacking immunogenicity, it acts as a 
polar solvent by the electrostatic attraction between its dipoles and the ion present (Chen et al., 2013). HWE has been used to investigate many bioactive properties of $C$. sinensis and the method can be altered to achieve greater yields or conduct a more thorough extraction by increasing the temperature and duration of heating. A basic HWE can be conducted by boiling whole fruiting bodies in water for 30 minutes, a process used by Marchbank et al. (2011) to identify gastric reparative properties of $C$. sinensis in murine models. Although HWE is commonly used, there is concern that such heat could denature some bioactive compounds, therefore solvent extractions have also been used to assist extraction yield (Liu et al., 2010) in addition to changes in pressure and variety of enzymes (Yan et al., 2014). Liu et al., (2010) attempted to improve extraction efficiency using water combined with ethanol in a $1: 1 \mathrm{v} / \mathrm{v}$ ratio. This approach was used to investigate $C$. sinensis protective properties against middle cerebral artery occlusion-induced focal cerebral ischemia in rats. Approximately, $240 \mathrm{ml}$ of water was heated to boiling for 3 hours with reflux, $80 \mathrm{ml}$ of water extract was then lyophilized giving a yield of $29 \%$, prior to reconstitution in $160 \mathrm{ml}$ of ethanol, ultimately yielding $3.7 \mathrm{~g}$ of dry powder. In their study it was found that different $C$. sinensis extractions resulted in a reduced serum antioxidant (lactate dehydrogenase) concentration compared to the rats which were not treated with any $C$. sinensis extract. Whereas other $C$. sinensis extracts significantly lower serum lactate dehydrogenase concentration (Liu et al., 2010). However the yield was no larger than other studies using a simpler hot water extraction with a shorter duration (Chen et al., 2013).

C. sinensis extractions can then be analysed via high-performance liquid chromatographymass spectrometry (HPLC-MS/MS), which is used routinely in the authentication and quality assessment of $C$. sinensis extracts (Hu et al., 2015). Once the compounds and concentrations present have been identified, extractions can be administered to larvae repeating experiments described in this research, to determine if certain extractions with varying concentrations of compounds have greater protective effects against a panel of pharmaceutical, microbial or toxin tests (using insect larvae as the host). As $C$. sinensis appears to have a positive impact on gastric tissues, it would be beneficial to further investigate the potential therapeutic effects extracts of C. sinensis has against gastric diseases such as IBD and Crohn's disease. A suitable advancement would be to administer $C$. sinensis extract to patients with gastric specific diseases, and monitor changes to their inflammatory markers such as IL-6 and colonic and intestinal tissue through endoscopy or biopsies. 
A further investigation would be to explore if $C$. sinensis or colostrum has any additive or synergistic effects with current pharmaceuticals in use, e.g., lansoprazole and aspirin. Patients with Crohn's disease are often treated with corticosteroids, however the long-term use of these are linked to higher mortality and complications such as Cushing's syndrome (cortisol dysregulation) therefore when prescribed, corticosteroid-sparing drugs are also given once resistance of dependence is seen (Al-Jaouni et al., 2002, Benchimol et al., 2008). To avoid this, corticosteroids are usually prescribed as an initial treatment plan then given as frequent repeated courses to manage symptoms (D'Haens et al., 2008). Due to the difficulties associated with treating Crohn's disease with corticosteroids it is essential to identify a better treatment plan where resistance is avoided, and remission is achieved more rapidly. C. sinensis extractions could be an alternative treatment to aid in reinforcing the gut structure and reducing permeability thus further protect the gut from pharmaceutical induced complications.

\subsubsection{Galleria mellonella as a biotoxin model}

This study produced substantial evidence to support G. mellonella larvae as an alternative model to rats and mice for some pharmaceutical, bacterial and marine toxins, all of which are encountered via an oral route and lead to gastric disorder. However, studies are required to assess the generalisability of G. mellonella as a model for toxins, as currently in the UK and EU, a validated in vivo model is absent (the mouse bioassay has been phase out for screening purposes, with the exception of neurotoxins). It is recognised among marine toxicology labs that the limitation of using sensitive in vitro and in chemico tools to screen toxins, is that only known lipphilic toxins are detected (diarrhoeic, azaspiracid), and they remain unsuitable for rare dangerous neurotoxins. Therefore, there is scope for an alternative in vivo model such as G. mellonella to help with screening, but also be further developed to identify toxin targets.

A further study to assess the sensitivity of G. mellonella larvae to distinguish between marine toxins would be to use pectenotoxins (PTXs). PTX are macrolactones created by dinoflagellates, e.g., Dinophysis, often found in the digestive glands of shellfish along with OA and are not associated with diarrhoea, however, appear to induce damage to the liver (Burgess and Shaw, 2001, Dominguez et al., 2010). Fourteen analogues of PTX have been described to date, with PTX-2 being the predominant precursor. Mouse toxicity assays have found large differences between mode of administration, with intraperitoneal injection having a much 
greater toxic effect than oral administration, however, few toxicokinetic studies are available (Sandvik et al., 2020). Unlink OA, PTX does not inhibit PP2A but does appear to effect filamentous actin (F-actin) and induce apoptosis with PTX analogues having diverse toxicity and symptom ranges (Espiña et al., 2008). A study utilising G. mellonella larva to obtain specific toxicokinetic values for the different analogues - in manner similar to the azaspiracids herein (Chapter 4).

The ultimate goal would be to have G. mellonella larvae approved an accepted assay for Food Standards to complement liquid chromatography/mass spectrometry (LC-MS/MS) monitoring programmes for shellfish toxins (and perhaps, agriculture-associated toxins like mycotoxins).

\section{Bibliography}

AASEN, J. A., ESPENES, A., HESS, P. \& AUNE, T. 2010. Sub-lethal dosing of azaspiracid1 in female NMRI mice. Toxicon, 56, 1419-1425.

AASEN, J. A., ESPENES, A., MILES, C. O., SAMDAL, I. A., HESS, P. \& AUNE, T. 2011. Combined oral toxicity of azaspiracid- 1 and yessotoxin in female NMRI mice. Toxicon, 57, 909-917.

ABAL, P., LOUZAO, M. C., FRAGA, M., VILARIÑO, N., FERREIRO, S., VIEYTES, M. R. \& BOTANA, L. M. 2017. Absorption and Effect of Azaspiracid-1 Over the Human Intestinal Barrier. Cellular Physiology and Biochemistry, 43, 136-146.

ABRÀMOFF, M. D., MAGALHÃES, P. J. \& RAM, S. J. 2004. Image processing with ImageJ. Biophotonics international, 11, 36-42.

ACHESON, D. \& ALLOS, B. M. 2001. Campylobacter jejuni Infections: Update on Emerging Issues and Trends. Clinical Infectious Diseases, 32, 1201-1206.

AGUWA, C. N. 1985. Incidence of gastric ulcers by indomethacin and piroxicam in rats. Archives of toxicology, 56, 212-213.

AKHGARI, A., HESHMATI, Z., AFRASIABI GAREKANI, H., SADEGHI, F., SABBAGH, A., SHARIF MAKHMALZADEH, B. \& NOKHODCHI, A. 2017. Indomethacin electrospun nanofibers for colonic drug delivery: In vitro dissolution studies. Colloids and Surfaces B: Biointerfaces, 152, 29-35.

AL-JAOUNI, R., SCHNEIDER, S. M., PICHE, T., RAMPAL, P. \& HÉBUTERNE, X. 2002. Effect of steroids on energy expenditure and substrate oxidation in women with crohn's disease. The American Journal of Gastroenterology, 97, 2843-2849.

AL-QARAWI, A. A., ABDEL-RAHMAN, H., ALI, B. H., MOUSA, H. M. \& EL-MOUGY, S. A. 2005. The ameliorative effect of dates (Phoenix dactylifera L.) on ethanol-induced gastric ulcer in rats. Journal of Ethnopharmacology, 98, 313-317.

ALlEGRA, E., TITBALL, R. W., CARTER, J. \& CHAMPION, O. L. 2018. Galleria mellonella larvae allow the discrimination of toxic and non-toxic chemicals. Chemosphere, 198, 469-472. 
ALLONSIUS, C. N., VAN BEECK, W., DE BOECK, I., WITTOUCK, S. \& LEBEER, S. 2019. The microbiome of the invertebrate model host Galleria mellonella is dominated by Enterococcus. Animal Microbiome, 1, 7 .

ALTINCICEK, B., LINDNER, M., LINDER, D., PREISSNER, K. \& VILCINSKAS, A. 2007. Microbial metalloproteinases mediate sensing of invading pathogens and activate innate immune responses in the lepidopteran model host Galleria mellonella. Infect Immun, 75.

ALTSCHUL, S. F., GISH, W., MILLER, W., MYERS, E. W. \& LIPMAN, D. J. 1990. Basic local alignment search tool. Journal of molecular biology, 215, 403-410.

AN, M. J., CHEON, J. H., KIM, S. W., PARK, J. J., MOON, C. M., HAN, S. Y., KIM, E. S., KIM, T. I. \& KIM, W. H. 2009. Bovine colostrum inhibits nuclear factor $\kappa \mathrm{B}$-mediated proinflammatory cytokine expression in intestinal epithelial cells. Nutrition Research, 29, 275-280.

ANDLAUER, W. \& FÜRST, P. 2002. Nutraceuticals: a piece of history, present status and outlook. Food Research International, 35, 171-176.

ANTONISSEN, G., MARTEL, A., PASMANS, F., DUCATELLE, R., VERBRUGGHE, E., VANDENBROUCKE, V., LI, S., HAESEBROUCK, F., VAN IMMERSEEL, F. \& CROUBELS, S. 2014. The impact of Fusarium mycotoxins on human and animal host susceptibility to infectious diseases. Toxins, 6, 430-452.

APPRILL, A., MCNALLY, S., PARSONS, R. \& WEBER, L. 2015. Minor revision to V4 region SSU rRNA 806R gene primer greatly increases detection of SAR11 bacterioplankton. Aquatic Microbial Ecology, 75, 129-137.

ARTEAGA BLANCO, L. A., CRISPIM, J. S., FERNANDES, K. M., DE OLIVEIRA, L. L., PEREIRA, M. F., BAZZOLLI, D. M. S. \& MARTINS, G. F. 2017. Differential cellular immune response of Galleria mellonella to Actinobacillus pleuropneumoniae. Cell and Tissue Research, 370, 153-168.

AUNE, T., ESPENES, A., AASEN, J. A. B., QUILLIAM, M. A., HESS, P. \& LARSEN, S. 2012. Study of possible combined toxic effects of azaspiracid-1 and okadaic acid in mice via the oral route. Toxicon, 60, 895-906.

BAGWE, S., THARAPPEL, L. J. P., KAUR, G. \& BUTTAR, H. S. 2015. Bovine colostrum: an emerging nutraceutical. Journal of Complementary and Integrative Medicine, 12, $175-185$.

BARBOSA, P., BERRY, D. \& KARY, C. K. 2014. Insect Histology: Practical Laboratory Techniques, John Wiley \& Sons.

BARNOY, S., GANCZ, H., ZHU, Y., HONNOLD, C. L., ZURAWSKI, D. V. \& VENKATESAN, M. M. 2017. The Galleria mellonella larvae as an in vivo model for evaluation of Shigella virulence. Gut microbes, 8, 335-350.

BASIVIREDDY, J., JACOB, M., RAMAMOORTHY, P., PULIMOOD, A. B. \& BALASUBRAMANIAN, K. A. 2003. Indomethacin-induced free radical-mediated changes in the intestinal brush border membranes. Biochemical Pharmacology, 65, 683-695.

BECATTINI, S., TAUR, Y. \& PAMER, E. G. 2016. Antibiotic-Induced Changes in the Intestinal Microbiota and Disease. Trends in Molecular Medicine, 22, 458-478.

BENCHIMOL, E. I., SEOW, C. H., STEINHART, A. H. \& GRIFFITHS, A. M. 2008. Traditional corticosteroids for induction of remission in Crohn's disease. Cochrane Database of Systematic Reviews.

BERGIN, D., REEVES, E. P., RENWICK, J., WIENTJES, F. B. \& KAVANAGH, K. 2005. Superoxide production in Galleria mellonella hemocytes: identification of proteins homologous to the NADPH oxidase complex of human neutrophils. Infection and immunity, 73, 4161-4170. 
BERVEN, G., SÆTRE, F., HALVORSEN, K. \& SEGLEN, P. O. 2001. Effects of the diarrhetic shellfish toxin, okadaic acid, on cytoskeletal elements, viability and functionality of rat liver and intestinal cells. Toxicon, 39, 349-362.

BIALOJAN, C. \& TAKAI, A. 1988. Inhibitory effect of a marine-sponge toxin, okadaic acid, on protein phosphatases. Specificity and kinetics. Biochemical Journal, 256, 283-290.

BJARNASON, I. \& TAKEUCHI, K. 2009. Intestinal permeability in the pathogenesis of NSAID-induced enteropathy. Journal of Gastroenterology, 44, 23-29.

BLASER, M. J., BERKOWITZ, I. D., LAFORCE, F. M., CRAVENS, J., RELLER, L. B. \& WANG, W.-L. L. 1979. Campylobacter enteritis: clinical and epidemiologic features. Annals of internal medicine, 91, 179-185.

BLEUMINK-PLUYM, N. M., VAN ALPHEN, L. B., BOUWMAN, L. I., WÖSTEN, M. M. \& VAN PUTTEN, J. P. 2013. Identification of a functional type VI secretion system in Campylobacter jejuni conferring capsule polysaccharide sensitive cytotoxicity. PLoS Pathog, 9, e1003393.

BODERO, M., HOOGENBOOM, R. L. A. P., BOVEE, T. F. H., PORTIER, L., DE HAAN, L., PEIJNENBURG, A. \& HENDRIKSEN, P. J. M. 2018. Whole genome mRNA transcriptomics analysis reveals different modes of action of the diarrheic shellfish poisons okadaic acid and dinophysis toxin-1 versus azaspiracid-1 in Caco-2 cells. Toxicology in Vitro, 46, 102-112.

BOGUŚ, M. I., LIGEZA-ŻUBER, M., POLAŃSKA, M. A., MOSIEWICZ, M., WŁÓKA, E. \& SOBOCINSKA, M. 2018. Fungal infection causes changes in the number, morphology and spreading ability of Galleria mellonella haemocytes. Physiological Entomology, 43, 214-226.

BOMBELLI, P., HOWE, C. J. \& BERTOCCHINI, F. 2017. Polyethylene bio-degradation by caterpillars of the wax moth Galleria mellonella. Current Biology, 27, R292-R293.

BOSNJAK, T., SOLBERG, R., HEMATI, P. D., JAFARI, A., KASSEM, M. \& JOHANSEN, H. T. 2019. Lansoprazole inhibits the cysteine protease legumain by binding to the active site. Basic \& Clinical Pharmacology \& Toxicology, 125, 89-99.

BRADFORD, G. H., GLORIA, A. B., JACK, R. L., SCOTT, B., LOU, D. I., JIANHUA, Z. \& VICTOR, M. D.-U. 2012. Integration of cellular bioenergetics with mitochondrial quality control and autophagy. Biological Chemistry, 393, 1485-1512.

BRODERICK, N. A. \& LEMAITRE, B. 2012. Gut-associated microbes of Drosophila melanogaster. Gut microbes, 3, 307-321.

BROWNE, N., HEELAN, M. \& KAVANAGH, K. 2013. An analysis of the structural and functional similarities of insect hemocytes and mammalian phagocytes. Virulence, 4, 597-603.

BROWNE, N. \& KAVANAGH, K. 2013. Developing the potential of using Galleria mellonella larvae as models for studying brain infection by Listeria monocytogenes. Virulence, 4, 271-272.

BROWNE, N., SURLIS, C. \& KAVANAGH, K. 2014. Thermal and physical stresses induce a short-term immune priming effect in Galleria mellonella larvae. Journal of Insect Physiology, 63, 21-26.

BRUNE, K. \& PATRIGNANI, P. 2015. New insights into the use of currently available nonsteroidal anti-inflammatory drugs. Journal of pain research, 8, 105.

BRYANT, A. E., BAYER, C. R., ALDAPE, M. J. \& STEVENS, D. L. 2015. The roles of injury and nonsteroidal anti-inflammatory drugs in the development and outcomes of severe group A streptococcal soft tissue infections. Current opinion in infectious diseases, 28, 231. 
BUCHON, N., BRODERICK, N. A., POIDEVIN, M., PRADERVAND, S. \& LEMAITRE, B. 2009. Drosophila intestinal response to bacterial infection: activation of host defense and stem cell proliferation. Cell host \& microbe, 5, 200-211.

BUENZ, E. J., BAUER, B. A., OSMUNDSON, T. W. \& MOTLEY, T. J. 2005. The traditional Chinese medicine Cordyceps sinensis and its effects on apoptotic homeostasis. Journal of Ethnopharmacology, 96, 19-29.

BUENZ, E. J., WEAVER, J. G., BAUER, B. A., CHALPIN, S. D. \& BADLEY, A. D. 2004. Cordyceps sinensis extracts do not prevent Fas-receptor and hydrogen peroxideinduced T-cell apoptosis. Journal of Ethnopharmacology, 90, 57-62.

BULUSHOVA, N. V., ELPIDINA, E. N., ZHUZHIKOV, D. P., LYUTIKOVA, L. I., ORTEGO, F., KIRILLOVA, N. E., ZALUNIN, I. A. \& CHESTUKHINA, G. G. 2011 a. Complex of digestive proteinases of Galleria mellonella caterpillars. Composition, properties, and limited proteolysis of Bacillus thuringiensis endotoxins. Biochemistry (Moscow), 76, 581-589.

BULUSHOVA, N. V., ZHUZHIKOV, D. P., LYUTIKOVA, L. I., KIRILLOVA, N. E., ZALUNIN, I. A. \& CHESTUKHINA, G. G. 2011b. Toxin-binding proteins isolated from yellow mealworm Tenebrio molitor and wax moth Galleria mellonella. Biochemistry (Moscow), 76, 202-208.

BURGESS, V. \& SHAW, G. 2001. Pectenotoxins - an issue for public health: a review of their comparative toxicology and metabolism. Environment international, 27, 275-283.

BUTT, T. M., COATES, C. J., DUBOVSKIY, I. M. \& RATCLIFFE, N. A. 2016. Entomopathogenic fungi: new insights into host-pathogen interactions. Advances in genetics. Elsevier.

BÜYÜKGÜZEL, E., BÜYÜKGÜZEL, K., SNELA, M., ERDEM, M., RADTKE, K., ZIEMNICKI, K. \& ADAMSKI, Z. 2013. Effect of boric acid on antioxidant enzyme activity, lipid peroxidation, and ultrastructure of midgut and fat body of Galleria mellonella. Cell biology and toxicology, 29, 117-129.

BÜYÜKGÜZEL, E., TUNAZ, H., STANLEY, D. \& BÜYÜKGÜZEL, K. 2007. Eicosanoids mediate Galleria mellonella cellular immune response to viral infection. Journal of Insect Physiology, 53, 99-105.

CAMPBELl, P. M., CAO, A. T., HINES, E. R., EAST, P. D. \& GORDON, K. H. J. 2008. Proteomic analysis of the peritrophic matrix from the gut of the caterpillar, Helicoverpa armigera. Insect Biochemistry and Molecular Biology, 38, 950-958.

CAO, Z., LEPAGE, K. T., FREDERICK, M. O., NICOLAOU, K. C. \& MURRAY, T. F. 2010. Involvement of Caspase Activation in Azaspiracid-Induced Neurotoxicity in Neocortical Neurons. Toxicological Sciences, 114, 323-334.

CAO, Z., LI, W., LIU, R., LI, X., LI, H., LIU, L., CHEN, Y., LV, C. \& LIU, Y. 2019. pH- and enzyme-triggered drug release as an important process in the design of anti-tumor drug delivery systems. Biomedicine \& Pharmacotherapy, 118, 109340.

CAPORASO, J. G., KUCZYNSKI, J., STOMBAUGH, J., BITTINGER, K., BUSHMAN, F. D., COSTELlO, E. K., FIERER, N., PENA, A. G., GOODRICH, J. K. \& GORDON, J. I. 2010. QIIME allows analysis of high-throughput community sequencing data. Nature methods, 7, 335-336.

CARD, R., VAUGHAN, K., BAGNALL, M., SPIROPOULOS, J., COOLEY, W., STRICKLAND, T., DAVIES, R. \& ANJUM, M. F. 2016. Virulence characterisation of Salmonella enterica isolates of differing antimicrobial resistance recovered from UK livestock and imported meat samples. Frontiers in microbiology, 7, 640.

CARVALHO, FREDERIC A., KOREN, O., GOODRICH, JULIA K., JOHANSSON, MALIN E. V., NALBANTOGLU, I., AITKEN, JESSE D., SU, Y., CHASSAING, B., WALTERS, WILLIAM A., GONZÁLEZ, A., CLEMENTE, JOSE C., CULLENDER, 
TYLER C., BARNICH, N., DARFEUILLE-MICHAUD, A., VIJAY-KUMAR, M., KNIGHT, R., LEY, RUTH E. \& GEWIRTZ, ANDREW T. 2012. Transient Inability to Manage Proteobacteria Promotes Chronic Gut Inflammation in TLR5-Deficient Mice. Cell Host \& Microbe, 12, 139-152.

CHAE, A., AITCHISON, A., DAY, A. S. \& KEENAN, J. I. 2017. Bovine colostrum demonstrates anti-inflammatory and antibacterial activity in in vitro models of intestinal inflammation and infection. Journal of Functional Foods, 28, 293-298.

CHAMOUN-EMANUELli, A. M., BRYAN, L. K., COHEN, N. D., TETRAULT, T. L., SZULE, J. A., BARHOUMI, R. \& WHITFIELD-CARGILE, C. M. 2019. NSAIDs disrupt intestinal homeostasis by suppressing macroautophagy in intestinal epithelial cells. Scientific reports, 9, 14534-14534.

CHAMPION, O. L., WAGLEY, S. \& TITBALL, R. W. 2016. Galleria mellonella as a model host for microbiological and toxin research. Virulence, 7, 840-845.

CHANDLER, J. A., LANG, J. M., BHATNAGAR, S., EISEN, J. A. \& KOPP, A. 2011. Bacterial communities of diverse Drosophila species: ecological context of a hostmicrobe model system. PLoS genet, 7, e1002272.

CHEN, P. X., WANG, S., NIE, S. \& MARCONE, M. 2013. Properties of Cordyceps Sinensis: A review. Journal of Functional Foods, 5, 550-569.

CHENG, T. O. 2007. The history of aspirin. Texas Heart Institute Journal, 34, 392.

CHERTKOVA, E. A., GRIZANOVA, E. V. \& DUBOVSKIY, I. M. 2018. Bacterial and fungal infections induce bursts of dopamine in the haemolymph of the Colorado potato beetle Leptinotarsa decemlineata and greater wax moth Galleria mellonella. Journal of Invertebrate Pathology, 153, 203-206.

CHOI, J., WHITTEN, M., CHO, M., LEE, K., KIM, M., RATCLIFFE, N. \& LEE, B. 2002. Calreticulin enriched as an early-stage encapsulation protein in wax moth Galleria mellonella larvae. Developmental \& Comparative Immunology, 26, 335-343.

COATES, C. J., LIM, J., HARMAN, K., ROWLEY, A. F., GRIFFITHS, D. J., EMERY, H. \& LAYTON, W. 2019. The insect, Galleria mellonella, is a compatible model for evaluating the toxicology of okadaic acid. Cell Biology and Toxicology, 35, 219-232.

CODDINGTON, E. J. \& CHAMBERLIN, M. E. 1999. Acid/base transport across the midgut of the tobacco hornworm, Manduca sexta. Journal of Insect Physiology, 45, 493-500.

COOLS, F., TORFS, E., AIZAWA, J., VANHOUTTE, B., MAES, L., CALJON, G., DELPUTTE, P., CAPPOEN, D. \& COS, P. 2019. Optimization and characterization of a Galleria mellonella larval infection model for virulence studies and the evaluation of therapeutics against Streptococcus pneumoniae. Frontiers in microbiology, 10, 311.

COPPENS, P., DA SILVA, M. F. \& PETTMAN, S. 2006. European regulations on nutraceuticals, dietary supplements and functional foods: a framework based on safety. Toxicology, 221, 59-74.

COSKUN, M., KAYIS, T., SULANC, M. \& OZALP, P. 2006. Effects of different honeycomb and sucrose levels on the development of greater wax moth Galleria mellonella larvae. International Journal of Agriculture and Biology, 8, 855-858.

CREE, B. A. C., SPENCER, C. M., VARRIN-DOYER, M., BARANZINI, S. E. \& ZAMVIL, S. S. 2016. Gut microbiome analysis in neuromyelitis optica reveals overabundance of Clostridium perfringens. Annals of Neurology, 80, 443-447.

CUtUli, M. A., PETRONIO PETRONIO, G., VERGAlito, F., MAGNIFICO, I., PIETRANGELO, L., VENDITTI, N. \& DI MARCO, R. 2019. Galleria mellonella as a consolidated in vivo model hosts: New developments in antibacterial strategies and novel drug testing. Virulence, 10, 527-541. 
CYTRYŃSKA, M., MAK, P., ZDYBICKA-BARABAS, A., SUDER, P. \& JAKUBOWICZ, T. 2007. Purification and characterization of eight peptides from Galleria mellonella immune hemolymph. Peptides, 28, 533-546.

D'HAENS, G., BAERT, F., VAN ASSCHE, G., CAENEPEEL, P., VERGAUWE, P., TUYNMAN, H., DE VOS, M., VAN DEVENTER, S., STITT, L., DONNER, A., VERMEIRE, S., VAN DE MIEROP, F. J., COCHE, J.-C. R., VAN DER WOUDE, J., OCHSENKÜHN, T., VAN BODEGRAVEN, A. A., VAN HOOTEGEM, P. P., LAMBRECHT, G. L., MANA, F., RUTGEERTS, P., FEAGAN, B. G. \& HOMMES, D. 2008. Early combined immunosuppression or conventional management in patients with newly diagnosed Crohn's disease: an open randomised trial. The Lancet, 371, 660667.

DASTI, J. I., TAREEN, A. M., LUGERT, R., ZAUTNER, A. E. \& GROß, U. 2010. Campylobacter jejuni: A brief overview on pathogenicity-associated factors and disease-mediating mechanisms. International Journal of Medical Microbiology, 300, 205-211.

DAVIS, P. F., GREENHILL, N. S., ROWAN, A. M. \& SCHOLLUM, L. M. 2007. The safety of New Zealand bovine colostrum: Nutritional and physiological evaluation in rats. Food and Chemical Toxicology, 45, 229-236.

DAVISON, G., MARCHBANK, T., MARCH, D. S., THATCHER, R. \& PLAYFORD, R. J. 2016. Zinc carnosine works with bovine colostrum in truncating heavy exerciseinduced increase in gut permeability in healthy volunteers. The American journal of clinical nutrition, 104, 526-536.

DE BRUYN, M., WIERSMA, V. R., HELFRICH, W., EGGLETON, P. \& BREMER, E. 2015. The Ever-Expanding Immunomodulatory Role of Calreticulin in Cancer Immunity. Frontiers in Oncology, 5, 35.

DESESSO, J. M. \& JACOBSON, C. F. 2001. Anatomical and physiological parameters affecting gastrointestinal absorption in humans and rats. Food and Chemical Toxicology, 39, 209-228.

DIETRICH, J., GRASS, I., GÜNZEL, D., HEREK, S., BRAEUNING, A., LAMPEN, A. \& HESSEL-PRAS, S. 2019. The marine biotoxin okadaic acid affects intestinal tight junction proteins in human intestinal cells. Toxicology in Vitro, 58, 150-160.

DOMINGUEZ, H. J., PAZ, B., DARANAS, A. H., NORTE, M., FRANCO, J. M. \& FERNÁNDEZ, J. J. 2010. Dinoflagellate polyether within the yessotoxin, pectenotoxin and okadaic acid toxin groups: Characterization, analysis and human health implications. Toxicon, 56, 191-217.

DOUNAY, A. \& FORSYTH, C. 2002. Okadaic acid: the archetypal serine/threonine protein phosphatase inhibitor. Current medicinal chemistry, 9, 1939-1980.

DUBOVSKII, I. M., GRIZANOVA, E. V., CHERTKOVA, E. A., SLEPNEVA, I. A., KOMAROV, D. A., VORONTSOVA, Y. L. \& GLUPOV, V. V. 2010. Generation of reactive oxygen species and activity of antioxidants in hemolymph of the moth larvae Galleria mellonella (L.) (Lepidoptera: Piralidae) at development of the process of encapsulation. Journal of Evolutionary Biochemistry and Physiology, 46, 35-43.

DUBOVSKIY, I. M., GRIZANOVA, E. V., WHITTEN, M. M., MUKHERJEE, K., GREIG, C., ALIKINA, T., KABILOV, M., VILCINSKAS, A., GLUPOV, V. V. \& BUTT, T. M. 2016. Immuno-physiological adaptations confer wax moth Galleria mellonella resistance to Bacillus thuringiensis. Virulence, 7, 860-870.

DUBOVSKIY, I. M., WHITTEN, M. M. A., KRYUKOV, V. Y., YAROSLAVTSEVA, O. N., GRIZANOVA, E. V., GREIG, C., MUKHERJEE, K., VILCINSKAS, A., MITKOVETS, P. V., GLUPOV, V. V. \& BUTT, T. M. 2013. More than a colour 
change: insect melanism, disease resistance and fecundity. Proceedings of the Royal Society B: Biological Sciences, 280.

DÖHLER, J. R. \& NEBERMANN, L. 2002. Bovine colostrum in oral treatment of enterogenic endotoxaemia in rats. Critical Care, 6, 536.

ECKBURG, P. B., BIK, E. M., BERNSTEIN, C. N., PURDOM, E., DETHLEFSEN, L., SARGENT, M., GILL, S. R., NELSON, K. E. \& RELMAN, D. A. 2005. Diversity of the Human Intestinal Microbial Flora. Science, 308, 1635.

EL AIDY, S., DERRIEN, M., AARDEMA, R., HOOIVELD, G., RICHARDS, S., DANE, A., DEKKER, J., VREEKEN, R., LEVENEZ, F. \& DORÉ, J. 2014. Transient inflammatory-like state and microbial dysbiosis are pivotal in establishment of mucosal homeostasis during colonisation of germ-free mice. Beneficial Microbes, 5, 67-77.

ELFSTRAND, L., LINDMARK-MÅNSSON, H., PAULSSON，M., NYBERG，L. \& ÅKESSON, B. 2002. Immunoglobulins, growth factors and growth hormone in bovine colostrum and the effects of processing. International Dairy Journal, 12, 879-887.

ELMI, A., DOREY, A., WATSON, E., JAGATIA, H., INGLIS, N. F., GUNDOGDU, O., BAJAJ-ELLIOTT, M., WREN, B. W., SMITH, D. G. E. \& DORRELL, N. 2018. The bile salt sodium taurocholate induces Campylobacter jejuni outer membrane vesicle production and increases OMV-associated proteolytic activity. Cellular Microbiology, 20, e12814.

ELMI, A., WATSON, E., SANDU, P., GUNDOGDU, O., MILLS, D. C., INGLIS, N. F., MANSON, E., IMRIE, L., BAJAJ-ELLIOTT, M., WREN, B. W., SMITH, D. G. E. \& DORRELL, N. 2012. <span class="named-content genus-species" id="named-content$1 ">$ Campylobacter jejuni</span> Outer Membrane Vesicles Play an Important Role in Bacterial Interactions with Human Intestinal Epithelial Cells. Infection and Immunity, 80, 4089.

EMERY, H., JOHNSTON, R., ROWLEY, A. F. \& COATES, C. J. 2019. Indomethacininduced gut damage in a surrogate insect model, Galleria mellonella. Archives of toxicology, 93, 2347-2360.

ENDO, Y. \& NISHITSUTSUJI-UWO, J. 1981. Mode of Action of Bacillus thuringiensis \&delta;-Endotoxin : Ultrastructural Changes of Midgut Epithelium of Pieris, Lymantria and Ephestia Larvae. Applied Entomology and Zoology, 16, 231-241.

ENGEL, P. \& MORAN, N. A. 2013. The gut microbiota of insects-diversity in structure and function. FEMS microbiology reviews, 37, 699-735.

EREN, A. M., MORRISON, H. G., LESCAULT, P. J., REVEILLAUD, J., VINEIS, J. H. \& SOGIN, M. L. 2015. Minimum entropy decomposition: unsupervised oligotyping for sensitive partitioning of high-throughput marker gene sequences. The ISME journal, 9, 968-979.

ESPIÑA, B., LOUZAO, M. C., ARES, I. R., CAGIDE, E., VIEYTES, M. R., VEGA, F. V., RUBIOLO, J. A., MILES, C. O., SUZUKI, T., YASUMOTO, T. \& BOTANA, L. M. 2008. Cytoskeletal toxicity of pectenotoxins in hepatic cells. British Journal of Pharmacology, 155, 934-944.

ESPÍN, J. C., GARCÍA-CONESA, M. T. \& TOMÁS-BARBERÁN, F. A. 2007. Nutraceuticals: Facts and fiction. Phytochemistry, 68, 2986-3008.

FEDHILA, S., BUISSON, C., DUSSURGET, O., SERROR, P., GLOMSKI, I. J., LIEHL, P., LERECLUS, D. \& NIELSEN-LEROUX, C. 2010. Comparative analysis of the virulence of invertebrate and mammalian pathogenic bacteria in the oral insect infection model Galleria mellonella. Journal of Invertebrate Pathology, 103, 24-29.

FERREIRO, S. F., VILARIÑO, N., CARRERA, C., LOUZAO, M. C., CANTALAPIEDRA, A. G., SANTAMARINA, G., CIFUENTES, J. M., VIEIRA, A. C. \& BOTANA, L. M. 
2016. Subacute cardiovascular toxicity of the marine phycotoxin azaspiracid-1 in rats. Toxicological Sciences, 151, 104-114.

FERREIRO, S. F., VILARIÑO, N., CARRERA, C., LOUZAO, M. C., SANTAMARINA, G., CANTALAPIEDRA, A. G., CIFUENTES, J. M., VIEIRA, A. C. \& BOTANA, L. M. 2017. Subacute immunotoxicity of the marine phycotoxin yessotoxin in rats. Toxicon, $129,74-80$.

FERREIRO, S. F., VILARIÑO, N., LOUZAO, M. C., NICOLAOU, K. C., FREDERICK, M. O. \& BOTANA, L. M. 2014. In vitro chronic effects on hERG channel caused by the marine biotoxin azaspiracid-2. Toxicon, 91, 69-75.

FIORENTINI, C., MATARRESE, P., FATTOROSSI, A. \& DONELLI, G. 1996. Okadaic acid induces changes in the organization of F-actin in intestinal cells. Toxicon, 34, 937-945.

FRANCHINI, A., MALAGOLI, D. \& OTTAVIANI, E. 2010. Targets and effects of yessotoxin, okadaic acid and palytoxin: A differential review. Marine drugs, 8, 658677.

FRANZETTI, E., CASARTELli, M., D'ANTONA, P., MONTALI, A., ROMANELLI, D., CAPPELlOZZA, S., CACCIA, S., GRIMALDI, A., DE EGUILEOR, M. \& TETTAMANTI, G. 2016. Midgut epithelium in molting silkworm: A fine balance among cell growth, differentiation, and survival. Arthropod Structure \& Development, 45, 368-379.

FREEDMAN, D. J., TACKET, C. O., DELEHANTY, A., MANEVAL, D. R., NATARO, J. \& CRABB, J. H. 1998. Milk Immunoglobulin with Specific Activity against Purified Colonization Factor Antigens Can Protect against Oral Challenge with Enterotoxigenic Escherichia coli. The Journal of Infectious Diseases, 177, 662-667.

FUCHS, B. B., O'BRIEN, E., EL KHOURY, J. B. \& MYLONAKIS, E. 2010. Methods for using Galleria mellonella as a model host to study fungal pathogenesis. Virulence, 1, 475-482.

FUREY, A., O'DOHERTY, S., O'CALLAGHAN, K., LEHANE, M. \& JAMES, K. J. 2010. Azaspiracid poisoning (AZP) toxins in shellfish: Toxicological and health considerations. Toxicon, 56, 173-190.

GARCÍA-RAYADO, G., NAVARRO, M. \& LANAS, A. 2018. NSAID induced gastrointestinal damage and designing GI-sparing NSAIDs. Expert Review of Clinical Pharmacology, 11, 1031-1043.

GIANNOUli, M., PALATUCCI, A. T., RUBinO, V., RUGGIERO, G., ROMANO, M., TRIASSI, M., RICCI, V. \& ZARRILLI, R. 2014. Use of larvae of the wax moth Galleria mellonella as an in vivo model to study the virulence of Helicobacter pylori. BMC microbiology, 14, 228.

GODSCHALK, P. C., KUIJF, M. L., LI, J., MICHAEL, F. S., ANG, C. W., JACOBS, B. C., KARWASKI, M.-F., BROCHU, D., MOTERASSED, A. \& ENDTZ, H. P. 2007. Structural characterization of Campylobacter jejuni lipooligosaccharide outer cores associated with Guillain-Barré and Miller Fisher syndromes. Infection and immunity, $75,1245-1254$.

GORNALL, A. G., BARDAWILL, C. J. \& DAVID, M. M. 1949. Determination of serum proteins by means of the biuret reaction. Journal of biological chemistry, 177, 751-766.

GREEN, L. F., BERGQUIST, P. R. \& BULLIVANT, S. 1980. The structure and function of the smooth septate junction in a transporting epithelium: the Malpighian tubules of the New Zealand glow-worm Arachnocampa luminosa. Tissue and Cell, 12, 365-381.

GRIDER, J. R., FOXX-ORENSTEIN, A. E. \& JIN, J.-G. 1998. 5-Hydroxytryptamine4 receptor agonists initiate the peristaltic reflex in human, rat, and guinea pig intestine. Gastroenterology, 115, 370-380. 
GRIZANOVA, E., DUBOVSKIY, I., WHITTEN, M. \& GLUPOV, V. 2014. Contributions of cellular and humoral immunity of Galleria mellonella larvae in defence against oral infection by Bacillus thuringiensis. Journal of invertebrate pathology, 119, 40-46.

GRIZANOVA, E. V., COATES, C. J., DUBOVSKIY, I. M. \& BUTT, T. M. 2019. Metarhizium brunneum infection dynamics differ at the cuticle interface of susceptible and tolerant morphs of Galleria mellonella. Virulence, 10, 999-1012.

GU, G.-S., REN, J.-A., LI, G.-W., YUAN, Y.-J., LI, N. \& LI, J.-S. 2015. Cordyceps sinensis preserves intestinal mucosal barrier and may be an adjunct therapy in endotoxininduced sepsis rat model: a pilot study. International journal of clinical and experimental medicine, 8, 7333.

HAGIWARA, C., TANAKA, M. \& KUDO, H. 2002. Increase in colorectal epithelial apoptotic cells in patients with ulcerative colitis ultimately requiring surgery. Journal of Gastroenterology and Hepatology, 17, 758-764.

HANNA, N., AHMED, K., ANWAR, M., PETROVA, A., HIATT, M. \& HEGYI, T. 2004. Effect of storage on breast milk antioxidant activity. Archives of Disease in Childhood - Fetal and Neonatal Edition, 89, F518.

HARRIS, N. V., WEISS, N. S. \& NOLAN, C. M. 1986. The role of poultry and meats in the etiology of Campylobacter jejuni/coli enteritis. American Journal of Public Health, 76, 407-411.

HECHTMAN, D., CYBULSKY, M., FUCHS, H., BAKER, J. \& GIMBRONE, M. 1991. Intravascular IL-8. Inhibitor of polymorphonuclear leukocyte accumulation at sites of acute inflammation. The Journal of Immunology, 147, 883-892.

HEDSTROM, L. 2002. Serine Protease Mechanism and Specificity. Chemical Reviews, 102, 4501-4524.

HELGESEN, M. The effect of diet on the gut microbiota in Strongylocentrotus droebachiensis. 2017.

HESS, P., TWINER, M. J., KILCOYNE, J. \& SOSA, S. 2015. Azaspiracid toxins: toxicological profile.

HIGUCHI, K., YODA, Y., AMAGASE, K., KATO, S., TOKIOKA, S., MURANO, M., TAKEUCHI, K. \& UMEGAKI, E. 2009. Prevention of NSAID-induced small intestinal mucosal injury: prophylactic potential of lansoprazole. Journal of clinical biochemistry and nutrition, 45, 125.

HJØRNEVIK, L. V., FRØYSET, A. K., GRØNSET, T. A., RUNGRUANGSAKTORRISSEN, K. \& FLADMARK, K. E. 2015. Algal toxin azaspiracid-1 induces early neuronal differentiation and alters peripherin isoform stoichiometry. Marine drugs, 13, 7390-7402.

HOFFMANN, J. A. 1995. Innate immunity of insects. Current Opinion in Immunology, 7, 410.

HOLMES, E., LI, J. V., ATHANASIOU, T., ASHRAFIAN, H. \& NICHOLSON, J. K. 2011. Understanding the role of gut microbiome-host metabolic signal disruption in health and disease. Trends in Microbiology, 19, 349-359.

HOOPER, L. V. \& GORDON, J. I. 2001. Commensal Host-Bacterial Relationships in the Gut. Science, 292, 1115.

HU, H., XIAO, L., ZHENG, B., WEI, X., ELLIS, A. \& LIU, Y.-M. 2015. Identification of chemical markers in Cordyceps sinensis by HPLC-MS/MS. Analytical and Bioanalytical Chemistry, 407, 8059-8066.

HUGHES, G. S., HEALD, D. L., BARKER, K. B., PATEL, R. K., SPILLERS, C. R., WATTS, K. C., BATTS, D. H. \& EULER, A. R. 1989. The effects of gastric $\mathrm{pH}$ and food on the pharmacokinetics of a new oral cephalosporin, cefpodoxime proxetil. Clinical Pharmacology \& Therapeutics, 46, 674-685. 
IGNASIAK, K. \& MAXWELL, A. 2018. Oxytetracycline reduces the diversity of tetracyclineresistance genes in the Galleria mellonella gut microbiome. BMC microbiology, 18, 228.

ITO, E., FREDERICK, M. O., KOFTIS, T. V., TANG, W., PETROVIC, G., LING, T. \& NICOLAOU, K. C. 2006. Structure toxicity relationships of synthetic azaspiracid-1 and analogs in mice. Harmful Algae, 5, 586-591.

ITO, E., SATAKE, M., OFUJI, K., HIGASHI, M., HARIGAYA, K., MCMAHON, T. \& YASUMOTO, T. 2002a. Chronic effects in mice caused by oral administration of sublethal doses of azaspiracid, a new marine toxin isolated from mussels. Toxicon, 40, 193-203.

ITO, E., SATAKE, M., OFUJI, K., KURITA, N., MCMAHON, T., JAMES, K. \& YASUMOTO, T. 2000. Multiple organ damage caused by a new toxin azaspiracid, isolated from mussels produced in Ireland. Toxicon, 38, 917-930.

ITO, E. \& TERAO, K. 1994. Injury and recovery process of intestine caused by okadaic acid and related compounds. Natural toxins, 2, 371-377.

ITO, E., YASUMOTO, T., TAKAI, A., IMANISHI, S. \& HARADA, K. 2002b. Investigation of the distribution and excretion of okadaic acid in mice using immunostaining method. Toxicon, 40, 159-165.

JIANG, H., VILCINSKAS, A. \& KANOST, M. R. 2010. Immunity in Lepidopteran Insects. In: SÖDERHÄLL, K. (ed.) Invertebrate Immunity. Boston, MA: Springer US.

JORJÃO, A. L., OLIVEIRA, L. D., SCORZONI, L., FIGUEIREDO-GODOI, L. M. A., CRISTINA A. PRATA, M., JORGE, A. O. C. \& JUNQUEIRA, J. C. 2018. From moths to caterpillars: Ideal conditions for Galleria mellonella rearing for in vivo microbiological studies. Virulence, 9, 383-389.

JOSEPH, W. 2014. Origins and activation of prophenoloxidases in the digestive tract of the cricket, Gryllus bimaculatus. Archives of insect biochemistry and physiology, 87, 95104.

KANGASSAlO, K., VALTONEN, T. M., ROFF, D., PÖLKKI, M., DUBOVSKIY, I. M., SORVARI, J. \& RANTALA, M. J. 2015. Intra- and trans-generational effects of larval diet on susceptibility to an entomopathogenic fungus, Beauveria bassiana, in the greater wax moth, Galleria mellonella. Journal of Evolutionary Biology, 28, 1453-1464.

KANOST, M. R. \& GORMAN, M. J. 2008. Phenoloxidases in insect immunity. Insect immunology, 1, 69-96.

KARA, H. E., TURAN, Y., ER, A., ACAR, M., TÜMAY, S. \& SINAN, S. 2014. PURIFICATION AND CHARACTERIZATION OF $\beta$-GLUCOSIDASE FROM GREATER WAX MOTH Galleria mellonella L. (LEPIDOPTERA: PYRALIDAE). Archives of Insect Biochemistry and Physiology, 86, 209-219.

KARABACAK, M., KANBUR, M., ERASLAN, G., SILIĞ, Y., SOYER SARICA, Z., TEKELI, M. Y. \& TAŞ, A. 2018. The effects of colostrum on some biochemical parameters in the experimental intoxication of rats with paracetamol. Environmental Science and Pollution Research, 25, 23897-23908.

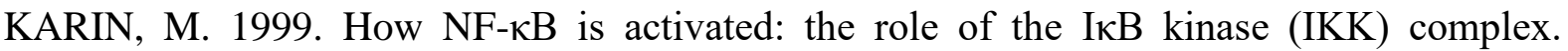
Oncogene, 18, 6867-6874.

KAUR, G., SOMAIYA, R., WASIM, M. \& BUTTAR, H. S. 2014. Cardioprotective effects of bovine colostrum against isoproterenol-induced myocardial infarction in rats. $J$ Pharmacol Toxicol, 9, 37-45.

KAVANAGH, K. \& REEVES, E. P. 2004. Exploiting the potential of insects for the in vivo pathogenicity testing of microbial pathogens. FEMS Microbiol, 28. 
KAZEK, M., KACZMAREK, A., WROŃSKA, A. K. \& BOGUŚ, M. I. 2020. Conidiobolus coronatus induces oxidative stress and autophagy response in Galleria mellonella larvae. PLOS ONE, 15, e0228407.

KELLY, A. F., MARTÍNEZ-RODRIGUEZ, A., BOVILL, R. A. \& MACKEY, B. M. 2003. Description of a "Phoenix" Phenomenon in the Growth of <em>Campylobacter jejuni</em> at Temperatures Close to the Minimum for Growth. Applied and Environmental Microbiology, 69, 4975-4978.

KETUDAT CAIRNS, J. R. \& ESEN, A. 2010. $\beta$-Glucosidases. Cellular and Molecular Life Sciences, 67, 3389-3405.

KHAN, Z., MACDONALD, C., WICKS, A. C., HOLT, M. P., FLOYD, D., GHOSH, S., WRIGHT, N. A. \& PLAYFORD, R. J. 2002. Use of the 'nutriceutical', bovine colostrum, for the treatment of distal colitis: results from an initial study. Alimentary Pharmacology \& Therapeutics, 16, 1917-1922.

KHAZAEINIA, T. \& JAMALI, F. 2003. A comparison of gastrointestinal permeability induced by diclofenac-phospholipid complex with diclofenac acid and its sodium salt. J Pharm Pharmaceut Sci, 6, 352-9.

KILCOYNE, J., NULTY, C., JAUFFRAIS, T., MCCARRON, P., HERVE, F., FOLEY, B., RISE, F., CRAIN, S., WILKINS, A. L., TWINER, M. J., HESS, P. \& MILES, C. O. 2014. Isolation, Structure Elucidation, Relative LC-MS Response, and in Vitro Toxicity of Azaspiracids from the Dinoflagellate Azadinium spinosum. Journal of Natural Products, 77, 2465-2474.

KIM, J. R., YEON, S. H., KIM, H. S. \& AHN, Y. J. 2002. Larvicidal activity against Plutella xylostella of cordycepin from the fruiting body of Cordyceps militaris. Pest Management Science: formerly Pesticide Science, 58, 713-717.

KIM, J. W., JEON, W. K. \& KIM, E. J. 2005. Combined effects of bovine colostrum and glutamine in diclofenac-induced bacterial translocation in rat. Clinical Nutrition, 24, 785-793.

KIM, Y., AHMED, S., STANLEY, D. \& AN, C. 2018. Eicosanoid-mediated immunity in insects. Developmental \& Comparative Immunology, 83, 130-143.

KIM, Y., KIM, M. J., HAN, K. S., IMM, J. Y., OH, S. \& KIM, S. H. 2009. Anticancer activity of lactoferrin isolated from caprine colostrum on human cancer cell lines. International Journal of Dairy Technology, 62, 277-281.

KLOEZEN, W., VAN HELVERT-VAN POPPEL, M., FAHAL, A. H. \& VAN DE SANDE, W. W. 2015. A Madurella mycetomatis grain model in Galleria mellonella larvae. PLoS neglected tropical diseases, 9, e0003926.

KOJIMA, S. \& RIFKIN, D. B. 1993. Mechanism of retinoid-induced activation of latent transforming growth factor- $\beta$ in bovine endothelial cells. Journal of Cellular Physiology, 155, 323-332.

KROCK, B., TILlMANN, U., TEBBEN, J., TREFAULT, N. \& GU, H. 2019. Two novel azaspiracids from Azadinium poporum, and a comprehensive compilation of azaspiracids produced by Amphidomataceae, (Dinophyceae). Harmful Algae, 82, 1-8.

KROCK, B., TILLMANN, U., WITT, M. \& GU, H. 2014. Azaspiracid variability of Azadinium poporum (Dinophyceae) from the China Sea. Harmful Algae, 36, 22-28.

KRYUKOV, V. Y., KRYUKOVA, N. A., TOMILOVA, O. G., VORONTSOVA, Y., CHERTKOVA, E., PERVUSHIN, A. L., SLEPNEVA, I., GLUPOV, V. V. \& YAROSLAVTSEVA, O. N. 2020. Comparative analysis of the immune response of the wax moth Galleria mellonella after infection with the fungi Cordyceps militaris and Metarhizium robertsii. Microbial Pathogenesis, 141, 103995.

KRYUKOV, V. Y., YAROSLAVTSEVA, O., SURINA, E., TYURIN, M., DUBOVSKIY, I. \& GLUPOV, V. 2015. Immune reactions of the greater wax moth, Galleria mellonella 
L.(lepidoptera, pyralidae) larvae under combined treatment of the entomopathogens Cordyceps militaris (L.: Fr.) Link and Beauveria bassiana (Bals.-Criv.) Vuill.(Ascomycota, Hypocreales). Entomological Review, 95, 693-698.

KURAISHI, T., BINGGELI, O., OPOTA, O., BUCHON, N. \& LEMAITRE, B. 2011. Genetic evidence for a protective role of the peritrophic matrix against intestinal bacterial infection in Drosophila melanogaster. Proceedings of the National Academy of Sciences, 108, 15966-15971.

KURODA, M., YOSHIDA, N., ICHIKAWA, H., TAKAGI, T., OKUDA, T., NAITO, Y., OKANOUE, T. \& YOSHIKAWA, T. 2006. Lansoprazole, a proton pump inhibitor, reduces the severity of indomethacin-induced rat enteritis. International journal of molecular medicine, 17, 89-93.

KWON, O. Y., LEE, J. S., CHOI, H. S., HONG, H. P., JANG, K.-H., PAEK, J. H., KANG, S. A. \& KO, Y. G. 2010. Antioxidant and anticytokine effects of bovine colostrum in intestinal ischemia/reperfusion injured rat model. Food Science and Biotechnology, 19, 1295-1301.

LAMPROU, I., MAMALI, I., DALLAS, K., FERTAKIS, V., LAMPROPOULOU, M. \& MARMARAS, V. J. 2007. Distinct signalling pathways promote phagocytosis of bacteria, latex beads and lipopolysaccharide in medfly haemocytes. Immunology, 121, 314-327.

LANAS, A. \& SOPEÑA, F. 2009. Nonsteroidal anti-inflammatory drugs and lower gastrointestinal complications. Gastroenterology Clinics, 38, 333-352.

LANE, N. J., DALLAI, R. \& ASHHURST, D. E. 1996. Structural macromolecules of the cell membranes and the extracellular matrices of the insect midgut. In: LEHANE, M. J. \& BILLINGSLEY, P. F. (eds.) Biology of the Insect Midgut. Dordrecht: Springer Netherlands.

LANGE, A., BEIER, S., HUSON, D. H., PARUSEL, R., IGLAUER, F. \& FRICK, J.-S. 2018a. Genome sequence of Galleria mellonella (greater wax moth). Genome announcements, 6.

LANGE, A., SCHÄFER, A., BENDER, A., STEIMLE, A., BEIER, S., PARUSEL, R. \& FRICK, J.-S. 2018b. Galleria mellonella: A Novel Invertebrate Model to Distinguish Intestinal Symbionts From Pathobionts. Frontiers in Immunology, 9.

LANGMAN, M., MORGAN, L. \& WORRALL, A. 1985. Use of anti-inflammatory drugs by patients admitted with small or large bowel perforations and haemorrhage. Br Med J (Clin Res Ed), 290, 347-349.

LARSEN, J. M. 2017. The immune response to Prevotella bacteria in chronic inflammatory disease. Immunology, 151, 363-374.

LASSUDRIE, M., HÉGARET, H., WIKFORS, G. H. \& DA SILVA, P. M. 2020. Effects of marine harmful algal blooms on bivalve cellular immunity and infectious diseases: A review. Developmental \& Comparative Immunology, 108, 103660.

LAWSON, A., LOGAN, J., O'NEILL, G., DESAI, M. \& STANLEY, J. 1999. Large-Scale Survey of CampylobacterSpecies in Human Gastroenteritis by PCR and PCR-EnzymeLinked Immunosorbent Assay. Journal of Clinical Microbiology, 37, 3860-3864.

LEE, Y. S., YUN, E. K., JANG, W. S., KIM, I., LEE, J. H., PARK, S. Y., RYU, K. S., SEO, S. J., KIM, C. H. \& LEE, I. H. 2004. Purification, cDNA cloning and expression of an insect defensin from the great wax moth, Galleria mellonella. Insect Molecular Biology, 13, 65-72.

LEY, R. E. 2016. Prevotella in the gut: choose carefully. Nature reviews Gastroenterology \& hepatology, 13, 69-70.

LEY, R. E., PETERSON, D. A. \& GORDON, J. I. 2006a. Ecological and Evolutionary Forces Shaping Microbial Diversity in the Human Intestine. Cell, 124, 837-848. 
LEY, R. E., TURNBAUGH, P. J., KLEIN, S. \& GORDON, J. I. 2006b. Microbial ecology: human gut microbes associated with obesity. Nature, 444, 1022.

LI, S. P., YANG, F. Q. \& TSIM, K. W. K. 2006. Quality control of Cordyceps sinensis, a valued traditional Chinese medicine. Journal of Pharmaceutical and Biomedical Analysis, 41, 1571-1584.

LIM, J., COATES, C. J., SEOANE, P. I., GARELNABI, M., TAYLOR-SMITH, L. M., MONTEITH, P., MACLEOD, C. L., ESCARON, C. J., BROWN, G. D. \& HALL, R. A. 2018. Characterizing the mechanisms of nonopsonic uptake of cryptococci by macrophages. The Journal of Immunology, 200, 3539-3546.

LIMAYE, A. Drishti: a volume exploration and presentation tool. Developments in X-ray Tomography VIII, 2012. International Society for Optics and Photonics, 85060X.

LINSER, P. J. \& DINGLASAN, R. R. 2014. Insect gut structure, function, development and target of biological toxins. Advances in insect physiology. Elsevier.

LIU, Y., ZHENG, J.-W., PENG, X.-C., LI, H.-Y., HUANG, L., LI, D.-W., LIU, J.-S. \& YANG, W.-D. 2020. Changes in colonic microbiotas in rat after long-term exposure to low dose of okadaic acid. Chemosphere, 254, 126874.

LIU, Z., LI, P., ZHAO, D., TANG, H. \& GUO, J. 2010. Protective effect of extract of Cordyceps sinensis in middle cerebral artery occlusion-induced focal cerebral ischemia in rats. Behavioral and Brain Functions, 6, 61.

LOH, J. M., ADENWALLA, N., WILES, S. \& PROFT, T. 2013. Galleria mellonella larvae as an infection model for group A streptococcus. Virulence, 4, 419-428.

MACDONALD, C. E., CALNAN, D. P., PODAS, T., JOHNSON, W. \& PLAYFORD, R. J. 1998. Clinical trial of, bovine colostrum for protection against NSAID-induced enteropathy. Gastroenterology, 114, A209.

MACDONALD, T., MORANT, S., ROBINSON, G., SHIELD, M., MCGILCHRIST, M., MURRAY, F. \& MCDEVITT, D. 1997. Association of upper gastrointestinal toxicity of non-steroidal anti-inflammatory drugs with continued exposure: cohort study. Bmj, 315, 1333-1337.

MAGOČ, T. \& SALZBERG, S. L. 2011. FLASH: fast length adjustment of short reads to improve genome assemblies. Bioinformatics, 27, 2957-2963.

MAGUIRE, R., DUGGAN, O. \& KAVANAGH, K. 2016. Evaluation of Galleria mellonella larvae as an in vivo model for assessing the relative toxicity of food preservative agents. Cell Biology and Toxicology, 32, 209-216.

MAGUIRE, R., KUNC, M., HYRSL, P. \& KAVANAGH, K. 2017. Caffeine administration alters the behaviour and development of Galleria mellonella larvae. Neurotoxicology and Teratology, 64, 37-44.

MAHMOOD, A., FITZGERALD, A. J., MARCHBANK, T., NTATSAKI, E., MURRAY, D., GHOSH, S. \& PLAYFORD, R. J. 2007. Zinc carnosine, a health food supplement that stabilises small bowel integrity and stimulates gut repair processes. Gut, 56, 168.

MAITY, P., BINDU, S., CHOUBEY, V., ALAM, A., MITRA, K., GOYAL, M., DEY, S., GUHA, M., PAL, C. \& BANDYOPADHYAY, U. 2008. Lansoprazole protects and heals gastric mucosa from non-steroidal anti-inflammatory drug (NSAID)-induced gastropathy by inhibiting mitochondrial as well as Fas-mediated death pathways with concurrent induction of mucosal cell renewal. Journal of Biological Chemistry, 283, 14391-14401.

MAK, P., ZDYBICKA-BARABAS, A. \& CYTRYŃSKA, M. 2010. A different repertoire of Galleria mellonella antimicrobial peptides in larvae challenged with bacteria and fungi. Developmental \& Comparative Immunology, 34, 1129-1136.

MANDATO, C. A., DIEHL-JONES, W. L., MOORE, S. J. \& DOWNER, R. G. 1997. The effects of eicosanoid biosynthesis inhibitors on prophenoloxidase activation, 
phagocytosis and cell spreading in Galleria mellonella. Journal of Insect Physiology, 43, 1-8.

MANFRIN, C., DREOS, R., BATTISTELLA, S., BERAN, A., GERDOL, M., VAROTTO, L., LANFRANCHI, G., VENIER, P. \& PALLAVICINI, A. 2010. Mediterranean Mussel Gene Expression Profile Induced by Okadaic Acid Exposure. Environmental Science \& Technology, 44, 8276-8283.

MARCHBANK, T., OJOBO, E., PLAYFORD, C. J. \& PLAYFORD, R. J. 2011. Reparative properties of the traditional Chinese medicine Cordyceps sinensis (Chinese caterpillar mushroom) using HT29 cell culture and rat gastric damage models of injury. British journal of nutrition, 105, 1303-1310.

MARMARAS, V. J. \& LAMPROPOULOU, M. 2009. Regulators and signalling in insect haemocyte immunity. Cellular Signalling, 21, 186-195.

MATSUI, H., SHIMOKAWA, O., KANEKO, T., NAGANO, Y., RAI, K. \& HYODO, I. 2011. The pathophysiology of non-steroidal anti-inflammatory drug (NSAID)-induced mucosal injuries in stomach and small intestine. Journal of clinical biochemistry and nutrition, 48, 107-111.

MATSUKAWA, Y., TOMITA, Y., NISHINARITA, S., HORIE, T., KATO, K., ARAKAWA, Y., KO, K., SHIMADA, H., NAKANO, M. \& KITAMI, Y. 1997. Efficacy of Lansoprazole against Peptic Ulcers Induced by Nonsteroidal Anti-Inflammatory Drugs: Endoscopic Evaluation of Ulcer Healing. Journal of international medical research, 25, 190-195.

MCCONNELL, E. L., BASIT, A. W. \& MURDAN, S. 2008. Measurements of rat and mouse gastrointestinal $\mathrm{pH}$, fluid and lymphoid tissue, and implications for in-vivo experiments. Journal of Pharmacy and Pharmacology, 60, 63-70.

MCLEAN, J., LIU, H.-N., MILETIC, D., WENG, Y. C., ROGAEVA, E., ZINMAN, L., KRIZ, J. \& ROBERTSON, J. 2010. Distinct biochemical signatures characterize peripherin isoform expression in both traumatic neuronal injury and motor neuron disease. Journal of Neurochemistry, 114, 1177-1192.

MICHIELAN, A., \#X, INC, \#XE0 \& , R. 2015. Intestinal Permeability in Inflammatory Bowel Disease: Pathogenesis, Clinical Evaluation, and Therapy of Leaky Gut. Mediators of Inflammation, 2015, 10.

MIGUEL-ALIAGA, I., JASPER, H. \& LEMAITRE, B. 2018. Anatomy and physiology of the digestive tract of Drosophila melanogaster. Genetics, 210, 357-396.

MOGHADDAM, M. R. B., TONK, M., SCHREIBER, C., SALZIG, D., CZERMAK, P., VILCINSKAS, A. \& RAHNAMAEIAN, M. 2016. The potential of the Galleria mellonella innate immune system is maximized by the co-presentation of diverse antimicrobial peptides. Biological chemistry, 397, 939-945.

MORTENSEN, N. P., KUIJF, M. L., ANG, C. W., SCHIELlERUP, P., KROGFELT, K. A., JACOBS, B. C., VAN BELKUM, A., ENDTZ, H. P. \& BERGMAN, M. P. 2009. Sialylation of Campylobacter jejuni lipo-oligosaccharides is associated with severe gastro-enteritis and reactive arthritis. Microbes and Infection, 11, 988-994.

MOUSAVI, S., BERESWILL, S. \& HEIMESAAT, M. M. 2020. Novel Clinical Campylobacter jejuni Infection Models Based on Sensitization of Mice to Lipooligosaccharide, a Major Bacterial Factor Triggering Innate Immune Responses in Human Campylobacteriosis. Microorganisms, 8, 482.

MOWLDS, P., COATES, C., RENWICK, J. \& KAVANAGH, K. 2010. Dose-dependent cellular and humoral responses in Galleria mellonella larvae following $\beta$-glucan inoculation. Microbes and infection, 12, 146-153.

MUKHERJEE, K., HAIN, T., FISCHER, R., CHAKRABORTY, T. \& VILCINSKAS, A. 2013a. Brain infection and activation of neuronal repair mechanisms by the human 
pathogen Listeria monocytogenes in the lepidopteran model host Galleria mellonella. Virulence, 4, 324-332.

MUKHERJEE, K., RAJU, R., FISCHER, R. \& VILCINSKAS, A. 2013b. Galleria mellonella as a model host to study gut microbe homeostasis and brain infection by the human pathogen Listeria monocytogenes. Yellow Biotechnology I. Springer.

MUKHERJEE, K. \& VILCINSKAS, A. 2014. Development and immunity-related microRNAs of the lepidopteran model host Galleria mellonella. BMC genomics, 15, 112.

MUKHERJEE, S. 2003. Diarrhea associated with lansoprazole. Journal of gastroenterology and hepatology, 18, 602-603.

MUKHOPADHYAY, S., PANDA, P. K., SINHA, N., DAS, D. N. \& BHUTIA, S. K. 2014. Autophagy and apoptosis: where do they meet? Apoptosis, 19, 555-566.

MUNDAY, R. 2013. Is protein phosphatase inhibition responsible for the toxic effects of okadaic acid in animals? Toxins, 5, 267-285.

MÉNDEZ-SALAZAR, E. O., ORTIZ-LÓPEZ, M. G., GRANADOS-SILVESTRE, M. D. L. Á., PALACIOS-GONZÁLEZ, B. \& MENJIVAR, M. 2018. Altered Gut Microbiota and Compositional Changes in Firmicutes and Proteobacteria in Mexican Undernourished and Obese Children. Frontiers in Microbiology, 9.

NAITO, M., FRIRDICH, E., FIELDS, J. A., PRYJMA, M., LI, J., CAMERON, A., GILBERT, M., THOMPSON, S. A. \& GAYNOR, E. C. 2010. Effects of Sequential $<$ em>Campylobacter jejuni</em> 81-176 Lipooligosaccharide Core Truncations on Biofilm Formation, Stress Survival, and Pathogenesis. Journal of Bacteriology, 192, 2182-2192.

NAPPI, A. J. \& OTTAVIANI, E. 2000. Cytotoxicity and cytotoxic molecules in invertebrates. BioEssays, 22, 469-480.

NATHAN, S. 2014. New to Galleria mellonella. Virulence, 5, 371-374.

NEHME, N. T., LIÉGEOIS, S., KELE, B., GIAMMARINARO, P., PRADEL, E., HOFFMANN, J. A., EWBANK, J. J. \& FERRANDON, D. 2007. A model of bacterial intestinal infections in Drosophila melanogaster. PLoS pathogens, 3.

NEMELKA, K. W., BROWN, A. W., WALlACE, S. M., JONES, E., ASHER, L. V., PATTARINI, D., APPLEBEE, L., GILLILAND, T. C., GUERRY, P. \& BAQAR, S. 2009. Immune response to and histopathology of Campylobacter jejuni infection in ferrets (Mustela putorius furo). Comparative medicine, 59, 363-371.

NEVILLE, K. E., BOSSE, T. L., KLEKOS, M., MILLS, J. F., WEICKSEL, S. E., WATERS, J. S. \& TIPPING, M. 2018. A novel ex vivo method for measuring whole brain metabolism in model systems. Journal of neuroscience methods, 296, 32-43.

NUGENT, S. G., KUMAR, D., RAMPTON, D. S. \& EVANS, D. F. 2001. Intestinal luminal $\mathrm{pH}$ in inflammatory bowel disease: possible determinants and implications for therapy with aminosalicylates and other drugs. Gut, 48, 571.

OFUJI, K., SATAKE, M., MCMAHON, T., SILKE, J., JAMES, K. J., NAOKI, H., OSHIMA, Y. \& YASUMOTO, T. 1999. Two analogs of azaspiracid isolated from mussels, Mytilus edulis, involved in human intoxication in Ireland. Natural Toxins, 7, 99-102.

OLSEN, R. J., WATKINS, M. E., CANTU, C. C., BERES, S. B. \& MUSSER, J. M. 2011. Virulence of serotype M3 Group A Streptococcus strains in wax worms (Galleria mellonella larvae). Virulence, 2, 111-119.

PANEL, E. C. 2008. Opinion of the scienti fi c panel on contaminants in the food chain on a request from the European commission on marine biotoxins in shell fi sh-okadaic acid and analogues. The EFSA Journal, 589, 1-62. 
PANIAGUA VOIROL, L. R., FRAGO, E., KALTENPOTH, M., HILKER, M. \& FATOUROS, N. E. 2018. Bacterial Symbionts in Lepidoptera: Their Diversity, Transmission, and Impact on the Host. Frontiers in Microbiology, 9.

PARADA, A. E., NEEDHAM, D. M. \& FUHRMAN, J. A. 2016. Every base matters: assessing small subunit rRNA primers for marine microbiomes with mock communities, time series and global field samples. Environmental Microbiology, 18, 1403-1414.

PAULUS, H. E. 1988. FDA arthritis advisory committee meeting: serious gastrointestinal toxicity of nonsteroidal antiinflammatory drugs; drug-containing renal and biliary stones; diclofenac and carprofen approved. Arthritis \& Rheumatism: Official Journal of the American College of Rheumatology, 31, 1450-1451.

PELIN, M., KILCOYNE, J., NULTY, C., CRAIN, S., HESS, P., TUBARO, A. \& SOSA, S. 2018a. Toxic equivalency factors (TEFs) after acute oral exposure of azaspiracid 1, -2 and -3 in mice. Toxicology Letters, 282, 136-146.

PELIN, M., KILCOYNE, J., NULTY, C., CRAIN, S., HESS, P., TUBARO, A. \& SOSA, S. 2018b. Toxic equivalency factors (TEFs) after acute oral exposure of azaspiracid 1,-2 and -3 in mice. Toxicology Letters, 282, 136-146.

PEREIRA, T. C., DE BARROS, P. P., FUGISAKI, L. R. D. O., ROSSONI, R. D., RIBEIRO, F. D. C., DE MENEZES, R. T., JUNQUEIRA, J. C. \& SCORZONI, L. 2018. Recent advances in the use of Galleria mellonella model to study immune responses against human pathogens. Journal of Fungi, 4, 128.

PERRON, N., TREMBLAY, E., FERRETTI, E., BABAKISSA, C., SEIDMAN, E. G., LEVY, E., MÉNARD, D. \& BEAULIEU, J.-F. 2013. Deleterious effects of indomethacin in the mid-gestation human intestine. Genomics, 101, 171-177.

PLAYFORD, R., FLOYD, D., MACDONALD, C., CALNAN, D., ADENEKAN, R., JOHNSON, W., GOODLAD, R. \& MARCHBANK, T. 1999. Bovine colostrum is a health food supplement which prevents NSAID induced gut damage. Gut, 44, 653-658.

PLAYFORD, R. J., MACDONALD, C. E., CALNAN, D. P., FLOYD, D. N., PODAS, T., JOHNSON, W., WICKS, A. C., BASHIR, O. \& MARCHBANK, T. 2001. Coadministration of the health food supplement, bovine colostrum, reduces the acute nonsteroidal anti-inflammatory drug-induced increase in intestinal permeability. Clinical Science, 100, 627-633.

PLAYFORD, R. J., MACDONALD, C. E. \& JOHNSON, W. S. 2000. Colostrum and milkderived peptide growth factors for the treatment of gastrointestinal disorders. The American Journal of Clinical Nutrition, 72, 5-14.

RAI, K., MATSUI, H., KANEKO, T., NAGANO, Y., SHIMOKAWA, O., UDO, J., HIRAYAMA, A., HYODO, I., INDO, H. P. \& MAJIMA, H. J. 2011. Lansoprazole inhibits mitochondrial superoxide production and cellular lipid peroxidation induced by indomethacin in RGM1 cells. Journal of Clinical Biochemistry and Nutrition, 49, 25-30.

RATCLIFFE, N. A. \& GAGEN, S. J. 1976. Cellular defense reactions of insect hemocytes in vivo: Nodule formation and development in Galleria mellonella and Pieris brassicae larvae. Journal of Invertebrate Pathology, 28, 373-382.

REEVES, E. P., LU, H., JACOBS, H. L., MESSINA, C. G., BOLSOVER, S., GABELLA, G., POTMA, E. O., WARLEY, A., ROES, J. \& SEGAL, A. W. 2002. Killing activity of neutrophils is mediated through activation of proteases by $\mathrm{K}+$ flux. Nature, 416, 291297.

REEVES, E. P., MESSINA, C., DOYLE, S. \& KAVANAGH, K. 2004. Correlation between gliotoxin production and virulence of Aspergillus fumigatus in Galleria mellonella. Mycopathologia, 158, 73-79. 
RENWICK, J., REEVES, E. P., WIENTJES, F. B. \& KAVANAGH, K. 2007. Translocation of proteins homologous to human neutrophil p47phox and p67phox to the cell membrane in activated hemocytes of Galleria mellonella. Developmental \& Comparative Immunology, 31, 347-359.

REUTER, B. K., CIRINO, G. \& WALLACE, J. L. 1994. Markedly reduced intestinal toxicity of a diclofenac derivative. Life Sciences, 55, PL1-PL8.

RIBEIRO, F. C., BARROS, P. P., ROSSONI, R. D., JUNQUEIRA, J. C. \& JORGE, A. O. C. 2016. Lactobacillus rhamnosus inhibits Candida albicans virulence factors in vitro and modulates immune system in Galleria mellonella. Journal of Applied Microbiology, 122, 201-211.

RIZZATTI, G., LOPETUSO, L. R., GIBIINO, G., BINDA, C. \& GASBARRINI, A. 2017. Proteobacteria: A Common Factor in Human Diseases. BioMed Research International, 2017, 9351507.

ROBERTSON , J., DOROUDCHI , M. M., NGUYEN , M. D., DURHAM , H. D., STRONG , M. J., SHAW , G., JULIEN , J.-P. \& MUSHYNSKI , W. E. 2003. A neurotoxic peripherin splice variant in a mouse model of ALS. Journal of Cell Biology, 160, 939949.

RODRÍGUEZ, J. A., THEODUlOZ, C., YÁÑEZ, T., BECERRA, J. \& SCHMEDAHIRSCHMANN, G. 2006. Gastroprotective and ulcer healing effect of ferruginol in mice and rats: Assessment of its mechanism of action using in vitro models. Life Sciences, 78, 2503-2509.

ROMÁN, Y., ALFONSO, A., LOUZAO, M. C., DE LA ROSA, L. A., LEIRA, F., VIEITES, J. M., VIEYTES, M. R., OFUJI, K., SATAKE, M., YASUMOTO, T. \& BOTANA, L. M. 2002. Azaspiracid-1, a potent, nonapoptotic new phycotoxin with several cell targets. Cellular Signalling, 14, 703-716.

ROSSI, R., DELL'AVERSANO, C., KROCK, B., CIMINIELlO, P., PERCOPO, I., TILLMANN, U., SOPRANO, V. \& ZINGONE, A. 2017. Mediterranean Azadinium dexteroporum (Dinophyceae) produces six novel azaspiracids and azaspiracid-35: a structural study by a multi-platform mass spectrometry approach. Analytical and Bioanalytical Chemistry, 409, 1121-1134.

ROWIN, J., XIA, Y., JUNG, B. \& SUN, J. 2017. Gut inflammation and dysbiosis in human motor neuron disease. Physiological Reports, 5, e13443.

ROWLEY, A. F. \& POWELL, A. 2007. Invertebrate immune systems-specific, quasi-specific, or nonspecific? The Journal of Immunology, 179, 7209-7214.

SALAMITOU, S., RAMISSE, F., BREHÉLIN, M., BOURGUET, D., GILOIS, N., GOMINET, M., HERNANDEZ, E. \& LERECLUS, D. 2000. The plcR regulon is involved in the opportunistic properties of Bacillus thuringiensis and Bacillus cereus in mice and insects. Microbiology, 146, 2825-2832.

SAMPSON, T. R., DEBELIUS, J. W., THRON, T., JANSSEN, S., SHASTRI, G. G., ILHAN, Z. E., CHALLIS, C., SCHRETTER, C. E., ROCHA, S. \& GRADINARU, V. 2016. Gut microbiota regulate motor deficits and neuroinflammation in a model of Parkinson's disease. Cell, 167, 1469-1480. e12.

SANDVIK, M., MILES, C. O., WILKINS, A. L. \& FÆSTE, C. 2020. In vitro hepatic biotransformation of the algal toxin pectenotoxin-2. Toxicon: X, 6, 100031.

SANYAL, S. N. \& KAUSHAL, N. 2005. Effect of two non-steroidal anti-inflammatory drugs, aspirin and nimesulide on the D-glucose transport and disaccharide hydrolases in the intestinal brush border membrane. Pharmacol Reports, 57, 833-839.

SATAKE, M., OFUJI, K., NAOKI, H., JAMES, K. J., FUREY, A., MCMAHON, T., SILKE, J. \& YASUMOTO, T. 1998. Azaspiracid, a new marine toxin having unique spiro ring 
assemblies, isolated from Irish mussels, Mytilus edulis. Journal of the American Chemical Society, 120, 9967-9968.

SATYARAJ, E., REYNOLDS, A., PELKER, R., LABUDA, J., ZHANG, P. \& SUN, P. 2013. Supplementation of diets with bovine colostrum influences immune function in dogs. British Journal of Nutrition, 110, 2216-2221.

SCALFARO, C., IACOBINO, A., NARDIS, C. \& FRANCIOSA, G. 2017. Galleria mellonella as an in vivo model for assessing the protective activity of probiotics against gastrointestinal bacterial pathogens. FEMS Microbiology Letters, 364.

SCHLOSS, P. D., GEVERS, D. \& WESTCOTT, S. L. 2011. Reducing the Effects of PCR Amplification and Sequencing Artifacts on 16S rRNA-Based Studies. PLOS ONE, 6, e27310.

SENIOR, N. J., BAGNALL, M. C., CHAMPION, O. L., REYNOLDS, S. E., LA RAGIONE, R. M., WOODWARD, M. J., SALGUERO, F. J. \& TITBALL, R. W. 2011. Galleria mellonella as an infection model for Campylobacter jejuni virulence. Journal of Medical Microbiology, 60, 661-669.

SEO, P. J., KIM, N., KIM, J.-H., LEE, B. H., NAM, R. H., LEE, H. S., PARK, J. H., LEE, M. K., CHANG, H., JUNG, H. C. \& SONG, I. S. 2012. Comparison of Indomethacin, Diclofenac and Aspirin-Induced Gastric Damage according to Age in Rats. Gut and liver, 6, 210-217.

SHAHED, A. R., KIM, S. I. \& SHOSKES, D. A. 2001. Down-regulation of apoptotic and inflammatory genes by Cordyceps Sinensis extract in rat kidney following ischemia/reperfusion. Transplantation Proceedings, 33, 2986-2987.

SHAHEEN, N., AHMED, S., AZHAR, I. \& HASAN, M. 2013. Analgesic, anti-inflammatory and antiemetic activities of Cleome scaposa DC. Phytopharmacology, 4, 106-113.

SHEEHAN, G., GARVEY, A., CROKE, M. \& KAVANAGH, K. 2018. Innate humoral immune defences in mammals and insects: The same, with differences ? Virulence, 9, 1625-1639.

SHIN, J. M. \& KIM, N. 2013. Pharmacokinetics and pharmacodynamics of the proton pump inhibitors. Journal of neurogastroenterology and motility, 19, 25-35.

SI, J., YOU, H. J., YU, J., SUNG, J. \& KO, G. 2017. Prevotella as a hub for vaginal microbiota under the influence of host genetics and their association with obesity. Cell host \& microbe, 21, 97-105.

SIDERI, M., TSAKAS, S., MARKOUTSA, E., LAMPROPOULOU, M. \& MARMARAS, V. J. 2008. Innate immunity in insects: Surface-associated dopa decarboxylase-dependent pathways regulate phagocytosis, nodulation and melanization in medfly haemocytes. Immunology, 123, 528-537.

SIGTHORSSON, G., CRANE, R., SIMON, T., HOOVER, M., QUAN, H., BOLOGNESE, J. \& BJARNASON, I. 2000. COX-2 inhibition with rofecoxib does not increase intestinal permeability in healthy subjects: a double blind crossover study comparing rofecoxib with placebo and indomethacin. Gut, 47, 527.

SLÁMA, K. \& LUKÁŠ́, J. 2011. Myogenic nature of insect heartbeat and intestinal peristalsis, revealed by neuromuscular paralysis caused by the sting of a braconid wasp. Journal of insect physiology, 57, 251-259.

SMECUOL, E., BAI, J. C., SUGAI, E., VAZQUEZ, H., NIVELONI, S., PEDREIRA, S., MAURIÑO, E. \& MEDDINGS, J. 2001. Acute gastrointestinal permeability responses to different non-steroidal anti-inflammatory drugs. $G u t, 49,650$.

SMITS, W. K., LYRAS, D., LACY, D. B., WILCOX, M. H. \& KUIJPER, E. J. 2016. Clostridium difficile infection. Nature Reviews Disease Primers, 2, 16020. 
SONG, Y., MALMUTHUGE, N., LI, F. \& GUAN, L. L. 2018. Colostrum feeding shapes the hindgut microbiota of dairy calves during the first $12 \mathrm{~h}$ of life. FEMS Microbiology Ecology, 95.

STACK, W., ATHERTON, J., HAWKEY, G., LOGAN, R. \& HAWKEY, C. 2002. Interactions between Helicobacter pylori and other risk factors for peptic ulcer bleeding. Alimentary pharmacology \& therapeutics, 16, 497-506.

STROBER, W. 2015. Trypan blue exclusion test of cell viability. Current protocols in immunology, 111, A3. B. 1-A3. B. 3.

SUGANO, K., KONTANI, T., KATSUO, S., TAKEI, Y., SAKAKI, N., ASHIDA, K., MIZOKAMI, Y., ASAKA, M., MATSUI, S. \& KANTO, T. 2012. Lansoprazole for secondary prevention of gastric or duodenal ulcers associated with long-term nonsteroidal anti-inflammatory drug (NSAID) therapy: results of a prospective, multicenter, double-blind, randomized, double-dummy, active-controlled trial. Journal of gastroenterology, 47, 540-552.

SUMM, O. \& EVERS, S. 2013. Mechanism of action of indomethacin in indomethacinresponsive headaches. Current pain and headache reports, 17, 327.

TAMBOLI, C. P., NEUT, C., DESREUMAUX, P. \& COLOMBEL, J. 2004. Dysbiosis in inflammatory bowel disease. Gut, 53, 1-4.

TANIGAWA, M., SUZUKI, C., NIWANO, K., KANEKATSU, R., TANAKA, H., HORIIKE, K., HAMASE, K. \& NAGATA, Y. 2016. Participation of D-serine in the development and reproduction of the silkworm Bombyx mori. Journal of insect physiology, 87, 2029.

TAYLOR, Z. W., BROWN, H. A., HOLDEN, H. M. \& RAUSHEL, F. M. 2017. Biosynthesis of Nucleoside Diphosphoramidates in Campylobacter jejuni. Biochemistry, 56, 60796082.

TILLMANN, U., JAÉN, D., FERNÁNDEZ, L., GOTTSCHLING, M., WITT, M., BLANCO, J. \& KROCK, B. 2017. Amphidoma languida (Amphidomatacea, Dinophyceae) with a novel azaspiracid toxin profile identified as the cause of molluscan contamination at the Atlantic coast of southern Spain. Harmful Algae, 62, 113-126.

TRES, F., TREACHER, K., BOOTH, J., HUGHES, L. P., WREN, S. A. C., AYLOTT, J. W. \& BURLEY, J. C. 2016. Indomethacin-Kollidon VA64 Extrudates: A Mechanistic Study of pH-Dependent Controlled Release. Molecular Pharmaceutics, 13, 1166-1175.

TSAI, C. J.-Y., LOH, J. M. S. \& PROFT, T. 2016. Galleria mellonella infection models for the study of bacterial diseases and for antimicrobial drug testing. Virulence, 7, 214-229.

TURNER, J. R. 2006. Molecular basis of epithelial barrier regulation: from basic mechanisms to clinical application. The American journal of pathology, 169, 1901-1909.

TWINER, M. J., EL-LADKI, R., KILCOYNE, J. \& DOUCETTE, G. J. 2012a. Comparative Effects of the Marine Algal Toxins Azaspiracid-1, -2, and -3 on Jurkat T Lymphocyte Cells. Chemical Research in Toxicology, 25, 747-754.

TWINER, M. J., HANAGRIFF, J. C., BUTLER, S., MADHKOOR, A. K. \& DOUCETTE, G. J. 2012b. Induction of Apoptosis Pathways in Several Cell Lines following Exposure to the Marine Algal Toxin Azaspiracid. Chemical Research in Toxicology, 25, 1493 1501.

UK, F. S. A. 2018. Biotoxin and phytoplankton monitoring. In: AGENCY, U. F. S. (ed.). UK Government.

URUAKPA, F. O., ISMOND, M. A. H. \& AKOBUNDU, E. N. T. 2002. Colostrum and its benefits: a review. Nutrition Research, 22, 755-767.

UWO, M. F., UI-TEI, K., PARK, P. \& TAKEDA, M. 2002. Replacement of midgut epithelium in the greater wax moth, Galleria mellonela, during larval-pupal moult. Cell and Tissue Research, 308, 319-331. 
VALLET-GELY, I., LEMAITRE, B. \& BOCCARD, F. 2008. Bacterial strategies to overcome insect defences. Nature Reviews Microbiology, 6, 302-313.

VAN DEN ELSEN, L. W. J., GARSSEN, J., BURCELIN, R. \& VERHASSELT, V. 2019. Shaping the Gut Microbiota by Breastfeeding: The Gateway to Allergy Prevention? Frontiers in Pediatrics, 7.

VAN DER VIJVER, R. J., VAN LAARHOVEN, C. J. H. M., LOMME, R. M. L. M. \& HENDRIKS, T. 2013. Diclofenac causes more leakage than naproxen in anastomoses in the small intestine of the rat. International Journal of Colorectal Disease, 28, 12091216.

VAN LEEUWEN, R. W. F., VAN GELDER, T., MATHIJSSEN, R. H. J. \& JANSMAN, F. G. A. 2014. Drug-drug interactions with tyrosine-kinase inhibitors: a clinical perspective. The Lancet Oncology, 15, e315-e326.

VAN PUTTEN, J. P. M., VAN ALPHEN, L. B., WÖSTEN, M. M. S. M. \& DE ZOETE, M. R. 2009. Molecular Mechanisms of Campylobacter Infection. In: SASAKAWA, C. (ed.) Molecular Mechanisms of Bacterial Infection via the Gut. Berlin, Heidelberg: Springer Berlin Heidelberg.

VILARIÑO, N. 2008. Marine toxins and the cytoskeleton: azaspiracids. The FEBS Journal, 275, 6075-6081.

VILARIÑO, N., NICOLAOU, K., FREDERICK, M. O., CAGIDE, E., ARES, I. R., LOUZAO, M. C., VIEYTES, M. R. \& BOTANA, L. M. 2006. Cell growth inhibition and actin cytoskeleton disorganization induced by azaspiracid-1 structure- activity studies. Chemical research in toxicology, 19, 1459-1466.

VOGEL, H., ALTINCICEK, B., GLÖCKNER, G. \& VILCINSKAS, A. 2011. A comprehensive transcriptome and immune-gene repertoire of the lepidopteran model host Galleria mellonella. BMC Genomics, 12, 308.

WAGLEY, S., BORNE, R., HARRISON, J., BAKER-AUSTIN, C., OTTAVIANI, D., LEONI, F., VUDDHAKUL, V. \& TITBALL, R. W. 2018. Galleria mellonella as an infection model to investigate virulence of Vibrio parahaemolyticus. Virulence, 9, 197-207.

WALLACE, J. L., ZAMUNER, S. R., MCKNIGHT, W., DICAY, M., MENCARELLI, A., DEL SOLDATO, P. \& FIORUCCI, S. 2004. Aspirin, but not NO-releasing aspirin (NCX-4016), interacts with selective COX-2 inhibitors to aggravate gastric damage and inflammation. American Journal of Physiology-Gastrointestinal and Liver Physiology, 286, G76-G81.

WALLER, D. A., THOMAS, N. W. \& SELF, T. J. 1988. Epithelial restitution in the large intestine of the rat following insult with bile salts. Virchows Archiv A, 414, 77-81.

WALTERS, W., HYDE, E. R., BERG-LYONS, D., ACKERMANN, G., HUMPHREY, G., PARADA, A., GILBERT, J. A., JANSSON, J. K., CAPORASO, J. G., FUHRMAN, J. A., APPRILL, A. \& KNIGHT, R. 2015. Improved Bacterial 16S rRNA Gene (V4 and V4-5) and Fungal Internal Transcribed Spacer Marker Gene Primers for Microbial Community Surveys. mSystems, 1, e00009-15.

WANG, L. \& LIGOXYGAKIS, P. 2006. Pathogen recognition and signalling in the Drosophila innate immune response. Immunobiology, 211, 251-261.

WANG, X., LU, H., SHAO, Y. \& ZONG, S. 2018. Morphological and ultrastructural characterization of the alimentary canal in larvae of Streltzoviella insularis (Staudinger)(Lepidoptera: Cossidae). Entomological Research.

WATANABE, H., HORIE, Y., TAKANOBU, H., KOSHIO, M., FLYNN, K., IGUCHI, T. \& TATARAZAKO, N. 2017. Medaka extended one-generation reproduction test evaluating 4-nonylphenol. Environmental toxicology and chemistry, 36, 3254-3266.

WHITTEN, M. M. A. \& COATES, C. J. 2017. Re-evaluation of insect melanogenesis research: Views from the dark side. Pigment Cell \& Melanoma Research, 30, 386-401. 
WOJDA, I. 2017. Immunity of the greater wax moth Galleria mellonella. Insect Science, 24, 342-357.

WOJDA, I., KOWALSKI, P. \& JAKUBOWICZ, T. 2009. Humoral immune response of Galleria mellonella larvae after infection by Beauveria bassiana under optimal and heatshock conditions. Journal of Insect Physiology, 55, 525-531.

XU, L., ZHANG, L., ZHANG, Y., SHENG, Q. \& ZHAO, A. 2011. Qualitative and quantitative comparison of hormone contents between bovine and human colostrums. International Dairy Journal, 21, 54-57.

YAMAGUCHI, Y., KAGOTA, S., NAKAMURA, K., SHINOZUKA, K. \& KUNITOMO, M. 2000. Antioxidant activity of the extracts from fruiting bodies of cultured Cordyceps sinensis. Phytotherapy Research, 14, 647-649.

YAN, J.-K., WANG, W.-Q. \& WU, J.-Y. 2014. Recent advances in Cordyceps sinensis polysaccharides: Mycelial fermentation, isolation, structure, and bioactivities: A review. Journal of Functional Foods, 6, 33-47.

YANG, M., ZOU, Y., WU, Z. H., LI, S. L. \& CAO, Z. J. 2015. Colostrum quality affects immune system establishment and intestinal development of neonatal calves. Journal of Dairy Science, 98, 7153-7163.

YEOMAN, C. J., HAN, Y., DODD, D., SCHROEDER, C. M., MACKIE, R. I. \& CANN, I. K. O. 2010. Chapter 1 - Thermostable Enzymes as Biocatalysts in the Biofuel Industry. Advances in Applied Microbiology. Academic Press.

YODA, Y., AMAGASE, K., KATO, S., TOKIOKA, S., MURANO, M., KAKIMOTO, K., NISHIO, H., UMEGAKI, E., TAKEUCHI, K. \& HIGUCHI, K. 2010. Prevention by lansoprazole, a proton pump inhibitor, of indomethacin-induced small intestinal ulceration in rats through induction of heme oxygenase-1. Journal of Physiology and Pharmacology, 61, 287.

ZARANTONELLI, M. L., HUERRE, M., TAHA, M.-K. \& ALONSO, J.-M. 2006. Differential role of lipooligosaccharide of Neisseria meningitidis in virulence and inflammatory response during respiratory infection in mice. Infection and immunity, 74, 5506-5512.

ZHANG, S. L., BAI, L., GOEL, N., BAILEY, A., JANG, C. J., BUSHMAN, F. D., MEERLO, P., DINGES, D. F. \& SEHGAL, A. 2017. Human and rat gut microbiome composition is maintained following sleep restriction. Proceedings of the National Academy of Sciences, 114, E1564-E1571. 


\section{Appendices}

\section{APPENDIX A}

\section{Optimising fixation G. mellonella larvae}

Healthy final instar larvae were purchased from Livefoods Direct Ltd. Larvae weighing between $250 \mathrm{mg}$ and $350 \mathrm{mg}$ were selected for fixation trials. Carnoy's fixative (60\% ethanol, $30 \%$ chloroform and 10\% glacial acetic acid v/v) (Barbosa et al., 2014) and 10\% unbuffered formalin fixative were trialled across 24,48 and 72 hours to determine the most suitable fixation period. Additionally, several methods of fixation were tested as follows: intrahaemocoelic injection of fixative, piercing larvae cuticle whilst submerged in fixative, placing larvae in fixative and a combination of the three methods. Fixed larvae were dehydrated in $100 \%$ ethanol for 24 hours before dissection. As a result, it was determined that the most appropriate way of fixation, was $10 \%$ unbuffered formalin of which $20 \mu 1$ was injected into each larva. Fixed insects were placed into $7 \mathrm{ml}$ of $10 \%$ formalin and pierced with a needle to allow deeper tissue penetration of fixative over 28-30 hours. This method had limited shrinkage and hardening while thoroughly fixing the larvae.

Table A1. Values of significance following a Tukey's multiple comparisons test for Galleria mellonella going through pupation after inoculation via force feeding (FF) or intrahaemocoelic injection (INJ) of indomethacin or PBS and the untreated control.

\begin{tabular}{|c|c|c|c|c|}
\hline 6 days post inoculation & $\begin{array}{l}\text { Mean } \\
\text { Diff. }\end{array}$ & $95.00 \%$ CI of diff. & Summary & $\begin{array}{l}\text { Adjusted } \\
\text { P Value }\end{array}$ \\
\hline $7.5 \mu \mathrm{g} /$ larvae FF vs. Control & -60 & -96.66 to -23.34 & $* * * *$ & $<0.0001$ \\
\hline $7.5 \mu \mathrm{g} / \mathrm{larvae}$ FF vs. $5 \mu \mathrm{g} / \mathrm{larvae}$ INJ & -46.67 & -83.32 to -10.01 & $* *$ & 0.0023 \\
\hline $\begin{array}{l}7.5 \mu \mathrm{g} / \mathrm{larvae} \text { FF vs. } 2.5 \mu \mathrm{g} / \mathrm{larvae} \\
\text { INJ }\end{array}$ & -40 & -76.66 to -3.342 & $*$ & 0.0195 \\
\hline $\begin{array}{l}7.5 \mu \mathrm{g} / \mathrm{larvae} \text { FF vs. } 0.5 \mu \mathrm{g} / \mathrm{larvae} \\
\text { INJ }\end{array}$ & -36.67 & -73.32 to -0.008652 & $*$ & 0.0499 \\
\hline $7.5 \mu \mathrm{g} /$ larvae FF vs. 5\% DMSO INJ & -40 & -76.66 to -3.342 & $*$ & 0.0195 \\
\hline $5 \mu \mathrm{g} /$ larvae FF vs. Control & -50 & -86.66 to -13.34 & $* * *$ & 0.0007 \\
\hline $5 \mu \mathrm{g} /$ larvae FF vs. $5 \mu \mathrm{g} /$ larvae INJ & -36.67 & -73.32 to -0.008652 & $*$ & 0.0499 \\
\hline $2.5 \mu \mathrm{g} /$ larvae FF vs. Control & -60 & -96.66 to -23.34 & $* * * *$ & $<0.0001$ \\
\hline $2.5 \mu \mathrm{g} / \mathrm{larvae}$ FF vs. $5 \mu \mathrm{g} / \mathrm{larvae}$ INJ & -46.67 & -83.32 to -10.01 & $* *$ & 0.0023 \\
\hline $\begin{array}{l}2.5 \mu \mathrm{g} / \mathrm{larvae} \text { FF vs. } 2.5 \mu \mathrm{g} / \mathrm{larvae} \\
\text { INJ }\end{array}$ & -40 & -76.66 to -3.342 & $*$ & 0.0195 \\
\hline $\begin{array}{l}2.5 \mu \mathrm{g} / \text { larvae FF vs. } 0.5 \mu \mathrm{g} / \mathrm{larvae} \\
\text { INJ }\end{array}$ & -36.67 & -73.32 to -0.008652 & $*$ & 0.0499 \\
\hline $2.5 \mu \mathrm{g} /$ larvae FF vs. $5 \%$ DMSO INJ & -40 & -76.66 to -3.342 & $*$ & 0.0195 \\
\hline $1 \mu \mathrm{g} /$ larvae FF vs. Control & -60 & -96.66 to -23.34 & $* * * *$ & $<0.0001$ \\
\hline $1 \mu \mathrm{g} /$ larvae FF vs. $5 \mu \mathrm{g} /$ larvae INJ & -46.67 & -83.32 to -10.01 & $* *$ & 0.0023 \\
\hline
\end{tabular}




\begin{tabular}{|c|c|c|c|c|}
\hline $1 \mu \mathrm{g} /$ larvae FF vs. $2.5 \mu \mathrm{g} / \mathrm{larvae}$ INJ & -40 & -76.66 to -3.342 & $*$ & 0.0195 \\
\hline $1 \mu \mathrm{g} /$ larvae FF vs. $0.5 \mu \mathrm{g} / \mathrm{larvae}$ INJ & -36.67 & -73.32 to -0.008652 & $*$ & 0.0499 \\
\hline $1 \mu \mathrm{g} /$ larvae FF vs. 5\% DMSO INJ & -40 & -76.66 to -3.342 & $*$ & 0.0195 \\
\hline $0.5 \mu \mathrm{g} /$ larvae FF vs. Control & -50 & -86.66 to -13.34 & $* * *$ & 0.0007 \\
\hline $0.5 \mu \mathrm{g} / \mathrm{larvae}$ FF vs. $5 \mu \mathrm{g} / \mathrm{larvae}$ INJ & -36.67 & -73.32 to -0.008652 & $*$ & 0.0499 \\
\hline $5 \%$ DMSO FF vs. Control & -46.67 & -83.32 to -10.01 & $* *$ & 0.0023 \\
\hline 7 days post inoculation & $\begin{array}{l}\text { Mean } \\
\text { Diff. }\end{array}$ & $95.00 \%$ CI of diff. & Summary & $\begin{array}{l}\text { Adjusted } \\
\text { P Value }\end{array}$ \\
\hline $7.5 \mu \mathrm{g} /$ larvae FF vs. Control & -46.67 & -83.32 to -10.01 & $* *$ & 0.0023 \\
\hline $\begin{array}{l}7.5 \mu \mathrm{g} / \text { larvae FF vs. } 2.5 \mu \mathrm{g} / \text { larvae } \\
\text { INJ }\end{array}$ & -40 & -76.66 to -3.342 & $*$ & 0.0195 \\
\hline $5 \mu \mathrm{g} /$ larvae FF vs. Control & -56.67 & -93.32 to -20.01 & $* * * *$ & $<0.0001$ \\
\hline $5 \mu \mathrm{g} /$ larvae FF vs. $7.5 \mu \mathrm{g} /$ larvae INJ & -40 & -76.66 to -3.342 & $*$ & 0.0195 \\
\hline $5 \mu \mathrm{g} /$ larvae FF vs. $2.5 \mu \mathrm{g} /$ larvae INJ & -50 & -86.66 to -13.34 & $* * *$ & 0.0007 \\
\hline $5 \mu \mathrm{g} /$ larvae FF vs. $1 \mu \mathrm{g} /$ larvae INJ & -40 & -76.66 to -3.342 & $*$ & 0.0195 \\
\hline $5 \mu \mathrm{g} /$ larvae FF vs. $0.5 \mu \mathrm{g} /$ larvae INJ & -36.67 & -73.32 to -0.008652 & $*$ & 0.0499 \\
\hline $5 \mu \mathrm{g} /$ larvae FF vs. $5 \%$ DMSO INJ & -40 & -76.66 to -3.342 & $*$ & 0.0195 \\
\hline $2.5 \mu \mathrm{g} /$ larvae FF vs. Control & -60 & -96.66 to -23.34 & $* * * *$ & $<0.0001$ \\
\hline $\begin{array}{l}2.5 \mu \mathrm{g} / \text { larvae FF vs. } 7.5 \mu \mathrm{g} / \text { larvae } \\
\text { INJ }\end{array}$ & -43.33 & -79.99 to -6.675 & $* *$ & 0.0069 \\
\hline $\begin{array}{l}2.5 \mu \mathrm{g} / \text { larvae FF vs. } 2.5 \mu \mathrm{g} / \text { larvae } \\
\text { INJ }\end{array}$ & -53.33 & -89.99 to -16.68 & $* * *$ & 0.0002 \\
\hline $2.5 \mu \mathrm{g} /$ larvae FF vs. $1 \mu \mathrm{g} /$ larvae INJ & -43.33 & -79.99 to -6.675 & $* *$ & 0.0069 \\
\hline $\begin{array}{l}2.5 \mu \mathrm{g} / \text { larvae FF vs. } 0.5 \mu \mathrm{g} / \text { larvae } \\
\text { INJ }\end{array}$ & -40 & -76.66 to -3.342 & $*$ & 0.0195 \\
\hline $2.5 \mu \mathrm{g} /$ larvae FF vs. 5\% DMSO INJ & -43.33 & -79.99 to -6.675 & $* *$ & 0.0069 \\
\hline $1 \mu \mathrm{g} /$ larvae FF vs. Control & -63.33 & -99.99 to -26.68 & $* * * *$ & $<0.0001$ \\
\hline $1 \mu \mathrm{g} /$ larvae FF vs. $7.5 \mu \mathrm{g} /$ larvae INJ & -46.67 & -83.32 to -10.01 & $* *$ & 0.0023 \\
\hline $1 \mu \mathrm{g} /$ larvae FF vs. $2.5 \mu \mathrm{g} /$ larvae INJ & -56.67 & -93.32 to -20.01 & $* * * *$ & $<0.0001$ \\
\hline $1 \mu \mathrm{g} /$ larvae FF vs. $1 \mu \mathrm{g} /$ larvae INJ & -46.67 & -83.32 to -10.01 & $* *$ & 0.0023 \\
\hline $1 \mu \mathrm{g} /$ larvae FF vs. $0.5 \mu \mathrm{g} /$ larvae INJ & -43.33 & -79.99 to -6.675 & $* *$ & 0.0069 \\
\hline $1 \mu \mathrm{g} /$ larvae FF vs. 5\% DMSO INJ & -46.67 & -83.32 to -10.01 & $* *$ & 0.0023 \\
\hline $0.5 \mu \mathrm{g} /$ larvae FF vs. Control & -53.33 & -89.99 to -16.68 & $* * *$ & 0.0002 \\
\hline $\begin{array}{l}0.5 \mu \mathrm{g} / \text { larvae FF vs. } 7.5 \mu \mathrm{g} / \text { larvae } \\
\text { INJ }\end{array}$ & -36.67 & -73.32 to -0.008652 & $*$ & 0.0499 \\
\hline $\begin{array}{l}0.5 \mu \mathrm{g} / \text { larvae FF vs. } 2.5 \mu \mathrm{g} / \text { larvae } \\
\text { INJ }\end{array}$ & -46.67 & -83.32 to -10.01 & $* *$ & 0.0023 \\
\hline $0.5 \mu \mathrm{g} /$ larvae FF vs. $1 \mu \mathrm{g} /$ larvae INJ & -36.67 & -73.32 to -0.008652 & $*$ & 0.0499 \\
\hline $0.5 \mu \mathrm{g} /$ larvae FF vs. $5 \%$ DMSO INJ & -36.67 & -73.32 to -0.008652 & $*$ & 0.0499 \\
\hline $5 \%$ DMSO FF vs. Control & -43.33 & -79.99 to -6.675 & $* *$ & 0.0069 \\
\hline $5 \%$ DMSO FF vs. $2.5 \mu \mathrm{g} /$ larvae INJ & -36.67 & -73.32 to -0.008652 & $*$ & 0.0499 \\
\hline Control vs. $5 \mu \mathrm{g} /$ larvae INJ & 40 & 3.342 to 76.66 & $*$ & 0.0195 \\
\hline
\end{tabular}

\begin{tabular}{lllll}
\hline $\mathbf{8}$ days post inoculation & $\begin{array}{l}\text { Mean } \\
\text { Diff. }\end{array}$ & $\mathbf{9 5 . 0 0 \%}$ CI of diff. & Summary & $\begin{array}{l}\text { Adjusted } \\
\text { P Value }\end{array}$ \\
\hline $7.5 \mu \mathrm{g} /$ larvae FF vs. Control & -40 & -76.66 to -3.342 & $*$ & 0.0195 \\
\hline $5 \mu \mathrm{g} / \mathrm{larvae}$ FF vs. Control & -53.33 & -89.99 to -16.68 & $* * *$ & 0.0002 \\
\hline
\end{tabular}




\begin{tabular}{|c|c|c|c|c|}
\hline $5 \mu \mathrm{g} /$ larvae FF vs. $7.5 \mu \mathrm{g} /$ larvae INJ & -43.33 & -79.99 to -6.675 & $* *$ & 0.0069 \\
\hline $5 \mu \mathrm{g} /$ larvae FF vs. $5 \mu \mathrm{g} /$ larvae INJ & -46.67 & -83.32 to -10.01 & $* *$ & 0.0023 \\
\hline $5 \mu \mathrm{g} /$ larvae FF vs. $2.5 \mu \mathrm{g} /$ larvae INJ & -46.67 & -83.32 to -10.01 & $* *$ & 0.0023 \\
\hline $5 \mu \mathrm{g} / \mathrm{larvae}$ FF vs. $1 \mu \mathrm{g} /$ larvae INJ & -40 & -76.66 to -3.342 & $*$ & 0.0195 \\
\hline $5 \mu \mathrm{g} /$ larvae FF vs. $0.5 \mu \mathrm{g} /$ larvae INJ & -36.67 & -73.32 to -0.008652 & $*$ & 0.0499 \\
\hline $5 \mu \mathrm{g} /$ larvae FF vs. $5 \%$ DMSO INJ & -36.67 & -73.32 to -0.008652 & $*$ & 0.0499 \\
\hline $2.5 \mu \mathrm{g} /$ larvae FF vs. Control & -53.33 & -89.99 to -16.68 & $* * *$ & 0.0002 \\
\hline $\begin{array}{l}2.5 \mu \mathrm{g} / \text { larvae FF vs. } 7.5 \mu \mathrm{g} / \text { larvae } \\
\text { INJ }\end{array}$ & -43.33 & -79.99 to -6.675 & $* *$ & 0.0069 \\
\hline $2.5 \mu \mathrm{g} /$ larvae FF vs. $5 \mu \mathrm{g} /$ larvae INJ & -46.67 & -83.32 to -10.01 & $* *$ & 0.0023 \\
\hline $\begin{array}{l}2.5 \mu \mathrm{g} / \text { larvae FF vs. } 2.5 \mu \mathrm{g} / \text { larvae } \\
\text { INJ }\end{array}$ & -46.67 & -83.32 to -10.01 & $* *$ & 0.0023 \\
\hline $2.5 \mu \mathrm{g} /$ larvae FF vs. $1 \mu \mathrm{g} /$ larvae INJ & -40 & -76.66 to -3.342 & $*$ & 0.0195 \\
\hline $\begin{array}{l}2.5 \mu \mathrm{g} / \mathrm{larvae} \text { FF vs. } 0.5 \mu \mathrm{g} / \mathrm{larvae} \\
\text { INJ }\end{array}$ & -36.67 & -73.32 to -0.008652 & $*$ & 0.0499 \\
\hline $2.5 \mu \mathrm{g} /$ larvae FF vs. $5 \%$ DMSO INJ & -36.67 & -73.32 to -0.008652 & $*$ & 0.0499 \\
\hline $1 \mu \mathrm{g} /$ larvae FF vs. Control & -53.33 & -89.99 to -16.68 & $* * *$ & 0.0002 \\
\hline $1 \mu \mathrm{g} / \mathrm{larvae}$ FF vs. $7.5 \mu \mathrm{g} /$ larvae INJ & -43.33 & -79.99 to -6.675 & $* *$ & 0.0069 \\
\hline $1 \mu \mathrm{g} /$ larvae FF vs. $5 \mu \mathrm{g} /$ larvae INJ & -46.67 & -83.32 to -10.01 & $* *$ & 0.0023 \\
\hline $1 \mu \mathrm{g} / \mathrm{larvae}$ FF vs. $2.5 \mu \mathrm{g} /$ larvae INJ & -46.67 & -83.32 to -10.01 & $* *$ & 0.0023 \\
\hline $1 \mu \mathrm{g} / \mathrm{larvae}$ FF vs. $1 \mu \mathrm{g} / \mathrm{larvae}$ INJ & -40 & -76.66 to -3.342 & $*$ & 0.0195 \\
\hline $1 \mu \mathrm{g} / \mathrm{larvae}$ FF vs. $0.5 \mu \mathrm{g} / \mathrm{larvae}$ INJ & -36.67 & -73.32 to -0.008652 & $*$ & 0.0499 \\
\hline $1 \mu \mathrm{g} / \mathrm{larvae}$ FF vs. 5\% DMSO INJ & -36.67 & -73.32 to -0.008652 & $*$ & 0.0499 \\
\hline $0.5 \mu \mathrm{g} /$ larvae FF vs. Control & -46.67 & -83.32 to -10.01 & $* *$ & 0.0023 \\
\hline $\begin{array}{l}0.5 \mu \mathrm{g} / \text { larvae FF vs. } 7.5 \mu \mathrm{g} / \mathrm{larvae} \\
\text { INJ }\end{array}$ & -36.67 & -73.32 to -0.008652 & $*$ & 0.0499 \\
\hline $0.5 \mu \mathrm{g} /$ larvae FF vs. $5 \mu \mathrm{g} /$ larvae INJ & -40 & -76.66 to -3.342 & $*$ & 0.0195 \\
\hline $\begin{array}{l}0.5 \mu \mathrm{g} / \mathrm{larvae} \text { FF vs. } 2.5 \mu \mathrm{g} / \mathrm{larvae} \\
\text { INJ }\end{array}$ & -40 & -76.66 to -3.342 & $*$ & 0.0195 \\
\hline 5\% DMSO FF vs. Control & -36.67 & -73.32 to -0.008652 & $*$ & 0.0499 \\
\hline
\end{tabular}

\begin{tabular}{lllll}
\hline 9 days post inoculation & $\begin{array}{l}\text { Mean } \\
\text { Diff. }\end{array}$ & $\begin{array}{l}\mathbf{9 5 . 0 0 \%} \text { CI of } \\
\text { diff. }\end{array}$ & Summary & $\begin{array}{l}\text { Adjusted } \\
\text { P Value }\end{array}$ \\
\hline $7.5 \mu \mathrm{g} /$ larvae FF vs. Control & -40 & -76.66 to -3.342 & $*$ & 0.0195 \\
\hline $5 \mu \mathrm{g} /$ larvae FF vs. Control & -53.33 & -89.99 to -16.68 & $* * *$ & 0.0002 \\
\hline $5 \mu \mathrm{g} /$ larvae FF vs. $7.5 \mu \mathrm{g} /$ larvae INJ & -46.67 & -83.32 to -10.01 & $* *$ & 0.0023 \\
\hline $5 \mu \mathrm{g} /$ larvae FF vs. $5 \mu \mathrm{g} /$ larvae INJ & -46.67 & -83.32 to -10.01 & $* *$ & 0.0023 \\
\hline $5 \mu \mathrm{g} /$ larvae FF vs. $2.5 \mu \mathrm{g} /$ larvae INJ & -43.33 & -79.99 to -6.675 & $* *$ & 0.0069 \\
\hline $5 \mu \mathrm{g} /$ larvae FF vs. $1 \mu \mathrm{g} /$ larvae INJ & -40 & -76.66 to -3.342 & $*$ & 0.0195 \\
\hline $5 \mu \mathrm{g} /$ larvae FF vs. $0.5 \mu \mathrm{g} /$ larvae INJ & -40 & -76.66 to -3.342 & $*$ & 0.0195 \\
\hline $2.5 \mu \mathrm{g} /$ larvae FF vs. Control & -50 & -86.66 to -13.34 & $* * *$ & 0.0007 \\
\hline $2.5 \mu \mathrm{g} /$ larvae FF vs. $7.5 \mu \mathrm{g} /$ larvae & -43.33 & -79.99 to -6.675 & $* *$ & 0.0069 \\
$\mathrm{INJ}$ & & & & 0.0069 \\
\hline $2.5 \mu \mathrm{g} /$ larvae FF vs. $5 \mu \mathrm{g} /$ larvae INJ & -43.33 & -79.99 to -6.675 & $* *$ & 0.0195 \\
\hline $2.5 \mu \mathrm{g} /$ larvae FF vs. $2.5 \mu \mathrm{g} /$ larvae & -40 & -76.66 to -3.342 & $*$ & \\
$\mathrm{INJ}$ & & & & \\
\hline $2.5 \mu \mathrm{g} /$ larvae FF vs. $1 \mu \mathrm{g} /$ /arvae INJ & -36.67 & -73.32 to - & $*$ & 0.0499 \\
\hline
\end{tabular}




\begin{tabular}{|c|c|c|c|c|}
\hline $\begin{array}{l}2.5 \mu \mathrm{g} / \text { larvae FF vs. } 0.5 \mu \mathrm{g} / \text { larvae } \\
\text { INJ }\end{array}$ & -36.67 & $\begin{array}{l}-73.32 \text { to }- \\
0.008652\end{array}$ & $*$ & 0.0499 \\
\hline $1 \mu \mathrm{g} /$ larvae FF vs. Control & -46.67 & -83.32 to -10.01 & ** & 0.0023 \\
\hline $1 \mu \mathrm{g} /$ larvae FF vs. $7.5 \mu \mathrm{g} /$ larvae INJ & -40 & -76.66 to -3.342 & $*$ & 0.0195 \\
\hline $1 \mu \mathrm{g} /$ larvae FF vs. $5 \mu \mathrm{g} /$ larvae INJ & -40 & -76.66 to -3.342 & $*$ & 0.0195 \\
\hline $1 \mu \mathrm{g} /$ larvae FF vs. $2.5 \mu \mathrm{g} /$ larvae INJ & -36.67 & $\begin{array}{l}-73.32 \text { to }- \\
0.008652\end{array}$ & $*$ & 0.0499 \\
\hline $0.5 \mu \mathrm{g} /$ larvae FF vs. Control & -46.67 & -83.32 to -10.01 & $* *$ & 0.0023 \\
\hline $\begin{array}{l}0.5 \mu \mathrm{g} / \text { larvae FF vs. } 7.5 \mu \mathrm{g} / \text { larvae } \\
\text { INJ }\end{array}$ & -40 & -76.66 to -3.342 & $*$ & 0.0195 \\
\hline $0.5 \mu \mathrm{g} /$ larvae FF vs. $5 \mu \mathrm{g} /$ larvae INJ & -40 & -76.66 to -3.342 & $*$ & 0.0195 \\
\hline $\begin{array}{l}0.5 \mu \mathrm{g} / \text { larvae FF vs. } 2.5 \mu \mathrm{g} / \text { larvae } \\
\text { INJ }\end{array}$ & -36.67 & $\begin{array}{l}-73.32 \text { to }- \\
0.008652\end{array}$ & $*$ & 0.0499 \\
\hline $5 \%$ DMSO FF vs. Control & -36.67 & $\begin{array}{l}-73.32 \text { to }- \\
0.008652\end{array}$ & $*$ & 0.0499 \\
\hline
\end{tabular}

\begin{tabular}{|c|c|c|c|c|}
\hline 10 days post inoculation & $\begin{array}{l}\text { Mean } \\
\text { Diff. }\end{array}$ & $\begin{array}{l}\text { 95.00\% CI of } \\
\text { diff. }\end{array}$ & Summary & $\begin{array}{l}\text { Adjusted } \\
\text { P Value }\end{array}$ \\
\hline $7.5 \mu \mathrm{g} /$ larvae FF vs. Control & -36.67 & $\begin{array}{l}-73.32 \text { to }- \\
0.008652\end{array}$ & $*$ & 0.0499 \\
\hline $5 \mu \mathrm{g} /$ larvae FF vs. Control & -53.33 & -89.99 to -16.68 & $* * *$ & 0.0002 \\
\hline $5 \mu \mathrm{g} /$ larvae FF vs. $7.5 \mu \mathrm{g} /$ larvae INJ & -46.67 & -83.32 to -10.01 & $* *$ & 0.0023 \\
\hline $5 \mu \mathrm{g} /$ larvae FF vs. $5 \mu \mathrm{g} /$ larvae INJ & -46.67 & -83.32 to -10.01 & $* *$ & 0.0023 \\
\hline $5 \mu \mathrm{g} /$ larvae FF vs. $2.5 \mu \mathrm{g} /$ larvae INJ & -46.67 & -83.32 to -10.01 & $* *$ & 0.0023 \\
\hline $5 \mu \mathrm{g} /$ larvae FF vs. $1 \mu \mathrm{g} /$ larvae INJ & -46.67 & -83.32 to -10.01 & $* *$ & 0.0023 \\
\hline $5 \mu \mathrm{g} /$ larvae FF vs. $0.5 \mu \mathrm{g} /$ larvae INJ & -40 & -76.66 to -3.342 & $*$ & 0.0195 \\
\hline $5 \mu \mathrm{g} /$ larvae FF vs. $5 \%$ DMSO INJ & -36.67 & $\begin{array}{l}-73.32 \text { to }- \\
0.008652\end{array}$ & $*$ & 0.0499 \\
\hline $2.5 \mu \mathrm{g} /$ larvae FF vs. Control & -50 & -86.66 to -13.34 & $* * *$ & 0.0007 \\
\hline $\begin{array}{l}2.5 \mu \mathrm{g} / \text { larvae FF vs. } 7.5 \mu \mathrm{g} / \text { larvae } \\
\text { INJ }\end{array}$ & -43.33 & -79.99 to -6.675 & $* *$ & 0.0069 \\
\hline $2.5 \mu \mathrm{g} /$ larvae FF vs. $5 \mu \mathrm{g} /$ larvae INJ & -43.33 & -79.99 to -6.675 & $* *$ & 0.0069 \\
\hline $\begin{array}{l}2.5 \mu \mathrm{g} / \text { larvae FF vs. } 2.5 \mu \mathrm{g} / \text { larvae } \\
\text { INJ }\end{array}$ & -43.33 & -79.99 to -6.675 & $* *$ & 0.0069 \\
\hline $2.5 \mu \mathrm{g} /$ larvae FF vs. $1 \mu \mathrm{g} /$ larvae INJ & -43.33 & -79.99 to -6.675 & $* *$ & 0.0069 \\
\hline $\begin{array}{l}2.5 \mu \mathrm{g} / \text { larvae FF vs. } 0.5 \mu \mathrm{g} / \text { larvae } \\
\text { INJ }\end{array}$ & -36.67 & $\begin{array}{l}-73.32 \text { to }- \\
0.008652\end{array}$ & $*$ & 0.0499 \\
\hline $1 \mu \mathrm{g} /$ larvae FF vs. Control & -36.67 & $\begin{array}{l}-73.32 \text { to }- \\
0.008652\end{array}$ & $*$ & 0.0499 \\
\hline $0.5 \mu \mathrm{g} /$ larvae FF vs. Control & -36.67 & $\begin{array}{l}-73.32 \text { to }- \\
0.008652\end{array}$ & $*$ & 0.0499 \\
\hline
\end{tabular}




\section{Novel use of insect larvae as a substitute model for indomethacin-induced gastric damage

\section{Introduction}

There is a constant need to develop alternative in vivo models to vertebrates for both ethical and financial reasons. The larvae of the greater wax-moth, Galleria mellonella, is one potential alternative that boasts low maintenance costs, thermotolerance to $37^{\circ} \mathrm{C}$, and highthroughput capacity. These larvae are used routinely as a 'surrogate' model for studying drug toxicity and microbial virulence [1, 2]. The incorporation of insect larvae in biomedicine is increasing in popularity, however, there remains a lack of knowledge regarding their use for studying gut pathobiology. Our aim was to assess the suitability of $G$. mellonello as a novel platform for characterising the gastrointestinalside effects of biopharmaceuticals.

\section{Methodology}

We administered the non-steroidal anti-inflammatory drug, indomethacin $[0-7.5$ $\mu \mathrm{g} / \mathrm{larva]}$ to $G$. mellonello via force-feeding and intrahaemocoelic injection (Fig. 1). The toxic side effects were assessed using midgut tissue histopathology and gastric permeability assays - in addition to monitoring survival, development, blood cell (haemocyte) numbers, and detoxification-associated enzyme activities [3].

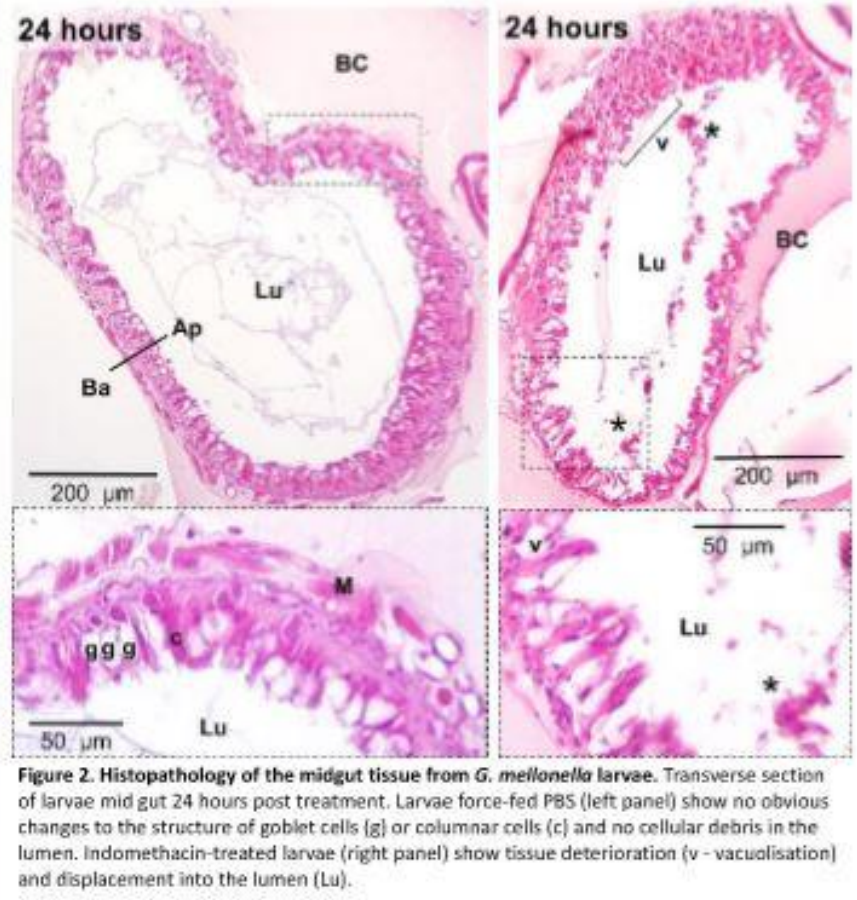

\section{Results/Discussion}

In the absence of indomethacin, the larval midgut is a uniform arrangement of epithelial and goblet cells, with an intact layer of visceral muscle beneath the basement membrane (BM) (Fig. 2). These features are similar to rodent intestines [4]. When larvae were force-fed indomethacin, clear signs of damage such as cell death and blistering were visible. The deterioration of the midgut promoted gut leakiness (Fig. 3). There was a significant increase in the number of latex microspheres in the haemolymph of larvae co-inoculated with indomethacin when compared to those force-fed PBS only.

Indomethacin-induced 'leakiness' of the intestine is well characterised in humans and rodents [5], and herein, we find these symptoms in G. mellonella larvae also. Our novel data support the use of insect larvae as a putative model for gut pathobiology.

Reterences
[1] Senioc, N. L. et at 12011). Journat of Medical Macrobiologry, 60151, 661.

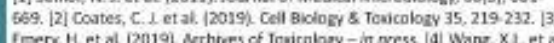
Emenc H. et al [2019]. Nechwes of Towicobogy - in press, [4] Ware, XL et al 6. R. et at. (1993), Oigestine Cisesses and Sciences, 38/31, 417-425

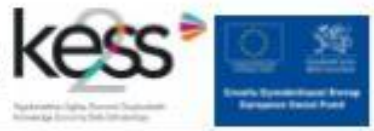

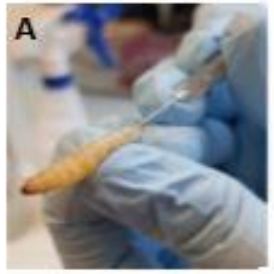

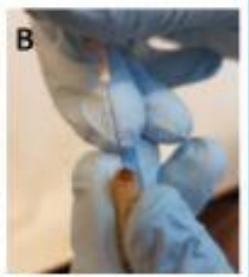

Figure 1. (A) intrahaemocoelic injection and (B) force feeding insect larvae with indomethacin
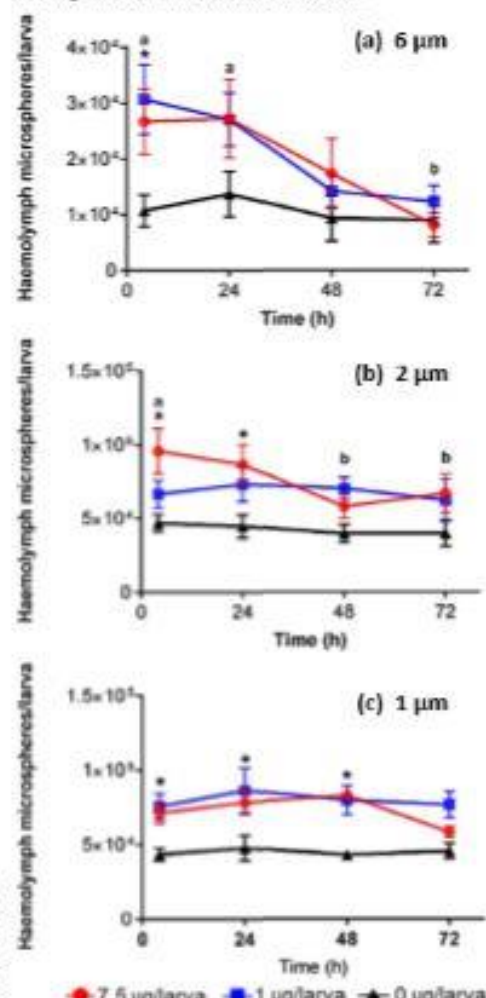

Figure 3. Gut permeability of G. mellanella larvae following force feeding of indomethacin. The number of 6 um $(a), 2$ in into the haemolymph were counted. An asterisk $\left({ }^{\circ}\right)$ and unshared letters $(x, y)$ represent significant differences $(\mathrm{P} \leq 0.05)$.

Acknowledgements

HE. is the recipient of a KESSZ PAO Scholaratig. Wo would the to

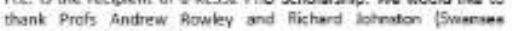
Unversingy for collaborstions

Figure A1. Poster presentation for FRAME. Presented at the FRAME 50th Anniversary Symposium, July 2019 at the University of Nottingham. 


\section{Authorship Declaration}

Paper 1: Indomethacin-induced gut damage in a surrogate insect model, Galleria mellonella Contributes to Chapter 2

The following people and institutions contributed to the publication of work undertaken as part of this thesis:

Author 1: Helena Emery, Swansea University. Lead author, carried out all experiments and data analysis.

Author 2: Professor Richard Johnston, Swansea University. Carried out microtomography scanning and provided methods for microtomography and provided training for 3D drishti.

Author 3: Professor Andrew Rowley, Swansea University. Provided training for histology, blind screening of histological slides and contributed to histology analysis.

Author 4: Dr Christopher Coates, Swansea University. Primary investigator; conceived the study, data analysis and figure preparation, co-wrote the manuscript, revised the manuscript.

We the undersigned agree with the above stated "proportion of work undertaken" for each of the above published peer-reviewed manuscripts contributing to this thesis:

Author 1: H Emery 24/08/2020

Author 2: Professor Richard Johnston 24/08/2020

Author 3: Professor Andrew Rowley 24/08/2020

Author 4: Dr Christopher Coates 24/08/2020 


\title{
Indomethacin-induced gut damage in a surrogate insect model, Galleria mellonella
}

\author{
Helena Emery' - Richard Johnston ${ }^{2}$ - Andrew F. Rowley' - Christopher J, Coates'C
}

Becelved: 1 May 2019/Accepted: 26 June 2019/ Published oniline: 3 July 2019

o The Author(s) 2019

\begin{abstract}
Indomethacin is a non-steroidal anti-inflammatory drug that causes gastric ulceration and increased 'leakiness' in rat models, and is used routinely as a toxicology assay to screen novel compounds for repair and restitution properties. We set out to establish conditions for indomethacin-induced gut damage in wax-moth (Galleria mellonella) larvac with a view to reducing the need for rodents in such experimentation. We administered indomethacin $(0.5-7.5 \mu \mathrm{g} / \mathrm{larva} ; 2-30 \mathrm{mg} / \mathrm{kg})$ to $G$. mellonella via intrahaemocoelic injection and gavage (force-feeding) and monitored survival and development, blood cell (haemocyte) numbers, and changes in gut permeability. Increased levels of gut leakiness were observed within the first 4 to $24 \mathrm{~h}$ by tracking fluorescent microspheres in the faeces and hacmolymph (blood equivalent). Additionally, we recorded varying levels of tissue damage in histological sections of the insect midgut, including epithelial sloughing and cell necrosis. Degeneration of the midgut was accompanied by significant increases in detoxification-associated activities (superoxide dismutase and glutathione-S-transferase). Herein, we present the first evidence that $G$. mellonella larvae force-fed indomethacin display broad symptoms of gastric damage similar to their rodent counterparts.
\end{abstract}

Keywords Innate immunity - Gastrointestinal damage - Histopathology - Rodent models - Gavage - Gut leakiness . Eicosanoids

\section{Introduction}

When considering more carefully our use of vertebrates in disease and toxicology experimentation, there is constant noed to develop alternative model systems in vitro, in vivo or in silico, One such in vivo alternative is the larvae of the greater wax-moth, Galleria mellonella. These insects are now used widespread as 'mini-hosts' for the investigation of microbial pathogenicity (Mowlds et al. 2010; Kloezen et al. 2015; Lim et al. 2018; Cools et al. 2019), screening of xenobiotics/toxins (Allegra et al. 2018; Coates et al. 2019) and functional

Flectronic supplementary material The online version of this article (https://doi.org/10.1007/s00204-019-02508-4) contains supplementary material, which is available to authorized users.

D. Christopher J, Costes

c.j.costes faswansea.ac, Lk

1 Department of Biosciences, College of Science, Swansea University, Swansea SA2 SPP, Wales, UK

2 Advanced Imaging Materials (AIM) Facility, Materials Research Centre, College of Fingineering, Swansea University, Swansea SA1 8HN, Wales, UK characterisation of virulence factors (Altincicek et al. 2007 Champion et al. 2016). Larvae of $\boldsymbol{G}$. mellonella are designated a non-animal technology (Allegra et al. 2018), which means there are fewer ethical restrictions and regulations compared to vertebrates. Additionally, practical advantages include low maintenance costs, thermal tolerance to $37^{\circ} \mathrm{C}$. case of use (accurate dosages) and high turnower - results can be obtained within $72 \mathrm{~h}$ in contrast to vertebrates. Although $G$. mellonella have been used to study the infectivity of gut pathogens such as Campylobacter jejuni (Senior et al. 2011), Listeria monocytogenes (Mukherjec et al. 2013a), Vibrio spp. (Wagley et al. 2018), Shigella spp. (Barnoy et al. 2017) and Salmonella enterica (Card et al. 2016), there remains a distinct lack of information regarding the alimentary canal of this insect and its role in pathogenesis.

From mouth to rectum, the digestive system of lepidopteran larvae (like $G$. mellonella) consists of three distinct regions: the foregut (stomatodacum), midgut (mesenteron) and hindgut (proctodacum) (Engel and Moran 2013; Linser and Dinglasan 2014). The midgut is the primary site of digestion and absorption in many insects, and importantly, it lacks the exoskeletal/chitin lining seen in the fore- and 
hind-guts. The basic tissue architecture of the midgut is similar to those found in the human intestine, such as epithelial arrangements of columnar cells and smooth septate junctions that control permeability - analogous to tight junctions (Green et al. 1980). The insect peritrophic matrix is the functional equivalent to the mammalian mucus layer, which acts as a barrier for the epithelial cells and impedes pathogen movement into the body cavity (i.e., the haemocoel) (Camphell et al. 2008; Kuraishi et al. 2011). Moreover, some microbial communities characterised in the midgut of $G$. mellonella are similar to those found in crypts of the human intestine (Mukherjee et al. 2013b).

Non-steroidal anti-inflammatory drugs (NSAIDs) are used extensively to alleviate pain by inhibiting the activities of cyclooxygenase isozymes (COX1, COX2), yet known side effects manifest in gastrointestinal injury (Playford et al. 1999; Brune and Patrignani 2015). In particular, indomethacin causes ulceration by inducing apoptosis, reducing gastric blood flow and activating innate immune cells (neutrophils), which all contribute to mucosal secretion, maintenance and defence (Marchbank et al. 2011; Matsui et al. 2011). Gastric complications arising from indomethacin exposure bave been developed into a standard rodent restraint/ulcer assay to screen novel compounds and health food supplements for putative therapeutic properties - tissue repair and restitution (Aguwa 1985; Playford et al. 1999, 2001; Mahmood et al. 2007). The adverse effects of indomethacin, notably inflammation, permeability and REDOX imbalance, have been studied thoroughly in humans and rodents (Basivireddy et al. 2003; Bjarnason and Takeuchi 2009; Sigthorsson et al. 2000; Perron el al. 2013). Therefore, we set out to interrogate the effects of indomethacin on the alimentary canal of $\boldsymbol{G}$, mellonella. First, we utilised two inoculation methods (intrahaemocoelic injection and gavage) to assess the relative toxicity of indomethacin in insect larvae across

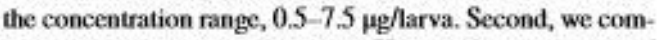
bined histopathology screening, X-ray microtomography/ microscopy (XRM) and enzyme activities to assess the integrity of the midgut tissues in the absence and presence of this NSAID. Indomethacin treatments were established by extrapolating from Marchbank et al. (2011), wherein a dose of $20 \mathrm{mg} / \mathrm{kg}$ was administered to induce gastric damage in rats - the equivalent being $5.0 \mu \mathrm{\mu g} / \mathrm{larva}$.

\section{Materlals and methods}

\section{Reagents}

Unless stated otherwise, all chemicals and reagents were sourced from Sigma Aldrich (Dorset UK) in their purest form listed. Green and blue fluorescent (latex) microspheres ranging from 0.5 to $6 \mu \mathrm{m}$ in diameter were purchased from Polysciences, Inc. (Fluoresbite ${ }^{\infty}$ ). Stock solutions of indomethacin were prepared in $5 \%$ dimethyl sulfoxide (DMSO; $\mathrm{v} / \mathrm{v}$ ) and diluted in filter-sterilized $(0.2 \mu \mathrm{m})$ phosphate-buffered saline (PBS) $\mathrm{pH} 7.4$

\section{Insects}

Larvae of $G$, mellonella (final instar) were purchased from Livefoods Direet Ltd (Sheffield UK). Upon arrival, each larva was inspected for signs of damage, infection and melanisation. Healthy larvae weighing between $0.25 \mathrm{~g}$ and $0.35 \mathrm{~g}$ were retained and stored at $15^{\circ} \mathrm{C}$ in the dark for no more than 7 days.

\section{Larval survival and pupation studies}

Larvae were assigned randomly to each treatment $(n=10$ per replicate) and placed in $90 \mathrm{~mm}$ petri dishes lined with Whatman filter paper and wood shavings from the commercial supplier. Indomethacin was administered by intrahaemocoelic injection (INJ) or force feeding (FF; gavage) using a sterile 27 -gauge hypodermic needle across the concentration range, 0 to $7.5 \mu \mathrm{g} / \mathrm{larva}$. The volume of each inoculum was standardised to $10 \mu \mathrm{L}$. The negative control consisted of PBS pH 7.4 containing 5\% dimethyl sulfoxide (DMSO). Post-inoculation, larvae were incubated at $30^{\circ} \mathrm{C}$ and assessed for mortality (response to prodding) and development (pupation events) for up to 10 days.

\section{Haemocyte counts and viability}

Larvae treated with indomethacin, PBS (containing $5 \%$ DMSO), or untreated (i.e., no inoculation) were assessed for haemocyte numbers within 4-and 72-hours post-inoculation. Insects were chilled on ice for $\sim 3 \mathrm{~min}$ prior to injection of $100 \mu \mathrm{L}$ anticoagulant $(15 \mathrm{mM} \mathrm{NaCl}, 155 \mathrm{mM}$ trisodium citrate, $30 \mathrm{mM}$ citric acid, $20 \mathrm{mM}$ disodium EDTA, pH 5.5). Larvae containing anti-coagulant were placed back on ice for $\mathbf{2} \mathrm{min}$ prior to piercing the integument above the head using a 27 -gauge hypodermic needle. Hacmolymph was collected into pre-chilled, sterile microcentrifuge tubes. For haemocyte viability, haemolymph was extracted in the absence of anticoagulant and mixed in a ratio of 1.5 with $0.4 \%$ trypan blue ( $w / v$ in PBS) and incubated at room temperature for 1 to $2 \mathrm{~min}$. In all cases, haemolymph samples were applied to an improved Neubauer hacmocytometer for cell enumeration using brightfield optics of a compound microscope. Two technical replicates were performed per sample. Haemocytes were considered dead when the cytoplasm stained positively for trypan-blue (Strober 2015). 


\section{Detoxification-associated assays}

Haemolymph was extracted (as stated above) and pooled from three larvae at $4,24,48$ and $72 \mathrm{~h}$ after being forcefed indomethacin. Haemolymph was centrifuged at $500 \times \mathrm{g}$ for $5 \mathrm{~min}$ at $4^{\circ} \mathrm{C}$ to pellet the haemocytes. Approximately, $2.5 \times 10^{5}$ haemocytes from each treatment/time point were added to the lysis buffer $(50 \mathrm{mM}$ potassium phosphate, $2 \mathrm{mM}$ EDTA, $1 \mathrm{mM}$ DTT and a proteinase inhibitor cocktail (Roche cOmplete ${ }^{\mathrm{TM}}$ Mini kit)) and centrifuged at $14,000 \times g$ for $10 \mathrm{~min}$ at $4^{\circ} \mathrm{C}$. Supernatants were retained and stored at $-80^{\circ} \mathrm{C}$. Midgut tissues from three larvac per treatment/time point were dissected out and washed in PBS. Approximately, $30 \mathrm{mg}$ of tissue was placed in $1 \mathrm{ml}$ lysis buffer and homogenised for $\sim 1 \mathrm{~min}$ (using a borosilicate mini glass homogeniser) prior to centrifugation and storage as described above. Protein concentrations of haemolymph and midgut samples were determined using the Biuret method with bovine serum albumin (BSA, $0-20 \mathrm{mg} / \mathrm{ml}$ ) as a standard (Gornall et al. 1949).

\section{Superoxide dismutase (SOD) activity}

SOD activity (EC 1.15.1.1) was determined using the method described by Dubovskiy et al. (2008). Briefly, $80 \mu \mathrm{L}$ of sample (haemolymph or homogenised midgut) was mixed with reaction solution ( $500 \mu \mathrm{L}$ PBS containing $70 \mu \mathrm{M}$ NBT, $125 \mu \mathrm{M}$ xanthine), followed by the addition of $20 \mu \mathrm{L}$ xanthine oxidase solution ( $5 \mathrm{mg} \mathrm{BSA}, 15 \mu \mathrm{L}$ xanthine oxidase (20 units/mL) per ml PBS), and incubated for $20 \mathrm{~min}$ at $28^{\circ} \mathrm{C}$. Total assay volume was $600 \mu \mathrm{L}$. Xanthine oxidase catalyses xanthine to produce superoxide anions $\left(\mathrm{O}_{2}^{-}\right)$, which reduce NBT to a formazan dye. The inhibition of NBT reduction is indicative of SOD activity, which is monitored spectrophotometrically at $\$ 60 \mathrm{~nm}$. SOD activity is presented as the increase in absorbance $(560 \mathrm{~nm})$ per min per mg protein.

\section{Glutathione S-transferase (GST) activity}

GST activity (EC 2.5.1.18) was determined using the method described by Dubovskiy et al. (2008). Briefly, $20 \mu \mathrm{L}$ of sample was mixed with $500 \mu \mathrm{L}$ GST assay solution (1 mM glutathione, $1 \mathrm{mM}$ 1-chloro-2,4-dinitrobenzene (DNCB) dissolved in PBS) and incubated at $28^{\circ} \mathrm{C}$ for $5 \mathrm{~min}$. The reaction of DNCB and glutathione is catalysed by GST, producing 5-(2,4-dinitrophenyl)-glutathionedetectable at $340 \mathrm{~nm}$. GST activity is presented as the increase in absorbance $(340 \mathrm{~nm}$ ) per min per $\mathrm{mg}$ protein.

\section{Gut permeability assessments}

Fluorescently tagged, carboxylate-modified latex microspheres $\left(1 \times 10^{6}\right)$ of $0.5 \mu \mathrm{m}, 1 \mu \mathrm{m}, 2 \mu \mathrm{m}$ and $6 \mu \mathrm{m}$ in diameter were resuspended in $10 \mu \mathrm{L}$ PBS (control) or $10 \mu \mathrm{L}$ indomethacin solution ( 1 or $7.5 \mu \mathrm{g}$ dose) to form co-inoculates (thereby avoiding piercing an insect twice). Larvae were force-fed $10 \mu \mathrm{L}$ of each co-inoculate and incubated at $30^{\circ} \mathrm{C}$ until haemolymph and faeces were collected from each treatment group at $4,24,48$ and $72 \mathrm{~h}$. Faeces were homogenised in $1 \mathrm{~mL}$ PBS $\mathrm{pH}$ 7.4. The number of microspheres in the haemolymph/faeces was enumerated using a fluorescent microscope (Olympus Bx43f).

\section{Histopathology of the insect alimentary canal}

Larvae force-fed indomethacin (7.5 $\mu \mathrm{g}$ per insect) or PBS (negative control) were killed at $4,24,48$ and $72 \mathrm{~h}$ postinoculation by intrahaemocoelic injection of $100 \mu \mathrm{L} 10 \%$ buffered formalin, immediately prior to submersion in the same solution for $24 \mathrm{~h}$. Larvae were cut into three sections, head, middle and posterior (anus), and stored in $70 \%$ ethanol prior to wax embedding. Briefly, each sample was dehydrated using an ethanol series, $70 \%, 80 \%$ and $90 \%$ for $1 \mathrm{~h}$ each, followed by $3 \times 1$ h $100 \%$ ethanol washes. Dehydrated samples were washed twice in HistoClear or HistoChoice (Sigma Aldrich) for $1 \mathrm{~h}$ each to remove any remaining residues. Samples were resuspended in 50:50 HistoChoice: paraffin wax for $1 \mathrm{~h}$ prior to complete wax embedding. Embedded samples were cut into $-6 \mu \mathrm{m}$ sections using a microtome, adhered to glass slides with egg albumin solution $(\sim 1 \% \mathrm{w} / \mathrm{v})$ and dried for $24 \mathrm{~h}$ before staining. Loaded slides were stained using Cole's hacmatoxylin and eosin (see Supp. Materials for further details).

\section{X-ray microtomography/microscopy of Galleria mellonella}

Larvae force-fed PBS were killed at $72 \mathrm{~h}$ and fixed with $10 \%$ formalin for $30 \mathrm{~h}$, followed by dehydration in $100 \%$ ethanol for 24 b. Samples were submerged in Lugol's iodine (PRO.LAB Diagnostics) for 2 weeks and washed with $70 \%$ ethanol prior to microscopy. Insect samples were analysed via $X$-ray microscopy (XRM) using a lab-based Zeiss Xradia 520 (Carl Zeiss XRM, Pleasanton, CA, USA) $X$-ray microscope attached to a CCD detector system with scintillator-coupled visible light optics, and tungsten transmission target. The specimen was placed in a plastic screw-top microcentrifuge tube and submerged in $75 \%$ ethanol to prevent the tissues from drying out. To achieve a higher resolution over the entire organism, the insect was imaged along its $\sim 25 \mathrm{~mm}$ length at high resolution, using an overlap-scan and stitching procedure including five 
individual scans, with $44 \%$ overlap between each scan. An $\mathrm{X}$-ray tube voltage of $80 \mathrm{kV}$ and a tube current of $87 \mu \mathrm{A}$ were used with an exposure of $1000 \mathrm{~ms}$ and a total of 3201 projections. An objective lens giving an optical magnification of 0.4 was selected with binning set to 2 , producing an isotropic voxel (3-D pixel) size of $8.5635 \mu \mathrm{m}$. The individual tomograms were reconstructed from 2-D projections and stitched into a single large volume using a Zeiss commercial software package (XMReconstructor, Carl Zeiss), utilising a cone-beam reconstruction algorithm based on filtered back-projection. XMReconstructor was also used to produce 2-D grey seale slices for subsequent analysis. Drishti and Drishti Paint software V2.6.4. (Limaye 2012) was employed to highlight regions of interest and digitally segment the alimentary canal.

\section{Data handling}

All data were gathered from experiments carried out on at least three independent occasions and are represented by mean values \pm standard error (see individual figure legends for sample sizes). D'Agostino and Pearson normality tests were performed, and if necessary, data were squareroot transformed. Results from survival studies were analysed using the log-rank (Mantel-Cox) test for comparing curves, whereas larval pupation levels, total haemocyte counts, cell deaths, and faecal/haemolymph microsphere loads were analysed using 2-or 3-way ANOVA with Tukey's multiple comparison tests in GraphPad Prism v7. A Pearson's Correlation test was employed to assess the relationship between microsphere size $(0.5-6 \mu \mathrm{m})$ and their presence in the haemolymph of control larvae. Enzyme assays, GST and SOD, were analysed in ' $R$ ' studio using General Liner Hypotheses (ghlt). In all cases, differences were considered significant when $P \leq 0.05$.

Histology slides were single-blind assessed using paired treatment $(n=13)$ and control $(n=13)$ samples from 4 to 72 h. A grading system (1-4) was used to categorise the extent of tissue damage(s) or lack thereof (sce Supp. Materials for descriptions). Drishti and Drishti Paint V2.6.4. (Limaye 2012) and ImageJ (Abràmoff et al. 2004) softwarc were used to process/present microscopy findings. Images were adjusted for colour balance and contrast/brightness only.

\section{Results}

\section{Evaluating the relative toxicity of indomethacin on Galleria mellonella}

\section{Survival}

Intrahaemocoelic injection of indomethacin had no measurable negative impacts on larval survival across the concentration range $0.5-7.5 \mu \mathrm{g} / \mathrm{larva}$ ( $0 \%$ mortality), which is the equivalent to $2-30 \mathrm{mg} / \mathrm{kg}$ in rodents. Oral administration of indomethacin (force-feeding) in excess of $2.5 \mu$ /larva led to a 7-10\% decline in survival within $72 \mathrm{~b}$ (Fig. 1) but was not found to be statistically significant overall (log-rank (Mantel-Cox) test; $X^{2}(6)=11.71, P=0.0688$ ). Further inspection of the survival curves revealed no statistical differences between the PBS/DMSO controls and either $2.5 \mu \mathrm{g} / \mathrm{larva}$ $\left(X^{2}(1)=2.034, P=0.1538\right)$ or 5-7.5 $\mu / /$ larva $\left(X^{2}(1)=3.105\right.$, $P=0.0780$ )

\section{Development}

Approximately $87 \%$ of untreated larvae transitioned into pupae after 6 days incubation at $30^{\circ} \mathrm{C}$ (experimental temperature) increasing to $97 \%$ by day 10 (Fig. 2). No more than $40 \%$ of $G$. mellonella force-fed indomethacin (0.5-7.5 $\mu \mathrm{g} /$

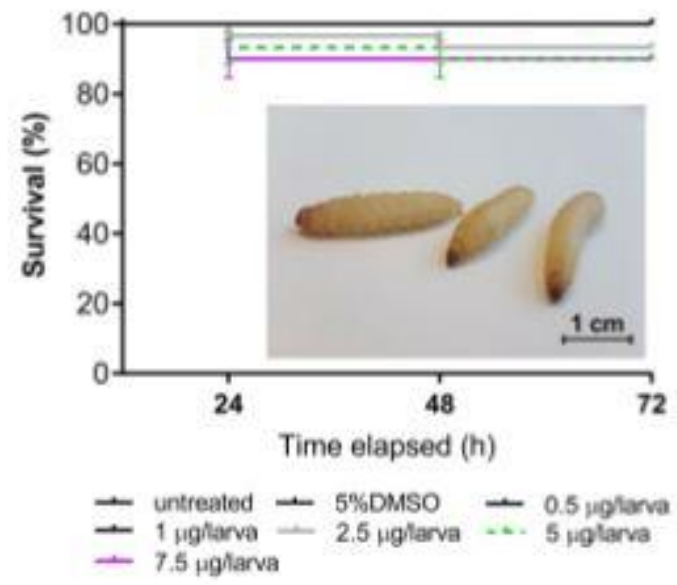

Fig. 1 Survival of Galleria mellonella larvae following force-feeding (gavape) of indomethacin, 0.5-7.5 $\mu$ /arva. Post inoculation, larvac were maintsined in darkness at $30^{\circ} \mathrm{C}$ for $72 \mathrm{~h}$. I arvae that were unresponsive to being rolled over or prodded were considered dead. val ues are expressed as mean \pm SE $(n=30$ per treatment, 210 in total). Inset: three healthy (untreated) $G$. mellonella larvae. It should be noted that direct injection of indomethacin or PBS with 5\% DMSO (control) did not lead to any insect mortality 

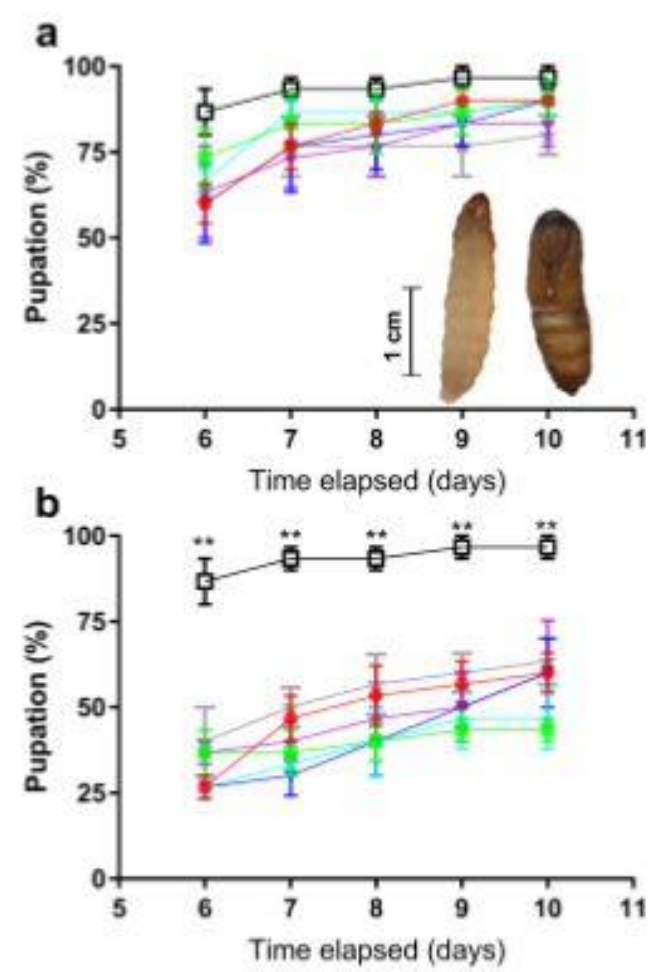

$+0.5 \mu \mathrm{g} * 5 \mu \mathrm{g}-7.5 \mu \mathrm{g}$
$+5 \%$ DMSO $* 2.5 \mu \mathrm{g}$
+ untreated $* 1 \mu \mathrm{g}$

Fi. 2 Development (pupation) of Galleria mellonella larvae following inoculation with indomethacin, $0.5-7.5 \mu$ /larva. I arvae reseived indomethacin via intrahaemocoelic injection (a) or force foeding (b) and were maintained subsexpesilly in darkness at $30{ }^{\circ} \mathrm{C}$ for 10 days. The number of larvae undergoing pupation was recorded. Inset (a): typical appearances of a healthy larva (left image) and a moth pupa (fight imase). Values are expressed as mean + SE $(n=30$ per treatment, 390 in total across both inoculation methods). Symbol: $* * P<0.01$ when comparing untreated to all other treatments at the respective time points. The negative control consisted of force feeding or injecting the insect with PBS containing 5\% DMSO

larva) or PBS ( $+5 \%$ DMSO, absent indomethacin) were observed pupating at day 6 . By day 10 , numbers of pupac increased to $80-90 \%$ for injected larvac (Fig. 2a) and $\leq 63 \%$ for force-fed larvae (Fig. 2b). Using a three-way ANOVA, we determined time (Days 6 and 10) and inoculation method (FF and INJ) to account for $38 \%(F(1,24)=50.02$ $P<0.0001)$ and $41 \%(F(1,24)=54.19, P<0.0001)$ of the variation, respectively. Less than $0.5 \%$ of the variation within the data can be attributed to treatment: PBS + 5\%DMSO (negative control), $1 \mu \mathrm{g}$ (low dose) and $7.5 \mu \mathrm{g}$ (high dose) indomethacin per larva $(F(2,24)=0.0833, P=0.92)$. Tukey's multiple comparison (post hoc) tests revealed statistical differences between the untreated insects and those force-fed indomethacin or PBS only (Fig. 2b), with no difference detected between mean values of injected insects at any time-point. These data suggest the route of administration alone can delay the onset of pupation, and it is unlikely due to indomethacin exposure.

\section{Circulating blood cells (haemocytes)}

To investigate further any potential non-target effects of indomethacin, we monitored immune cell (hacmocyte) numbers and levels of cell death within the insect haemolymph (i.e., blood). Total hacmocyte counts varied litule in untreated and PBS ( $+5 \%$ DMSO) control insects over the 72 -h experimental period, $2.5-3 \times 10^{6} / \mathrm{mL}$ and $2.7-3.7 \times 10^{6} \%$ $\mathrm{mL}$, respectively (Fig. 3). At $24 \mathrm{~h}$ post-inoculation, all concentrations of indomethacin injected into the haemolymph led to substantial increases in baemocyte numbers (up to $\left.5 \times 10^{6} / \mathrm{mL}\right)$ compared to the untreated/control $\left(<2.8 \times 10^{6} /\right.$ mL; Fig. 3a). Force-feeding indomethacin led to increased circulating haemocyte numbers at the highest dose of $7.5 \mu \mathrm{g} /$ larva only $\left(4.4 \times 10^{6} / \mathrm{mL}\right.$; Fig. 3 b). In all cases, haemocyte numbers returned to control levels by 48 h. A 2-way ANOVA revealed treatment to account for $15-19 \%$ of the variation within the data $(\mathrm{INJ}-\boldsymbol{F}(4,40)=4.488, P=0.0043$; FF $F(4,40)=2.988, P=0.030)$. Time accounted for $\angle 5 \%$ of the variation in force-fed insects (Time, $F(3,40)=1.033$, $\boldsymbol{P}=0.3884)$ but $\sim 30 \%$ for injected insects (Time, $\boldsymbol{F}(3$, $40)=11.84, P<0.0001)$. These data likely reflect the unimpeded exposure of baemocytes to indomethacin when it is administered directly into the haemocoel (body cavity), whereas the gut presents a natural barrier when administered via force-feeding.

Concerning cytotoxicity, proportions of hacmocytes staining positively for trypan-blue (i.e., dead or dying) ranged from 7 to $12 \%$ for the duration of the experimentregardless of the inoculation method used or dose of indomethacin (Fig. 3; Supp. Table 1).

\section{Characterising the effects of indomethacin on the midgut of Galleria mellonella}

\section{Alimentary canal mapping}

Using X-ray microtomography, we mapped the alimentary canal and integumentary musculature of $G$. mellonella larvae $(n=3)$ over their entire $\sim 25 \mathrm{~mm}$ lengths with a resolution of $8.6 \mu \mathrm{m}$ (Fig. $4 \mathrm{a}-\mathrm{c}$ ). Like most insects, the alimentary canal can be sub-categorised into three regions: foregut, midgut and hindgut (Fig. $4 \mathrm{~b}$ ). The midgut is 

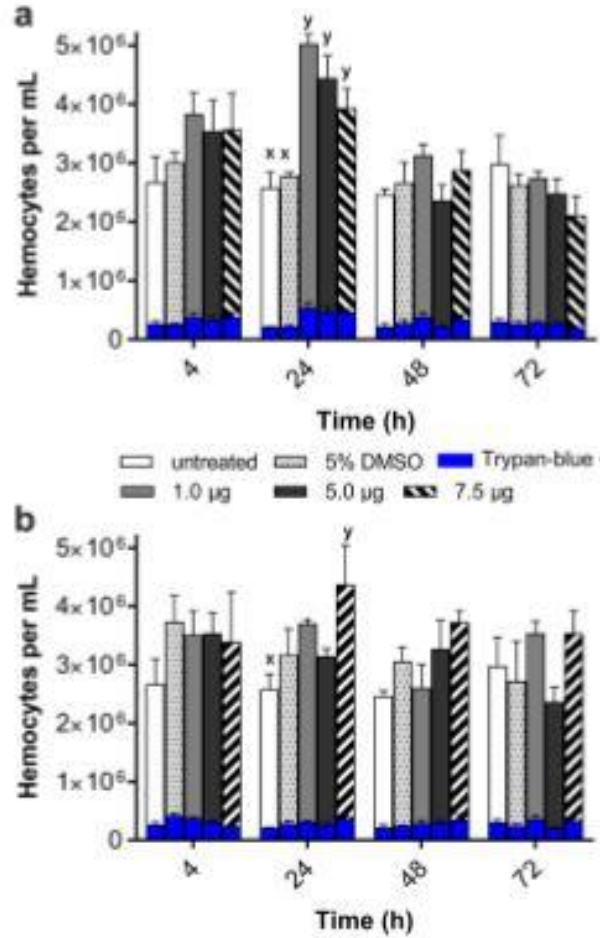

Hg. 3 Haemocyte responses of Galleria mellonella larvae following noculation of indomethacin, 1-75 ye/larva. Larvae feceived indomethacin via intrahaemocoelic injection (a) or force feeding (b) and were maintained subsecuently in darkness at 30 " $\mathrm{C}$ for 3 days. Total haemocyte counts were performed, and haemocyte viability was determined using the trypan blue exclusion assay (desal cell numbers are represented by the blue bars in $\mathbf{a}$ and $\mathbf{b}$ ). Values are presented as the mean $+\mathrm{SE}(n=36$ per treatment, 324 in total across both inoculation methods). Unshared letters $(\boldsymbol{x}, \mathrm{y})$ represent sienificant differences $(P \leq 0.05)$ determined by They's multiple comparison tests. The negative control coosisted of force feeding or injecting the insect with PBS containing 5\% DMSO (colour ngure online)

distinguishable from the fore- and hind-guts due to the absence of a cuticle lining, and our measurements indicate that the midgut tissues make-up $>50 \%$ of the alimentary canal $(n=3)$ in this species. The midgut is arranged into pleats or folds of columnar epithelial cells and goblet cells that produce and maintain the peritrophic matrix (functional equivalent to the human mucus layer). Visceral (striated) muscles surround the gut tissues (anchoring the cellular arrangements in place) and represent the final layer between the gut contents and the haemolymph.

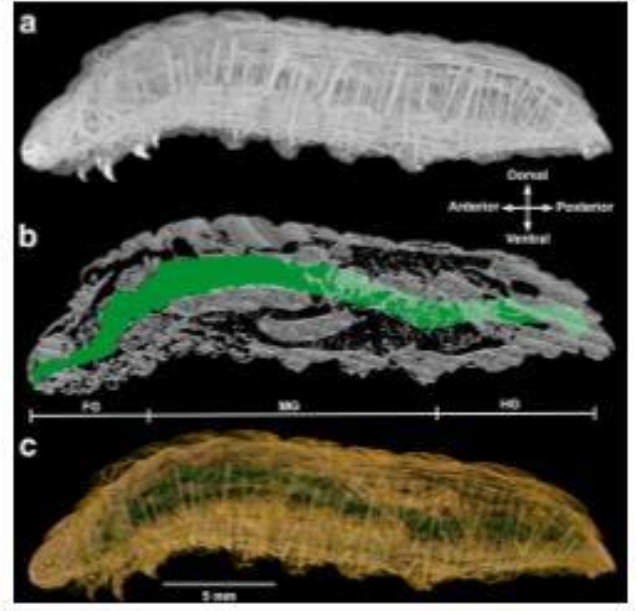

Fig. 4 X-ray microtomography of Galleria mellonella, a 3-dimen sional rexder of a representative insect larva stained with Lugol's iodine. Striated muscle fibres are distributed throughout the insectmuscles are altached to the integument and run through the cuticle tayers. b The entire alimentary canal of the insect larva was highlighted by manually inspecting and colouring 1014 stices using Drishti software (L imaye 2012). FG, foregut; MG, midgut; HD, hindgut. e Combined 3-dimensional render with the inlegument architec ture coloured yellow and the alimentary canal coloured green

\section{Gut permeability}

Indomethacin and NSAIDs broadly increase gastrointestinal 'leakiness' in humans and rodents, with tissue damage located in the small intestine and colon (Smecuol et al. 2001). In order to test whether indomethacin caused similar pathological symptoms in the insect alimentary canal, we force-fed $G$. mellonella inocula containing indomethacin ( 0 , 1 or $7.5 \mu \mathrm{g} /$ larva) and a selection of microspheres, 0.5-6 $\mu \mathrm{m}$ in diameter (Fig. 5). In the absence of indomethacin (PBS $[+5 \%$ DMSO] only), the majority of $6 \mu \mathrm{m}$ and $2 \mu \mathrm{m}$ spheres (36-58\%) made their way down the alimentary canal and were defecated within 24 h (Fig. 5a, b), whereas $1 \mu \mathrm{m}$ spheres $(33 \%)$ were released later at $48 \mathrm{~h}$ (Fig. 5c). The presence of indomethacin ( 1 or $7.5 \mu \mathrm{g} / \mathrm{larva}$ ) led to substantial increases in the number of microspheres $(0.5-6 \mu \mathrm{m})$ detected in the haemolymph (blood) and concomitantly fewer were recovered from faeces. At 4 and $24 \mathrm{~h}$ post-indomethacin treatment, $2.7-3.1 \times 10^{4}(6 \mu \mathrm{m})$ spheres leaked from the gut into the hacmolymph in comparison to the control, $1.0-1.4 \times 10^{4}$ (Fig. Sa). Notably, the presence of microspheres in the hacmolymph was recognised by phagocytic haemocytes and subsequently internalised (Supp. Figure 1). Independent of microsphere size, indomethacin exacerbated 
Fig. 6 Gross histopatbology of the midgut tissues from control and indomethacin-treated

Galleria mellonella Photo-

micrographs depict transverse sections of the midgut archillecture at $4,24,48$ and $72 \mathrm{~h}$ anter force-feeding PBS (controtupper panels) or indomethacin (7.5 up-lower panels). Black boures (broken lines) are boxes (broken lines) are used to highlight mag of interest. Ap, apical; Ba.

basolaleral; BB, brush border; $\mathrm{BC}$, body cavity; bl, blebbing/ blistering of the cells; BM.

basement membrane; $c$, colum nar epithetial ceil; g. goblet

cell Lu, lamen; $M$, muscle; $v$. vacuole. An asterisk (*) denotes cellular damage and displacement into the lumen, and black arrows point to haemocytes (immune cells) within the body cavily (baemocoel). The negative control consisted of force feeding the insect with PBS

$(+5 \%$ DMSO $)$ in the abseace of indomethacin (0 He/sarva)
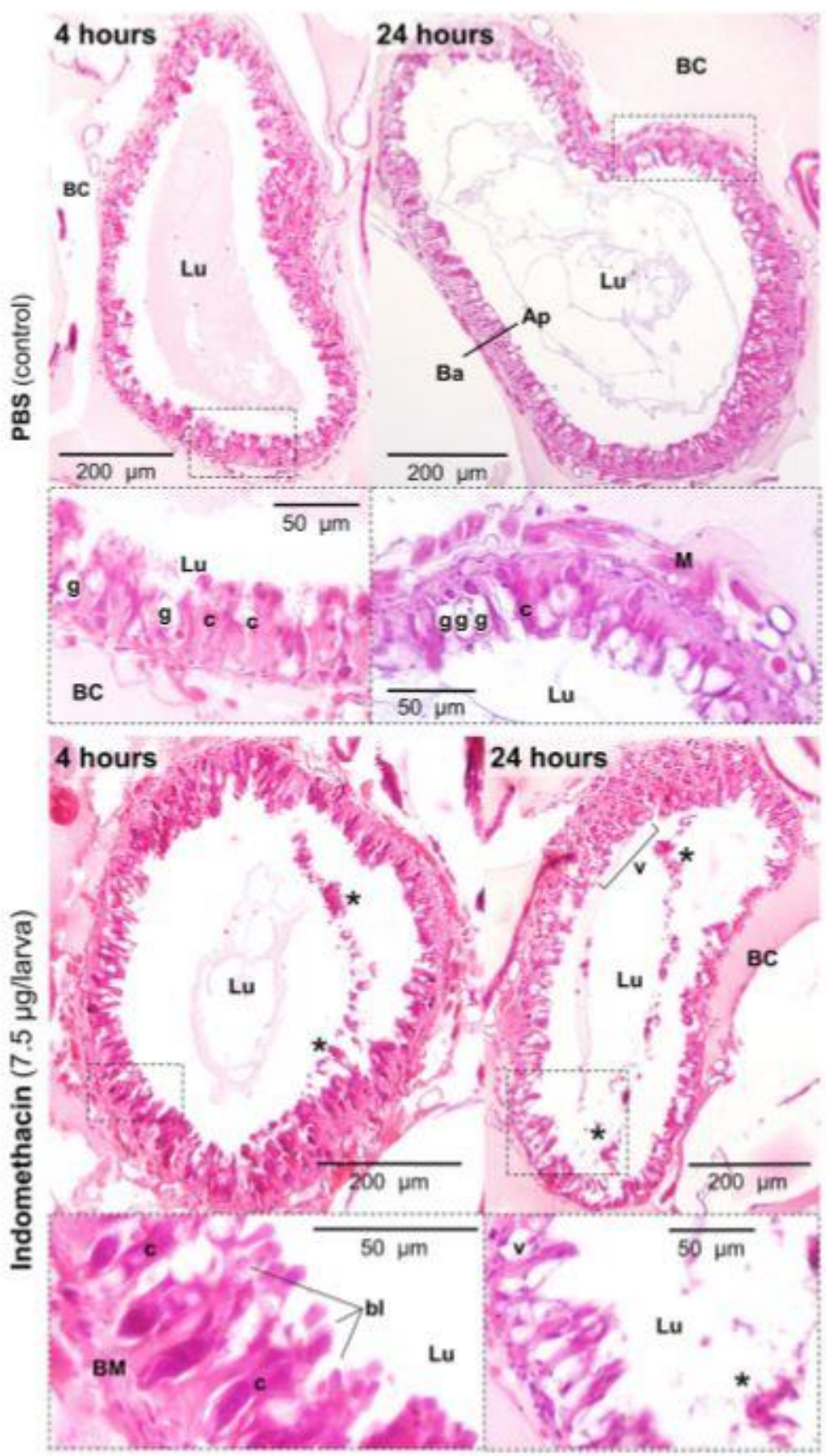

Q) Springer 

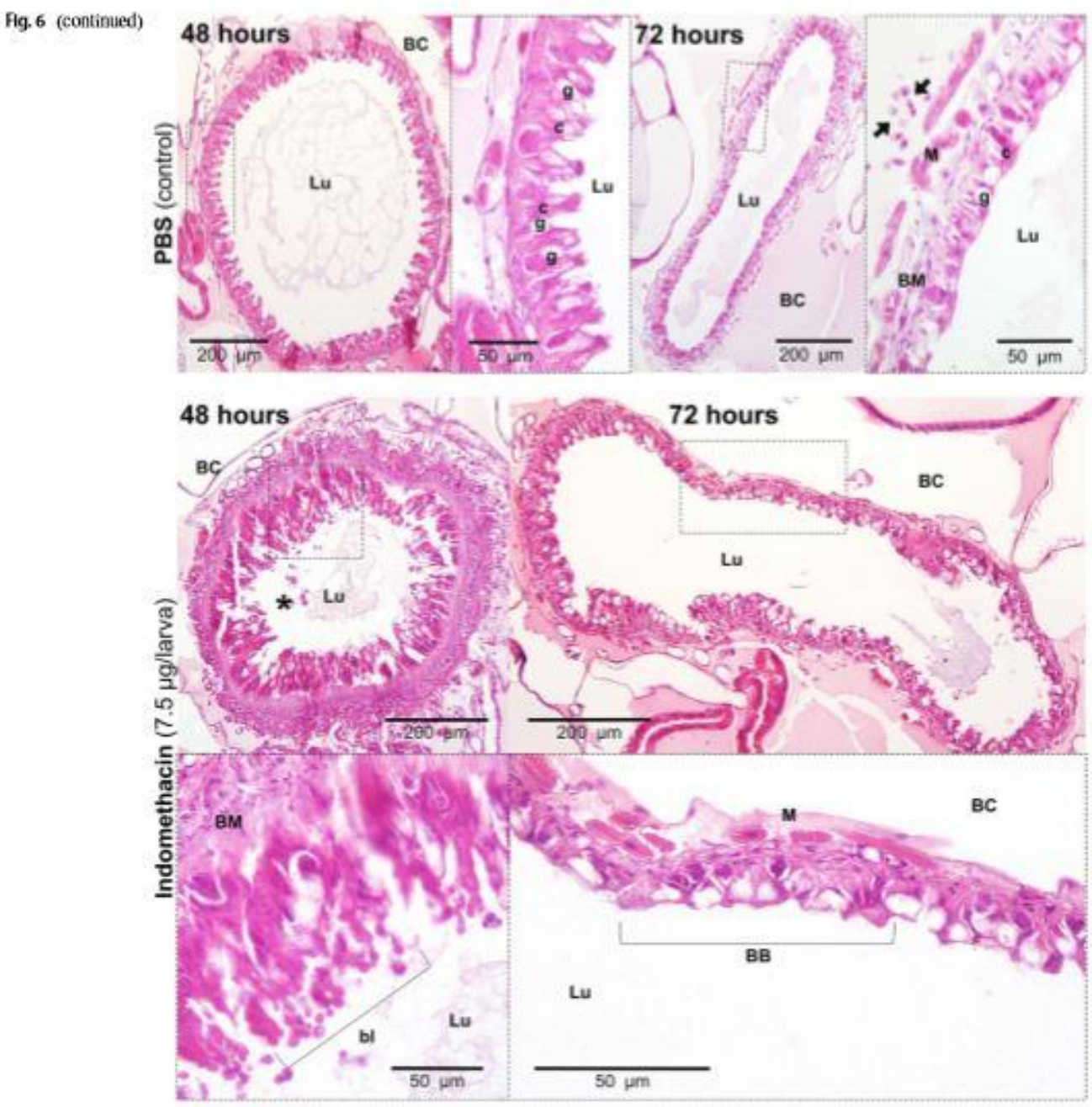

Uniform cellular arrangements of epithelial and goblet cells were observed, in addition to an intact basement membrane and visceral (striated) muscle layer. All control slides were considered grade 1 ( $0-2$ discrete changes), with the exception of a single section at the 48 -h time point that was assigned grade 2 (Supp. Figure 4). Following indomethacin treatment, varying levels of tissue damage and midgut degradation were visible within $448 \mathrm{~h}$ of all insects surveyed (Fig. 6- lower panels). Tissue slides were assigned damage grades 2 to 4 at $4 \mathrm{~h}$, and grades $2 / 3$ at 24 and 48 h (Supp.
Figure 4) - representing at least five aherrations per slide (discrete, localised changes) to $>50 \%$ compromised tissue (global damage; Fig. 7). Deterioration of the larval gut manifested as sloughed epithelial cells, increased vacuolisation, partial/complete displacement of the gut lining into the lumen, cellular debris (or potential apoptotic bodies), membrane blistering/blebbing, nuclear condensation (pyknosis) and fragmentation (karyhorrhexis) (Figs, 6, 7). By $72 \mathrm{~h}$, the majority of slides were graded 1 and 2 (Supp. Figure 4), which suggests the tissue is being repaired. 


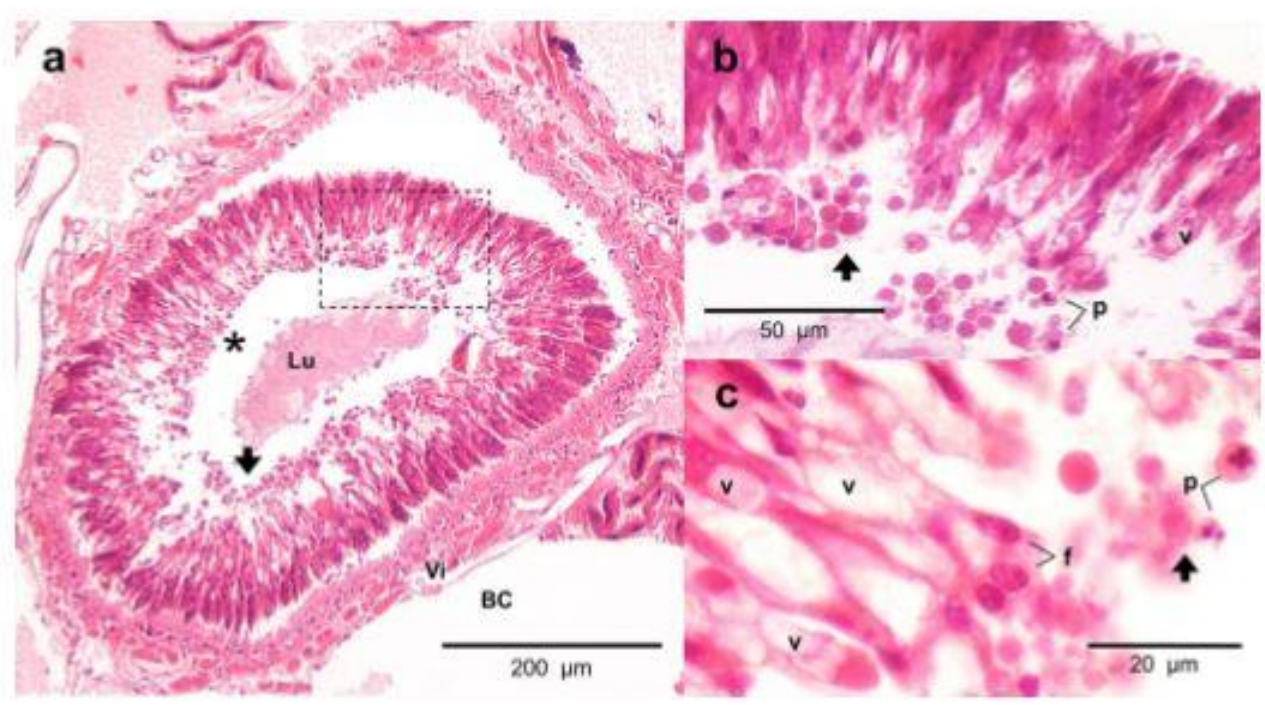

Fig. 7 Hissopathology of compromised midgat tissues from Giellerio mellomella force fed $(7.5$ pos) indomethacio, a Transwerse section of the larval midgut at 4 h post-treatment. The photomicrograph displays severe global tissue damage, where almost the entife epithelium has dissociated (sloughed) from the hasement membrane and visceral (Vi) mascle. The black arrows (all panels) signify the large number of

Fg. 8 Detoxification-associated activities within Galleria mellonella following forceeeding of indoenethasin, 0-7.5 ug/larva. Glutathione S-transterase aclivily was determined in the midgut (a) and haenoly be (b) by the by the change in 5-(2,4-dinitropheny glutachivee accumulation (A340 am). Superoxide dismulase activity was determine in the midgut (c) and haemoyempt (d) vis the inhibition of NIBT reduction (AS60 $\mathrm{mm})$. Data are presented as floating hars (min, max) with mean lines shown ( $n=36$ per trestment, 180 in total; 3 insects were pooled at each time point). Unshared letters (a, b) represen: statistical differences $(P<000)$ as determined by Tukey's mult ple comparison lests. The nega tive coatrol consisted of force reeding the insects with PBS +5\% DMSO in the absence of indoenethacin (0 ug/arva)

a

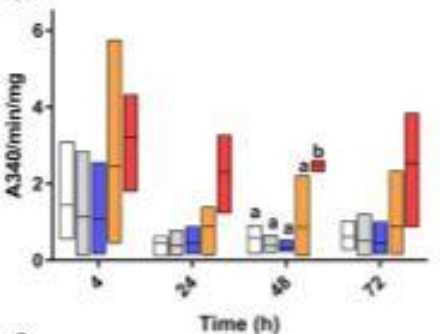

c

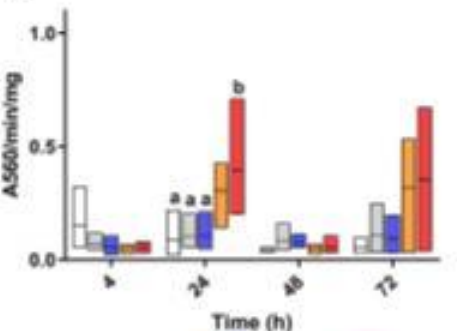

d
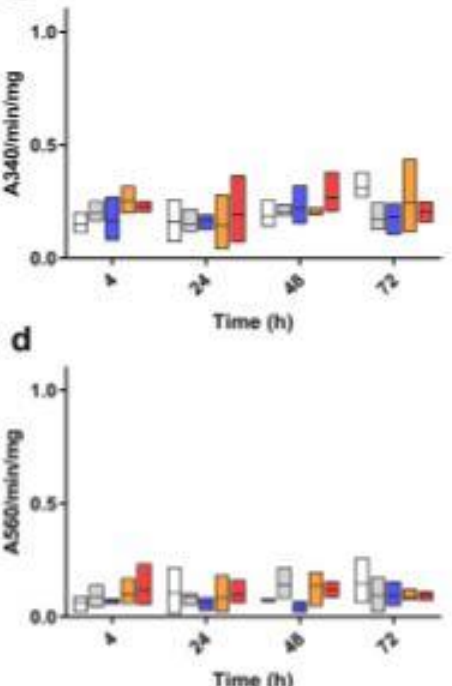

$\square$ untreated 1 uglana $\square 7.5$ polane 5\% DN65 $\square$ splane

Q Springer 


\section{Gut repair (detoxification)}

Administration of indomethacin by force feeding led to significant increases in glutathione- $S$-transferase $(F(4$, $40)=7.35, P=0.0002)$ and superoxide dismutase (F ( 4 , $40)=2.635 P=0.0481$ ) activities within the gut, but not in the haemolymph (GST; $F(4,40)=0.5449, P=0.704$ and SOD; $F(4,40)=0.801, P=0.515 ; F i g .8)$. I arvae that were inoculated with $7.5 \mu \mathrm{g}$ indomethacin demonstrated consistently higher levels of GST activity in the gut, 2.3-3.2 Abs $[340 \mathrm{~nm}] / \mathrm{min} / \mathrm{mg}$, when compared to all other treatments/controls and timepoints (Fig. 8a), whereas SOD activity peaked at 24 and $72 \mathrm{~h}, \sim 0.4 \mathrm{Abs}[560 \mathrm{~nm} / \mathrm{min} / \mathrm{mg}$ (Fig. 8c). A two-way ANOVA revealed time to be a sig. nificant factor regarding SOD activity $(F(3,40)=4.266$ $P=0.0105$ ), but this was not the case for GST ( $F(3$, $40)=2.584, P=0.067$ ). These patterns of enzyme activ ity complement the restored nature of the midgut tissues seen at $72 \mathrm{~h}$ in the histology (Fig. 6). Detoxification-associated activities in the haemolymph remained below 0.32 Abs [340 nm $/ / \mathrm{min} / \mathrm{mg}$ for GST and 0.15 Abs[560 nm]/ min/mg for SOD (Fig. 8b, d) - indicating strongly that the adverse effects of indomethacin were restricted to the gut

\section{Discussion}

We assessed the relative toxicity of indomethacin in insect larvae via gavage (force-feeding) and intrahaemocoelic injection across a concentration range relevant to rodent models, $2-30 \mathrm{mg} / \mathrm{kg}(0.5-7.5 \mu \mathrm{g} / \mathrm{larva})$, and found negligible side effects in terms of survival, development or immune cytotoxicity (Figs. 1, 2, 3). When foree-fed, larvae displayed broad symptoms of injury to the alimentary canal (Figs. 5 , $6,7,8)$, e.g., a threefold increase in gut permeability and tissue degradation. We present considerable evidence that the integrity of the midgut is compromised by indomethacin within 4 to $24 \mathrm{~h}$, causing sufficient damage to activate repair/detoxification mechanisms (GST and SOD). The onse of indomethacin-associated gut leakiness has also been recorded within $24 \mathrm{~h}$ in humans and mice, alongside ulceration and epithelial cell shrinkage (Playford el al. 1999, 2001, Bjarnason and Takeuchi 2009). Our combined use of X-ray microtomography and wax histology provides novel insight into the gross anatomy of the $G$. mellonella digestive tracthighlighting the suitability of the midgut for comparative pathobiology (Figs, 4, 5, 6, 7, Supp. Figure 2).

Indomethacin is a broad inhibitor of cyclooxygenase isozymes (COX1 and $\mathrm{COX} 2)$, which are responsible for the initiation of prostaglandin synthesis $\left(\mathrm{PGG}_{2}\right)$, and ultimately, the maintenance of inflammatory programmes and gastrointestinal mucosa (reviewed by Brune and Patrignani 2015). In a previous study by Büyukgüuzel et al. $(2007)$, eicosanoid presence in the haemolymph was deemed essential for mediating nodule formation - a cellular defence reaction - during viremia. When they injected $50 \mu \mathrm{g}$ of indomethacin into the haemocoel, there was a significant reduction in the number of nodules formed. We did not notice a reduction in the phagocytic capacity of haemocytes in insects forcefed $7.5 \mu \mathrm{g}$ indomethacin and latex microspheres (Supp. Figure 1), which is also in contrast to earlier findings by Mandato et al. (1997). The authors reported on a reduction in the phagocytic index of $G$. mellonella hacmocytes when exposed to $10 \mu \mathrm{M}$ indomethacin in vitro (Mandato et al. 1997). A likely explanation for this discrepancy is that the majority of force-fed indomethacin remains in the midgut of our insects, despite the microspheres leaking into the surrounding haemolymph (where they are targeted by hacmocytes; Supp. Figure 1). The immune cells of insects, namely the hacrnocytes, share many mechanistic and structural similarities with the innate immune cells of vertebrates, including pathogen recognition receptor signalling and phagocytosis-associated respiratory burst (Renwick et al 2007; Browne et al. 2013; Butt et al. 2016). Mandato et al (1997) and Büyükgüzel et al. (2007) describe the immuneinterference of indomethacin, and in addition to our observations of indomethacin-induced gut impairment, we consider that eicosanoid-like signalling should be added to the list of functional similarities between the innate immune systems of insects and vertebrates.

\section{Galleria mellonella as an alternative animal model}

The fruit fly Drosophila melanogaster is a superior genetic resource and over the past decade has been manipulated to gain novel insight into stem cell fate, immunity, antibiosis and homeostasis in the gut (Buchon et al. 2009; Chandler et al. 2011; Broderick and Lemaitre 2012; Miguel-Aliags et al. 2018). Only recently, the genome of $G$. mellonelle was made available (Lange et al. 2018a), yet it stands unannotated. Furthermore, despite wax-moth larvae being used widespread as a screening tool for novel therapeutics, path ogenicity, and toxicology (reviewed by Tsai et al. 2016), there remains a historical lag in molecular resources with the exception of some transcriptomic and miRNA data (Vogel et al. 2011; Mukherjee and Vilcinskas 2014). The financial and ethical incentives for using insect larvae over rodents and zebrafish are attractive, and so, we propose that vertebrates could be replaced partially for gut pathobiology. Waxmoth larvae are larger than traditional models like nematodes and drosophilids - this has two distinct advantages: (1) accurate doses can be administered orally (force-feeding), and (2) gram quantities of gut tissue can be obtained easily for downstream processing. Unlike other insect orders, the midgut of lepidopteran larvae represent the majority of tissue along the alimentary canal (mouth to anus; Fig. 4) 
and contains a specialised cell type, namely the goblet cell (Fig. 6), which is also found in the human intestine (Engel and Moran 2013; Linser and Dinglasan 2014).

The most common larval inoculation technique is intrahaemocoelic injection of test compounds/microbes; however, force-feeding $G$. mellonella (i.e., gavage) is an emerging practice. When screening common food preservatives (c.g., potassium nitrate), Maguire et al. (2016) obtained comparable toxicology data $\left(\mathrm{LD}_{\mathrm{so}}\right)$ between insect larvae, human cell lines (HEp-2) and rats. We recently provided evidence that larvac can also be used to assess the lethality and putative immune-toxicological effects of shellfish poisoning toxins (e.g., okadaic acid) at FDA-regulated levels in contaminated foods (Coates et al. 2019). Okadaic acid that was force-fed to insects disrupted midgut bomeostasis, leading to detrimental kevels of lipid peroxidation (malondialdehyde accumulation) in a dose-dependent manner-resembling symptoms found often in the standardised mouse bioassay. Lange et al. (2018b) proved that $G$. mellonella could differentiate between an enteric symbiont (Bacteroides vulgatus) and pathobiont (Escherichia coli), and mount a strong immune response involving reaction oxygen/nitrogen species. Oral administration of the pathogen stimulated the up-regulation of immune-recognition genes in insects (e.g. apolipophorin III) and mice (e.g., Cd14) alike. Interestingly, oral exposure of $G$. mellonella larvae to caffeine led to elevated levels of theobromine and theophylline in the hacmolymph - suggesting that caffeine metabolism in this insect is similar to the process in mammals (Maguire et al. 2017).

A better understanding of $G$. mellonella's alimentary canal should assist insecticide development (e.g., boric acid and biopesticides, Büyükgüzel et al. 2013; Grizanova et al. 2014) as lepidopteran insects represent a sizeable number of devastating agricultural pests (c.g., Spodoptera littoralis; Linser and Dinglasan 2014). A key difference between the digestive systems of vertebrates and insects is the presence of phenoloxidase (PO) enzymes (Whitten and Coates 2017). Phenoloxidases are responsible for the early processing of pigment precursors (quinones) into melanin, which plays several roles in development and immunity. Insect faeces are melanised upon release, and although the function of this is unclear, presumably it is due to gut phenoloxidase-activitic: and their oxidising/nitrosative by-products maintaining resident microbial populations from over-growing (Whitten and Coates 2017). The gut microbiomes of insects are diverse and tend to be species specific-influenced invariably by diet and environmental factors (Engel and Moran 2013). Few studies have focussed on the $G$. mellonella gut microbiome, yet representatives of the Bacteroidetes, Firmicutes and Proteobacteria are homologous to the biota on microvilli of the human intestinal crypts (Mukherjec et al. 2013b; Dubovskiy et al. 2016). Further work is needed to profile the residents of the insect gut, including fungi, viruses and Archaea. This is a timely topic, as wax-moth larvae are capable of degrading plastic (polypropylene; Bombelli et al. 2017) - likely facilitated by the microbial consortium of their alimentary canal.

\section{Concluding remarks}

We investigated the physiological effects of indomethacin on $G$. mellonella, providing compelling evidence that indomethacin exposure leads to tissue damage, cell death, gut leakiness, and REDOX imbalance in insect larvae. This mimics closely the pathological symptoms of their roden counterparts. We describe the functional/structural similarities of lepidopteran midgut tissues to those found in regions of the human gastrointestinal tract. Our data reinforce the use of $G$, mellonella as a surrogate toxicology model, with a focus on screening nutraceuticals and food additives.

Acknowledgements This research has been released as a pre-prin (Fmery et al. 2019) and is ayailable at biokriy; hitps//www bion ivorefoontent10.1101/606319v1. We would like to thank Mrs Sophie Malkin (Bluefish Technical Omcer, Swansea University) for ber assistance with histology preparations, Dr Christopher B Cunningham (Swansea University) for helpful discussions, and Elizabeth Evans and Ria Mitchell (AIM Facility) for helpful tips with image data segmentation.

Author contribution CJC conceived/designed all the experiments. HI performed the experiments with assistance from $\mathrm{CJC}, \mathrm{AFR}$ and $\mathrm{RJ}$. HI collated the data. $\mathrm{HE}$ and CJC analysed and interpreted the data. CJC prepared the manuscript, with input from $\mathrm{HI}, \mathrm{AFR}$ and $\mathrm{RJ}$.

Funding Finnancial support was secured throush the Furopean Socia Fund (ESF) KFSS2 scheme, and supplemented via start-up funds (COllege or Science, Swansea University) assigned to CrC. HE is the recipient of a KFSS2 PhD schotarship, which is co-sponsored by Mr Joh Rolls (The Golden Dairy Ldd.). AFR is part-funded through the join BBSRCNNERC Aquaculture Collaboration Hub UK (BB/P017215/1). The X-ray work is supported by the Advanced Imasing of Mater als (AIM) Facility (1920 FilsRC Grant No. HP/MO28267/1) and the GS through the Furopean Union's Coovergence programme administered by the Welsh Government.

\section{Compliance with ethical standards}

Ethics statement This study was approwed by the College of Science (Swansea University) research ethics committee (SU-ethics-1202.18/470).

Conflict of Interest The authors declare that they have no conflict of interest.

Open Access This article is distributed under the terms of the Creatiwe Commons Attribution 4.0 Internationsl I. icense (hitpd/ferealiveco mmons,org/licenses/by $/ 4.0$ ), which permils unestricted use, distribution, and reproduction in any medium, provided you give appropriate credit to the original author(s) and the source, provide a link to the Crealive Commons license, and indicale ir changes were made.

\section{Q Springer}




\section{References}

Abramot MD, Magalhăes PJ, Ram SJ (2004) Imsge processing with Imagel. Biophot Int 11(7):36-42

Aguwa CN (1985) Incidence of gastric ulcers by indomethacin and piroxicam in rats. Arch Toxion 56(3)-212-213

Allegra E, Tithall RW, Carter J, Champion OL. (2018) Galleria mellonella larvae allow the discrimination of toxic and non-foxic chemicals. Chemosphere 198:469-472

Altincicek B, L.inder M, Linder D, Preissner KT, Vilcinskas A (2007) Microbial metalloproteinases mediate sensing of invading pathogens and activate innste immune responses in the lepidopteran model host Gaileria mellonella Infect Immun 75(1)-175-183

Barnoy S, Gancz, H, Zhu Y, Hoonold CL, Zurawski DV, Venkateca MM (2017) The Galleria mellonella larvae as an in vivo model for evaluation of Shigella virulence. Gut Microbe 8(4):335-350

Basivireddy J, Jacob M, Ramamoorthy P, Pulimood AB, Balasabra manian KA (2003) Indomethacin induced free radical-mediated changes in the intestinal brush border membranes. Biochen Phar macol 65(4):683-69s

Bjarnason I, Takeuchi K (2009) Intestinal permeability in the pathopen esis of NSAID-induced enterope hy. J Gastroenterol 44(19)-23-29

Bombelli P, Howe CJ, Berlocchini F (2017) Polyethylene bio-degradation by caterpillars of the wax moth Galleria mellonella. Cur Biol 27(8)-R292-R293

Broderick NA, Lemaitre B (2012) Gut-associaled microbes of Dros ophila melanogasser. Gut Microbe 3(4):307-321

Browne N, Heelan M, Kavanagh K (2013) An analysis of the structura and functional similarities of insect hemocytes and mammalian phasocytes. Virulence $4(7): 597-60$ ?

Brune K, Patrignani P (2015) New insights into the use of currently available non-steroidal anti-inflammatory drugs. J Pain Res 8:105-118. bstps://doi.org/10.2147/JPR_575160

Buchoe N, Broderick NA, Poidevin M, Pradervand S, Lemaitre B (2009) Drosophila intestinal response to bacterial infection: activation of host defense and stem cell proliferation. Cell Host Microbe 5(2):200-211

Butt TM, Coates CJ, Duborskiy IM, Ratcliffe NA (2016) Fintomopathogenic fungi: new insights into host-patbogen interactions. Adv Genet 94:307-364

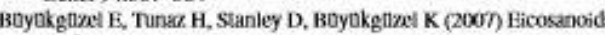
mediate Galleria mellonella cellular immune response to Vira infection. J Insect Physiol 53(1)-99-105

Boyakgtwel E, Baynkoazel K, Snela M, Frdem M, Radike K, Ziemnicki K, Adamski Z (2013) Elrect of boric acid on antioxi. dant enzyme activity, lipid peroxidation, and ultrastructure of midgat and fat body of Galleria mellonella. Cell Biol Toxicol 29(2):117-129

Campbell PM, Cao NT, Hines FR, Fast PD, Gordon KH (2008) Proteomic analysis of the peritrophic matrix from the gut of the caterpillar, Helicoverpa armigera. Insect Biochem Mol Bich $38(10): 950-958$

Card R, Vaughan K, Bagnall M, Spiropoulos J, Cooley W, Strickland Tet al. (2016) Virulence characterisation of Salmonella enterica isolates of differing antimicrobial resistance reoowered from UK livestock and imported meat samples. Front Microbiol 7:640

Champion Ol., Wagley S, Titball RW (2016) Galleria mellonella as a model host for microbiological and toxin research. Virulence $7(7): 840-845$

Chandler JA, I ang JM, Bhatnspar S, Eisen JA, Kopp A (2011) Bacte rial communities of diverse Drosophila species: ecolosical con text of a host-microbe modet system. PLoS Genet 7(9):e1002z72

Costes C, Lim J, Harman K, Rowley Ar?, Grimths D, Emery H, Lay ton W (2019) The insect, Galleria mellonella, is a compatibje model for evaluating the toxicology of okadaic acid. Cell Biol Toxicol 35(3):219-232

Cools $\mathrm{F}$ Torfs E, Aizawa J, vanhoutte B, Maes $\mathrm{L}$, Caljon G et al. (2019) Optimization and characterization of a Galleria mellomello larval infection model for virulence studies and the evaluation of therapeutics against Srreptococcus pneumoniae. Froat Microbiol 10:311. htips:/doi. ong/10.3389/micb.2019.00311

Dubovskiy IM, Martemyanow VV, Woronlsova YI, Rantala MJ, Gry zamova FV, Glupor VV (2008) Eflect of bacterial infection on antioxidant activity and lipid peroxidation in the mideut of Galle ria mellomella $\mathrm{L}$, Iarvae (Lepidopters, Pyralidae). Comp Biochem Physiol C 148(1):1-5

Dubovskiy IM, Grizanova EV, Whitten MM, Mukherjee K et al. (2016) Immuno-physiokogical adaptations confer wax moth Gatleria mellonella resistance to Bacillus thuringiensis. Viruleace $7(8): 860-870$

Engel P, Moran NA (2013) The gut microbiota of insects-diversity in structure and function. FEMS Microbiol Rev 37(5)699-735

Gornall AG, Bardrwill CJ, David MM (1949) Determination of serum proteins by means of the biuret reaction. $J$ Biol Chem $177(2): 751-766$

Green LF, Bergquist PR, Bullivant S (1980) The structure and function of the smooth septate junction in a transporting epithelium: the Malpighian tubules of the New Zealand glow-worm Aractinocampa luminosa. Tissue Cell 12(2):365-381

Grizanova EV, Duhorskiy IM, Whitten MMA, Glupow VV (2014) Contributions of cellular and humoral immunity of Galleria melLonella larvae in defence against oral infection by Bacillus thur ingiensis. J Invertbe Pathol 119:40-46

Kloezen W, van Helvert-van Poppel M, Fahal AH, van de Sande wW (2015) A Madurella myceromats grain model in Galleria mel lonella Larvae. PloS Neglect Trop D 9(7):e0003926

Kuraishti T, Binggeli O, Opota O, Buchon N, Lemaitre B (2011) Genetic evidence for a proxective fole of the peritrophic matrix against intestinal bacterial inlection in Drosophila melanogaster. Proc Nat Acad Sci 108(38): 15966-15971

Lange A, Beier S, Huson DH, Parusel R, Iglauer F, Fick IS (2018a) Genome sequence of Galleria mellonella (greater wax moth). Genome Announc 6(2) se01220-001317. https://oi.org/10.1128 genomeA.01220-17

Lano A, Schbfer A, Bender A, Sleimle A, Beier S, parusel R, Frick IS (2018b) Galleria mellonellos a novel invertebrate model to distinguish intestinal symbionts from pathobionts. Froot Immunol 9:2114. https://doi.org/10.3389/12mmu.2018.02114

Lim J, Coates C., Seoane PL, Garelnabi M, Taylor-Smith LM et al (2018) Characterizing the mechanisens of nonopsonic uptake of Cryptacracci by macrophases. J Immunol 200(10):3539-3546. hittps:/0biorg/10.4099/jimmanol.1700790

Limaye A (2012) Drishti: a volume exploration and presentation tool. In: Developments in X-ray Tomogranhy VIII. International Sociefy for Optics and Photonics (Vol. 8506, p. 85060X)

Linser PJ, Dinglasan RR (2014) Insect gut structure, function, development and target of biological toxins. Adv Insect Physiol 47:1-37

Maguire R, Duggan O, Kavanagh K (2016) Evaluation of Gulleria melLonella larvae as an in vivo model for assessing the relative toxicity of food preservative anents. Cell Biol Toxicol 32(3)-209-216 tion a larvas. Neurotoxicol Teratol 64:37-44

Mahmood A, Fitrgerald A, Marchbank T, Ntatsaki E, Murray D, Ghosh S, Playford RJ (2007) Zine carnosine, a bealth food supplement that stabilises small bowel integrity and stimulates gut repair processes. Gut 56(2):168-175

Mandato CA, Diehi-Jones WI., Moore SJ, Downer RG (1997) The effects of eicosanoid biosynthesis inhibitors on prophenoloxidase 
activation, phagocylosis and cell spreading in Galleria mellonella J Insect Physiol 43(1):1-8

Marchbank T, Ojobo E, Playford CJ, Playford RJ (2011) Reparative properties of the traditional Chinese medicine Condyceps sinensis (Chinese caterpillar mushroom) using HT29 cell culture and ra gastric damage models of injury. Br J Nutr I05(9): 1303-1310

Matsui H, Shimokawa O, Kancko T, Nagano Y, Rai K, Hyodo I (2011) The pathophysiology of non-steroidal anti-infammatory drus (NSAID)-induced mucosal injuries in stomach and small intestine. J Clinical Biochem Nutr 48(2):107-111

Mipuel-Aliags I, Jasper H, I emaitre B (2018) Anatomy and physiology of the digestive tract of Drosophila melanogaster. Genetics 210(2):357-396

Mowids P, Coates C, Rerwick J, Kavanagh K (2010) Dose-dependen cellular and humoral responses in Galleria mellonella larvae following \&-glucan inoculation. Microbes fnfect 12(2):146-153

Mukherjee K, Vilcinskas A (2014) Development and immunity-related microRNAs of the lepidopteran model host Galleria mellonella. BMC Genomics 15(1):705

Mukherjee K, Hain T, Fischer R, Chakrahorty T, Vilcinakas A (2013a) Brain infection and activation of neuroasl repair mechanisms by the human pathogen Lisseria monocynogenes in the lepidopteran model host Galleria mellonella. Virulence 4(4):324-332

Mukherjee K, Raju R, Fischer R, Vilcinskas A (2013b) Galleria mellonella as a model host to study gut microbe homeostasis and brain infection by the human pathogen I isteria monacyiogrnes. In: Vilcinskas A (ed) Yellow biolechnology I. Springer, Rerlin, Heideltherg, pp 27-39

Perron N, Tremblay R, Ferretti F, Bahakissa C, seidman Fet al (2013) Deleterious effects of indomethacin in the mid-gestation human intestine. Gienomics 101(3):171-177

Payford RJ, Hoyd DN, MacDonald CE, Cainan DP, Adenekan RO et al (1999) Bovine colostrum is a bealth food supplement which prevents NSAID induceal gut damage. Gut 44(5):653-658

Playford RJ, MacDonsid CF, Calnan DP, Flowd DN, Podas $T$ et al (2001) Co-administration of the health food supplement, bovine colostrum, reduces the acute non-steroidal anti-innammatory drug-induced increase in intestinal permeability. Clin Sci 100(6):627-633

kerawick J, Roeves FP. Wientjes FB, Kavanagh K (2007) Translocition of proteins homologous to human neutrophil p47phox and p67phox to the cell membrane in activated hemocyles or Galleria mellanella. Dev Comp Immunol 31(4)-347-359

Senior NJ, Bagnall MC, Champion OI Reynolds SE, La Ragione RM ef al (2011) Galleria mellonella \&s an infection model for Campy. lobacter jejani Virulence. J Med Microbiol 60(5):661-669

Sigthorson G, Crane R, Simon T, Hoover M, Quan H, Bolognese J, Bjarnason I (2000) COX 2 inhibition with rofecoxib does not increase intestinal permeability in healthy subjects: a double blint crossover sudy comparing forecox bo with placelo and indomechacin. $47(4): 527-532$

Smecaol R, Bai XC, Sugai E, Varqugez H, Niveloni S et al (2001) Acute gastrointestinal permeability responses to different non-steroidai anti-inllammatory druss. Gut 49(5):650-655

Strober W (2015) Trypan bloe exclusion test of cell viability. Curr Protoc Immunol. htps://doi.org/10.1002/0471142735. ima03bs111

Txai CJY, 1 .sh JMS, Proft T (2016) Gialleria mellowella infection mod els for the study or bacterial diseases and for antimicrobial drug testing. Virulence 7(3):214-229

Vogel H, Altincicek B, Glockner G, Vilcinskas A (2011) A compre hensive transcriptome and immune-gene repertoire of the lepidopteran model host Galleria mellomella. BMC Genomics 12(1):30

Wagley S, Borne R, Harrison J, Baker-Austin C, Ottaviani D et al (2018) Galleria mellonella as an infaction model to investioate virulence of $V$ ibrio parahomolyous. Virutence $9(1): 197-207$ esis research: views from the dark side. Pigment Cell Melanoma Res $30(4)-386-401$

Publisher's Note Springer Nature remains neutral with regard to furisdictiongl claims in peblishod mans and institutiongl aflitiations. 


\section{APPENDIX B}

\section{Assessing the potential toxicity of other pharmaceuticals}

There was no significant difference in survival between treatments or concentrations compared to control or other treatments. Larvae force fed the highest dose of Lansoprazole (10 $\mu \mathrm{g} / \mathrm{larva})$ had a $3 \%$ increase in morbidity by 72 hours (figure B1a), those force-fed with $10 \mu \mathrm{g} /$ larva of Aspirin had on average a 16\% increase in mortality whereas the highest doses of Aspirin (25 $\mu \mathrm{g} /$ larva) wasn't quantifiably different to the control (figure B1b). All doses of Diclofenac salt had between 3-7\% increase in mortality (figure B1c) compared to the control however this wasn't statistically significant when conducting a Tukey's multiple comparisons test.

Larvae that were force fed had an overall reduction in development compared to the control, however this was a smaller effect with larvae inoculated with Lansoprazole as the only significantly different for larvae inoculated with $2.5 \mu \mathrm{g} /$ larva Lansoprazole 6 days post inoculation ( $\mathrm{P}=0.0033)$ (figure $\mathrm{B} 2 \mathrm{a}$ and Table $\mathrm{B} 1$ ). This is possibly due to Lansoprazole being a protective and restorative pharmaceutical for gastric damage and at the lower dose, did not aid in healing potential physical damage caused by the inoculation method as quickly. On the other hand, both NSAID's, Aspirin and Diclofenac salt had significantly reduced development from Day 6 onwards compared to the untreated control (figure B2 b and c). Similarly, to the indomethacin development study, neither Aspirin nor Diclofenac salt inoculation effects appear to be concentration dependent. The lowest rate of development when force fed Diclofenac salt was observed at a concentration $5 \mu \mathrm{g} /$ larva with an average of $50 \%$ of larvae in pupation by day 10 compared to the control of $96 \%$ undergoing pupation $(\mathrm{P}=0.0009)$ (figure B2c) however this isn't significantly different when compared to other concentrations or PBS where 10 days post inoculation $76 \%$ of larvae were in metamorphosis (table B1 and figure B2). Larvae force fed Aspirin demonstrated the lowest rate of pupation with 56\% undergoing by day 10 in those inoculated with $2.5 \mu \mathrm{g} /$ larva $(2.5 \mu \mathrm{g} /$ larva /control $(\mathrm{P}=0.0113))$. Although not statistically significant, at the highest concentration of Aspirin $(25 \mu \mathrm{g} / \mathrm{larva})$ pupation was $7 \%$ higher on average than larvae inoculated with $2.5 \mu \mathrm{g} / \mathrm{larva}$. 

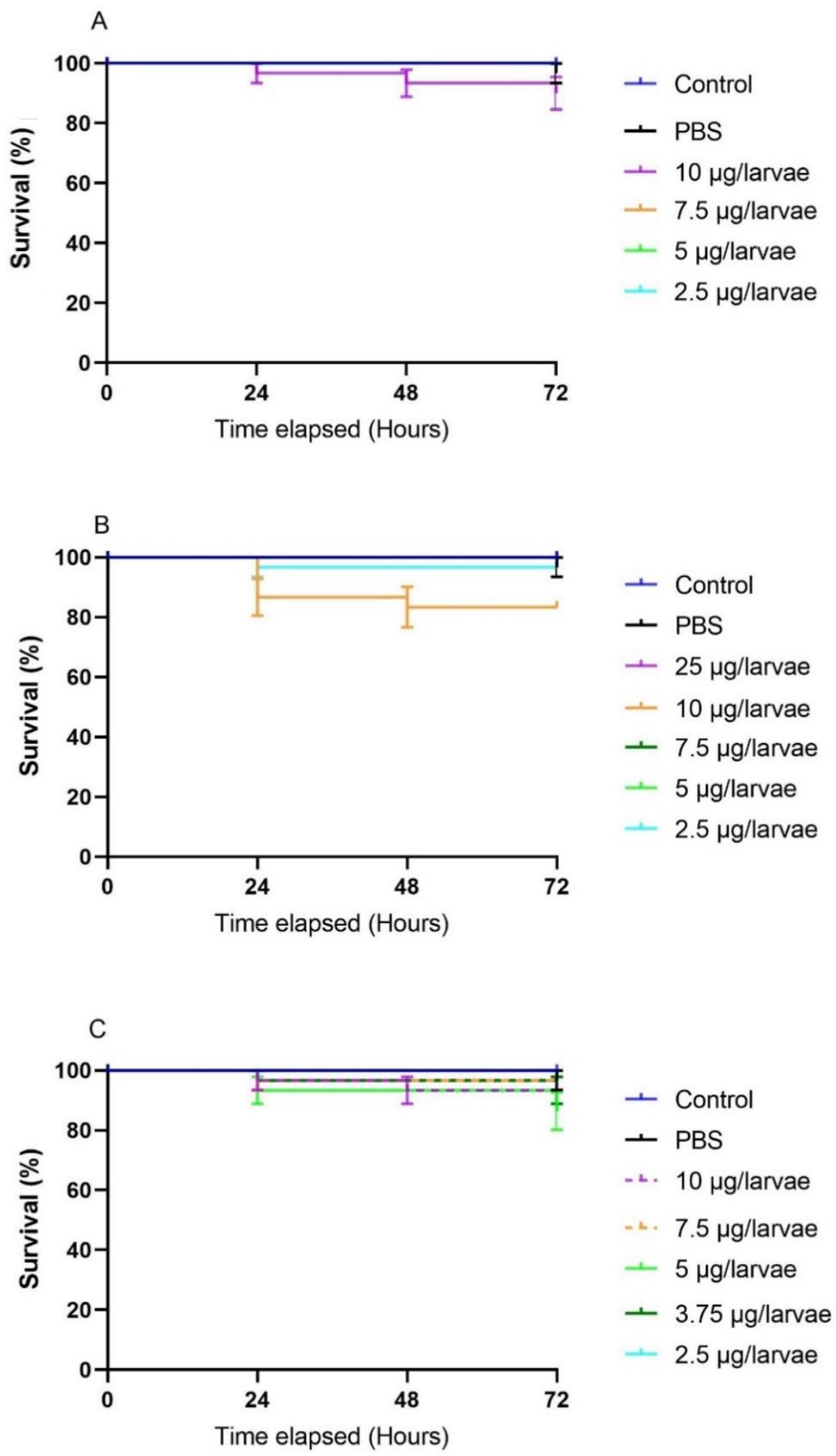

Figure B1 Survival of Galleria mellonella larvae after force feeding Lansoprazole (a), Aspirin (b) Diclofenac salt (c) $25-2.5 \mu \mathrm{g} /$ larva. Larvae were anesthetised on ice prior to inoculation and kept at $30{ }^{\circ} \mathrm{C}$ in darkness for 72 hours. Larvae were deemed dead when no longer responded to being rolled or touched. Values stated are mean $\pm \mathrm{SE}(\mathrm{n}=30$ per treatment, 480 in total). 

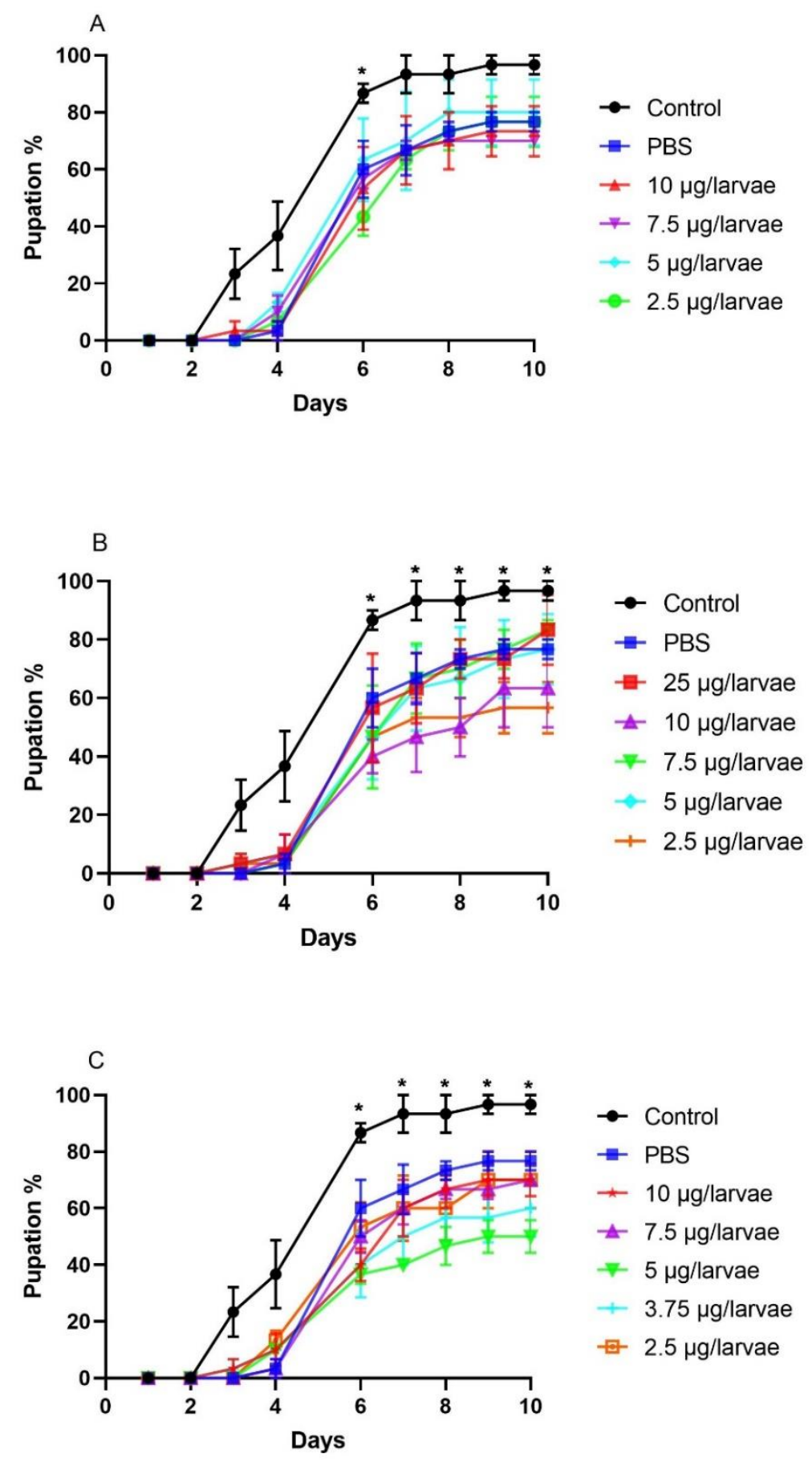

Figure B2. Galleria mellonella larval pupation 0-10 days post force feeding of a rage of 2.5$25 \mu \mathrm{g} /$ larva of Lansoprazole (A and B), Aspirin (C and D) and Diclofenac salt (E and ). Larvae were maintained at $30{ }^{\circ} \mathrm{C}$ in darkness for 10 days post inoculation. The number of larvae undergoing pupation was recorded. Values are expressed as mean $\pm \mathrm{SE}(\mathrm{n}=30$ per treatment, 480 in total). Symbol: ${ }^{*} \mathrm{P}<0.03$ when comparing untreated to all other treatments at the respective time points. The negative control consisted of force feeding or injecting the insect with PBS containing 5\% DMSO. 
Larvae inoculated with indomethacin had large variation of $6 \mu \mathrm{m}$ spheres found in the faeces for all treatments. However, larvae which were reared on $10 \%$ C. sinensis had a peak of $(87,500$ microspheres/larva) $6 \mu \mathrm{m}$ microspheres in the faeces 24 hours post inoculation, whereas other indomethacin force fed larvae peaked at 48 hours. Furthermore, larvae which were reared on

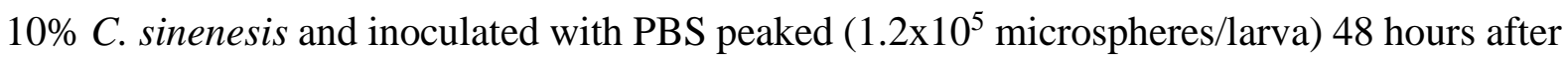
inoculation with whereas all other larvae inoculated with PBS had a gradual increase from 448 hours followed by a peak at 72 hours (Figure B3). This could indicate that the $C$. sinensis enhanced diet increase the rate of defecation in larvae (possibly through increased gut motility). 
A
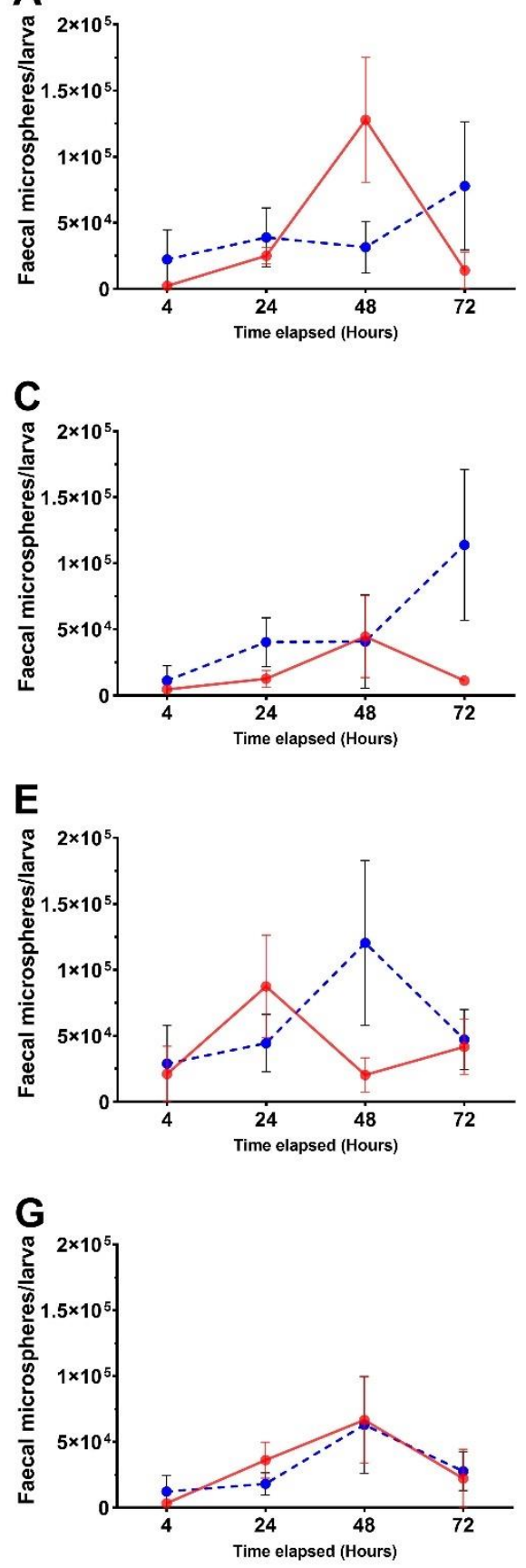

Indomethacin $(7.5 \mu \mathrm{g} / \mathrm{larva})$
B

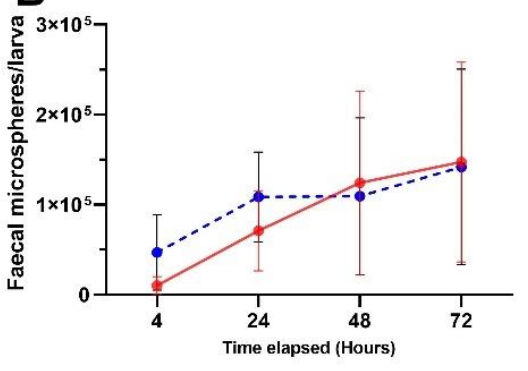

D

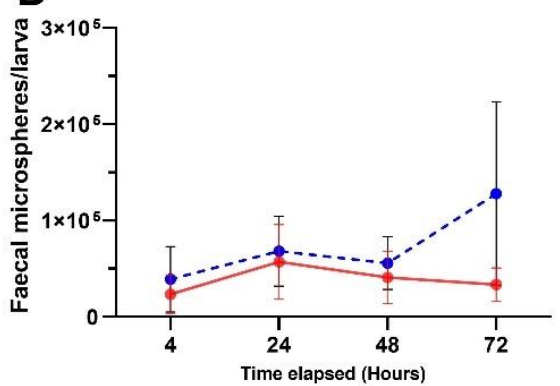

$\mathbf{F}$

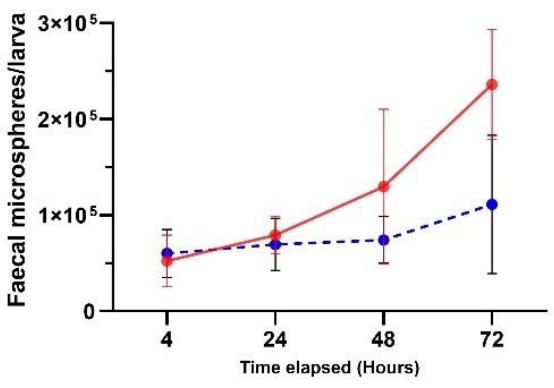

$\mathrm{H}$

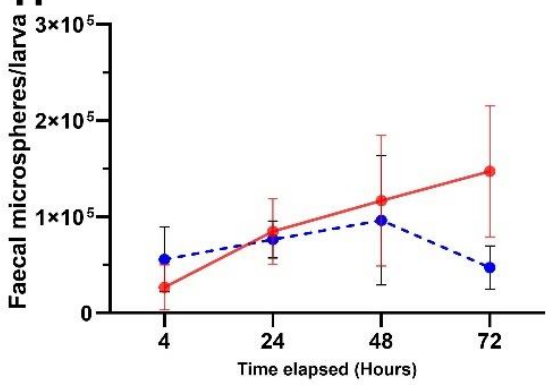

PBS (5\% DMSO)

Figure B3. Gut permeability of Galleria mellonella larvae reared on normal (A and B), nutraceutical supplemented food; $10 \%$ Colostrum $(\mathrm{C}$ and $\mathrm{D}), 10 \%$ C. sinensis $(\mathrm{E}$ and $\mathrm{F})$ and $5 \%$ C. sinensis with 5\% colostrum $(\mathrm{G}$ and $\mathrm{H})$ following force feeding of indomethacin, $7.5 \mu \mathrm{g} /$ larva. Permeability was determined by the number of $6 \mu \mathrm{m}$ (A, C, E and G), $2 \mu \mathrm{m}(\mathrm{B}$, $\mathrm{D}, \mathrm{F}$ and $\mathrm{H}$ ) spheres found in the faeces between 4 and $72 \mathrm{~h}$ post-inoculation. Each larva were co-inoculated with $1 \times 10^{6}$ microspheres and indomethacin $(7.5 \mu \mathrm{g} / \mathrm{larva})$ or PBS and incubated in the dark at $30^{\circ} \mathrm{C}$. Data represent the mean $\pm \mathrm{SE}(n=192,768$ in total across both microsphere sizes). The negative control consisted of force-feeding the insect with PBS $(+5 \%$ DMSO) in the absence of indomethacin ( $0 \mu \mathrm{g} /$ larva). 
Table B1. Values of significance following a Tukey's multiple comparison test of Galleria mellonella going through pupation after force feeding Lansoprazole, Aspirin or Diclofenac salt comparing effects of the concentrations at 6-10 days post inoculation.

\begin{tabular}{|c|c|c|c|c|}
\hline Tukey's multiple comparisons test & $\begin{array}{l}95.00 \% \text { CI of } \\
\text { diff. }\end{array}$ & Summary & $\begin{array}{l}\text { Adjusted } \\
\text { Value }\end{array}$ & $\mathbf{P}$ \\
\hline \multicolumn{4}{|l|}{ Day 6} & \\
\hline Control vs. Lansoprazole $2.5 \mu \mathrm{g} /$ larva & 7.870 to 78.80 & $* *$ & 0.0033 & \\
\hline Control vs. Aspirin $10 \mu \mathrm{g} /$ larva & 11.20 to 82.13 & $* * *$ & 0.0009 & \\
\hline Control vs. Aspirin $7.5 \mu \mathrm{g} /$ larva & 4.537 to 75.46 & $*$ & 0.0113 & \\
\hline Control vs. Aspirin $5 \mu \mathrm{g} /$ larva & 4.537 to 75.46 & $*$ & 0.0113 & \\
\hline Control vs. Aspirin $2.5 \mu \mathrm{g} /$ larva & 4.537 to 75.46 & $*$ & 0.0113 & \\
\hline Control vs. Diclofenac $10 \mu \mathrm{g} /$ larva & 11.20 to 82.13 & $* * *$ & 0.0009 & \\
\hline Control vs. Diclofenac $7.5 \mu \mathrm{g}$ /larva & 1.204 to 72.13 & $*$ & 0.0345 & \\
\hline Control vs. Diclofenac $5 \mu \mathrm{g} /$ larva & 14.54 to 85.46 & $* * *$ & 0.0002 & \\
\hline Control vs. Diclofenac $3.75 \mu \mathrm{g} /$ larva & 11.20 to 82.13 & $* * *$ & 0.0009 & \\
\hline
\end{tabular}

\begin{tabular}{llll}
\hline Day 7 & & & \\
\hline Control vs. Aspirin $10 \mu \mathrm{g} /$ larva & 11.20 to 82.13 & $* * *$ & 0.0009 \\
\hline Control vs. Aspirin $2.5 \mu \mathrm{g} /$ larva & 4.537 to 75.46 & $*$ & 0.0113 \\
\hline Control vs. Diclofenac $5 \mu \mathrm{g} /$ larva & 17.87 to 88.80 & $* * *$ & $<0.0001$ \\
\hline Control vs. Diclofenac $3.75 \mu \mathrm{g} /$ larva & 7.870 to 78.80 & $* *$ & 0.0033 \\
\hline
\end{tabular}

\begin{tabular}{llll}
\hline Day 8 & & & \\
\hline Control vs. Aspirin $10 \mu \mathrm{g} /$ larva & 7.870 to 78.80 & $* *$ & 0.0033 \\
\hline Control vs. Aspirin $2.5 \mu \mathrm{g} /$ larva & 4.537 to 75.46 & $*$ & 0.0113 \\
\hline Control vs. Diclofenac $5 \mu \mathrm{g} /$ larva & 11.20 to 82.13 & $* * *$ & 0.0009 \\
\hline Control vs. Diclofenac $3.75 \mu \mathrm{g} /$ larva & 1.204 to 72.13 & $*$ & 0.0345 \\
\hline & & & \\
\hline Day 9 & & & \\
\hline Control vs. Aspirin $2.5 \mu \mathrm{g} /$ larva & 4.537 to 75.46 & $*$ & \\
\hline & & & 0.0113 \\
\hline Day 10 & & & 0.0113 \\
\hline Control vs. Aspirin $2.5 \mu \mathrm{g} /$ larva & 4.537 to 75.46 & $*$ & 0.0009 \\
\hline Control vs. Diclofenac $5 \mu \mathrm{g} /$ larva & 11.20 to 82.13 & $* * *$ & 0.0345 \\
\hline Control vs. Diclofenac $3.75 \mu \mathrm{g} /$ larva & 1.204 to 72.13 & $*$ & \\
\hline
\end{tabular}


Table B2. Faeces weight from G. mellonella larvae reared on nutraceutical-enhanced foods and co-inoculated with $6 \mu \mathrm{m}$ microspheres and indomethacin $(n=36,108$ in total)

\begin{tabular}{|c|c|c|c|c|c|}
\hline $6 \mu \mathrm{m}$ & $4 \mathrm{hr}$ & $24 \mathrm{hr}$ & $48 \mathrm{hr}$ & $72 \mathrm{hr}$ & $\begin{array}{l}\text { Total faeces } \\
\text { /larvae (mg) }\end{array}$ \\
\hline $\begin{array}{l}\text { Standard food and } \\
\text { Indomethacin }\end{array}$ & 0.49 & 1.00 & 0.51 & 0.53 & 2.53 \\
\hline $\begin{array}{l}5 \% \text { Colostrum and } \\
\text { Indomethacin }\end{array}$ & 0.35 & 1.02 & 0.51 & 0.49 & 2.37 \\
\hline $\begin{array}{l}\text { 5\% C. sinensis and } \\
\text { Indomethacin }\end{array}$ & 0.70 & 0.90 & 0.66 & 0.75 & 3.02 \\
\hline $\begin{array}{l}5 \%+5 \% \text { combined } \\
\text { and Indomethacin }\end{array}$ & 0.77 & 1.28 & 0.73 & 0.58 & 3.36 \\
\hline $\begin{array}{c}\text { Standard food and } \\
\text { PBS }\end{array}$ & 0.58 & 0.82 & 0.40 & 0.36 & 2.17 \\
\hline $\begin{array}{c}5 \% \text { Colostrum and } \\
\text { PBS }\end{array}$ & 0.52 & 1.19 & 0.86 & 0.57 & 3.13 \\
\hline $\begin{array}{c}5 \% \text { C. sinensis and } \\
\text { PBS }\end{array}$ & 0.80 & 1.16 & 0.77 & 0.75 & 3.48 \\
\hline $\begin{array}{c}5 \%+5 \% \text { combined } \\
\text { and PBS }\end{array}$ & 0.82 & 0.93 & 0.47 & 0.50 & 2.71 \\
\hline
\end{tabular}


Table B3 a. Faeces weight from G. mellonella larvae reared on nutraceutical-enhanced foods and co-inoculated with $2 \mu \mathrm{m}$ microspheres and indomethacin. Data is expressed as weight of faeces/larvae, $(n=36,108$ in total $)$

\begin{tabular}{|c|c|c|c|c|c|}
\hline $2 \mu \mathrm{m}$ & $4 \mathrm{hr}$ & $24 \mathrm{hr}$ & $48 \mathrm{hr}$ & $72 \mathrm{hr}$ & $\begin{array}{l}\text { Total faeces } \\
\text { /larvae (mg) }\end{array}$ \\
\hline $\begin{array}{l}\text { Standard food and } \\
\text { Indomethacin }\end{array}$ & 0.35 & 1.26 & 0.60 & 0.88 & 3.08 \\
\hline $\begin{array}{l}5 \% \text { Colostrum and } \\
\text { Indomethacin }\end{array}$ & 0.70 & 0.91 & 0.43 & 0.62 & 2.66 \\
\hline $\begin{array}{l}5 \% \text { C. sinensis and } \\
\text { Indomethacin }\end{array}$ & 0.26 & 1.09 & 0.42 & 0.90 & 2.66 \\
\hline $\begin{array}{c}5 \%+5 \% \text { combined and } \\
\text { Indomethacin }\end{array}$ & 0.76 & 1.41 & 0.51 & 0.87 & 3.55 \\
\hline Standard food and PBS & 0.94 & 0.61 & 0.48 & 0.28 & 2.31 \\
\hline $5 \%$ Colostrum and PBS & 1.22 & 1.06 & 0.60 & 0.80 & 3.69 \\
\hline $5 \%$ C. sinensis and PBS & 0.86 & 1.16 & 0.65 & 2.52 & 5.19 \\
\hline $\begin{array}{c}5 \%+5 \% \text { combined and } \\
\text { PBS }\end{array}$ & 1.29 & 1.12 & 0.68 & 0.94 & 4.02 \\
\hline
\end{tabular}


Table B2 b. Statistical significance using Tukey's multiple comparisons test of faeces weight from G. mellonella larvae reared on nutraceutical-enhanced foods and co-inoculated with $2 \mu \mathrm{m}$ microspheres and indomethacin

\begin{tabular}{|c|c|c|c|}
\hline & Mean Diff. & $95.00 \%$ CI of diff. & $\begin{array}{l}\text { Adjusted } \mathbf{P} \\
\text { Value }\end{array}$ \\
\hline $\begin{array}{l}\text { Standard food and Indomethacin vs. } \\
\text { 5\% C. sinensis and PBS }\end{array}$ & -1.647 & -3.289 to -0.004590 & $0.0489 *$ \\
\hline $\begin{array}{c}5 \% \text { Colostrum and Indomethacin vs. } \\
5 \% \text { C. sinensis and PBS }\end{array}$ & -1.907 & -3.549 to -0.2646 & $0.0121 *$ \\
\hline $\begin{array}{c}5 \%+5 \% \text { combined and } \\
\text { Indomethacin vs. } 5 \% \text { C. sinensis and } \\
\text { PBS }\end{array}$ & -1.657 & -3.299 to -0.01459 & $0.0465 *$ \\
\hline $\begin{array}{l}\text { Standard food and PBS vs. 5\% C. } \\
\text { sinensis and PBS }\end{array}$ & -2.243 & -3.885 to -0.6013 & $0.0016 * *$ \\
\hline $\begin{array}{l}\text { 5\% Colostrum and PBS vs. 5\% C. } \\
\text { sinensis and PBS }\end{array}$ & -1.723 & -3.365 to -0.08126 & $0.0331 *$ \\
\hline
\end{tabular}

Table B3. Statistically significant results following a Tukey's multiple comparison test of larvae movement, following force feeding (FF) or injection (INJ) of $1 \times 10^{6}$ or $3 \times 10^{6}$ C. jejuni or PBS or the untreated control (NT)

\begin{tabular}{|c|c|c|c|c|}
\hline Time Elapsed (Hours) & $\begin{array}{l}\text { Mean } \\
\text { Diff. }\end{array}$ & $95.00 \%$ CI of diff. & Summary & Adjusted P Value \\
\hline \multicolumn{5}{|l|}{24} \\
\hline Normal NT vs. Normal FF $1 \times 10^{6}$ & 1.333 & 0.2013 to 2.465 & $* *$ & 0.0039 \\
\hline Normal NT vs. Normal FF $3 \times 10^{6}$ & 1.867 & 0.7347 to 2.999 & $* * * *$ & $<0.0001$ \\
\hline Normal NT vs. Colostrum FF 3x10 & 1.3 & 0.1680 to 2.432 & $* *$ & 0.0062 \\
\hline Normal NT vs. Colostrum INJ 3x10 & 1.7 & 0.5680 to 2.832 & $* * * *$ & $<0.0001$ \\
\hline Normal NT vs. $C$. sinensis FF $3 \times 10^{6}$ & 1.267 & 0.1347 to 2.399 & $* *$ & 0.0097 \\
\hline Normal NT vs. C. sinensis INJ $3 \times 10^{6}$ & 1.533 & 0.4013 to 2.665 & $* * *$ & 0.0002 \\
\hline Normal NT vs. Both FF $1 \times 10^{6}$ & 1.433 & 0.3013 to 2.565 & $* * *$ & 0.0009 \\
\hline Normal NT vs. Both FF $3 \times 10^{6}$ & 2.2 & 1.068 to 3.332 & $* * * *$ & $<0.0001$ \\
\hline Normal NT vs. Both INJ $3 \times 10^{6}$ & 1.667 & 0.5347 to 2.799 & $* * * *$ & $<0.0001$ \\
\hline Normal PBS FF vs. Normal FF 1x10 & 1.333 & 0.2013 to 2.465 & $* *$ & 0.0039 \\
\hline Normal PBS FF vs. Normal FF $3 \times 10^{6}$ & 1.867 & 0.7347 to 2.999 & $* * * *$ & $<0.0001$ \\
\hline Normal PBS FF vs. Colostrum FF 3x10 & 1.3 & 0.1680 to 2.432 & $* *$ & 0.0062 \\
\hline Normal PBS FF vs. Colostrum INJ 3x10 & 1.7 & 0.5680 to 2.832 & $* * * *$ & $<0.0001$ \\
\hline Normal PBS FF vs. C. sinensis FF $3 \times 10^{6}$ & 1.267 & 0.1347 to 2.399 & $* *$ & 0.0097 \\
\hline Normal PBS FF vs. C. sinensis $\mathrm{INJ} 3 \times 10^{6}$ & 1.533 & 0.4013 to 2.665 & $* * *$ & 0.0002 \\
\hline Normal PBS FF vs. Both FF $1 \times 10^{6}$ & 1.433 & 0.3013 to 2.565 & $* * *$ & 0.0009 \\
\hline
\end{tabular}




\begin{tabular}{|c|c|c|c|c|}
\hline Normal PBS FF vs. Both FF $3 \times 10^{6}$ & 2.2 & 1.068 to 3.332 & $* * * *$ & $<0.0001$ \\
\hline 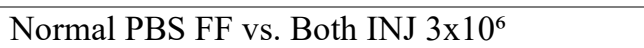 & 1.667 & 0.5347 to 2.799 & $* * * *$ & $<0.0001$ \\
\hline Normal FF $1 \times 10^{6}$ vs. Normal PBS INJ & -1.333 & -2.465 to -0.2013 & $* *$ & 0.0039 \\
\hline Normal FF 1x10 16 vs. Colostrum NT & -1.2 & -2.332 to -0.06799 & * & 0.0227 \\
\hline Normal FF $1 \times 10^{6}$ vs. Colostrum PBS INJ & -1.2 & -2.332 to -0.06799 & $*$ & 0.0227 \\
\hline Normal FF $1 \times 10^{6}$ vs. C. sinensis NT & -1.367 & -2.499 to -0.2347 & $* *$ & 0.0024 \\
\hline Normal FF $1 \times 10^{6}$ vs. C. sinensis $\mathrm{PBS}$ INJ & -1.3 & -2.432 to -0.1680 & $* *$ & 0.0062 \\
\hline Normal FF 1x106 vs. Both NT & -1.333 & -2.465 to -0.2013 & $* *$ & 0.0039 \\
\hline Normal FF $1 \times 10^{6}$ vs. Both PBS FF & -1.333 & -2.465 to -0.2013 & $* *$ & 0.0039 \\
\hline Normal FF $1 \times 10^{6}$ vs. Both PBS INJ & -1.333 & -2.465 to -0.2013 & $* *$ & 0.0039 \\
\hline Normal FF $3 \times 10^{6}$ vs. Normal PBS INJ & -1.867 & -2.999 to -0.7347 & $* * * *$ & $<0.0001$ \\
\hline Normal FF $3 \times 10^{6}$ vs. Normal INJ $1 \times 10^{6}$ & -1.2 & -2.332 to -0.06799 & $*$ & 0.0227 \\
\hline Normal FF 3x10 $10^{6}$ v. Colostrum NT & -1.733 & -2.865 to -0.6013 & $* * * *$ & $<0.0001$ \\
\hline Normal FF 3x10 6 vs. Colostrum PBS FF & -1.433 & -2.565 to -0.3013 & $* * *$ & 0.0009 \\
\hline Normal FF 3x10 ${ }^{6}$ vs. Colostrum PBS INJ & -1.733 & -2.865 to -0.6013 & $* * * *$ & $<0.0001$ \\
\hline Normal FF $3 \times 10^{6}$ vs. C. sinensis NT & -1.9 & -3.032 to -0.7680 & $* * * *$ & $<0.0001$ \\
\hline Normal FF $3 \times 10^{6}$ vs. C. sinensis PBS FF & -1.533 & -2.665 to -0.4013 & $* * *$ & 0.0002 \\
\hline Normal FF $3 \times 10^{6}$ vs. C. sinensis $\mathrm{PBS}$ INJ & -1.833 & -2.965 to -0.7013 & $* * * *$ & $<0.0001$ \\
\hline Normal FF $3 \times 10^{6}$ vs. Both NT & -1.867 & -2.999 to -0.7347 & $* * * *$ & $<0.0001$ \\
\hline Normal FF $3 \times 10^{6}$ vs. Both PBS FF & -1.867 & -2.999 to -0.7347 & $* * * *$ & $<0.0001$ \\
\hline Normal FF $3 \times 10^{6}$ vs. Both PBS INJ & -1.867 & -2.999 to -0.7347 & $* * * *$ & $<0.0001$ \\
\hline Normal PBS INJ vs. Colostrum FF 3x10 & 1.3 & 0.1680 to 2.432 & $* *$ & 0.0062 \\
\hline Normal PBS INJ vs. Colostrum INJ 3x10 & 1.7 & 0.5680 to 2.832 & $* * * *$ & $<0.0001$ \\
\hline Normal PBS INJ vs. C. sinensis FF $3 \times 10^{6}$ & 1.267 & 0.1347 to 2.399 & $* *$ & 0.0097 \\
\hline Normal PBS INJ vs. C. sinensis INJ $3 \times 10^{6}$ & 1.533 & 0.4013 to 2.665 & $* * *$ & 0.0002 \\
\hline Normal PBS INJ vs. Both FF 1x10 & 1.433 & 0.3013 to 2.565 & $* * *$ & 0.0009 \\
\hline Normal PBS INJ vs. Both FF $3 \times 10^{6}$ & 2.2 & 1.068 to 3.332 & $* * * *$ & $<0.0001$ \\
\hline Normal PBS INJ vs. Both INJ 3×10 & 1.667 & 0.5347 to 2.799 & $* * * *$ & $<0.0001$ \\
\hline Normal INJ $1 \times 10^{6}$ vs. Both FF $3 \times 10^{6}$ & 1.533 & 0.4013 to 2.665 & $* * *$ & 0.0002 \\
\hline 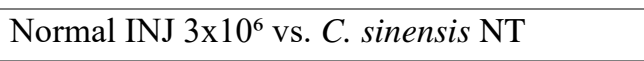 & -1.133 & -2.265 to -0.001326 & $*$ & 0.0493 \\
\hline Colostrum NT vs. Colostrum FF 3x10 & 1.167 & 0.03466 to 2.299 & $*$ & 0.0337 \\
\hline Colostrum NT vs. Colostrum INJ 3x10 & 1.567 & 0.4347 to 2.699 & $* * *$ & 0.0001 \\
\hline Colostrum NT vs. C. sinensis FF $3 \times 10^{6}$ & 1.133 & 0.001326 to 2.265 & $*$ & 0.0493 \\
\hline Colostrum NT vs. $C$. sinensis INJ $3 \times 10^{6}$ & 1.4 & 0.2680 to 2.532 & $* *$ & 0.0015 \\
\hline Colostrum NT vs. Both FF $1 \times 10^{6}$ & 1.3 & 0.1680 to 2.432 & $* *$ & 0.0062 \\
\hline Colostrum NT vs. Both FF $3 \times 10^{6}$ & 2.067 & 0.9347 to 3.199 & $* * * *$ & $<0.0001$ \\
\hline 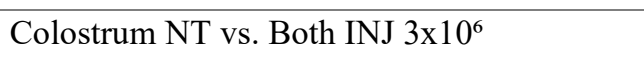 & 1.533 & 0.4013 to 2.665 & $* * *$ & 0.0002 \\
\hline $\begin{array}{l}\text { Colostrum PBS FF vs. Colostrum INJ } \\
3 \times 10^{6}\end{array}$ & 1.267 & 0.1347 to 2.399 & $* *$ & 0.0097 \\
\hline Colostrum PBS FF vs. Both FF $3 \times 10^{6}$ & 1.767 & 0.6347 to 2.899 & $* * * *$ & $<0.0001$ \\
\hline 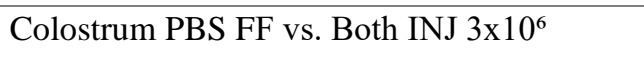 & 1.233 & 0.1013 to 2.365 & $*$ & 0.015 \\
\hline Colostrum FF $1 \times 10^{6}$ vs. Both FF $3 \times 10^{6}$ & 1.433 & 0.3013 to 2.565 & $* * *$ & 0.0009 \\
\hline $\begin{array}{l}\text { Colostrum FF 3x106 vs. Colostrum PBS } \\
\text { INJ }\end{array}$ & -1.167 & -2.299 to -0.03466 & $*$ & 0.0337 \\
\hline Colostrum FF $3 \times 10^{6}$ vs. C. sinensis $\mathrm{NT}$ & -1.333 & -2.465 to -0.2013 & $* *$ & 0.0039 \\
\hline $\begin{array}{l}\text { Colostrum FF } 3 \times 10^{6} \text { vs. C. sinensis PBS } \\
\text { INJ }\end{array}$ & -1.267 & -2.399 to -0.1347 & $* *$ & 0.0097 \\
\hline Colostrum FF $3 \times 10^{6}$ vs. Both NT & -1.3 & -2.432 to -0.1680 & $* *$ & 0.0062 \\
\hline
\end{tabular}




\begin{tabular}{|c|c|c|c|c|}
\hline Colostrum FF $3 \times 10^{6}$ vs. Both PBS FF & -1.3 & -2.432 to -0.1680 & $* *$ & 0.0062 \\
\hline Colostrum FF $3 \times 10^{6}$ vs. Both PBS INJ & -1.3 & -2.432 to -0.1680 & $* *$ & 0.0062 \\
\hline $\begin{array}{l}\text { Colostrum PBS INJ vs. Colostrum INJ } \\
\mathbf{3 x 1 0}^{6}\end{array}$ & 1.567 & 0.4347 to 2.699 & $* * *$ & 0.0001 \\
\hline $\begin{array}{l}\text { Colostrum PBS INJ vs. C. sinensis FF } \\
3 \times 10^{6}\end{array}$ & 1.133 & 0.001326 to 2.265 & $*$ & 0.0493 \\
\hline $\begin{array}{l}\text { Colostrum PBS INJ vs. C. sinensis INJ } \\
3 \times 10^{6}\end{array}$ & 1.4 & 0.2680 to 2.532 & $* *$ & 0.0015 \\
\hline Colostrum PBS INJ vs. Both FF 1x10 & 1.3 & 0.1680 to 2.432 & $* *$ & 0.0062 \\
\hline Colostrum PBS INJ vs. Both FF $3 \times 10^{6}$ & 2.067 & 0.9347 to 3.199 & $* * * *$ & $<0.0001$ \\
\hline Colostrum PBS INJ vs. Both INJ $3 \times 10^{6}$ & 1.533 & 0.4013 to 2.665 & $* * *$ & 0.0002 \\
\hline Colostrum INJ $1 \times 10^{6}$ vs. Both FF $3 \times 10^{6}$ & 1.267 & 0.1347 to 2.399 & $* *$ & 0.0097 \\
\hline Colostrum INJ 3x10 ${ }^{6}$ vs. $C$. sinensis NT & -1.733 & -2.865 to -0.6013 & $* * * *$ & $<0.0001$ \\
\hline $\begin{array}{l}\text { Colostrum INJ } 3 \times 10^{6} \text { vs. C. sinensis PBS } \\
\text { FF }\end{array}$ & -1.367 & -2.499 to -0.2347 & $* *$ & 0.0024 \\
\hline $\begin{array}{l}\text { Colostrum INJ } 3 \times 10^{6} \text { vs. C. sinensis PBS } \\
\text { INJ }\end{array}$ & -1.667 & -2.799 to -0.5347 & $* * * *$ & $<0.0001$ \\
\hline Colostrum INJ $3 \times 10^{6}$ vs. Both NT & -1.7 & -2.832 to -0.5680 & $* * * *$ & $<0.0001$ \\
\hline Colostrum INJ 3x10 vs. Both PBS FF & -1.7 & -2.832 to -0.5680 & $* * * *$ & $<0.0001$ \\
\hline Colostrum INJ $3 \times 10^{6}$ vs. Both PBS INJ & -1.7 & -2.832 to -0.5680 & $* * * *$ & $<0.0001$ \\
\hline C. sinensis $\mathrm{NT}$ vs. C. sinensis FF $3 \times 10^{6}$ & 1.3 & 0.1680 to 2.432 & $* *$ & 0.0062 \\
\hline C. sinensis $\mathrm{NT}$ vs. C. sinensis $\mathrm{INJ} 3 \times 10^{6}$ & 1.567 & 0.4347 to 2.699 & $* * *$ & 0.0001 \\
\hline C. sinensis $\mathrm{NT}$ vs. Both FF $1 \times 10^{6}$ & 1.467 & 0.3347 to 2.599 & $* * *$ & 0.0005 \\
\hline C. sinensis NT vs. Both FF $3 \times 10^{6}$ & 2.233 & 1.101 to 3.365 & $* * * *$ & $<0.0001$ \\
\hline C. sinensis NT vs. Both INJ $3 \times 10^{6}$ & 1.7 & 0.5680 to 2.832 & $* * * *$ & $<0.0001$ \\
\hline C. sinensis PBS FF vs. C. sinensis INJ $3 \times 10^{6}$ & 1.2 & 0.06799 to 2.332 & $*$ & 0.0227 \\
\hline C. sinensis PBS FF vs. Both FF $3 \times 10^{6}$ & 1.867 & 0.7347 to 2.999 & $* * * *$ & $<0.0001$ \\
\hline C. sinensis PBS FF vs. Both INJ $3 \times 10^{6}$ & 1.333 & 0.2013 to 2.465 & $* *$ & 0.0039 \\
\hline C. sinensis FF $1 \times 10^{6}$ vs. Both FF $3 \times 10^{6}$ & 1.333 & 0.2013 to 2.465 & $* *$ & 0.0039 \\
\hline C. sinensis FF $3 \times 10^{6}$ vs. C. sinensis $\mathrm{PBS}$ INJ & -1.233 & -2.365 to -0.1013 & $*$ & 0.015 \\
\hline C. sinensis FF $3 \times 10^{6}$ vs. Both NT & -1.267 & -2.399 to -0.1347 & $* *$ & 0.0097 \\
\hline C. sinensis FF $3 \times 10^{6}$ vs. Both PBS FF & -1.267 & -2.399 to -0.1347 & $* *$ & 0.0097 \\
\hline C. sinensis FF $3 \times 10^{6}$ vs. Both PBS INJ & -1.267 & -2.399 to -0.1347 & $* *$ & 0.0097 \\
\hline $\begin{array}{l}\text { C. sinensis PBS INJ vs. C. sinensis INJ } \\
3 \times 10^{6}\end{array}$ & 1.5 & 0.3680 to 2.632 & *** & 0.0003 \\
\hline C. sinensis PBS INJ vs. Both FF $1 \times 10^{6}$ & 1.4 & 0.2680 to 2.532 & $* *$ & 0.0015 \\
\hline C. sinensis PBS INJ vs. Both FF $3 \times 10^{6}$ & 2.167 & 1.035 to 3.299 & $* * * *$ & $<0.0001$ \\
\hline C. sinensis PBS INJ vs. Both INJ $3 \times 10^{6}$ & 1.633 & 0.5013 to 2.765 & $* * * *$ & $<0.0001$ \\
\hline C. sinensis $\mathrm{INJ} 1 \times 10^{6}$ vs. Both FF $3 \times 10^{6}$ & 1.433 & 0.3013 to 2.565 & $* * *$ & 0.0009 \\
\hline C. sinensis $\mathrm{INJ} 3 \times 10^{6}$ vs. Both NT & -1.533 & -2.665 to -0.4013 & $* * *$ & 0.0002 \\
\hline C. sinensis INJ $3 \times 10^{6}$ vs. Both PBS FF & -1.533 & -2.665 to -0.4013 & $* * *$ & 0.0002 \\
\hline C. sinensis INJ $3 \times 10^{6}$ vs. Both PBS INJ & -1.533 & -2.665 to -0.4013 & $* * *$ & 0.0002 \\
\hline Both NT vs. Both FF 1x10 & 1.433 & 0.3013 to 2.565 & $* * *$ & 0.0009 \\
\hline Both NT vs. Both FF $3 \times 10^{6}$ & 2.2 & 1.068 to 3.332 & $* * * *$ & $<0.0001$ \\
\hline Both NT vs. Both INJ $3 \times 10^{6}$ & 1.667 & 0.5347 to 2.799 & $* * * *$ & $<0.0001$ \\
\hline Both PBS FF vs. Both FF $1 \times 10^{6}$ & 1.433 & 0.3013 to 2.565 & $* * *$ & 0.0009 \\
\hline Both PBS FF vs. Both FF $3 \times 10^{6}$ & 2.2 & 1.068 to 3.332 & $* * * *$ & $<0.0001$ \\
\hline Both PBS FF vs. Both INJ $3 \times 10^{6}$ & 1.667 & 0.5347 to 2.799 & $* * * *$ & $<0.0001$ \\
\hline Both FF $1 \times 10^{6}$ vs. Both PBS INJ & -1.433 & -2.565 to -0.3013 & $* * *$ & 0.0009 \\
\hline
\end{tabular}




\begin{tabular}{|c|c|c|c|c|}
\hline Both FF $3 \times 10^{6}$ vs. Both PBS INJ & -2.2 & -3.332 to -1.068 & $* * * *$ & $<0.0001$ \\
\hline Both FF $3 \times 10^{6}$ vs. Both INJ $1 \times 10^{6}$ & -1.333 & -2.465 to -0.2013 & $* *$ & 0.0039 \\
\hline Both PBS INJ vs. Both INJ 3x106 & 1.667 & 0.5347 to 2.799 & $* * * *$ & $<0.0001$ \\
\hline \multicolumn{5}{|l|}{48} \\
\hline Normal NT vs. Normal FF $1 \times 10^{6}$ & 1.733 & 0.6013 to 2.865 & $* * * *$ & $<0.0001$ \\
\hline Normal NT vs. Normal FF $3 \times 10^{6}$ & 2.133 & 1.001 to 3.265 & $* * * *$ & $<0.0001$ \\
\hline Normal NT vs. Normal INJ $3 \times 10^{6}$ & 1.5 & 0.3680 to 2.632 & $* * *$ & 0.0003 \\
\hline 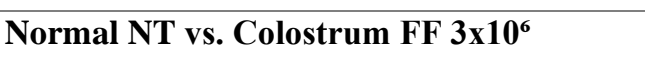 & 1.5 & 0.3680 to 2.632 & $* * *$ & 0.0003 \\
\hline Normal NT vs. Colostrum INJ 3x10 & 2.333 & 1.201 to 3.465 & $* * * *$ & $<0.0001$ \\
\hline Normal NT vs. C. sinensis FF $3 \times 10^{6}$ & 1.367 & 0.2347 to 2.499 & $* *$ & 0.0024 \\
\hline Normal NT vs. $C$. sinensis INJ $3 \times 10^{6}$ & 1.8 & 0.6680 to 2.932 & $* * * *$ & $<0.0001$ \\
\hline Normal NT vs. Both FF $1 \times 10^{6}$ & 1.6 & 0.4680 to 2.732 & $* * * *$ & $<0.0001$ \\
\hline Normal NT vs. Both FF $3 \times 10^{6}$ & 2.767 & 1.635 to 3.899 & $* * * *$ & $<0.0001$ \\
\hline Normal NT vs. Both INJ 1×10 & 1.233 & 0.1013 to 2.365 & $*$ & 0.015 \\
\hline Normal NT vs. Both INJ $3 \times 10^{6}$ & 1.967 & 0.8347 to 3.099 & $* * * *$ & $<0.0001$ \\
\hline Normal PBS FF vs. Normal FF 1x10 & 1.733 & 0.6013 to 2.865 & $* * * *$ & $<0.0001$ \\
\hline Normal PBS FF vs. Normal FF $3 \times 10^{6}$ & 2.133 & 1.001 to 3.265 & $* * * *$ & $<0.0001$ \\
\hline 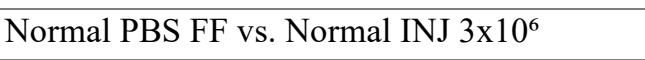 & 1.5 & 0.3680 to 2.632 & $* * *$ & 0.0003 \\
\hline Normal PBS FF vs. Colostrum FF 3x106 & 1.5 & 0.3680 to 2.632 & $* * *$ & 0.0003 \\
\hline Normal PBS FF vs. Colostrum INJ 3x10 & 2.333 & 1.201 to 3.465 & $* * * *$ & $<0.0001$ \\
\hline Normal PBS FF vs. C. sinensis FF $3 \times 10^{6}$ & 1.367 & 0.2347 to 2.499 & $* *$ & 0.0024 \\
\hline Normal PBS FF vs. C. sinensis INJ $3 \times 10^{6}$ & 1.8 & 0.6680 to 2.932 & $* * * *$ & $<0.0001$ \\
\hline Normal PBS FF vs. Both FF $1 \times 10^{6}$ & 1.6 & 0.4680 to 2.732 & $* * * *$ & $<0.0001$ \\
\hline Normal PBS FF vs. Both FF $3 \times 10^{6}$ & 2.767 & 1.635 to 3.899 & $* * * *$ & $<0.0001$ \\
\hline Normal PBS FF vs. Both INJ 1x10 & 1.233 & 0.1013 to 2.365 & $*$ & 0.015 \\
\hline Normal PBS FF vs. Both INJ 3x106 & 1.967 & 0.8347 to 3.099 & $* * * *$ & $<0.0001$ \\
\hline Normal FF 1x10 vs. Normal PBS INJ & -1.733 & -2.865 to -0.6013 & $* * * *$ & $<0.0001$ \\
\hline Normal FF 1x10 ${ }^{6}$ vs. Colostrum NT & -1.6 & -2.732 to -0.4680 & $* * * *$ & $<0.0001$ \\
\hline Normal FF 1x10 ${ }^{6}$ vs. Colostrum PBS FF & -1.3 & -2.432 to -0.1680 & $* *$ & 0.0062 \\
\hline Normal FF $1 \times 10^{6}$ vs. Colostrm PBS INJ & -1.6 & -2.732 to -0.4680 & $* * * *$ & $<0.0001$ \\
\hline Normal FF $1 \times 10^{6}$ vs. C. sinensis NT & -1.767 & -2.899 to -0.6347 & $* * * *$ & $<0.0001$ \\
\hline Normal FF $1 \times 10^{6}$ vs. C. sinensis PBS FF & -1.4 & -2.532 to -0.2680 & $* *$ & 0.0015 \\
\hline Normal FF $1 \times 10^{6}$ vs. C. sinensis $\mathrm{PBS}$ INJ & -1.7 & -2.832 to -0.5680 & $* * * *$ & $<0.0001$ \\
\hline Normal FF $1 \times 10^{6}$ vs. Both NT & -1.733 & -2.865 to -0.6013 & $* * * *$ & $<0.0001$ \\
\hline Normal FF $1 \times 10^{6}$ vs. Both PBS FF & -1.733 & -2.865 to -0.6013 & $* * * *$ & $<0.0001$ \\
\hline Normal FF 1x106 vs. Both PBS INJ & -1.733 & -2.865 to -0.6013 & $* * * *$ & $<0.0001$ \\
\hline Normal FF $3 \times 10^{6}$ vs. Normal PBS INJ & -2.133 & -3.265 to -1.001 & $* * * *$ & $<0.0001$ \\
\hline Normal FF $3 \times 10^{6}$ vs. Normal INJ $1 \times 10^{6}$ & -1.133 & -2.265 to -0.001326 & $*$ & 0.0493 \\
\hline Normal FF 3x10 16 vs. Colostrum NT & -2 & -3.132 to -0.8680 & $* * * *$ & $<0.0001$ \\
\hline Normal FF 3x10 6 vs. Colostrum PBS FF & -1.7 & -2.832 to -0.5680 & $* * * *$ & $<0.0001$ \\
\hline Normal FF 3x10 6 vs. Colostrum FF $1 \times 10^{6}$ & -1.3 & -2.432 to -0.1680 & $* *$ & 0.0062 \\
\hline Normal FF $3 \times 10^{6}$ vs. Colostrm PBS INJ & -2 & -3.132 to -0.8680 & $* * * *$ & $<0.0001$ \\
\hline Normal FF $3 \times 10^{6}$ vs. Colostrum INJ 1x10 & -1.133 & -2.265 to -0.001326 & $*$ & 0.0493 \\
\hline Normal FF $3 \times 10^{6}$ vs. C. sinensis $\mathrm{NT}$ & -2.167 & -3.299 to -1.035 & $* * * *$ & $<0.0001$ \\
\hline Normal FF $3 \times 10^{6}$ vs. C. sinensis $\mathrm{PBS} F \mathrm{FF}$ & -1.8 & -2.932 to -0.6680 & $* * * *$ & $<0.0001$ \\
\hline
\end{tabular}




\begin{tabular}{|c|c|c|c|c|}
\hline Normal FF $3 \times 10^{6}$ vs. C. sinensis $\mathrm{FF} 1 \times 10^{6}$ & -1.133 & -2.265 to -0.001326 & $*$ & 0.0493 \\
\hline Normal FF $3 \times 10^{6}$ vs. $C$. sinensis $\mathrm{PBS}$ INJ & -2.1 & -3.232 to -0.9680 & $* * * *$ & $<0.0001$ \\
\hline Normal FF $3 \times 10^{6}$ vs. C. sinensis INJ $1 \times 10^{6}$ & -1.2 & -2.332 to -0.06799 & $*$ & 0.0227 \\
\hline Normal FF $3 \times 10^{6}$ vs. Both NT & -2.133 & -3.265 to -1.001 & $* * * *$ & $<0.0001$ \\
\hline Normal FF $3 \times 10^{6}$ vs. Both PBS FF & -2.133 & -3.265 to -1.001 & $* * * *$ & $<0.0001$ \\
\hline Normal FF $3 \times 10^{6}$ vs. Both PBS INJ & -2.133 & -3.265 to -1.001 & $* * * *$ & $<0.0001$ \\
\hline Normal PBS INJ vs. Normal INJ $3 \times 10^{6}$ & 1.5 & 0.3680 to 2.632 & $* * *$ & 0.0003 \\
\hline 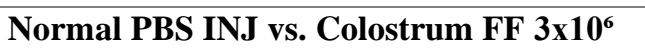 & 1.5 & 0.3680 to 2.632 & $* * *$ & 0.0003 \\
\hline Normal PBS INJ vs. Colostrum INJ 3x10 & 2.333 & 1.201 to 3.465 & $* * * *$ & $<0.0001$ \\
\hline Normal PBS INJ vs. $C$. sinensis $\mathrm{FF} 3 \times 10^{6}$ & 1.367 & 0.2347 to 2.499 & $* *$ & 0.0024 \\
\hline Normal PBS INJ vs. $C$. sinensis INJ $3 \times 10^{6}$ & 1.8 & 0.6680 to 2.932 & $* * * *$ & $<0.0001$ \\
\hline Normal PBS INJ vs. Both FF $1 \times 10^{6}$ & 1.6 & 0.4680 to 2.732 & $* * * *$ & $<0.0001$ \\
\hline Normal PBS INJ vs. Both FF $3 \times 10^{6}$ & 2.767 & 1.635 to 3.899 & $* * * *$ & $<0.0001$ \\
\hline Normal PBS INJ vs. Both INJ $1 \times 10^{6}$ & 1.233 & 0.1013 to 2.365 & $*$ & 0.015 \\
\hline Normal PBS INJ vs. Both INJ $3 \times 10^{6}$ & 1.967 & 0.8347 to 3.099 & $* * * *$ & $<0.0001$ \\
\hline $\begin{array}{l}\text { Normal INJ 1x10 } 6 \text { vs. Colostrum INJ } \\
3 \times 10^{6}\end{array}$ & 1.333 & 0.2013 to 2.465 & $* *$ & 0.0039 \\
\hline Normal INJ $1 \times 10^{6}$ vs. Both FF $3 \times 10^{6}$ & 1.767 & 0.6347 to 2.899 & $* * * *$ & $<0.0001$ \\
\hline Normal INJ 3x106 vs. Colostrum NT & -1.367 & -2.499 to -0.2347 & $* *$ & 0.0024 \\
\hline Normal INJ 3x10 ${ }^{6}$ vs. Colostrm PBS INJ & -1.367 & -2.499 to -0.2347 & $* *$ & 0.0024 \\
\hline Normal INJ $3 \times 10^{6}$ vs. C. sinensis NT & -1.533 & -2.665 to -0.4013 & $* * *$ & 0.0002 \\
\hline Normal INJ $3 \times 10^{6}$ vs. C. sinensis PBS FF & -1.167 & -2.299 to -0.03466 & $*$ & 0.0337 \\
\hline Normal INJ $3 \times 10^{6}$ vs. C. sinensis $\mathrm{PBS}$ INJ & -1.467 & -2.599 to -0.3347 & $* * *$ & 0.0005 \\
\hline Normal INJ $3 \times 10^{6}$ vs. Both NT & -1.5 & -2.632 to -0.3680 & $* * *$ & 0.0003 \\
\hline Normal INJ $3 \times 10^{6}$ vs. Both PBS FF & -1.5 & -2.632 to -0.3680 & $* * *$ & 0.0003 \\
\hline Normal INJ $3 \times 10^{6}$ vs. Both FF $3 \times 10^{6}$ & 1.267 & 0.1347 to 2.399 & $* *$ & 0.0097 \\
\hline Normal INJ 3x106 vs. Both PBS INJ & -1.5 & -2.632 to -0.3680 & $* * *$ & 0.0003 \\
\hline Colostrum NT vs. Colostrum FF 3x10 & 1.367 & 0.2347 to 2.499 & $* *$ & 0.0024 \\
\hline Colostrum NT vs. Colostrum INJ 3x10 & 2.2 & 1.068 to 3.332 & $* * * *$ & $<0.0001$ \\
\hline Colostrum NT vs. C. sinensis FF $3 \times 10^{6}$ & 1.233 & 0.1013 to 2.365 & $*$ & 0.015 \\
\hline Colostrum NT vs. C. sinensis INJ 3x10 & 1.667 & 0.5347 to 2.799 & $* * * *$ & $<0.0001$ \\
\hline Colostrum NT vs. Both FF $1 \times 10^{6}$ & 1.467 & 0.3347 to 2.599 & $* * *$ & 0.0005 \\
\hline Colostrum NT vs. Both FF $3 \times 10^{6}$ & 2.633 & 1.501 to 3.765 & $* * * *$ & $<0.0001$ \\
\hline Colostrum NT vs. Both INJ $3 \times 10^{6}$ & 1.833 & 0.7013 to 2.965 & $* * * *$ & $<0.0001$ \\
\hline $\begin{array}{l}\text { Colostrum PBS FF vs. Colostrum INJ } \\
3 \times 10^{6}\end{array}$ & 1.9 & 0.7680 to 3.032 & $* * * *$ & $<0.0001$ \\
\hline Colostrum PBS FF vs. C. sinensis INJ $3 \times 10^{6}$ & 1.367 & 0.2347 to 2.499 & $* *$ & 0.0024 \\
\hline Colostrum PBS FF vs. Both FF 1x106 & 1.167 & 0.03466 to 2.299 & $*$ & 0.0337 \\
\hline 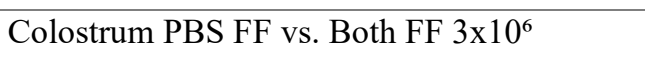 & 2.333 & 1.201 to 3.465 & $* * * *$ & $<0.0001$ \\
\hline 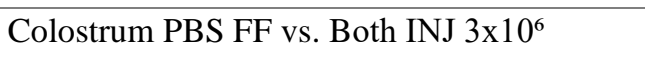 & 1.533 & 0.4013 to 2.665 & $* * *$ & 0.0002 \\
\hline $\begin{array}{l}\text { Colostrum FF 1x10 }{ }^{6} \text { vs. Colostrum INJ } \\
3 \times 10^{6}\end{array}$ & 1.5 & 0.3680 to 2.632 & $* * *$ & 0.0003 \\
\hline Colostrum FF $1 \times 10^{6}$ vs. Both FF $3 \times 10^{6}$ & 1.933 & 0.8013 to 3.065 & $* * * *$ & $<0.0001$ \\
\hline 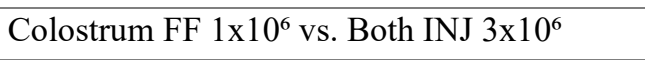 & 1.133 & 0.001326 to 2.265 & $*$ & 0.0493 \\
\hline $\begin{array}{l}\text { Colostrum FF 3x10 vs. Colostrm PBS } \\
\text { INJ }\end{array}$ & -1.367 & -2.499 to -0.2347 & $* *$ & 0.0024 \\
\hline Colostrum FF $3 \times 10^{6}$ vs. C. sinensis $\mathrm{NT}$ & -1.533 & -2.665 to -0.4013 & $* * *$ & 0.0002 \\
\hline Colostrum FF $3 \times 10^{6}$ vs. C. sinensis $\mathrm{PBS} F \mathrm{FF}$ & -1.167 & -2.299 to -0.03466 & $*$ & 0.0337 \\
\hline
\end{tabular}




\begin{tabular}{|c|c|c|c|c|}
\hline $\begin{array}{l}\text { Colostrum FF } 3 \times 10^{6} \text { vs. C. sinensis PBS } \\
\text { INJ }\end{array}$ & -1.467 & -2.599 to -0.3347 & $* * *$ & 0.0005 \\
\hline Colostrum FF $3 \times 10^{6}$ vs. Both NT & -1.5 & -2.632 to -0.3680 & $* * *$ & 0.0003 \\
\hline Colostrum FF $3 \times 10^{6}$ vs. Both PBS FF & -1.5 & -2.632 to -0.3680 & $* * *$ & 0.0003 \\
\hline Colostrum FF $3 \times 10^{6}$ vs. Both FF $3 \times 10^{6}$ & 1.267 & 0.1347 to 2.399 & $* *$ & 0.0097 \\
\hline Colostrum FF $3 \times 10^{6}$ vs. Both PBS INJ & -1.5 & -2.632 to -0.3680 & $* * *$ & 0.0003 \\
\hline $\begin{array}{l}\text { Colostrm PBS INJ vs. Colostrum INJ } \\
3 \times 10^{6}\end{array}$ & 2.2 & 1.068 to 3.332 & $* * * *$ & $<0.0001$ \\
\hline Colostrm PBS INJ vs. $C$. sinensis FF $3 \times 10^{6}$ & 1.233 & 0.1013 to 2.365 & $*$ & 0.015 \\
\hline Colostrm PBS INJ vs. $C$. sinensis INJ $3 \times 10^{6}$ & 1.667 & 0.5347 to 2.799 & $* * * *$ & $<0.0001$ \\
\hline Colostrm PBS INJ vs. Both FF $1 \times 10^{6}$ & 1.467 & 0.3347 to 2.599 & $* * *$ & 0.0005 \\
\hline Colostrm PBS INJ vs. Both FF $3 \times 10^{6}$ & 2.633 & 1.501 to 3.765 & $* * * *$ & $<0.0001$ \\
\hline Colostrm PBS INJ vs. Both INJ $3 \times 10^{6}$ & 1.833 & 0.7013 to 2.965 & $* * * *$ & $<0.0001$ \\
\hline $\begin{array}{l}\text { Colostrum INJ } 1 \times 10^{6} \text { vs. Colostrum INJ } \\
3 \times 10^{6}\end{array}$ & 1.333 & 0.2013 to 2.465 & $* *$ & 0.0039 \\
\hline Colostrum INJ $1 \times 10^{6}$ vs. Both FF $3 \times 10^{6}$ & 1.767 & 0.6347 to 2.899 & $* * * *$ & $<0.0001$ \\
\hline Colostrum INJ 3x106 vs. C. sinensis NT & -2.367 & -3.499 to -1.235 & $* * * *$ & $<0.0001$ \\
\hline $\begin{array}{l}\text { Colostrum INJ 3x106 vs. C. sinensis PBS } \\
\text { FF }\end{array}$ & -2 & -3.132 to -0.8680 & $* * * *$ & $<0.0001$ \\
\hline $\begin{array}{l}\text { Colostrum INJ } 3 \times 10^{6} \text { vs. C. sinensis FF } \\
1 \times 10^{6}\end{array}$ & -1.333 & -2.465 to -0.2013 & $* *$ & 0.0039 \\
\hline $\begin{array}{l}\text { Colostrum INJ } 3 \times 10^{6} \text { vs. C. sinensis PBS } \\
\text { INJ }\end{array}$ & -2.3 & -3.432 to -1.168 & $* * * *$ & $<0.0001$ \\
\hline $\begin{array}{l}\text { Colostrum INJ } 3 \times 10^{6} \text { vs. C. sinensis INJ } \\
1 \times 10^{6}\end{array}$ & -1.4 & -2.532 to -0.2680 & $* *$ & 0.0015 \\
\hline Colostrum INJ $3 \times 10^{6}$ vs. Both NT & -2.333 & -3.465 to -1.201 & $* * * *$ & $<0.0001$ \\
\hline Colostrum INJ $3 \times 10^{6}$ vs. Both PBS FF & -2.333 & -3.465 to -1.201 & $* * * *$ & $<0.0001$ \\
\hline Colostrum INJ $3 \times 10^{6}$ vs. Both PBS INJ & -2.333 & -3.465 to -1.201 & $* * * *$ & $<0.0001$ \\
\hline C. sinensis $\mathrm{NT}$ vs. C. sinensis FF $3 \times 10^{6}$ & 1.4 & 0.2680 to 2.532 & $* *$ & 0.0015 \\
\hline C. sinensis $\mathrm{NT}$ vs. $C$. sinensis $\mathrm{INJ} 3 \times 10^{6}$ & 1.833 & 0.7013 to 2.965 & $* * * *$ & $<0.0001$ \\
\hline C. sinensis NT vs. Both FF $1 \times 10^{6}$ & 1.633 & 0.5013 to 2.765 & $* * * *$ & $<0.0001$ \\
\hline C. sinensis NT vs. Both FF $3 \times 10^{6}$ & 2.8 & 1.668 to 3.932 & $* * * *$ & $<0.0001$ \\
\hline C. sinensis NT vs. Both INJ $1 \times 10^{6}$ & 1.267 & 0.1347 to 2.399 & $* *$ & 0.0097 \\
\hline C. sinensis NT vs. Both INJ $3 \times 10^{6}$ & 2 & 0.8680 to 3.132 & $* * * *$ & $<0.0001$ \\
\hline C. sinensis $\mathrm{PBS}$ FF vs. C. sinensis $\mathrm{INJ} 3 \times 10^{6}$ & 1.467 & 0.3347 to 2.599 & $* * *$ & 0.0005 \\
\hline C. sinensis $\mathrm{PBS}$ FF vs. Both FF $1 \times 10^{6}$ & 1.267 & 0.1347 to 2.399 & $* *$ & 0.0097 \\
\hline C. sinensis PBS FF vs. Both FF $3 \times 10^{6}$ & 2.433 & 1.301 to 3.565 & $* * * *$ & $<0.0001$ \\
\hline C. sinensis PBS FF vs. Both INJ $3 \times 10^{6}$ & 1.633 & 0.5013 to 2.765 & $* * * *$ & $<0.0001$ \\
\hline C. sinensis FF $1 \times 10^{6}$ vs. Both FF $3 \times 10^{6}$ & 1.767 & 0.6347 to 2.899 & $* * * *$ & $<0.0001$ \\
\hline C. sinensis FF $3 \times 10^{6}$ vs. C. sinensis $\mathrm{PBS}$ INJ & -1.333 & -2.465 to -0.2013 & $* *$ & 0.0039 \\
\hline C. sinensis FF $3 \times 10^{6}$ vs. Both NT & -1.367 & -2.499 to -0.2347 & $* *$ & 0.0024 \\
\hline C. sinensis FF $3 \times 10^{6}$ vs. Both PBS FF & -1.367 & -2.499 to -0.2347 & $* *$ & 0.0024 \\
\hline C. sinensis FF $3 \times 10^{6}$ vs. Both FF $3 \times 10^{6}$ & 1.4 & 0.2680 to 2.532 & $* *$ & 0.0015 \\
\hline C. sinensis FF $3 \times 10^{6}$ vs. Both PBS INJ & -1.367 & -2.499 to -0.2347 & $* *$ & 0.0024 \\
\hline $\begin{array}{l}\text { C. sinensis PBS INJ vs. C. sinensis INJ } \\
3 \times 10^{6}\end{array}$ & 1.767 & 0.6347 to 2.899 & $* * * *$ & $<0.0001$ \\
\hline C. sinensis PBS INJ vs. Both FF $1 \times 10^{6}$ & 1.567 & 0.4347 to 2.699 & $* * *$ & 0.0001 \\
\hline C. sinensis $\mathrm{PBS}$ INJ vs. Both FF $3 \times 10^{6}$ & 2.733 & 1.601 to 3.865 & $* * * *$ & $<0.0001$ \\
\hline C. sinensis $\mathrm{PBS}$ INJ vs. Both INJ $1 \times 10^{6}$ & 1.2 & 0.06799 to 2.332 & $*$ & 0.0227 \\
\hline C. sinensis PBS INJ vs. Both INJ $3 \times 10^{6}$ & 1.933 & 0.8013 to 3.065 & $* * * *$ & $<0.0001$ \\
\hline
\end{tabular}




\begin{tabular}{|c|c|c|c|c|}
\hline C. sinensis INJ $1 \times 10^{6}$ vs. Both FF $3 \times 10^{6}$ & 1.833 & 0.7013 to 2.965 & $* * * *$ & $<0.0001$ \\
\hline C. sinensis INJ $3 \times 10^{6}$ vs. Both NT & -1.8 & -2.932 to -0.6680 & $* * * *$ & $<0.0001$ \\
\hline C. sinensis INJ $3 \times 10^{6}$ vs. Both PBS FF & -1.8 & -2.932 to -0.6680 & $* * * *$ & $<0.0001$ \\
\hline C. sinensis INJ $3 \times 10^{6}$ vs. Both PBS INJ & -1.8 & -2.932 to -0.6680 & $* * * *$ & $<0.0001$ \\
\hline Both NT vs. Both FF $1 \times 10^{6}$ & 1.6 & 0.4680 to 2.732 & $* * * *$ & $<0.0001$ \\
\hline Both NT vs. Both FF $3 \times 10^{6}$ & 2.767 & 1.635 to 3.899 & $* * * *$ & $<0.0001$ \\
\hline Both NT vs. Both INJ $1 \times 10^{6}$ & 1.233 & 0.1013 to 2.365 & $*$ & 0.015 \\
\hline Both NT vs. Both INJ $3 \times 10^{6}$ & 1.967 & 0.8347 to 3.099 & $* * * *$ & $<0.0001$ \\
\hline Both PBS FF vs. Both FF $1 \times 10^{6}$ & 1.6 & 0.4680 to 2.732 & $* * * *$ & $<0.0001$ \\
\hline Both PBS FF vs. Both FF $3 \times 10^{6}$ & 2.767 & 1.635 to 3.899 & $* * * *$ & $<0.0001$ \\
\hline Both PBS FF vs. Both INJ $1 \times 10^{6}$ & 1.233 & 0.1013 to 2.365 & $*$ & 0.015 \\
\hline Both PBS FF vs. Both INJ $3 \times 10^{6}$ & 1.967 & 0.8347 to 3.099 & $* * * *$ & $<0.0001$ \\
\hline Both FF $1 \times 10^{6}$ vs. Both FF $3 \times 10^{6}$ & 1.167 & 0.03466 to 2.299 & $*$ & 0.0337 \\
\hline Both FF $1 \times 10^{6}$ vs. Both PBS INJ & -1.6 & -2.732 to -0.4680 & $* * * *$ & $<0.0001$ \\
\hline Both FF $3 \times 10^{6}$ vs. Both PBS INJ & -2.767 & -3.899 to -1.635 & $* * * *$ & $<0.0001$ \\
\hline Both FF $3 \times 10^{6}$ vs. Both INJ $1 \times 10^{6}$ & -1.533 & -2.665 to -0.4013 & $* * *$ & 0.0002 \\
\hline Both PBS INJ vs. Both INJ 1x10 & 1.233 & 0.1013 to 2.365 & $*$ & 0.015 \\
\hline \multicolumn{5}{|l|}{72} \\
\hline Normal NT vs. Normal FF $1 \times 10^{6}$ & 1.833 & 0.7013 to 2.965 & $* * * *$ & $<0.0001$ \\
\hline Normal NT vs. Normal FF $3 \times 10^{6}$ & 2.2 & 1.068 to 3.332 & $* * * *$ & $<0.0001$ \\
\hline Normal NT vs. Normal INJ $1 \times 10^{6}$ & 1.133 & 0.001326 to 2.265 & $*$ & 0.0493 \\
\hline Normal NT vs. Normal INJ $3 \times 10^{6}$ & 1.8 & 0.6680 to 2.932 & $* * * *$ & $<0.0001$ \\
\hline Normal NT vs. Colostrum FF 3x10 & 1.5 & 0.3680 to 2.632 & $* * *$ & 0.0003 \\
\hline Normal NT vs. Colostrum INJ 3x10 & 2.467 & 1.335 to 3.599 & $* * * *$ & $<0.0001$ \\
\hline Normal NT vs. C. sinensis FF $1 \times 10^{6}$ & 1.167 & 0.03466 to 2.299 & $*$ & 0.0337 \\
\hline Normal NT vs. C. sinensis FF $3 \times 10^{6}$ & 1.533 & 0.4013 to 2.665 & $* * *$ & 0.0002 \\
\hline Normal NT vs. C. sinensis INJ $3 \times 10^{6}$ & 1.933 & 0.8013 to 3.065 & $* * * *$ & $<0.0001$ \\
\hline Normal NT vs. Both FF $1 \times 10^{6}$ & 1.833 & 0.7013 to 2.965 & $* * * *$ & $<0.0001$ \\
\hline Normal NT vs. Both FF $3 \times 10^{6}$ & 2.767 & 1.635 to 3.899 & $* * * *$ & $<0.0001$ \\
\hline Normal NT vs. Both INJ $1 \times 10^{6}$ & 1.533 & 0.4013 to 2.665 & $* * *$ & 0.0002 \\
\hline Normal NT vs. Both INJ 3x10 & 2.167 & 1.035 to 3.299 & $* * * *$ & $<0.0001$ \\
\hline Normal PBS FF vs. Normal FF $1 \times 10^{6}$ & 1.833 & 0.7013 to 2.965 & $* * * *$ & $<0.0001$ \\
\hline Normal PBS FF vs. Normal FF $3 \times 10^{6}$ & 2.2 & 1.068 to 3.332 & $* * * *$ & $<0.0001$ \\
\hline Normal PBS FF vs. Normal INJ $1 \times 10^{6}$ & 1.133 & 0.001326 to 2.265 & $*$ & 0.0493 \\
\hline Normal PBS FF vs. Normal INJ $3 \times 10^{6}$ & 1.8 & 0.6680 to 2.932 & $* * * *$ & $<0.0001$ \\
\hline Normal PBS FF vs. Colostrum FF 3x10 & 1.5 & 0.3680 to 2.632 & $* * *$ & 0.0003 \\
\hline Normal PBS FF vs. Colostrum INJ 3x10 & 2.467 & 1.335 to 3.599 & $* * * *$ & $<0.0001$ \\
\hline Normal PBS FF vs. C. sinensis FF $1 \times 10^{6}$ & 1.167 & 0.03466 to 2.299 & $*$ & 0.0337 \\
\hline Normal PBS FF vs. C. sinensis FF $3 \times 10^{6}$ & 1.533 & 0.4013 to 2.665 & $* * *$ & 0.0002 \\
\hline Normal PBS FF vs. C. sinensis INJ $3 \times 10^{6}$ & 1.933 & 0.8013 to 3.065 & $* * * *$ & $<0.0001$ \\
\hline Normal PBS FF vs. Both FF $1 \times 10^{6}$ & 1.833 & 0.7013 to 2.965 & $* * * *$ & $<0.0001$ \\
\hline Normal PBS FF vs. Both FF 3x10 & 2.767 & 1.635 to 3.899 & $* * * *$ & $<0.0001$ \\
\hline Normal PBS FF vs. Both INJ $1 \times 10^{6}$ & 1.533 & 0.4013 to 2.665 & $* * *$ & 0.0002 \\
\hline Normal PBS FF vs. Both INJ 3x106 & 2.167 & 1.035 to 3.299 & $* * * *$ & $<0.0001$ \\
\hline Normal FF $1 \times 10^{6}$ vs. Normal PBS INJ & -1.833 & -2.965 to -0.7013 & $* * * *$ & $<0.0001$ \\
\hline
\end{tabular}




\begin{tabular}{|c|c|c|c|c|}
\hline Normal FF 1x10 vs. Colostrum NT & -1.7 & -2.832 to -0.5680 & $* * * *$ & $<0.0001$ \\
\hline Normal FF 1x10 ${ }^{6}$ vs. Colostrum PBS FF & -1.433 & -2.565 to -0.3013 & $* * *$ & 0.0009 \\
\hline Normal FF 1x106 vs. Colostrm PBS INJ & -1.7 & -2.832 to -0.5680 & $* * * *$ & $<0.0001$ \\
\hline Normal FF $1 \times 10^{6}$ vs. C. sinensis $\mathrm{NT}$ & -1.7 & -2.832 to -0.5680 & $* * * *$ & $<0.0001$ \\
\hline Normal FF $1 \times 10^{6}$ vs. C. sinensis $\mathrm{PBS}$ FF & -1.6 & -2.732 to -0.4680 & $* * * *$ & $<0.0001$ \\
\hline Normal FF $1 \times 10^{6}$ vs. C. sinensis $\mathrm{PBS}$ INJ & -1.8 & -2.932 to -0.6680 & $* * * *$ & $<0.0001$ \\
\hline Normal FF $1 \times 10^{6}$ vs. Both NT & -1.833 & -2.965 to -0.7013 & $* * * *$ & $<0.0001$ \\
\hline Normal FF $1 \times 10^{6}$ vs. Both PBS FF & -1.833 & -2.965 to -0.7013 & $* * * *$ & $<0.0001$ \\
\hline Normal FF $1 \times 10^{6}$ vs. Both PBS INJ & -1.833 & -2.965 to -0.7013 & $* * * *$ & $<0.0001$ \\
\hline Normal FF $3 \times 10^{6}$ vs. Normal PBS INJ & -2.2 & -3.332 to -1.068 & $* * * *$ & $<0.0001$ \\
\hline Normal FF 3x10 $10^{6}$ v. Colostrum NT & -2.067 & -3.199 to -0.9347 & $* * * *$ & $<0.0001$ \\
\hline Normal FF 3x10 $10^{6}$ s. Colostrum PBS FF & -1.8 & -2.932 to -0.6680 & $* * * *$ & $<0.0001$ \\
\hline Normal FF $3 \times 10^{6}$ vs. Colostrm PBS INJ & -2.067 & -3.199 to -0.9347 & $* * * *$ & $<0.0001$ \\
\hline Normal FF 3x10 vs. Colostrum INJ $1 \times 10^{6}$ & -1.2 & -2.332 to -0.06799 & $*$ & 0.0227 \\
\hline Normal FF $3 \times 10^{6}$ vs. $C$. sinensis $\mathrm{NT}$ & -2.067 & -3.199 to -0.9347 & $* * * *$ & $<0.0001$ \\
\hline Normal FF $3 \times 10^{6}$ vs. C. sinensis $\mathrm{PBS}$ FF & -1.967 & -3.099 to -0.8347 & $* * * *$ & $<0.0001$ \\
\hline Normal FF $3 \times 10^{6}$ vs. C. sinensis PBS INJ & -2.167 & -3.299 to -1.035 & $* * * *$ & $<0.0001$ \\
\hline Normal FF $3 \times 10^{6}$ vs. C. sinensis INJ $1 \times 10^{6}$ & -1.2 & -2.332 to -0.06799 & $*$ & 0.0227 \\
\hline Normal FF $3 \times 10^{6}$ vs. Both NT & -2.2 & -3.332 to -1.068 & $* * * *$ & $<0.0001$ \\
\hline Normal FF $3 \times 10^{6}$ vs. Both PBS FF & -2.2 & -3.332 to -1.068 & $* * * *$ & $<0.0001$ \\
\hline Normal FF $3 \times 10^{6}$ vs. Both PBS INJ & -2.2 & -3.332 to -1.068 & $* * * *$ & $<0.0001$ \\
\hline Normal PBS INJ vs. Normal INJ 1x10 & 1.133 & 0.001326 to 2.265 & $*$ & 0.0493 \\
\hline Normal PBS INJ vs. Normal INJ $3 \times 10^{6}$ & 1.8 & 0.6680 to 2.932 & $* * * *$ & $<0.0001$ \\
\hline Normal PBS INJ vs. Colostrum FF 3x10 & 1.5 & 0.3680 to 2.632 & $* * *$ & 0.0003 \\
\hline Normal PBS INJ vs. Colostrum INJ 3x10 & 2.467 & 1.335 to 3.599 & $* * * *$ & $<0.0001$ \\
\hline Normal PBS INJ vs. C. sinensis FF $1 \times 10^{6}$ & 1.167 & 0.03466 to 2.299 & $*$ & 0.0337 \\
\hline Normal PBS INJ vs. C. sinensis FF $3 \times 10^{6}$ & 1.533 & 0.4013 to 2.665 & $* * *$ & 0.0002 \\
\hline Normal PBS INJ vs. C. sinensis INJ $3 \times 10^{6}$ & 1.933 & 0.8013 to 3.065 & $* * * *$ & $<0.0001$ \\
\hline Normal PBS INJ vs. Both FF $1 \times 10^{6}$ & 1.833 & 0.7013 to 2.965 & $* * * *$ & $<0.0001$ \\
\hline Normal PBS INJ vs. Both FF $3 \times 10^{6}$ & 2.767 & 1.635 to 3.899 & $* * * *$ & $<0.0001$ \\
\hline Normal PBS INJ vs. Both INJ $1 \times 10^{6}$ & 1.533 & 0.4013 to 2.665 & $* * *$ & 0.0002 \\
\hline 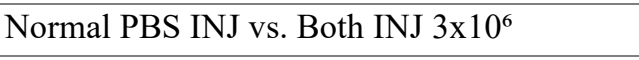 & 2.167 & 1.035 to 3.299 & $* * * *$ & $<0.0001$ \\
\hline $\begin{array}{l}\text { Normal INJ } 1 \times 10^{6} \text { vs. Colostrum INJ } \\
3 \times 10^{6}\end{array}$ & 1.333 & 0.2013 to 2.465 & $* *$ & 0.0039 \\
\hline Normal INJ $1 \times 10^{6}$ vs. Both NT & -1.133 & -2.265 to -0.001326 & $*$ & 0.0493 \\
\hline Normal INJ $1 \times 10^{6}$ vs. Both PBS FF & -1.133 & -2.265 to -0.001326 & $*$ & 0.0493 \\
\hline Normal INJ $1 \times 10^{6}$ vs. Both FF $3 \times 10^{6}$ & 1.633 & 0.5013 to 2.765 & $* * * *$ & $<0.0001$ \\
\hline Normal INJ 1x106 vs. Both PBS INJ & -1.133 & -2.265 to -0.001326 & $*$ & 0.0493 \\
\hline Normal INJ 3x10 6 vs. Colostrum NT & -1.667 & -2.799 to -0.5347 & $* * * *$ & $<0.0001$ \\
\hline Normal INJ 3x10 ${ }^{6}$ vs. Colostrum PBS FF & -1.4 & -2.532 to -0.2680 & $* *$ & 0.0015 \\
\hline Normal INJ 3x10 6 vs. Colostrm PBS INJ & -1.667 & -2.799 to -0.5347 & $* * * *$ & $<0.0001$ \\
\hline Normal INJ $3 \times 10^{6}$ vs. C. sinensis NT & -1.667 & -2.799 to -0.5347 & $* * * *$ & $<0.0001$ \\
\hline Normal INJ $3 \times 10^{6}$ vs. C. sinensis PBS FF & -1.567 & -2.699 to -0.4347 & $* * *$ & 0.0001 \\
\hline Normal INJ $3 \times 10^{6}$ vs. C. sinensis PBS INJ & -1.767 & -2.899 to -0.6347 & $* * * *$ & $<0.0001$ \\
\hline Normal INJ $3 \times 10^{6}$ vs. Both NT & -1.8 & -2.932 to -0.6680 & $* * * *$ & $<0.0001$ \\
\hline Normal INJ $3 \times 10^{6}$ vs. Both PBS FF & -1.8 & -2.932 to -0.6680 & $* * * *$ & $<0.0001$ \\
\hline Normal INJ $3 \times 10^{6}$ vs. Both PBS INJ & -1.8 & -2.932 to -0.6680 & $* * * *$ & $<0.0001$ \\
\hline
\end{tabular}




\begin{tabular}{|c|c|c|c|c|}
\hline Colostrum NT vs. Colostrum FF 3x10 & 1.367 & 0.2347 to 2.499 & $* *$ & 0.0024 \\
\hline Colostrum NT vs. Colostrum INJ 3x10 & 2.333 & 1.201 to 3.465 & $* * * *$ & $<0.0001$ \\
\hline Colostrum NT vs. $C$. sinensis FF $3 \times 10^{6}$ & 1.4 & 0.2680 to 2.532 & $* *$ & 0.0015 \\
\hline Colostrum NT vs. $C$. sinensis INJ 3x10 & 1.8 & 0.6680 to 2.932 & $* * * *$ & $<0.0001$ \\
\hline Colostrum NT vs. Both FF $1 \times 10^{6}$ & 1.7 & 0.5680 to 2.832 & $* * * *$ & $<0.0001$ \\
\hline Colostrum NT vs. Both FF $3 \times 10^{6}$ & 2.633 & 1.501 to 3.765 & $* * * *$ & $<0.0001$ \\
\hline Colostrum NT vs. Both INJ 1x106 & 1.4 & 0.2680 to 2.532 & $* *$ & 0.0015 \\
\hline Colostrum NT vs. Both INJ $3 \times 10^{6}$ & 2.033 & 0.9013 to 3.165 & $* * * *$ & $<0.0001$ \\
\hline $\begin{array}{l}\text { Colostrum PBS FF vs. Colostrum INJ } \\
3 \times 10^{6}\end{array}$ & 2.067 & 0.9347 to 3.199 & $* * * *$ & $<0.0001$ \\
\hline Colostrum PBS FF vs. C. sinensis FF $3 \times 10^{6}$ & 1.133 & 0.001326 to 2.265 & $*$ & 0.0493 \\
\hline Colostrum PBS FF vs. C. sinensis INJ $3 \times 10^{6}$ & 1.533 & 0.4013 to 2.665 & $* * *$ & 0.0002 \\
\hline Colostrum PBS FF vs. Both FF 1x10 & 1.433 & 0.3013 to 2.565 & $* * *$ & 0.0009 \\
\hline Colostrum PBS FF vs. Both FF $3 \times 10^{6}$ & 2.367 & 1.235 to 3.499 & $* * * *$ & $<0.0001$ \\
\hline Colostrum PBS FF vs. Both INJ $1 \times 10^{6}$ & 1.133 & 0.001326 to 2.265 & $*$ & 0.0493 \\
\hline Colostrum PBS FF vs. Both INJ 3x106 & 1.767 & 0.6347 to 2.899 & $* * * *$ & $<0.0001$ \\
\hline $\begin{array}{l}\text { Colostrum FF } 1 \times 10^{6} \text { vs. Colostrum INJ } \\
3 \times 10^{6}\end{array}$ & 1.367 & 0.2347 to 2.499 & $* *$ & 0.0024 \\
\hline Colostrum FF $1 \times 10^{6}$ vs. Both FF $3 \times 10^{6}$ & 1.667 & 0.5347 to 2.799 & $* * * *$ & $<0.0001$ \\
\hline $\begin{array}{l}\text { Colostrum FF } 3 \times 10^{6} \text { vs. Colostrm PBS } \\
\text { INJ }\end{array}$ & -1.367 & -2.499 to -0.2347 & $* *$ & 0.0024 \\
\hline Colostrum FF $3 \times 10^{6}$ vs. C. sinensis NT & -1.367 & -2.499 to -0.2347 & $* *$ & 0.0024 \\
\hline Colostrum FF $3 \times 10^{6}$ vs. C. sinensis $\mathrm{PBS}$ FF & -1.267 & -2.399 to -0.1347 & $* *$ & 0.0097 \\
\hline $\begin{array}{l}\text { Colostrum FF } 3 \times 10^{6} \text { vs. C. sinensis PBS } \\
\text { INJ }\end{array}$ & -1.467 & -2.599 to -0.3347 & $* * *$ & 0.0005 \\
\hline Colostrum FF $3 \times 10^{6}$ vs. Both NT & -1.5 & -2.632 to -0.3680 & $* * *$ & 0.0003 \\
\hline Colostrum FF $3 \times 10^{6}$ vs. Both PBS FF & -1.5 & -2.632 to -0.3680 & $* * *$ & 0.0003 \\
\hline Colostrum FF $3 \times 10^{6}$ vs. Both FF $3 \times 10^{6}$ & 1.267 & 0.1347 to 2.399 & $* *$ & 0.0097 \\
\hline Colostrum FF $3 \times 10^{6}$ vs. Both PBS INJ & -1.5 & -2.632 to -0.3680 & $* * *$ & 0.0003 \\
\hline $\begin{array}{l}\text { Colostrm PBS INJ vs. Colostrum INJ } \\
3 \times 10^{6}\end{array}$ & 2.333 & 1.201 to 3.465 & $* * * *$ & $<0.0001$ \\
\hline Colostrm PBS INJ vs. C. sinensis FF $3 \times 10^{6}$ & 1.4 & 0.2680 to 2.532 & $* *$ & 0.0015 \\
\hline Colostrm PBS INJ vs. C. sinensis INJ $3 \times 10^{6}$ & 1.8 & 0.6680 to 2.932 & $* * * *$ & $<0.0001$ \\
\hline Colostrm PBS INJ vs. Both FF $1 \times 10^{6}$ & 1.7 & 0.5680 to 2.832 & $* * * *$ & $<0.0001$ \\
\hline Colostrm PBS INJ vs. Both FF $3 \times 10^{6}$ & 2.633 & 1.501 to 3.765 & $* * * *$ & $<0.0001$ \\
\hline Colostrm PBS INJ vs. Both INJ 1x10 & 1.4 & 0.2680 to 2.532 & $* *$ & 0.0015 \\
\hline 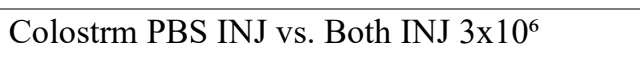 & 2.033 & 0.9013 to 3.165 & $* * * *$ & $<0.0001$ \\
\hline $\begin{array}{l}\text { Colostrum INJ } 1 \times 10^{6} \text { vs. Colostrum INJ } \\
3 \times 10^{6}\end{array}$ & 1.467 & 0.3347 to 2.599 & $* * *$ & 0.0005 \\
\hline Colostrum INJ $1 \times 10^{6}$ vs. Both FF $3 \times 10^{6}$ & 1.767 & 0.6347 to 2.899 & $* * * *$ & $<0.0001$ \\
\hline Colostrum INJ $1 \times 10^{6}$ vs. Both INJ $3 \times 10^{6}$ & 1.167 & 0.03466 to 2.299 & $*$ & 0.0337 \\
\hline Colostrum INJ $3 \times 10^{6}$ vs. C. sinensis NT & -2.333 & -3.465 to -1.201 & $* * * *$ & $<0.0001$ \\
\hline 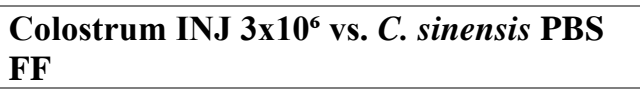 & -2.233 & -3.365 to -1.101 & $* * * *$ & $<0.0001$ \\
\hline $\begin{array}{l}\text { Colostrum INJ 3x10 vs. C. sinensis FF } \\
1^{6} 10^{6}\end{array}$ & -1.3 & -2.432 to -0.1680 & $* *$ & 0.0062 \\
\hline $\begin{array}{l}\text { Colostrum INJ 3x10 vs. C. sinensis } \mathrm{PBS} \\
\text { INJ }\end{array}$ & -2.433 & -3.565 to -1.301 & $* * * *$ & $<0.0001$ \\
\hline $\begin{array}{l}\text { Colostrum INJ } 3 \times 10^{6} \text { vs. } C \text {. sinensis INJ } \\
1 \times 10^{6}\end{array}$ & -1.467 & -2.599 to -0.3347 & $* * *$ & 0.0005 \\
\hline Colostrum INJ $3 \times 10^{6}$ vs. Both NT & -2.467 & -3.599 to -1.335 & $* * * *$ & $<0.0001$ \\
\hline
\end{tabular}




\begin{tabular}{|c|c|c|c|c|}
\hline Colostrum INJ $3 \times 10^{6}$ vs. Both PBS FF & -2.467 & -3.599 to -1.335 & $* * * *$ & $<0.0001$ \\
\hline Colostrum INJ $3 \times 10^{6}$ vs. Both PBS INJ & -2.467 & -3.599 to -1.335 & $* * * *$ & $<0.0001$ \\
\hline C. sinensis $\mathrm{NT}$ vs. C. sinensis FF $3 \times 10^{6}$ & 1.4 & 0.2680 to 2.532 & $* *$ & 0.0015 \\
\hline C. sinensis $\mathrm{NT}$ vs. C. sinensis $\mathrm{INJ} 3 \times 10^{6}$ & 1.8 & 0.6680 to 2.932 & $* * * *$ & $<0.0001$ \\
\hline C. sinensis $\mathrm{NT}$ vs. Both FF $1 \times 10^{6}$ & 1.7 & 0.5680 to 2.832 & $* * * *$ & $<0.0001$ \\
\hline C. sinensis NT vs. Both FF $3 \times 10^{6}$ & 2.633 & 1.501 to 3.765 & $* * * *$ & $<0.0001$ \\
\hline C. sinensis $\mathrm{NT}$ vs. Both INJ $1 \times 10^{6}$ & 1.4 & 0.2680 to 2.532 & $* *$ & 0.0015 \\
\hline C. sinensis NT vs. Both INJ $3 \times 10^{6}$ & 2.033 & 0.9013 to 3.165 & $* * * *$ & $<0.0001$ \\
\hline C. sinensis $\mathrm{PBS}$ FF vs. C. sinensis FF $3 \times 10^{6}$ & 1.3 & 0.1680 to 2.432 & $* *$ & 0.0062 \\
\hline C. sinensis PBS FF vs. C. sinensis INJ $3 \times 10^{6}$ & 1.7 & 0.5680 to 2.832 & $* * * *$ & $<0.0001$ \\
\hline C. sinensis PBS FF vs. Both FF $1 \times 10^{6}$ & 1.6 & 0.4680 to 2.732 & $* * * *$ & $<0.0001$ \\
\hline C. sinensis PBS FF vs. Both FF $3 \times 10^{6}$ & 2.533 & 1.401 to 3.665 & $* * * *$ & $<0.0001$ \\
\hline C. sinensis PBS FF vs. Both INJ $1 \times 10^{6}$ & 1.3 & 0.1680 to 2.432 & $* *$ & 0.0062 \\
\hline C. sinensis PBS FF vs. Both INJ $3 \times 10^{6}$ & 1.933 & 0.8013 to 3.065 & $* * * *$ & $<0.0001$ \\
\hline C. sinensis $\mathrm{FF} 1 \times 10^{6}$ vs. C. sinensis $\mathrm{PBS}$ INJ & -1.133 & -2.265 to -0.001326 & $*$ & 0.0493 \\
\hline C. sinensis FF $1 \times 10^{6}$ vs. Both NT & -1.167 & -2.299 to -0.03466 & $*$ & 0.0337 \\
\hline C. sinensis FF $1 \times 10^{6}$ vs. Both PBS FF & -1.167 & -2.299 to -0.03466 & $*$ & 0.0337 \\
\hline C. sinensis FF $1 \times 10^{6}$ vs. Both FF $3 \times 10^{6}$ & 1.6 & 0.4680 to 2.732 & $* * * *$ & $<0.0001$ \\
\hline C. sinensis FF $1 \times 10^{6}$ vs. Both PBS INJ & -1.167 & -2.299 to -0.03466 & $*$ & 0.0337 \\
\hline C. sinensis $\mathrm{FF} 3 \times 10^{6}$ vs. C. sinensis $\mathrm{PBS}$ INJ & -1.5 & -2.632 to -0.3680 & $* * *$ & 0.0003 \\
\hline C. sinensis FF $3 \times 10^{6}$ vs. Both NT & -1.533 & -2.665 to -0.4013 & $* * *$ & 0.0002 \\
\hline C. sinensis FF $3 \times 10^{6}$ vs. Both PBS FF & -1.533 & -2.665 to -0.4013 & *** & 0.0002 \\
\hline C. sinensis $\mathrm{FF} 3 \times 10^{6}$ vs. Both FF $3 \times 10^{6}$ & 1.233 & 0.1013 to 2.365 & $*$ & 0.015 \\
\hline C. sinensis FF $3 \times 10^{6}$ vs. Both PBS INJ & -1.533 & -2.665 to -0.4013 & $* * *$ & 0.0002 \\
\hline $\begin{array}{l}\text { C. sinensis } \mathrm{PBS} \text { INJ vs. C. sinensis INJ } \\
3 \times 10^{6}\end{array}$ & 1.9 & 0.7680 to 3.032 & $* * * *$ & $<0.0001$ \\
\hline C. sinensis PBS INJ vs. Both FF $1 \times 10^{6}$ & 1.8 & 0.6680 to 2.932 & $* * * *$ & $<0.0001$ \\
\hline C. sinensis PBS INJ vs. Both FF $3 \times 10^{6}$ & 2.733 & 1.601 to 3.865 & $* * * *$ & $<0.0001$ \\
\hline C. sinensis $\mathrm{PBS}$ INJ vs. Both INJ $1 \times 10^{6}$ & 1.5 & 0.3680 to 2.632 & $* * *$ & 0.0003 \\
\hline C. sinensis PBS INJ vs. Both INJ $3 \times 10^{6}$ & 2.133 & 1.001 to 3.265 & $* * * *$ & $<0.0001$ \\
\hline C. sinensis INJ $1 \times 10^{6}$ vs. Both FF $3 \times 10^{6}$ & 1.767 & 0.6347 to 2.899 & $* * * *$ & $<0.0001$ \\
\hline C. sinensis INJ $1 \times 10^{6}$ vs. Both INJ $3 \times 10^{6}$ & 1.167 & 0.03466 to 2.299 & $*$ & 0.0337 \\
\hline C. sinensis INJ $3 \times 10^{6}$ vs. Both NT & -1.933 & -3.065 to -0.8013 & $* * * *$ & $<0.0001$ \\
\hline C. sinensis INJ $3 \times 10^{6}$ vs. Both PBS FF & -1.933 & -3.065 to -0.8013 & $* * * *$ & $<0.0001$ \\
\hline C. sinensis INJ $3 \times 10^{6}$ vs. Both PBS INJ & -1.933 & -3.065 to -0.8013 & $* * * *$ & $<0.0001$ \\
\hline Both NT vs. Both FF 1x10 & 1.833 & 0.7013 to 2.965 & $* * * *$ & $<0.0001$ \\
\hline Both NT vs. Both FF $3 \times 10^{6}$ & 2.767 & 1.635 to 3.899 & $* * * *$ & $<0.0001$ \\
\hline Both NT vs. Both INJ $1 \times 10^{6}$ & 1.533 & 0.4013 to 2.665 & $* * *$ & 0.0002 \\
\hline Both NT vs. Both INJ $3 \times 10^{6}$ & 2.167 & 1.035 to 3.299 & $* * * *$ & $<0.0001$ \\
\hline Both PBS FF vs. Both FF 1x10 & 1.833 & 0.7013 to 2.965 & $* * * *$ & $<0.0001$ \\
\hline Both PBS FF vs. Both FF $3 \times 10^{6}$ & 2.767 & 1.635 to 3.899 & $* * * *$ & $<0.0001$ \\
\hline Both PBS FF vs. Both INJ $1 \times 10^{6}$ & 1.533 & 0.4013 to 2.665 & $* * *$ & 0.0002 \\
\hline Both PBS FF vs. Both INJ $3 \times 10^{6}$ & 2.167 & 1.035 to 3.299 & $* * * *$ & $<0.0001$ \\
\hline Both FF $1 \times 10^{6}$ vs. Both PBS INJ & -1.833 & -2.965 to -0.7013 & $* * * *$ & $<0.0001$ \\
\hline Both FF $3 \times 10^{6}$ vs. Both PBS INJ & -2.767 & -3.899 to -1.635 & $* * * *$ & $<0.0001$ \\
\hline Both FF $3 \times 10^{6}$ vs. Both INJ $1 \times 10^{6}$ & -1.233 & -2.365 to -0.1013 & $*$ & 0.015 \\
\hline Both PBS INJ vs. Both INJ $1 \times 10^{6}$ & 1.533 & 0.4013 to 2.665 & $* * *$ & 0.0002 \\
\hline
\end{tabular}


Table B4. Statistically significant results following a Tukey's multiple comparison test of larvae melanisation, following force feeding (FF) or injection (INJ) of $1 \times 10^{6}$ or $3 \times 10^{6}$ C. jejuni or PBS or the untreated control (NT)

\begin{tabular}{|c|c|c|c|c|}
\hline Time Elapsed (Hours) & Mean Diff. & $95.00 \%$ CI of diff. & Summary & $\begin{array}{l}\text { Adjusted P } \\
\text { Value }\end{array}$ \\
\hline \multicolumn{5}{|l|}{24} \\
\hline Normal NT vs. Normal FF $1 \times 10^{6}$ & 0.8667 & 0.01755 to 1.716 & $*$ & 0.0384 \\
\hline Normal NT vs. Normal FF $3 \times 10^{6}$ & 1.3 & 0.4509 to 2.149 & $* * * *$ & $<0.0001$ \\
\hline Normal NT vs. Colostrum INJ 3x10 & 1.167 & 0.3175 to 2.016 & $* * *$ & 0.0001 \\
\hline Normal NT vs. $C$. sinensis $\mathrm{FF} 3 \times 10^{6}$ & 0.8667 & 0.01755 to 1.716 & $*$ & 0.0384 \\
\hline Normal NT vs. $C$. sinensis INJ $3 \times 10^{6}$ & 1 & 0.1509 to 1.849 & $* *$ & 0.0039 \\
\hline Normal NT vs. Both FF $1 \times 10^{6}$ & 1.033 & 0.1842 to 1.882 & $* *$ & 0.0021 \\
\hline Normal NT vs. Both FF $3 \times 10^{6}$ & 1.6 & 0.7509 to 2.449 & $* * * *$ & $<0.0001$ \\
\hline 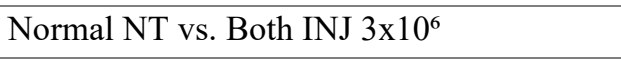 & 1.033 & 0.1842 to 1.882 & $* *$ & 0.0021 \\
\hline Normal PBS FF vs. Normal FF $1 \times 10^{6}$ & 0.8667 & 0.01755 to 1.716 & $*$ & 0.0384 \\
\hline Normal PBS FF vs. Normal FF $3 \times 10^{6}$ & 1.3 & 0.4509 to 2.149 & $* * * *$ & $<0.0001$ \\
\hline $\begin{array}{l}\text { Normal PBS FF vs. Colostrum INJ } \\
3 \times 10^{6}\end{array}$ & 1.167 & 0.3175 to 2.016 & $* * *$ & 0.0001 \\
\hline Normal PBS FF vs. C. sinensis FF $3 \times 10^{6}$ & 0.8667 & 0.01755 to 1.716 & $*$ & 0.0384 \\
\hline Normal PBS FF vs. C. sinensis INJ $3 \times 10^{6}$ & 1 & 0.1509 to 1.849 & $* *$ & 0.0039 \\
\hline Normal PBS FF vs. Both FF $1 \times 10^{6}$ & 1.033 & 0.1842 to 1.882 & $* *$ & 0.0021 \\
\hline Normal PBS FF vs. Both FF $3 \times 10^{6}$ & 1.6 & 0.7509 to 2.449 & $* * * *$ & $<0.0001$ \\
\hline Normal PBS FF vs. Both INJ 3x106 & 1.033 & 0.1842 to 1.882 & $* *$ & 0.0021 \\
\hline Normal FF $1 \times 10^{6}$ vs. Normal PBS INJ & -0.8667 & -1.716 to -0.01755 & $*$ & 0.0384 \\
\hline Normal FF $1 \times 10^{6}$ vs. C. sinensis NT & -0.8667 & -1.716 to -0.01755 & $*$ & 0.0384 \\
\hline Normal FF $1 \times 10^{6}$ vs. C. sinensis $\mathrm{PBS}$ INJ & -0.8667 & -1.716 to -0.01755 & $*$ & 0.0384 \\
\hline Normal FF $1 \times 10^{6}$ vs. Both NT & -0.8667 & -1.716 to -0.01755 & $*$ & 0.0384 \\
\hline Normal FF $1 \times 10^{6}$ vs. Both PBS FF & -0.8667 & -1.716 to -0.01755 & $*$ & 0.0384 \\
\hline Normal FF 1x106 vs. Both PBS INJ & -0.8667 & -1.716 to -0.01755 & $*$ & 0.0384 \\
\hline Normal FF $3 \times 10^{6}$ vs. Normal PBS INJ & -1.3 & -2.149 to -0.4509 & $* * * *$ & $<0.0001$ \\
\hline Normal FF 3x10 vs. Colostrum NT & -1.2 & -2.049 to -0.3509 & $* * * *$ & $<0.0001$ \\
\hline Normal FF 3x10 ${ }^{6}$ vs. Colostrum PBS FF & -1 & -1.849 to -0.1509 & $* *$ & 0.0039 \\
\hline Normal FF 3x106 vs. Colostrm PBS INJ & -1.2 & -2.049 to -0.3509 & $* * * *$ & $<0.0001$ \\
\hline Normal FF $3 \times 10^{6}$ vs. C. sinensis NT & -1.3 & -2.149 to -0.4509 & $* * * *$ & $<0.0001$ \\
\hline Normal FF $3 \times 10^{6}$ vs. C. sinensis PBS FF & -1.1 & -1.949 to -0.2509 & $* * *$ & 0.0005 \\
\hline Normal FF $3 \times 10^{6}$ vs. C. sinensis PBS INJ & -1.3 & -2.149 to -0.4509 & $* * * *$ & $<0.0001$ \\
\hline Normal FF $3 \times 10^{6}$ vs. C. sinensis INJ $1 \times 10^{6}$ & -0.9 & -1.749 to -0.05088 & $*$ & 0.0227 \\
\hline Normal FF 3x106 vs. Both NT & -1.3 & -2.149 to -0.4509 & $* * * *$ & $<0.0001$ \\
\hline
\end{tabular}




\begin{tabular}{|c|c|c|c|c|}
\hline Normal FF $3 \times 10^{6}$ vs. Both PBS FF & -1.3 & -2.149 to -0.4509 & $* * * *$ & $<0.0001$ \\
\hline Normal FF $3 \times 10^{6}$ vs. Both PBS INJ & -1.3 & -2.149 to -0.4509 & $* * * *$ & $<0.0001$ \\
\hline $\begin{array}{l}\text { Normal PBS INJ vs. Colostrum INJ } \\
3 \times 10^{6}\end{array}$ & 1.167 & 0.3175 to 2.016 & $* * *$ & 0.0001 \\
\hline Normal PBS INJ vs. C. sinensis FF $3 \times 10^{6}$ & 0.8667 & 0.01755 to 1.716 & $*$ & 0.0384 \\
\hline Normal PBS INJ vs. C. sinensis INJ $3 \times 10^{6}$ & 1 & 0.1509 to 1.849 & $* *$ & 0.0039 \\
\hline Normal PBS INJ vs. Both FF $1 \times 10^{6}$ & 1.033 & 0.1842 to 1.882 & $* *$ & 0.0021 \\
\hline Normal PBS INJ vs. Both FF $3 \times 10^{6}$ & 1.6 & 0.7509 to 2.449 & $* * * *$ & $<0.0001$ \\
\hline Normal PBS INJ vs. Both INJ $3 \times 10^{6}$ & 1.033 & 0.1842 to 1.882 & $* *$ & 0.0021 \\
\hline Normal INJ $1 \times 10^{6}$ vs. Both FF $3 \times 10^{6}$ & 1.133 & 0.2842 to 1.982 & $* * *$ & 0.0003 \\
\hline Normal INJ $3 \times 10^{6}$ vs. Both FF $3 \times 10^{6}$ & 0.9 & 0.05088 to 1.749 & $*$ & 0.0227 \\
\hline Colostrum NT vs. Colostrum INJ 3x10 & 1.067 & 0.2175 to 1.916 & $* *$ & 0.0011 \\
\hline Colostrum NT vs. $C$. sinensis INJ $3 \times 10^{6}$ & 0.9 & 0.05088 to 1.749 & $*$ & 0.0227 \\
\hline Colostrum NT vs. Both FF $1 \times 10^{6}$ & 0.9333 & 0.08422 to 1.782 & $*$ & 0.013 \\
\hline Colostrum NT vs. Both FF $3 \times 10^{6}$ & 1.5 & 0.6509 to 2.349 & $* * * *$ & $<0.0001$ \\
\hline Colostrum NT vs. Both INJ $3 \times 10^{6}$ & 0.9333 & 0.08422 to 1.782 & $*$ & 0.013 \\
\hline $\begin{array}{l}\text { Colostrum PBS FF vs. Colostrum INJ } \\
3 \times 10^{6}\end{array}$ & 0.8667 & 0.01755 to 1.716 & $*$ & 0.0384 \\
\hline Colostrum PBS FF vs. Both FF $3 \times 10^{6}$ & 1.3 & 0.4509 to 2.149 & $* * * *$ & $<0.0001$ \\
\hline Colostrum FF $1 \times 10^{6}$ vs. Both FF $3 \times 10^{6}$ & 1.1 & 0.2509 to 1.949 & $* * *$ & 0.0005 \\
\hline $\begin{array}{l}\text { Colostrm PBS INJ vs. Colostrum INJ } \\
3 \times 10^{6}\end{array}$ & 1.067 & 0.2175 to 1.916 & $* *$ & 0.0011 \\
\hline $\begin{array}{l}\text { Colostrm PBS INJ vs. C. sinensis INJ } \\
3 \times 10^{6}\end{array}$ & 0.9 & 0.05088 to 1.749 & $*$ & 0.0227 \\
\hline Colostrm PBS INJ vs. Both FF $1 \times 10^{6}$ & 0.9333 & 0.08422 to 1.782 & $*$ & 0.013 \\
\hline Colostrm PBS INJ vs. Both FF $3 \times 10^{6}$ & 1.5 & 0.6509 to 2.349 & $* * * *$ & $<0.0001$ \\
\hline Colostrm PBS INJ vs. Both INJ $3 \times 10^{6}$ & 0.9333 & 0.08422 to 1.782 & $*$ & 0.013 \\
\hline Colostrum INJ $1 \times 10^{6}$ vs. Both FF $3 \times 10^{6}$ & 0.9 & 0.05088 to 1.749 & $*$ & 0.0227 \\
\hline 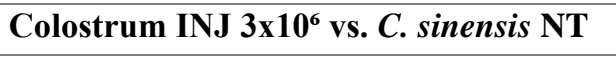 & -1.167 & -2.016 to -0.3175 & $* * *$ & 0.0001 \\
\hline $\begin{array}{l}\text { Colostrum INJ } 3 \times 10^{6} \text { vs. C. sinensis PBS } \\
\text { FF }\end{array}$ & -0.9667 & -1.816 to -0.1175 & $* *$ & 0.0072 \\
\hline $\begin{array}{l}\text { Colostrum INJ } 3 \times 10^{6} \text { vs. } C \text {. sinensis } \mathrm{PBS} \\
\text { INJ }\end{array}$ & -1.167 & -2.016 to -0.3175 & $* * *$ & 0.0001 \\
\hline Colostrum INJ $3 \times 10^{6}$ vs. Both NT & -1.167 & -2.016 to -0.3175 & $* * *$ & 0.0001 \\
\hline Colostrum INJ $3 \times 10^{6}$ vs. Both PBS FF & -1.167 & -2.016 to -0.3175 & $* * *$ & 0.0001 \\
\hline Colostrum INJ $3 \times 10^{6}$ vs. Both PBS INJ & -1.167 & -2.016 to -0.3175 & $* * *$ & 0.0001 \\
\hline C. sinensis $\mathrm{NT}$ vs. C. sinensis $\mathrm{FF} 3 \times 10^{6}$ & 0.8667 & 0.01755 to 1.716 & $*$ & 0.0384 \\
\hline C. sinensis $\mathrm{NT}$ vs. C. sinensis INJ $3 \times 10^{6}$ & 1 & 0.1509 to 1.849 & $* *$ & 0.0039 \\
\hline C. sinensis NT vs. Both FF $1 \times 10^{6}$ & 1.033 & 0.1842 to 1.882 & $* *$ & 0.0021 \\
\hline C. sinensis $\mathrm{NT}$ vs. Both FF $3 \times 10^{6}$ & 1.6 & 0.7509 to 2.449 & $* * * *$ & $<0.0001$ \\
\hline C. sinensis NT vs. Both INJ $3 \times 10^{6}$ & 1.033 & 0.1842 to 1.882 & $* *$ & 0.0021 \\
\hline C. sinensis PBS FF vs. Both FF $3 \times 10^{6}$ & 1.4 & 0.5509 to 2.249 & $* * * *$ & $<0.0001$ \\
\hline C. sinensis FF $1 \times 10^{6}$ vs. Both FF $3 \times 10^{6}$ & 1.067 & 0.2175 to 1.916 & $* *$ & 0.0011 \\
\hline $\begin{array}{l}\text { C. sinensis FF } 3 \times 10^{6} \text { vs. C. sinensis } \mathrm{PBS} \\
\text { INJ }\end{array}$ & -0.8667 & -1.716 to -0.01755 & $*$ & 0.0384 \\
\hline C. sinensis FF $3 \times 10^{6}$ vs. Both NT & -0.8667 & -1.716 to -0.01755 & $*$ & 0.0384 \\
\hline C. sinensis FF $3 \times 10^{6}$ vs. Both PBS FF & -0.8667 & -1.716 to -0.01755 & $*$ & 0.0384 \\
\hline C. sinensis FF $3 \times 10^{6}$ vs. Both PBS INJ & -0.8667 & -1.716 to -0.01755 & $*$ & 0.0384 \\
\hline $\begin{array}{l}\text { C. sinensis } \mathrm{PBS} \text { INJ vs. C. sinensis INJ } \\
3 \times 10^{6}\end{array}$ & 1 & 0.1509 to 1.849 & $* *$ & 0.0039 \\
\hline
\end{tabular}




\begin{tabular}{|c|c|c|c|c|}
\hline C. sinensis PBS INJ vs. Both FF $1 \times 10^{6}$ & 1.033 & 0.1842 to 1.882 & $* *$ & 0.0021 \\
\hline C. sinensis PBS INJ vs. Both FF $3 \times 10^{6}$ & 1.6 & 0.7509 to 2.449 & $* * * *$ & $<0.0001$ \\
\hline C. sinensis PBS INJ vs. Both INJ $3 \times 10^{6}$ & 1.033 & 0.1842 to 1.882 & $* *$ & 0.0021 \\
\hline C. sinensis INJ $1 \times 10^{6}$ vs. Both FF $3 \times 10^{6}$ & 1.2 & 0.3509 to 2.049 & $* * * *$ & $<0.0001$ \\
\hline C. sinensis INJ $3 \times 10^{6}$ vs. Both NT & -1 & -1.849 to -0.1509 & $* *$ & 0.0039 \\
\hline C. sinensis INJ $3 \times 10^{6}$ vs. Both PBS FF & -1 & -1.849 to -0.1509 & $* *$ & 0.0039 \\
\hline C. sinensis INJ $3 \times 10^{6}$ vs. Both PBS INJ & -1 & -1.849 to -0.1509 & $* *$ & 0.0039 \\
\hline Both NT vs. Both FF $1 \times 10^{6}$ & 1.033 & 0.1842 to 1.882 & $* *$ & 0.0021 \\
\hline Both NT vs. Both FF $3 \times 10^{6}$ & 1.6 & 0.7509 to 2.449 & $* * * *$ & $<0.0001$ \\
\hline Both NT vs. Both INJ $3 \times 10^{6}$ & 1.033 & 0.1842 to 1.882 & $* *$ & 0.0021 \\
\hline Both PBS FF vs. Both FF $1 \times 10^{6}$ & 1.033 & 0.1842 to 1.882 & $* *$ & 0.0021 \\
\hline Both PBS FF vs. Both FF $3 \times 10^{6}$ & 1.6 & 0.7509 to 2.449 & $* * * *$ & $<0.0001$ \\
\hline Both PBS FF vs. Both INJ $3 \times 10^{6}$ & 1.033 & 0.1842 to 1.882 & $* *$ & 0.0021 \\
\hline Both FF $1 \times 10^{6}$ vs. Both PBS INJ & -1.033 & -1.882 to -0.1842 & $* *$ & 0.0021 \\
\hline Both FF $3 \times 10^{6}$ vs. Both PBS INJ & -1.6 & -2.449 to -0.7509 & $* * * *$ & $<0.0001$ \\
\hline Both FF $3 \times 10^{6}$ vs. Both INJ $1 \times 10^{6}$ & -1.1 & -1.949 to -0.2509 & $* * *$ & 0.0005 \\
\hline Both PBS INJ vs. Both INJ $3 \times 10^{6}$ & 1.033 & 0.1842 to 1.882 & $* *$ & 0.0021 \\
\hline \multicolumn{5}{|l|}{48} \\
\hline Normal NT vs. Normal FF $1 \times 10^{6}$ & 1.2 & 0.3509 to 2.049 & $* * * *$ & $<0.0001$ \\
\hline Normal NT vs. Normal FF $3 \times 10^{6}$ & 1.5 & 0.6509 to 2.349 & $* * * *$ & $<0.0001$ \\
\hline Normal NT vs. Normal INJ 3x106 & 1.033 & 0.1842 to 1.882 & $* *$ & 0.0021 \\
\hline Normal NT vs. Colostrum FF 3x10 & 1 & 0.1509 to 1.849 & $* *$ & 0.0039 \\
\hline Normal NT vs. Colostrum INJ 3x10 & 1.7 & 0.8509 to 2.549 & $* * * *$ & $<0.0001$ \\
\hline Normal NT vs. C. sinensis FF $3 \times 10^{6}$ & 0.9 & 0.05088 to 1.749 & $*$ & 0.0227 \\
\hline Normal NT vs. C. sinensis INJ $3 \times 10^{6}$ & 1.2 & 0.3509 to 2.049 & $* * * *$ & $<0.0001$ \\
\hline Normal NT vs. Both FF $1 \times 10^{6}$ & 1.067 & 0.2175 to 1.916 & $* *$ & 0.0011 \\
\hline Normal NT vs. Both FF $3 \times 10^{6}$ & 1.967 & 1.118 to 2.816 & $* * * *$ & $<0.0001$ \\
\hline Normal NT vs. Both INJ 3x106 & 1.3 & 0.4509 to 2.149 & $* * * *$ & $<0.0001$ \\
\hline Normal PBS FF vs. Normal FF $1 \times 10^{6}$ & 1.2 & 0.3509 to 2.049 & $* * * *$ & $<0.0001$ \\
\hline Normal PBS FF vs. Normal FF 3x10 & 1.5 & 0.6509 to 2.349 & $* * * *$ & $<0.0001$ \\
\hline Normal PBS FF vs. Normal INJ 3x10 & 1.033 & 0.1842 to 1.882 & $* *$ & 0.0021 \\
\hline Normal PBS FF vs. Colostrum FF 3x10 & 1 & 0.1509 to 1.849 & $* *$ & 0.0039 \\
\hline $\begin{array}{l}\text { Normal PBS FF vs. Colostrum INJ } \\
3 \times 10^{6}\end{array}$ & 1.7 & 0.8509 to 2.549 & $* * * *$ & $<0.0001$ \\
\hline Normal PBS FF vs. $C$. sinensis FF $3 \times 10^{6}$ & 0.9 & 0.05088 to 1.749 & $*$ & 0.0227 \\
\hline Normal PBS FF vs. C. sinensis INJ $3 \times 10^{6}$ & 1.2 & 0.3509 to 2.049 & $* * * *$ & $<0.0001$ \\
\hline Normal PBS FF vs. Both FF $1 \times 10^{6}$ & 1.067 & 0.2175 to 1.916 & $* *$ & 0.0011 \\
\hline Normal PBS FF vs. Both FF $3 \times 10^{6}$ & 1.967 & 1.118 to 2.816 & $* * * *$ & $<0.0001$ \\
\hline Normal PBS FF vs. Both INJ 3x10 & 1.3 & 0.4509 to 2.149 & $* * * *$ & $<0.0001$ \\
\hline Normal FF $1 \times 10^{6}$ vs. Normal PBS INJ & -1.2 & -2.049 to -0.3509 & $* * * *$ & $<0.0001$ \\
\hline Normal FF 1x10 ${ }^{6}$ vs. Colostrum NT & -1.1 & -1.949 to -0.2509 & $* * *$ & 0.0005 \\
\hline Normal FF 1x10 ${ }^{6}$ vs. Colostrum PBS FF & -0.9 & -1.749 to -0.05088 & $*$ & 0.0227 \\
\hline Normal FF 1x10 vs. Colostrm PBS INJ & -1.1 & -1.949 to -0.2509 & $* * *$ & 0.0005 \\
\hline Normal FF $1 \times 10^{6}$ vs. C. sinensis NT & -1.2 & -2.049 to -0.3509 & $* * * *$ & $<0.0001$ \\
\hline Normal FF $1 \times 10^{6}$ vs. C. sinensis PBS FF & -1.1 & -1.949 to -0.2509 & $* * *$ & 0.0005 \\
\hline Normal FF $1 \times 10^{6}$ vs. $C$. sinensis PBS INJ & -1.2 & -2.049 to -0.3509 & $* * * *$ & $<0.0001$ \\
\hline
\end{tabular}




\begin{tabular}{|c|c|c|c|c|}
\hline Normal FF $1 \times 10^{6}$ vs. Both NT & -1.2 & -2.049 to -0.3509 & $* * * *$ & $<0.0001$ \\
\hline Normal FF $1 \times 10^{6}$ vs. Both PBS FF & -1.2 & -2.049 to -0.3509 & $* * * *$ & $<0.0001$ \\
\hline Normal FF $1 \times 10^{6}$ vs. Both PBS INJ & -1.2 & -2.049 to -0.3509 & $* * * *$ & $<0.0001$ \\
\hline Normal FF $3 \times 10^{6}$ vs. Normal PBS INJ & -1.5 & -2.349 to -0.6509 & $* * * *$ & $<0.0001$ \\
\hline Normal FF $3 \times 10^{6}$ vs. Normal INJ $1 \times 10^{6}$ & -0.8667 & -1.716 to -0.01755 & $*$ & 0.0384 \\
\hline Normal FF 3x10 ${ }^{6}$ vs. Colostrum NT & -1.4 & -2.249 to -0.5509 & $* * * *$ & $<0.0001$ \\
\hline Normal FF 3x10 ${ }^{6}$ vs. Colostrum PBS FF & -1.2 & -2.049 to -0.3509 & $* * * *$ & $<0.0001$ \\
\hline $\begin{array}{l}\text { Normal FF 3x10 }{ }^{6} \text { vs. Colostrum FF } \\
1 \times 10^{6}\end{array}$ & -0.9333 & -1.782 to -0.08422 & $*$ & 0.013 \\
\hline Normal FF $3 \times 10^{6}$ vs. Colostrm PBS INJ & -1.4 & -2.249 to -0.5509 & $* * * *$ & $<0.0001$ \\
\hline Normal FF $3 \times 10^{6}$ vs. C. sinensis NT & -1.5 & -2.349 to -0.6509 & $* * * *$ & $<0.0001$ \\
\hline Normal FF $3 \times 10^{6}$ vs. C. sinensis $\mathrm{PBS} F \mathrm{FF}$ & -1.4 & -2.249 to -0.5509 & $* * * *$ & $<0.0001$ \\
\hline Normal FF $3 \times 10^{6}$ vs. $C$. sinensis $\mathrm{FF} 1 \times 10^{6}$ & -0.9 & -1.749 to -0.05088 & $*$ & 0.0227 \\
\hline Normal FF $3 \times 10^{6}$ vs. C. sinensis PBS INJ & -1.5 & -2.349 to -0.6509 & $* * * *$ & $<0.0001$ \\
\hline Normal FF $3 \times 10^{6}$ vs. C. sinensis INJ $1 \times 10^{6}$ & -0.9667 & -1.816 to -0.1175 & $* *$ & 0.0072 \\
\hline Normal FF 3x10 vs. Both NT & -1.5 & -2.349 to -0.6509 & $* * * *$ & $<0.0001$ \\
\hline Normal FF $3 \times 10^{6}$ vs. Both PBS FF & -1.5 & -2.349 to -0.6509 & $* * * *$ & $<0.0001$ \\
\hline Normal FF $3 \times 10^{6}$ vs. Both PBS INJ & -1.5 & -2.349 to -0.6509 & $* * * *$ & $<0.0001$ \\
\hline 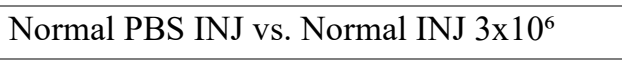 & 1.033 & 0.1842 to 1.882 & $* *$ & 0.0021 \\
\hline $\begin{array}{l}\text { Normal PBS INJ vs. Colostrum FF } \\
3 \times 10^{6}\end{array}$ & 1 & 0.1509 to 1.849 & $* *$ & 0.0039 \\
\hline $\begin{array}{l}\text { Normal PBS INJ vs. Colostrum INJ } \\
3 \times 10^{6}\end{array}$ & 1.7 & 0.8509 to 2.549 & $* * * *$ & $<0.0001$ \\
\hline Normal PBS INJ vs. C. sinensis FF $3 \times 10^{6}$ & 0.9 & 0.05088 to 1.749 & $*$ & 0.0227 \\
\hline Normal PBS INJ vs. C. sinensis $\mathrm{INJ} 3 \times 10^{6}$ & 1.2 & 0.3509 to 2.049 & $* * * *$ & $<0.0001$ \\
\hline Normal PBS INJ vs. Both FF $1 \times 10^{6}$ & 1.067 & 0.2175 to 1.916 & $* *$ & 0.0011 \\
\hline Normal PBS INJ vs. Both FF $3 \times 10^{6}$ & 1.967 & 1.118 to 2.816 & $* * * *$ & $<0.0001$ \\
\hline 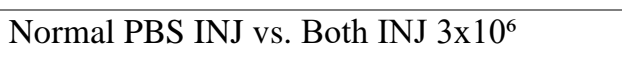 & 1.3 & 0.4509 to 2.149 & $* * * *$ & $<0.0001$ \\
\hline $\begin{array}{l}\text { Normal INJ } 1 \times 10^{6} \text { vs. Colostrum INJ } \\
3 \times 10^{6}\end{array}$ & 1.067 & 0.2175 to 1.916 & $* *$ & 0.0011 \\
\hline Normal INJ $1 \times 10^{6}$ vs. Both FF $3 \times 10^{6}$ & 1.333 & 0.4842 to 2.182 & $* * * *$ & $<0.0001$ \\
\hline Normal INJ 3x10 16 vs. Colostrum NT & -0.9333 & -1.782 to -0.08422 & $*$ & 0.013 \\
\hline Normal INJ 3x10 ${ }^{6}$ vs. Colostrm PBS INJ & -0.9333 & -1.782 to -0.08422 & $*$ & 0.013 \\
\hline Normal INJ $3 \times 10^{6}$ vs. C. sinensis $\mathrm{NT}$ & -1.033 & -1.882 to -0.1842 & $* *$ & 0.0021 \\
\hline Normal INJ $3 \times 10^{6}$ vs. C. sinensis PBS FF & -0.9333 & -1.782 to -0.08422 & $*$ & 0.013 \\
\hline Normal INJ $3 \times 10^{6}$ vs. C. sinensis $\mathrm{PBS}$ INJ & -1.033 & -1.882 to -0.1842 & $* *$ & 0.0021 \\
\hline Normal INJ 3x106 vs. Both NT & -1.033 & -1.882 to -0.1842 & $* *$ & 0.0021 \\
\hline Normal INJ $3 \times 10^{6}$ vs. Both PBS FF & -1.033 & -1.882 to -0.1842 & $* *$ & 0.0021 \\
\hline Normal INJ $3 \times 10^{6}$ vs. Both FF $3 \times 10^{6}$ & 0.9333 & 0.08422 to 1.782 & $*$ & 0.013 \\
\hline Normal INJ 3x106 vs. Both PBS INJ & -1.033 & -1.882 to -0.1842 & $* *$ & 0.0021 \\
\hline Colostrum NT vs. Colostrum FF 3x10 & 0.9 & 0.05088 to 1.749 & $*$ & 0.0227 \\
\hline Colostrum NT vs. Colostrum INJ 3x10 & 1.6 & 0.7509 to 2.449 & $* * * *$ & $<0.0001$ \\
\hline Colostrum NT vs. $C$. sinensis INJ 3x10 & 1.1 & 0.2509 to 1.949 & $* * *$ & 0.0005 \\
\hline Colostrum NT vs. Both FF $1 \times 10^{6}$ & 0.9667 & 0.1175 to 1.816 & $* *$ & 0.0072 \\
\hline Colostrum NT vs. Both FF $3 \times 10^{6}$ & 1.867 & 1.018 to 2.716 & $* * * *$ & $<0.0001$ \\
\hline Colostrum NT vs. Both INJ $3 \times 10^{6}$ & 1.2 & 0.3509 to 2.049 & $* * * *$ & $<0.0001$ \\
\hline $\begin{array}{l}\text { Colostrum PBS FF vs. Colostrum INJ } \\
3 \times 10^{6}\end{array}$ & 1.4 & 0.5509 to 2.249 & $* * * *$ & $<0.0001$ \\
\hline
\end{tabular}




\begin{tabular}{|c|c|c|c|c|}
\hline $\begin{array}{l}\text { Colostrum PBS FF vs. C. sinensis INJ } \\
3 \times 10^{6}\end{array}$ & 0.9 & 0.05088 to 1.749 & $*$ & 0.0227 \\
\hline Colostrum PBS FF vs. Both FF $3 \times 10^{6}$ & 1.667 & 0.8175 to 2.516 & $* * * *$ & $<0.0001$ \\
\hline Colostrum PBS FF vs. Both INJ $3 \times 10^{6}$ & 1 & 0.1509 to 1.849 & $* *$ & 0.0039 \\
\hline $\begin{array}{l}\text { Colostrum FF 1x10 vs. Colostrum INJ } \\
3 \times 10^{6}\end{array}$ & 1.133 & 0.2842 to 1.982 & $* * *$ & 0.0003 \\
\hline Colostrum FF $1 \times 10^{6}$ vs. Both FF $3 \times 10^{6}$ & 1.4 & 0.5509 to 2.249 & $* * * *$ & $<0.0001$ \\
\hline $\begin{array}{l}\text { Colostrum FF 3x10 vs. Colostrm PBS } \\
\text { INJ }\end{array}$ & -0.9 & -1.749 to -0.05088 & $*$ & 0.0227 \\
\hline Colostrum FF $3 \times 10^{6}$ vs. $C$. sinensis $\mathrm{NT}$ & -1 & -1.849 to -0.1509 & $* *$ & 0.0039 \\
\hline $\begin{array}{l}\text { Colostrum FF } 3 \times 10^{6} \text { vs. C. sinensis } \mathrm{PBS} \\
\text { FF }\end{array}$ & -0.9 & -1.749 to -0.05088 & $*$ & 0.0227 \\
\hline $\begin{array}{l}\text { Colostrum FF } 3 \times 10^{6} \text { vs. C. sinensis PBS } \\
\text { INJ }\end{array}$ & -1 & -1.849 to -0.1509 & $* *$ & 0.0039 \\
\hline Colostrum FF $3 \times 10^{6}$ vs. Both NT & -1 & -1.849 to -0.1509 & $* *$ & 0.0039 \\
\hline Colostrum FF $3 \times 10^{6}$ vs. Both PBS FF & -1 & -1.849 to -0.1509 & $* *$ & 0.0039 \\
\hline Colostrum FF $3 \times 10^{6}$ vs. Both FF $3 \times 10^{6}$ & 0.9667 & 0.1175 to 1.816 & $* *$ & 0.0072 \\
\hline Colostrum FF $3 \times 10^{6}$ vs. Both PBS INJ & -1 & -1.849 to -0.1509 & $* *$ & 0.0039 \\
\hline $\begin{array}{l}\text { Colostrm PBS INJ vs. Colostrum INJ } \\
3 \times 10^{6}\end{array}$ & 1.6 & 0.7509 to 2.449 & $* * * *$ & $<0.0001$ \\
\hline $\begin{array}{l}\text { Colostrm PBS INJ vs. C. sinensis INJ } \\
3 \times 10^{6}\end{array}$ & 1.1 & 0.2509 to 1.949 & $* * *$ & 0.0005 \\
\hline Colostrm PBS INJ vs. Both FF $1 \times 10^{6}$ & 0.9667 & 0.1175 to 1.816 & $* *$ & 0.0072 \\
\hline Colostrm PBS INJ vs. Both FF $3 \times 10^{6}$ & 1.867 & 1.018 to 2.716 & $* * * *$ & $<0.0001$ \\
\hline Colostrm PBS INJ vs. Both INJ $3 \times 10^{6}$ & 1.2 & 0.3509 to 2.049 & $* * * *$ & $<0.0001$ \\
\hline $\begin{array}{l}\text { Colostrum INJ } 1 \times 10^{6} \text { vs. Colostrum INJ } \\
3 \times 10^{6}\end{array}$ & 0.9333 & 0.08422 to 1.782 & $*$ & 0.013 \\
\hline 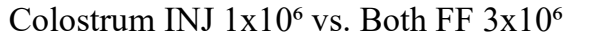 & 1.2 & 0.3509 to 2.049 & $* * * *$ & $<0.0001$ \\
\hline Colostrum INJ 3x106 vs. C. sinensis NT & -1.7 & -2.549 to -0.8509 & $* * * *$ & $<0.0001$ \\
\hline $\begin{array}{l}\text { Colostrum INJ } 3 \times 10^{6} \text { vs. } C \text {. sinensis PBS } \\
\text { FF }\end{array}$ & -1.6 & -2.449 to -0.7509 & $* * * *$ & $<0.0001$ \\
\hline $\begin{array}{l}\text { Colostrum INJ } 3 \times 10^{6} \text { vs. } C \text {. sinensis FF } \\
1 \times 10^{6}\end{array}$ & -1.1 & -1.949 to -0.2509 & $* * *$ & 0.0005 \\
\hline $\begin{array}{l}\text { Colostrum INJ } 3 \times 10^{6} \text { vs. } C \text {. sinensis } \mathrm{PBS} \\
\text { INJ }\end{array}$ & -1.7 & -2.549 to -0.8509 & $* * * *$ & $<0.0001$ \\
\hline $\begin{array}{l}\text { Colostrum INJ } 3 \times 10^{6} \text { vs. C. sinensis INJ } \\
1 \times 10^{6}\end{array}$ & -1.167 & -2.016 to -0.3175 & $* * *$ & 0.0001 \\
\hline Colostrum INJ $3 \times 10^{6}$ vs. Both NT & -1.7 & -2.549 to -0.8509 & $* * * *$ & $<0.0001$ \\
\hline Colostrum INJ $3 \times 10^{6}$ vs. Both PBS FF & -1.7 & -2.549 to -0.8509 & $* * * *$ & $<0.0001$ \\
\hline 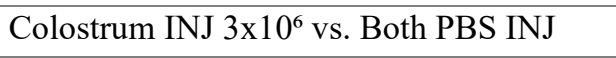 & -1.7 & -2.549 to -0.8509 & $* * * *$ & $<0.0001$ \\
\hline Colostrum INJ $3 \times 10^{6}$ vs. Both INJ $1 \times 10^{6}$ & -0.8667 & -1.716 to -0.01755 & $*$ & 0.0384 \\
\hline C. sinensis $\mathrm{NT}$ vs. C. sinensis $\mathrm{FF} 3 \times 10^{6}$ & 0.9 & 0.05088 to 1.749 & $*$ & 0.0227 \\
\hline C. sinensis $\mathrm{NT}$ vs. C. sinensis INJ $3 \times 10^{6}$ & 1.2 & 0.3509 to 2.049 & $* * * *$ & $<0.0001$ \\
\hline C. sinensis NT vs. Both FF $1 \times 10^{6}$ & 1.067 & 0.2175 to 1.916 & $* *$ & 0.0011 \\
\hline C. sinensis NT vs. Both FF $3 \times 10^{6}$ & 1.967 & 1.118 to 2.816 & $* * * *$ & $<0.0001$ \\
\hline C. sinensis NT vs. Both INJ $3 \times 10^{6}$ & 1.3 & 0.4509 to 2.149 & $* * * *$ & $<0.0001$ \\
\hline $\begin{array}{l}\text { C. sinensis } \mathrm{PBS} \text { FF vs. } C \text {. sinensis INJ } \\
3 \times 10^{6}\end{array}$ & 1.1 & 0.2509 to 1.949 & $* * *$ & 0.0005 \\
\hline C. sinensis $\mathrm{PBS}$ FF vs. Both FF $1 \times 10^{6}$ & 0.9667 & 0.1175 to 1.816 & $* *$ & 0.0072 \\
\hline C. sinensis PBS FF vs. Both FF $3 \times 10^{6}$ & 1.867 & 1.018 to 2.716 & $* * * *$ & $<0.0001$ \\
\hline C. sinensis PBS FF vs. Both INJ $3 \times 10^{6}$ & 1.2 & 0.3509 to 2.049 & $* * * *$ & $<0.0001$ \\
\hline C. sinensis FF $1 \times 10^{6}$ vs. Both FF $3 \times 10^{6}$ & 1.367 & 0.5175 to 2.216 & $* * * *$ & $<0.0001$ \\
\hline
\end{tabular}




\begin{tabular}{|c|c|c|c|c|}
\hline $\begin{array}{l}\text { C. sinensis FF } 3 \times 10^{6} \text { vs. C. sinensis } \mathrm{PBS} \\
\text { INJ }\end{array}$ & -0.9 & -1.749 to -0.05088 & $*$ & 0.0227 \\
\hline C. sinensis $\mathrm{FF} 3 \times 10^{6}$ vs. Both $\mathrm{NT}$ & -0.9 & -1.749 to -0.05088 & $*$ & 0.0227 \\
\hline C. sinensis FF $3 \times 10^{6}$ vs. Both PBS FF & -0.9 & -1.749 to -0.05088 & $*$ & 0.0227 \\
\hline C. sinensis FF $3 \times 10^{6}$ vs. Both FF $3 \times 10^{6}$ & 1.067 & 0.2175 to 1.916 & $* *$ & 0.0011 \\
\hline C. sinensis FF $3 \times 10^{6}$ vs. Both PBS INJ & -0.9 & -1.749 to -0.05088 & $*$ & 0.0227 \\
\hline $\begin{array}{l}\text { C. sinensis PBS INJ vs. C. sinensis INJ } \\
3 \times 10^{6}\end{array}$ & 1.2 & 0.3509 to 2.049 & $* * * *$ & $<0.0001$ \\
\hline C. sinensis PBS INJ vs. Both FF $1 \times 10^{6}$ & 1.067 & 0.2175 to 1.916 & ** & 0.0011 \\
\hline C. sinensis PBS INJ vs. Both FF $3 \times 10^{6}$ & 1.967 & 1.118 to 2.816 & $* * * *$ & $<0.0001$ \\
\hline C. sinensis PBS INJ vs. Both INJ $3 \times 10^{6}$ & 1.3 & 0.4509 to 2.149 & $* * * *$ & $<0.0001$ \\
\hline C. sinensis INJ $1 \times 10^{6}$ vs. Both FF $3 \times 10^{6}$ & 1.433 & 0.5842 to 2.282 & $* * * *$ & $<0.0001$ \\
\hline C. sinensis INJ $3 \times 10^{6}$ vs. Both NT & -1.2 & -2.049 to -0.3509 & $* * * *$ & $<0.0001$ \\
\hline C. sinensis INJ $3 \times 10^{6}$ vs. Both PBS FF & -1.2 & -2.049 to -0.3509 & $* * * *$ & $<0.0001$ \\
\hline C. sinensis INJ $3 \times 10^{6}$ vs. Both PBS INJ & -1.2 & -2.049 to -0.3509 & $* * * *$ & $<0.0001$ \\
\hline Both NT vs. Both FF $1 \times 10^{6}$ & 1.067 & 0.2175 to 1.916 & $* *$ & 0.0011 \\
\hline Both NT vs. Both FF $3 \times 10^{6}$ & 1.967 & 1.118 to 2.816 & $* * * *$ & $<0.0001$ \\
\hline Both NT vs. Both INJ 3x10 & 1.3 & 0.4509 to 2.149 & $* * * *$ & $<0.0001$ \\
\hline Both PBS FF vs. Both FF $1 \times 10^{6}$ & 1.067 & 0.2175 to 1.916 & $* *$ & 0.0011 \\
\hline Both PBS FF vs. Both FF $3 \times 10^{6}$ & 1.967 & 1.118 to 2.816 & $* * * *$ & $<0.0001$ \\
\hline Both PBS FF vs. Both INJ $3 \times 10^{6}$ & 1.3 & 0.4509 to 2.149 & $* * * *$ & $<0.0001$ \\
\hline Both FF $1 \times 10^{6}$ vs. Both FF $3 \times 10^{6}$ & 0.9 & 0.05088 to 1.749 & $*$ & 0.0227 \\
\hline Both FF $1 \times 10^{6}$ vs. Both PBS INJ & -1.067 & -1.916 to -0.2175 & $* *$ & 0.0011 \\
\hline Both FF $3 \times 10^{6}$ vs. Both PBS INJ & -1.967 & -2.816 to -1.118 & $* * * *$ & $<0.0001$ \\
\hline Both FF $3 \times 10^{6}$ vs. Both INJ $1 \times 10^{6}$ & -1.133 & -1.982 to -0.2842 & $* * *$ & 0.0003 \\
\hline Both PBS INJ vs. Both INJ $3 \times 10^{6}$ & 1.3 & 0.4509 to 2.149 & $* * * *$ & $<0.0001$ \\
\hline \multicolumn{5}{|l|}{72} \\
\hline Normal NT vs. Normal FF $1 \times 10^{6}$ & 1.267 & 0.4175 to 2.116 & $* * * *$ & $<0.0001$ \\
\hline Normal NT vs. Normal FF $3 \times 10^{6}$ & 1.567 & 0.7175 to 2.416 & $* * * *$ & $<0.0001$ \\
\hline 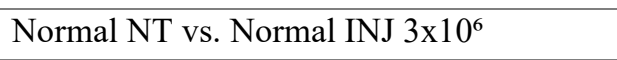 & 1.3 & 0.4509 to 2.149 & $* * * *$ & $<0.0001$ \\
\hline Normal NT vs. Colostrum FF $3 \times 10^{6}$ & 1 & 0.1509 to 1.849 & $* *$ & 0.0039 \\
\hline Normal NT vs. Colostrum INJ 3x10 & 1.733 & 0.8842 to 2.582 & $* * * *$ & $<0.0001$ \\
\hline Normal NT vs. C. sinensis FF $3 \times 10^{6}$ & 1 & 0.1509 to 1.849 & $* *$ & 0.0039 \\
\hline Normal NT vs. C. sinensis INJ $3 \times 10^{6}$ & 1.267 & 0.4175 to 2.116 & $* * * *$ & $<0.0001$ \\
\hline Normal NT vs. Both FF $1 \times 10^{6}$ & 1.233 & 0.3842 to 2.082 & $* * * *$ & $<0.0001$ \\
\hline Normal NT vs. Both FF $3 \times 10^{6}$ & 2.067 & 1.218 to 2.916 & $* * * *$ & $<0.0001$ \\
\hline Normal NT vs. Both INJ $1 \times 10^{6}$ & 1.1 & 0.2509 to 1.949 & $* * *$ & 0.0005 \\
\hline 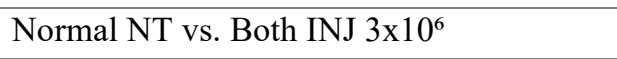 & 1.4 & 0.5509 to 2.249 & $* * * *$ & $<0.0001$ \\
\hline Normal PBS FF vs. Normal FF $1 \times 10^{6}$ & 1.267 & 0.4175 to 2.116 & $* * * *$ & $<0.0001$ \\
\hline Normal PBS FF vs. Normal FF $3 \times 10^{6}$ & 1.567 & 0.7175 to 2.416 & $* * * *$ & $<0.0001$ \\
\hline Normal PBS FF vs. Normal INJ 3x10 & 1.3 & 0.4509 to 2.149 & $* * * *$ & $<0.0001$ \\
\hline Normal PBS FF vs. Colostrum FF 3x10 & 1 & 0.1509 to 1.849 & $* *$ & 0.0039 \\
\hline $\begin{array}{l}\text { Normal PBS FF vs. Colostrum INJ } \\
3 \times 10^{6}\end{array}$ & 1.733 & 0.8842 to 2.582 & $* * * *$ & $<0.0001$ \\
\hline Normal PBS FF vs. C. sinensis FF $3 \times 10^{6}$ & 1 & 0.1509 to 1.849 & $* *$ & 0.0039 \\
\hline Normal PBS FF vs. $C$. sinensis INJ $3 \times 10^{6}$ & 1.267 & 0.4175 to 2.116 & $* * * *$ & $<0.0001$ \\
\hline Normal PBS FF vs. Both FF $1 \times 10^{6}$ & 1.233 & 0.3842 to 2.082 & $* * * *$ & $<0.0001$ \\
\hline
\end{tabular}




\begin{tabular}{|c|c|c|c|c|}
\hline Normal PBS FF vs. Both FF $3 \times 10^{6}$ & 2.067 & 1.218 to 2.916 & $* * * *$ & $<0.0001$ \\
\hline Normal PBS FF vs. Both INJ $1 \times 10^{6}$ & 1.1 & 0.2509 to 1.949 & $* * *$ & 0.0005 \\
\hline Normal PBS FF vs. Both INJ $3 \times 10^{6}$ & 1.4 & 0.5509 to 2.249 & $* * * *$ & $<0.0001$ \\
\hline Normal FF $1 \times 10^{6}$ vs. Normal PBS INJ & -1.267 & -2.116 to -0.4175 & $* * * *$ & $<0.0001$ \\
\hline Normal FF 1x10 ${ }^{6}$ vs. Colostrum NT & -1.167 & -2.016 to -0.3175 & $* * *$ & 0.0001 \\
\hline Normal FF 1x10 $10^{6}$ vs. Colostrum PBS FF & -0.9667 & -1.816 to -0.1175 & $* *$ & 0.0072 \\
\hline Normal FF 1x106 vs. Colostrm PBS INJ & -1.167 & -2.016 to -0.3175 & $* * *$ & 0.0001 \\
\hline Normal FF $1 \times 10^{6}$ vs. $C$. sinensis $\mathrm{NT}$ & -1.167 & -2.016 to -0.3175 & $* * *$ & 0.0001 \\
\hline Normal FF $1 \times 10^{6}$ vs. C. sinensis $\mathrm{PBS} F \mathrm{FF}$ & -1.067 & -1.916 to -0.2175 & $* *$ & 0.0011 \\
\hline Normal FF $1 \times 10^{6}$ vs. C. sinensis PBS INJ & -1.267 & -2.116 to -0.4175 & $* * * *$ & $<0.0001$ \\
\hline Normal FF $1 \times 10^{6}$ vs. Both NT & -1.267 & -2.116 to -0.4175 & $* * * *$ & $<0.0001$ \\
\hline Normal FF $1 \times 10^{6}$ vs. Both PBS FF & -1.267 & -2.116 to -0.4175 & $* * * *$ & $<0.0001$ \\
\hline Normal FF $1 \times 10^{6}$ vs. Both PBS INJ & -1.267 & -2.116 to -0.4175 & $* * * *$ & $<0.0001$ \\
\hline Normal FF $3 \times 10^{6}$ vs. Normal PBS INJ & -1.567 & -2.416 to -0.7175 & $* * * *$ & $<0.0001$ \\
\hline Normal FF $3 \times 10^{6}$ vs. Normal INJ $1 \times 10^{6}$ & -1.067 & -1.916 to -0.2175 & $* *$ & 0.0011 \\
\hline Normal FF 3x10 ${ }^{6}$ vs. Colostrum NT & -1.467 & -2.316 to -0.6175 & $* * * *$ & $<0.0001$ \\
\hline Normal FF 3x10 $1{ }^{6}$ vs. Colostrum PBS FF & -1.267 & -2.116 to -0.4175 & $* * * *$ & $<0.0001$ \\
\hline Normal FF 3x106 vs. Colostrm PBS INJ & -1.467 & -2.316 to -0.6175 & $* * * *$ & $<0.0001$ \\
\hline Normal FF $3 \times 10^{6}$ vs. C. sinensis NT & -1.467 & -2.316 to -0.6175 & $* * * *$ & $<0.0001$ \\
\hline Normal FF $3 \times 10^{6}$ vs. C. sinensis PBS FF & -1.367 & -2.216 to -0.5175 & $* * * *$ & $<0.0001$ \\
\hline Normal FF $3 \times 10^{6}$ vs. C. sinensis $\mathrm{FF} 1 \times 10^{6}$ & -0.9 & -1.749 to -0.05088 & $*$ & 0.0227 \\
\hline Normal FF $3 \times 10^{6}$ vs. C. sinensis PBS INJ & -1.567 & -2.416 to -0.7175 & $* * * *$ & $<0.0001$ \\
\hline Normal FF $3 \times 10^{6}$ vs. C. sinensis INJ $1 \times 10^{6}$ & -0.9667 & -1.816 to -0.1175 & $* *$ & 0.0072 \\
\hline Normal FF $3 \times 10^{6}$ vs. Both NT & -1.567 & -2.416 to -0.7175 & $* * * *$ & $<0.0001$ \\
\hline Normal FF $3 \times 10^{6}$ vs. Both PBS FF & -1.567 & -2.416 to -0.7175 & $* * * *$ & $<0.0001$ \\
\hline Normal FF $3 \times 10^{6}$ vs. Both PBS INJ & -1.567 & -2.416 to -0.7175 & $* * * *$ & $<0.0001$ \\
\hline 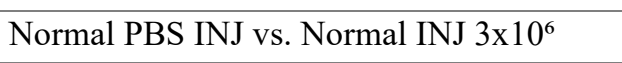 & 1.3 & 0.4509 to 2.149 & $* * * *$ & $<0.0001$ \\
\hline $\begin{array}{l}\text { Normal PBS INJ vs. Colostrum FF } \\
3 \times 10^{6}\end{array}$ & 1 & 0.1509 to 1.849 & $* *$ & 0.0039 \\
\hline $\begin{array}{l}\text { Normal PBS INJ vs. Colostrum INJ } \\
3 \times 10^{6}\end{array}$ & 1.733 & 0.8842 to 2.582 & $* * * *$ & $<0.0001$ \\
\hline Normal PBS INJ vs. C. sinensis FF $3 \times 10^{6}$ & 1 & 0.1509 to 1.849 & $* *$ & 0.0039 \\
\hline Normal PBS INJ vs. C. sinensis INJ $3 \times 10^{6}$ & 1.267 & 0.4175 to 2.116 & $* * * *$ & $<0.0001$ \\
\hline Normal PBS INJ vs. Both FF $1 \times 10^{6}$ & 1.233 & 0.3842 to 2.082 & $* * * *$ & $<0.0001$ \\
\hline Normal PBS INJ vs. Both FF $3 \times 10^{6}$ & 2.067 & 1.218 to 2.916 & $* * * *$ & $<0.0001$ \\
\hline Normal PBS INJ vs. Both INJ $1 \times 10^{6}$ & 1.1 & 0.2509 to 1.949 & $* * *$ & 0.0005 \\
\hline Normal PBS INJ vs. Both INJ 3x106 & 1.4 & 0.5509 to 2.249 & $* * * *$ & $<0.0001$ \\
\hline $\begin{array}{l}\text { Normal INJ } 1 \times 10^{6} \text { vs. Colostrum INJ } \\
3 \times 10^{6}\end{array}$ & 1.233 & 0.3842 to 2.082 & $* * * *$ & $<0.0001$ \\
\hline Normal INJ $1 \times 10^{6}$ vs. Both FF $3 \times 10^{6}$ & 1.567 & 0.7175 to 2.416 & $* * * *$ & $<0.0001$ \\
\hline 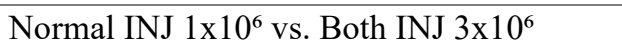 & 0.9 & 0.05088 to 1.749 & $*$ & 0.0227 \\
\hline Normal INJ 3x10 6 vs. Colostrum NT & -1.2 & -2.049 to -0.3509 & $* * * *$ & $<0.0001$ \\
\hline $\begin{array}{l}\text { Normal INJ 3x106 vs. Colostrum PBS } \\
\text { FF }\end{array}$ & -1 & -1.849 to -0.1509 & $* *$ & 0.0039 \\
\hline Normal INJ 3x10 ${ }^{6}$ vs. Colostrm PBS INJ & -1.2 & -2.049 to -0.3509 & $* * * *$ & $<0.0001$ \\
\hline Normal INJ $3 \times 10^{6}$ vs. C. sinensis NT & -1.2 & -2.049 to -0.3509 & $* * * *$ & $<0.0001$ \\
\hline Normal INJ $3 \times 10^{6}$ vs. C. sinensis PBS FF & -1.1 & -1.949 to -0.2509 & $* * *$ & 0.0005 \\
\hline Normal INJ $3 \times 10^{6}$ vs. C. sinensis $\mathrm{PBS}$ INJ & -1.3 & -2.149 to -0.4509 & $* * * *$ & $<0.0001$ \\
\hline
\end{tabular}




\begin{tabular}{|c|c|c|c|c|}
\hline Normal INJ $3 \times 10^{6}$ vs. Both NT & -1.3 & -2.149 to -0.4509 & $* * * *$ & $<0.0001$ \\
\hline Normal INJ $3 \times 10^{6}$ vs. Both PBS FF & -1.3 & -2.149 to -0.4509 & $* * * *$ & $<0.0001$ \\
\hline Normal INJ $3 \times 10^{6}$ vs. Both PBS INJ & -1.3 & -2.149 to -0.4509 & $* * * *$ & $<0.0001$ \\
\hline Colostrum NT vs. Colostrum FF $3 \times 10^{6}$ & 0.9 & 0.05088 to 1.749 & $*$ & 0.0227 \\
\hline Colostrum NT vs. Colostrum INJ 3x10 & 1.633 & 0.7842 to 2.482 & $* * * *$ & $<0.0001$ \\
\hline Colostrum NT vs. C. sinensis FF $3 \times 10^{6}$ & 0.9 & 0.05088 to 1.749 & $*$ & 0.0227 \\
\hline Colostrum NT vs. $C$. sinensis INJ 3x10 & 1.167 & 0.3175 to 2.016 & $* * *$ & 0.0001 \\
\hline Colostrum NT vs. Both FF 1x106 & 1.133 & 0.2842 to 1.982 & $* * *$ & 0.0003 \\
\hline Colostrum NT vs. Both FF $3 \times 10^{6}$ & 1.967 & 1.118 to 2.816 & $* * * *$ & $<0.0001$ \\
\hline Colostrum NT vs. Both INJ 1x10 & 1 & 0.1509 to 1.849 & $* *$ & 0.0039 \\
\hline Colostrum NT vs. Both INJ 3x106 & 1.3 & 0.4509 to 2.149 & $* * * *$ & $<0.0001$ \\
\hline $\begin{array}{l}\text { Colostrum PBS FF vs. Colostrum INJ } \\
3 \times 10^{6}\end{array}$ & 1.433 & 0.5842 to 2.282 & $* * * *$ & $<0.0001$ \\
\hline $\begin{array}{l}\text { Colostrum PBS FF vs. C. sinensis INJ } \\
3 \times 10^{6}\end{array}$ & 0.9667 & 0.1175 to 1.816 & $* *$ & 0.0072 \\
\hline Colostrum PBS FF vs. Both FF $1 \times 10^{6}$ & 0.9333 & 0.08422 to 1.782 & $*$ & 0.013 \\
\hline Colostrum PBS FF vs. Both FF $3 \times 10^{6}$ & 1.767 & 0.9175 to 2.616 & $* * * *$ & $<0.0001$ \\
\hline Colostrum PBS FF vs. Both INJ $3 \times 10^{6}$ & 1.1 & 0.2509 to 1.949 & $* * *$ & 0.0005 \\
\hline $\begin{array}{l}\text { Colostrum FF } 1 \times 10^{6} \text { vs. Colostrum INJ } \\
3 \times 10^{6}\end{array}$ & 1 & 0.1509 to 1.849 & $* *$ & 0.0039 \\
\hline Colostrum FF $1 \times 10^{6}$ vs. Both FF $3 \times 10^{6}$ & 1.333 & 0.4842 to 2.182 & $* * * *$ & $<0.0001$ \\
\hline $\begin{array}{l}\text { Colostrum FF 3x10 vs. Colostrm PBS } \\
\text { INJ }\end{array}$ & -0.9 & -1.749 to -0.05088 & $*$ & 0.0227 \\
\hline Colostrum FF $3 \times 10^{6}$ vs. $C$. sinensis $\mathrm{NT}$ & -0.9 & -1.749 to -0.05088 & $*$ & 0.0227 \\
\hline $\begin{array}{l}\text { Colostrum FF } 3 \times 10^{6} \text { vs. C. sinensis PBS } \\
\text { INJ }\end{array}$ & -1 & -1.849 to -0.1509 & $* *$ & 0.0039 \\
\hline Colostrum FF $3 \times 10^{6}$ vs. Both NT & -1 & -1.849 to -0.1509 & $* *$ & 0.0039 \\
\hline Colostrum FF $3 \times 10^{6}$ vs. Both PBS FF & -1 & -1.849 to -0.1509 & $* *$ & 0.0039 \\
\hline Colostrum FF $3 \times 10^{6}$ vs. Both FF $3 \times 10^{6}$ & 1.067 & 0.2175 to 1.916 & $* *$ & 0.0011 \\
\hline Colostrum FF $3 \times 10^{6}$ vs. Both PBS INJ & -1 & -1.849 to -0.1509 & $* *$ & 0.0039 \\
\hline $\begin{array}{l}\text { Colostrm PBS INJ vs. Colostrum INJ } \\
3 \times 10^{6}\end{array}$ & 1.633 & 0.7842 to 2.482 & $* * * *$ & $<0.0001$ \\
\hline Colostrm PBS INJ vs. C. sinensis FF $3 \times 10^{6}$ & 0.9 & 0.05088 to 1.749 & $*$ & 0.0227 \\
\hline $\begin{array}{l}\text { Colostrm PBS INJ vs. C. sinensis INJ } \\
3 \times 10^{6}\end{array}$ & 1.167 & 0.3175 to 2.016 & $* * *$ & 0.0001 \\
\hline Colostrm PBS INJ vs. Both FF $1 \times 10^{6}$ & 1.133 & 0.2842 to 1.982 & $* * *$ & 0.0003 \\
\hline Colostrm PBS INJ vs. Both FF $3 \times 10^{6}$ & 1.967 & 1.118 to 2.816 & $* * * *$ & $<0.0001$ \\
\hline Colostrm PBS INJ vs. Both INJ $1 \times 10^{6}$ & 1 & 0.1509 to 1.849 & $* *$ & 0.0039 \\
\hline 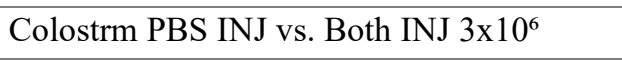 & 1.3 & 0.4509 to 2.149 & $* * * *$ & $<0.0001$ \\
\hline $\begin{array}{l}\text { Colostrum INJ } 1 \times 10^{6} \text { vs. Colostrum INJ } \\
3 \times 10^{6}\end{array}$ & 0.9667 & 0.1175 to 1.816 & $* *$ & 0.0072 \\
\hline Colostrum INJ $1 \times 10^{6}$ vs. Both FF $3 \times 10^{6}$ & 1.3 & 0.4509 to 2.149 & $* * * *$ & $<0.0001$ \\
\hline Colostrum INJ $3 \times 10^{6}$ vs. C. sinensis NT & -1.633 & -2.482 to -0.7842 & $* * * *$ & $<0.0001$ \\
\hline $\begin{array}{l}\text { Colostrum INJ } 3 \times 10^{6} \text { vs. } C \text {. sinensis } \mathrm{PBS} \\
\text { FF }\end{array}$ & -1.533 & -2.382 to -0.6842 & $* * * *$ & $<0.0001$ \\
\hline $\begin{array}{l}\text { Colostrum INJ 3x10 vs. C. sinensis FF } \\
1^{6} 10^{6}\end{array}$ & -1.067 & -1.916 to -0.2175 & $* *$ & 0.0011 \\
\hline $\begin{array}{l}\text { Colostrum INJ 3x10 vs. C. sinensis } \mathrm{PBS} \\
\text { INJ }\end{array}$ & -1.733 & -2.582 to -0.8842 & $* * * *$ & $<0.0001$ \\
\hline $\begin{array}{l}\text { Colostrum INJ } 3 \times 10^{6} \text { vs. } C \text {. sinensis INJ } \\
1^{1 \times 10^{6}}\end{array}$ & -1.133 & -1.982 to -0.2842 & $* * *$ & 0.0003 \\
\hline
\end{tabular}




\begin{tabular}{|c|c|c|c|c|}
\hline Colostrum INJ $3 \times 10^{6}$ vs. Both NT & -1.733 & -2.582 to -0.8842 & $* * * *$ & $<0.0001$ \\
\hline Colostrum INJ $3 \times 10^{6}$ vs. Both PBS FF & -1.733 & -2.582 to -0.8842 & $* * * *$ & $<0.0001$ \\
\hline Colostrum INJ $3 \times 10^{6}$ vs. Both PBS INJ & -1.733 & -2.582 to -0.8842 & $* * * *$ & $<0.0001$ \\
\hline C. sinensis $\mathrm{NT}$ vs. $C$. sinensis $\mathrm{FF} 3 \times 10^{6}$ & 0.9 & 0.05088 to 1.749 & $*$ & 0.0227 \\
\hline C. sinensis $\mathrm{NT}$ vs. C. sinensis $\mathrm{INJ} 3 \times 10^{6}$ & 1.167 & 0.3175 to 2.016 & $* * *$ & 0.0001 \\
\hline C. sinensis NT vs. Both FF $1 \times 10^{6}$ & 1.133 & 0.2842 to 1.982 & $* * *$ & 0.0003 \\
\hline C. sinensis NT vs. Both FF $3 \times 10^{6}$ & 1.967 & 1.118 to 2.816 & $* * * *$ & $<0.0001$ \\
\hline C. sinensis NT vs. Both INJ $1 \times 10^{6}$ & 1 & 0.1509 to 1.849 & $* *$ & 0.0039 \\
\hline C. sinensis NT vs. Both INJ $3 \times 10^{6}$ & 1.3 & 0.4509 to 2.149 & $* * * *$ & $<0.0001$ \\
\hline $\begin{array}{l}\text { C. sinensis } \mathrm{PBS} \text { FF vs. } C \text {. sinensis INJ } \\
3 \times 10^{6}\end{array}$ & 1.067 & 0.2175 to 1.916 & $* *$ & 0.0011 \\
\hline C. sinensis $\mathrm{PBS}$ FF vs. Both FF $1 \times 10^{6}$ & 1.033 & 0.1842 to 1.882 & $* *$ & 0.0021 \\
\hline C. sinensis PBS FF vs. Both FF $3 \times 10^{6}$ & 1.867 & 1.018 to 2.716 & $* * * *$ & $<0.0001$ \\
\hline C. sinensis $\mathrm{PBS}$ FF vs. Both INJ $1 \times 10^{6}$ & 0.9 & 0.05088 to 1.749 & $*$ & 0.0227 \\
\hline C. sinensis PBS FF vs. Both INJ $3 \times 10^{6}$ & 1.2 & 0.3509 to 2.049 & $* * * *$ & $<0.0001$ \\
\hline C. sinensis FF $1 \times 10^{6}$ vs. Both FF $3 \times 10^{6}$ & 1.4 & 0.5509 to 2.249 & $* * * *$ & $<0.0001$ \\
\hline $\begin{array}{l}\text { C. sinensis FF } 3 \times 10^{6} \text { vs. C. sinensis } \mathrm{PBS} \\
\text { INJ }\end{array}$ & -1 & -1.849 to -0.1509 & $* *$ & 0.0039 \\
\hline C. sinensis FF $3 \times 10^{6}$ vs. Both NT & -1 & -1.849 to -0.1509 & $* *$ & 0.0039 \\
\hline C. sinensis FF $3 \times 10^{6}$ vs. Both PBS FF & -1 & -1.849 to -0.1509 & $* *$ & 0.0039 \\
\hline C. sinensis FF $3 \times 10^{6}$ vs. Both FF $3 \times 10^{6}$ & 1.067 & 0.2175 to 1.916 & $* *$ & 0.0011 \\
\hline C. sinensis FF $3 \times 10^{6}$ vs. Both PBS INJ & -1 & -1.849 to -0.1509 & $* *$ & 0.0039 \\
\hline $\begin{array}{l}\text { C. sinensis } \mathrm{PBS} \text { INJ vs. } C \text {. sinensis INJ } \\
3 \times 10^{6}\end{array}$ & 1.267 & 0.4175 to 2.116 & $* * * *$ & $<0.0001$ \\
\hline C. sinensis $\mathrm{PBS}$ INJ vs. Both FF $1 \times 10^{6}$ & 1.233 & 0.3842 to 2.082 & $* * * *$ & $<0.0001$ \\
\hline C. sinensis PBS INJ vs. Both FF $3 \times 10^{6}$ & 2.067 & 1.218 to 2.916 & $* * * *$ & $<0.0001$ \\
\hline C. sinensis PBS INJ vs. Both INJ $1 \times 10^{6}$ & 1.1 & 0.2509 to 1.949 & $* * *$ & 0.0005 \\
\hline C. sinensis PBS INJ vs. Both INJ $3 \times 10^{6}$ & 1.4 & 0.5509 to 2.249 & $* * * *$ & $<0.0001$ \\
\hline C. sinensis INJ $1 \times 10^{6}$ vs. Both FF $3 \times 10^{6}$ & 1.467 & 0.6175 to 2.316 & $* * * *$ & $<0.0001$ \\
\hline C. sinensis INJ $3 \times 10^{6}$ vs. Both NT & -1.267 & -2.116 to -0.4175 & $* * * *$ & $<0.0001$ \\
\hline C. sinensis INJ $3 \times 10^{6}$ vs. Both PBS FF & -1.267 & -2.116 to -0.4175 & $* * * *$ & $<0.0001$ \\
\hline C. sinensis INJ $3 \times 10^{6}$ vs. Both PBS INJ & -1.267 & -2.116 to -0.4175 & $* * * *$ & $<0.0001$ \\
\hline Both NT vs. Both FF $1 \times 10^{6}$ & 1.233 & 0.3842 to 2.082 & $* * * *$ & $<0.0001$ \\
\hline Both NT vs. Both FF $3 \times 10^{6}$ & 2.067 & 1.218 to 2.916 & $* * * *$ & $<0.0001$ \\
\hline Both NT vs. Both INJ $1 \times 10^{6}$ & 1.1 & 0.2509 to 1.949 & $* * *$ & 0.0005 \\
\hline Both NT vs. Both INJ $3 \times 10^{6}$ & 1.4 & 0.5509 to 2.249 & $* * * *$ & $<0.0001$ \\
\hline Both PBS FF vs. Both FF $1 \times 10^{6}$ & 1.233 & 0.3842 to 2.082 & $* * * *$ & $<0.0001$ \\
\hline Both PBS FF vs. Both FF $3 \times 10^{6}$ & 2.067 & 1.218 to 2.916 & $* * * *$ & $<0.0001$ \\
\hline Both PBS FF vs. Both INJ $1 \times 10^{6}$ & 1.1 & 0.2509 to 1.949 & $* * *$ & 0.0005 \\
\hline Both PBS FF vs. Both INJ $3 \times 10^{6}$ & 1.4 & 0.5509 to 2.249 & $* * * *$ & $<0.0001$ \\
\hline Both FF $1 \times 10^{6}$ vs. Both PBS INJ & -1.233 & -2.082 to -0.3842 & $* * * *$ & $<0.0001$ \\
\hline Both FF $3 \times 10^{6}$ vs. Both PBS INJ & -2.067 & -2.916 to -1.218 & $* * * *$ & $<0.0001$ \\
\hline Both FF $3 \times 10^{6}$ vs. Both INJ $1 \times 10^{6}$ & -0.9667 & -1.816 to -0.1175 & $* *$ & 0.0072 \\
\hline Both PBS INJ vs. Both INJ $1 \times 10^{6}$ & 1.1 & 0.2509 to 1.949 & $* * *$ & 0.0005 \\
\hline 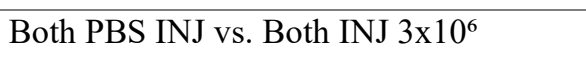 & 1.4 & 0.5509 to 2.249 & $* * * *$ & $<0.0001$ \\
\hline
\end{tabular}




\section{APPENDIX C}

Table C1. Average weight of faeces from $G$. mellonella larvae after force feeding AZA 1,2 or 3.

Time elapsed

(hours)

\begin{tabular}{llllll}
\hline & 5 & 12.5 & 25 & 37.5 & 50 \\
& ng/larva & ng/larva & ng/larva & ng/larva & ng/larva \\
\hline $\mathbf{4}$ & 0.23 & 0.21 & 0.17 & 0.20 & 0.18 \\
\hline $\mathbf{2 4}$ & 0.78 & 0.76 & 0.77 & 0.27 & 0.41 \\
\hline $\mathbf{4 8}$ & 1.26 & 1.07 & 1.18 & 0.48 & 0.64 \\
\hline
\end{tabular}

Time elapsed

AZA 2

(hours)

\begin{tabular}{llllll}
\hline & 5 & 12.5 & 25 & 37.5 & 50 \\
& ng/larva & ng/larva & ng/larva & ng/larva & ng/larva \\
\hline $\mathbf{4}$ & 0.18 & 0.17 & 0.17 & 0.13 & 0.16 \\
\hline $\mathbf{2 4}$ & 0.85 & 0.76 & 0.44 & 0.74 & 0.53 \\
\hline $\mathbf{4 8}$ & 1.316667 & 1.178148 & 1.08 & 1.136667 & 0.926667 \\
\hline
\end{tabular}

Time elapsed (hours)

\begin{tabular}{llllll}
\hline & 20 & 50 & 100 & 150 & 300 \\
& ng/larva & ng/lava & ng/larva & ng/larva & \begin{tabular}{l} 
ng/larva \\
\hline $\mathbf{4}$
\end{tabular} \\
\hline $\mathbf{2 4}$ & 0.26 & 0.13 & 0.09 & 0.10 & 0.05 \\
\hline $\mathbf{4 8}$ & 0.79 & 0.40 & 0.16 & 0.04 & 0.17 \\
\hline
\end{tabular}

Time elapsed

\section{Control}

(hours)

\begin{tabular}{lll}
\hline & $\begin{array}{l}\text { PBS }+2 \% \\
\text { Ethanol }\end{array}$ & Untreated \\
\hline $\mathbf{4}$ & 0.15 & 0.86 \\
\hline $\mathbf{2 4}$ & 0.75 & 1.22 \\
\hline $\mathbf{4 8}$ & 1.21 & 1.43 \\
\hline
\end{tabular}


Table C2. Statistical significance of faeces weight from G. mellonella larvae after force feeding AZA 1, 2 or 3.

\begin{tabular}{|c|c|c|c|c|}
\hline \multicolumn{5}{|l|}{ Time elapsed (hours) } \\
\hline 24 & $\begin{array}{l}\text { Mean } \\
\text { Diff. }\end{array}$ & $95.00 \%$ CI of diff. & Summary & $\begin{array}{l}\text { Adjusted } \\
\text { P Value }\end{array}$ \\
\hline $\begin{array}{l}\text { AZA1 } 5 \text { ng/larva vs. AZA3 } 100 \\
\text { ng/larva }\end{array}$ & 0.6233 & 0.01684 to 1.230 & $*$ & 0.0374 \\
\hline $\begin{array}{l}\text { AZA1 } 5 \text { ng/larva vs. AZA3 } 150 \\
\text { ng/larva }\end{array}$ & 0.74 & 0.1335 to 1.346 & $* *$ & 0.0039 \\
\hline $\begin{array}{l}\text { AZA1 } 5 \text { ng/larva vs. AZA3 } 300 \\
\text { ng/larva }\end{array}$ & 0.6133 & 0.006839 to 1.220 & $*$ & 0.0445 \\
\hline $\begin{array}{l}\text { AZA1 } 12.5 \text { ng/larva vs. AZA3 } \\
150 \text { ng/larva }\end{array}$ & 0.7167 & 0.1102 to 1.323 & $* *$ & 0.0063 \\
\hline $\begin{array}{l}\text { AZA1 } 25 \text { ng/larva vs. AZA3 } \\
100 \text { ng/larva }\end{array}$ & 0.61 & 0.003505 to 1.216 & $*$ & 0.0471 \\
\hline $\begin{array}{l}\text { AZA1 } 25 \text { ng/larva vs. AZA3 } \\
150 \text { ng/larva }\end{array}$ & 0.7267 & 0.1202 to 1.333 & $* *$ & 0.0051 \\
\hline $\begin{array}{l}\text { AZA1 } 37.5 \mathrm{ng} / \mathrm{larva} \text { vs. } \\
\text { Untreated }\end{array}$ & -0.75 & -1.356 to -0.1435 & $* *$ & 0.0031 \\
\hline AZA1 50 ng/larva vs. Untreated & -0.61 & -1.216 to -0.003505 & $*$ & 0.0471 \\
\hline $\begin{array}{l}\text { AZA2 } 5 \text { ng/larva vs. AZA3 } 100 \\
\text { ng/larva }\end{array}$ & 0.69 & 0.08351 to 1.296 & $*$ & 0.0108 \\
\hline $\begin{array}{l}\text { AZA2 } 5 \text { ng/larva vs. AZA3 } 150 \\
\text { ng/larva }\end{array}$ & 0.8067 & 0.2002 to 1.413 & $* * *$ & 0.0009 \\
\hline $\begin{array}{l}\text { AZA2 } 5 \text { ng/larva vs. AZA3 } 300 \\
\text { ng/larva }\end{array}$ & 0.68 & 0.07351 to 1.286 & $*$ & 0.0131 \\
\hline $\begin{array}{l}\text { AZA2 } 12.5 \text { ng/larva vs. AZA3 } \\
150 \text { ng/larva }\end{array}$ & 0.7133 & 0.1068 to 1.320 & $* *$ & 0.0067 \\
\hline $\begin{array}{l}\text { AZA2 } 37.5 \text { ng/larva vs. AZA3 } \\
150 \text { ng/larva }\end{array}$ & 0.6967 & 0.09017 to 1.303 & $* *$ & 0.0094 \\
\hline $\begin{array}{l}\text { AZA3 } 20 \text { ng/larva vs. AZA3 } \\
100 \text { ng/larva }\end{array}$ & 0.63 & 0.02351 to 1.236 & $*$ & 0.0332 \\
\hline $\begin{array}{l}\text { AZA3 20 ng/larva vs. AZA3 } \\
150 \text { ng/larva }\end{array}$ & 0.7467 & 0.1402 to 1.353 & $* *$ & 0.0034 \\
\hline $\begin{array}{l}\text { AZA3 } 20 \text { ng/larva vs. AZA3 } \\
300 \text { ng/larva }\end{array}$ & 0.62 & 0.01351 to 1.226 & $*$ & 0.0396 \\
\hline AZA3 $50 \mathrm{ng} / \mathrm{larva}$ vs. Untreated & -0.62 & -1.226 to -0.01351 & $*$ & 0.0396 \\
\hline $\begin{array}{l}\text { AZA3 } 100 \text { ng/larva vs. } \\
\text { Untreated }\end{array}$ & -0.86 & -1.466 to -0.2535 & $* * *$ & 0.0003 \\
\hline AZA3 150 ng/larva vs. PBS & -0.71 & -1.316 to -0.1035 & $* *$ & 0.0072 \\
\hline $\begin{array}{l}\text { AZA3 } 150 \text { ng/larva vs. } \\
\text { Untreated }\end{array}$ & -0.9767 & -1.583 to -0.3702 & $* * * *$ & $<0.0001$ \\
\hline $\begin{array}{l}\text { AZA3 } 300 \text { ng/larva vs. } \\
\text { Untreated }\end{array}$ & -0.85 & -1.456 to -0.2435 & $* * *$ & 0.0003 \\
\hline \multicolumn{5}{|l|}{48} \\
\hline $\begin{array}{l}\text { AZA1 } 5 \text { ng/larva vs. AZA1 } 150 \\
\text { ng/larva }\end{array}$ & 0.7733 & 0.1668 to 1.380 & $* *$ & 0.0019 \\
\hline $\begin{array}{l}\text { AZA1 } 5 \text { ng/larva vs. AZA1 } 300 \\
\text { ng/larva }\end{array}$ & 0.62 & 0.01351 to 1.226 & $*$ & 0.0396 \\
\hline $\begin{array}{l}\text { AZA1 } 5 \text { ng/larva vs. AZA3 } 150 \\
\text { ng/larva }\end{array}$ & 0.9333 & 0.3268 to 1.540 & $* * * *$ & $<0.0001$ \\
\hline
\end{tabular}




\begin{tabular}{|c|c|c|c|c|}
\hline $\begin{array}{l}\text { AZA1 } 5 \text { ng/larva vs. AZA3 } 300 \\
\text { ng/larva }\end{array}$ & 0.8233 & 0.2168 to 1.430 & $* * *$ & 0.0006 \\
\hline $\begin{array}{l}\text { AZA1 } 12.5 \text { ng/larva vs. AZA3 } \\
150 \text { ng/larva }\end{array}$ & 0.75 & 0.1435 to 1.356 & $* *$ & 0.0031 \\
\hline $\begin{array}{l}\text { AZA1 } 12.5 \text { ng/larva vs. AZA3 } \\
300 \text { ng/larva }\end{array}$ & 0.64 & 0.03351 to 1.246 & $*$ & 0.0278 \\
\hline $\begin{array}{l}\text { AZA1 } 25 \text { ng/larva vs. AZA1 } \\
150 \text { ng/larva }\end{array}$ & 0.7 & 0.09351 to 1.306 & $* *$ & 0.0088 \\
\hline $\begin{array}{l}\text { AZA1 } 25 \text { ng/larva vs. AZA3 } \\
150 \text { ng/larva }\end{array}$ & 0.86 & 0.2535 to 1.466 & $* * *$ & 0.0003 \\
\hline $\begin{array}{l}\text { AZA1 } 25 \text { ng/larva vs. AZA3 } \\
300 \text { ng/larva }\end{array}$ & 0.75 & 0.1435 to 1.356 & $* *$ & 0.0031 \\
\hline $\begin{array}{l}\text { AZA1 } 25 \text { ng/larva vs. AZA2 } \\
21.5 \text { ng/larva }\end{array}$ & -0.6933 & -1.300 to -0.08684 & $*$ & 0.0101 \\
\hline $\begin{array}{l}\text { AZA1 } 37.5 \text { ng/larva vs. AZA2 } \\
37.5 \text { ng/larva }\end{array}$ & -0.6533 & -1.260 to -0.04684 & $*$ & 0.0217 \\
\hline $\begin{array}{l}\text { AZA1 } 37.5 \text { ng/larva vs. AZA3 } \\
20 \text { ng/larva }\end{array}$ & -0.6667 & -1.273 to -0.06017 & $*$ & 0.0169 \\
\hline AZA1 37.5 ng/larva vs. PBS & -0.73 & -1.336 to -0.1235 & $* *$ & 0.0048 \\
\hline $\begin{array}{l}\text { AZA1 } 37.5 \text { ng/larva vs. } \\
\text { Untreated }\end{array}$ & -0.9433 & -1.550 to -0.3368 & $* * * *$ & $<0.0001$ \\
\hline $\begin{array}{l}\text { AZA1 } 50 \text { ng/larva vs. AZA2 } 5 \\
\text { ng/larva }\end{array}$ & -0.68 & -1.286 to -0.07351 & $*$ & 0.0131 \\
\hline AZA1 50 ng/larva vs. Untreated & -0.79 & -1.396 to -0.1835 & $* *$ & 0.0013 \\
\hline $\begin{array}{l}\text { AZA2 } 5 \text { ng/larva vs. AZA3 } 150 \\
\text { ng/larva }\end{array}$ & 0.9933 & 0.3868 to 1.600 & $* * * *$ & $<0.0001$ \\
\hline $\begin{array}{l}\text { AZA2 } 5 \text { ng/larva vs. AZA3 } 300 \\
\text { ng/larva }\end{array}$ & 0.8833 & 0.2768 to 1.490 & $* * *$ & 0.0001 \\
\hline $\begin{array}{l}\text { AZA2 } 12.5 \text { vs. AZA3 } 150 \\
\text { ng/larva }\end{array}$ & 0.8533 & 0.2468 to 1.460 & $* * *$ & 0.0003 \\
\hline $\begin{array}{l}\text { AZA2 } 12.5 \text { ng/larva vs. AZA3 } \\
300 \text { ng/larva }\end{array}$ & 0.7433 & 0.1368 to 1.350 & $* *$ & 0.0036 \\
\hline $\begin{array}{l}\text { AZA2 } 25 \text { ng/larva vs. AZA3 } \\
150 \text { ng/larva }\end{array}$ & 0.7567 & 0.1502 to 1.363 & $* *$ & 0.0027 \\
\hline $\begin{array}{l}\text { AZA2 } 25 \text { ng/larva vs. AZA3 } \\
300 \text { ng/larva }\end{array}$ & 0.6467 & 0.04017 to 1.253 & $*$ & 0.0246 \\
\hline $\begin{array}{l}\text { AZA2 } 37.5 \text { ng/larva vs. AZA3 } \\
150 \text { ng/larva }\end{array}$ & 0.8133 & 0.2068 to 1.420 & $* * *$ & 0.0008 \\
\hline $\begin{array}{l}\text { AZA2 } 37.5 \text { ng/larva vs. AZA3 } \\
300 \text { ng/larva }\end{array}$ & 0.7033 & 0.09684 to 1.310 & $* *$ & 0.0082 \\
\hline $\begin{array}{l}\text { AZA3 } 20 \text { ng/larva vs. AZA3 } \\
150 \text { ng/larva }\end{array}$ & 0.8267 & 0.2202 to 1.433 & $* * *$ & 0.0006 \\
\hline $\begin{array}{l}\text { AZA3 } 20 \text { ng/larva vs. AZA3 } \\
300 \text { ng/larva }\end{array}$ & 0.7167 & 0.1102 to 1.323 & $* *$ & 0.0063 \\
\hline $\begin{array}{l}\text { AZA3 } 100 \text { ng/larva vs. } \\
\text { Untreated }\end{array}$ & -0.6933 & -1.300 to -0.08684 & $*$ & 0.0101 \\
\hline AZA3 150 ng/larva vs. PBS & -0.89 & -1.496 to -0.2835 & $* * *$ & 0.0001 \\
\hline $\begin{array}{l}\text { AZA3 } 150 \text { ng/larva vs. } \\
\text { Untreated }\end{array}$ & -1.103 & -1.710 to -0.4968 & $* * * *$ & $<0.0001$ \\
\hline AZA3 300 ng/larva vs. PBS & -0.78 & -1.386 to -0.1735 & $* *$ & 0.0016 \\
\hline $\begin{array}{l}\text { AZA3 } 300 \text { ng/larva vs. } \\
\text { Untreated }\end{array}$ & -0.9933 & -1.600 to -0.3868 & $* * * *$ & $<0.0001$ \\
\hline
\end{tabular}




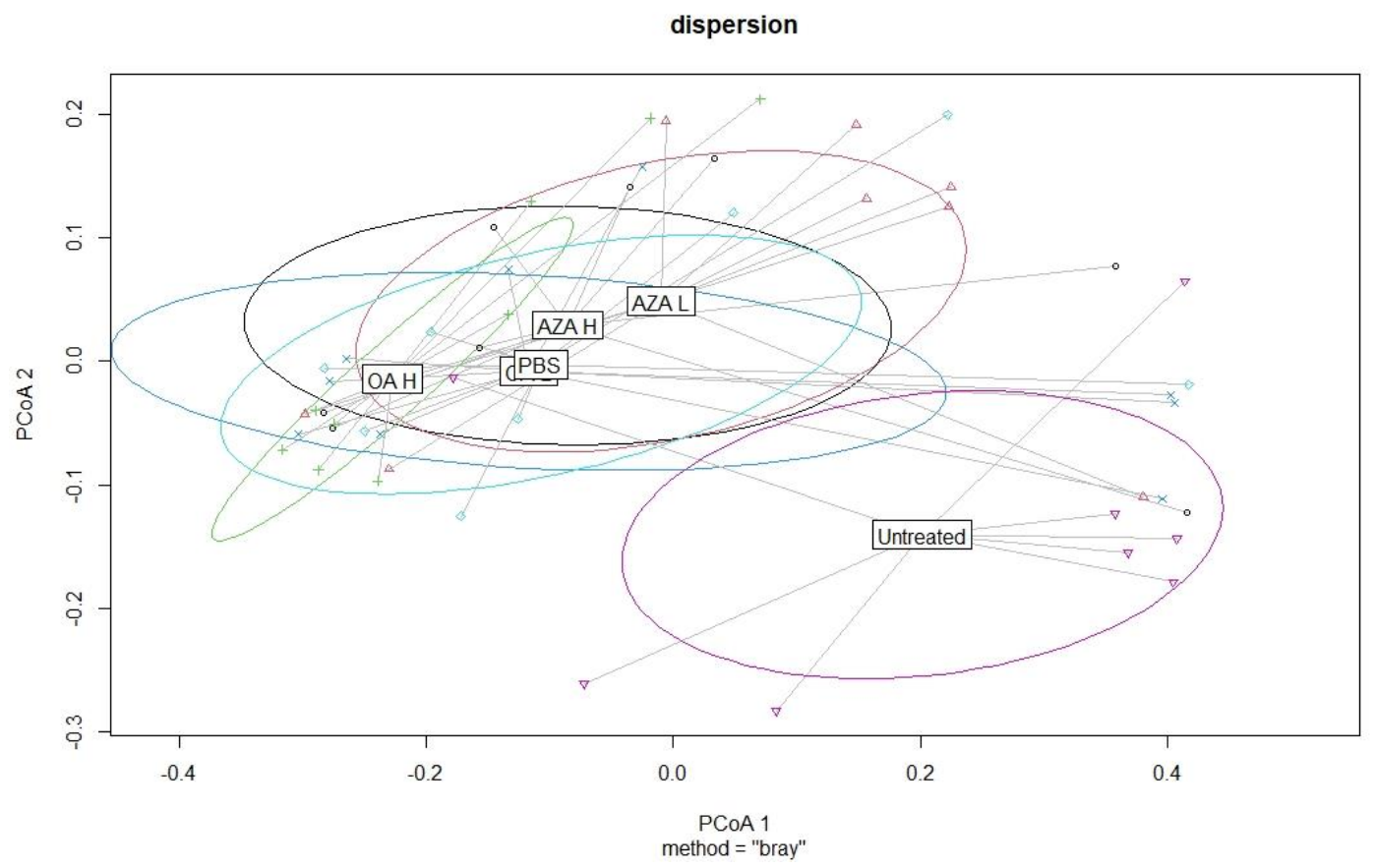

Figure C1. Dispersion plot of the merged timepoint of Untreated (U) vs AZA1, OA, and PBS.

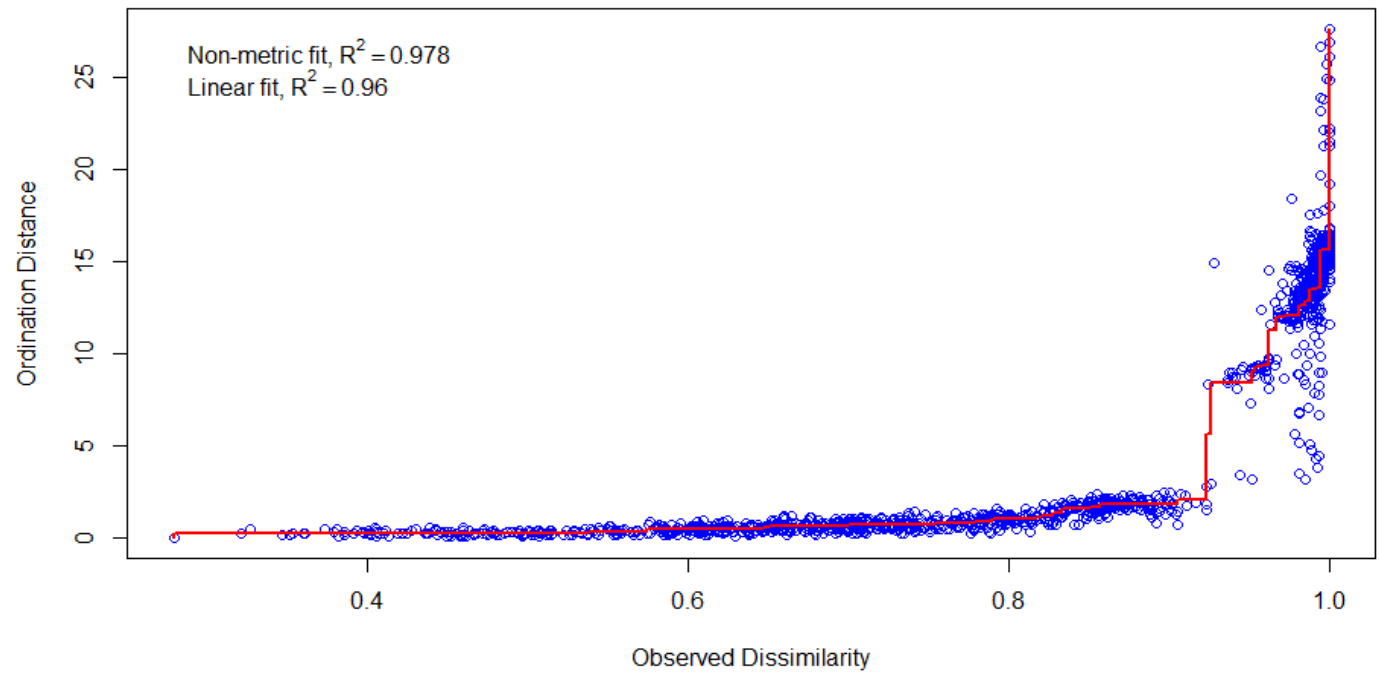

Figure C2. Stress plot of the merged timepoint of Untreated (U) vs AZA1, OA, and PBS. 


\section{Appendix D}

Contributions to the following research article:

Cell Biol Toxicol (2019) 35:219-232

https://doi.org/0.1007/s10565-018-09448-2

ORIGINAL ARTICLE

\section{The insect, Galleria mellonella, is a compatible model for evaluating the toxicology of okadaic acid}

Christopher J. Coates 19 - Jenson Lim .

Katie Harman - Andrew F. Rowley *

David J. Griffiths - Helena Emery - Will Layton

Abstract The polyether toxin, okadaic acid, cause diarthetic shellfish poisoning in humans. Despite extensive research into its cellular targets using rodent models, we know little about its putative effect(s) on innate immunity. We inoculated larvae of the greater wax moth, Galleria mellonella, with physiologically relevant doses of okadaic acid by direct injection into the haemocoel (body cavity) and/or gavage (force-feeding). We monitored larval survival and employed a range of cellular and biochemical assays to assess the potential hamful effects of okadaic acid. Okadaic acid at concentrations $\geq 75 \mathrm{ng} / \mathrm{larva}(\geq 242 \mu \mathrm{g} / \mathrm{kg}$ ) led to significant reductions in larval survival $(>65 \%)$ and circulating haemocyte (blood cell) numbers ( $>50 \%$ ) within $24 \mathrm{~h}$ post-inoculation. In the haemolymph, okadaic acid reduced haemocyte viability and increased phenoloxidase activities. In the midgut, okadaic acid induced oxidative damage as determined by increases in superoxide dismutase activity and levels of malondialdehyde (i.e. lipid peroxidation). Our observa-

Electronic supplementary material The online version of this article (hutps:/doiorg/10,1007/s10565-018-09448-2) contain supplementary material, which is available to auhborized users.

C. J. Coates (A) + K. Haman + A. F. Rowley -

D. J. Griftiths - H. Emery - W, Layton

Department of Biosciences, College of Science, Swansea

University, Swansea, Wales SA2 8PP, UK

e-mail: c.j.coates@iswansea.ac,uk

J. Lim

Biological and Environmental Sciences, Faculty of Natumal

Sciences, University of Stirling, Stirling, Scotand FK9 4LA, UK tions of insect larvae correspond broadly to data published using rodent models of shellfish-poisoning toxidrome, including complementary $\mathrm{LD}_{50}$ values: $206-242 \mu \mathrm{g} / \mathrm{kg}$ in mice, $\sim 239 \mu \mathrm{g} / \mathrm{kg}$ in $G$. mellonella. These data support the use of this insect as a surrogate model for the investigation of marine toxins, which offers distinct ethical and financial incentives.

Keywords Haemocytes - Innate immunity- Oxidative stress - Phenoloxidase - Shellfish-poisoning syndrome . Immunotoxicology

\section{Abbreviations}

DSP Dianhetic shellfish poisoning

OA Okadaic acid

PBS Phosphate-buffered saline

MDA Malondialdehyde

PO Phenoloxidase

SDS Sodium dodecyl sulphate

SOD Superoxide dismutase

\section{Introduction}

Diarrhetic shellfish poisoning (DSP) is one of several recognised shellfish-poisoning syndromes including amnesic, neurotoxic, and paralytic. Upon consumption of bivalves, crustaceans, sea urchins, and finfish contaminated with the polyether toxin, okadaic acid $\left(\mathrm{C}_{44} \mathrm{H}_{6 \times} \mathrm{O}_{13}\right)$. DSP manifests as nausea, vomiting. diarrhoea, abdominal cramps, and chills (Vale and 
Ethics approval number: SU-Ethics-Student-120218/470

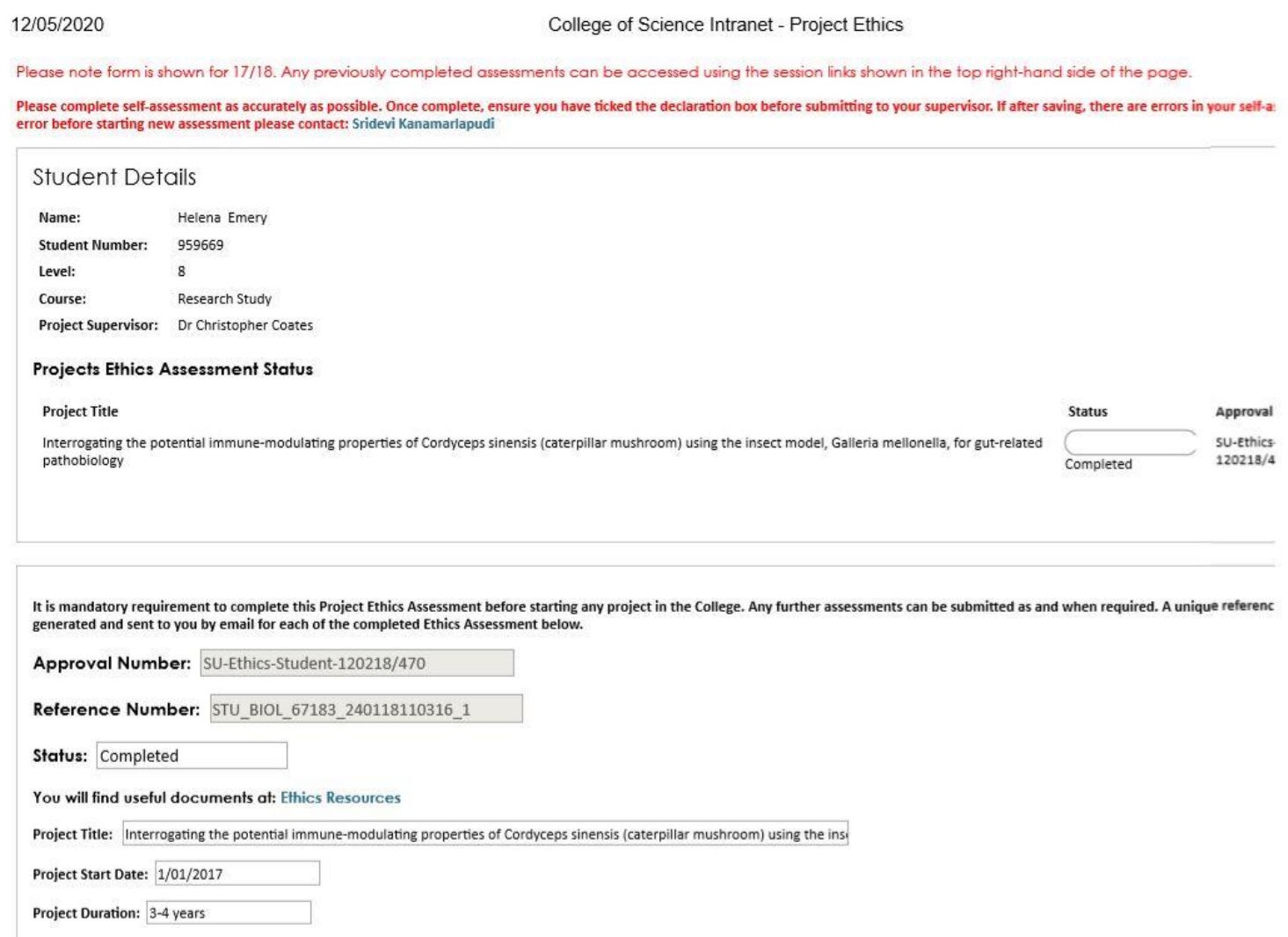


Risk assessments, a full list provided as a PDF.

HEALTH \& SAFETY

IECHYD A DIOGELWCH

\title{
RISK ASSESSMENT OF AN ACTIVITY INVOLVING DELIBERATE WORK WITH MICROORGANISMS
}

\begin{abstract}
This risk assessment form should be used to assist in the assessment of risks from an activity involving deliberate work with an infectious of harmful biological agent. The aim of the assessment is to identify those at risk from infection or other harm and the measures required to eliminate or control the risks to human health and the environment to an acceptable level.
\end{abstract}

\begin{tabular}{|l|l|}
\hline \multicolumn{2}{|l|}{ SECTION 1: PROJECT INFORMATION } \\
\hline 1.1 Principal Investigator/Academic Supervisor \\
\hline Name & College \\
\hline Dr Christopher Coates & Science, Department of Biosciences \\
\hline 1.2 Person undertaking this risk assessment (if different from above) \\
\hline Name & College \\
\hline Helena Emery (PGR) & Science, Department of Biosciences \\
\hline 1.3 Project title &
\end{tabular}

Using the insect model, Galleria mellonella, for gut-related pathobiology

1.4 Brief overview of the work (in layman's terms)

Challenging insect larvae (Galleria mellonella) with bacteria known to cause, or associated with, gastric damage. Using these insect larvae provides a well-established, ethical alternative to rodent testing.

The aim is to induce damage using bacteria (Mycobacterium avium (subspecies) paratuberculosis, Campylobacter jejuni) via two inoculation methods (intrahaemocoelic injection, gavage). Experimental larvae will then be monitored for survivorship and gut restitution, and 'treated' with colostrum, mushroom extract (Cordyceps sinensis) and common anti-ulcer drugs. In this instance, we are using the insect as a screening tool by following well-established protocols in our lab and the published literature (e.g., Coates et al., 2018; Cell Biology \& Toxicology; Lim, Coates et al., Journal of Immunology; Senior et al., 2011).

\section{References:}

Coates, C. J., Lim, J., Harman, K., Rowley, A. F., Griffiths, D. J., Emery, H., \& Layton, W. (2018). The insect, Galleria mellonella, is a compatible model for evaluating the toxicology of okadaic acid. Cell Biology and Toxicology, 1-14.

Lim, J., Coates, C. J., Seoane, P. I., Garelnabi, M., Taylor-Smith, L. M., Monteith, P., ... \& May, R. C. (2018). Characterizing the Mechanisms of Nonopsonic Uptake of Cryptococci by Macrophages. The Journal of Immunology, ji1700790.

Senior, N. J., Bagnall, M. C., Champion, O. L., Reynolds, S. E., La Ragione, R. M., Woodward, M. J., ... \& Titball, R. W. (2011). Galleria mellonella as an infection model for Campylobacter jejuni virulence. Journal of medical microbiology, 60(5), 661-669. 


\begin{tabular}{|c|c|c|c|}
\hline \multicolumn{4}{|c|}{ 2.1 List microorganisms deliberately used } \\
\hline Name of microorganism & $\begin{array}{l}\text { Mycobacterium } \\
\text { avium (subspecies) } \\
\text { paratuberculosis }\end{array}$ & Campylobacter jejuni & Clostridium difficile \\
\hline $\begin{array}{l}\text { Identified as human pathogen } \\
\text { on ACDP } \text { list }^{1}\end{array}$ & Yes & Yes & Yes \\
\hline If yes please state hazard group & 2 & 2 & 2 \\
\hline $\begin{array}{l}\text { If not on ACDP list, is there any } \\
\text { evidence to support the } \\
\text { microorganism may present a } \\
\text { risk to human health }\end{array}$ & $\begin{array}{l}\text { Click or tap here to } \\
\text { enter text. }\end{array}$ & $\begin{array}{l}\text { Click or tap here to } \\
\text { enter text. }\end{array}$ & $\begin{array}{l}\text { Click or tap here to } \\
\text { enter text. }\end{array}$ \\
\hline $\begin{array}{l}\text { Normal routes of human } \\
\text { infection }\end{array}$ & $\begin{array}{l}\square \text { Inhalation } \\
\bigotimes \text { Oral/ingestion } \\
\square \text { Mucocutaneous } \\
\square \text { Percutaneous } \\
\square \text { Via vector (e.g. } \\
\text { insect) } \\
\square \text { Allergen }\end{array}$ & $\begin{array}{l}\square \text { Inhalation } \\
\bigotimes \text { Oral/ingestion } \\
\square \text { Mucocutaneous } \\
\square \text { Percutaneous } \\
\square \text { Via vector (e.g. } \\
\text { insect) } \\
\square \text { Allergen }\end{array}$ & $\begin{array}{l}\square \text { Inhalation } \\
\bigotimes \text { Oral/ingestion } \\
\square \text { Mucocutaneous } \\
\square \text { Percutaneous } \\
\square \text { Via vector (e.g. } \\
\text { insect) } \\
\square \text { Allergen }\end{array}$ \\
\hline $\begin{array}{l}\text { Multiplicity of infection if } \\
\text { known (i.e. number of } \\
\text { organisms required to establish } \\
\text { an infection) }\end{array}$ & N/A & N/A & N/A \\
\hline $\begin{array}{l}\text { Consequence of infection to } \\
\text { humans }\end{array}$ & $\begin{array}{l}\text { Suspected cause of } \\
\text { Johne's disease in } \\
\text { ruminants and } \\
\text { linked to Crohn's } \\
\text { disease in humans }\end{array}$ & $\begin{array}{l}\text { Localised infection } \\
\text { causing diarrhoea. } \\
\text { Treatment with } \\
\text { antibiotics may be } \\
\text { required in severe } \\
\text { infection or those } \\
\text { immunocompromised. } \\
\text { In some cases, } \\
\text { infection can result in } \\
\text { irritable bowel } \\
\text { syndrome. }\end{array}$ & $\begin{array}{l}\text { Infects the bowel } \\
\text { resulting in } \\
\text { diarrhoea. } \\
\text { Treatment with } \\
\text { antibiotics may be } \\
\text { required. Serious } \\
\text { infection can damage } \\
\text { the bowel requiring } \\
\text { surgery. }\end{array}$ \\
\hline $\begin{array}{l}\text { Is the microorganism a } \\
\text { specified animal pathogen } \\
\left(\text { SAPO }^{2}\right)\end{array}$ & No & No & No \\
\hline $\begin{array}{l}\text { If yes please state SAPO hazard } \\
\text { group }\end{array}$ & Choose an item. & Choose an item. & Choose an item. \\
\hline $\begin{array}{l}\text { Detail of any other harm the } \\
\text { microorganism may pose to the }\end{array}$ & None & None & None \\
\hline
\end{tabular}


Section 3: Experimental Procedures

3.1 Description of experimental procedures:

(Brief details, also indicate any non-standard laboratory operations and any procedures that might require specific control measures e.g. use of sharps, generation of aerosols, in vivo work)

$>$ All experimental work will be carried out in the recently refurbished CAT2 facility, Wallace 044 .

$>$ A standardised inoculum of each bacterium will be prepared (exponential phase) in sterile phosphate buffered saline (PBS).

$>$ Each insect larvae is weighed, inspected for visual signs of infection/damage (i.e., melanisation) and placed in a petri dish ( $n=10$ per replicate) lined with Whatman filter paper and wood shavings (provided by commercial supplier)

>Larvae are chilled on ice for 2-5 minutes (immobilises insects) prior to the administration of bacteria using a 26/27 gauge needle. The larvae can be held with sterile forceps to reduce the risk of accidental puncture of skin. Insects can be inoculated by intrahaemocoelic injection, or by force feeding (gavage). $>$ Each insect receives 10-20 $\mu \mathrm{L}$ inoculum. Negative controls are given PBS. Sharps will be disposed of in the appropriate containers.

$>$ Experimental G. mellonella are incubated between 30 and $37^{\circ} \mathrm{C}$ for up to 10 days in the dark (depending on the experiment).

$>$ At 4, 24, 48 and 72 hours post-treatment, survivorship, pupation and melanisation are monitored by following a well-established 'health index' for this insect (Loh et al., 2013)

$>$ In some instances, insects will be co-treated with an anti-inflammatory (anti-ulcer) drug, colostrum or extracts from the medicinal fungus, Cordyceps sinensis, in attempts to gauge putative repair and restitution properties

$>$ Additionally, some insects will be sacrificed by fixation (for wax histology) or dissected on ice to remove the midgut for nucleic and amino acid (DNA, RNA, protein) extractions.

$>$ Any contaminated tissues will be bagged and frozen, autoclaved and disposed of following university guidelines and procedures.

Loh, J. M., Adenwalla, N., Wiles, S., \& Proft, T. (2013). Galleria mellonella larvae as an infection model for group A streptococcus. Virulence, 4(5), 419-428.

3.2 Quantities used and frequency of use:

This information will enable you to determine the likelihood of exposure and therefore the risks from this particular activity. Please indicate maximum culture volumes at any time shown as multiples of flask volumes to give an idea of scale.

Max. volume per

culture/sample

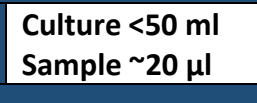

Max. volume per experiment:

$<1 \mathrm{ml}$ on each

Frequency of experiments

10 Galleria for each bacterium, per replicate

Survivorship:

40x Galleria for M. avium paratuberculosis (ATCC BAA-968)

40x Galleria for $C$. jejuni (ATCC BAA-224)

40x Galleria for $C$. difficile (ATCC 51695)

40x Galleria for PBS (negative control)

Gut dissections/wax histology:

30x Galleria for M. avium paratuberculosis

30x Galleria for $C$. jejuni

30x Galleria for $C$. difficile

30x Galleria for PBS (negative control)

(these samples may be split 50:50 between injected and force-fed) 


\section{Section 4: MeAsures to Prevent or Control Exposure \\ Preventing exposure \\ 4.1 Could a less hazardous substance (or form of the substance) be used instead? \\ (If it can, then it should be used or justification be given here why it is not being used. COSHH requires substitution with less hazardous materials wherever possible, but there may be good reasons for not using them.)}

Only known hazardous substance will be the bacterial inocula required for infection. Recent evidence suggests that substituting pathogenic gut bacteria with 'symbiotic' bacteria (e.g., Bacteroides vulgatus; Lange et al., 2018) leads to differential innate immune responses of the insect host, therefore less hazardous alternatives are not suitable here.

Lange, A., Schäfer, A., Bender, A., Steimle, A., Beier, S., Parusel, R., \& Frick, J. S. (2018). Galleria mellonella: a novel invertebrate model to distinguish intestinal symbionts from pathobionts. Frontiers in immunology, 9 .

Controlling exposure

4.2 Containment Level - what containment level is required for this work with regard to COSHH/SAPO?

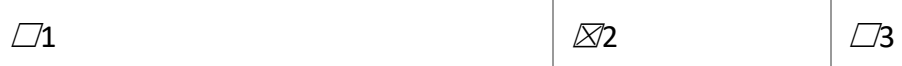

CL3 only - application for derogation from the following controls (list if relevant and justify)

Click or tap here to enter text.

Premises where this work will be carried out

\begin{tabular}{|l|l|l|}
\hline Building & Laboratories & Containment level \\
\hline Wallace & $\mathbf{0 4 4}$ & $\mathbf{2}$
\end{tabular}

Will the work be segregated from others not involved in the work and if not, how will they be informed of the hazards? : 


\begin{abstract}
Work will be segregated. Bacterial cultures and infected insects will be stored separately from other work. Two dedicated incubators $\left(30 / 37^{\circ} \mathrm{C}\right)$ will be used exclusively for this. Appropriate warning signs are in place on entrance to the lab, and signage will be placed on incubators whilst experiments are running.
\end{abstract}

One (of two) microbiological safety cabinets will be used to work with the gut-related pathogens (a sign to reflect this will be on display).

\title{
4.3 Engineering Controls (Containment \& Ventilation) \\ a) Is a microbial safety cabinet (or isolator for in vivo work) required? These must be used for activities generating potentially infectious aerosols or splashes.

\begin{tabular}{l|l} 
ХYES $\square$ NO & Class: $\square$ I $\bigotimes$ II $\square$ III \\
\hline If required, what processes require its use? & Preparation of bacterial inocula for in vivo work \\
\hline Specify other local ventilation control measures considered appropriate (e.g. downdraft table, isolator):
\end{tabular}

N/A

b) Will centrifugation be used?

$\square$ YES $\triangle$ NO

If yes, will buckets and rotors be sealed?

If yes, where will buckets or rotors be opened?

If yes, how will spillages in the centrifuge be dealt with?

c) Will incubators be used?

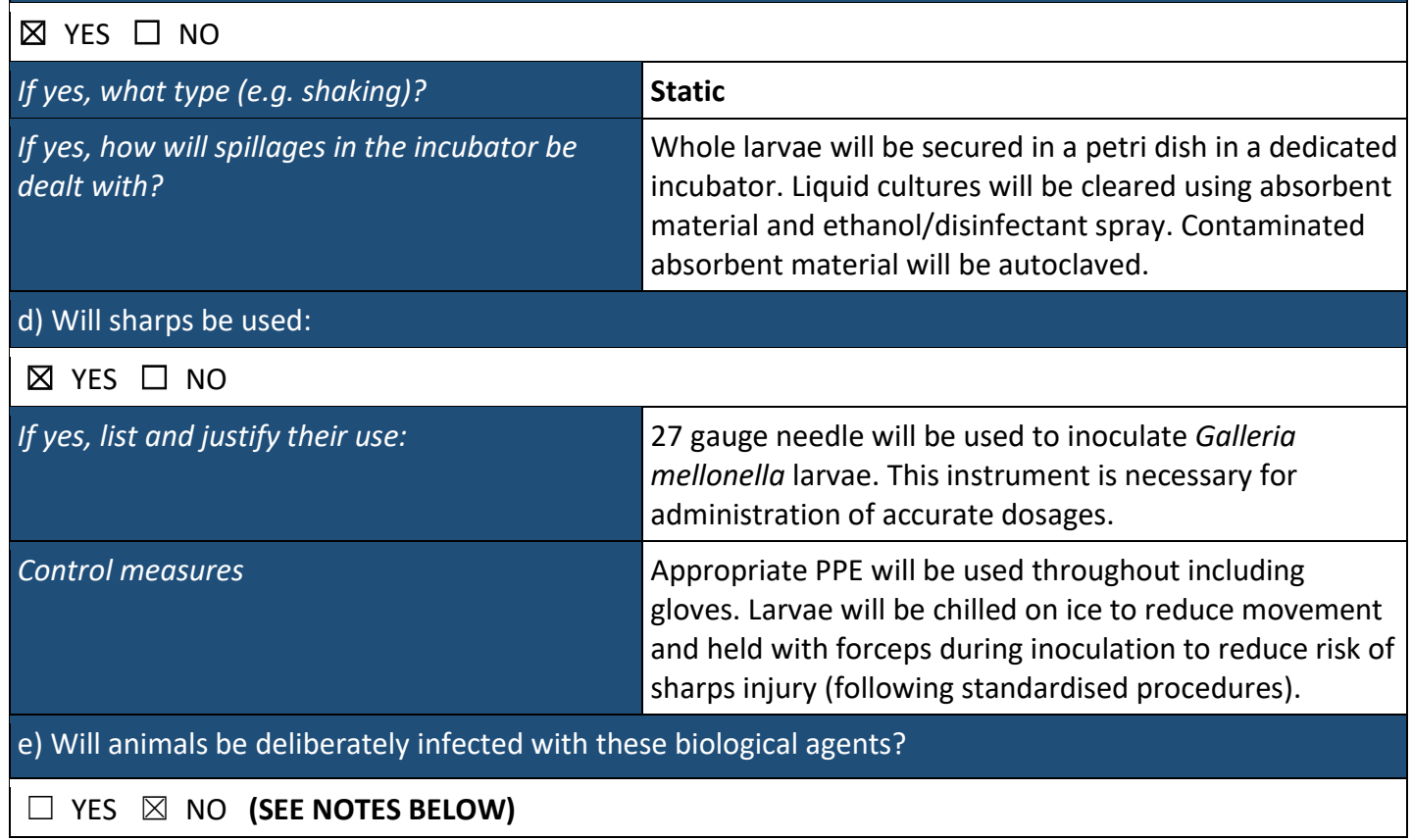


If yes, describe the procedure, control measures and whether shedding of infectious agents by animals is expected?
Galleria mellonella are considered a non-animal technology (NAT) in the context of UK animal use regulations (ASPA) where procedures on this species are not regulated. However, insects will be deliberately infected and shedding of infectious material may occur via faces in larvae force-fed bacteria. In this instance, the faeces and contaminated larvae, petri dish and filter paper will be collected when the experiment is completed, bagged and stored frozen $\left(-20^{\circ} \mathrm{C}\right.$ to $\left.-80^{\circ} \mathrm{C}\right)$ until autoclaving following the university procedures. Infected insects will be stored separately in a dedicated static incubator.

\begin{tabular}{|c|c|c|c|}
\hline Lab coat & Gloves & Eye or face (specify if yes) & Other (specify) \\
\hline QYES & $\triangle$ YES $\square$ NO & $\square$ YES $\square$ NO & \multirow[t]{3}{*}{ Click or tap here to enter text } \\
\hline Details: & Details: & Details: & \\
\hline Howie Lab Coat & Nitrile gloves & $\begin{array}{l}\text { Click or tap here to enter } \\
\text { text. }\end{array}$ & \\
\hline
\end{tabular}

4.5 Transportation

a) How will viable material be transported within the laboratory?

Bacterial cultures will be prepared in Corning (vented screw-cap) conical flasks. Infected insects will be transported in petri dishes secured with tape.

b) How will viable material be transported locally outside the laboratory?

No reagent or living organism will leave the laboratory during experimentation.

In some instances, fixed insects will be transported in $\mathbf{7 0 \%}$ ethanol to the histology suite for wax embedding (023, Wallace)

c) Will viable material be shipped anywhere (off campus)? YES $\triangle N O$

If yes, what will be shipped?

If yes, how will this be shipped (e.g. Category Click or tap here to enter text.

A, Category B, Exempt, Non-hazardous)?

\subsection{Waste disposal procedures:}

(Disinfectants, concentrations, exposure times, autoclaving procedures, incinerator procedures, include any animal related wastes.)

\begin{tabular}{|c|c|c|}
\hline Waste & Decontamination method (include details on efficacy) & $\begin{array}{l}\text { Disposal route } \\
\text { e.g. drain/incineration/landfill }\end{array}$ \\
\hline Liquid waste & $\begin{array}{l}\text { Bacterial cultures will be inactivated on site using a } \\
\text { disinfectant (bleach, Virkon S } 2 \% \text { solution for } 1 \text { hour) } \\
\text { prior to autoclaving }\end{array}$ & Drain, once deactivated \\
\hline Solid waste & $\begin{array}{l}\text { All Galleria mellonella larvae will be frozen prior to } \\
\text { autoclaving }\end{array}$ & $\begin{array}{l}\text { Following University procedures } \\
\text { Incineration once deactivated }\end{array}$ \\
\hline Sharp waste & $\begin{array}{l}\text { Decontaminated with bleach/Virkon } 2 \% \text { solution for } 1 \\
\text { hour and into sharps bin. }\end{array}$ & Following University procedures \\
\hline
\end{tabular}


* Virkon ${ }^{\mathrm{TM}} \mathrm{S}$ is the breakthrough disinfectant formulation that defines on farm biosecurity. A $2 \%$ solution has been powerful, proven performance against over 500 strains of viruses, bacteria and fungi including Foot and Mouth Disease (FMD), Avian Influenza, Salmonella and Campylobacter, Virkon ${ }^{\mathrm{TM}} \mathbf{S}$ is selected by governments worldwide for Emergency Disease Control.

Alternatively, a $2 \%$ Biocleanse solution can be used too.

Biocleanse $\left(\right.$ Teknon $\left.{ }^{\circledR}\right)$ has powerful bactericidal and virucidal properties. Biocleanse concentrate is free from sodium hypochlorite, phosphates and enzymes and effectively removes blood, fat, proteins, grease and other organic and non-organic contaminants whilst simultaneously disinfecting the treated surface. It is safe to use on ferrous and non-ferrous metals, ceramics, glassware and plastics. Biocleanse kills E. coli, Salmonella, Listeria, Candida and Penicillium at $1 \%$ dilution and MRSA at $2 \%$ dilution. Efficacy against C. difficile, Avian Flu, HIV and $\mathrm{HBV}$ is proven at $5 \%$ dilution. In addition, under COSHH Regulations, the product does not require a maximum exposure limit and when diluted to normal use concentrations, is not irritating to skin.

\begin{tabular}{l}
4.7 Emergency procedures \\
(spillages - if not covered by local rules/standard operating procedure) Remember to take into account \\
route of exposure \\
\hline Inside primary containment (if relevant e.g. MSC, isolator) \\
\hline $2 \%$ Virkon, followed by $70 \%$ alcohol \\
\hline Outside primary containment but within the laboratory (secondary containment) \\
\hline $2 \%$ Virkon, followed by $70 \%$ alcohol \\
\hline Outside secondary containment (if relevant): \\
\hline Click or tap here to enter text. \\
\hline Other procedures (e.g. following any kind of accidental exposure, needlestick etc.): \\
\hline Click or tap here to enter text.
\end{tabular}

\section{Section 5: Personnel and Health Issues}

5.1 Vaccination

For ACDP 2 or above human pathogens - to be completed by Occupational Health

Is an effective vaccination available for any of the pathogens associated with this work?

No

5.2 Is health surveillance/health clearance required?

\begin{tabular}{l|l}
\hline Staff and postgraduate research students & $\square$ YES $\$ NO \\
\hline Taught students (undergrad and MSc) & $\square$ YES $\$ NO (initial Health clearance only)
\end{tabular}

5.3 Identify any particular groups of workers who may be at increased risk from this material: (for

example pregnant workers, young persons under 18, disabled workers, those with pre-existing disease that increases susceptibility.)

Immuno-compromised (antibiotic regime, HIV+)

Anyone who might have compromised resistance to disease for any reason should seek advice from the University Occupational Health Service regarding the need for additional precautions.

5.4 Information, instruction and training

Describe the training that will be given to all those affected (directly or indirectly) by the work activity.

044 Laboratory training and inductions will be provided Dr Christopher Coates and/or Dr Almudena OrtizUrquiza. If concerns are raised regarding pre-existing conditions, advice will be sought from University

Occupational Health Service. 


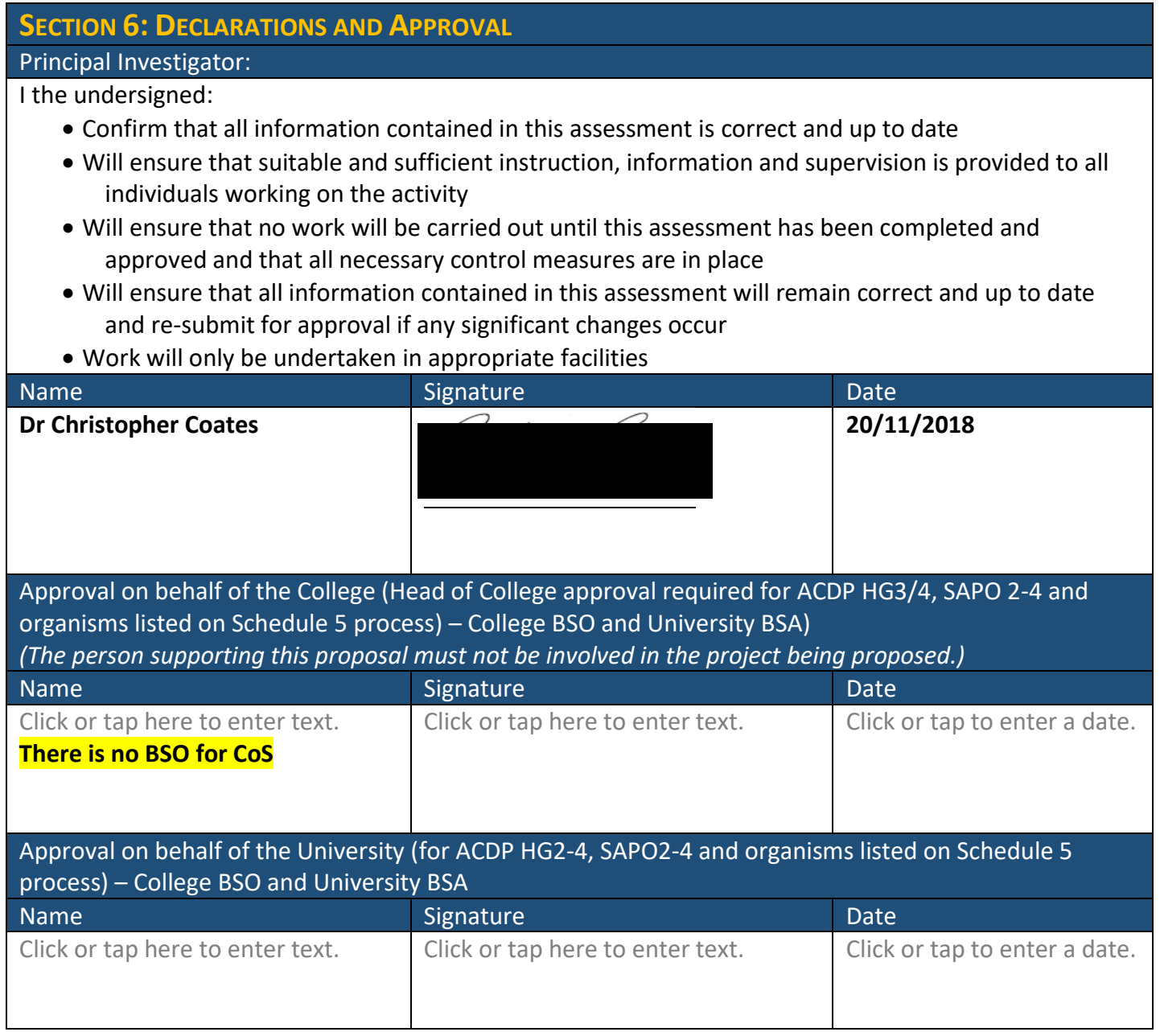

Additional information:

Laboratory 044 has restricted access at the discretion of Dr Christopher Coates and Dr Almudena Ortiz. This lab is equipped with a Salto electronic lock.

A designated autoclave is located in the Wallace Building room 001 . This autoclave is regularly validated using a 12-point thermocouple technique. Staff technicians keep records of the validation. Once autoclaved the waste will be place in tiger bags and disposed into the autoclave skip situated in the car park between Margam and ILS1.

There is a designated lidded bin to store and transport double-bagged waste from lab 044 to the autoclave. Autoclave facilities are in the same building.

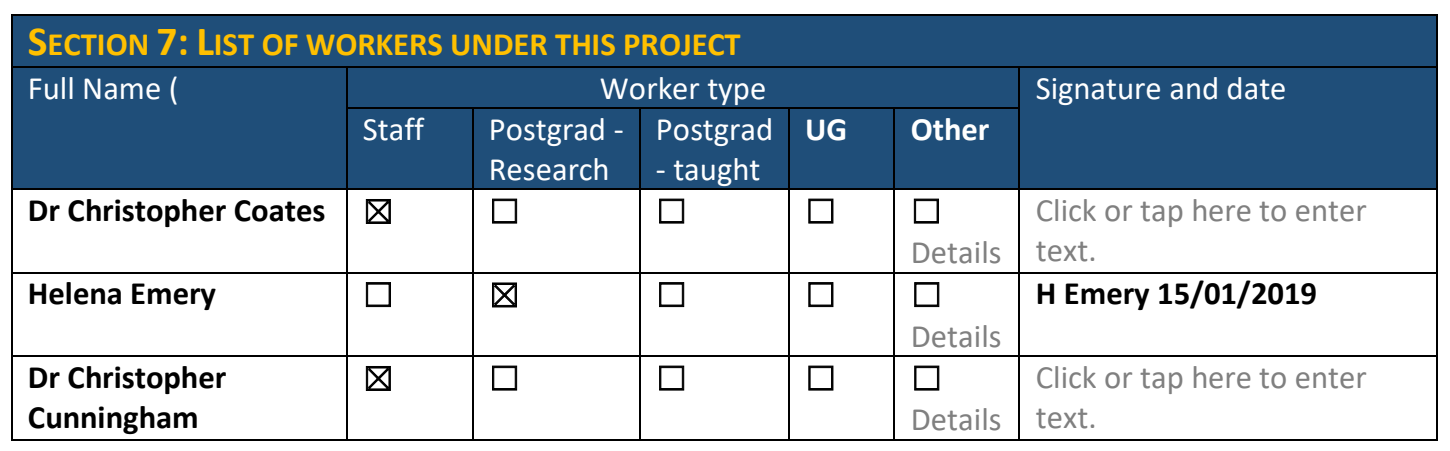


Bioscience and Geography Protocol Risk Assessment Form (Expand or contract fields, or append additional sheets as required; insert NA if not applicable)

\begin{tabular}{|c|c|c|c|c|}
\hline Protocol \#1 & \multicolumn{4}{|c|}{ Title: Injecting Trypan blue $(0.4 \%)$ into G. mellonella } \\
\hline $\begin{array}{l}\text { Associated Protocols } \\
\text { H............................... }\end{array}$ & \multicolumn{4}{|c|}{$\begin{array}{l}\text { Description: } \\
\text { Injecting and force feeding } 20 \mu \mathrm{l} \text { of trypan blue }(0.4 \% \text { solution) into } G \text {. } \\
\text { mellonella gut and haemocoel }\end{array}$} \\
\hline \multicolumn{5}{|c|}{$\begin{array}{l}\text { Location: Laboratory } 123 \text { Wallace Building } \\
\text { circle which Bioscience and Geography Local Rules apply- } \\
\text { Bent Field Genetie-Manipulation Laboratory }\end{array}$} \\
\hline \multicolumn{5}{|c|}{ Identify here risks and control measures for work in this environment, additional to Local Rules } \\
\hline Chemicals & Quantity & Hazards & $\begin{array}{c}\text { Category } \\
(\mathbf{A}, \mathbf{B}, \mathbf{C}, \mathrm{D})^{*}\end{array}$ & $\begin{array}{l}\text { Exp. } \\
\text { Sc }\end{array}$ \\
\hline Trypan Blue $(0.4 \%)$ & $0.4 \mathrm{~g}$ in $100 \mathrm{ml}$ & Potential carcinogen & A & 2 \\
\hline \multicolumn{2}{|c|}{$\begin{array}{ll} & \text { Hazard Category (known or potential) } \\
\text { A (e.g. carcinogen/teratogen/mutagen) } \\
\text { B (e.g. v.toxic/toxic/explosive/pyrophoric) } \\
\text { C (e.g. harmful/irritant/corrosive/high } \\
\text { flammable/oxidising) } \\
\text { D (e.g. non classified) }\end{array}$} & \multicolumn{3}{|c|}{$\begin{array}{l}\text { Exposure Potential Circle the highest Exposure } \\
\text { Score above. Use this to calculate the exposure } \\
\text { potential for the entire protocol (see handbook). } \\
\text { Indicate this value below. } \\
\text { Low }\end{array}$} \\
\hline \multicolumn{5}{|c|}{$\begin{array}{l}\text { Primary containment (of product) :- Sealed (darkened) plastic/glass flask } \\
\text { Storage conditions and maximum duration :- cool, well-ventilated place }\end{array}$} \\
\hline \multicolumn{5}{|c|}{ Secondary containment (of protocol):- Open bench } \\
\hline \multicolumn{5}{|c|}{ Disposal:- SU hazardous waste disposal } \\
\hline \multicolumn{5}{|c|}{ Identify other control measures (circle or delete) - nitrile gloves and safety glasses, laboratory coat } \\
\hline \multicolumn{5}{|c|}{ Justification and controls for any work outside normal hours } \\
\hline \multicolumn{5}{|c|}{ Emergency procedures:- spillage clearance (soak up with inert absorbent material and dispose as bazardous waste) } \\
\hline \multicolumn{5}{|c|}{ Supervision/training for worker (circle) } \\
\hline None required & \multicolumn{4}{|c|}{ Already trained Training required Superviged always } \\
\hline \multicolumn{5}{|c|}{$\begin{array}{l}\text { Declaration I declare that I have assessed the hazards and risks associated with my wogk and will toke appropriate measures to } \\
\text { decrease these risks, as far as possible elininating them, and will monitor the effectiveness of these risk control measures. }\end{array}$} \\
\hline \multicolumn{5}{|c|}{ Name \& counter-signature of } \\
\hline \multicolumn{5}{|c|}{ Date of first reassessment } \\
\hline
\end{tabular}




\begin{tabular}{|c|c|c|c|c|}
\hline Protocol \#2 & \multicolumn{4}{|c|}{ Title: Making Carnoy's fixative solution } \\
\hline Associated Protocols & \multicolumn{4}{|c|}{$\begin{array}{l}\text { Description: } \\
\text { Making the Carnoy's fixative solution to be used for fixing Galleria } \\
\text { mellonella. }\end{array}$} \\
\hline \multicolumn{5}{|c|}{$\begin{array}{l}\text { Location: Laboratory } 123 \text { Wallace building } \\
\text { circle which Bioscience and Geography Local Rules apply - } \\
\text { Bent-Field Genetie-Manipulatien Laboratory }\end{array}$} \\
\hline \multicolumn{5}{|c|}{ Identify here risks and control measures for work in this environment, additional to Local Rules } \\
\hline Chemicals & Quantity & Hazards & $\begin{array}{c}\text { Category } \\
(\mathbf{A}, \mathbf{B}, \mathbf{C}, \mathbf{D})^{*}\end{array}$ & $\begin{array}{l}\text { Exp. } \\
\text { Score }\end{array}$ \\
\hline Chloroform $100 \%$ & $30 \mathrm{ml}$ & $\begin{array}{l}\text { Skin irritant, harmful if } \\
\text { swallowed, serious eye irritation, } \\
\text { toxic if inhaled, may cause } \\
\text { drowsiness or dizziness, } \\
\text { suspected of causing cancer, } \\
\text { suspected of damaging fertility or } \\
\text { the unborn child, causes damage } \\
\text { to organs through prolonged or } \\
\text { repeated exposure }\end{array}$ & A & 6 \\
\hline Ethanol $100 \%$ & $60 \mathrm{ml}$ & $\begin{array}{l}\text { Highly flammable liquid or } \\
\text { vapour, causes eye irritation }\end{array}$ & $\mathrm{C}$ & 6 \\
\hline Acetic acid (glacial) & $10 \mathrm{ml}$ & $\begin{array}{l}\text { Flammable liquid and vapour, } \\
\text { causes severe skin burns and eye } \\
\text { damage }\end{array}$ & B & 4 \\
\hline \multicolumn{2}{|c|}{$\begin{array}{l}\text { Hazard Category (known or potential) } \\
\text { A (e.g. carcinogen/teratogen/mutagen) } \\
\text { B (e.g. v.toxic/toxic/explosive/pyrophoric) } \\
\text { C (e.g. harmful/irritant/corrosive/high } \\
\text { flammable/oxidising) } \\
\text { D (e.g. non classified) }\end{array}$} & \multicolumn{3}{|c|}{$\begin{array}{l}\text { Exposure Potential Circle the highest Exposure } \\
\text { Score above. Use this to calculate the exposure } \\
\text { potential for the entire protocol (see handbook). } \\
\text { Indicate this value below. } \\
\text { Medium }\end{array}$} \\
\hline \multicolumn{5}{|c|}{ Primary containment (of product) :- Sealed glass flask } \\
\hline \multicolumn{5}{|c|}{$\begin{array}{l}\text { Storage conditions and maximum duration :- cool, dry, well-ventilated place. Contains flammable } \\
\text { liquids - storage in flame tamer in Laboratory } 123 .\end{array}$} \\
\hline \multicolumn{5}{|c|}{ Secondary containment (of protocol):- Fume hood/cupboard } \\
\hline \multicolumn{5}{|c|}{ Disposal:- SU hazardous waste disposal } \\
\hline \multicolumn{5}{|c|}{$\begin{array}{l}\text { Identify other control measures (circle or delete) - nitrile gloves, laboratory coat, safety glasses and a } \\
\text { mask when handling chloroform }\end{array}$} \\
\hline \multicolumn{5}{|c|}{ Justification and controls for ariy work outside normal hours } \\
\hline
\end{tabular}

\begin{tabular}{|l|}
\hline disposal If necessary use trained response staff. \\
\hline $\begin{array}{l}\text { Supervision/training for worker (circle) } \\
\text { None required } \quad \text { Already trained } \quad \text { Training required }\end{array}$ \\
$\begin{array}{l}\text { Declaration I declare that I have assessed the hazards and risks associated with my work and will take appropriate measures to } \\
\text { decrease these risks, as far as possible eliminating then, and will monitor the effectiveness of these risk control measures. } \\
\text { Name \& signature of worker.... } \\
\text { Name \& counter-signature of } s 2\end{array}$ \\
\hline Date of first reassessment
\end{tabular}


Bioscience and Geography Protocol Risk Assessment Form

(Expand or contract fields, or append additional sheets as required; insert NA if not applicable)

\begin{tabular}{|l|l|}
\hline Protocol \#3 & Title: Paraffin Wax galleria fixing \\
\hline $\begin{array}{l}\text { Associated Protocols } \\
\text { \#........................... }\end{array}$ & $\begin{array}{l}\text { Description: } \\
\text { Submerge Galleria mellonella in melted paraffin wax to fix the larvae for } \\
\text { histology tests }\end{array}$ \\
\hline
\end{tabular}

Location: Laboratory 123 Wallace building

circle which Bioscience and Geography Local Rules apply -

Bent Field Genetie-Manipulation Laboratory Offiee/Faeility Radieisetepe

Identify here risks and control measures for work in this environment, additional to Local Rules

\begin{tabular}{|l|l|l|l|l|}
\hline \multicolumn{1}{|c|}{ Chemicals } & \multicolumn{1}{|c|}{ Quantity } & Hazards & $\begin{array}{c}\text { Category } \\
(\mathbf{A}, \mathbf{B}, \mathbf{C}, \mathbf{D})^{*}\end{array}$ & $\begin{array}{c}\text { Exp. } \\
\text { Score }\end{array}$ \\
\hline Paraffin wax & $50 \mathrm{~g}$ & N/A & D & 4 \\
\hline
\end{tabular}

\begin{tabular}{|l|l|} 
Hazard Category (known or potential) & Exposure Potential Circle the highest Exposure
\end{tabular}

A (e.g. carcinogen/teratogen/mutagen) Score above. Use this to calculate the exposure

B (e,g, v,toxic/toxic/explosive/pyrophoric) potential for the entire protocol (see handbook).

C (e.g. harmful/irritant/corrosive/high Indicate this value below. flammable/oxidising)

D (e.g. non classified)

Low

Primary containment (of product) :- Sealed flask

Storage conditions and maximum duration :- cool, dry, well-ventilated place

Secondary containment (of protocol):- Open bench

Disposal:- SU hazardous waste disposal - burnt in chemical incinerator with an afterburner and scrubber.

Identify other control measures (circle or delete) - nitrile gloves, laboratory coat and safety glasses

Justification and controls for any work outside normal hours

Emergency procedures:- spillage clearance (if melted soak up with inert absorbent material and dispose as hazardous waste. If solid sweep up and dispose in SU waste disposal)

Burn with melted paraffin wax (run surplus amounts of cold water over affected area and seek first aid)

Supervision/training for worker (circle)

None required Alreaty trained Trainingrequired Stpervised always

Declaration I declare that I have assessed the hazards and risks associated with my work and will take appropriate measures to decrease these risks, us far as poscible eliminating them, and will monitor the effectiveness of these risk control measures.

Name \& signature of worker

Name \& counter-signature of s 


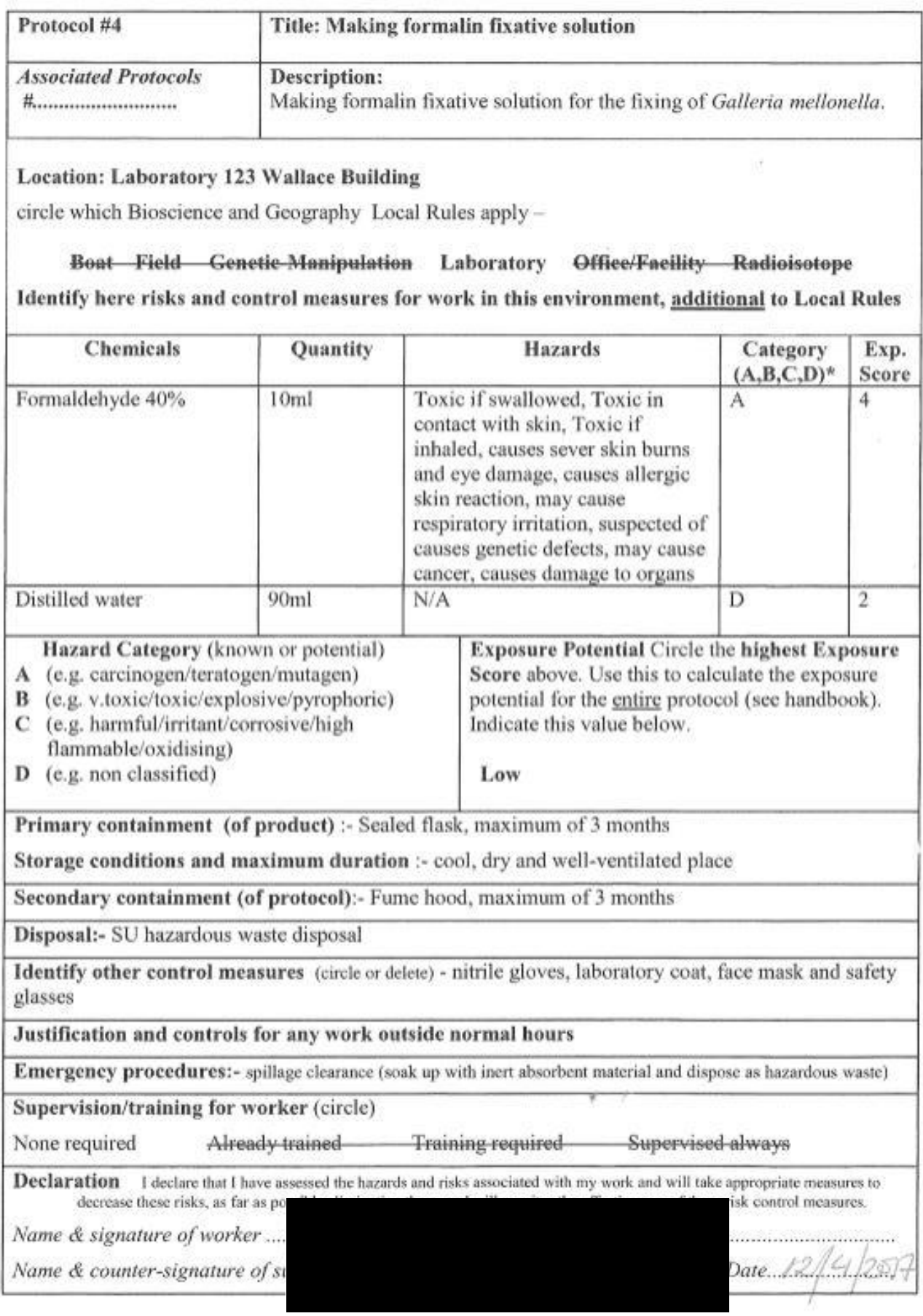




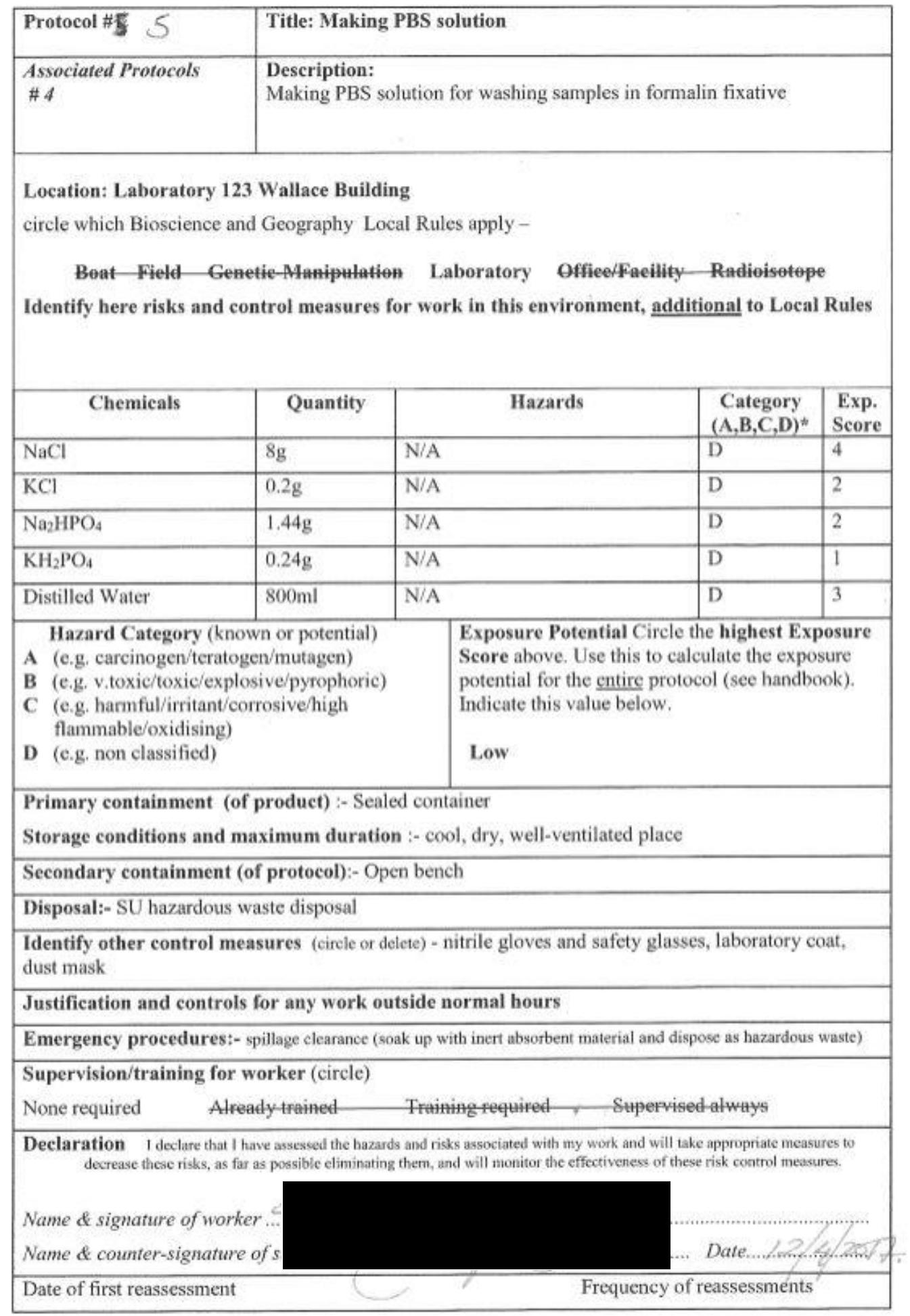

